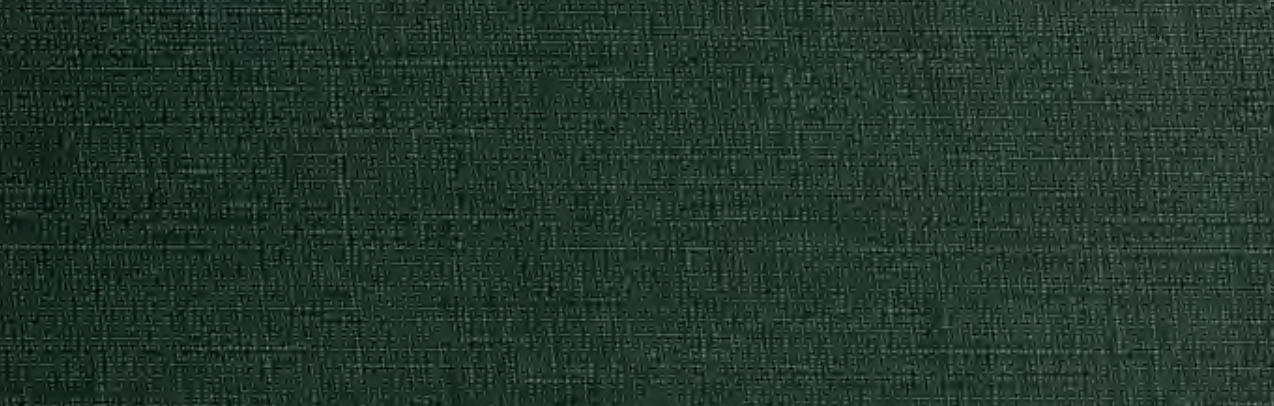




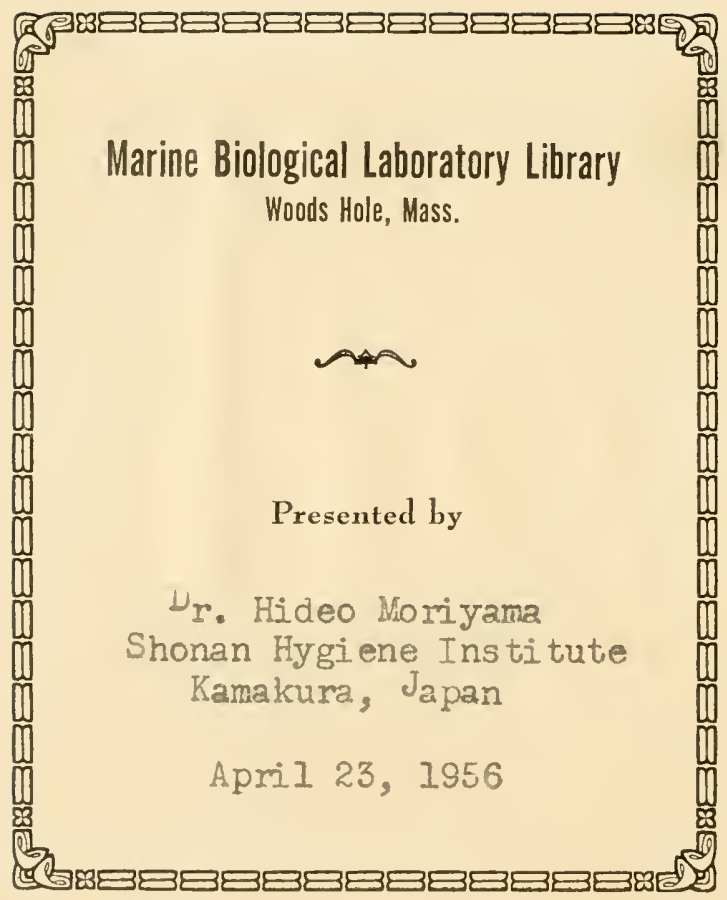




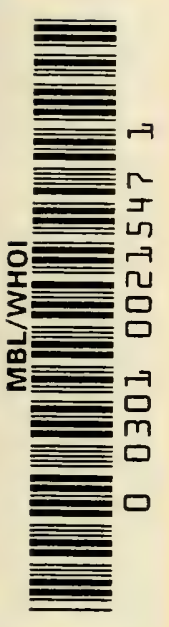



THE NATURE OF VIRUSES

AND

THE ORIGIN OF LIFE 



\title{
THE NATURE OF VIRUSES
} AND

\section{THE ORIGIN OF LIFE}

\author{
BY \\ HIDEO MORIYAMA \\ Shonan Hygiene Institute, Kamakura, Japan
}


PUBLISHED BY

Shonan Hygiene Institute, Kamakura, Japan

SOLE AGENCY

Igaku-Shoin Company, Limited

20, Hongo-6, Bunkyo-ku, Tokyo, Japan

Printed in Japan by Kato-Bunmei-Sha Printing Co., Tokyo 


\section{P REF A CE}

If one were possessed with the geocentric theory, innumerable astronomical facts ever known would appear to him to be extremely confused. At present an immense number of phenomena concerning living matter have been investigated and almost every detail of them has been fully explored. However, the author felt that they might mostly be not duly understood, so that biology might appear only an enormous accumulation of facts not to be entitled to be called a "science." Where numbers of related facts are fully understood and arranged orderly on system there may emerge a "science."

Indeed, most of numberless biological phenomena known appear to be extremely complicated and incomprehensible. Such a chaos might have prevailed the brain of the geocentrist. The Copernican theory may be needed in order for biology to become a "science."

The author felt that he found a theory by which all the life phenomena might be explained most simply and by which every known fact without exception might be arranged on system orderly. The theory was founded on the numerous facts found by the author and his collaborator, Dr. Ohashi, and has been advanced, as he believes, by applying modern researches carried out by other workers especially in virology.

The manner whereby the theory has developed as well as the way in which numerous biological phenomena are arranged orderly according to the theory are described in this book. The more fundamental parts of the theory are treated in detail in the sister volume of this book, "Immunity."

It is very regrettable that the author's concept may not be fully expressed in these two books, because English is not his mother tongue and besides he was unfortunately never blessed with any aptitude for languages.

Hideo Moriyama.

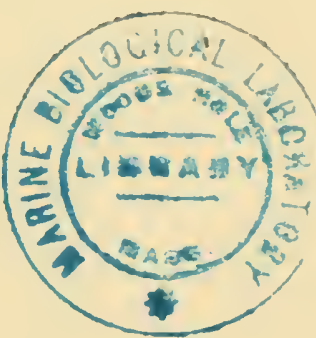



Preface

\section{PART. I \\ INTRODUCTION TO VIRUSES}

CHAPTER I. VIRUS PARTICLES. . . . . . . . . . . . . 3

1. The Key to Solving the Riddle of Life . . . . . . . . . . . 3

2. Bacterial Viruses . . . . . . . . . . . . . . . . 4

3. Virus Particles as Denaturase. . . . . . . . . . . . . 6

CHAPTER II. THE SIZE OF VIRUS PARTICLES. . . . . . . . . . 9

1. Relation between the Kind of Host Cells and the Size of Virus Particles 9

2. Difference in the Resistance of Virus due to Particle Size . . . . . 11

3. Water Quantity Combining with Virus Particles .......... 15

CHAPTER III. THE ACTIVITY OF VIRUS PARTICLES . . . . . . . 20

1. The Difference in Phage Activity due to the Cultivating Condition of Host Bacterria . . . . . . . . . . . . . 20

2. The Effect of Difference in Bacterial Strains on Phage Activity . . . 21

3. The Activity of Vaccinia Virus . . . . . . . . . . . . 23

CHAPTER IV. THE TRANSMISSION OF PROTEIN CHANGE . . . . . 25

1. Multiplicable Denaturase . . . . . . . . . . . . . . 25

2. Similarity between Rennin and Viruses. . . . . . . . . . 26

3. The Infection of Denaturation . . . . . . . . . . . . 28

CHAPTER V. THE SUMMARY OF PART I. . . . . . . . . 31

REFERENCES ........................... 34

\section{PART II}

THE FUNDAMENTAL STRUCTURE OF PROTOPALSM AND OF VIRUSES

CHAPTER I. THE STRUCTURE AND ACTION OF PROTOPLASM . . . 39

1. The Structure of Protoplasm. . . . . . . . . . . . . . 39

2. The Action of Protoplasm. . . . . . . . . . . . 43

CHAPTER II. POWERFUL FORCE, GENERATED BY PROTEIN POLIMERIZATION. . . . . . . . . . . . . . . . 45

1. Water Molecule Layer Surrounding Virus Particles. . . . . . . . . 45

2. The Force Generated by Polimerization. . . . . . . . . . . . 48 
CHAPTER III. THE PROPAGATION OF TRANSNATURATION

AND THE MULTIPLICATION OF VIRUSES. . . . . . . . . . . . . . 52

1. Propagation of Transnaturation in Protoplasm by Viruses. . . . . . 52

2. Spread of Denaturation in Red Blood Cells . . . . . . . . . . . . 54

CHAPTER IV. THE CRYSTALLINITY OF VIRUSES. . . . . . . . . . 58

1. The Fusion of Particles. . . . . . . . . . . . . . . . 58

2. The Expulsion of Lipids . . . . . . . . . . . . . . . . 59

3. The Length of Crystalline Virus Particles . . . . . . . . . . . . 62

4. Various Shapes of Plant Viruses . . . . . . . . . . . . 66

CHATER V. FINER STRUCTURE OF VIRUS PARTICLES AND ITS SIGNIFICANCE. . . . . . . . . . . . . . . 68

1. Decomposition and Fusion of Elementary Bodies of Protoplasm. . . . 68

2. Virus Particles Containing Lipids. . . . . . . . . . . . . . . 70

3. The Size of Virus Particles as Coagulated Elementary Bodies . . . . 74

CHAPTER VI. THE SHAPE OF VIRUS PARTICLES. . . . . . . . . . . 77

1. Tailed and Filamentous Virus Particles. . . . . . . . . . . . . . 77

2. Particles Elusive in Electron Micrography . . . . . . . . . . 82

CHAPTER VII. THE MODE OF VIRUS MULTIPLICATION . . . . . . 87

1. Multiplication of Phage within Bacterial Cell Protoplasm. . . . . . . 87

2. The Difference in Nucleic Acid Content between Virus and Host Cell

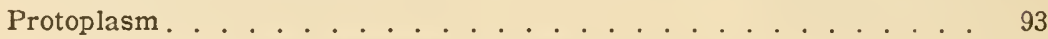

3. The Mode of Production of Virus Structure . . . . . . . . . . . 95

CHAPTER VIII. REPLICATION OF VIRUS PATTERN. . . . . . . . . . 99

1. Structural Constitution of Viruses . . . . . . . . . . . . . . . . . 99

2. Change of Viruses Following Their Combination with Host Cells . . 101

3. Essential Factors for the Spread of the Structural Change Caused by a Virus. . . . . . . . . . . . . . . . 104

CHAPTER IX. VIRUS AND NUCLEIC ACIDS . . . . . . . . . . 108

1. Nucleic Acids in Virus Particles . . . . . . . . . . . . . . 108

2. The Action of Nucleic Acids . . . . . . . . . . . . . . 111

CHAPTER X. THE SUMMARY OF PART II . . . . . . . . . . 116

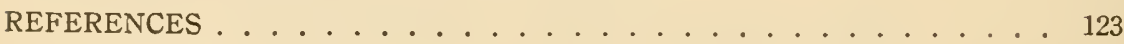

PART III

THE EVOLUTION OF VIRUSES AND THE GENERATION OF THE SECONDARY ORGANISMS

CHAPTER I. THE ORIGIN OF VIRUSES. . . . . . . . . . . . . . . . 129

1. The Generation of Phage. . . . . . . . . . . . . . . . . 129

2. The Reason for the Phage Production from Healthy Bacteria . . . 132

3. Non-Pathogenic Viruses. . . . . . . . . . . . . . . . 136

4. Latent Viruses . . . . . . . . . . . . . . . . . . . 139 
CHAPTER II. THE GENERATION OF VIRUSES.

-1. Changes in the Structure of Protoplasm Protein Leading to Virus

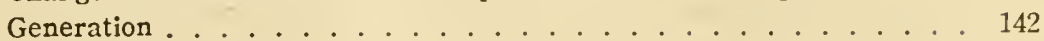

2. Environmental Change and Virus Generation. . . . . . . . . . 143

3. The Seasonal Change in the Virus Infection . . . . . . . . . . 146

CHAPTER III. IMPERFECT VIRUSES ... . . . . . . . . . . 151

1. Eczema or Allergic Dermatitis . . . . . . . . . . . . 151

2. Cancers. . . . . . . . . . . . . . . . 152

3. The Maintenance of Assimilase Action in Protoplasm Fragments. . 154

CHAPTER IV. THE VARIABILITY OF VIRUSES . . . . . . . . . . 158

1. The Inheritance of Altered Structure. . . . . . . . . . . 158

2. The Variation of Newly Produced Viruses . . . . . . . . . . . 160

3. Increase and Decrease in the Virulence of Viruses. . . . . . . . 163

CHAPTER V. FIXED VIRUSES. . . . . . . . . . . . . 165

1. Immunity against Viruses . . . . . . . . . . . . . 165

2. The Fixation of Viruses . . . . . . . . . . . . . 167

CHAPTER VI. DEVELOPMENT FROM VIRUSES TO ORGANISMS . . . 167

1. Rickettsiae . . . . . . . . . . . . . . . 169

2. Indisputable Organisms . . . . . . . . . . . . . . 170

CHAPTER VII. CAUSES OF THE EVOLUTION OF VIRUSES . . . . . 173

1. Development of Instincts Indispensable for Organisms . . . . . . . 173

2. Difficult Situation of Newly Generated Viruses to Continue Their Existence. . . . . . . . . . . . . . 175

CHAPTER VIII. VIRUSES AND INSECTS . . . . . . . . . . . 177

1. The Multiplication of Viruses in Insects . . . . . . . . . . . 177

2. The Reversibility of Protein Structure . . . . . . . . . . . 178

3. Heritable Changed Structure . . . . . . . . . . . . . . 181

4. The Cause of the Fixation of Viruses . . . . . . . . . . . . . 184

CHAPTER IX. THE REJUVENATION OF VIRUSES . . . . . . . . . . 189

1. The Transmission of Viruses by Insects . . . . . . . . . . . . 189

2. The Mechanims of Rejuvenation . . . . . . . . . . . . 190

3. Various Means for Rejuvenation . . . . . . . . . . . . . 195

CHAPTER X. THE SECONDARY ORGANISMS. . . . . . . . . . 198

1. Parasitism and Commensalism . . . . . . . . . . . 198

2. Important Significance of Parasitism . . . . . . . . . . . 200

3. The Limit of the Secondary Organisms. . . . . . . . . . 203

4. Inclusion Bodies and Metamorphosis . . . . . . . . . . 206

CHAPTER XI. THE SUMMARY OF PART III . . . . . . . . . 209

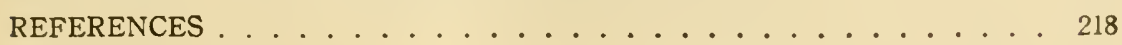




\section{PART IV}

\section{THE GENERATION OF THE PRIMARY ORGANISMS AND THE FUNDAMENTAL PRINCIPLES OF LIFE PHENOMENA}

CHAPTER I. THE MATRIX FOR THE GENERATION OF THE PRIMARY ORGANISMS......................... 223

1. The Sedimentation of Globulin and Lipids in the Primeval Oceans . . 223

2. The Properties of Artificial Cells. . . . . . . . . . . 225

3. The Evolution of the Primitive Organisms . . . . . . . . . 228

CHAPTER II. THE PATTERN OF PROTOPLASM . . . . . . . . 231

1. Protoplasm as a Mixed Crystal. . . . . . . . . . . . 231

2. The Evolution of Protein Molecules . . . . . . . . . . . 233

3. The Reason for the Presence of Optically Active Amino Acids in Protoplasm . . . . . . . . . . . . . . . 234

CHAPTER III. THE NATURE OF GENES . . . . . . . . . . . . 237

1. The Generation of Genes. . . . . . . . . . . . 237

2. The Structure of Genes ................... 238

3. The Size of Genes . . . . . . . . . . . . . . . 241

CHAPTER IV. THE SUPREMACY OF GENES . . . . . . . . . . . 244

1. The Type of Nucleic Acids and Its Biological Significance . . . . . 244

2. Plasmagenes . . . . . . . . . . . . . . . 248

CHAPTER V. RELATIONSHIP OF GENES TO ENZYMES . . . . . . 251

1. The Nature of Enzymes . . . . . . . . . . . . . 251

2. The Relationship between Genes and Enzymes . . . . . . . . 252

CHAPTER VI. FACTORS INTERFERING WITH GENES. . . . . . . 257

1. Hormones. . . . . . . . . . . . . . . . . 257

2. Inorganic Salts . . . . . . . . . . . . . . 259

CHAPTER VII. SEXUAL REPRODUCTION AND REJUVENESCENCE . . 267

1. The Origin of Sexual Reproduction. . . . . . . . . . . 267

2. Rejuvenescence of Microorganisms by Making Use of the Sexual Reproduction of the Host. . . . . . . . . . . . 271

CHAPTER VIII. REJUVENESCENCE OF MICROORGANISMS WITHOUT SEXUAL REPRODUCTION .......... 275

1. The Microorganisms Parasitic on Insects. . . . . . . . . 275

2. The Rejuvenescence of Tubercle Bacillus. . . . . . . . . 276

3. The Significance of Filtrable Forms of Bacteria . . . . . . . 279

CHAPTER IX. METABOLISM . . . . . . . . . . 284

1. Energy Requirement by Extremely Primitive Organisms . . . . . 284

2. Nucleic Acid as Energy Donor . . . . . . . . . . . . . . 288 
3. Dimensions of the Mass of Assimilase with Special Reference to

CHAPTER X. THE CHANGE OF PROTOPLASM STRUCTURE AND THE REVELATION OF LIFE PHENOMENA . . . . . . 298

1. The Oscillation of Protoplasm Structure . . . . . . . . . . 298

2. Resorption and Excretion. . . . . . . . . . . . 300

3. The Movement of Organisms. . . . . . . . . . . . . 304

4. The Mechanism of Blood Coagulation. . . . . . . . . . . 309

5. The Mechanism of Mitosis . . . . . . . . . . . . . . 312

CHAPTER XI. THE SUMMARY OF PART IV . . . . . . . . . 318

REFERENCES . . . . . . . . . . . . . . . . . 328

\section{PART V \\ THE NATURE OF EVOLUTION}

CHAPTER I. THE THEORY OF MEMORY. . . . . . . . . . 335

1. The Faculty of Protein to Memorize Its Structure . . . . . . . 335

2. Training Effect and the Oblivion of Memory. . . . . . . . . 339

3. The Cause of Aging . . . . . . . . . . . . . 343

CHAPTER II. ONTOGENY . . . . . . . . . . . . . 347

1. The Principle of Individual Development . . . . . . . . . 347

2. The Mechanism of Regeneration . . . . . . . . . . . 349

3. The Formation of Organs. . . . . . . . . . . . . 351

4. The Significance of Fertilization . . . . . . . . . . . 358

CHAPTER III. THE DEVELOPMENT OF CANCERS . . . . . . . . 361

1. The Reduction of Protoplasm to Primitive Structure . . . . . . . . 361

2. The Predisposition to Cancer. . . . . . . . . . . . 364

CHAPTER IV. THE MANNER OF THE GRADUAL CHANGE OF GENES 367

1. The Explanation of the Biogenetic Law or the Theory of Recapitulation 367

2. The Liberation of Active Groups. . . . . . . . . . . . . 368

3. The Establishment of the Strong Reversibility in Gene Structure. . . 373

CHAPTER V. GRADUAL ALTERATION OF GENE AND ITS RELATIONSHIP TO MUTATION . . . . . . . 375

1. Orthogenesis . . . . . . . . . . . . . . . 375

2. Phage as a Free Gene . . . . . . . . . . . . . . 376

3. The Production of Fitted Characters by Adaptation . . . . . . . 379

CHAPTER VI. SELECTION AND ISOLATION . . . . . . . . . 382

1. The Effect of the Selection. . . . . . . . . . . . . . 382

2. The Effect of Isolation and the Origin of Species. . . . . . . . 385

CHAPTER VII. THE MECHANISM OF ADAPTATION. . . . . . . . 387

1. The Adaptation as a Reversible Change . . . . . . . . . . . 387

2. Dauermodifikation .................... . . . 388

3. Various Types of Adaptation. . . . . . . . . . . . . . 389 
CHAPTER VIII. THE INHERITANCE OF ACQUIRED CHARACTERS . . 394

1. The Forgetting of the Original Structure. . . . . . . . . . . . 394

2. The Transmission of the Acquired Pattern to Germ Cells . . . . . 397

3. The Effect of Use and Disuse . . . . . . . . . . . . 400

CHAPTER IX. EVOLUTION AND MUTATION. . . . . . . . . . 402

1. The Significance of Mutation . . . . . . . . . . . . . 402

2. Genic Changes due to Chemical Agents. . . . . . . . . 403

CHAPTER X. THE MECHANISM OF EVOLUTION . . . . . . . . 407

1. The Evolution without Natural Selection . . . . . . . . 407

2. The Evolution of Evolution Mechanism. . . . . . . . . 408

3. The Survival of the Fittest. . . . . . . . . . . . . 410

4. The Degeneration of Organs and Atavism . . . . . . . . 411

CHAPTER XI. THE CHANGE IN HUMAN CHARACTER BY ENVIRONMENT ................ 414

1. Climate and Mankind. ........................ 414

2. The Inheritance of Habitude . . . . . . . . . . . 416

CHAPTER XII. THE EVOLUTION OF MANKIND AND ITS FUTURE. • 419

1. The Orthogenesis of Protein Molecules........... 419

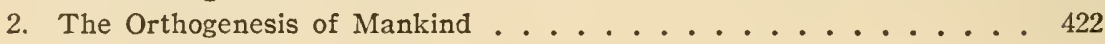

3. The Cause of the Orthogenesis of Man. . . . . . . . . . 427

4. Viruses, the Fatal Enemy of Man ............ 431

CHAPTER XIII. THE SUMMARY OF PART V. .......... 440 REFERENCES . ..................... 451 


\section{PART I}

\section{INTRODUCTION TO VIRUSES}

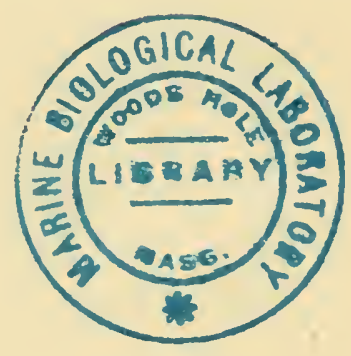





\section{CHAPTER I \\ VIRUS PARTICLES}

\section{The Key to Solving the Riddle of Life}

There may be no riddle so fascinating and so difficult as that of life. Around us, - on the land, in the sea, under the ground, or in the air, life is wriggling, jostling, thronging in every possible shape. What is living? Even to think about this question is the very manifestation of life itself. A great number of inquisitive human beings have tried and even now are trying to pierce its mystery. Viruses are the key to solve this fascinating riddle. Without this key it may be impossible to open the mysterious chamber of life and, accordingly, to find out the nature of viruses is indispensable for the solution of the riddle.

It has long been known that the majority of infectious diseases are caused by microorganisms such as bacteria and protozoa. There are, however, many infectious diseases not originated by microorganisms observable under the ordinary microscope, and such infectious diseases appear to be rather more numerous. Among these are common cold, measles, and mumps, the diseases by which almost every one of us is affected. Smallpox, influenza, trachoma, yellow fever, poliomyelitis, many types of encephalitis, rabies, etc., are also counted among them. Not only human beings but also many other creatures, for instance, domestic animals, insects, and vegetables, are also infected with them.

The agents producing such diseases are now known to pass through bacteria-proof filters, and are called filtrable viruses or shortly viruses.

The characteristic features of viruses are firstly their too small size as compared with other pathogenic microbes, and secondly their inability to grow in vitro with ordinary bacteriological method; for their multiplication are needed always living cells, in which only they can grow. For the multplication, however, usually a virus needs a certain kind of living cells; that is, certain cells of a certain creature are necessary for the multiplication. This fact is of great importance, since it reminds us of the relation between an enzyme and its substrate. Thus, the relationship between a virus and the cells in which it can grow appears similar to that between cliastase and starch, or between pepsin and protein. These enzymes, however, cannot multiply by affecting the respective substrate in contrast to viruses which can mul- 
tiply in the cells.

To the belief of the writer, viruses are a kind of enzymes, and the protoplasm protein of the cells is their substrate, and when the protein is affected by a virus, an active structure or structures are produced which can act as the virus; this change of the protein is to spread through the protoplasm as a chain reaction. The reason why the writer has reached such a conclusion will be mentioned step by step.

\section{Bacterial Viruses}

The virus which affects bacteria is called phage. Since there are numerous kinds of bacteria and, moreover, since a certain strain of bacteria is generally affected by a number of different viruses, the kind of phage should be very numerous. Phage has formerly been regarded by many workers as a special kind of viruses or virus-like agent, not as a true virus, but now it has become evident that it is a typical virus having no peculiar property dissimilar to the ordinary. As we could ascertain this fact long ago, we chose phage as our object of virus researches. Besides phage, our experiments were carried out with vaccinia virus and the experimental results obtained with this virus were well consistent, without exception, with those obtained with phage, and vice versa.

Since viruses affect living cells only, it is a rather difficult task to investigate into their function. Phage, however, affects bacteria which can easily be cultivated and handled in vitro, so that the employment of phage as an experimental object offers a vast advantage.

When bacterial cells are infected with a phage they will generally be dissolved into minute particles and such particles carry the virus activity; the particles may, therfore, in turn affect other normal cells causing the latter again to dissolve into minute particles. In such a way phage may multiply.

The chief reason for which viruses were regarded as a microorganism might be dependent on their multiplicability but, besides this, their particulate nature might have a share with it. As will be mentioned later, the fact that viruses exist in minute particles involves a striking significance.

A high speed centrifuge is commonly used to isolate virus, but as we unfortunately had no such an apparatus we contrived a chemical method for the isolation. Thus, we were able to make virus particles sedimentable by an ordinary centrifuge by taking advantate of the fact that the virus particles have the property to agglutinate at a weakly acid $\mathrm{pH}(1)$. Although no fundamental differences seemed to exist in their property between particles obtained by a high speed centrifuge 
and those isolated by our method, there is a good reason to believe that the former particles are apparently a portion of the latter having peculiar properties as will be fully discussed in a later chapter. Our application of such a method in virus investigation might enable us to succeed in finding facts other researchers might have failed to observe.

Although the optimal agglutination $\mathrm{pH}$ varied to more or less extent with varying kind of viruses, it was found to be about 5.5 with vaccinia and also with many plant viruses, and 4.6 with phage. This $\mathrm{pH}$ was markedly influenced by both the kind and the concentration of inorganic salts present in the media. If due regard was not paid to the salt, occasionally virus particles were isolated in an entirely inactivated state, whereas application of distilled water containing no salt yielded usually good results. It should be noted in this connection that sometimes Ringer's solution proved to be better than distilled water while physiological saline solution proved unfavourable (2) (3). Ringer's solution contains, besides $\mathrm{NaCl}$, minute quantities of other salts, especially that of $\mathrm{Ca}$, which may play a rôle in this case as will fully be discussed later.

When a desirable precipitate cannot be obtained by a mere adjustment of a virus solution to its optimal $\mathrm{pH}$, the addition of alcohol at about thirty per cent is recommended, thereby viruses may become readily sedimentable, although alcohol tends to inactivate the virus.

The substances isolated by our method are the aggregate of minute particles composed of a protein and lipids. In order to prove these particles to be the virus itself, we carried out a series of experiments from various points of view. A number of reports, however, have been made afterwards by some workers informing that considerable results were obtained by a similar chemical method(4)(5)(6)(7). They used mainly methanol instead of ethanol, and manipulations were always carried out at extremely low temperatures which must have been very effective to prevent the virus from inactivation.

It is most important, however, that such particles, composed of a protein and lipids, are never peculiar to viruses, but that they can be obtained from normal cells having no concern with viruses. For example, bacterial culture lysed by a phage may give rise to a precipitate by a proper addition of acetic acid, and the precipitate thus produced is composed of the particles with phage activity, whereas similar particles with no virus activity can be isolated from normal bacterial cells ground up mechanically or lysed by proper chemicals. The particles never have virus activity when the cells are decomposed by agents other than viruses (8).

Whether viruses are involved or not, the yield of the particles by our method is so high that almost the total protoplasm appears to have 
been changed into such particles and isolated as such. According to our experiment, for example, phage particles weighing about $1 \times 10^{-7} \gamma$ in the dry state were obtained per cell of $E$. coli, whereas the dry weight of a single cell was estimated to be $1.1 \times 10^{-7} \gamma(9)$. Since the dry weight of a single coli-phage particle obtained by us was measured to be about $1 \times 10^{-9} \gamma$, some $10^{2}$ particles would be produced from a single bacterial cell, if its whole protoplasm was changed into phage particles. On the other hand, Delbrück estimated the number of phage particles produced from a single cell to be 100 to 300 , and designated as "burst size" (10); this figure seems to be in accord with our estimation. Likewise in the case of vaccinia virus, from the infected skin tissue of a rabbit or of a calf are obtained amounts of particles so large that the whole protoplasm of the tissue seems to have been converted into the particles. In addition, it can actually be observed under the microscope by the dark field illumination that the cells are fully packed with virus-like particles (11).

From these facts we have concluded that the protoplasm has the property to be readily changed into minute particles by physical or chemical effects and that when produced by a virus the particles are endowed with the virus activity. Our theory of the virus was based on the fact above stated that virus-like particles can be obtained from the normal cells, but most workers seemed to pay little attention to this important fact when it was first found by us. In recent years, however, this became generally accepted and at present particles are called "normal components" or "healthy particles" (12), although no workers seem as yet to value this fact so high as we do.

When the normal particles were first recoginzed, the writer expressed the opinion that the relationship between the particles and the virus might be comparable to that between serum globulins and the antibody. This belief has become at present still much firmer to the writer; the reason will be made clear by degrees with the progress of the description.

\section{Virus Particles as Denaturase}

Under the microscope by dark field illumination, normal particles, even when not liberated from the cell, can be observed in the cell itself. When cells, such as those of the rabbit brain or liver, are pressed between cover-glasses and observed, almost the total cell-body appears to be decomposed by the mechanical pressure into minute particles.

Phage particles can be seen under the microscope, but in the growing young cells no virus-like particles may be observed. However, if bacteria are treated with such chemicals as a salt of heavy metal, 
alcohol, acetic acid, etc., or exposed to heat or ground up mechanically, particles will appear in the cell, and sometimes some of them will be discharged and found outside the cell-body (13). Under the microscope this phenomenon appears to be just a coagulation of the protoplasm into minute particles. When phage is added instead of such a chemical or physical aggression, the bacterial cell may undergo a similar change with the production of minute particles. Even without any particular aggression, an incubation of coli-bacteria for a prolonged period may lead to the production of particles inside the cell body. Since the bacteria in which particles have so appeared are not motile, they may be in a dead state. At any rate, it should be borne in mind that the particles produced by any other causes than phage never possess the phage action and that only the particles which appear in the presence of phage are provided with the action.

Lepeschkin (13) emphasized the fact that the protoplasm, in general, coagulates into minute bodies on the exposure to chemical or physical agents, and that when the coagulation is still reversible, the particle formation is not so distinct, but that when a stimulus sufficient to kill the cells is given, the particles are vigorously formed, the total protoplasm being coagulated into minute bodies. Sometimes minute particle thus formed are set free from the cells and exist in the surrounding medium. According to him, such coagulation of the protoplasm of a spirogyra-cell caused by a pressure of the cover-glass will spread successively to another part of the protoplasm until the total is coagualted into minute particles; sometimes the coagulation will be transmitted to another cell, not limited to a single cell.

In view of these facts, the writer began to form the opinion that viruses were a sort of denaturase of protoplasm protein, and that the denaturation or the change in the protein structure would spread successively in the protoplasm as a chain reaction, followed by the coagulation of the protoplasm, and that a group or a structure concerning the virus action would be produced on the structural change or the denaturation of the protoplasm protein. As pointed out already, the characteristic feature of viruses exists in the fact that they cannot multiply without living cells. This fact may lead to the view that for the generation of the structure capable of acting as a virus, the protoplasm protein is needed to be in a "living state".

As stated above, virus-like particles can be obtained from normal cells. This is, however, not always the case; for example, while virus particles may be readily isolated from the rabbit skin tissue affected by vaccinia, the isolation is impossible from the normal skin. It is also known that papilloma virus particles can be separated from the infected skin tissue regardless of the failure of the isolation of similar particles 
from the normal healthy skin. It is evident, therefore, that no viruslike particles are present in the normal skin tissue. The writer was able, however, to ascertain that, on the injection of toxic agencies such as staphylococcal toxin or sapotoxin, the akin tissue would give rise to particles similar to vaccinia virus which could readily be separated as in the case of virus infection. Thus virus-like particles can also be produced by a proper stimulus, without virus, in the cell in which no particles can be detected normally. In the case of the skin tissue, therefore, it may be said that protein capable of coagulating into minute bodies is produced by the effect of a virus which provides to the protein with the virus activity. 


\section{CHAPTER II}

\section{THE SIZE OF VIRUS PARTICLES}

\section{Relationship between the Kind of Host Cells and the Size of Virus Particles}

A vast number of researches have been carried out concerning the size of viruses, presumably mainly because viruses were believed to be a type of microbes. If enzymes were similarly considered to be a kind of microbes, profound attentions might have likewise been paid to their particle, or molecular, sizes. However, of course, since no one ever regarded them as microbes, little attention was paid to their sizes; nontheless, it is known that enzymes such as succinooxidase and cytochrome exist in a particulate state like a virus (14), although other enzymes such as pepsin, trypsin, and urease may be in a molecular state of the globulin type. As will be stated later, there are other enzymes or enzyme-like agents which should be regarded as having a particulate nature as do viruses, indicating that the particulate nature is never a characteristic feature belonging to viruses only. However, on account of the combination of this particulate nature with other characteristics which are the very image of microbes, the size of viruses is apt to be regarded as being strictly specific to the sort of viruses.

If virus particles are nothing but the protoplasm protein coagulated into particles, it may be impossible for a virus to have always a uniform size specific to the virus. It should be noted in this connection that even protein molecules are known at present to have no uniform size. Thus, recent electrophoretic studies have indicated that $\beta$-lactoglobulin, which had been regarded as an excellent example of a protein, pure in view of their electrophoretic mobility, sedimentation constant, and solubility, is polydisperse in a weakly acid solution, although appears homogeneous at $\mathrm{pH} 8$ (15).

Since viruses were generally isolated by the application of centrifugal force, and since, as a rule, particles sedimentable at a given revolution rate were separated and regarded as the only virus, it might be a matter of course that the preparations thus obtained were composed of similar sized particles. Thus in former days and occasionally even now each kind of viruses is likely to be regarded as possessing its specific, uniform size, and some workers went so far as to calculate their "molecular 
weight". At present, however, their inhomogeneity both in size and shape appears to have become generally realized.

All the particles present, regardless of their different properties, are to be isolated by our chemical method and consequently virus preparations obtained by our method consist in most cases of particles of a variety of sizes. It is, however, worthy of note that the particle size seems to be governed mainly by the sort of cells from which the particles have been isolated.

Thus, normal particles from E. coli were estimated to have an average diameter of about $0.1 \mu$ in the dry state, whereas similar sized particles were isolated from the bacterial culture lysed by a phage, that is, the phage particles proved to have the size of the normal particles (16). Again, vaccinia virus particles, obtained from the rabbit skin infected with the virus, were found much larger in size than the bacterial particles, being a little less than $0.3 \mu$, whilst the particles isolated from the skin tissue rendered inflammatory by toxic agents other than the virus possessed a similar large size. On the other hand, when isolated from the rabbit testicles infected with the same vaccinia virus, the virus particles were found to be of a much smaller diameter, being about $0.2 \mu$, which was proved in turn to be the size of particles obtained from the normal testicles (17).

In the case of vaccinia, however, some difference appeared to be present in sizes between particles isolated from an infected rabbit testicle and those from a normal testicle, but to a degree of insignificance. Also in the case of coli-phage, the difference due to phage strains sometimes was found in particle size, though also usually insignificant.

The presence of the above mentioned remarkable difference in virus size due to the difference in host cells seems to be of very importance in the consideration of the virus nature. Besides us, some workers appear to have already paid attention to this remarkable fact. For instance, Bawden and Crook found that the size of potato virus $\mathrm{X}$ varies remarkably with the kind of tissue extracts from infected plant, although no great difference is detected in their infectivity (18). Levaditi also stated that the poliomyelitis virus isolated from mice appeared much larger than that isolated from monkeys (19). A strain of coli phage was reported to exhibit different properties including particle size when cultivated in different media (20), suggesting the presence of different properties in bacterial protoplasm protein which varies with the media in which they were cultivated. Moreover, mouse pneumonitis virus preparations were found to show significant difference in size when isolated from different sources, $i$. $e$., from mouse or rat lung or from the yolk sack of the embryonated hen's eggs (21). 
It is generally believed that vaccinia virus particles are much greater in size than influenza virus particles, but according to an electron micrographic study by Wyckoff (22) particles similar in diameter to those of influenza have been seen developing from certain cells of chick embryo inoculated with vaccinia.

At present, the virus size is usually determined by electron micrographs, but as we were unable to make use of this method, the particle size was measured by us in the following way: The average dry weight of a single virus particle was first estimated under the cardiod-ultramicroscope in Beobachtungskammer by counting the number of particles in a virus protein solution of a known concentration, and subsequently the diameter of the particles was calculated from this dry weight.

Since the diameter is a function of the cube root of the weight, a considerable accuracy should be expected in the values thus obtained. By this method the writer estimated vaccinia virus from rabbit or calf skin tissue to be about $0.25 \mu$ (17); this appears agreed well with the value estimated by electron microscope and reported by other workers.

At any rate, it seems probable that the particle size is in the main subjected to the host cell in which the virus was produced. If viruses are to be produced from the protoplasm, it should naturally be expected that not only particle size but also many other properties are more or less governed by the host cell. In fact, it is actually shown that even the chemical composition of a virus appears sometimes to vary with the source from which it was isolated.

Thus, the work of Knight on influenza virus indicated that the virus particles isolated from mouse lungs or from embryonated hen's eggs possess an antigenic moiety characteristic of the respective host, although they seem to be homogeneous, electrochemically and in sedimentation behaviours (23). It was also confirmed that chicken tumour virus bears a resemblance in its chemical composition as well as in the immunólogical property to normal particles isolated from the chicken tissue (24) (25). With equine encephalomyelitis virus a similar fact is known: The virus isolated from embryonated hen's eggs resembles remarkably in the immunochemical behaviour to the normal component (26) (27). Polson and Wyckoff, investigating 17 kinds of amino acids, found that there was no significant difference in the chemical composition between $E$. coli and its phage (28).

\section{Difference in the Resistance of Virus due to Particle Size}

Virus preparations obtained by our method appear in the main to consist of homogeneous particles under the microscope, but if examined carefully it will be revealed that this is not the case. We were able to 
show that the activity for a unit mass of phage or vaccinia virus protein varies with the particle size and that larger sized particles are more active than smaller ones. Using collodion membranes having various pore diameters, we demonstrated that particles capable of passing through a membrane of a smaller pore diameter have a much

Table 1

Filtrability of Virus Proteins

\begin{tabular}{|c|c|c|c|c|c|}
\hline & & $\begin{array}{l}\text { Virus protein } \\
\text { isolated from } \\
\text { vaccine pulp }\end{array}$ & $\begin{array}{l}\text { Virus protein } \\
\text { isolated from } \\
\text { rabbit testicle }\end{array}$ & $\begin{array}{l}\text { Coli- } \\
\text { phage, } \\
\text { No. I. }\end{array}$ & $\begin{array}{l}\text { Coli- } \\
\text { phage, } \\
\text { No. II. }\end{array}$ \\
\hline $\left.\begin{array}{l}\text { Filtrability } \\
\text { through } \\
\text { Berkefeld V. }\end{array}\right\}$ & $\begin{array}{c}\text { Protein } \\
\text { Virus action }\end{array}$ & $\begin{array}{c}1 / 100 \\
1 / 100-1 / 1,000\end{array}$ & $\begin{array}{l}1 / 5 \\
1 / 10\end{array}$ & $\begin{array}{c}\text { almost } \\
\text { total } \\
, "\end{array}$ & $\begin{array}{c}\text { almost } \\
\text { total } \\
,,\end{array}$ \\
\hline $\begin{array}{l}\text { Filtrability } \\
\text { through } \\
\text { collodion } \\
\text { membrane of } \\
0.9 \mu \text { average } \\
\text { pore diame- } \\
\text { ter }\end{array}$ & $\begin{array}{c}\text { Protein } \\
\text { Virus action }\end{array}$ & $\begin{array}{l}12 \% \\
1 / 10\end{array}$ & $\begin{array}{c}\text { almost total } \\
,,\end{array}$ & - & $\square$ \\
\hline $\left.\begin{array}{l}\text { Filtrability } \\
\text { through } \\
\text { collodion } \\
\text { membrane of } \\
0.58 \mu \text { ave- } \\
\text { rage pore } \\
\text { diameter }\end{array}\right\}$ & $\begin{array}{c}\text { Protein } \\
\text { Virus action }\end{array}$ & $\begin{array}{l}\text { less than } 1 \% \\
1 / 1,000-1 / 10,000\end{array}$ & $\begin{array}{l}16 \% \\
1 / 10\end{array}$ & $\begin{array}{l}61 \% \\
49 \%\end{array}$ & $\begin{array}{l}84 \% \\
45 \%\end{array}$ \\
\hline $\begin{array}{l}\text { Filtrability } \\
\text { through } \\
\text { collodion } \\
\text { membrane of } \\
0.34 \mu \text { average } \\
\text { pore diame- } \\
\text { ter }\end{array}$ & $\begin{array}{c}\text { Protein } \\
\text { Virus action }\end{array}$ & $=$ & $\begin{array}{l}\text { less than } 1 \% \\
1 / 1,000-1 / 10,000\end{array}$ & $\begin{array}{l}7.1 \% \\
2 \%\end{array}$ & $\begin{array}{l}9.5 \% \\
3 \%\end{array}$ \\
\hline
\end{tabular}

About $0.1 \%$ virus protein water solution $(\mathrm{pH} 7.5)$ was used for the filtra-

tion. The protein quantity was measured nephelometrically.

less activity as shown in Table 1 . This can be clearly shown with phage as its activity can be precisely expressed in numerical values. Some workers were liable to consider that viruses were regularly provided with extremelly small sizes, and occasionally they even insisted that larger particles were only a concomitance, but this fact indicates that rather the reverse is the case (16).

The difference in the activity attributable to the difference in the particle size was found with phage to be very striking. The ratio of the activity of phage protein for a single particle, which could not pass through $0.58 \mu$ membrane, to the activity of the protein which could 
pass through $0.34 \mu$ membrane was calculated from the experimental results indicated in Table 1 , and estimated to be about $50-100: 1$. On the assumption that the average size was proportional to the pore diameter of the membrane, and that the larger particles were larger than the smaller ones by $1.5 \times 1.5$, the calculation was made as follows:

The activity of the larger-particle phage for a single particle:

the activity of the smaller-particle phage for a single particle $=(100-45) \times(1.5 \times 1.5)^{3} /(100-84): 3 / 9.5=100: 1$.

This was obtained from the data of phage II in the Table, while from the data of phage I it was calculated as $50: 1$. This showed that the ratio of the number of the active large particles to the total number of the large-sized particles was about 50-100 times as great as the radio calculated with the small-sized particles. This may be attributed to the labile property of the small sized particles; as shown in

Table 2

Relation between the Size of Phage Protein Partice and Its Resistance to Heat

\begin{tabular}{|c|c|c|c|c|}
\hline & & $\begin{array}{l}\text { Number of } \\
\text { active phage } \\
\text { particles per } \\
\text { cc. before } \\
\text { heating }\end{array}$ & $\begin{array}{l}\text { Number of } \\
\text { active phage } \\
\text { particles per } \\
\text { cc. after hea- } \\
\text { ting }\left(50^{\circ} \mathrm{C} \text {. }\right. \\
\text { for } 1 \text { hour) }\end{array}$ & $\begin{array}{l}\text { Rate of decrease } \\
\text { in number of } \\
\text { active phage par- } \\
\text { ticles after hea- } \\
\text { ting }\end{array}$ \\
\hline Before heating & $\begin{array}{l}\text { Phage I } \\
\text { Phage II }\end{array}$ & $\begin{array}{l}2.6 \times 10^{7} \\
4.6 \times 10^{7}\end{array}$ & $\begin{array}{l}2.4 \times 10^{7} \\
4.2 \times 10^{7}\end{array}$ & $\begin{array}{l}1: 1 \\
1: 1\end{array}$ \\
\hline $\begin{array}{l}\text { Protein capable } \\
\text { of passing } \\
\text { through } 0.54 \mu \\
\text { membrane }\end{array}$ & $\left\{\begin{array}{l}\text { Phage I } \\
\text { Phage II }\end{array}\right.$ & $\begin{array}{l}1.1 \times 10^{7} \\
2.2 \times 10^{7}\end{array}$ & $\begin{array}{l}5.6 \times 10^{6} \\
9.4 \times 10^{6}\end{array}$ & $\begin{array}{l}1: 2 \\
1: 2\end{array}$ \\
\hline $\begin{array}{l}\text { Protein capable } \\
\text { of passing } \\
\text { through } 0.34 \mu \\
\text { membrane }\end{array}$ & $\begin{array}{l}\text { Phage I } \\
\text { Phage II }\end{array}$ & $\begin{array}{l}8.2 \times 10^{5} \\
1.1 \times 10^{6}\end{array}$ & $\begin{array}{l}7.8 \times 10^{4} \\
4.8 \times 10^{4}\end{array}$ & $\begin{array}{l}1: 11 \\
1: 23\end{array}$ \\
\hline
\end{tabular}

Table 2, the resistance of phage to heat varied remarkably with the particle size, and the larger the size the greater the resistance; in other words, phage protein existing in a larger particle was more stable to heat than that in a smaller particle, showing that the property of the phage protein varied with particle size.

As described already, viruses are considered to have the faculty to cause a structural change in the protoplasm protein, thereby the protein can acquire the virus function. If the protein coagulates into too small particles on such a change, active structures in the particle may be labile and readily inactivated. There is a good reason, as will be described in a later chapter, to assume that virus protein has to exist 
in a state of the particle of over a certain size in order to exhibit the virus action and that the action is stronger as the particle becomes larger.

The unstability of smaller sized particles could be proved by the writer also with vaccinia virus protein. Galloway and Elford found that foot-and-mouth disease virus became more unstable after "purification" through a collodion membrane having a small pore diameter (29). This fact indicates that the small sized particles of this virus are also more unstable than the larger ones. Tobacco mosaic virus particles appear likewise to have various sizes. Bawden and Pirie (30) have suggested that the small particles may be virus that has become noninfective without losing serological activity or that they are incompletely formed or mal-formed virus particles, although it has been claimed by a number of workers that particles are $280 \mathrm{~m} \mu$ long, and that such are only the virus. Crook and Sheffield (31) found that a virus preparation containing only particles shorter than $280 \mathrm{~m} \mu$ had even some infectivity. Takahashi and Rawlings (32) also stated that particles shorter than $225 \mathrm{~m} \mu$ could be infective.

Before the universal application of electron microscope, the size of virus was usually measured by collodion membranes; the size was estimated from the average pore diameter of the most porous membrane that prevents the passage of the virus particles. Thus, it should naturally follow that the virus will show the smallest diameter when measured by this method. In short, there seems little doubt that the particle size estimated by collodion membranes is of the smallest particle in which the virus protein can preserve its activity; in other words, when the protoplasm protein is coagulated into particles smaller than those of this size the virus activity may fail to be revealed.

By electron micrographs the particle size of various phage strains has been estimated and reported to be a little less than $0.1 \mu$, while by the filtration method some phage was previously reported to be so small as having a diameter of the order of $0.01 \mu$. Also from our experimental data, phage appeared sometimes to retain its activity even when the protein existed in so small a particle as of this order (16).

Of the smallest viruses known are those of mouth-and-foot disease and of poliomyelitis, and both of which are accepted to be also of the order of $0.01 \mu$. This may be ascribed to their stable nature, capable of retaining their activity even when decomposed to such a small size. According to Andrewes and Horstmann (33) the viruses that are pathogenic to animals and known to have very small sizes show a high resistance against a variety of chemicals.

At present, the sizes of many viruses are mostly measured by electron miroscope, and it has been recognized that the sizes thus measured are larger than those estimated from the filtrability as is 
expected from the view point above mentioned. However, it should be borne in mind that the particles shown in electron micrographs are in the desiccated state, while those treated with the membrane are in a hydrated state combining with a great quantity of water as will be described in the next section, and as a consequence the membrane method must give a much greater value if the experiment is carried out with the same particle. But the results obtained were entirely reverse, indicating the presence of the striking difference in the particle size between the average sized particle and the smallest that narrowly retains the activity.

\section{Water Quantity Combining with Virus Particles}

Virus particles shown in electron micrographs are in the dry state, not in the hydrated state in which they are naturally existing, and hence, at least in this respect, they fail to reveal the true feature of virus particles. According to the writer's findings, when suspended in water, virus particles are combined with the quantity of water ten times as much as its dry weight, and always being accompanied by this combined water (34). The evidences found by the writer from which the conclusion was derived are as follows:

Firstly, when the virus particles agglutinated in a weakly acid solution were centrifuged and sedimented by an ordinary centrifuge for a long period of time and the water content of the precipitate thus sedimented was measured at intervals during the course of centrifugation, then it was found that the water content of the sediment diminished rapidly at the beginning of the centrifugation but that at later

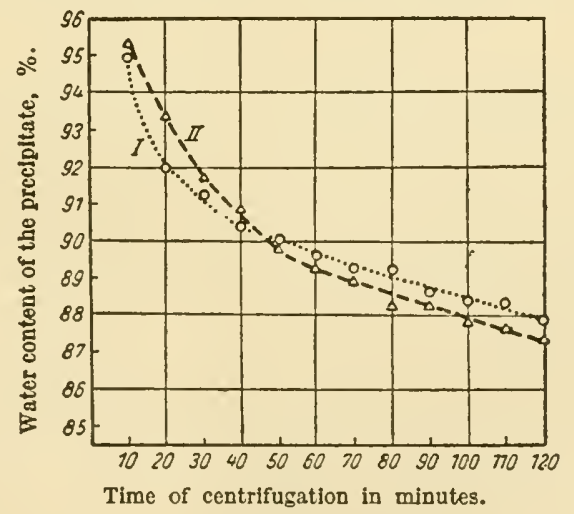

Fig. 1. Cenrifugal precipitation of coli-phage protein particles. I : Phage-protein, sample No. 1. II : Phage-protein, sample No. II. 
periods the diminution rate became constant and the water content would decrease rectilinearly. If we assume that the decrease in the water content with a constant rate is due to the squeezing out of the water contained in the particles and that the rapid decrease at the beginning of the centrifugation with an inconstant rate is due to the pressing out of the water interposing among particles, the water content of the particles can be estimated by measuring the content at the period when the decreasing rate will become constant. In this manner, with both vaccinia and phage, it was estimated to be about 90 per cent. In Fig. 1 was shown the experimental result obtained with phage particles. Such a result was obtained without any concern with the virus activity, no differences being found between virus particles and the normal ones. In the case of plant particles, after a certain period of centrifugation the water content of the precipitate tended to become constant as shown in Fig. 2. Accordingly, in such a case the water content can be estimated more accurately than in other cases.

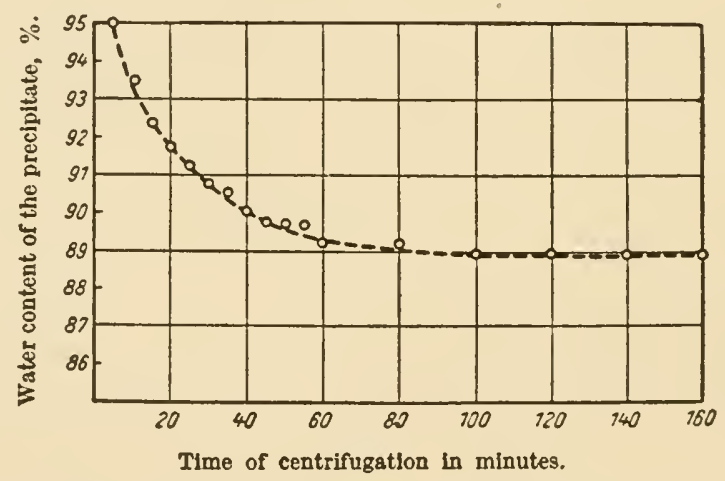

Fig. 2. Centrifugal precipitation of vegetable protein particles from the leaves of $B$. chinensis L. var. oleifera Makino.

Secondly, it was calculated from the sugar-insoluble space of the particle that approximately $10 \mathrm{cc}$. of water was combining with $1 \mathrm{~g}$. of dry particles; the sugar-insoluble space of the particle was calculated from the concentration of a sugar in the solution in which the virus particles were suspended. The sugar concentration was measured after a known quantity of the sugar had been fully dissolved in a known volume of the virus suspension from which the particles were subsequently centrifuged off. Experimental data obtained with vaccinia virus particles are shown in Fig. 3.

Thirdly, by counting the particle number under the ultramicroscope the average diameter of dry particle was calculated to be about $0.25 \mu$ 
with vaccinia virus isolated form calf skin, and about $0.1 \mu$ with coliphage, whereas by measuring the filtrability through collodion membranes the average sized particle of the vaccinia virus and of phage was estimated to be respectively in diameter about $0.5 \mu$, and about $0.2 \mu$. If the particles are always associated with the quantity of water 10 times as great as its dry weight, the diameter will be estimated to be a little more than two times greater.

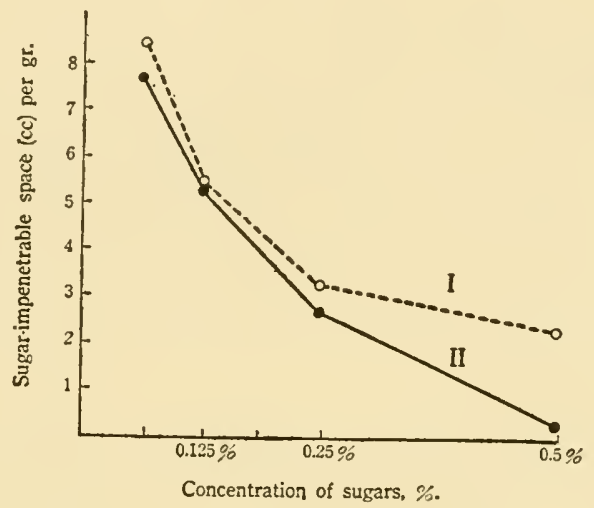

Fig. 3. Sugar-impenetrable space of vaccinia virus particles.

I : Glucose, II : Fructose.

For these reasons it was concluded that the virus particles in a water solution would combine with the water about 10 times as much as the dry weight. This appears to hold true generally for protoplasm protein particles without reference to virus action.

On investigating the space of vaccinia particles non-penetrable by sugar or protein, McFarlane et al. (35) concluded that the particles are surrounded by a considerably thick layer of water molecules which fail to act as the solvent and which combine with the particles firmly enough to sediment with them. At present, the writer believes that their conclusion is proper, though at first the writer assumed that the particles were in an extremely swelled state on absorbing all the estimated quantity of water, an assumption which must be unreasonable. As will be mentioned later there are many evidences to suggest that the majority of the estimated water quantity is present outside the particles forming a thick layer, and only a small portion of it being absorbed by them as claimed by the above authors. If the water quantity combines firmly with the particle also in the case of filtration through collodion membrane, the diameter will naturally appear to be approximately two times as great as that of the dry particle. 
The decrease in the sugar-insoluble space, as indicated in Fig. 3, when the concentrations of the sugar are higher, may be due to the penetration of the sugar into the water layer. On the thickness of this water layer as well as on the force which attracts the water molecules around the particles, a due discussion will be made in a later chapter. This force appears to play an important part in the accomplishment of the virus action, a matter which will likewise be fully discussed later.

To estimate the water quantity in virus particles usually the specific gravity is at first to be measured. For this purpose a certain amount of a substance is dissolved into a virus suspension, and the change raised thereby in the sedimentation rate of the particles is investigated. Sugars or inorganic salts dissolved for such a purpose, however, will penetrate into the particle when the concentrations are high as indicated in the experiment in Fig. 3. Consequently, the specific gravity estimated by such a method may be erroneous; presumably the obtainable values are too large, and accordingly the water contents are too small.

A linear relation is expected between the sedimentation rates and the concentrations of a dissolved substance, if the dissolved substance do not penetrate into the water which is associating with the particle. However, this is not the case, and the sedimentation rate is not a linear function of the density of the medium (36). In additon, a slurinkage may occur in the particle when the density of the medium is high (37). In order to avoid this shrinkage, Sharp et al. (38) used a protein solution of a low osmotic pressure, and estimated the water content of influenza virus $A$ and $B$ and also the swine type to be respectively 52 , 34.4 , and 43.3 per cent. They claimed that the relation between the sedimentation rate and the solvent density fell on a straight line when protein solutions were used; but it may be unreasonable to assume that the protein, regardless of its concentration, can dissolve only into the water layer outside the particle without penetrating into the water present inside. Hence, the reported values do not seem to the writer to be legitimate.

According to Schachmann and Lauffer (39), the specific gravity of tobacco mosaic virus particles was calculated to be 1.13 in serum albumin solutions, while 1.27 in sucrose solutions, indicating, also in the case of the plant virus which is known to have peculiar properties distinct from usual animal viruses, sugars appear to penetrate more readily than do proteins. They calculated the water content of the tobacco mosaic virus to be 65 per cent from the specific gravity in the protein solutions. From X-ray measurements, Pirie (40) concluded that the virus is normally associated with about its own weight of 
water. It is worthy of note that this water was claimed to exist in forming a water layer several molecules thick surrounding the particle as in the case of vaccinia virus, since X-ray pattern from the internal structure of the particles was found similar for dried virus and for virus in solution.

Not only protoplasm protein particles, but also usual protein molecules in solution seem to be combined generally with great quantities of water. From X-ray analysis Crowfoot (41) estimated the water contents of crystalline haemoglobin and lactoglobulin to be respectively 46.6 and 40.8 per cent. Bull (42) stated that a crystalline protein combined so much water as 83 per cent in the saturated water vapor.

There seems no doubt that the majority of water quantity, proved by the writer to exist in the association with the virus particle, is present outside the particle in forming a thick water layer, although the true water content, $i$. $e$, the water present inside the particle must also be considered. Thus the diameter of the hydrated particle should be much greater than that of the desiccated one. Nevertheless, viruses were previously called ultramicroscopic pathogens and their invisible nature was looked upon even as one of their characteristics. In fact, vaccinia virus particles, isolated by our method, whose diameter even in the dry state is so large as $0.25 \mu$ are never visible under the ordinary microscope if examined in a neutral solution, but usually will become visible when the $\mathrm{pH}$ of the solution is adjusted to a weakly acid $\mathrm{pH}$ near the isoelectric point and stained properly with fuchsin water solution.

This remarkable property of the virus may probably be due to the thick water layer surrounding the particles as well as the true high water content. The fact that the particles become visible at a weakly acid $\mathrm{pH}$ may be ascribed to the minimum swelling at the isoelectric point.

Bacterial cells infected with phage are known to swell up and become invisible before bursting into phage particles. We could likewise ascertain this phenomenon (9) which may be attributed to the water molecules sttracted by the lyophylic groups which may be liberated in the course of the coagulation or the denaturation of the protoplasm by phage infection. 


\section{CHAPTER III}

\section{THE ACTIVITY OF VIRUS PARTICLES}

\section{The Difference in Phage Activity due to the Cultivating Condition of Host Bacteria}

The demonstration of active phage is usually achieved by using an agar plate on which a proper dilution of phage-containing filtrate is spread with the host bacteria. When active phage particles are present in the filtrate, there will appear on the agar plate a number of small circular areas of clearing in which no bacterial growth occurs. These areas are called plaques. A plaque is regarded as produced by a single phage particle which affects surrounding bacteria to cause the lysis, and therefore the number of active particles can be estimated by counting the number of plaques produced by a given quantity of the phage-containing solution.

As mentioned already, the protoplasm protein particles liberated from the bacterial cells which have been affected by a phage appear to be able to act as the virus. However, some of the particles seem to act as such under appropriate conditions only; if all the particles could exhibit the phage action, it would occur only in extremely rare occasions.

It was found by Ohashi (43) that the ratio of the number of particles able to act as active phage on an agar plate to that capable of multiplying in a broth varies over a wide range with the filtrate examined. He distributed a given quantity of a diluted phage filtrate, in which one or less particle can be contained, into 20 tubes containing bacterial broth suspension, and incubated them for a certain period of time. From the number of the tubes, in which the phage multiplication failed to occur, and that of the total, he calculated statistically the number of the active particles present. The number of active particles thus calculated, however, never agreed with that estimated by counting the number of the plaques produced on the agar plate on which a certain volume of the same filtrate has been spread with the bacteria.

Ohashi examined about 40 different filtrates and found that the ratio, so far as the examined filtrates were concerned, could vary from about 0.1 to 3,000 . This ratio was inclined in the majority of cases to be smaller than 1.0 , with about 80 per cent of the total samples the ratio being found to be smaller than 1.0 , indicating that the phage particles, 
in general, exhibited their action easier in a broth than on an agar plate.

Since the ratio can vary in such a wide range, it seems quite impossible to calculate the absolute number of active phage particles; we can only know the relative number of particles which exhibit their action under a definite condition. It is a well known fact that even the number of plaques that may appear on an agar plate varies with the property of the agar plate. There may exist phage particles which can exhibit their action neither in a broth nor on an agar plate.

This fact must be of the utmost importance, giving warning to virus researchers to be very prudent in estimating the activity of some virus samples. If all the phage particles, the ratio of which is 3,000 as in a sample above cited, can act as the active phage on an agar plate and the activity of such particles are measured in a broth, then it will appear as if only one of 3,000 particles had the activity; one would commit a great mistake, if one concluded from such a result that only 0.03 per cent of the particles were the true phage, the remaining 99.97 per cent being "impurities".

\section{The Effect of the Dif ference in Bacterial Strains on Phage Activity}

The writer discussed above the difference in the phage activity due to the conditions under which a certain strain of bacteria are affected by phage. If similar observations were made with various bacterial strains, much greater differences in the phage activity would be revealed.

On endeavouring to isolate phage preparations possessing the activity as high as possible, we succeeded in obtaining the phage sample whose minimal quantity required for producing a plaque was $6.8 \times 10^{-16}$ g.; a certain strain of $E$. coli was used in this experiment (2). On the other hand, the average dry weight of a single particle of this preparation was estimated to be about $1 / 2 \times 10^{-15} \mathrm{~g}$.; hence almost all the produced particles could be considered as possessing the phage activity. The average dry weight was estimated by the method already described. This indicates that all the particles obtained by our method can reveal, under a proper condition, the phage action, a conclusion which is of a profound significance from various points of view as will be mentioned later.

The experimental result from which this remarkable conclusion was derived was obtained by using a certain strain of $E$. coli, which was highly sensitive to the phage; but if other strains of bacteria were used, utterly different results would be obtained. For axample, the phage strain used in this experiment could infect a strain of typhoid 
and of dysentery bacillus as well as the strain of coli, which was very sensitive to the phage as above mentioned, from which it had been produced, while the activity of the phage sample proved to vary strikingly among these strains of bacteria, and the ratio of the number of plaques produced by a given quantity of the sample was found to be as follows:

$1: 0.4: 0.001=$ coli $:$ typhoid : dysentery.

Therefore, if the typhoid or the dysentery bacillus strain was used instead of the coli strain, the minimal quantity required for producing a plaque would be estimated as $10 / 4 \times 6.8 \times 10^{-16} \mathrm{~g}$. with the typhoid, and $1,000 \times 6.8 \times 10^{-16} \mathrm{~g}$. with the dysentery bacillus. Hence, it would be said that the phage sample which could reveal the high activity on the coli strain was able to act on the dysentery strain merely as a very weak phage, only one particle out of 1,000 being capable of acting as a phage (44).

Plaques would never show uniform properties, but differ strikingly in size and shape even when the plaques were produced on an agar plate with the same phage sample and with the same strain of bacteria, showing that each phage particle had each individuality. The above mentioned difference in the plaque number due to the difference in the host bacteria may be attributed to this individuality, but it appears possible that more than one particle may be required for the production of one plaque if phage is not strong enough or host cells are not sensitive enough to the phage so as a single particle can infect a bacterial cell.

In addition to the function as a virus, some phage particles exhibit a faculty of depriving the bacteria of their viability. In a study on this faculty, we have found that many particles are necessary, in order to deprive the viability, to combine with a single bacterial cell when the cell is rather resistant, although a single paricle seems sufficient for a sensitive cell (45) (46). It has been reported that also many particles are needed to infect a single cell if the phage is inactivated to a proper degree by ultraviolet irradiation, wherein the higher the irradiation the larger number of particles are required (47) (48). Moreover, the phage particles treated by ultraviolet irradiation to be reduced inactive, is found to hasten the lysis of the heavily irradiated $E$. coli, which are already inclined to autolysis (49). Similar evidence has been presented also with influenza virus; the virus particles irradiated by ultraviolet light or heated to $50^{\circ} \mathrm{C}$ can act as the virus, if great many particles affect a small number of host cells (50). Further, it has been claimed that the infectivity of mouse-pox virus is enhanced remarkably when it is added with a vast number of the virus particles inactivated by ultraviolet irradiation (51).

In view of these facts it may safely be concluded that either when 
the virus actitity is relatively low or when the resistance of the host cell is relatively high, more than one particle are necessary to infect a single host cell. In addition, there are many reasons to suppose that the degree of protoplasm change is directly proportional to the quantity of agents causing the change (46) as will be shown later where a discussion is made on the mechanism of haemolysis.

\section{The Activity of Vaccinia Virus}

The vaccinia virus, with which we carried out a series of experiments, did unfortunately not exhibit a high activity upon rabbit skin. The number of infectious particles present in a virus sample isolated by us was so small that the ratio of the number of infective particles to that of the total was found, when examined with rabbit skin, to be of the order of $1: 1,000$. Nevertheless, it may not be proper to conclude from this fact that our virus sample was highly impure. The skin of the rabbits which we employd showed individually different susceptibility to the virus, that is, the susceptibility varied with the individuality of the rabbit. Therefore, it might be possible to change the ratio if we made experiment with other rabbits more susceptible to the virus or with some appropriate tissues other than the rabbit skin.

Nakamura and Ohfuji (52) found that a minute amount of the vaccinia could much easier be detected when injected into rabbit testicles than when injected into its skin; they proved under the microscope the existence of the infection in the testicles by the histopathological changes. Nakamura and Fukumura (53) have also confirmed that the infective titre of the emulsion of cells which had been infected with the vaccinia virus which they possessed, as measured by the intracutaneous injection into rabbit, was $1 \times 10^{4}$ to $1 \times 10^{5}$, while as measured by the above method of the intratesticular injection, $1 \times 10^{3}$ to $1 \times 10^{3}$. Consequently, if the infective titre of the virus particle, all of which were assumed to be active in the testicles, was measured by the intracutaneous injection, it would appear, as a matter of course, as if only one particle amongst 1,000 to 10,000 were the true virus. It is, however, not reasonable to consider that all strains of vaccinia are always so highly infective to testicles. For example, since the vaccinia isolated by Smadel et al (54) was claimed to be so effective on the skin that almost each particle was able to produce the infection, it cannot be expected that such a vaccinia is so much more infective to testicles. On the contrary, the existence of strains of vaccinia may be possible which is more infective to skin than to testicles. Moreover, the existence of vaccinia particles which can neither exhibit their action in testicles nor in skin 
tissues though able to infect another proper tissue may also be possible.

At any rate, as mentioned above, host cells of a high resistance may be infected only with a great number of virus particles, a conclusion which can also be reached with plant viruses. Thus, the minimum infective quantity of tobacco mosaic virus particles is said to be usually of the order of $10^{-9} \mathrm{~g}$, which can be reduced to $10^{-11}$ to $10^{-12} \mathrm{~g}$, if very sensitive host plants are adopted (55). However, even in this latter case the minimum quantity is equivalent to the virus particles more than $10^{6}$, so that there seems no doubt that an extremely large number of virus particles are required for the establishment of the infection.

The active unit of plant viruses such as tobacco mosaic virus is commonly estimated from the number of local lesions produced on the leaf on which a given quantity of a virus sample has been rubbed. The numbers of lesions, however, are known to vary with each plant, and even with one and the same plant various results are obtained with different leaves. On this account, in the routine method a test sample and the standard are applied symmetrically on the same leaf. It is only natural that the discordance becomes greater if the species of plants are not the same. For example, potato virus $Y$ can affect tobacco plant in. such a high dilution of $1 / 10,000$, but fails to infect potato plant unless the dilution is lower than $1 / 500$ (55). 


\section{CHAPTER IV}

\section{THE TRANSMISSION OF PROTEIN CHANGE}

\section{Multiplicable Denaturase}

Living cells are essential for the multiplication of viruses. Viruses can multiply only in the living cells. Viruses may cause a change in the structure of protoplasm protein of host cells; as a result the protein may be endowed autocatalitically with the virus activity. In this respect viruses may be looked upon as a kind of denaturase. However, they are, if so, by no means the denaturase in a narrow sense. An enzyme capable of converting a native protein into a "denatured" state is usually termed denaturase, but the change in the protoplasm protein due to viruses never appears to be a "denaturation", and hence viruses, if necessary, should be called "transnaturase" instead of denaturase. If it was impertinent to regard viruses as an enzyme, it would be considered at least as an agent capable of causing a transnaturation in the protoplasm protein, There are, however, many reasons to regard viruses as a kind of enzymes, a full account of which will be given in a later chapter.

Each virus is antigenically specific. Thus, animals infected or treated experimentally with a virus give rise to an antibody capable of reacting specifically with the virus. The virus is deprived of its activity by the antibody.

The appearance of virus-antigenicity in the host cell protein following a virus infection may be ascribed to the occurrence of a specific structural change in the protoplasm protein by the virus action, and owing to the change the cells may fall into a pathological condition. Thus viruses can be regarded as multiplicable transnaturase of protoplasm protein.

Knignt (56) showed the existence of a difference in the amino acid composition between the influenza virus particles and those separated from the normal hen's embryo from which the virus particles were isolated, indicating that the change of the protoplasm protein due to the virus can effect the amino acid composition. According to Andreae and Thompson (57) chromatogram of healthy and leaf-roll infected potato tubers revealed a striking and consistent difference in the occurrence of tryrtophane and tyrosine. Rafelson et al. (58) found that the presence of Theiler's virus stimulated the incorporation of radioactive carbon from glucose into most of the amino acids of minced 
mouse brain, but that it inhibited the incorporation of glucose fragment into lysine and histidine, the amounts of these two amino acids being reduced in the virus-infected tissues, whereas the amount of the other amino acids was unchanged, suggesting that the virus multiplication was intimately associated with the change in the amount of these two amino acids.

No difference in the amino acid composition was found between particles of phage and its host bacterial cells (28), but it may be unreasonable to conclude from this finding that they are entirely identical in the chemical composition, since the finding may merely show that there was no chemical difference large enough to be detected by ordinary chemical methods. If there exists an antigenical difference at all, there should also be a chemical difference between a virus and the protoplasm protein of host cells.

The structural change of the protoplasm protein due to a virus may spread in the protoplasm as a chain reaction, since the virus does proliferate in the protoplasm. This chain reaction may be brought about by the protein which has been changed structurally by the virus and which may affect in turn, as a newly formed virus, the protoplasm protein surrounding it.

Fischer (59) claimed that in coagulating blood a coagulating agent is produced and that such an agent produced in a blood plasm can cause the coagulation of another plasm in which a similar coagulating agent will in turn be produced, thus the agent multiplying indefinitely. However, the agent cannot be detected in the blood plasm already having finished the coagulation. Fischer interpreted this fact as based upon the mutual saturation of the liberated active groups with the completion of the coagulation. It may not be impossible to regard a virus as such a coagulant, by which the protoplams protein is coagulated into minute particles, in which active groups capable of acting as the virus liberated during the coagulation remain unchanged.

The coagulation of blood may occur by a physico-chemical change in the plasm protein. In a similar way the coagulation of protoplasm protein into virus particles may be a result of the structural change by the virus, and hence it may be said that minute body formation is only a result of the virus multiplication, never the essential feature of the virus.

\section{Similarity between Rennin and Viruses}

Many phenomena concerning viruses appear to be readily explained, as we have seen above, if viruses are regarded as denaturase or trans- 
naturase of the protoplasm protein. On the other hand, rennin is an enzyme which is known as a typical denaturase, and actually there seems to be a close similarity in their nature between this enzyme and viruses.

Casein from milk is coagulated by this enzyme, and its coagulating action has been described as a conversion of caseinogen into casein or of casein into paracasein. Rennin seems to cause in casein molecules a physico-chemical change leading to the coagulation. Haurowitz (60) considered that rennin causes a slight unfolding of the peptide chains of casein, by which polar or ionic groups of the casein molecules are rendered mutually accessible to one another and are thereby able to form intramolecular salt like bonds. A similar argument may be made with viruses, that is, the action of a virus consists in the conversion of virogen or provirus into the virus; the precursor, of course, must be the normal protoplasm protein, in which polar groups may be liberated by the unfolding of the peptide chains, thereby protoplasm may be coagulated into minute particles and the rearrangement of the peptide chains following this unfolding may lead to the appearance of the virus. activity.

According to the writer's study (61) rennin is similar to viruses in its physical and chemical nature, and its activity is associated with virus-like particles composed of a protein and lipids like viruses. So far as his study confirmed, rennin was stable in such virus-like particles, which become extremely labile when decomposed into smaller particles just as in viruses. Moreover, the enzyme particles isolated by the writer proved to bear a striking resemblance to viruses such as vaccinia and phage in many other respects, that is, in the behaviour towards inorganic salts and $\mathrm{pH}$ changes of the solution, and also in the agglutinability at a weakly acid $\mathrm{pH}$; its isolation was achieved by applying this latter property as with viruses. It is a well known fact that some plant viruses can be crystallized, while rennin was likewise prepared in a crystalline form (62). Such a crystallizable character is never in discordant with its particulate nature, as will be discussed later in another chapter.

Between the enzyme and viruses, however, there exists an essential difference; namely the protoplasm protein coagulated by a virus into minute particles can exhibit the virus action, whereas coagulated casein fails to act as the enzyme. Nevertheless, an evidence can be presented that casein molecules that are being coagulated by rennin can promote the coagulation of other molecules. Thus, the length of time for the coagulation of milk by rennin was found to be governed not only by the concentration of rennin, but also by that of milk. The milk solutions of a series of concentrations, to each of which was 
added the same quantity of rennin, were expected to coagulate the sooner, the lesser the concentration, but the result was entirely reverse ; for example, of the two solutions of milk powder having different concentrations, i. e. 5 per cent and 0.15 per cent, the former coagulated approximately 10 times as faster as did the latter if the rennin was not added too much. This phenomenon can be best explained by assuming that the coagulation is a chain reaction, that is, the casein molecules having been changed to some extent by rennin can affect other intact molecules to accelerate the change, which should occur the more readily the higher the concentration of casein.

\section{The Infection of Denaturation}

The inactivation change of phage particles by its antiserum appears likewise to infect other intact particles to some extent as shown in Table 3 (63). Namely, the same paradox phenomenon was observed as in the case of rennin and milk, showing that the inactivation occurred the more markedly the higher the concentration of phage particles. Thus,

Table 3

Activity of Antiserum and of Tannin upon Phage

\begin{tabular}{|c|c|c|c|c|c|c|c|}
\hline \multirow[b]{2}{*}{ Antiserum } & $\begin{array}{l}\text { Antiserum }(1: 100) \text {, cc. } \\
\text { Phage solution }(0.01 \%) \text { cc. } \\
\text { Water, cc. }\end{array}$ & $\begin{array}{l}1 \\
1 \\
0\end{array}$ & $\begin{array}{c}1 \\
1 / 2 \\
1 / 2\end{array}$ & $\begin{array}{c}1 \\
1 / 4 \\
3 / 4\end{array}$ & $\begin{array}{c}1 \\
1 / 8 \\
7 / 8\end{array}$ & $\begin{array}{c}1 \\
1 / 16 \\
15 / 16\end{array}$ & $\begin{array}{c}1 \\
1 / 32 \\
31 / 32\end{array}$ \\
\hline & $\begin{array}{l}\text { Ratio }(\%) \text { of the number } \\
\text { of remaining active } \\
\text { particles to the total } \\
\text { particle number mixed, } \\
3 \text { hours after the mixing } \\
\text { of above } 3 \text { components }\end{array}$ & 0.51 & 1.6 & 4.3 & 5.0 & 5.2 & 4.5 \\
\hline \multirow[b]{2}{*}{ Tannin } & $\begin{array}{l}\text { Tannin }(0.016 \%), \text { cc. } \\
\text { Phage solution }(0.01 \%) \text { cc. } \\
\text { Water, cc. }\end{array}$ & $\begin{array}{l}1 \\
1 \\
0\end{array}$ & $\begin{array}{c}1 \\
1 / 2 \\
1 / 2\end{array}$ & $\begin{array}{c}1 \\
1 / 4 \\
3 / 4\end{array}$ & $\begin{array}{c}1 \\
1 / 8 \\
7 / 8\end{array}$ & $\begin{array}{c}1 \\
1 / 16 \\
15 / 16\end{array}$ & $\begin{array}{c}1 \\
1 / 32 \\
31 / 32\end{array}$ \\
\hline & $\begin{array}{l}\text { Ratio }(\%) \text { of the number } \\
\text { of remaining active } \\
\text { particles to the total } \\
\text { particle number mixed, } \\
3 \text { hours after the mixing } \\
\text { of above } 3 \text { components }\end{array}$ & 5.1 & 6.7 & 8.4 & 8.4 & 8.0 & 5.1 \\
\hline
\end{tabular}

when the concentration of phage particles is high enough to make the particles come into contact with each other, the denaturation of a particle by the antiserum may successively be transmitted to other particles. 
The inactivation of phage by its antibody can be regarded as a result of a kind of denaturation (46). When tannic acid was used instead of the antiserum a similar phenomenon was likewise observed, although not so conspicuous as in the antiserum as indicated in Table 3 ; thus, the denaturation by tannic acid seemed to be less infectious. Also with $\mathrm{HgCl}_{2}$ the same could occur to a certain extent as seen in Table 4 (64) (65). Again, heat denaturation of a protein was believed by

$$
\begin{aligned}
& \text { Table } 4 \\
& \text { Infection of Inactivation of Phage } \\
& \text { I. Infection of phage inactivation due to } \mathrm{HgCl}_{2}
\end{aligned}
$$

\begin{tabular}{l|c|c|c|c|c}
\hline \multicolumn{1}{c|}{ Phage concentration (\%) } & 0.01 & 0.005 & 0.0025 & 0.00125 & 0.000625 \\
\hline $\begin{array}{l}\text { Ratio (\%) of the number of remaining } \\
\text { active particles to the total particles } \\
\text { added, } 30 \text { min. at } 37^{\circ} \mathrm{C} \text {, after the } \\
\text { addition of } \mathrm{HgCl}_{2} \text { at the concentration } \\
\text { of } \mathrm{N} / 200 .\end{array}$ & 2.8 & 3.7 & 4.2 & 4.9 & 3.9 \\
\hline
\end{tabular}

II. Infection of phage inactivation due to heat

\begin{tabular}{l|c|c|c|c|c}
\hline Phage concentration (\%) & 0.05 & 0.025 & 0.0125 & 0.00625 & 0.003125 \\
\hline $\begin{array}{l}\text { Ratio (\%) of the number of remaining } \\
\text { active particles to the total particles } \\
\text { present at first, after } 30 \mathrm{~min} \text { of }\end{array}$ & 0.7 & 3 & 4 & 5 & 12 \\
heating at $65^{\circ} \mathrm{C}$.
\end{tabular}

some workers to be infectious (66) (67), whereas according to the study of the writer heat denaturation of phage also appeared to be so as indicated in Table 4 , where the higher the phage concentration, the more striking the inactivation (65).

Bawden and Pirie (68) showed that the rate of inactivation of tomato bushy stunt virus by freezing is increased by the increase in the concentration of the virus; this might also be caused by the infection. Furthermore, according to Kassanis and Kleczkowski (69) the ratio of inhibitor, such as ribonuclease, to tobacco mosaic virus to neutralize the infectivity decreased as the concentration of the virus in. creased; that is, the higher the concentration of the virus the smaller is the amount of inhibitor needed to neutralize a given weight of the virus.

All these evidences suggest that the protein denaturation is generally infectious, and there seems little doubt that this infection occurs most distinctly in the protoplasm, where an elaborate mechanism is probably provided for the occurrence of the infection. As already discussed, in the blood plasm the infection seems to occur readily, whilst, 
since the blood may be looked upon as a kind of free protoplasm or at least as a physiological fluid similar to the protoplasm, there should also exist in it a sort of the mechanism in favour of the infection.

Lepeschkin (13) found that the granule coagulation of the protoplasm of a spirogyra cell caused by a pressing of the cover glass spread successively to another part of the protoplasm until the total mass would be coagulated into minute particles. Such a coagulation was found occasionally to be transmitted to another cell, never being confined to the single cell. According to Bünning (70) a cutting of an allium cell caused the coagulation of the protoplasm at the cutting site and after the coagulation was spread throughout the whole protoplasm, it was transmitted to the surrounding cells. The spreading velocity was 0.09 to $0.13 \mathrm{~mm}$. per min. according to him, and about $0.12 \mathrm{~mm}$. in spirogyra cell according to Lepeschkin. Thus in the protoplasm does occur the infection of the coagulation or denaturation in such a striking manner, and this must be one of the most important characteristics of the protoplasm. Viruses may be unable to multiply without this character of protoplasm. Presumably, not only viruses but also all the life phenomena may depend upon this character, a concept which will be considered in great detail in the next Part. 


\section{CHAPTER V \\ SUMMARY OF PART I}

There are a great number of infectious diseases caused by agents evidently different from ordinary microorganisms. Such pathogens are known as viruses. Viruses are usually of sizes much smaller than ordinary pathogenic microbes and can pass through bacteria-proof filters, and accordingly sometimes called "filtrable" viruses. Like usual pathogenic microorganisms, viruses multiply in the body of organisms they affected, but for their multiplication living cells are always necessary. Generally, a virus tends to proliferate solely in the cells of a certain kind of a certain organism.

A virus which affects bacteria is named phage. Phage was formerly regarded as a special type of viruses or a virus-like agent, but we could not find any essential differences between phage and vaccinia virus, one of the typical viruses. Our studies were made chiefly with these two viruses, and a theory as regards the nature of viruses has been put forwards.

The activity of both phage and vaccinia virus is carried by particulate protein which can be agglutinated at an isoelectric point of a weak acid. Separation of the activity from the particles is impossible and therefore the particles are regarded as viruses themselves. No essential differences seem present between the particles prepared by our isoelectric precipitation method and those isolated by other workers by means of usual ultracentrifuge; the latter particles appear in most cases to be a portion of the former. Since we can prove that almost all the phage particles isolated by our method occasionally exhibit the virus action, it is unreasonable to regard our sample as having always a great quantity of "impure" particles.

Such particles are not peculiar to viruses, but can be isolated from normal cells without any association with virus. In general, the protoplasm appears to have the property to be coagulated or disintegrated into minute particles by physical or chemical effects, but only those which have been produced by a virus are endowed with the virus action.

The yield of virus particles by our method is so great that almost the total protoplasm of the cell affected by a virus is considered to 
be converted into the particles. This is also the case with normal particles having no connection with virus.

The size of virus particles is mainly determined by the kind of cells from which they have been derived. Vaccinia virus particles isolated from the calf or rabbit skin is evidently different in size from those isolated from the rabbit testicle, the former being much larger than the latter. Again, phage particles are even much smaller than the vaccinia virus isolated from the testicle, while the normal bacteria are disintegrated into particles of a small size entirely similar to that of phage. It seems probable that viruses, not only in size but also in immunological and even in chemical properties, are sujected to the cells from which they were produced.

The water quantity combining with the protoplams particle is estimated to be so great as approximately ten fold of its dry weight, no matter whether the particle is produced by a virus or not.

Usually virus particles consist of a protein and lipids like the normal protoplasm. This may only be a natural result if virus particles are nothing but the particles produced by coagulation of the protoplasm.

The number of active particles in a phage sample is revealed to be different with the condition under which the number is measured even when the same strain of host bacteria is used. If various kinds of host bacteria are used the difference becomes very striking. This may be attributed to the different phage-susceptibility of the bacteria, varying with the environmental condition as well as with the strain.

A strain of bacteria with an extremely high susceptibility for a phage may be infected under a very suitable condition by a single particle of the phage, though usually more than one particle seem to be required for the infection. This holds true also for other viruses such as vaccinia and some plant viruses.

In addition, each virus particle may have each individuality and accordingly even in one and the same sample, particles with various properties may be involved. Therefore, the estimation of the absolute titre of a virus sample seems to be extremely difficult, if not entirely impossible.

It is worthy of note that a cell generally tends to be disintegrated into similar sized particles whose size seems to be determined by the kind of the cell. However, there are usually some differences in size even among the virus particles produced from the same kind of cells, and smaller particles prove to be much more unstable than larger ones. 
This may partially account for the fact that viruses exist commonly in particles having sizes larger than a certain value; if their particle size are too small, viruses may be unable to exist as such because of their extremely unstable property.

Virus particles are immunologically distinct from the normal particles isolated from the healthy cells. The antibody against a virus can specifically react with the virus particles, whereas the antibody against the protoplasm protein isolated from the normal host cells usually exhibit no action upon the virus particles isolated from the corresponding cells. Thus there seems no doubt that a virus is provided with a chemical group or groups capable of acting as an antigen different from the normal protoplasm protein of the host cells. Actually, certain differences are occasionally proved between them even in the amino acid composition.

Viruses appear to provide to the protoplams protein of the cell they affected, in changing the property of the protoplasm, with a chemical group or groups which are present in their own configuration. Hence, viruses can be regarded as a kind of denaturase, or more adequately "transnaturase", of the protoplasm protein. When the protoplasm protein of host cells is furnished with such chemical groups through "transnaturation" by a virus, the protein may acquire the ability to act as the virus.

Protein denaturation is considered, in general, to be infectious to a certain extent, that is, a denaturating change occurring in a protein molecule tends to infect other intact molecules, and thus the denaturation spreads as a chain reaction. Such an infection seems to occur always strikingly in the protoplasm. Denaturation or coagulation arising in a portion of the protoplasm can spread promptly to the total protoplasm and occasionally even to that of surrounding cells.

Similar chain reaction may occur also in the protoplasm of the host cells infected with a virus resulting in the appearance of a chemical group or groups specific to the virus; thus the virus may multiply.

The probable manner in which a virus structure is replicated in the protoplasm protein and the mechanism by which the replicated structure is transmitted successively in the protoplasm will be described in detail in the next Part. 


\section{REFERENCES}

(1) Moriyama, H. and Ohashi, S. : J. Shanghai Sci. Inst., (4), 63, 1939.

(2) Moriyama, H. and Ohashi, S.: Archiv Virusforsch., (1), 571, 1940.

(3) Moriyama, H. and Ohashi, S.: J. Shanghai Sci. Inst., (5), 189, 1941.

(4) Brumfield, H. P., et al.: Proc. Soc. Exp. Biol. \& Med., (68), 410, 1948.

(5) Pollard, M. and Finegold, M. S.: Texas Repts. Biol. \& Med., (6), 200, 1948.

(6) Cox, H. R., et al.: J. Imm., (56), 149, 1947.

(7) Wahl, R. and Blum-Emerique, L.: Ann. Inst. Pasteur, (76), 103, 1949.

(8) Moriyama, H. and Ohashi, S. : J. Shanghai Sci. Inst., (3), 155, 161, 1937.

(9) Moriyama, H. and Ohashi, S. : J. Shanghai Sci. Inst., (3), 329, 1938.

(10) Delbrüick, M. : J. Bact., (50), 131, 1945.

(11) Moriyama, H.: J. Shanghai Sci. Inst., (3), 135, 141, 1937.

(12) Wyckoff, R. W. G.: Advances in Protein Chem., (6), 1, 1951.

(13) Lepeschkin, W. W.: Zellnekrobiosis u. Protoplasmatod, Berlin, 1937.

(14) Hogeboom, G. H., et al. : J. Biol. Chem., (165), 615, 1946.

(15) Tristram, G. R. : Advances in Proteinchem., (5), 1949.

(16) Moriyama, H. and Ohashi, S.: J. Shanghai Sci. Inst., (3), 317, 1938.

(17) Moriyama, H. : J. Shanghai Sci. Inst., (3), 239, 1938.

(18) Bawden, F. C. and Crook, E. M. : Brit. J. Exp. Path., (28), 403, 1947.

(19) Levaditi, C.: Compt. Rend. Soc. Biol., (136), 96, 1942.

(20) Hook, A. E., et al.: J. Biol. Chem., (165), 241, 1946.

(21) Cosslett, V. E., et al. : Brit. J. Exp. Path., (31), 454, 1950.

(22) Wyckoff, R. W. G. : J. Imm., (70), 187, 1953.

(23) Knight, C. A. : J. Exp. Med., (83), 11, 281, 1946.

(24) Kabat, E. A. and Furth, J. : J. Exp. Med., (71), 55, 1940.

(25) Claude, A.: Science, (91), 77, 1940.

(26) Engel, L. L. and Randall, R.: J. Imm., (55), 331, 1947.

(27) Colowick, S. P., et al.: J. Biol. Chem., (168), 583, 1947.

(28) Polson, A., and Wyckoff, W. G. : Science, (108), 501, 1948.

(29) Galloway, I. A. and Elford, W. J. : Brit. J. Exp. Path., (17), 187, 1936.

(30) Bawden, F. C. and Pirie, N. W.: Brit. J. Exp. Path., (26), 277, 1945.

(31) Crook, E. M. and Sheffield, F. M. L. : Brit. J. Exp. Path., (27), 328, 1946.

(32) Takahashi, W. N. and Rawlings, T. E.: Phytopath., (38), 297, 1948.

(33) Andrewes, G. H. and Horstmann, D. M. : J. Gen. Microbiol., (3), 297, 1949.

(34) Moriyama, H. : Archiv Virusforsch., (1), 273, 1939.

(35) McFarlane, A. S., et al. : Brit. J. Exp. Path., (20), 485, 1939.

(36) Lauffer, M. A., et al.: Advances in Enzymology, (9), 171, 1949.

(37) Moriyama, H.: Archiv Virusforsch., (1), 422, 1940.

(38) Sharp, D. G., et al. : J. Biol. Chem., (159), 29, 1945.

(39) Schachmann, H. K. and Lauffer, M. A. : J. Amer. Chem. Soc., (71), 536, 1949.

(40) Pirie, N. W.: Advances in Enzymology, (1), 5, 1945.

(41) Crowfoot, D.: Chem. Revs., (28), 215, 1941.

(42) Bull, H. B.: J. Amer, Chem. Soc., (66), 1499, 1944.

(43) Ohashi, S.: J. Shanghai Sci. Inst., (4), 259, 1939.

(44) Moriyama, H. and Ohashi, S. : J. Shanghai Sci. Inst., (5), 189, 1941.

(45) Moriyama, H. and Ohashi, S.: J. Shanghai Sci. Inst., (4), 39, 1938. 
(46) Moriyama, H.: Immunity (sister volume of this book), in press.

(47) Luria, S. E. : Proc. Nat. Acad. Sci., (33), 253, 1947.

(48) Luria, S. E. and Dulbecco, R. : Genetics, (34), 93, 1949.

(49) Anderson, T. F.: J. Cellular Comp. Physiol., (25), 1, 1945.

(50) Henle, W. and Liu, O. C.: J. Exp. Med., (94), 305, 1951.

(51) Andrewes, C. H. and Elford, W. J. : Brit. J. Exp. Path., (28), 278, 1947.

(52) Nakamura, Y. and Ohfuji, M.: Med. J. Hokkaido Imp. Univ., (2), 475, 1925

(53) Nakamura, Y. and Fukumura, M. : Ditto, (16), 329, 1938.

(54) Smadel, J. E., et al.: J. Exp. Med., (70), 379, 1939.

(55) Bawden, F. C.: Plant Viruses and Virus Diseases, 1950.

(56) Kniglit, C. A. : J. Exp. Med., (86), 125, 1947.

(57) Andreae, W. A. and Thompson, K. L. : Nature, (166), 72, 1950.

(58) Rafelson, M. E., et al.: J. Biol. Chem., (193), 205, 1951.

(59) Fischer, A.: Bioch. Z., (279), 108, 1935.

(60) Haurowitz, F.: Chemistry and Biology of Proteins, New York, 203, 1950.

(61) Moriyama, H.: Arch. Virusforsch., (1) 510, 1940; (2), 71, 1941.

(62) Berridge, N. J.: Biochem. J., (39), 179, 1943.

(63) Moriyama, H. and Ohashi, S.: Z. Imm., (99), 282, 1941.

(64) Moriyama, H. and Ohashi, S.: Arch. Virusforsch., (2), 205, 1941.

(65) Moriyama, H. and Ohashi, S.: Z. Imm., (99), 419, 1941.

(66) Fischer, A.: Z. physik. Chem., A, (176), 260, 1936.

(67) Rondoni, P.: Z. physiol. Chem., (254), 207, 1938.

(68) Bawden, F. C., and Pirie, N. W.: Bioch. J., (37), 70, 1943.

(69) Kassanis, B. and Kleczkowski, A. : J. Gen. Microbiol., (2), 143, 1948.

(70) Bünning, E.: Bot. Arch.. (15), 4. 1926. 



\section{PART iI}

THE FUNDAMENTAL STRUCTURE OF PROTOPLASM AND OF VIRUSES 



\section{CHAPTER I}

\section{THE STRUCTURE AND FUNCTION OF PROTOPLASM}

\section{The Structure of Protoplasm.}

Life phenomena are always associated with the protoplasm; indeed, where there is no protoplasm there is no life. The protoplasm is the principal unit of life. As discussed in the former Part the protoplasm is likewise indispensable for virus multiplication. Accordingly, in order to solve the riddle of life as well as to study the nature of viruses, detailed knowledges regarding the structure of protoplasm are of the utmost importance.

Bensley (1) proposed the opinion that the basic constituents of protoplasm are of a fibrous structure, and at present this view is generally held by the majority of workers. Thus, the predominant opinion held by authors in this field seems essentially as follows: The protoplasm is composed of parallel alignment of fibrillar elements made of bundles of thread-like molecules, and the parallel fibres are joined by lateral bonds. Wyckoff has actually observed such fibrillar structures in protoplasm by electron micrographs, and suggested that fibrillar macromolecules may be the essential components of protoplasm (2).

The writer has reached a similar conclusion as to the protoplasm structure through detailed studies on the nature of viruses and on various fundamental phenomena of life as will be descrided later. So far as our studies have reached, minute particles produced by the disintegration of protoplasm are composed of proteins and lipids without reference to the association or non-association of virus activity; the proteins are demonstrated to have the characters of euglobulin. In the writer's opinion, in the protoplasm the molecules of these proteins are made into parallel fibres in their stretched thread-like form with lipids interposed among them.

It is an established fact that the protoplasm mainly consists of proteins and lipids, which the latter usually constitute about one third of the total cell mass. The lipid contents of mitochondria and microsomes, both of which are the important granular elements of protoplasm, have been reported to be about 24 and 40 per cent, respectively. It has been found that the lipids in these cell inclusions occur in a firm chemical association with the rest of the structure, since they 
cannot be removed even by prolonged extraction with organic solvents, such as ether or benzene, unless the complex is severely disrupted (3).

There are, on the other hand, a great number of evidences that the protoplasm is composed of granules. As described in the previous part, protopiasm is readily decomposed into virus-like particles. On the administration of a proper stimulus it will begin to coagulate into minute particles at the site of stimulation. The coagulation thus induced will be successively transmitted to other parts.

Thus, on the one hand, protoplasm appears to be composed of fibrillar elements, while on the other hand, it appears to be granules in the nature. Actually many granular elements such as mitochondria and microsomes are known to be present in abundance in the protoplasm, and hence a series of workers postulated the corpuscular theories of protoplasm, insisting on its granular nature; among them Bütschli's foam structure or honeycomb theory is well known. Frey-Wyssling insisted, however, that all hypothesis as regards plasm structure which postulate distinct submicroscopic particles, such as granules, droplets, alveoles, and ultramicrons, must be discarded as being corpuscular theories (4).

Although these two concepts regarding protoplasm structure may seem incompatible with each other, in the opinion of the writer they are never contradictory. According to the writer's theory the structure of protoplasm is as follows: In protoplasm, extended thread-like protein molecules of globulin nature make up bundles, and such bundles or corpuscles composed of parallel alignment of thread-like protein molecules represent the unit component of protoplasm. The writer has proposed the name "elementary body of protoplasm" to this unit. Lipids may be interposed among the protein threads as indicated by I in Fig. 4. If protein molecules combine directly with one another, a solid crystal may result; but owing to this lipid interposition, the elementary body may be able to exist in a liquid crystalline state.

Such elementary bodies or bundles of protein threads constitute the protoplams in forming in tern a parallel array joined by a loose junction end to end as well as side by side, so that, protopasm is on the whole also a kind of liquid crystal. The elementary body may be liable to be decomposed into several thinner bundles, $i$. $e$., "elementary bundles" as shown in Fig. 5 ; in other words, several elementary bundles form a thicker bundle, $i$. $e$. an elementary body.

The extended protoplasm proteins tend to be contracted when certain physical or chemical effects are given as stimuli. Elementary bodies may be coagulated into separate particles following such contraction of protoplasm proteins.

On account of the orderly association of protein molecules just 


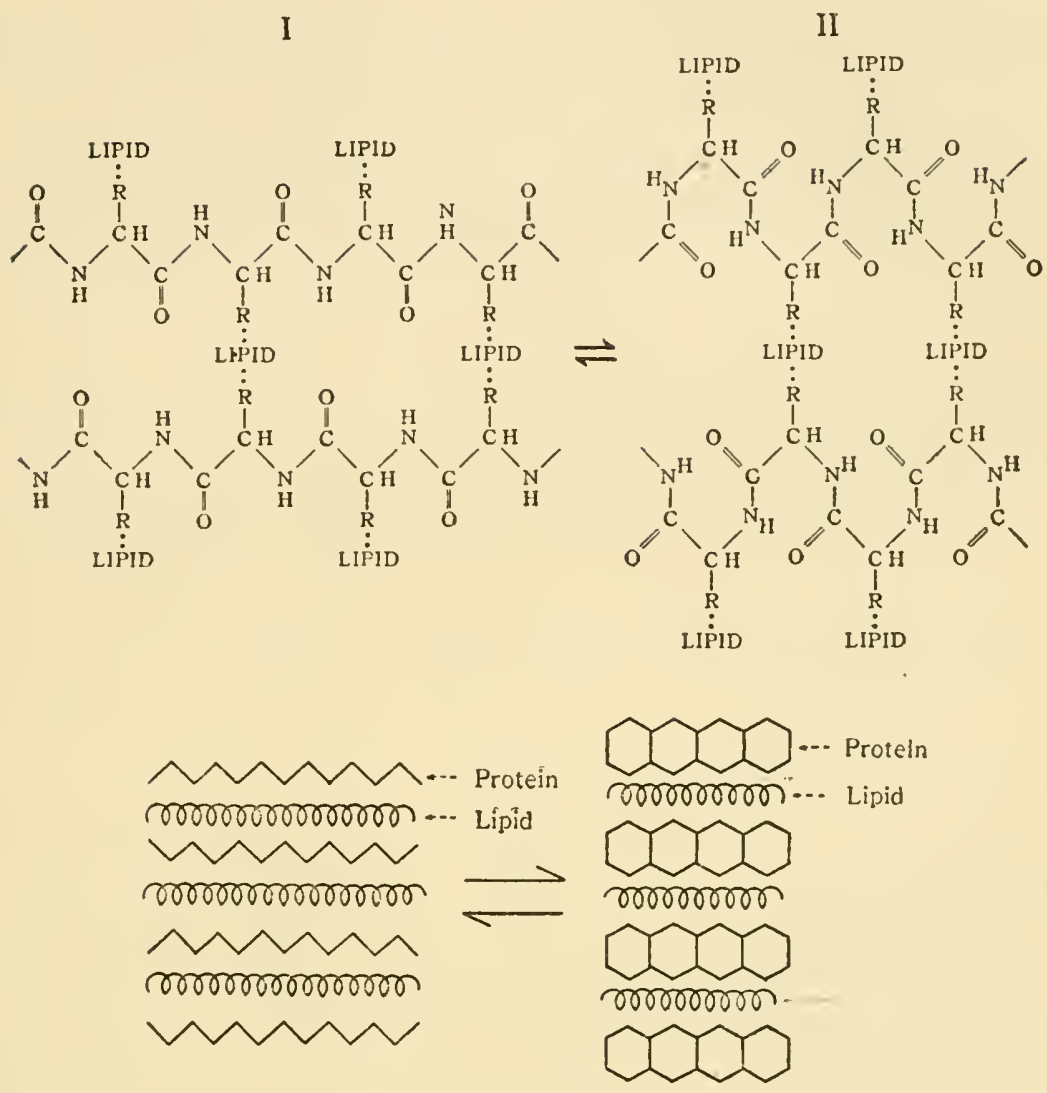

Fig. 4. Reversible coagulation of protoplasm
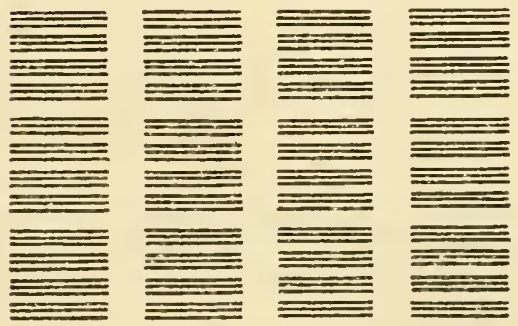

Elementary bodies

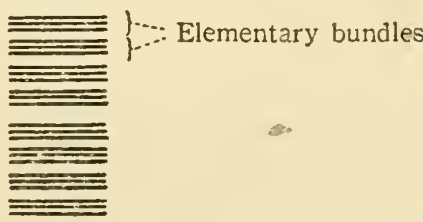

Fig. 5. Structure of protoplasm. Hundreds of stretched protein molecules form a bundle (elementary bundle) in parallel alignment. Each several of such elementary bundles in a parallel association in turn form an elementary body. Between the protein molecules lipids are inserted as shown in Fig. 4. 
described, the coagulation may spread successively in the protoplasm as a chain reaction, a contraction of the proteins at a site of protoplasm becoming in turn a stimulus on the other proteins or on the elementary bodies surrounding them (Fig. 6). Such coagulation or contraction of the elementary bodies is, as a rule, reversible, and when agents having caused the change, $i$. e., stimuli, are removed, they can resume their former state.

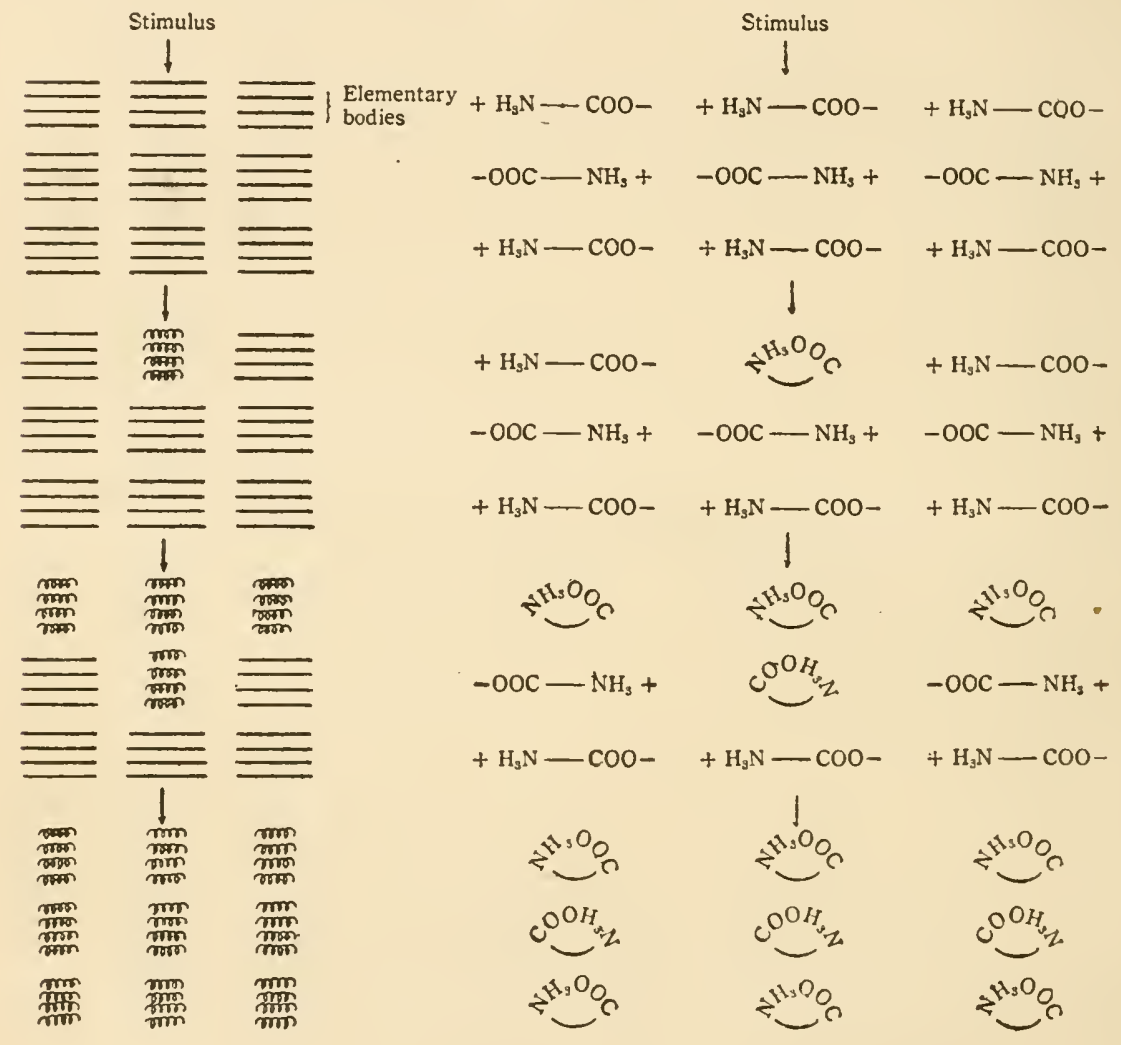

Fig. 6. Transmissible coagulation of protoplasm. In the left, the manner in which elementary bodies are coagulated into minute bodies, and in the right, the manner in which polar groups of protein molecules undergo mutual association are illustrated.

This may be the principal feature of the irritability of protoplasm. The contraction of elementary bodies joined with one another along the long axis may account for the various functions of protoplasm, such as changes in cell shape, the activity and direction of cytoplasmic 
current, and the formations of spindle fibres which pull daughter chromosomes. Detailed discussions on these phemomena will be made in part IV.

Many particles known to be present physiologically, such as mitochondria and microsomes referred to above, may be a kind of elementary body itself or its combined products, existing normally in a coagulated state. It may, however, be unreasonable to consider that such particles are always present in a coagulated state. According to Ris and Mirsky (5), the living interphase nucleus is optically homogeneous, chromosomal structures appearing after injury and after treatment with most histological fixations. In the living nucleus the chromosomes are present in a greatly extended state, filling the entire nucleus homogeneously; upon injury the chromosomes condensed and become visible, suggesting that the visible chromosomes are the conglomerates of coagulated elementary bodies.

The reasons wherefore the writer has formed the above mentioned opinion as regards the protoplasm structure may become comprehended gradually as the description proceeds.

\section{The Action of Protoplasm}

Analogy present between the protein synthesis and crystal growth has for a long time arrested attention of a number of workers. Haurowitz considered protein synthesis or protoplasm growth as follows (6): The template is formed in protoplasm by a protein molecule identical with the protein to be formed, but present in an expanded state, the amino acids which form the building stones of the replica are adsorbed tyrosine to the tyrosyl residues of the template, arginine to arginyl, and the other amino acids likewise to the corresponding residues, in analogy to the process of crystallization, only molecules which are the same as the molecules forming the crystal are adsorbed to the surface of the crystal.

As above mentioned, according to the writer's theory the protoplasm is in itself a crystal, but usually protoplasm fails to show the double refraction, a characteristic of crystals, although occasionally it shows this property especially when it is streaming. This may be attributed to a partial contraction of proteins resulting in some irregularity in the alignment of elementary bodies. There are good many reasons to suppose, as will be mentioned later, that protoplasm protein molecules in the normal protoplasm are not completely stretched, but exist in a partially contracted state. Anyhow, the protoplasm can be looked upon as a type of liquid crystals and protein synthesis can 
be regarded as the growth of this crystal, wherein each amino acid may be adsorbed to the corresponding site to produce the perfect protoplasm proteins as suggested by Haurowitz. Even a complete protein molecule having a configuration siightly different from that of the protoplasm protein may sometimes be adsorbed, and after being changed into the configuration same as that of the protoplasm protein by the spatial rearrangement of its polar groups, may be fused into the protoplasm as in the manner shown in Fig. 7.

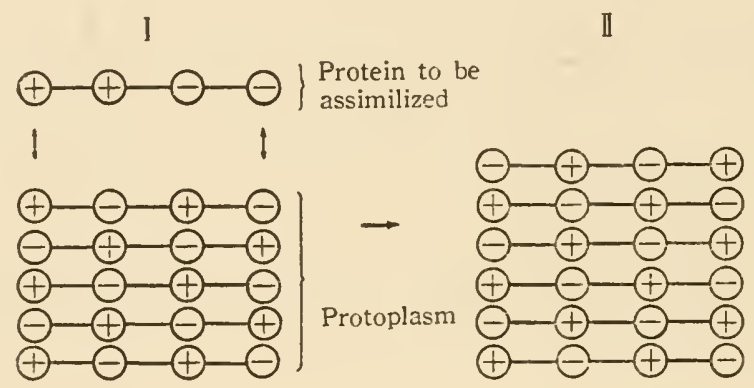

Fig. 7. Diagrammatic illustration of assimilase action of protoplasm.

I; The arrangement of polar groups in the protein to be assimilized is different from that of the protoplasm in the sites shown by arrows.

II : The protein is fused into the protoplasm following the rearrangement of polar groups.

In this respect, the writer regards protoplasm as a kind of enzymes, clesignated as "assimilase". As will be discussed in the next chapter, the action of this assimilase is considered as arising from the polymerization of the same protein molecules. A powerful physicochemical force may be generated in the assimilase by the spatial arrangement of polar groups piled up by the polymerization of proteins, a force which can cause the rearrangement of the polar forces in the adsorbed protein. 


\section{CHAPTER II}

\section{POWERFUL FORCE GENERATED BY PROTEIN POLYMERIZATION.}

\section{Water-Molecule Layer Surrounding Virus Particles}

As previously mentioned (Part 1, Chapter III), protoplasm particles including viruses combine with so much water amounts as approximately ten times of their dry weight, and its greater part seems to exist in forming a thick layer of molecules around the particle. Next we shall consider as regards the thickness of the water layer as well as the cause by which the layer is produced.

The relation between the sugar-insoluble space of the protein particle and the sugar concentration of the solution in which the particle is suspended was already shown in Fig. 3 in Part I. As clearly indicated in this Fig. the sugar-insoluble space, as a rule, increases remarkably as the sugar concentration decreases, and it may be deduced from the curve in this figure, that the space will become nearly 20 as the sugar concentration approaches 0 . In fact, the greatest value obtained in the writer's various experimental studies on the space was about 20 . If the space was 20 and the total water amount was contained within the particle, the water content of the particle would be 95 per cent; but it is unreasonable to consider that the particle could swell to such an unusual extent.

Moreover, the fact that the curve in Fig. 3 ascends remarkably as the sugar concentration decreases can most reasonably be explained by assuming that the water layer cannot, as a rule, act as a solvent, but that the combined water molecules pass gradually into the ordinary water molecules as they exist in more outer layer, so that the suger can penetrate furthur into the layer as the concentration increases. Water molecules may likewise possibly be absorbed into the particle itself, and the absorbed water molecules existing inside the particle may be similar in their physicochemical character to those existing in inner layer, although it may be possible that the former molecules are more marked in their inability to act as a solvent. Hence the sugar may even penetrate into the particle when its concentration is extremely high.

It seems very difficult, if not impossible, to estimate the water amount inside the particle. Since sugar may penetrate into the particle 
when its concentration is high, the estimation is impossible by measuring the space incapable of dissolving a sugar.

The routine method of determination of the water content by measuring the particle density, which is estimated from the sedimentation rate of the particle in various concentrations of substances, such as sugar, protein, or inorganic salt, also may fail to give any indubitable value, since the substances may penetrate into the particle when the concentration is high as just pointed out.

For such reasons the writer has been unable to estimate the water amount actually contained in the particle. However, since the ordinary virus particles can be regarded as the elementary bodies of protoplasm, their water content should be similar to that of protoplasm itself whose water content is generally accepted to be about 75 per cent. Now, therefore, argument will be advanced on assuming the water content to be 70 per cent, a little less than that of protoplasm.

As a particle should increase in diameter approximately by 1.6 times when a dry particle absorbes water until its water content becomes 70 per cent, vaccinia virus particle whose diameter is $0.25 \mu$ in dry state will become $0.4 \mu$ on water absorption to this extent. If water is further adsorbed on this swelled particle and the total amount of combined water reaches as great as 10 times its dry weight, the diameter must increase again by 1.5 times. In this state, the thickness of the water layer is calculated to be $0.09 \mu$, or $900 \mathrm{~A}$ : in this calculation, the density of the dry particle is taken as 1.33 , a value which was previously obtained by the writer (7).

According to the writer's estimation, the combined water amount was similar without reference to the kind of virus. For example, the amount of combined water was estimated to be similar both with vaccinia virus and phage, whereas the particle of the former was found to be greater in diameter as much as 3 times than that of the latter (8). This must be a noteworthy fact, because, if the thickness of the water layer was the same, the combined water amount of phage particle should be far greater than that of vaccinia virus particle, but the fact is that the amount is found to be the same, showing that the layer becomes thinner as the particle becomes smaller.

In the same way as with the vaccinia virus particle the thickness of the water layer of phage particle is calculated to be $300 \mathrm{~A}$ on the assumption that the diameter of the dry particle is $80 \mathrm{~m} \mu$ and its density is 1.45 ; also this value of density was previously estimated by us (9).

The water molecules forming the layer with such a thickness are considered to behave themselves always in association with the particle. The sugar-insoluble space, as above mentioned, was sometimes 
estimated to be so great as 20 . In such a case the layer must have a thickness much greater than that estimated above, and hence it seems possible that even the water molecules existing at a distance even greater than $900 \mathrm{~A}$ apart from the particle surface have a property somewhat different from that of the ordinary water.

Estimations are made above on the assumption that the water content inside the particle is 70 per cent, but there are no great alterations if it is assumed to be either 65 or 75 per cent: with vaccinia virus particle it is calculated to be 1,100 and $800 \mathrm{~A}$, respectively; with phage 350 and $270 \mathrm{~A}$.

The thickness of the water layer thus appears to vary with the size of the particle in the direct proportion to the diameter. This must result in the fact that the quantity of the combined water is identical regardless of the particle size.

Rothen (10) reached to a similar conclusion in his studies on the "long range force" acting between an antigen and its antibody. According to him, the force becomes greater and can reach longer distances as the thickness of the antigen molecule layer increases. This long range force may cause the attraction of the water molecules around the virus particle. Since this force is specific as claimed by Rothen, it seems highly probable that viruses can act upon the host cells through this long range force. If so, the action is expected to become greater with the increasing particle size. This, as is argued in the following, seems actually the case.

Long range interaction seems to occur also in a solution of tobacco mosaic virus, where the molecules assume regular positions at intervals as great as 1,000 A (11), a finding which should be expected from the existence of the thick water layer. It has been reported that even in haemoglobin crystals molecular layers of the protein seem to be separated by water to a distance of $65 \mathrm{~A}$; 30 per cent of the water of haemoglobin crystals is so firmly bound that it is not available as a solvent for small ions (12).

Rothen (13) insists upon the existence of the long range force acting several hundreds $\mathrm{A}$ between an antigen and antibody and between an enzyme and its substrate, while a number of workers are disinclined to accept his view. It is, however, never an unusual phenomenon that various particles or granules in physiologically active cells exert their influence on each other even when the distance between them is considerably great and can take positions in a mutual connection. Since this seems also true in vitro observation, the writer is likewise firmly convinced of its existence. 


\section{The Force Generated by Polymerization}

It is probably true to say that the thick water layer is attracted onto the surface of virus particles by Rothen's long range force. But it must be an important question: what is the nature of the force?

Prior to answering this question, Rothen's experiment referred to above should be discussed in detail. He used bovine serum albumin as the antigen and homologous rabbit antiserum as the antibody, and found that the thickness of molecules of the antibody attracted by the layer of the antigen molecules became greater as the number of the antigen monolayers increased. When the antigen was a single monolayer the thickness of the antibody was found to be about $30 \mathrm{~A}$, while when antigen was piled up in 8 layers it became $230 \mathrm{~A}$.

A possible explanation of this phenomenon is that the antibodyattracting force of the antigen may result from its polymerization and that the force may consist of electrostatic force arising from the polar groups of the protein. If a molecule can be compared to an electric cell, the force may be expected to become greater on the polymerization or on piling up. The difference in the pattern of arrangement of polar groups may account for the specificity of the force.

Water molecules may be attracted by such a pattern of polar groups, positive pole of the molecule being attracted by the negative pattern, and negative pole by the positive pattern, so that the water layer thus formed should have in itself the pattern of polar forces. If water molecules are thus successively attracted in accordance with the pattern, forming a thikness of, say, 1,000 A, the outside of the most outer layer also may have the pattern. In such a case, it may be said that Rothen's long range force can reach a distance of 1,000 A. However, as illustrated in Fig. 8, since the arrangement of water molecules may become the more irregular as they exist in the more outer layer, the pattern will become more incomplete with the increasing distance. It has been reported that the long range force failed to be demonstrated if egg albumin was used as antigen instead of bovine albumin. This might be due to the failure of the protein to polymerize regularly.

Haurowitz (14) studied the reaction between the atoxyl-azo protein, containing As in various proportions, and its antibody, and found that the antibody-attracting force of the antigen seems to increase with the increasing number of As molecules combined with the protein; when As quantity is very great a single molecule of the antigen can combine with even 50 molecules of the antibody. This result may be expected if the polymerization of the protein is enhanced with the increasing As content, wherein As-containing groups being piled up through the 
polymerization. When antigen molecule increases in size, molecules of antibody combining with it seem generally to increase in number; for example, according to Heidelberger and Kendall (15), while a single

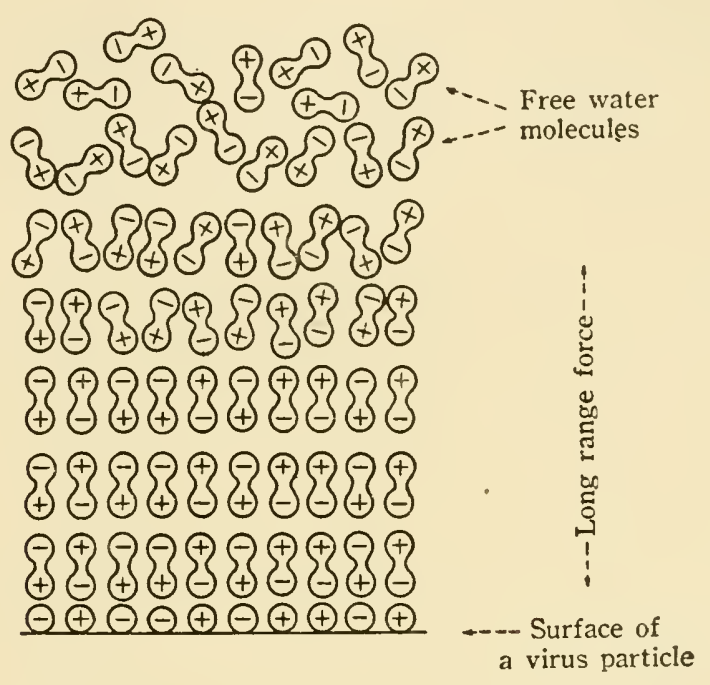

Fig. 8. Diagram of the long range force.

molecule of serum albumin as antigen can combine only 6 molecules of the antibody, thyroglobulin which is known to have molecular weight about 10 times as great as that of serum albumin can combine 60 molecules. The increase in the molecular size of antigen can be looked upon as the result of polymerization of unit molecules with the piling up of the specific active groups. The accumulation of the monomolecular layers of antigen in the experiment of Rothen may be regarded as the increase of the molecular size.

The fact that virus activities are carried by particles having sizes greater than a certain value may come from the requirement of polymerization of protein molecules for the activities. If the polymerization degree is low, though sufficient for acting as a virus, the virus may be able to exhibit only a weak action and in addition unstab!e and liable to be inactivated.

According to our study, as described in Part I, Chapter III, smallsized phage particles have the activity much less than that of largesized particles, and 50 or 100 small-sized particles seem to be compatible in their activity to a single large-sized particle. This fact may be attributed to the instability of the small-sized particles, but it seems a 
more probable explanation that most of the small-sized particles cannot act as the virus because of the low polymerization degree. Phage particles of extremely small sizes were occasionally found to produce only very small plaques showing their weak activity.

In the studies on the interaction of large and small haemoagglutinating particles of Newcastle disease virus with red cells, Grenoff and Henle ( $15 \mathrm{a}$ ) have found that whereas large particles were readily adsorbed onto red cells, only relatively small amounts of small particles appear to combine with red cells under the same conditions, and moreover, large particles cause haemolysis whereas small ones lack this property.

It is well known that antigenic substances are always large molecules, of molecular weight of the order of 10,000 or more, and consequently being usually colloidal in solution. This shows that, in order to act as antigens, antigenic substances must be in molecular state of considerable sizes as in viruses, although the sizes required for antigens are not so large as those of the viruses. Polysaccharides isolated from bacteria are generally non-antigenic, but when adsorbed on particulate substances, such as collodion particles or kaolin, they can act as antigens. Again, albumose, though usually non-antigenic, can produce antibody if adsorbed on colloidal aluminium hydroxide. Also some lipids extracted from various organisms are not antigenic per se, but when injected into an animal after mixed with swine serum, can cause the antibody production.

These non-antigenic substances may acquire the antigen-ability by the polymerization on the surface of colloidal particles on which they are adsorbed, thereby the physicochemical force capable of acting as the active group of antigens may become greater. They may possibly act as antigens even when no such polymerization occurs, if non-specific colloidal particles injected with them can induce some disturbances in the protoplasm configuration of the antibody-forming cell, on which the mixtures or complexes are adsorbed, in assisting the formation of the replica corresponding to the antigenic pattern in the protoplasm. In the opinion of the writer antigens like viruses act as templates to produce replicas in the protoplasm (22). There are many evidences that a non-specific disturbance in the protoplasm caused by factors other than viruses may exert a favourable influence upon a virus to infect the cell; a detailed account of this will be given later.

Enzymes are another agent for whose function a similar large molecular state may be necessary. A group of enzymes, known as conjugated ones, consists of two components, $i$. e. coenzyme and apoenzyme; the former in itself has no enzymatic action and has a low molecular weight, but on combination with the latter the activity of the 
enzyme develops, and this latter is the protein moiety of a colloidal nature, possessing also no enzymatic activity. The development of enzymatic activity on the combination of the two components may be chiefly due to the high molecular weight of the complex so formed. Likewise in this case some disturbances in the substrate configuration caused by the non-specific or partially specific physicochemical force of the apoenzyme molecule, resulting from the polymerization, may participate in the development of enzymatic action.

It is known that enzyme molecules or, in general, protein molecules in a solution tend to associate or polymerize into larger molecules or aggregates. This property may contribute to the exhibition of enzymatic action. In spite of their protein nature, viruses are usually not affected by proteolytic enzymes. This may be accounted for by the higher and firmer polymerization of viruses than enzymes. Even if enzymes function as such on polymerization, the degree of polymerization may be insignificant as compared with that of viruses.

From a number of facts mentioned above it may be conciuded that the increase in molecular weight, or the polymerization of molecules, seems to make the physicochemical force arising from the configuration more effective. Life phenomena may be intimately correlated to such forces coming from polymerization. Peculiar properties belonging to the so-called colloidal substances may presumably depend upon such forces. Since all the living bodies consist of colloids, the life phenomena should be based upon these colloidal substances, and therefore life phenomena may be said to be developed by the long range forces. 


\section{CHAPTER III}

\section{THE PROPAGATION OF TRANSNATURATION AND THE MULTIPLICATION OF VIRUSES}

\section{Propagation of Transnaturation in Protoplasm by Viruses}

In the opinion of the writer, virus particles are, either in their construction or in their character, identical with elementary bodies of protoplasm and consequently are a kind of assimilase. When protoplasm is disintegrated into elementary bodies and in these bodies the original structure of the protoplasm is retained, the bodies can act as assimilase existing in a state of minute particles.

Such an elementary body can act upon another assimilase, changing the structure of the latter to be identical with it; that is, it can exert the "assimilase" action upon another assimilase if the latter is weaker in action. An elementary body, however, shares no faculty to synthesize from amino acids or from their components a protein which possesses the same structure as that of the protein of which the elementary body is composed. In other words, viruses can "assimilize" other assimilases whose action is weaker than that of their own, but cannot proliferate in media containing no cells by synthesizing the protein.

Thus, when a virus affects cell protoplasm whose assimilase acticn is weaker than that of the virus, the protoplasm will be changed to become identical with the virus, as shown in Fig. 9. The change may start at the site where the virus contacts with the protoplasm and may propagate successively to other parts until finally the spatial arrangement of polar groups in the whole protoplasm protein is altered to become identical with that of the virus, being followed occasionally by the coagulation of protoplasm into elementary bodies. If the protoplasm is disintegrated into coagulated elementary bodies, these bodies may be looked upon as virus particles. This is the principal way in which the viruses multiply.

Thus, since for the protoplasm to become identical in its structure with a virus is to make the virus multiply in it, particle formation is never essential for the multiplication, and accordingly viruses are produced in the protoplasm irrespective of the occurrence or nonoccurrence of decomposition of the protoplasm into minute particles. 
The assimilase action, as discussed in the previous chapter, arises from the orderly polymerization of protein molecules of the same structure, and hence, when the polymerization is incomplete and loose, a

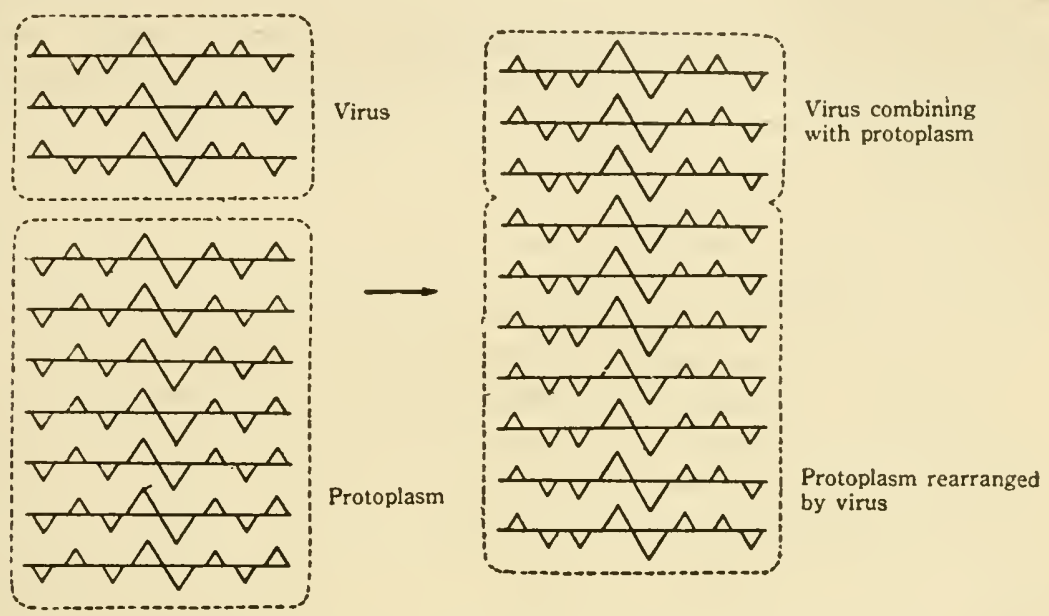

Fig. 9. The mode of multiplication of virus in protoplasm.

virus particle may fail to act as a typical virus, only being capable of disturbing the structure of the protoplasm without inducing exact replica. Such an incomplete virus particle may sometimes be able to exhibit its virus action, if many particles are put together to be stabilized in its structure or to be united to cooperate. Luria (16) found a peculiar phenomenon that phage is produced when bacterium is infected with many particles of phage inactivated by ultraviolet light. In addition it has been reported that a great number of mouse pox virus having been inactivated and being unable to multiply can enhance the virus action of a minute quantity of the intact virus (17). Moreover, evidences are known that a virus which has been inactivated to a certain degree is capable of developing some effect upon the protoplasm of host cells though fails to produce the replica. For example, phage particle inactivated by ultravio.et ray can promote the lysis of bacteria due to irradiation, thereby no virus multiplication taking place (18), and again phage particles inactivated by X-ray irradiation is still able to lyse and kill sensitive bacteria (19).

Physiologically active agents such as enzymes and toxins may be similar to viruses in their action, but so perfect a polymerization as in viruses, if needed, may not be necessary for their function, as the action of a template is never needed in these agents. 


\section{Spread of Denaturation in Red Blood Cells}

There are numerous evidences that various physicochemical changes can spread readily in the protoplasm. Almost all the life phenomena may presumably depend upon the transmission of the changes, which cannot be accomplished unless lipids are interposed among protein molecules rendering the protoplasm protein freely movable. On this account lipids may be regarded as an essential component of the protoplasm.

In this section some detailed discussion will be made on the haemolysis, which can be looked upon as one of the phenomena resulting from such a transmission of protoplasm change, in the hope that it may throw some light on the mecharism of the transmission.

Red blood cells consist of haemoglobin and stroma, which the latter is a substance sedimentable from water solution of lysed cells by passing $\mathrm{CO}_{2}$ into the solution. According to our study, stroma separated from haemoglobin is a particulate substance similar to viruses consisting of a protein and lipids (20). When acetic acid is added instead of $\mathrm{CO}_{2}$ to the lysed solution to adjust its $\mathrm{pH}$ to about 5.5 , it can be sedimented like virus particles and isolated by an ordinary centrifuge. This shows that the stroma thus obtained is nothing but the coagulated elementary bodies of the blood cells. Red cells seem, therefore, to be composed of such elementary bodies to which a great quantity of haemoglobin is adsorbed. Mitchson (21) has claimed that the thickness of the membrane of the human red cell is $0.5 \mu$, and that since this would occupy about 55 per cent of the volume of an intact cell, a red cell is largely made up of such a thick membrane containing haemoglobin. This thick "membrane" may be the stroma, which is the protoplasm itself, and which can be decomposed into virus like particles, $i$. e., into coagulated elementary bodies.

When a physicochemical change is induced in this stroma to a certain degree, the stroma may become unable to maintain the adsorbed haemoglobin and thus haemolysis may follow. If so, the changing degree of stroma will be traced in detail by the liberated haemoglobin amount.

If the protoplasm structure is just as already described, haemolysis may take place in the following way: When a haemolytic agent combines with a red cell to exert an influence upon the protoplasm, a change will occur in the site of the combination of the agent and will spread successively in the protoplasm; when the change reaches to a certain degree haemoglobin will begin to escape from the protoplasm. In fact, experimental results obtained with haemolytic agents, such as 
saponin, $\mathrm{HgCl}_{2}$, and antiserum with complement, agreed well with this reasoning.

Firstly, it was confirmed that there was a certain incubation period between the addition of haemolytic agent and the commencement of haemolysis. This incubation period is expected from the above reasoning. Secondarily, it was found that this period is inversely proportional to the concentration of the agent, showing that the changing rate of stroma is directly proportional to the concentration. This fact may indicate that the haemolytic agent acts as a kind of catalyzer; if the blood cell protoplasm undergoes a change in a manner described above following the combination with the agent, the degee of the change should be directly proportional to the amount of the agent combining with it, and hence the haemolytic agent may appear in its action to be a sort of catalyzer.

The action of the haemolytic agents accordingly is comparable to that of partially inactivated viruses, which fail to produce replica but capable of disturbing the protoplasm structure. Thus, the fact that weak virus particles cannot act as the virus unless many particles are put together may be explained in this respect; namely, the protoplasm change caused by the virus is proportional to the virus amount combining with it, so that even a weak virus may overcome the protoplasm if many particles are present.

In this connection a more detailed discussion will be made on the experimental results obtained with saponin and rabbit blood cells. The relationship between saponin concentration and the time required for the haemolysis completion was found to be as indicated in Table 5 .

\section{Table 5 .}

Ralation between the Concentration of Saponin and the Time Required for the Completion of Hemolysis by the Saponin Concentration.

\begin{tabular}{|c|c|c|c|c|c|c|c|}
\hline $\begin{array}{l}\text { Concentration } \\
\text { of saponin, \%. } \\
\text { (C). }\end{array}$ & 0.1 & $\frac{\overbrace{\frac{N}{x}}}{\dot{0}}$ & $\overbrace{\frac{N}{x}}^{N}$ & $\frac{m}{\sqrt{N} \mid m}$ & 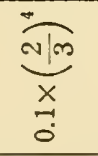 & $\overbrace{\frac{x}{0}}^{\frac{n}{0} / m}$ & $\overbrace{\frac{N}{x}}^{0}$ \\
\hline $\begin{array}{c}\text { Time required for } \\
\text { the completion of } \\
\text { hemolysis, sec. } \\
(t) \text {. }\end{array}$ & 71 & 84 & 109 & 142 & 230 & 420 & 1020 \\
\hline $1 / t, \quad(K)$. & 0.0141 & 0.0119 & 0.0092 & 0.0072 & 0.00 .43 & 0.0024 & 0.0010 \\
\hline
\end{tabular}

Saponin haemolysis is very fitted for such observations as the haemolysis is completed in short periods if once begins to occur.

In this table the time interval between saponin addition and the 
lysis completion is shown, but as the lysis completes immediately after its commencement, time cited here can be regarded as the time required for the lysis commencement. Therefore, incubating time required for the lysis commencement can be said to become the longer, the lower the saponin concentration.

Now, consideration will be made as regards the following equation representing the first order reaction: $1 / t \cdot \ln a /(a-x)=k$, where $a$ is the quantity of reacting substance existing at the outset, and $x$ is the reaction product in the time $i$; that is, in the case of haemolysis, $a$ is the protoplasm quantity to. be changed and $x$ is the protoplasm amount changed in the time $t$.

Since haemolysis is to be completed when $a /(a-x)$ becomes a certain value, this should be constant irrespective of the saponin concentration and hence can be designated as $k^{\prime}$, then we have:

$$
1 / t \cdot \ln k^{\prime}=k \text { or } 1 / t=k / \ln k^{\prime}=K \text {. }
$$

Thus, $1 / t$ is shown to be directly proportional to the velocity constant. On the other hand, the relation between $1 / t$, or $K$, and saponin concentration is indicated in Fig. 10, where it is shown that $K$ tends to be a linear function of the saponin concentration. Since $K$ is directly

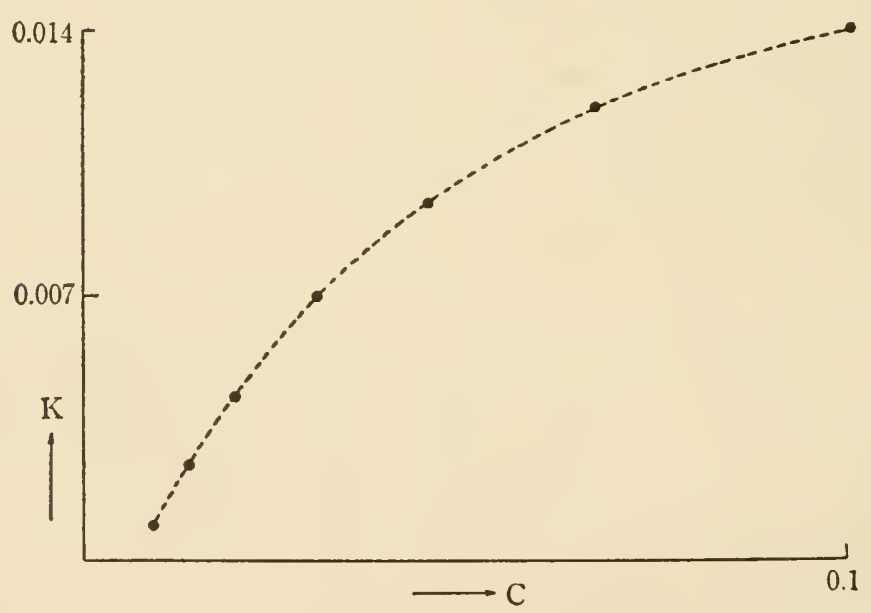

Fig. 10. Relation between the concentration of saponin (C) and the velocity of hemolytic reaction $(\mathrm{K})$. See Table 5 .

proportional to the velocity constant as just mentioned, this indicates that the velocity constant also tends to be a linear function of the saponin concentration. However, the effect of the saponin concentration becomes the lesser as it becomes the higher as clearly shown in 
Fig. 10; this may be due to the decrease in the effect of saponin per unit mass when its great amounts are adsorbed to a blood cell.

Experimental results obtained with saponin were thus fairly demonstrable, because haemolysis was completed in a short period; however, with $\mathrm{HgCl}_{2}$ the situation was somewhat different because the lysis by this salt needed an extremely long period, though similar results could be obtained. Likewise with antiserum and complement, the same proved to be true.

So far as our studies concern, at least some bacterial haemolysins are of a particulate nature like viruses, and may cause a change in the blood cell by a physicochemical force arising from their specific configuration as in the case of viruses; the changes induced by haemolysin may propagate through the protoplasm to cause haemolysis (22). Such a change, however, fails to cause the production of replica corresponding to the specific pattern of the haemolysin, so that the lysins cannot multiply in red cells and therefore they are not viruses.

Bacterial lysis caused by a phage can, in like manner, be explained by the assumption that the association among elementary bodies in the bacterial cell is loosened by the protoplasm change leading to the liberation of elementary bodies which are usually endowed with virus action by the change. It is known that when a bacterial cell is affected by a great excess of phage particles, no active particle is produced. This may result from a too rapid decomposition of the protoplasm to be endowed with the pattern exactly identical with that of the phage. Even in the case of lysis by a proper number of phage particles, the virus cannot multiply in the absence of certain nutrients or in the presence of metabolic "inhibitors" (23) presumably because of a similar failure of the replica formation. 


\section{CHAPTER IV}

\section{THE CRYSTALLINITY OF VIRUSES}

\section{The Fusion of Particles}

Virus-like particles can be isolated also from healthy plants by the same procedure by which the particles are isolated from animal tissues. We carried out experiments with plant materials such as leaves of cucumber (Cucumis sativus, L.), kidney-bean plant (Phaseolus vulgaris, L.), tomato plant (Solanum lycopersicum, L.), poplar (Poplulus nigra, L.), spindle-tree (Evonynus japonicus, Thumb.), dandelion ( $T a$ raxacum Melongena, L.) and petals of flowers of bindweed (Catystegia subvolubilis, Don.) (24).

Virus-like particles were recovered without exception from all these normal plant materials. In the case of tomato plant, we used leaves infected with mosaic virus as well as healthy ones, and were able to isolate similar particles from both materials. Berkefeld-filtrates of these plant saps diluted with water were added with acetic acid and precipitates produced thereby were separated by means of ordinary centrifuge. In the majority of cases the optimum precipitating point was found at a more acid side than in the case of animal materials, and particles obtained were proved to be usually smaller than those of animals.

Plant particles thus obtained shared a peculiar property not present in particles of either animal or bacterial origin, and tended to aggregate into larger particles or bodies. For example, particles of dandelion did form coccoid bodies of about $3 \mu$ in diameter when left at $\mathrm{pH}$ 4.0 for several days, and particles of cucumber aggregated to bacillary bodies which sometimes further developed into large homogeneous protozoa-like bodies. It is a noteworthy fact that in such bodies original particles entirely disappeared, but on the addition of dilute alkali, the particles would reappear and occasionally the homogeneous bodies would be broken down into the particles.

The fusion of particles into such larger masses was never observed with animal or bacterial particles. Some of such large masses would appear as if they were crystals, but no double refraction was demonstrated. Occasionally uniform large bodies were formed immediately after the precipitation of particles by acetic acid; in such cases it could be clearly seen under the microscope by dark field illumination that they were composed of minute particles which, however, when allowed 
to stand for a long period, disappeared gradually in fusing homogeneously.

These facts may be interpreted as flollows: Plant elementary bodies which have been coagulated in the course of isolating procedure can solve the coagulation, when stand at the isoelectric point, in liberating the polar groups which subsequently can make the particles associate with one another to form large masses which may be looked upon as the protoplasm itself. In other words, the coagulated elementary bodies can solve the coagulation to unite with one another, thereby they may be arranged orderly as in the original protoplasm, thus recovering the structure of the protoplasm itself. Therefore, the fusion of particles to larger bodies may be regarded as their return to the original protoplasm mass. At the isoelectric point $\mathrm{COOH}$ groups of the protein can coexist with the $\mathrm{NH}_{3}$ groups, probably contributing to the fusion of the particles.

The fact that such fusion can take place with plant particles, whereas this is not the case with animal ones, suggests that plant particles can readily solve their coagulation and can combine with one another while animal particles fail to do so. On the other hand, this may account for the difficulty of plant protoplasm to be coagulated into minute particles. Whereas animal particles can readily be precipitated only by the shift of the $\mathrm{pH}$ to its isoelectric point, plant particles occasionally fail to be obtained by this process; the addition of salts such as ammonium sulphate or sodium chloride is required for the complete precipitation. Thus, in the case of plant materials, mechanical grind may not be sufficient for the coagulation of elementary bodies. It is actually known that plant virus particles such as those of tomato bushy stunt, tobacco necrosis, southern bean mosaic and turnip yellow mosaic are soluble even at the isoelectric point and fail to precipitate (25).

\section{The Expulsion of Lipids}

The property of plant particles to fuse into homogeneous masses may be closely related to their crystallinity. Homogeneous masses appear sometimes as if they were crystals, but if produced by the mere addition of acetic acid, they never proved to be optically anisotropic when observed through crossed Nicol prisms, whereas precipitates with double refraction were sometimes formed when ammonium sulphate at $1 / 3$ saturation was added together with acetic acid; that is, crystals were formed when ammonium sulphate was added.

Among the plants serving as our experimental materials, the doubly refracting property was most distinctly observed with precipitates ob- 
tained by this procedure from cucumber, tomato, and egg-plant, while from dandelion and poplar were formed precipitates, the greater part of which was composed of minute particles without double refraction, only a small part presenting the refraction; and from the other plants doubly refracting precipitates were never obtained.

From both tomato and egg-plant were sometimes obtained typically crystalline masses with a definite shape and a sheen more intense than that of the precipitates without double refraction, whereas in some other cases only sediments were yielded appearing as though the minute particles themselves were exhibiting birefringency, 'the doubly refracting particles appearing to be so small.

The nitrogen content of such crystalline masses or particles was found to be approximately 16 per cent, the usual $\mathrm{N}$ value for ordinary proteins, showing that there was no room for the presence of lipids, and in fact no lipids could be recovered from such particles. By these facts the writer was convinced that the lipids in non-coagulated elementary bodies suspended in a plant sap were expelled from the bodies by the addition of ammonium sulphate and that consequently protein molecules were enabled to combine directly with one another in their expanded state to become doubly refracting.

The absence of doubly refracting property in the homogeneous masses produced by the mere addition of acetic acid may depend on the presence of lipids which may allow the protein molecules to partially shrink or contract in an irregular manner. The homogeneous masses without double refraction were actually proved to contain large amounts of lipids as does usual protoplasm. When once coagulated, animal elementary bodies, unlike plant particles, may hardly solve, if any, their coagulation. This may account for their inability to form larger homogeneous masses not to speak of crystals by the addition of ammonium sulphate.

If the protoplasm protein of plant cells affected by a virus can be obtained in such a crystalline form in which the protein structure to act as the virus is retained without being damaged by the elimination of lipids, the virus may be said to have been isolated in a crystalline form. The crystallinity is therefore by no means a characteristic of protoplasm protein having virus action. Crystals were actually obtained from healthy leaves as above described.

Since protoplasm protein is to be endowed with a virus action through the structural alteration by the virus, some differences may be expected in the crystalline form between "healthy" and "pathologic" crystals so that a crystal peculiar to a virus may be obtained from a plant affected by the virus. Bawden and Pirie (26) were able to crystallize tomato bushy stunt virus as rhombic dodecahedra, whereas we 
could isolate a similar crystal from apparently healthy leaves of tomato plant. According to our experiments, such a crystal was obtained only from the young leaves iri spring, in summer no crystalline mass being isolated even from infected leaves.

There should be a physicochemical association between protoplasm protein and lipids, so that the expulsion of lipids may have considerable influences upon the protein. When treated with ether at laboratory temperature, both vaccinia virus and phage were readily inactivated, and Japanese encephalitis virus protein isolated by our method was deprived even of the complement binding faculty. Ammonium sulphate may cause the expulsion of lipids either by damaging the combination between the protein and lipids or by enhancing the direct, mutual combining force of proteins. Whether the cause may be, there seems little doubt that the salt can expel the lipids out of some plant elementary bodies. Vaccinia virus or phage particles were immediately inactivated by the addition of the salt, showing the strong effect of the salt upon the virus protein. The fact that some plant viruses can be isolated in a crystalline form by the addition of this salt may therefore indicate the remarkable stability of the virus protein. There are, however, ample evidences, as will be described later, to show that the expulsion of lipids may occur during the infection of plants with a virus prior to the treatment with ammonium sulphate, which may only promote the aggregation of proteins.

At any rate, the fact that some plant virus proteins can retain their action even in a crystalline form, in which no lipids are contained, must be of the utmost importance in the consideration of the nature of virus action, since this fact shows that no lipid is necessary for the virus action. Accordingly, the virus action must be attributed to the purely polymerized protein molecules. The virus inactivation on the removal of lipids, as already pointed out, may be, therefore, caused by some alteration in the protein structure occurring as a result of the lipid elimination. Hence, the presence of lipids may be responsible only for the maintenance of the characteristic liquid crystalline state of protoplasm. If protoplasm consisted of proteins polymerized without intermixing lipids and existed in a sort of solid crystalline state, various complicated life phenomena would never be revealed. However, since the action of a virus consists in the function as a template, lipids are never needed for the action, but necessary for the protoplasm to develop a change responding to the template. Lipids are indispensable for producing a replica but not for acting as the template.

That lipids have no concern with the virus action may be evident also from the inconstancy of the lipid contents. Of animal viruses, that of papilloma has been claimed to have so little quantity of lipids 
as 1.5 per cent (27), whereas equine encephalitis virus contains so much as 50 per cent (28). Usually a considerable quantity of cholesterol is present in the lipids of vaccinia virus particles; however, Hoagland et al. (29) have shown that cholesterol can be removed from the particles by extraction in the cold with ether without affecting virus activity. According to our experiments, as above mentioned, inactivation occurred if the viruses were treated with ether at a laboratory temperature, whereas Harris succeeded in eliminating lipids at $-65^{\circ} \mathrm{C}$. from dessicated rabies virus with petroleum ether and found that the virus became stable by this procedure (30).

It should naturally be expected that viruses become stable on the lipid removal, since lipids may render the protein molecules easily changeable; when lipids are removed the protein molecules may be enabled to combine directly to form a stable structure. The remarkably stable nature of crystalline viruses may be explained in this respect. A virus may, however, become the more estranged from living matter, the more the lipids are eliminated, since life can appear only on the stage of instability.

\section{The Length of Crystalline Virus Particles}

Some plant viruses are isolated by ultracentrifugation in a form of minute rod-shaped particles containing no lipids. Such particles are not artefacts, because they can be demonstrated in infected leaves themselves, and the sap itself shows the anisotropy of flow. From electron micrographs the length of a particle of tobacco-mosaic virus was estimated to be about $0.3 \mu$, and the width about $15 \mathrm{~m} \mu$. Its "molecular weight" was calculated to be 40,000,000 (31).

It is a noteworthy fact that the particles of various strains of tobacco-mosaic virus are proved in electron micrographs to be similar in both size and shape regardless of the presence of the profound differences in their chemical composition. In discussing this fact Stanley stated that despite the differences in composition some general directive force appears to be effective during the construction of these virus particles (32). Is it really necessary to postulate such a mysterious being as a general directive force?

The majority of workers considered that there are striking differences between plant and animal viruses, since the plant viruses are isolated in rod-shaped particles consisting of a protein containing no lipid, whereas the latter viruses in particles having complex chemical compositions containing lipids, never being isolated in rod-shaped particles unlike the former. It is, however, known that some animal viruses can 
be separated in the same rod-shaped particles.

Insects are generally affected by a group of viruses and the disease is called polyhedrosis. Viruses causing polyhedrosis are claimed to have also rod-like shapes similar to some plant viruses. For instance, according to Bergold (33). the virus particles of silk-worm polyhedrosis are $288 \mathrm{~m} \mu$ in length (tobacco-mosaic virus is believed to have a length of $280 \mathrm{~m} \mu$ ), and $40 \mathrm{~m} \mu$ in width, and consist of a nucleoprotein having a high $\mathrm{P}$ content; tobacco-mosaic virus has a similar chemical composition as referred to later.

Such rod-shaped particles composed of nucleoprotein are not only found in silk-worm, but also in many other insects infected with polyhedrosis. For example, the virus particles of polyhedrosis of the gypsymoth caterpillar (Porthetria dispar, L.) are $41 \times 360 \mathrm{~m} \mu$; western tent caterpillar (Malacosoma pluviale, Dyar.), $40 \times 350 \mathrm{~m} \mu$; california oakworm (Phryganidia california, Pack.), $30 \times 270 \mathrm{~m} \mu$; western yellow-stripped armworm (Prodenia praefica, Grote), $50 \times 270 \mathrm{~m} \mu$; spruce budworm (Christoneura fumiferana, Clem.), $28 \times 260 \mathrm{~m} \mu$ (34).

The term polyhedrosis arises from the peculiar shape of inclusion bodies appearing in cells of infected insects and it is stated that such polymorphic inclusion bodies, in which viruses are contained, are themselves doubly refracting.

For what reasons do some viruses exist in such rod-shaped particles of a similar length not only in plants but also in insects? Is it true that a certain general directive force as Stanley suggested is operating in the course of virus production?

This fact, however, can readily be explained from the writer's view previously described as regards the protoplasm structure and the mechanism of virus reproduction. According to the writer's view rodshaped particles are no more than the elementary bodies from which lipids have been eliminated. Lipid expulsion may occur because of the structural change of protoplasm protein caused by a virus, as the protein becomes thereby incapable of retaining the lipids. The length of the particles, therefore, must be the length of an expanded elementary body itself whose length in turn must be the length of protoplasm protein molecule in a stretched form. As will be shown later, this length appears to have no relation to the presence of nucleic acids.

If we assume that the molecular weight of protoplasm, like that of the ordinary euglobulin, is 150,000 and that the distance between the two amino acid residues is $0.34 \mathrm{~m} \mu$, and that molecular weight around a single residue is 130 , the length of a stretched thread-like molecule is calculated to be approximately $0.4 \mu$. This should be the length of an expanded elementary body and, accordingly, the length of a rod-shaped virus particle. The value thus estimated, however, is 
a little longer than $0.3 \mu$ or $280 \mathrm{~m} \mu$, a value regarded as the length of a tobacco mosaic virus particle, although some insect viruses have been claimed to have a length near $0.4 \mu$ as described above.

Since the virus particles observed in electron micrographs are in the dry state, some shrinkage should be expected in them. It has been shown that protein crystals such as those of oxyhaemoglobin or insulin undergo shrinkage on the dessication. In methaemoglobin and $\beta$-lactoglobulin even so remarkable a shrinkage as up to 50 per cent is proved (35) (36).

"Molecular weight" of tobacco mosaic virus has been claimed, as mentioned above, to be $40,000,000$; such a high molecular weight cannot be attained unless approximately 200 globulin molecules with molecular weight of 150,000 are put together in a bundle even when a considerable amount of nucleic acids is intermingled. The bundles are considered to be originated from "elementary bundles", already described, from which lipids have been eliminated, not directly from elementary bodies themselves which tend to be decomposed into the elementary bundles.

As stated above, insect virus particles are reported to be in length approximately $0.3 \mu$ like some plant viruses, whilst in width they appear generally to be about two times as thick as plant viruses. The elementary bundles of insects may be said, therefore, to be thicker than those of plants, that is, in insects far greater number of protein molecules appear to be united to make an elementary bundle than in plants.

According to the kind of cells, protoplasm protein molecules may tend to form very long rods or filaments through end-to-end association. In such a case, virus particles may have lengths several times as long as $0.3 \mu$. The virus particles of latent mosaic of tomato have been claimed to be $525 \mathrm{~m} \mu$ in length (33); this may be an example of endto-end combination of two molecules. According to Takahashi (37) Brassica nigra virus is about $0.7 \mu$ long; this may also be two molecular long.

On the contrary, if the molecules split into shorter fragments, the particle should naturally become shorter. Whereas the length of tobacco-mosaic virus protein is generally believed to be $280 \mathrm{~m} \mu$, some workers are disinclined to accept the existence of such definite sized particle. For example, Crook and Sheffield (38) concluded from electron micrographs of tobacco mosaic virus that the particle size varies according to the method of preparation, showing no presence of basis for assigning a length. Bawden and Pirie (39) stated likewise that the average length of the particles in a virus preparation is determined by both the past history and present environment of the preparation, and that it is a compromise between the forces leading to end-to-end adhe- 
sion and those, such as thermal agitation, that tend to break this adhesion, breakable rod, when broken, leaving polar ends which can join together again.

As already mentioned, plant protoplasm proteins show a high tendency to unite with one another, while at the same time it is known that the process of purification causes aggregation of the particles initially present in the fresh infective sap. In connection with this fact, Bawden (25) stated that since the available evidence on tobaccomosaic virus and virus $\mathrm{X}$ suggests that any process that concentrates them or that removes contaminations from them is likely to cause agglutination, agglutination may be indispensable from a high degree of purity, and claims to have isolated homogeneous, unagglutinated particles may be equivalent to claiming incomplete purification. At present it seems generally accepted that tobacco-mosaic virus particles can occasionally exist in unusually long threads under the electron microscope. Stahmann et al. (40) found that Wisconsin pea-streak virus particles, on standing in distilled water for several weeks, associated endto-end and side-to-side to produce bundles in parallel alignment which were often coiled about each other.

Alteration in the particle lengths appears to occur not only in vitro but also in the plant cells in situ. On an electron microscope study of tobacco-mosaic virus extracted from pulp and juice after various periods of infection, Takahashi and Rawling (41) found that when the virus is extracted from finely macerated pulp and juice at the $\mathrm{pH}$ of plant juice, the proportion of short particles increases between the 4 th and 16th days after infection. They claimed that the short particles did not result from fracture of longer particles during maceration. Moreover, according to Black et al. (42), electron micrographs of sections through tobacco leaves infected with tobacco-mosaic virus sometimes showed in the cytoplasm of the diseased cells thread-like filaments much longer than $280 \mathrm{~m} \mu$, indicating the natural occurrence of end-to-end association of the rods.

Joly (43) showed by the method of streaming double refraction, that the prolonged cooling of tobacco-mosaic virus produces a partially reversible shortning of the virus particles. Furthermore, according to. Schramm (44), in alkaline buffers, tobacco-mosaic virus particles dissociated into smaller components having molecular weight of about 360,000 as compared with about $40,000,000$ for the original intact protein, but readjustment of the solution to $\mathrm{pH} 5$ yielded a material practically indistinguishable from the original native protein in molecular weight and shape, in crystal form and electrophoretic behaviour.

Thus, there seems no doubt that at least tobacco-mosaic virus particles can on the one hand split into smaller particles and on the other 
hand can form longer threads through end-to-end association. It seems possible, however, that thread-like particles of approximately $0.3 \mu$ in length are the most common and stable. Knight and Oster (45) claimed that the most majority of virus particles (20 to 40 per cent) fall into a group size of $15 \times 280 \mathrm{~m} \mu$ and less than 5 per cent being extraordinarily short or long. Rawling et al. (46) also published the opinion that the majority of particles are about $0.3 \mu$ in length, the longer particles being aggregations of these and the shorter units being due to breakage of some of the rods during the drying of the specimen before examination in the electron microscope.

\section{Various Shapes of Plant Viruses}

Many plant virus particles appear, as mentioned above, to be rodshaped, but this never holds true for all the plant viruses. It is generally accepted that the particles of plant viruses such as those of southern bean mosaic, tomato bushy stunt, tobacco necrosis, and turnip yellow mosaic, are of globular form having diameters of approximately $30 \mathrm{~m} \mu$ (32) (47). If virus particles are produced from elementary bodies of protoplasm on the alteration in its structure by a virus, there should be an intimate correlation between the rod-shaped particles and these globular ones.

In the study on the effect of high frequency sound vibrations on tobacco-mosaic virus, Oster (48) found that the vibrations break the virus rods, reducing the length first to $140 \mathrm{~m} \mu$, then to $70 \mathrm{~m} \mu$ until at the end of 64 minutes of continuous exposure to the vibrations one eighth of the original length being the most common. If the molecular weight of the unit protein forming the rod is 150,000 , then the particles having one eighth of the original length will be composed of protein fragments whose molecular weight is approximately 20,000 ; this value appears to be close to the molecular weight of Svedberg's unit, 17,600. If protein of certain cells was liable to break down into such units by the disturbance in its structure on the infection with a virus and thereby if the virus action was retained in these fragments, then the virus would be obtained as such extremely minute particles.

Oster suggested that only particles of $280 \mathrm{~m} \mu$ in length were infectious since a falling off of infectivity seemed concomitant with the breaking down of the original rods. However, as discussed in the previous Part, even with tobacco-mosaic virus there are evidences that the particles broken to a certain extent can retain some infectivity. If protoplasm proteins in some plant cells affected by certain viruses other than tobacco-mosaic virus were readily decomposable to smaller mole- 
cules and if disturbances, which might be brought about by the decomposition in the protein structure concerning the virus action, was insignificant, then minute particles produced by the decomposition would have the virus action.

Purified virus particles of both Rattle disease of tobacco and stemmottle disease of potato were found under the electron microscope to show two peaks in the distribution curves for particle length, one at about $150 \mathrm{~m} \mu$ and the other at about $300 \mathrm{~m} \mu$ (49). This can be interpreted as indicating that in such a case decomposition to half was liable to take place.

With certatn viruses whose particles are extremely liable to decompose into fragments, needle-shaped crystals like those of tobacco-mosaic virus may fail to develop on the addition of ammonium sulphate. In fact, the above mentioned plant viruses of globular particles, such as those of southern bean mosaic and tomato bushy stunt, have never been isolated in needles, but yielded rather in spherical crystals such as rhombic plate, hexagonal prisms, or octahedra.

The crystal shape may be effected by the degree or the manner of decomposition of the rods. Bawden (25) stated that the causes which determine the crystal form are unknown, and that if inactive sap is divided and the two parts purified separately, one sometimes crystallized in one form and the second in another, and that if dodecahedral crystals are dissolved and then recrystallized, bipyramids or circular laminae may be produced. According to Markham and Smith (50) when turnip yellow mosaic virus is crystallized from salt octahedra are produced, whereas when crystallized from alcohol long prisms are yielded. 


\section{CHAPTER V}

\section{FINER STRUCTURE OF VIRUS PARTICLES AND ITS SIGNIFICANCE}

\section{Decomposition and Fusion of Elementary Bodies of Protoplasm}

As mentioned in the previous Chapter, some plant viruses tend to reveal themselves in rod-shaped particles or sometimes in extremely small globular particles which can be regarded as the fragments of the rod. An important characteristic of these particles is that they contain no lipids, unlike many other viruses which exist in spherical bodies having never yet been obtained in crystalline forms.

According to our experiments, phage particles or phage-like particles isolated from normal bacteria contain a considerable amount of lipids, and their average diameter appears to be approximately $0.1 \mu$ (24) (9). In this connection, it should be noted that from many plant materials particles similar in size and composition to these bacterial particles were obtained. The writer was convinced that such particles are the coagulated elementary body of protoplasm itself. It can easily be calculated that a single rod of tobacco-mosaic virus having the size of $15 \times 280 \mathrm{~m} \mu$ cannot make such a particle even if it contains some lipids, unless about a dozen of the rods are put together. On the other hand, since, according to our estimation, the dry weight of a single phage particle is of the order of $1 \times 10^{-15} \mathrm{~g}$, the number of protein molecules in a single particle is estimated to be of the order of $3 \times 10^{3}$, and "molecular weight" of a particle of this size is calculated to be about $480,000,000$, a value which also indicates that about a dozen of tobacco-mosaic virus particles whose molecular weight is $40,000,000$ can only form a single particle of the phage.

For such a reason the writer has formed the opinion that the elementary body of protoplasm of certain cells tend to decompose into about a dozen of elementary bundles; rod-shaped viruses as above mentioned are considered to be such bundles from which lipids have been eliminated.

Thus, several hundreds of extended protoplasm protein molecules may associate in parallel alignment to make a bundle, $i . e$, an elementary bundle, and a dozen of the bundles, in turn, in parallel association form a unit particle, i.e., an elementary body. The establishment 
of such a structure may be achieved by a basic physicochemical property belonging naturally to the protoplasm protein. The writer was actually able to confirm in vitro that some plant protein of globulin nature does possess the property of forming virus-like particles. According to his investigation, no particulate protein was contained in Merck's ricin preparation, but when its water solution was added with ethanol at 30 per cent, its $\mathrm{pH}$ being adjusted to 5.5 by the addition of acetic acid, and left in an ice box for several days, then the protein molecules in the preparation together with some lipids sedimented in forming virus-like particles; these particles would further unite with one another to form homogeneous protoplasm-like body, if they were left at laboratory temperature after being isolated from the original solution by centrifugation and subsequently suspended in a weakly acid solution at the isoelectric point. When pressed mechanically or added with alkali, the homogeneous protoplasm-like body thus formed would be decomposed again into virus-like particles (51).

This important finding strongly suggests that at least some proteins with globulin nature possess a basic character to polymerize into virus-like particles, which in turn tend of fuse into a protoplasm-like body. But since the association among the particles in such a body may not be firm enough, the body may be disposed to disintegrate again into the particles. Elementary bodies and the protoplasm can be regarded as such particles and the body, respectively. Since this fact is the utmost important and the most fundamental, on the basis of which the writer's theory as regards the protoplasm structure have been developed, we shall have occasion later to discuss more in detail on this subject.

Svedberg and Pederson (52) postulated the term proteon to designate a native protein unit incapable of dividing into still smaller units having the native protein character. According to them, every native protein may be regarded as a system of such proteons. The conception, that a protein particle is not a mere conglomerate of proteons but an orderly aggregate or a polymerization, arose from their finding that in many cases, where ultracentrifugal studies show that a protein is uniform in size and shape under well defined external conditions, the protein in question is capable of dissociating into subunits when the environmental conditions are modified. It may perhaps be permissible to say, that the formation of a virus-like particle or its decomposition into unit molecules is only the manifestation of one of the basic characters of proteins.

Whereas haemocyanin has long been regarded as one of the best examples of dissociable complex, Brohult (53) found that its depolymerization is a function of both $\mathrm{pH}$ and salt concentration, the depolyme- 
rization increasing as the latter increases. Insulin was also thought as a reversibly dissociable system, its unit molecular weight being believed to be 6,000 (54).

An interesting fact was found with insect viruses that the viruses are isolated not only in the form of rod-shaped particles as above described but also in much thicker rods each of which appears to be composed of a number of the usual rods fusing together in parallel alignment. For example, in the case of polyhedrosis of the gypsy-moth caterpillar such extremely thick rods as $160 \times 415 \mathrm{~m} \mu$. were found in addition to the usual rods of $41 \times 360 \mathrm{~m} \mu$. In the case of nun-moth caterpillar most particles were proved so thick as appearing almost globular (34). Such a thick globular rod may be interpreted as the elementary body itself from which only lipids have been eliminated. The thinner usual rods or needles should be regarded as produced on the dissociation of such thick rods.

These thick rods have proved in general to be much longer than the usual thin rods or needles, a fact which may depend upon lesser degrees of the shrinkage due to the desiccation owing to their great thickness. Williams and Steere (55) have found in electron micrographs that also tobacco-mosaic virus particles exist in forming thick bundles in the juices from infected tobacco leaves; if the specimens are washed with one or two drops of distilled water before drying and shadowing with uranium, the bundles disintergrate into single needles.

\section{Virus Particles Containing Lipids}

As above discussed, some plant virus particles or rods can be regarded as elementary bundles whose structure has been altered by a virus and from which subsequently lipids have been expelled. The formation of similar particles may not be impossible if adequate physical or chemical effects other than viruses, which may be able to cause some disturbances in the protoplasm structure to expel the lipids, are applied to the protoplasm.

It is, however, generally believed that no virus-like rods can be isolated from normal plant tissues. Nevertheless, according to our investigation as shown already minute particles with double refraction could be obtained from various normal plant materials when the saps were treated with ammonium sulphate. It was uncertain whether or not such particles having double refraction were aggregates of the rods, but there seemed no doubt that the lipids could be expelled by the action of ammonium sulphate, since the particles contained no lipids. 
It appears to the writer rather strange that virus-like rods are generally believed to be never produced without virus, although we are unable to determine whether or not adequate stimuli other than a virus can give rise to the production of virus-like rods in plant tissues, as we unfortunately have neither the electron microscope nor ultracentrifuge. The above mentioned fact, however, that particles or bodies with double refraction are produced by the addition of ammonium sulphate suggests the possibility of rod formation without virus.

Yamafuji and his collaborators (56) claimed that they could produce tobacco-mosaic and polyhedrosis virus in plant leaves and silkworms respectively by application of such chemicals as $\mathrm{H}_{2} \mathrm{O}_{2}$ or hydroxylamine. It is a question whether or not viruses themselves were actually produced, but at least it seems certain that some chemicals could cause the expulsion of lipids to form virus-like bodies or aggregates. They attempted to connect oxidation process with this apparent virus formation, but it should be remembered that oxidizing agents, in general, exhibit profound effects upon protoplasm structure and that the general mutagenic activity of peroxide in microorganisms seems fairly established (57). Mutagenic activity of some agents can be considered to be based upon their ability to produce a structural change in nucleoproteins in protoplasm as discussed later. Therefore, if some agents could raise, like viruses, certain changes in protoplasm protein to break the combination of the protein with lipids, virus-like rods containing no lipids would be produced. According to a more recent report by Yamafuji et al. ( 57 a) polyhedral virus crystals formed chemically in silk-worm larvae exhibit the same electrophoretic pattern as those produced naturally by infection.

It seems to be believed by a number of workers that certain plant viruses, at least tobacco-mosaic viruses, are rod-shaped particles, and that such rods or particles are the only feature of the viruses. However, the writer holds the opinion that the virus particles without lipids are rather an exceptional existence even in tobacco-mosaic viruses, but that like many animal viruses these plant viruses usually exist in particles containing lipids. If the combination with the lipids was not broken by the virus infection, elementary bodies would coagulate in their natural state even in the plant cells and would come out as virus particles.

We failed to obtain any particles or aggregates having double refraction from infected tomato leaves in summer even when ammonium sulphate was applied, but could isolate in abundance only particles containing considerable quantities of lipids as in the case of animal viruses. Bawden (25) stated that the yield of plant viruses varies 
remarkably according to the season where the expriments are carried out. For example, the yield of tomato busy stunt virus in summer is only about one fifteenth of that in winter; with tobacco necrosis this is found to be only about one twentieth. This fact may indicate that in summer the combination with lipids is so firm that their expulsion is hardly possible.

According to Nixon and Watson (58) the sap from sugar beat plants infected with beat yellow virus contains at least two kinds of specific particles, and one of these is the rod which can be seen with the electron microscope, the other probably occurring in a much higher concentration, appearing to be spherical. They stated that possibly the rods are an alternative form of this spherical particles, which can be found in large quantities in clarified sap from healthy plants. This finding is what should be expected from the writer's view just mentioned. Furthermore, on an electron microscopic study. of tobacco-mosaic virus and some 35 other viruses, Johnson (59) found some rod-like particles with tobacco-mosaic virus and with several other viruses, but failed to find any rod in another large group of virus-infected plants. He suggested that the rod-like particles in question may be a result of disease rather than the ultimate causal unit.

It should be noted in this connection that only extremely small quantities of virus particles are usually yielded from infected plant saps. For example, the yield of tobacco-necrosis virus particles from a litre of infective bean's sap is stated to be only $0.001 \mathrm{~g}$. ; the yield of tobacco-mosaic virus proteins from infective tobacco plant sap appears to be the highest, but only $2.0 \mathrm{~g}$. (25).

Wildman and Bonner (60), on an electrophoretic study of plant virus proteins, found that a distinct fraction appeared in the sap following the infection of plants with tobacco-mosaic virus; the fraction was detectable after 4 days of infection, and after 20 days became very conspicuously. If the expulsion of lipids took place in the protoplasm proteins, there would appear a peculiar fraction detectable in the electrophoretic pattern, and therefore if the lipids expulsion failed to occur, the detection of virus formation would not be accomplished by this method. Actually in the sap of Turkish tobacco infected with curly-top virus no distinct fraction was demonstrated in the pattern; it should be realized that this virus has not ever been isolated in a crystalline form, indicating that lipid-expulsion cannot occur with this virus.

Insect viruses causing polyhedrosis can be isolated on the one hand, as described in a previous chapter, in rods similar to those of some plant viruses, whereas on the other hand, it has been reported that there 
are remarkable differences between the virus isolated from polyhedral bodies and that from the blood. For instance, polyhedrosis virus having a rod-shape isolated by Bergold (33) from polyhedral bodies was sedimented completely by ultracentrifugation at 10,000 r.p.m. and its "molecular weight" was calculated to be $916 \times 10^{6}$, while the virus particles isolated by Glaser and Stanley (61) from the infected insect blood was not sedimented at 10,000 r.p.m. but only at 27,000 r.p.m. and its "molecular weight" was estimated to be about $3,000,000$. Moreover, the virus from the blond was readily inactivated in solutions of more acid than $\mathrm{pH} 5$, whilst rod-shaped virus from polyhedral bodies was so stable as to stand unchanged for 24 hours at $\mathrm{pH} 2.0$; and again no rod-shaped particles similar to the virus was found in normal insects, whilst globular particles similar to those of the virus isolated from the blood were obtained from normal ones (62). These facts strongly suggest that the particles from the blood are nothing but the coagulated elementary bodies containing lipids, just as in usual animal viruses.

The expulsion of lipids appears to occur not only immediately after or during the change of the protoplasm protein by virus infection, but sometimes may occur gradually even after the change. The particles present in plant saps infected with tobacco-mosaic virus were separated into various fractions by Bawden and Pirie (63). The most rapidly sedimenting fractions consisted mostly of rod-shaped nucleoprotein, whilst only about a half of the total material in the most slowly sedimenting fraction was virus nucleoproteins; these fractions showed no anisotropy of flow and had low infectivity. Electron micrographs of the most slowly sedimenting fractions showed particles most of which are little longer than they were wide, while with increasing sedimentation rate the numbers of obvious rods increased. All the fractions were unstable and rapidly passed into forms that sedimented rapidly, showed intense anisotropy of flow, the change being most striking in the preparations that previously sedimented most slowly. Electron micrographs showed that these changes were accompanied by an increase in the number of rods and in their average length.

This fact can be interpreted as the gradual occurrence of lipid expulsion in vitro. Since the formation of rod-like particles never means the generation of viruses, it is only natural that no increase in the infectivity was observed despite the increase in the number of rods.

Bawden (26) cited another interesting fact: When concentrated salt-free solutions of a tobacco-necrosis virus are kept at $0^{\circ} \mathrm{C}$, crystallization occurs and thick triclinic prisms and the hexagonal, or pseudo- 
hexagonal, plates are produced. These begin to form after a few days, but growth continues for months and will give rise to single crystals several milimeters long. In this case the preparations lost most of their infectivity by the time crystallisation was completed, indicating the occurrence of some change in the protein structure, during the lipid expulsion, unfavourable for the virus activity. As previously discussed, the expulsion of lipids may be caused by changes of the protein structure generally unfavorable for the virus activity, though the virus may become more stable after the expulsion. In the above example of tobacco-mosaic virus, it has also been stated that the formation of rods was never followed by the in. crease in infectivity, but on the contrary by the reduction by about one-half.

The presence of an intimate correlation between the viruses and such rods or crystals could be demonstrated by the fact that both rods and crystals had the serological property specific to the respective virus. In general, the serological property of a virus is more stable than the infectivity, so that in the case above cited only the infectitivity seems to be lost, the serological property being left infact.

In the writer's opinion (22), virus particles having the property of the coagulated elementary bodies, if the coagulation is complete, fail ${ }^{\circ}$ to show the agglutinability by their antibody, because of the disappearance of the active polar groups on the folding of the protein molecules through the coagulation. However, if the protein molecules are unfolded and expanded, the reactive groups will reappear with the recovery of the agglutinability. Such an unfolding results from the expulsion of lipids, and as a consequence the rod formation is accompanied by the appearance of the agglutinability, whereas since serological property of plant viruses is usually recognized by the presence of the agglutinability, the appearance of the property is occasionally thought even to be the production of the virus protein itself, thus making the matter fall into the most confusion. It is stated that the formation of rods, in the above cited case of tomato-mosaic virus preparations, was actually accompanied by the revelation of the serological property.

\section{The Size of Virus Particles as Coagulated Elementary Bodies}

Among the virus particles isolated by our method those of phage are found to be the smallest and the dry particles are estimated to 
have the diameter of approximately $0.1 \mu$; normal bacterial particles are also of this size.

Particles obtained from various normal and infected plant material appears in the main to be of the same order of size, although their sizes seem far more inhomogeneous than those of phage, whilst animal particles are generally larger and their diameters appear mostly of the order of $0.2 \mu$. Vaccinia virus recoverable from skin tissues is found to be the largest, its diameter being estimated to be about $0.3 \mu(7)$.

From these facts the writer has formed the opinion that the size of the coagulated elementary body, corresponding to the plant-virus rods having the length of $0.3 \mu$, may be about $0.1 \mu$, in diameter. The diameter of various phage particles in electron micrographs haye been reported by a number of workers to be little less than $0.1 \mu$. This may be the size of a single elementary body fully coagulated in the dry state. Thus, an elementary body may be considered to undergo a considerable shrinkage, thereby being reduced in length to about one third to become a body with a diameter of the order of $0.1 \mu$, although in the expanded state it is about $0.3 \mu$ in length.

If the property of elementary bodies varied according to the kind of cells, some tending to combine end-to-end with every two bodies while the others with every three bodies, and if side-by-side combination corresponding to the end-to-end combination occurred on their coagulation into cuboidal bodies, then the diameters of the coagulated bodies would be about $0.2 \mu$ and $0.3 \mu$, respectively; but when side-byside combination failed to occur in response to the end-to-end associa tion, various intermediately sized particles would result.

Molluscum contagiosum virus particles have been found by Rake and Black (64) to be present plentifully in the inclusion bodies; under the electron microscope they proved to be brick shaped and $389 \times 272 \mathrm{~m} \mu$ in size, consisting of unit particles of $100 \times 83 \mathrm{~m} \mu$. According to the writer's view these unit particles must be the coagulated elementary bodies. Many other examples can be cited to show that viruses of large particles appear to be composed of smaller units like this virus.

If such a view is legitimate, particles smaller than $0.1 \mu$ must be the decomposition products of the unit particle. When coagulation occurred after the elementary body had been disintegrated into elementary bundles which could in turn undergo further decomposition, various extremely small particles would be yielded.

As already pointed out, phage particles isolated by our method are not of a uniform size and when the particles are the smaller, they are the more unstable and at the same time the activity is the lesser; this would only be a natural result if the virus activity arose 
from the polymerization of the protein molecules. Rothen (65) studied the influence of $\alpha$-particles on the antigenic property of bovine albumin and found that the albumin monolayer, when subjected to bombardment of $\alpha$-particles from a polonium source, completely lost in less than 30 minutes its property of combining with homologous antibody, while with a double layer 45 minutes were needed, and with three double layers or six monolayers even 135 minutes were not sufficient to inactivate the property. 


\section{CHAPTER VI}

\section{THE SHAPE OF VIRUS PARTICLES}

\section{Tailed and Filamentous Virus Particles}

It is a well known fact that some phage has a tail like a tadpole. If an elementary bundle in an elementary body failed to shrink, while the other remaining bundles coagulated into a body, a virus particle having a tail would be produced. The inability of shrinking of a certain elementary bundle may be attributed to its peculiar chemical composition; if such a peculiar composition occurred in a certain bundle of the body following the infection with some strain of the virus, each virus particle produced would have a tail.

Since the structural pattern induced in the protoplasm-protein by a virus is governed by the kind of virus, it should naturally occur that phages of some kind have a tail while others not. Not only the production of a tail but also the degree of the shrinkage or that of the decomposition or of the association of elementary bodies should more or less be influenced by the pattern of the virus, so that particle size would be subjected to a certain extent to the kind of viruses even when the cells affected are of a similar type, although the size is chiefly determined, as already stated, by the cells from which the virus is produced. According to our study (66), the particles of vaccinia virus recovered from infected rabbit testicles are a little smaller than the normal particles from noninfected testicles.

Glaser and Wyckoff (62) stated that normal particles from silkworms are rather more inhomogeneous in the sedimentation rate than the virus particles isolated from the worms infected with polyhedrosis. This may indicate that the elementary bodies of the silk-worm, when affected by the virus, tend to decompose more extensively than the normal bodies. Isolation of tailed particles from normal bacteria seems to have never been reported. This may show that the occurrence of the peculiar structural change causing inability of shrinking in a certain elementary bundle leading to the tail prodiction cannot be induced without certain phages.

It has been reported that in the presence of proflavine bacterial cells infected with phage underwent lysis without producing active phage particles (67). The bacteria which usually produced tailed particles would yield, in the presence of proflavine, lysates consisting 
mostly of tailless elements though often a small number of tailed particles were contained. The tailless particles would not be adsorbed by phage sensitive bacteria. This shows that in these particles the virus pattern was either absent or incomplete. The presence of tail may indicate the existence of complete virus pattern. An evidence was shown to suggest that nucleic acid content, if any, in these tailless incomplete particles was much lower than that of complete active particles. The tail formation may thus be attributed to high nucleic acid content which may render some elementary bundle unable to shrink.

As is well known, tailed particles are never confined to phage. For example, Newcastle disease viruses sometimes prove to have a tail, though more occasionally they are found in filamentous forms, which are believed by the writer to be elementary bundles or elementary bodies in an unfolded state. If some elementary bundle was left in this unfolded state, whilst the others formed a coagulated body on folding or shrinking, a tail would be produced as in the case of phage. According to Bang (68) Newcastle disease virus becomes filamentous when brought into hypertonic solutions of sodium chloride, whilst becomes spherical in water or 0.8 per cent salt solution. It is worthy of note that this change is reversible; no loss of infectivity is detected during the change.

Poliomyelitis virus may present another example: Bourdillon (69) claimed that electron micrographs of this virus show filaments of $15 \mathrm{~m} \mu$ wide, purified preparations readily agglutinating lineally and showing anisotropy of flow. In these and other respects they resemble closely some plant virus preparations, though filaments of animal viruses seem far more tender than those of plant viruses, a fact which may chiefly be attributed to the presence of lipids. There are also many reports informing of the filamentous forms of influenza virus. Avian erythromyeloblastic leucosis virus is likewise stated to be sometimes tailed particles (70). Studies of dengue fever virus by electron mircoscope has shown that the virus is thread-like particles, 42 to $46 \mathrm{~m} \mu$ in width and 175 to $220 \mathrm{~m} \mu$ in length (71).

Filaments of viruses such as those of Newcastle disease and polyomyelitis may be the unfolded elementary bundles or their end-toend association products, since their width is reported to be similar to that of tobacco-mosaic virus, whereas, in some cases at least, this does not seem to hold for influenza virus, because electron micrographs of influenza virus show that the filaments are far thicker in width than those of the former viruses, seggesting that the filaments of influenza virus are the elementary bodies themselves coagulated in forming a thread by end-to-end association. Actually many workers 
believe that the filaments can undergo segmentation into virus particles (72) (73).

If protoplasm has a structure just as the writer postulated, it will only naturally follow that viruses have such various forms. Although a phage is customarily regarded as having a definite size and shape, a variety of irregular and incomplete forms was shown in the untreated bacterial cultures lysed by a phage, and even filaments were found in a culture obtained at a low temperature (74). Observations made by Chapman (75) in electron micrographs of a certain strain of phage revealed various shapes of phage or phage-like particles. including not only the familiar type of a body with a single tail, but also dumbbell-shaped particles with or without tails, or various shaped bodies with one or more tails at each end, as well as the filaments or rod-shaped particles.

Hoyle (76), in his study of chorio-allantoic membrane of chick embryo infected with influenza virus by means of dark-field optical microscopy, suggested that elementary infectious units of influenza may be cellular fragments. On the other hand, Wyckoff (77) showed in electron micrographs of the same materials similarly extruded and detached bits of cytoplasm mainly consisting of filaments which in turn tends to segregate into spheres. These views and observations are of course consistent with the writer's findings and conceptions.

If host cells are not injured and broken up following virus infection, but can remain in their unchanged, complete forms, then the coagulated elementary bodies, which may be able to act as separate virus particles if freed from the cells, must exist in the protoplasm in a regular arrangement, because the protoplasm consists of elementary bodies so arranged. It has actually been found in electron micrographs that virus or virus-like particles are arranged regularly in a crystal pattern in bacterial or some plant cells affected by respective viruses (78). In a similar way Straus (79) showed that virus-like particles in a equally regular arrangement are present in protoplasm of papilloma cells.

Particles of various shapes are found also in normal cells without concerning any virus infection (80), indicating that protoplasm protein, in general, has the property to change its shape in various ways.

Wyckoff (81), in his study by electron micrographs on the formation of the particles of influenza virus, has confirmed that there is no essential morphological difference between particles arising from "healthy" cells and virus particles coming from cells infected with influenza. They both resemble bits of cellular cytoplasm rather than extraneous objects growing and multiplying at the expense of the cell. He stated that, if influenza virus particles are fragments of a 
diseased cell, as electron micrographs seem to suggest, it becomes hard to view them as organized invaders which multiplied within and at the expense of the diseased cell.

Besides myosin, a second protein, called actin, is extracted from muscles; this protein exists in a water solution in globular forms but salt in high concentrations converts the particulate actin into a fibrous form, which in turn is reverted to the globular form by removing the salt by dialysis (82). Entirely the same phenomenon is observed with Newcastle disease virus as stated above. Likewise with insulin an analogous finding has been reported; namely, this protein can be converted into filaments with a width about $15 \mathrm{~m} / \mathrm{like}$ tobacco-mosaic virus, and this filaments again can aggregate into globular forms (83). Lundgren (84) considered that filaments are produced by the unfolding of globular proteins and the aligning of polypeptide chains. In addition, electron micrographs of myosin, actomyosin, tropomyosin, and fibrin show that all these proteins have the common property to give fibrils of varying length and thickness (85).

The concept of the writer described thus far as regards the shape and size of viruses is diagrammatically shown in Fig. 11.

The ability of globular proteins to spread in the form of fibrils or filaments is also fairly shown in the readiness with which proteins spread and form the so-called surface films; the spreading is especially easily done in concentrated salt solutions and the film can be compressed immediately after spreading and occupy the same area at the point of minimum compressibility. Again, a change of potein called denaturation is thought generally as an unfolding of globular proteins into polypeptide chains. For example, it is reported that ovalbumin when denaturated in the presence of urea becomes 500 to $700 \mathrm{~A}$ in length (86).

An adequate system may be provided in the protoplasm for the readily occurrence of such a reversible change, $i$. $e$. stretching and shrinking, of the proteins. The majority of workers seem to pay attention mainly to the elementary bodies in a stretching state, forming the opinion that the protoplasm consists of fibrils, whilst others may attach importance to the elementary bodies in a coagulated state, advancing the globular theories of protoplasm. Usually viruses are in a coagulated state, but under certain environmental conditions they may stretch themselves to form filaments.

Sharp (87) has made studies on the "purified" virus of avian erythro-myeloblastic leucosis under varying conditions of electron micrography, and found that when preparations of the virus were allowed to dry in the presence of salt, the particles exhibited extreme pleomorphism, varying from filaments to sperm shapes, while the 
particles of the virus dried without salt were uniformly spheroidal, though variable in size, the average diameter being about $120 \mathrm{~m} \mu$. On this finding he suggested that the true form of the virus is spheroidal, the others being artefacts. Similar assumptions have been postulated
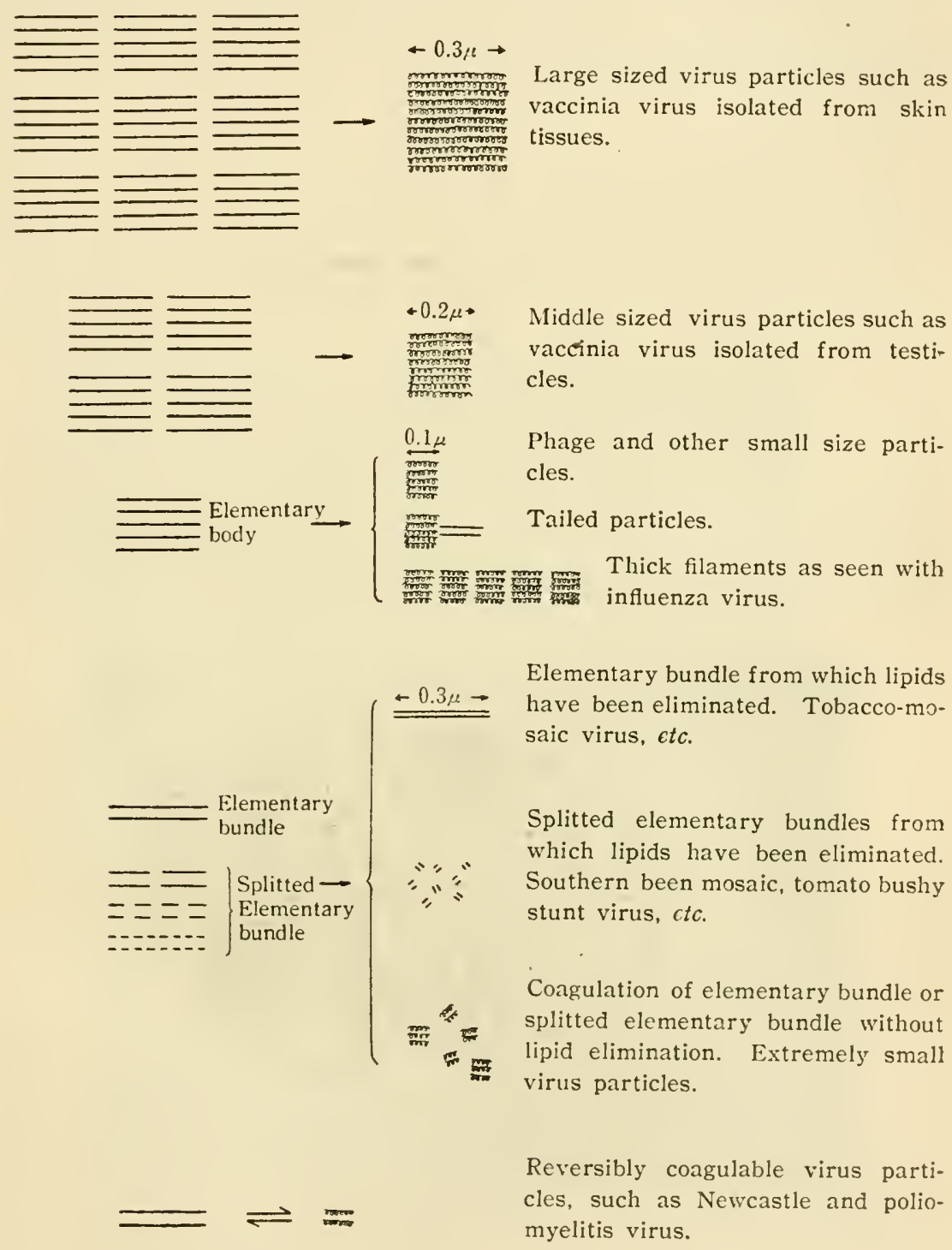

Fig. 11. Diagram of various shapes and sizes of virus particles. 
also by a number of other workers with other viruses, but such views would of course be improper. On the contrary, Chu et al. (88) considered the filamentous forms of influenza virus as a stage in the multiplication of the virus, the spheroid possibly representing the mature "resting" virus, which lengthens in the host cell to a filamentous entity and fragments transversely to form the spheroid again, a view which may also be unreasonable.

\section{Particles Elusive in Electron Micrography}

Angulo and his collaborators (89) examined various materials from a patient with tropical treponematosis, a non-viral disease, with electron microscope and found particles of filamentous forms resembling the virus of influenza and Newcastle disease as well as various globular paticles like those of influenza, poliomeylitis or pox group. Moreover, Murphy and Bang (90) have shown in electron micrographs that in a normal chick chorio-allantoic membrane cell "normal" filaments are seen everywhere. They demonstrated how much normal filaments resemble those of influenza. If the nature of virus particles is actually as hitherto discussed, such findings will be only natural. Nevertheless, rather strange to say, it seems commonly believed that particles peculiar to viruses are proved only with virus-infected tissues, the

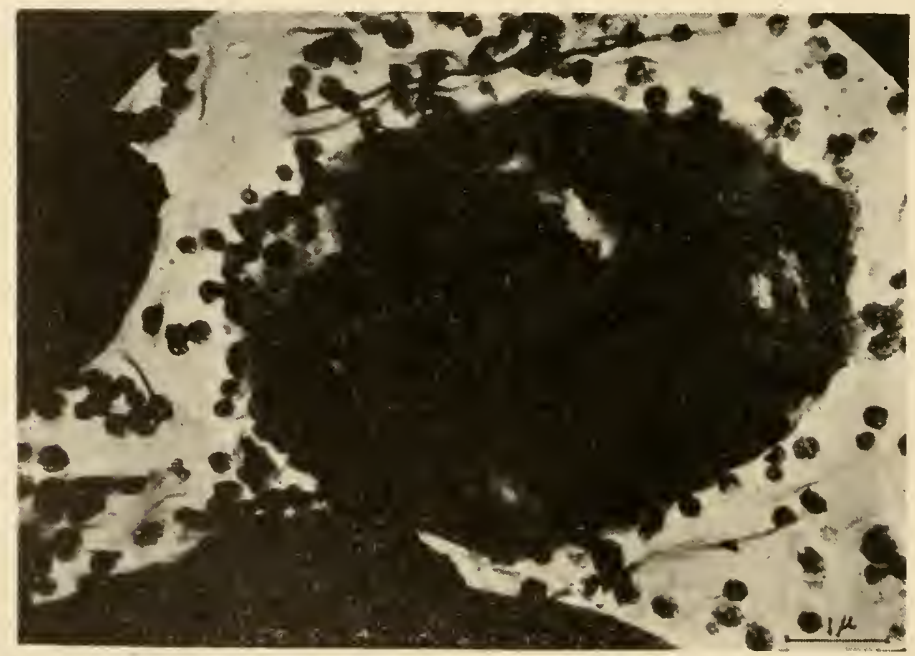

Fig. 12. Virus-like particles in a red blood cell. Blood cell is photographed in Ringer's solution. Some particles appear to have a tail like a tadpoie. 
main reason for which is descussed in this section. The reason for the production of virus particles having peculiar shape and size corresponding to a given virus was considered already.

According to our dark-field microscopic observation, the protoplasm of red blood cells also consists of virus-like particles, and whose diameter was estimated to be about $0.2 \mu$ (91). These particles, however, fail to reveal their true features in electron micrographs unless examined under proper conditions. The writer had the fortunate opportunity to investigate a great number of electron micrographs of red blood cells taken by members of the physiological laboratory in Yokohama Medical College and was able to confirm that the particles were revealed if Ringer's solution was used as indicated in Fig. 12. But when physiological saline solution was used no particles were shown in the micrographs as indicated in Fig. 13. Even when Ringer's solu-

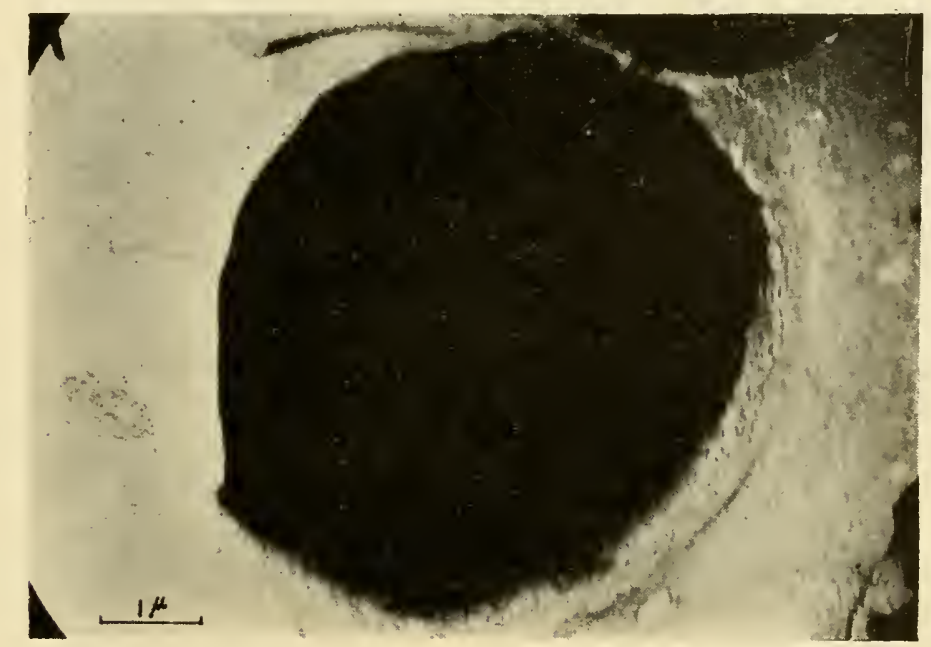

Fig. 13. A red blood cell photographed in physiological saline. No virus-like particles are seen.

tion was used, if a little deleterious manipulation was added to the cells, the particles became much smaller as shown in Fig. 14.

We noticed that samples of a certain unstable phage were occasionally severely inactivated during the isolating procedure by our method when physiological saline solution or distilled water was used, while this was prevented by Ringer's solution (91). This may be attributed to the divalent cations chiefly those of calcium present in Ringer's solution. A.dams (92) stated that the addition of $10^{-5} \mathrm{M}$. concentration of divalent cations, such as $\mathrm{Ca}$ or $\mathrm{Mg}$, markedly reduces the rate of 
inactivation of a certain strain of phage. Moreover, according to Sharp et al. (93) the equine encephalomyelitis virus, dried from suspensions of low salt content or washed on the film with water, appears in

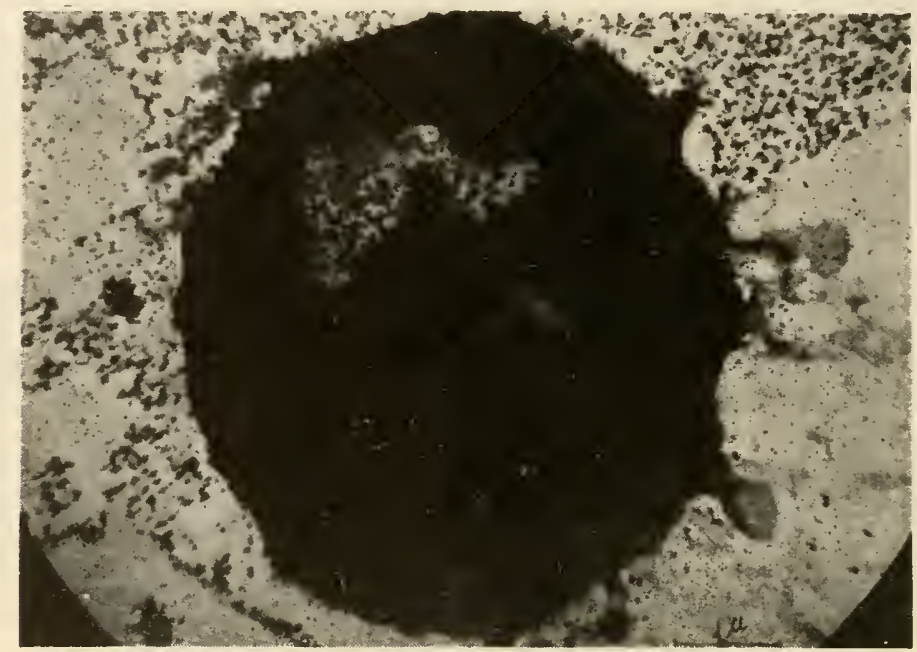

Fig. 14. Virus-like particles of extremely small size in a red blood cell. Partially hemolysed cell by hypotonic saline solution is photographed in Ringer's solution.

electron micrographs as round images with low contrast, whilst the contrast increases greatly in the presence of calcium ions. Also with images of influenza virus, it was noted that the periphery becomes well defined in the presence of small amounts of calcium.

Thus it is thought that elementary bodies, whether they have a virus action or not, are liable to be disintegrated or become elusive in the electron micrographs, a tendency which can be prevented by the presence of divalent cations. The divalent ions in these cases may stabilize the polymerization of proteins, thereby viruses can be saved from inactivation.

* However, if it is true that only virus particles can be caught in the micrographs, whilst normal particles cannot, then it would be attributed to some elementary bodies which have become stable enough by virus infection to stand the manipulation of the micrography because of an alteration in the chemical structure due to the virus. Usually cells may multiply more rapidly when affected by a virus, and since the enhancement in the multiplicability appears to be accompanied by the increase in the nucleic acid content in the cell, some elementary bodies would come to contain more nucleic acid following virus infection. Presumably this is the main reason for which "virus particles" 
are commonly caught well in micrographs.

On the one hand, it seems generally accepted that nucleic acid contents are increased when protein synthesis is to develop vigorously for the rapid multiplication of the cells; on the other hand, there is a good reason to consider, as will be detailed in a later chapter, that nucleic acids are capable of stabilizing the structure of protein polymers and thereby the character of elementary bodies for acting as a virus is intensified. The production of a tail in the phage particle may also be attributed to the high content of nucleic acid, which may cause some proteins to be left in a stretched form.

As already mentioned, virus-like particles are actually absent in the normal skin tissue, although virus particles may develop in it on the infection with a certain virus. Virus-like particles can be produced, however, in the skin tissue when a proper stimulus other than viruses is applied. Therefore, even when particles never present in rormal tissues are found in some pathological tissues, it cannot be said that the particles have a definite connection with viruses. The success of Angulo et al. referred to above, in demonstrating various virus-like particles may be attributed to their investigation with cells undergoing a change by the infection with the microorganism which may develop a change like that induced by a virus.

Epestein ct al. (94) have reported that between 24 and 72 hours after inoculation of the virus of foot-and-mouth disease into guinea pigs, within which interval generalization of the disease reached its height and the red blood cells were found infective for normal guinea pigs, particles with mean diameter of $246 \mathrm{~m} \mu$ were found in the red cells by the electron microscope. They claimed that the particles were absent in normal control material. However, their particles appear to be similar in size to those indicated in Fig. 12. If there was no mistake in their demonstration of the particles solely during certain periods after the infection, it should be interpreted as that the particles become during the periods stable enough to reveal their complete figures under the condition of their examination.

According to electron microscopic studies of Wyckoff (2) the protoplasm of young actively growing cells of $E$. coli does not contain filaments or particles but old cells commonly have the spherical macromolecules embedded in a matrix of filaments. There are many good reasons to believe that the structure of proteins becomes, as a rule, more stable and rigid as they grow older, a full account of which will be given later in Part $\mathrm{V}$.

Under the electron microscope, materials to be examined must be brought into an unusual dried state because of the vacuum, and at the same time must be exposed to a high temperature by the bombard- 
ment of electrons, so that particles have to stand this hardship to show their true figure without being decomposed.

If the particles are disintegrated to fragments of a size beyond the resolving power of the electron microscope, nothing will be photographed as in the case of blood cells shown in Fig. 13. However, if fragments remain within the limit of resolution, their demonstration under the electron microscope may be possible as indicated in Fig. 14. In such a case virus particles may be thought to be extremely small. Furthermore, it may be possible that the particles present in the cell surface are sometimes fused into a homogeneous membrane-like mass, wherefore the so-called cell-membrane may be photographed (95). Some workers claimed that even virus particles occasionally show a membrane-like structure on the surface, which may likewise be a dissolution product of protein molecules present on the surface. 


\section{CHAPTER VII}

\section{THE MODE OF VIRUS MULTIPLICATION}

\section{Multiplication of Phage within Bacterial Cell Protoplasm}

Many facts above cited have provided the basis on which the writer has formed the theory as regards the structure of protoplasm; meanwhile it may be said that these facts are supporting the theory strongly, since they can be explained finely by the theory. On the other hand, a vast number of facts have also been known concerning the multiplication of viruses. Now, it must be mentioned how these facts are to be interpreted by the writer's theory.

Phage seems to be the virus with which the most profound and most numerous investigations have ever been made, whereas some conclusions reached as to its multiplication by a number of workers appear to be inconsistent with what is expected from the theory above mentioned.

According to the writer's opinion, viruses are produced by the rearrangement of polar groups in protoplasm protein, and therefore it must be impossible that viruses are synthesized directly without concerning protoplasm protein. Nevertheless, some workers reached a remarkable conclusion that phage could be formed directly from components in the culture medium. For instance, a series of workers in this field, such as Cohen and Putnam (96) (97), concluded that the majority of elements in phage, such as phosphorus, were directly originated from those contained in the culture medium. According to Kozloff (98) about 75 to 95 per cent of phage protein nitrogen came from the medium after infection, while remaining 5 to 25 per cent were provided by bacteria. The writer is convinced, however, that these conclusions are entirely incorrect; the reasons are given in the following.

There are many evidences that only young bacteria are affected by phage, aged bacteria being commonly insusceptible, and thus phage can multiply, as a rule, only in young cells. First, the erroneous conclusions might be reached because of neglecting of this fact; namely since phage was produced by young growing bacteria which were rapidly synthesizing the protoplasm from the components in the culture media, the phage would be mistaken as being formed directly 
from the components.

The property of preferably affecting young hosts is not confined to phage, but it seems common with other viruses. For example, as is well known, rapidly developing plants are liable to be readily infected by virus diseases, while aged leaves having ceased growing are seldom infected. The fact that the chick embryo is used for cultivation of a great number of viruses may be mainly due to its juvenile character, for as Woodruff and Goodpasture (99) have suggested, although many viruses multiply in the chick embryo, they fail to grow in the adult chicken.

In order to obtain effectively large amounts of phage, we made a small number of bacteria multiply in the culture medium with a proper amount of phage. During the multiplication of bacteria in the presence of phage, newly produced protoplasm are imparted with the structure of phage. If phage is added to the culture of bacteria which have already ceased to grow, usually no phage develops. Again, if bacteria are spread with a small number of phage particles on agar plate, a number of minute round areas, in which no bacterial growth is occurring are produced, which correspond to each particle present at first. The phage present at first would multiply by affecting and lysing the surrounding bacteria to which it is attached and the newly produced phage in turn would affect the surrounding bacteria in a chain reaction to increase the clearing area. The area or plaque rapidly increases in size when bacteria are multiplying, but when their growth stops the increase also comes to a halt, indicating that growing cells only are affected and lysed by phage. When bacteria cease growing and become aged, the phage fails to affect and to lyse them, so that no further increase occurs in plaque size (100).

Fong (101) claimed that the phage-producing ability of streptococci is activated when the cocci are cultivated in a favourable environment for a rapid growth. Such activated cells, preserved at $5^{\circ} \mathrm{C}$. in Lock's solution, are able to produce phage abundantly and rapidly when affected by phage, but this phage-producing capacity is quickly lost when the cells are suspended in distilled water and are exposed to $44^{\circ} \mathrm{C}$. for 15 minutes. The activated state in this example must be the "young" state, and this state can be kept unchanged at the low temperature in Lock's solution, but the cells may grow "old" rapidly when exposed to $44^{\circ} \mathrm{C}$. in distilled water. Kopper (102) has actually found certain outstanding difference between old and young cells of colon bacteria. According to him, the transformation of old into young cells takes place during the lag phase of growth and necessarily involves a great number of physiological changes. The rejuvenating effect was most pronounced with glucose, but also was noted with a 
number of salts of carboxylic acids, peptone, and culture broths. Mixtures of equal parts of $\mathrm{NaCl}$ and $\mathrm{KCL}$ and low concentrations of $\mathrm{Na}$ azide and 2.4-dinitrophenol, respectively, were found to exert a pronounced inhibitory effect in this process (103).

Thus young growing cells seem to have peculiar protoplasm structure which is fitted for phage production, and accordingly if phage was present in bacterial cultures growing rapidly, as soon as the protoplasm proteins were synthesized from the components in the culture medium, the newly formed proteins would be changed into the phage proteins by the rearrangement of the polar groups, so that the components would seem to be converted directly into phage.

On the other hand, it is believed that bacteria are deprived of their viability by the infection with phage. If this were true, the components in the medium would have failed to enter the bacterial cells after the infection, and hence phage itself would have to take the components if these were found in the phage. However, this assumption is not correct, because the loss of the viability occurs only when bacteria are affected by a phage on an agar plate. In broth culture it seems not to take place at least in a manner so striking as on plate and moreover even on an agar plate only a peculiar phage can reveal the growth-inhibiting property, which is therefore not essential for phage. The phage particles isolated and purified by our method always fail to develop the growth-inhibiting property even when it can exhibit the property before the purification. This interesting fact is discussed in detail in the other book (22).

As described in Chapter VI in Part I, the activity of phage varies with the conditions under which the host bacteria are cultivated. The viability and the degree of lysis of bacteria after the infection by phage vary in a similar way. Thus, bacteria may lose the viability when infected with some phage on an agar plate as just mentioned, while this is not the case in a broth culture. In additon, on an agar plate bacteria affected by phage usually undergo a complete lysis while in a broth this does not necessarily follow. For example, according to our observation a certain strain of $E$. coli fails to undergo lysis in broth by the infection with a strain of phage. The difference between agar and broth culture may be ascribed to the degree of contact with air during the phage infection, since in the case of broth culture the greater the surface area of the culture, phage multiplies the more rapidly and abundantly (91). The plaque-formation would never result if the lysis and the growth inhibition failed to occur on an agar plate.

The fact that phage in broth may not only fail to inhibit the growth of bacteria but occasionally even can promote it before the 
occurrence of lysis is shown in Fig. 15 (104). As already mentioned, host cells usually multiply more rapidly on the infection with a virus; also in this respect phage presents no exception. However, it must be emphasized that a certain phage, which can promote in broth the bacterial growth in such a manner, can render the bacteria nonviable on agar plate.

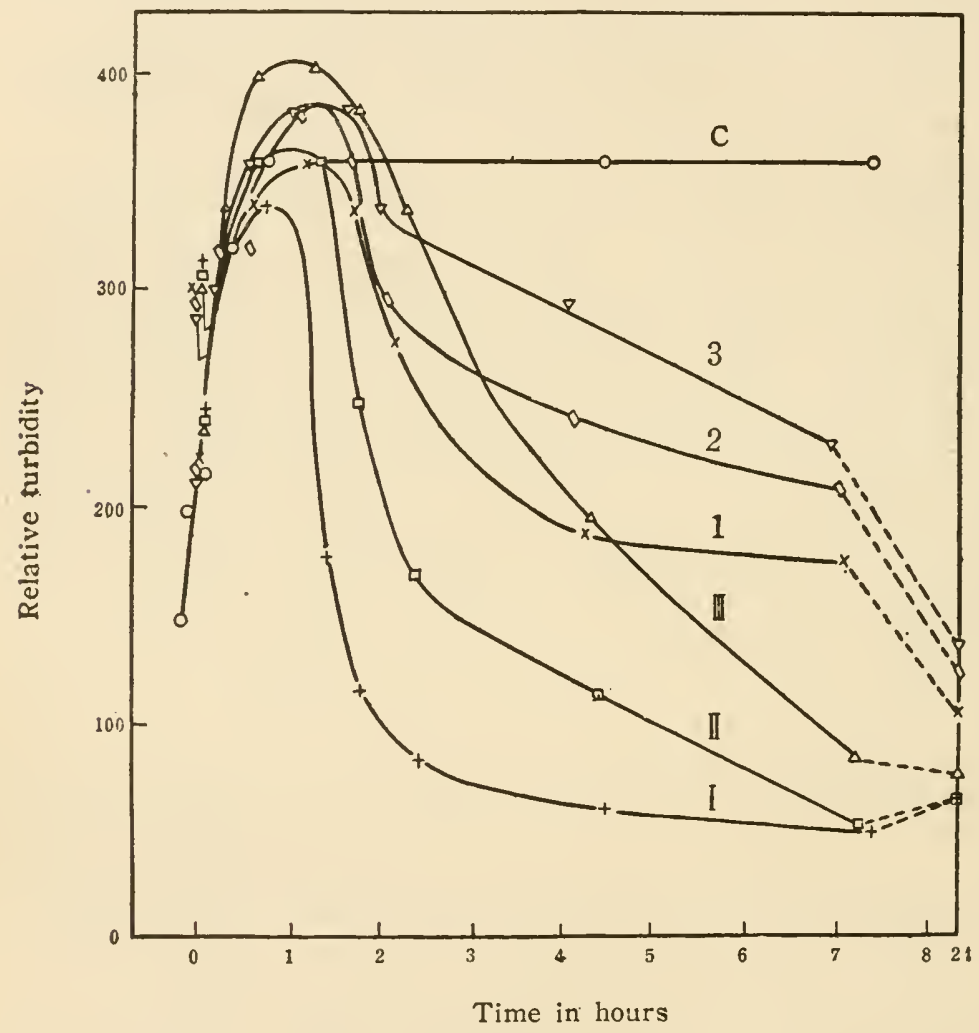

Fig. 15. Clearing of the bacterial suspension by phages of both large and small plaques.

Strain: B. dysenteriae (KA 1).

The broth-suspension of the bacteria (number of the viable bacteria: $1 \times$ $10^{7} / \mathrm{cc}$.), to which the plage filtrate has been added, is placed in a water bath at $37^{\circ} \mathrm{C}$. and the clearing degrees are observed nephelometrically with Pulfrich-photometer.

I, II and III are the suspensions to which the large plaque phage (average plaque diameter $; 2,6 \mathrm{~mm}$.) has been added so that the concentrations of the active particles become $I \times 10^{7}, 1 \times 10^{6}$ and $1 \times 10^{5} / \mathrm{cc}$. I, 2 and 3 are the suspensions to which the small plaque phage (average plaque diameter; $0,5 \mathrm{~mm}$.) has been added, particle concentrations being equal to I, II, and III respectively. $\mathrm{C}$ is the control suspension to which no phage has been added. 
Usually the viability of bacteria is examined by plating on agar and the bacteria incapable of forming colonies are regarded as having no viability or being dead. This might be another main reason for which the erroneous conclusion was reached. Cohen attributed the rapid increase in the amount of both protein nitrogen and nucleic acid in the bacterial culture infected with phage to the multiplication of phage itself, but this may show nothing but the rapid growth of bacteria after the infection.

Kozloff $e t a l$. in the above cited chemical studies of virus reproduction, stated that the percentage of the phage protein nitrogen derived from the bacteria was inversely proportional to the time of incubation and to the number of virus particles produced per bacterial cell. This may only be a natural result, since the prolonged period of incubation and increased number of virus particles per bacterial cell should be accompanied by the increased number of newly developed bacterial cells. They appeared to take into consideration only the bacteria which had been present at the beginning to reach the erroneous conclusion.

Such unreasonable conclusions seem, however, to be reached only with phage. A study on the uptake of radioactive phosphorus by influenza virus by Graham and McClelland (105) has shown that there is no direct exchange between the virus and phosphorus. It has been known for a long time that the increase of a plant virus is accompanied by a decrease in normal protein. For example, Wildman et al (106) made electrophoretic analysis of cytoplasmic protein solutions isolated from tobacco plants at different intervals after inoculation with tobacco-mosaic virus, and found that a predominant normal portion decreased as the virus protein increased by approximately the same amounts, suggesting that the virus protein is synthesized at the direct expense of the main protein component.

In an experiment with $\mathrm{P}^{32}$-labeled host bacteria, Putnam et al (107) have reported that from 60 to 90 per cent of the phage $P$ is derived from bacterial nucleic acid, and concluded that bacterial nucleic acid is the chief precursor of phage nucleic acid. It is remarkable that even with phage and bacteria such rather reasonable conclusion was reached, although the small portion of $\mathrm{P}$ had yet to be ascribed to that contained in the culture media.

According to Stent and Maaloe (108) on the average 58 per cent of the phosphorus is host derived in the first 15 per cent of the phage particles formed, while the average over the total yield indicates only 30 per cent of the phage phosphorus to the host derived. This result again suggests the multiplication of the bacteria after the infection. Labaw (109) found that the amount of bacteriophage phosphorus 
apparently derived from the medium after the infection varies from nearly zero to twice that originally in the bacterial host, depending on the strain of bacteriophage, that is, the bacterial phosphorus comprises on the average from nealy 100 per cent to about 30 per cent of the total phosphorus in the bacteriophage. It should be emphasized that this difference was attributed to the strain of phage. If a certain strain of phage could render the bacteria utterly non-viable after the infection, 100 per cent of the phage phosphorus would appear to result from the bacterial phosphorus.

Even if a small portion of phosphorus did appear to come from the medium in an experiment carried out with some bacteria actually reduced non-viable, it would be impossible to conclude that the small portion of the phage nucleoprotein was directly synthesized from the materials present in the medium, because the exchange of the elements or groups between the medium and the cell is considered to be possible without the net synthesis of the protein (110). In the theory of the writer, for the multiplication of a virus, the rearrangement of polar groups in the protoplasm protein so as to correspond to the pattern of the virus is to be raised. This rearrangement should be transmitted in the protoplasm as a wave which may consist in the shift of polar groups, and thus a type of transpeptidation may take place. It is conceivable that the elements or groups in the medium surrounding the cell may come to be involved in such transpeptidation and may be found in the virus particles produced.

On studying the propagation of poliomyelitis in monkey testicular tissue, Scherer and Syverton (111) have concluded that the amount of virus in the tissue was as much as tenfold greater than that in the liquid of the same cultures, and that viral production was evidently earlier and was detected for a longer period of time in the tissue than in the liquid phase. This should only be a natural result if the virus is produced in the protoplasm; the virus is to be liberated in the fluid after the protoplasm is decomposed. According to a study by Ackermann and Kunitz (112) on the intracellular distribution of herpes virus in embryonic liver, a significant amount of the virus was found bound to the mitochondria by an intimate attachment and the concept was advanced that these particles function in the development of virus. However, since mictochondria are a sort of granules of protoplasm with a rigid structure, if the protoplasm is decomposed intc particles after being changed into virus structure, the virus activity should naturally be retained markedly in mitochondria. 


\section{The Difference in Nucleic Acid Content between Virus and Host Cell Protoplasm}

There should be, and actually is, an intimate relation between virus and its host cell protoplasm in chemical compositions, but, on the other hand, there are claims that considerable differences are present between them. The differences are believed to be found in the main in both the kind and content of nucleic acids.

According to the chemical analysis of a strain of phage of $E$. coli by Csaky et al. (113) the virus consists of nucleic acids about 40 per cent, while the host bacteria only about 5 per cent. Such a striking difference may be mainly attributed to the isolation of peculiar particles with high nucleic acid content which are regarded as sole phage particles, because the particles with high nucleic acid content appear to sediment more rapidly than those containing a little or no nucleic acids. According to Markham and Smith (50) the purified preparation of turnip-yellow mosaic virus protein separates into two parts on the application of ultracentrifugation; the bottom component contains nucleic acid, while the top is nucleic acid free, although these two components have the same electrophoretic mobility and isoelectric point, and both crystallizing in the same crystalline forms.

Phosphorus content in a phage preparation isolated by our method was found to be 1.28 per cent, so that nucleic acid content in this preparation at most could not be greater than 13 per cent. This value is much lower as compared with that of phage particles isolated by means of ultracentrifuge by a number of workers. It must be borne in mind that all the particles present in the culture filtrate are to be isolated by our method, while this is not the case with the isolation by the ultracentrifugation. Again, the protoplasm, in general, contains a considerable amount of lipids, about one third of it being lipids. The phage particles obtained by our method likewise show a high content of lipids and also its one third was proved to be lipids, whereas lipid content of phage obtained by means of ultracentrifugation has been reported to be very low; according to Csaky et al. it was only about 1 per cent. The lower is the lipid content, the sedimentation rate may be the greater, and the reverse may hold true for nucleic acids.

Extremely small yields of virus particles by means of ultracentrifugation are also indicating that the particles isolated by this method are only a portion of the particles produced by the decomposition of the protoplasm on the infection. 
For example, the yield of phage by Csaky et al, from 1501 . of culture filtrate, was $60 \mathrm{mg}$, while by our method the same yield would be easily raised from only one 1 . Thus the yield by means of ultracentrifugations is of the order of one hundredth of that obtained by our method, and only particles containing nucleic acids in rich amount and lipids in poor are presumably separated as virus particles by the ultracentrifugation.

However, there is indeed a good reason for supposing that the particles with high content of nucleic acid and therefore those with high sedimentation rate are the virus particles with high activity, and thus when the others have no or little activity such particles only can reveal a high virus activity, the reason for which is discussed in detail in the next Chapter.

From what has been mentioned above it may be reasonable to conclude that the high content of nucleic acids in virus particles isolated by the ultracentrifugation is attributed to the separation of particles with high sedimentation rate. There is, however, another important reason for the high content of nucleic acids in virus particles. Gratia et al. (114), comparing the composition of the particles obtained by the ultracentrifugation from tissue extracts of the silk worm, infected with polyhedrosis virus, with those obtained from noninfected worms, found that particles isolated from healthy silk worms contained only ribonucleic acid, whereas the particles isolated from infected worms contained, in addition, desoxyribonucleic acid.

Moreover, it is generally recognized that bacteria infected with phage synthesize much greater desoxyribonucleic acid than do normal bacteria. Price (115) stated that normal staphylococci release neither ribonuclec acid nor desoxyribonucleic acid into the medium, whereas infected cells release both kinds of nucleic acids. Rafelson et al. (116) found that the uptake of $\mathrm{P}^{3}$ : by the minced, young mouse brain is markedly stimulated by the in vitro infection and growth of Theiler's virus in the minced tissue. They believed that the effect of the virus on the $\mathrm{P}^{32}$ uptake by the tissue may be explained by the increase of ribonucleic acid synthesis in the infective tissue. Saenz and Taylor (117) found a similar phenomenon with chick embryo ard influenza virus.

On the one hand cells infected with viruses are generally increased in their propagating power. Even in the case of bacteria affected by phage this is sometimes true as above stated. Thus, although bacterial cells are commonly subjected to lysis when affected by phage, a proper amount of phage is occasionally capable of promoting the proliferation of bacteria. On the other hand, as is generally accepted, rapid propagation of cells is generally accompanied by the increase in 
the nucleic acid content. It has been well established that nucleic acid concentration is much higher in rapidly growing embryonic tissue than in the corresponding adult tissue. The virus infection, therefore, should lead to the increase in the nucleic acid content of the protoplasm. If such protoplasm is coagulated into particles after being endowed with virus structure, virus particles containing nucleic acid in rich amount must be formed.

As already described, virus-like particles are never recovered from normal, untreated rabbit skin tissue, whereas when the tissue is injected with staphylococcus toxin or sapotoxin, the tissue becomes inflamed and swelled just as in the case of vaccinia virus injection, and particles containing nucleic acids like those of vaccinia virus are isolated in large quantities. If the apparent relation of nucleic acids to virus multiplication is merely due to the rapid growth of the cells there should be no change in the nucleic acid content when virus multiplication occurs in the cells which were preliminarily rendered nonviable. Actually, Price (115) claimed that when phage proliferates in the cells whose viability was lost on penicillin treatment, there occurs neither increase in the nucleic acid content nor change in the amount ratio of desoxyribonucleic to ribonucleic acid.

\section{The Mode of Production of Virus Structure}

As mentioned in Part $\mathrm{I}$, the relation between a virus and its host cell protoplasm is considered to be analogous to that between an enzyme and its substrate. A virus can combine with host cell protoplasm through the complementarily arranged polar groups distributed between them just as does an enzyme with it substrate.

To begin with, virus particles adsorbed to host protoplasm through such polar groups should unfold its coiled structure to show its full pattern. It is an established fact that globular protein molecules can readily spread in monomolecular films on the intermediate surface. The spreading of virus particle on the protoplam surface into a state corresponding to such a monomolecular film, may be caused by some physicochemical influence coming from the protoplasm, in this spreading certain amino acids or proteins with low molecular weights as well as inorganic salts may play an important role.

The unfolding of globular proteins including virus particles to a state of filaments is brought about, as already pointed out, by the presence of inorganic salts in proper concentrations. At the same time, there are a number of evidences that proteins with low molecular weights like albumin can exhibit the same effect upon the spreading 
(22). Thus, the solubility of horse serum globulin is not increased by the presence of pseudoglobulin, but increased by the addition of serum albumin (118). This may be interpreted as due to the unfolding of globulin molecules owing to the presence of albumin. In addition to albumin, various amino acids have shown to increase the solubility of euglobulin (119). As is well known, globulin is soluble in the presence of inorganic salts which as just mentioned have the spreading action. Serum globulin becomes sedimentable as the serum is dialyzed. In a similar way phage particles can be rendered easily precipitable by the dialysis of the culture filtrate.

The writer found that serum euglobulin, if isolated by our isoelectric precipitation method, exists in forming virus-like particles. According to the writer's opinion (22), the polymerization product of this serum euglobulin, if present in the spreading form, can act as complement in immunological reactions, but not so in the coagulated, virus-like particles, which the latter can be expanded on the addition of serum protein with an albumin nature termed end-piece or factor C'2, while the coagulated euglobulin particle is named mid-piece or factor C'1, so that on the addition of end-piece to mid-piece, $i$. $e$. the coagulated euglobulin particle, the complement activity may arise.

A well known virus-like factor of pneumococcus capable of transmitting the peculiar character of the coccus, from which it was separated, to another strain of coccus is needed for its function a component present in serum or serous fluids, which can be supplied by a fraction of bovine serum albumin (120).

As detailed already, protoplasm undergoes coagulation into minute particles on the application of stimulus. This coagulation spreads as a chain reaction in the protoplasm, but reversible in so far as the protoplasm remains "alive." The unfolding of such coagulated protoplasm may be possible, if certain low-molecular substances are present in the cell protoplasm for their unfolding.

Phage particles can be seen under the microscope by dark-ground illumination. However, if adsorbed onto bacterial surface they would become always invisible (9), probably due to the unfolding of the particles on the cell surface. In order to show the structure acting as the template, viruses should be required to unfold the peptide chains. If the physicochemical effect resulting from the spatial arrangement of polar groups revealed by the unfolding of a virus particle is stronger than that of the protoplasm, the spatial distribution of polar groups in the protoplasm may be changed by both the electrostatic repulsion and attraction so as to correspond to the pattern of virus. The change thus induced in the protoplasm structure must be the virus multiplication itself. 
On the contrary, if the protoplasm exhibits a stronger effect than the virus, the latter will be altered to become idential with the former in its structure and will be fused into it. In such a case the virus may be said to be assimilized by the cell, while in case of the occurrence of virus multiplication the protoplasm may be said to be assimilized by the virus.

In a study of a strain of phage adsorbed to a resistant bacterial mutant, Henry and Henry (121) found that the phage disappears without exerting any detectable effect on the host. Even in the same sample of virus particles, some virus particles may get the upper hand of the cells and can induce replica in them, while some other particles may fail to prevail over them because of their weak structure. The same may hold true for host cells, and some cells with high susceptibility may be assimilized by a virus, while others with less susceptibility may not be effected by the same virus.

If the polypeptide chains of protoplasm protein are in a stretched state and in a protein moleclue thus stretched, which is situated on the surface of the protoplasm, the rearrangement of polar groups is raised by a virus, which has adsorbed onto the protein, in such a manner that the spatial arrangement of polar groups in the protein becomes complementary to that of the virus, then the protein thread situated in the next place is in turn to rearrange its polar groups, thus the transmission of the viral pattern being established. In such a transmission, the pattern must be alternately reverse, that is, the replica is to be produced in every second chain as shown in Fig. 7 and 9. However, if the viral template possesses a pattern in which every two polar forces having opposite signs are always arranged in a symmetrical position as indicated in these Figs., the replica, if turred over, must be equal to the template.

If the template protein has not such a regular structure, the replica is naturally different from the template. It is, however, hardly conceivable that such two types of patterns are actually induced in the protoplasm.

On the other hand, Bergmann (122) claimed that a regular repetition is present in the amino acid sequence of a protein. If polar groups are so arranged in a polypeptide chain as to make the replica equal to the turned-over template, such a regular repetition in the sequence should be expected. It is believed by a number of workers that amino acid residues in the protein molecules are not arranged in a random fashion (85) (110). If there exists a regular repetition the same two protein molecules can combine with each other since a turned-over molecule reveals the complementary structure to the other. There are indeed many evidences that protein molecules of the same 
origin and the same kind have a strong tendency to combine with one another.

The combination between virus and the host cell must be raised by complementarily arranged polar groups between them, whilst there appears to exist a common antigenic structure or structures between a virus and the host cell protoplasm as mentioned in Part I, indicating that the complementary pattern is attributed to such common structures. Thus, a virus may be able to combine readily with a cell if the cell and the virus share a structure or structures in common.

The structures common to a virus and the host give rise to the combining force between them, whereas when they are drawn near by the combining force, uncommon structures present between them may exert a mutual electrostatic repulsion, and if the virus has a structure strong enough to rearrange the structure of the host cell protoplasm, the virus can assimilize the cell. Viruses must have such strong structures not present in the host cell protoplasm. 


\section{CHAPTER VIII \\ REPLICATION OF VIRUS PATTERN}

\section{Structural Constitution of Viruses}

It is a generally accepted fact that viruses can be inactivated without being deprived of their specific antigenicity. This suggests that more delicate, unstable part of the structure is required for the virus action than for the immunological activity.

Since virus particles even in the coagulated state can combine with their host cell, it is evident that the structure concerning the specific combination can exhibit its effect even when the virus are in the folded state. Such a structure may belong to the backbone structure of the protein and may be concerned in the immunological activity of the virus, whilst external, unstable groups which are revealed on the unfolding may be involved in the action of the virus as the template.

Such unstable groups may involve the repulsive force of the virus against the host cell protoplasm in contrast to the stable structures which may give rise to the specific combining force. On account of the disappearance of the repulsive force following the folding of the particle, thereby only the combining force being left to exhibit its effect, the virus may be able to combine easily with the host cell.

In the writer's theory (22), antibodies are produced in entirely the same way as viruses. However, in antibodies fine structures corresponding to those of antigens fail to be induced, only gross structures complementary to the determinant grougs of antigens being produced, so that antigens do not multiply as do viruses. In other words, antigens and antibodies share in common with the gross structures, but not with the fine structures. Accordingly, antigen and antibody can combine with one another but the uncommon fine structures may repulse mutually after the combination; thus immunological reaction such as precipitin reaction may follow. On the other hand, since parent viruses and newly produced daughter viruses are identical even in fine structures, they combine with one another as do antigen and antibody, but without mutual repulsion, whereas virus and host cell share in common with the gross structures, but not with the fine structures, so that the relation between antigen and antibody is comparable to that between virus and host cell.

In short, there are two kinds of structures in the virus, the one is 
stable, being involved in the combining force and able to develop the force even when the virus is in a folded, coagulated state, while the other is unstable, concerned in the repulsive force and revealed only when the particle unfolds. The virus action may be determined mainly by the latter structure against which the replica is to be croduced in the host protoplasm, although the former structure also may have a great influence upon the protoplasm in which the complementarily shaped structure is strengthened and specialized by the combination (22).

According to Anderson (123) phage particles require some amino acids especially tryprophan for the combination with the host cell. These "adsorption cofactors" appear to combine reversibly with the virus particles. This indicates that even for the development of the combining force are needed some factors or conditions.

A study by Hoyle (124) on the production of the complement-fixing antigens in the various phases of influenza virus infection shows that these antigens, which carry virus specificity without virus activity, increase in amount before virus activity appears. Moreover, it has been well acknowledged that at the beginning of the phage infection of bacteria, large amounts of antigenic substance are formed without being accompanied by the production of infective phage (125.) Presumably the structure formed at the beginning is incomplete, so that at first only the antigenic property may appear, but subsequently, when complete structure is accomplished, virus activity may arise. Thus it may be said that the rearrangement of polar groups is established gradually step by step, first only gross structures being formed and then finer ones added leading to the appearance of virus activity.

It has been shown that there occurs, after the inoculation of a virus, a latent period during which the virus fails to multiply, according to the virus employed the duration of this period ranging from 6 hours with influenza virus to 24 hours with mumps virus (126) (127). After such a latent period, there exists an interval during which the amount of virus in infected tissue increases rapidly. During this latent period only the formation of gross structures are probably being advanced.

Magnus (128) has reported an interesting study on the "incomplete virus": Undiluted allantoic fluid of chick embryo infected by influenza virus was used as inoculum and the amount of both agents concerning haemoagglutination and esg-infection produced after the inoculation were examined, and it was found that an increasing dissociation between the two agents occurred in the course of passages, infectivity decreasing markedly as compared to haemoagglutinin. Magnus considered that this was due to the production of non-infectious haemoag- 
glutinins, that is, "incomplete virus", possibly representing an immature form of the virus. His calculations indicated that only one of 10,000 particles was infective in the third passage. In this case, if diluted allantoic fluids were used instead of undiluted ones and a proper amount of virus particles was inoculated, complete virus was produced instead of the incomplete one. As previously stated, large sized particles, as a rule, involve strong and complete virus, and further decomposition of the particles into smaller fragments may result in the loss or decrease in the infectivity; on the other hand, it has been reported that this "incomplete virus" is smaller in size than the normal infective virus particles (126) (130).

The above finding shows that the complete structure of virus fails to be formed even after a prolonged period if environmental conditions. are not suitable. It has been reported that there occurs no multiplication of phage when the number of virus particles applied exceeds greatly the number of bacterial cells exposed, although the lysis of bacteria takes place (131). This likewise may indicate the production of incomplete virus. Moreover, Shlesinger (132) showed that when mouse brain is inoculated with a non-neurotropic strain of virus, production of complement fixing antigen and haemoagglutinin occurred but there was no production of infective virus. The yield of incomplete virus was proportional to the amount of infectious virus originally inoculated.

\section{Change of Viruses Following Their Combination with Host Cells}

Doermann and Anderson (123) have followed the production of a phage in host bacterial cells by disrupting the cells with intense sonic vibration at intervals after the infection, and have found that, up to 12 minutes after the infection, the disruption of the cells destroys 'their ability to form plaques, although free phage particles are highly resistant to such a treatment. They concluded from this evidence that in the freshly formed complex the virus is in a state sensitive to sonic vibration and that at the beginning of the infection the infecting virus particles has lost through some mysterious transformation its resistance to sonic vibration.

As above stated, viruses when combined with cells should have to unfold their structure in order to show their full pattern which is to be replicated in the host cells. However, in the unfolded form viruses may be labile unlike the virus in the coagulated coiled up state. Thus the phage may become extremely sensitive to sonic vibration on the combination with bacteria. 
We were able to demonstrate that phage and vaccinia virus are most stable in water containing no salt; under such a condition their folding is considered to be most complete (133) (134). Inorganic salts except those of divalent cations, such as calcium and magnesium, always render the phage more susceptible to heat. As was already discussed, divalent cations can cause virus particles to become rigid and stable, whereas salts of monovalent cations may cause their unfolding.

The disappearance of virus in host cells on the infection has been confirmed also with influenza virus. According to Isaacs and Edney (135), influenza virus injected into allantoic sac is mostly adsorbed by the chorio-allantoic membrane, but only less than 1 per cent of the inoculum is recovered from ground supsensions, recovering of the virus being reduced further by 10 fold, if membranes are incubated before grinding. Such a difficulty of recovering may naturally result, when the virus undergoes a change into an unstable stretched form on the surface of host celis so as to be inactivated during the isolating manipulation. According to Rountree (136) phage particles adsorbed onto host cells lose first its infectivity, followed by its antigenicity, and then ensues a period, from 12 to 19 minutes after the infection, during which neither infective phage nor antigen can be recovered from cells.

There are, however, evidences suggesting that viruses are destroyed on the infection in situ not during the isolating procedure. For instance, in biochemical studies of phage reproduction, Kozloff (137) claimed that all phage particles are partially broken down upon adsorption on the host cells; up to 80 per cent of the desoxyribonucleic acid and protein nitrogen of the adsorbed virus are decomposed during virus reproduction to a various small fragments.

This suggest that the adsorbed virus particles because of their unstable, stretched form may be influenced considerably by the assimilase action of the host cell protoplasm so as to be decomposed, although some of them may be able in the long run assimilize the protoplasm. In the writer's concept, as will be detailed in Part IV, Chapter V, when two protein molecules having different structures are held together, they exert to one another physicochemical influences due.to the different structure, and when the effect of the one is stronger enough to decompose the other, the former is called the proteolytic enzyme of the latter. In this respect, the host protoplasm may be said to act as an enzyme upon some of the adsorbed virus. Jesaitis and Goebel (138) have isolated water soluble, somatic antigen from a strain of dysenteria bacillus. This substance was found to inactivate all phages to which the bacillus is susceptible, and they (139) held the opinion that this substance serves as the receptor for phages which attack this bacillus. According to the theory of the writer, such a somatic antigen is noth- 
ing but the bacterial protoplasm protein which can combine with the phage through structures shared in common between the phage and the bacillus; the shared structures may be called receptors.

The protoplasm protein of susceptible cells should have weaker structural effect than the virus in order that the viral pattern is replicated in the cells. However, if some of the proteins acquire rigid structure through the combination with certain substances, say with polysaccharides, which make the protein structures rigid, then the proteins can act as virus inhibitor having the receptor for the virus. The virus which happens to combine with such rigid structures may be splitted since the virus has to be changed into labile, stretched form on the surface. The above cited claim that all phage particles are broken down upon the adsorption on the host cell suggests that the cell membrane may be mostly composed of protein complexes having such rigid structures. Form the point of this view it should be a natural result that the demonstration of the initial infecting virus particles is impossible in the cell macerates in which cell components acting as the inhibitor may be present abundantly.

Weidel (139a) has shown in his studies with bacterial membrane preparations that the interaction of intact bacteriophage with the host-cell membranes results in a disintegration of the membranes. This shows that the structure of the membrane was weaker than that of the virus. On the other hand, according to Barrington and Kozloff $(139 \mathrm{~b})$, electron micrographs taken at zero time and after incubation showed that adsorption of phage was accompanied by the disintegration of the virus and the conversion of the membrane to a granular residue, indicating clearly that at least some of the phage particles combined with the host cell were decomposed together with the cell membrane. In studying the interaction of $\mathrm{P}^{33}$-labelled phage and host cells, Mackal and Kozloff $(139 \mathrm{c})$ have found that a portion of the virus $\mathrm{P}$ was released into the medium in the process. But this release did not occur after adsorption to heat-killed bacteria. This may be attributed to the derangement of the host cell structure by heating resulting in the loss of rigid structure becoming unable to decompose the virus.

The virus particles may be decomposed on the cell surface into nucleic acids and proteins. The nucleic acids or their constituents thus formed appear to be incorporated into bacterial protoplasm whilst the proteins seem to be never utilized by the host cells. Thus, by infecting $E$. coli with $\mathrm{P}^{32}$ labeled phage, several workers have demonstrated that 30 to 50 per cent of the phage nucleic acid label is incorporated in the resulting "progeny". An equal high per cent to progeny transfer has been reported when the parental nucleic acid contained $C^{14}$ labeled 
purines (140). However, Hershy and Chase (141) showed that after infection with $\mathrm{S}^{35}$ labeled phage most of the sulphur containing phage protein could be stripped from the bactrial surface without interfering with phage production. The resulting phage contained little or no $S^{33}$, indicating that the protein fragments fails to be utilized by the bacteria in contrast to the nucleic acid constituent.

These findings are interpreted by some workers as indicating that phage is decomposed on the cell surface into protein and nucleic acid fragments which the latter only penetrate into cells to act as the template. In the opinion of the writer, however, the decomposition of the virus should be accompanied by the destruction of the virus activity, so that decomposed nucleic acid cannot act as the template, only serving as the cell constituents required for the cell growth. The virus particles which can achieve their purpose of reproduction should have complete structures.

Recently, Mackal and Kozloff (139 c) have actually confirmed that the material found in the progeny is largely, if not entirely, due to the use of fragments of the parent nucleic acid in the synthesis of the progeny nucleic acid, and they concluded that most of the transfer of parent material of phage to progeny is unessential for the reproduction process.

The viral pattern may be replicated on the cell surface on which the extended virus is attached and the replicated pattern may be transmitted to the inner side of the cell. However, it is conceivable that the virus itself can penetrate into the cell to impress its pattern directly to genes or gene-like particles, because accoräing to Coons et al. (142) various proteins injected intraveneously into mice were clearly demonstrated in the unaltered form in the nucleus of certain cells, often even in higher concentrations than in the cytoplasm. Similar penetration of viral nucleoproteins into cells may be possible.

\section{Essential Factors for the Spread of the Structural Change Caused by a Virus}

The first replica will be produced only in the portion of protoplasm with which a virus directly combines, no matter whether it be cytoplasm or a nuclear mass. The replica thus formed, however, is to be transmitted throughout the whole protoplasm. For the occurrence of such a transmission the association among the protein molecules in the protoplasm should not be too strong, as otherwise each protein molecule may be unable to change its structure independently of others even for a short period, whilst there must be associations among them in- 
timate enough to make them in the end identical with one another in their structure. Such an ideal association cannot be established but for the interposition of lipids among the protein molecules. This must be the main reason for the presence of lipids in the protoplasm.

On the other hand, as for viruses lipids are not only unnecessary, but unfavourable since viruses which may easily change their structure as does the usual protoplasm may commonly be unable to act as a strong virus. Rigid, unchangeable structure should be necessary for a virus to act as the template, since the action consists in the ability of changing the structure of the opponent. The great resistance of crystalline plant viruses to chemicals or heat may be attributed to the absence of lipids.

Even with the same content of lipids, the mode of association or constellation of protein molecules may effect the manner of the transmission of change in the protoplasm. The general susceptibility of young cells to viruses may be dependent upon the flexibility of the young protoplasm protein. There are good reasons to suppose that newly generated proteins possess a property to be easily altered in the structure as will be discussed in Part IV. The phage susceptibility of young bacteria can be retained for long periods at a low temperature, although immediately lost at a higher temperature, indicating that the flexibility of young proteins can be preserved at a low temperature (101).

If elementary bodies of protoplasm are irreversibly coagulated, viruses cannot multiply in the protoplasm, because the protoplasm in such a state may surely be inadequate for the production of replicas as well as for the transmission of the change. The irreversible coagulation must mean the death of protoplasm. But if protoplasm is killed without such a coagulation, the multiplication of viruses may not be impossible in the dead protoplasm. It has been established by a number of workers that phage can multiply in bacteria which have been renciered non-viable by chemical or physical agents such as penicillin, mustard-gas, formaldehyde, and ultraviolet rays. Similar phenomena have been proved also with other viruses. For example, chick embryos killed by prolonged storage at room temperature or at $4^{\mathrm{c}} \mathrm{C}$ are capable of supporting the multiplication of influenza virus upon incubation at $35 \mathrm{C}$ (143). The growth of Shope's papilloma virus is also possible in the cells sterilized by ultraviolet irradiation (144). According to Weiss (145), feline pneumonitis virus, a typical virus of the psittacosislymphogranuloma group, was cultivated for 20 passages in the yolk sacs of dead chick embryos; the rate of its growth was claimed to be almost similar to that in living embryos.

Multiplication of viruses in such dead cells is expected to be never 
accompanied by any nucleic acid change, since the multiplication should only be the rearrangement of polar groups in the protoplasm. In fact, Price (146) has confirmed that, during phage multiplication in bacteria, killed by penicillin, no change in nulcleic acids can be found. This shows that the nucleic acid change is not indispensable for virus proliferation.

The cell multiplication has to be accompanied by the protein synthesis for which great quantities of energy should be needed. In the above examples host cells are regarded as being dead because of their inability to multiply, which may be attributed to the destruction of energy-supplying system. If so, the fact that virus multiplication is achieved in dead cells, suggests that little or no energy, accordingly no protein synthesis, is required for the rearrangement of polar groups leading to the virus multiplication.

On the other hand, it has been reported that, in contrast to penicillin, streptomycin reduces bacteria on killing them incapable of prcducing phage. (147) (148). In this case, streptomycin may give rise in the protoplasm to a change thereby the rearrangement of polar groups due to the virus becomes impossible. While penicillin reveals no phage-inactivating ability, streptomycin can inactivate the virus, showing its strong action in disturbing the structure of the assimilase. In a similar manner the structure of bacterial cell protoplasm may be so disturbed as to be rendered incapable of performing the rearrangement. The inability of penicillin to inactivate phage indicates that penicillin has no such a strong disturbing faculty, although it can "kill" the bacteria presumably by destroying the energy-supplying system necessary for protein synthesis. If some host cells are "killed" in the same way by a certain agent other than penicillin, virus can multiply even in them.

Bacteria killed by ultraviolet-irradiation can produce phage, but the virus grows slower, and yields a smaller number of new particles (149). This suggests that the protoplasm structure is so changed by the killing action as to become somewhat unfavourable for the replication of the virus pattern. However, in order to suppress completely the ability of the cell to support the virus growth, enormous hits of the irradiation are required to reduce to half the number of bacteria capable. of inhibiting phage after infection (150).

It has been reported that chick embryo tissue cultivated for 13 . days in Hank's balanced salt solution prior to infection lost its ability to suport the growth of psittacosis virus, but this capacity could be restored by the addition of beef embryo extract at the time of infection $(150 \mathrm{a}) . \quad$ A significant amount of virus was adsorbed to the tissue cultivated under this condition without addition of beef embryo extract, indicating that the failure of virus to grow was not due to 
failure of virus to attack to and invade the cells. Presumably, the culture under the peculiar condition reduced the protoplasm structure of the cell inadequate to be impressed by the viral pattern. It may be said that the tissue cells under this culture condition may fall into a state comparable to that of the "aged" bacteria, while the cells may be rejuvenated by the addition of beef embryo extract to become capable of achieving the rearrangement of polar groups in response to the viral pattern.

To sum up, a virus can multiply in a cell even when the cell is in a "dead" state, if the rearrangement of polar groups in the cell protoplasm responding to the viral pattern is possible, while even viable cell cannot support the viral growth if the cell is unable to respond the viral pattern.

The virus activity may be determined by the spatial arrangement of polar groups, which in turn may be chiefly dependent upon the manner of distribution of amino acid residues, so that certain differences in the amino acid composition may be found between host cell protoplasm and the virus derived from it. As already pointed out this is said sometimes true. If a change like transamination or transpertidation took place in the protoplasm when its structure was enforced to alter in response to the template of the virus, some amino acids would appear as if they were newly synthesized. It may be unreasonable, therefore, to conclude that virus was newly synthesized from amino acids even when the amino acid composition of the virus was demonstrated to be evidently different from that of the protoplasm of host cells. 


\section{CHAPTER IX}

\section{VIRUSES AND NUCLEIC ACIDS}

\section{Nucleic Acids in Virus Particles}

At present it is generally believed that viruses are nucleoproteins, and nucleic acids are customarily considered as being indispensable for viruses. However, if writer's opinion so far discussed as regards the viruses is legitimate, it may be said that nucleic acids are not always necessary for the virus action. Since the template of viruses to be replicated in the host protoplasm should be involved in the structure of globulin-like proteins, there appears to be no need to assume nucleic acids as being indispensable for the template action.

In his splendid book "Plant Viruses and Virus Diseases" Bawden stated as follows: "In spite of the wide differences between the properties of the individual viruses so far purified, it would be rash to assume that they form a random and representative sample of the whole group. Because these are chemically similar, it can almost be taken for granted that some other viruses are also nucleoproteins, but to assume that all are would be decidedly premature. The methods of isolation so far used may be acting as a selective agency, succeeding only with those that are nucleoproteins."

If nucleic acids were essential components for viruses, their content and kind would be expected to be constant to a certain extent, but the fact is that they are unusually variable. Among many viruses studied so far phage appears to have the highest content of nucleic acid, and by Taylor (151) it has been estimated to be so high as nearly 50 per cent, whereas Newcastle disease virus has been reported to contain a quantity of nonlipid phosphorus equivalent to only about 1 per cent nucleic acid (152). Chemical compositions of various viruses are shown in Table 6; also from this Table it is evident that there are great differences in nucleic acids both in kind and content.

The writer has assumed as already stated that in the usual virus partcles as well as in the protoplasm, stretched peptide chains of protein molecules having the nature of euglobulin are arranged in parallel alignment, and lipids being inserted among the peptide chains. Nucleic acids, if contained any, are considered to be inserted, like the lipids, among the stretched protein molecules. X-ray analysis of tobacco-mosaic 
virus by Pfankuch (153) did not indicate a concentration of the comparatively dense nucleic acid in any particular part of the virus particle, and it was suggested that the virus may consist of either a long

Table 6.

Chemical Composition of Viruses.

After Beard, J. W. : J. Imm., Vol. 58, 49, 19.13.

\begin{tabular}{|c|c|c|c|c|c|c|c|c|c|c|c|c|c|}
\hline & \multicolumn{4}{|c|}{ Whole complex } & \multicolumn{4}{|c|}{ Lipid } & \multicolumn{5}{|c|}{ Nonlipid } \\
\hline & \multirow{2}{*}{ C } & \multirow{2}{*}{$\mathrm{N}$} & \multirow{2}{*}{$\mathrm{P}$} & \multirow{2}{*}{ 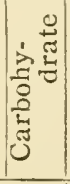 } & \multirow{2}{*}{$\stackrel{\text { సี }}{\stackrel{0}{0}}$} & \multirow{2}{*}{ 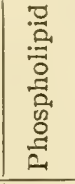 } & \multirow{2}{*}{$\mid$\begin{tabular}{c|}
-0 \\
0 \\
0 \\
0 \\
0 \\
0 \\
0 \\
0 \\
0 \\
0
\end{tabular}} & \multirow{2}{*}{ 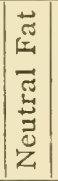 } & \multirow{2}{*}{ 预 } & \multirow{2}{*}{$\begin{array}{c}\frac{a}{0} \\
\stackrel{0}{0} \\
0 \\
2\end{array}$} & \multirow{2}{*}{ 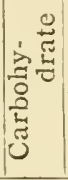 } & \multicolumn{2}{|c|}{$\begin{array}{l}\text { Nucleic } \\
\text { Acid }\end{array}$} \\
\hline & & & & & & & & & & & & 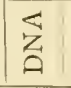 & 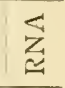 \\
\hline $\begin{array}{l}\text { Broth Bacterio- } \\
\text { phage } . . . \cdots \cdots . . . . . .\end{array}$ & 42.0 & 13.5 & 54.84 & 13.6 & 2.6 & 0 & 0 & 2.6 & 97.4 & 50.6 & 13.1 & 40.3 & 3. 6.6 \\
\hline $\begin{array}{l}\text { Synthetic Medium } \\
\text { Bacteriophage...... }\end{array}$ & 42.3 & 13.3 & 5.22 & 211.7 & 1.8 & 0 & 0 & & 30.2 & 52.4 & 11.2 & 44.6 & 1.3 \\
\hline Vaccinia .................. & 33.7 & 15.3 & 0.57 & 2.8 & 5.7 & 2.2 & 1.4 & 42. & 94.0 & 89.0 & 2.8 & 5.6 & \\
\hline Papilloma .............. & 49.6 & 15.0 & 0.94 & $\begin{array}{l}4 \\
4\end{array}$ & 1.5 & & & & 98.5 & 90.0 & & 8.7 & \\
\hline 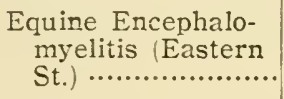 & 62.2 & 7.7 & 2.2 & 4.0 & 54.1 & 35.0 & 13.8 & 39.6 & 53.0 & 49.1 & 7.2 & & 4.4 \\
\hline $\begin{aligned} \text { Influenza } & A\langle\text { PRS } \\
\text { Strain) } & \cdots \ldots . . . . . . . .\end{aligned}$ & 53.2 & 10.0 & 0.97 & 12.5 & 23.4 & 11.3 & 7.0 & 5.1 & 77.5 & 65.0 & 7.3 & 1.5 & ? \\
\hline 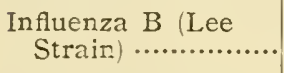 & 52.7 & 9.7 & 0.94 & 13.1 & 22.4 & 11.2 & 3.7 & 7.2 & 76.4 & 63.6 & 9.4 & 1.2 & ? \\
\hline Swine Influenza... & 51.4 & 9.0 & 0.87 & 10.0 & 24.0 & 10.7 & 5.7 & 7.7 & 77.6 & 67.6 & 10.0 & + & $?$ \\
\hline $\begin{array}{l}\text { Broth Me } \\
\text { Bacteriu }\end{array}$ & 49.1 & 13.2 & 2.72 & $212 \cdot 5$ & 7.75 & $5 \quad 7.75$ & 0 & 0 & 92.3 & 67.9 & 12.5 & 5.2 & 19.1 \\
\hline $\begin{array}{l}\text { Synthetic Medi } \\
\text { Bacterium } \cdots\end{array}$ & 49.0 & 13.2 & 2.66 & 11.6 & 9.11 & 9.11 & 0 & 0 & 90.9 & 67.7 & 11.6 & 2.4 & 20.9 \\
\hline $\begin{array}{l}\text { Normal Chick Em- } \\
\text { bryo Component... }\end{array}$ & 55.3 & 9.5 & 3.2 & 7.0 & 35.0 & 23.4 & 6.5 & 6.9 & 66.5 & 40.7 & 9.5 & & 10.6 \\
\hline
\end{tabular}

All values are per cent dry weight of the whole complex.

protein chain with nucleic acid side groups, or a regular arrangement of alternate nucleic acid and protein residues. The fact that tobaccomosaic virus is readily decomposed into smaller fragments also supports the view that nucleic acids are distributed in a homogeneous dissipation in the virus particles.

According to Schramm (154), tobacco-mosaic virus particles dissociate in alkaline solutions into two components, one of them free of nucleic acid, but having the same molecular weight as the other contaning nucleic acid. Readjustment of the solutions containing either one of them to $\mathrm{pH} 5$, yielded particles practically indistinguishable from the 
native protein particles in size and shape. Again, the action of nuclease leads to the cleavage of the bond between protein and nucleic acid and to a loss of biological activity, but the shape of the particles is not altered by nucleasc. It seems evident, therefore, that the shape and size of the virus particle are determined by the protein, nucleic acid apparently having no concern with it. On the other hand, it is stated that the molecular weight of desoxyribonucleic acid is 1.5 or 3.7 million; the length of this thread-like molecule is about $500 \mathrm{~m} \mu$. (155). Such a macro-molecule might be an artifact, yielded during the isolating procedure, as nucleic acids may have a great tendency to undergo polymerization.

Jordan (156) stated that all values of the molecular weight of nucleic acids, which have been determined from isolated sedimentation and diffusion constant determinations, must be regarded as unreliable. It is by no means certain that solutions of nucleic acids at the concentrations normally studied are molecularly disperse. The presence of divalent cations have shown to cause considerable aggregations, and it has been shown that the variation of light transmission with concentration shows a critical region at a concentration of 0.003 per cent which is regarded as an indication that aggregation commences at this concentration.

Monné (157) expressed the opinion that protoplasm is constructed of fibrils which consist of ribonucleic acid and nucleic acid free-sections regularly alternating with each other. Pederson (158) claimed that conjugated proteins containing non-protein components have a structure in which non-protein components are inserted like cement among bricks of protein molecules; thus these latter combining indirectly with one another through the inserted substances. Schmidt (159) considered likewise that in chromosomes nucleic acids are interposed like cement among the polypeptide chains of proteins.

Nucleic acids have long been known to possess the property to combine preferably with various proteins; owing to this property they were frequently used as precipitating agent of proteins. Even when no precipitate is formed, the formation of compounds between nucleic acids and neutral proteins is proved by a drop in the osmotic pressure of the dissolved protein and also by the loss of the high viscosity (160).

If protein molecules are cemented by nucleic acid, the alteration of the protein structure may scarcely occur. In fact, Carter and Greenstein (151) reported on a protective effect of desoxyribonucleic acid upon the heat coagulation of egg albumin. Nucleic acids may serve as an agent to prevent free motions of the peptide chains in the protoplasm, in contrast to lipids which may operate as a lubricating oil to facilitate the change of polypeptide chains. 


\section{The Action of Nucleic Acids}

When the assimilase activity of protoplasm is inferior to that of a virus, the virus can multiply in the protoplasm. On the other hand, the superiority in this activity on the virus part should arise from its rigid structure. Hence, if nucleic acids are inserted among protein molecules to make its structure rigid, the virus will become stable enough at least not to be assimilized by other assimilases.

Even in this respect alone, particles containing nucleic acids are expected to be an assimilase stronger than those containing no acids. Furthermore, the assimilase activity itself will become stronger, if more rigidly and more regularly are held together the protein molecules by nucleic acid, since the assimilase action is considered to be raised from the regular array of protein molecules.

The purified preparation of turnip-yellow mosaic virus has been found by Markham and Smith (50) to consist of two parts, about 80 per cent of particles containing nucleic acid and 20 per cent of a nucleic acid-free ones; this latter apparently non-infective, but share many properties of the infective ones containg nucleic acid. Thus, the both have similar electrophoretic mobility and form mixed crystals, and appear to contain the same antigens and to react fully with virus antisera. X-ray measurements on crystals of the two kinds of particles indicate that those containing nucleic acid are slightly smaller, suggesting that nucleic acid may hold the particles in a tighter mass. The separation of these two fractions is accomplished by ultracentrifugation, the bottom component being the protein containing nucleic acid. This greater sedimentation rate of the bottom component may result from the presence of nucleic acid, which holds tightly the protein molecules, while in the particles containing no nucleic acid, the combination among protein molecules may be loose, thus being not easily sedimentable.

The failure in proving the virus activity in this fraction may likewise be attributable to this slackness of the combination; however, it may be impossible to conclude that this fraction had always no virus activity, since it might not entirely be impossible to demonstrate its infectivity, if a suitable host with a high susceptibility was used under a proper environmental condition.

According to Heriott (162) a phage preparation which have been rendered nucleic acid free by "osmotical shocking" can still be adsorbed onto the host cells, prevent their multiplication and even lyse them, although the phage can reveal only less than 1 per cent of its original infectivity, a fact which may indicate that nucleic acid is not 
essential for the virus activity. Moreover, on studying the mammary cancer of mice, Barnum and Huseby (163) have obtained by high-speed centrifugation lipoprotein particles almost devoid of nucleic acid. These particles possessed cancer-producing poteintialities which were equal to the original nucleic acid containing preparation of the lactating gland. Maaloe and Symonds (164) indicated that in both premature and ordinary lysates of bacteria infected by phage there are non-infective particles containing sulfur but no nucleic acid. These particles adsorb on bacteria and precipitate with antiserum in a way similar to typical phage. However, they are somewhat lighter and have no appreciable killing effect on bacteria.

Price (165) has found that the activity of a phage preparation was increased by 20 to 30 fold by the addition of nucleic acid. This fact can be interpreted as indicating that some phage particles incapable of developing the virus activity bacause of the looseness in their structure acquire the rigidity on the combination with the nucleic acid it with the revelation of the virus activity. The nucleic acid used in this experiment was ribonucleic acid isolated from host bacteria or yeasts, whereas the nucleic acid contained in phage preparations is usually not ribonucleic but desoxyribonucleic acid which is regarded by some workers as having the special relation to the virus. This shows, however, that ribonucleic acid can contribute to the phage activity like desoxyribonucleic acid.

Bacteria infected with a phage are usually disintegrated into minute particles which can be isolated by our isoelectric precipitation method. It was found that only one out of 100 or 1,000 particles thus isolated shared the virus activity, and that as already described most of the activity belonged to larger particles, smaller ones being unstable, most of which existing in non-infective state. This fact may indicate that, even if nucleic acid content was equal, larger particles could develop the activity with more easiness than smaller ones. However, since the distribution of nucleic acids in bacterial protoplasm is not uniform, each particle produced should be different in nucleic acid content, and hence, even when the particles were equal in size, only some particles with higher nucleic acid content would be able to develop the virus activity. Furthermore, it may be possible that some phage can exist in larger particles because of their high content of nucleic acid which prevents the further decomposition of the particles into smaller ones.

Anyhow, particles with high contents of nucleic acid may show greater tendencies to act as viruses, and since such particles have greater sedimentation rates, only those sedimentable rapidly by ultracentrifugation would likely be regarded as the sole viruses. 
The coli-phage particles isolated by our method contain nucleic acids at the most only 15 per cent and even one third of the particie is composed of lipids, whereas those isolated by ultracentrifugation have been reported to contain so much nucleic acid as 40 per cent, or more, and lipids only about 1 per cent (113). Moreover, the yield by means of the ultracentrifuge is only of the order of one hundredth of the yield by our method, and accordingly it is evident that particles having a peculiar property only are to be obtained by ultracentrifugation. Such peculiar particles would be able to act as the virus even when other particles failed to do so. It must, however, be emphasized that almost all the particles isolated by our method can sometimes reveal phage activity when examined under most suitable conditions.

Polyhedrosis rirus, as already mentioned, is isolated, from polyhedral body, in needle shaped particles containing a large quantity of desoxyribonucleic acid, but it has been reported that such particles are contained in the polyhedral body only about 3 to 5 per cent; the most part of the body consists of proteins with a low phosphorus content having no virus activity (33).

The particles separated from normal, healthy plant leaves such as those of tobacco and tomato have been reported to contain little or no nucleic acid. The fact that they are so unstable as to be decomposed by trypsin is apparently due to this scantiness of nucleic acid and also to the high content of lipids. The probable reasons for the presence of nucleic acid in rich amount in the particles isolated from infected leaves are already considered. Virus particles are commonly not affected by proteolytic enzymes, indicating that the particles are stronger in their structure than the enzymes. Living protoplasm is likewise usually not affected by enzymes. However, when its regular configuration is damaged to become unable to act as the assimilase it may be called dead, and at the same time it will become digestable by enzymes. Normal plant particles are presumably in this dead state as their configuration would be disturbed severely during the preparing manipulation on account of their looseness in structure because of the lack of nucleic acid and so they may be split by trypsin in contrast to usual virus particles.

Some bacteria produce agents termed haemolysins which act on red blood cells to cause lysis. So far as our studies have reached, haemolysins appear to exist in virus-like particles and also their action, like that of viruses, consists in causing a disturbance in the protoplasm structure of blood cells. However, in contrast to viruses, haemolysins fail to multiply. This is possibly due to their inability to produce an exact replica in the protoplasm, that is, haemolysins may cause only a structural disturbance instead of producing the 
replica (22).

An interesting fact has been found by Hosoya et al. (166) concerning streptococcus haemolysin. When haemolytic streptococcus collected by centrifugation were shaken with 10 per cent nucleic acid solution for 20 minutes at $37^{\circ} \mathrm{C}$, strong haemolytic power was found in the solution after the bacteria were removed by centrifugation, whereas no haemolysin activity was obtained if the bacteria were washed with saline. Also powerful haemolysin was obtained when nucleic acid was added to the culture media. This fact can be explained in the same way as the fact, above cited, found by Price concerning a phage; namely, the virus-like particles produced by the bacteria are endowed with rigid structure by the nucleic acid to become capable of exerting strong disturbing action on the blood cells.

We were unable to separate haemolytic action of staphylococcus haemolysin from virus-like particles containing lipids; haemolytic action would readily be inactivated when the particles were precipitated at the isoelectric point, whereas Wittler and Pillemer (167) have succeeded in the purification of the haemolysin by isolating it from the culture filtrate on precipitating at $\mathrm{pH} 4.0$ in 15 per cent methanol at $-5^{\circ} \mathrm{C}$. The expulsion of lipids to some extent seems thus possible as in the case of some viruses if the treatment is made at low temperatures.

As already discussed, the protoplasm can be regarded as polymerization products of protein molecules possessing certain spatial arrangements of polar groups; the pattern of the spatial arrangement may differ with the kind of protoplasm or with the kind of cells, and each kind of cells may have its specific pattern. In the protoplasm of a certain cell, not only protein molecules but also other components, such as nucleic acids, lipids, and sugars, have to be arranged so as to be subjected to the specific pattern. Since the pattern of a cell is to be changed into the viral pattern following the infection with the virus, it may be expected that nucleic acid composition in a host cell is deviated from the normal when the acid is synthesized in response to the pattern of the protoplasm of the cell infected with a virus; the viral pattern is to be replicated in the protoplasm so that the pattern of the nucleic acid synthesized by the infected protoplasm must have the same viral pattern. In agreement with this view it has been claimed that nucleotide composition of a viral nucleic acid is characteristic of the kind of the kind of virus; in other words, the nucleic acid compositions of unrelated viruses are demonstrably different, whereas those of strains of a virus are indistinguishable (168). Moreover, it has been reported that phage DNA can be distinguished by its hydroxymethylcytosine content from bacterial DNA which contains 
cytosine; viral DNA increases and bacterial DNA is destroyed in infected bacteria (169). Again, it has been found that the DNA of certain strains of phage contains no cytosine; instead, they contain a hitherto unrecognized pyrimidine, while other viruses contain no this pyrimidine (170).

Since the structure of a nucleic acid polymer may be rigid, even when some virus-like particles, or elementary bodies of protoplasm, consist mostly of nucleic acids, only a small particle being proteins, the specific pattern may exist unimpared in the particles. In an extreme case where all the protein molecules are eliminated, particles thus consisting of nucleic acid only may not be impossible still to retain the pattern to act as the template. There seems to be an evidence that this may be possible as mentioned in the next Part.

As regards nucleic acids there are still many other problems to be discussed, but they will be reserved for Part IV where the nature of genes is to be considered in detail. 


\section{CHAPTER $\mathrm{X}$ \\ THE SUMMARY OF PART II}

The essential component of the protoplasm is a protein of globulin nature, molecules of which exist in parallel alignment in a stretched thread-like form, associating with one another in a regular array by a physicochemical force. Lipids are other important components, which are inserted among the protein molecules. Owing to the lipids, protoplasm can exhibit properties of a liquid, and protein molecules existing in it can change freely their structure; at the same time protoplasm itself can possess the specific configuration.

If the protoplasm is such a system consisting of molecules of protein and lipid, each of which associating regularly with one another to form a certain structure, it will be expected that a change arising at a site of the protoplasm will spread to other parts. Actually this appears to be the case; for example, it is known that, when an adecitate stimulus is applied to the protoplasm, there occurs at the site of stimulation a coagulation of protoplasm into minute particles, which spreads succesively through the whole protoplasm.

The protoplasm coagulation into such minute particles can be explained on the assumption that the thread-like protein molecules exist in forming bundles in parallel alignment, each bundle being formed by several hundreds of threads. This bundle is assumed to be the unit of protoplasm, and termed "elementary body of protoplasm." Lipids are inserted among protein molecules in this unit body, and the protoplasm is composed of these bodies which are arranged in parallel array, combining loosely with one another by a physicochemical force.

The thread-like protein molecules forming the body will contract or fold when a stimulus is given, so that the protoplasm as a whole coagulated into the unit particles by the stimulus. Since this coagulation of protoplasm, that is, the folding or contraction of the protein molecules, is reversible, the folded or contracted molecules will restore the stretched form when the stimulus is removed, whereby the protoplasm recovers its original state.

On the other hand, as regards its function the protoplasm is a 
kind of enzymes, which may be designated "assimilase," and is capable of adsorbing certain protein molecule having a structure somewhat different from that of its own protein constituent, thercby the structure of the protein is changed by the rearrangement in its spatial distribution of polar groups to be made identical with that of the protoplasm, thus the protein being incorporated into the protoplasm. Not only protein molecules but also amino acids are adsorbed onto the protoplasm surface to be adapted to the specific arrangement of its polar groups, and subsequently they are fused into the protoplasm; thus the protein is synthesized and the growth of protoplasm is accomplished. Protoplasm growth, therefore, occurs in the same way as a crystal growth, and hence cell multiplication can be looked upon as the growth of the protoplasm, a type of crystal. In fact, protoplasm is considered as a kind of liquid crystal, since it is a liquid with a definite structure.

\section{2}

The assimilase action of protoplasm apparently arises from the polymerization of protein molecules of an equal structure, and the action consists in the faculty to cause the rearrangement of polar groups of the proteins to be fused. Proteins in a separate, single molecular state fail to exhibit the effect which may arise from its specific arrangement of polar groups, whilst if many molecules with an equal arrangement polymerize in a regular way, the effect is considered to become stronger and as a result can exert its influence upon other proteins. This effect appears to a certain extent to be directly proportional to the number of polymerized molecules, and probably resulting from a kind of electrostatic force.

Virus particles are estimated to be combining with so much water amounts as 10 times the dry weight of the particles. The greater part of the molecules of the water are apparently present in forming a thick layer around the surface of the particle, and the thickness of the layer is calculated to be directly proportional to the particle diameter.

The force attracting the water molecules onto the surface of a virus particle is possibly identical with the force of the virus to produce its replica in the host cells. Virus particles may show a tendency to act as the stronger virus as the particle increases in size.

The fact confirmed by Rothen that the thickness of molecules of antibody attracted by the monolayers of the antigenic protein is directly proportional to the number of the piled-up monolayers of the 
antigen, provides likewise an evidence that the force arising from tile specific arrangement of polar groups may increase in the direct proportion to the degree of polymerization. It is known that the number of antibody molecules combining with the antigen increases as the latter molecule increases in size, and it is also accepted that both enzymes and antigens have to be in colloidal states in order to exert their action; in all these facts seems to be involved the force coming from the polymerization. The peculiar property of colloid may also be closely connected with the same force.

The fact that viruses are the particles of more than certain sizes may indicate the necessity of polymerization of virus proteins to a certain degree for the development of the activity.

If the protoplasm is disintegrated into its elementary bodies, and if in the bodies the original structure of the protoplasm is retained, the bodies themselves also can act as an assimilase. Viruses are regarded as a kind of the assimilase existing in such minute bodies.

However, the assimilase, when present in the form of free elementary bodies, is much less active than the original protoplasm, and is unable to incorporate either amino acids or foreign protein molecules, only being capable of assimilizing a certain protoplasm having a weaker assimilase action.

If the assimilase action of a virus is stronger than that of the protoplasm of a cell to which the virus is adsorbed, the arrangement of the polar groups of the protoplasm will be so changed as to become identical with that of the virus. Since this change may be transmitted throughout the protoplasm, the whole protoplasm protein may be changed finally to have the identical arrangement. If the protoplasm is disintegrated into coagulated elementary bodies by this change, the bodies may be regarded as virus particles. This is the way in which viruses multiply.

The mode of change of the protoplasm of red blood cells can be studied by investigating the mechanism of haemolysis. An incubation period required for the commencement of haemolysis after addition of a haemolytic agent can be regarded as the period during which a change, secondarily induced by the haemolytic agent, spreads throughout the protoplasm, $i$. e., the stroma of the red cell, to reduce the protoplasm incapable of retaining the haemoglobin which is being held by the stroma.

A similar incubation period is found commonly between the addi- 
tion of a phage and the disintegration of the bacteria, the lysis, which may be caused by a change of the protoplasm due to the virus disrupting the mutual association of elementary bodies.

It is found that the rate of change in the blood cell is roughly proportional to the concentration of a haemolytic agent, showing that the protoplasm undergoes a change which tends to be directly proportional to the amount of the haemolytic agent combining with the cell. Also in the case of a virus and its host cell, the degree of influence of the virus upon the host cell is apparently proportional to the number of the virus particles adsorbed to it. Thus a weak virus fails to infect the host cell when the number of the particles is small, but can get the upper hand of the cell if many particles affect in union.

Bacterial haemolysins, usually present in virus-like particles, may affect the blood cells in the same way as viruses affect the host cells. Thus, haemolysins may disturb the structure of the protoplasm of the blood cell to cause a change which spreads throughout the cell; as a result the association between the haemoglobin and the protoplasm is damaged leading to haemolysis. This change, however, cannot give rise to so precise a rearrangement as to produce the very replica of the haemolysin structure, so that haemolysins are not viruses.

Viruses, like haemolysins, may sometimes disintegrate the protoplasm of host cells without producing the replica. If phage or influenza virus particles are added to respective host cells in a great excess, little or no virus is produced, although thereby the host cells are injured severely.

\section{4}

Since virus particles are no more than elementary bodies of protoplasm that have been liberated from the cell, their chemical composition should be similar to that of the protoplasm, mainly composing of proteins and lipids. However, some plant viruses unlike protoplasm are composed of proteins only. This presumably results from the lipid elimination which might occur on the destruction of the combination between protein and lipids due to the disturbance by the virus. Such plant viruses are, therefore, isolated usually in the form of particles which can be regarded either as lipid-free elementary bodies themselves or as their split products, $i$. $e$., thinner bundles or minute globular particles without lipids.

Since thread-like protein molecules of the protoplasm may fail to contract when lipids are eliminated, the plant viruses without lipid may be bundles of protein threads. Usually hundreds of threads appear to form a single bundle, whose length, therefore, must be the 
length of a globulin molecule stretching in its polypeptide chain.

Such lipid-free particles, however, do not represent the only feature of the plant viruses, but they may rather be a peculiar feature. Thus, also in plant viruses coagulated elementary bodies themselves can be recognized as the most common virus particles as in animal viruses. In addition, also the elimination of lipid itself is by no. means peculiar to plant viruses; various polyhedrosis viruses of insects are isolated in lipid-free, needle-shaped particles similar to certain plant viruses both in shape and length.

Ammonium sulphate can sometimes expel the lipid from the plant protoplasm, so that crystals like those of some viruses are occasionally obtained from normal plant saps by treating with the salt.

In healthy plant cells the combination between the protein and lipid appears to be so firm that lipid elimination fails to occur of itself without the action of a certain virus, and consequently the lipid-free needles are customarily believed as the only feature of the virus. It seems possible, however, to produce virus-like lipid-free particles in plant or insect tissues by the application of some proper agents other than viruses.

The action of viruses may arise from the pattern of the protein molecules constituting the particles, and the effect of the pattern may be strengthened by the polymerization of the protein. Lipids are not necessary for the virus action, but indispensable for the protoplasm to freely change in its configuration when infected by a virus. Owing to the lipids, proteins can change their structure in the protoplasm, the change being transmitted throughout the cell, thereby the structure specific to a virus is given rise to. The free motions of protein molecules in the protoplasm owing to the presence of lipids must be theessential picture of the life itself.

The bundles of protoplasm-protein threads appear to have the property to associate end-to-end to form unusually long filaments. As a consequence certain plant viruses may occasionally reveal themselves in long filaments. At the same time, the bundle tends to split into shorter fragments, being accompanied, as a rule, by either a loss or decrease in the virus activity. It seems probable, however, that some plant virus proteins are so brittle that they can split into extremely small fragments without great disturbance in their structure and consequently without loss of its activity. In such a case, viruses. can be obtained in extremely small particles. A number of plant 
viruses are actually believed to be so small particles as their diameter are of the order of $0.03 \mu$ or less.

This is probably true also for the virus particles from which lipids are not eliminated. Thus, if coagulated fragments yielded by the split of elementary body retained the virus activity, the virus in question would be said to be very small particles. On the other hand, if every several elementary bodies were coagulated into a larger body on the combination with one another, the virus would reveal itself as a large particle, and if a number of elememtary bodies associated end-to-end and subsequently coagulated, thread-like particles would be produced.

Protoplasm proteins may be able to accomplish a reversible contraction not only in the protoplasm but also outside the cell, so that certain viruses may be globular under some environments while filaments under others. If some proteins in an elementary body were left in the stretched state while most molecules were coagulated or folded, then the so-called tailed-virus would appear.

The origin of various shapes and sizes of virus particles can thus be explained by the above concept concerning the protoplasm structure. At the same time it should be expected from the same concept that there may neither be shape nor size essential to virus particles. Various virus-like particles can be obtained from cells having no concern with viruses.

Nevertheless, it seems to be an established fact that particles which fail to be found in the normal cells can frequently be demonstrated in virus-infected cells by electron micrographs. Considerable changes may take place in the metabolic activity of cells when they are affected by viruses, resulting in the production of a different pattern in their chemical composition, especially in both amount and kind of nucleic acids. On account of such a change particles may become stable enough not to be disintegrated under the electron beam so that they are photographed fairly by the electron microscope. Thus particles appearing not present in the normal protoplasm will be raised on the infection with certain viruses.

According to our investigation under the microscope by dark-field illumination, red blood corpuscles, like other cells, are disintegrated into virus-like particles on the application of adequate injurious effects. But such particles cannot be photographed by the electron microscope unless Ringer's solution is used, and even with Ringer's solution the particles are sometimes disintegrated into much smaller ones and photographed as such; the use of saline instead of Ringer's solution may result in the failure of catching any particles in the electron micographs. Thus certain particles which do exist actually cannot be 
photographed unless brought under proper conditions, and occasionally even revealed in shapes entirely different from the actual ones.

\section{6}

Viruses are commonly believed in chemical nature to be nucleoproteins. The writer claims, however, that the pattern of virus-template is originally determined by the structure of protoplasm protein of a globulin nature, nucleic acids having nothing to do with the pattern. The effect of the template is enhanced by the polymerization of the protein molecules, but if nucleic acids are inserted among these molecules, the polymerization products, $i$. $e$., the virus particles, may become stable and rigid in the configuration so that the template action may be strengthened. In the particles without nucleic acid the mutual combination of the protein molecules is loose, and consequently the structure of particles are so unstable as to be readily destroyed and in addition the templating action itself is insignificant.

On the other hand, when nucleic acids are contained in the particle to make its structure rigid, the particle may become more easily sedimentable, so that by means of the ultracentrifuge particles containing nucleic acid in rich amount may be readily isolated, and again since the virus activity is mainly to be retained in these particles for the reason just mentioned, only particles containing nucleic acids in rich amount may come, as a natural result, to be considered as viruses.

Lipids, in contrast to nucleic acids, if contained in the particle, may render the structure easily changeable, and consequently may have an unfavourable influence upon the viruses against their action.

Since the function of viruses is to act as the template, lipids are not only unnecessary but rather deleterious, whereas for the protoplasm in which the replica is to be produced lipids are indispensable. In short, the free motion of protein structure in the protoplasm, a motion which is considered to be essential for the development of life phenomena, can be achieved only by the presence of lipids, whilst the motion may be hindered by nucleic acids. Therefore, particles containing great quantities of nucleic acids but little or no lipids are fitted to act as the template, so that such particles are liable to be regarded mainly as viruses. 


\section{REFERENCES}

(1) Benseley, R. R. : Anat. Rec., (72), 315, 1938.

(2) Wyckoff, R. W. C.: The Science in Progress, IV, 203, 1950; Advances in Protein Chem., (6), 1, 1951.

(3) Claude, A.: Advances in Protein Chem., (5), 423, 1949.

(4) Frey-Wyssling, A.: Submicroscopic Morphology of Protoplasm and Its Derivatives, London, 1947.

(5) Ris, H., and Mirsky, A. E. : J. Gen. Physiology, (32), 489, 1949.

(6) Haurowitz, F.: Chemistry and Biology of Proteins, New York, 1950.

(7) Moriyama, H.: J. Shanghai Sci. Inst., (3), 239, 1938.

(8) Mor!yama, H.: Arch. Virusforschung, (1), 273, 1939.

(9) Moriyama, H., and Ohashi, S. : J. Shanghai Sci. Inst., (3), 317, 1938.

(10) Rothen, A.: J. Biol. Chem., (163), 345, 1946; Science, (102), 446, 1945.

(11) Bernal, J. D. : Trans. Faraday Soc., (42) B, 1, 1946.

(12) Lundgren, H. P. and Ward, W. H.: Ann. Rev. Bioch., (18), 115, 1949.

(13) Rothen, A.: Growth, (11), 195, 1947.

(14) Haurowitz, F.: Z. physiol. Chem., (246), 23, 1936.

(15) Heidelberger, M., and Kendall, F. E. : J. Exp. Med., (62), 467, 697, 1935.

(15a) Granoff, A. and Henle, W.: J. Imm., (72), 329, 1954.

(16) Luria, S. E.: Proc. Nat. Acad. Sci., (33), 253, 1947.

(17) Andrewes, C. H., and Elford, W. J. : Brit. J. Exp. Path., (128), 278, 1947.

(18) Anderson, T. F.: J. Cellular Comp. Physiol., (25), 1, 194 ग̃.

(19) Watson, J. D. : J. Bact., (60), 697, 1950.

(20) Moriyama, H., and Ohashi, S.: J. Shanghai Sci. Inst., (3), 341, 1938.

(21) Mitchison, J. M. : Nature, (116), 348, 1950.

(22) Moriyama, H. : Immunity (sister volume of this book), in press

(23) Watson, J. D. : J. Bact., (60), 697, 1950.

(24) Moriyama, H., and Ohashi, S. : J. Shanghai Sci. Inst., (4), 17. 1938.

(25) Bawden, F. C.: Plant Viruses and Virus Diseases, III. Edition, 1950.

(26) Bawden, F. C., and Pirie, N. W. : Brit. J. Exp. Path., (19), 66, 1938.

(27) Taylor. A. R., ct al.: J. Infec. Dis., (71), 110, 1942.

(28) Taylor, A. R., ct al.: J. Infec. Dis., (72), 31, 1943.

(29) Hoagland, C. L., et al. : J. Exp. Med., (71), 737, 1940.

(30) Harris, D. L. : Science, (108), 158, 1948.

(31) Smith, K. M.: Recent Advances in Plant Viruses, London, 1951.

(32) Stanley. W. M., and Lauffer, M. A.: Viral and Rickettsial Infection of Man, Edited by Rivers, 18, 1948.

(33) Bergold, G. : Z. Naturforsch., (26), 122, 1947.

(34) Steinhaus, E. A. : Bact. Revs., (13), 203, 1949.

(35) Perutz, M. F. : Nature, (150), 324, 1942.

(36) Crowfoot, D., and Riley, D. E. : Nature, (141), 521, 1938.

(37) Takahashi, W. N.: Amer. J. Bot., (36), 533, 1949.

(38) Crook, E. M., and Sheffield, F. M. L. : Brit, J. Exp. Path., (27), 5, 1946.

(39) Bawden, F. C., and Pirie, N. W.: Brit. J. Exp. Path., (26), 294, 1945.

(40) Stahmann, M. A., ct al. : Phytopath., (40), 999, 1950.

(41) Takahashi, W. N., and Rawling, T. E. : Phytopath., (38), 279, 1948. 
(42) Black, L. M. : et al.: Proc. Soc. Exp. Biol. \& Med., (73), 119, 1950.

(43) Joly, M.: Bull. Soc. Chim. Biol., (30), 404, 1948.

(44) Schramm, G.: Naturwiss., (31), 94, 1943.

(45) Knight, C. A., and Oster, G.: Arch. Biochem., (15), 289, 1947.

(46) Rawling, T. E., et. al. : Amer. J. Bot., (36), 533, 1946.

(47) Takahashi, W. N., and Rawling, T. E.: Amer. J. Bot., (34), 271, 1947.

(48) Oster, G.: J. Gen. Physiol., (31), 89, 1947.

(49) Want, J. P. H., and Rozendall, A.: Tijdschr. Plantenziekten, (54), 134, 1948,

(50) Markham, R., and Smith, K. M. : Parasitology, (39), 330, 1949.

(51) Moriyama, H.: Kagaku, (11), 482, 1941.

(52) Svedberg, T., and Peterson, K. D. : Ultracentrifuge, Londan, 1940.

(53) Brohult, S.: J. Phys. and Colloid Chem., (51), 206, 1947.

(54) Gutfreud, H.: Bioch. J., (42), 544, 1948.

(55) Williams R. C., and Steere, R. L.: Science, (109), 308, 1949.

(56) Yamafuji, R., et al.: Bioch. Z., (318), 101, 1947; Enzymologia, (14), 24, 1950.

(57) Tatum, E. L., and Perkins, D. D.: Ann. Rev. Microbiology, (4), 134, 1950.

(57a) Yamafuji, K. et al: Enzymologia, (15), 327, 1953.

(58) Nixon, H. L. and Watson, M. A.: Nature, (168), 523. 1951.

(59) Johnson, J.: Phytopath., (41), 78, 1951.

(60) Wildman, S. G., and Bonner, J.: Scientific Monthly, (70), 337, 1950.

(61) Glaser, R. W., and Stanley, W. M.: J. Exp. Med., (77), 451, 1943.

(62) Glaser, R. W., and Wyckoff, R. W. G. : Proc. Soc. Exp. Biol. \& Med., (37), 503, 1937.

(63) Bawden, F. C., and Pirie, N. W. : Brit. J. Exp. Path., (26), 277, 1945.

(64) Rake, G., and Blank, H.: J. Invert. Dermat., (15), 81, 1950.

(65) Rothen, A.: J. Biol. Chem., (172), 841, 1948.

(66) Moriyama, H., and Ohashi, S.: J. Shanghai Sci. Inst., (4), 63, 1939.

(67) Luria, S. E. : The Second Symp. of Soc. for Gen. Microbiology, The Nature of Virus Multiplication, 1953, 99.

(68) Bang, F. B.: J. Exp. Med., (88), 251, 1948.

(69) Bourdillon, J. : Arch. Biochem., (3): 285, 1944.

(70) Beard, D., et al.: Proc. Soc. Exp. Biol. \& Med., (75), 533. 1950.

(71) Reagan. R. L., and Bruckner, A. L. : J. Bact,, (64), 233, 1952.

(72) Murphy, J. S., et al.: Proc. Soc. Exp. Biol. \& Med., (73), 596, 1950.

(73) Eddy, B. E., and Wyckoff, R. W. G.: Proc. Soc. Exp. Biol. \& Med., (75), 290, 1950.

(74) Edwards, D. F., and Wyckoff, R. W. G.: Proc. Soc. Exp. Biol. \& Med., (64), 16, 1947.

(75) Chapman, C., et al.: J. Bact., (61), 261, 1951.

(76) Hoyle, L. : J. Hyg., (48), 277, 1950.

(77) Wyckoff, R. W. G.: Experimentia, (6), 66, 1950.

(78) Wyckoff, R. W. G.: Proc. Soc. Exp. Biol. \& Med., (71), 144, 1949.

(79) Straus, M. J., et al.: Proc. Soc. Exp. Biol. \& Med., (72), 46, 1949.

(80) Gard, S. : Acta Med. Scandinav. Supp., (144), 1, 1943.

(81) Wyckoff, R. W. G.: J. Imm., (70), 187, 1953.

(82) Szent-Györgyi, A.: J. Colloid Sci., (1), 1, 1946.

(83) Wauch, D. F.: J. Amer. Chem. Soc., (70), 1850, 1948. 
(84) Lundegren, H. P.: Silk J., (23), 32, 1946.

(85) Bailey, K., and Sanger, F.: Ann. Rev. Bioch., (20), 103, 1951.

(86) Foster, J. F., and Samsa, E. G.: J. Amer. Chem. Soc., (73), 5388, 1951.

(87) Sharp, D. G., et al.: J. Bact., (63), 152, 1952.

(88) Chu, C. M., et al.: Lancet, (1), 602, 1949.

(89) Angulo, J. J., et al.: J. Bact., (60), 129, 1950.

(90) Murphy, J. S., and Bang, F. B. : J. Exp. Med., (95), 259, 1952.

(91) Moriyama, H., and Ohashi, S.: Arch. Virusforsch., (1), 571, 1940.

(92) Adams, M. H.: J. Gen. Physiol., (32), 579, 1949.

(93) Sharp, D. G., et al. : J. Biol. Chem.: (142), 193, 1942.

(94) Epstein, B., et al.: J. Exp. Med., (94), 171, 1951.

(95) Wolpers, C. : Naturwiss., (29), 416, 1941.

(96) Cohen, S. S.: J. Biol. Chem., (174), 281, 295, 1948.

(97) Putnam, F. W., and Kozloff, L. M.: Science, (108), 386, 1948.

(98) Kozloff, L. M., et al.: J. Biol. Chem., (188), 101, 1951.

(99). Woodruff, A. M. : Goodpasture, E. W.: Amer. J. Path., (7), 209, 1931.

(100) Moriyama, H., and Ohashi, S.: J. Shanghai Sci. Inst., (4), 39, 1938.

(101) Fong, J.: J. Gen. Physiol., (31), 473, 1948,

(102) Kopper, P. H. : Jour, Bact., (63), 639, 1952.

(103) Kopper, P. H.: Jour, Bact., (67), 507, 1954,

(104) Ohashi, S.: J. Shanghai Sci, Inst., (4), 151, 1939.

(105) Graham, A. F. : McClelland, L. : Canadian J. Res. Sect. E., (28), 121, 1950.

(106) Wildman, S. G., et al. : J. Biol. Chem., (180), 985, 1949.

(107) Putnam, F. M., et al.: J. Biol. Chem., (199), 177, 1952.

(108) Stent, G. S., and Maaloe, O. : Bioch. et Biophys. Acta, (10), 55, 1953.

(109) Labaw, L. W.: Jour. Bact., (66), . 429, 1953.

(110) Chantrenne, H.: The Nature of Virus Multiplication, The Second Symp. of the Soc. for Gen. Microbiology, Cambridge, 1953, 1.

(111) Scherer, W. F., and Syverton, J. T: : J. Exp. Med., (96), 369, 1952.

(112) Ackermann, W. W., and Kurtz, H. : J. Exp. Med., (96), 151, 1952.

(113) Csáky, T. Z., et al.: J. Biol. Chem., (185), 311, 1950.

(114) Gratia, A., et al.: Compt. rend. Soc. biol., (12), 35, 1947.

(115) Price, W. H.: J. Gen. Physiol, (33), 17, 1949.

(116) Raferson, M. E., et al.: J. Biol. Chem., (181), 583, 1949.

(117) Saenz, A. C., and Taylor, R. M. : J. Imm., (63), 331, 1949.

(118) Gronwall, A. : : Chem. Zentr., (11), 525, 1943.

(119) Wunderly, C.: Helv. chim. Acta, (25), 1053, 1942.

(120) Hotchkiss; R. D., and Ephrussi-Taylor, H. :' Federation Proc., (10), 200, 1951.

(121) Henry, J. E., and Henry, R. J.: ' J. Bact., (52), 481, 529, 1946.

(122) Bergmann, .M., and Niemann, J.: J. Biol. Chem., (118), 301, 1937; (122), $577,1938$.

(123) Anderson, T. F.: Research in Med. Science, 1, New-York, 1950.

(124) Hoyle, L. : Brit. J. Exp. Path., (29), 390, 1948.

(125) Rountree, P. M. : Nature, (168), 34, 1951.

(126) Henle, W., et al.: J. Exp. Med., (86), 423, 1947.

(127) Ginsberg, H. S., and Horsefall, F. L. : J. Exp. Med., (90), 393, 1949.

(128) Magnus, P.: Acta Path. et Microbiol., Scand., (28), 278, 1951. 
(129) Gard, S., and von Magnus, P.: Arkiv f. Kemi. Min. och. Geol., (24), B. No. 8, 1947.

(130) Harig, M., and Bernkoff, H. : J. Imm., (65), 585, 1950.

(131) Delbrück, M.: J. Gen. Physiol., (23), 643, 1940.

(132) Schlesinger, R. W.: Proc. Soc. Exp. Biol. Med., (74), 541, 1950.

(133) Ohashi, S.: J. Shanghai Sci. Inst., (3), 279, 1938.

(134) Moriyama, H.: J. Shanghai Sci. Inst., (3), 199, 1938.

(135) Isaacs, A., and Edney, M. : Aust. J. Exp. Biol. \& Med. Sci., (28), 635, 1950.

(136) Rountree, P. M. : Brit. J. Exp. Path., (32), 341, 1951.

(137) Kozloff, L. M. : J. Biol. Chem., (194), 83, 1952.

(138) Jesaitis, M. A., and Goebel, W. F. : J. Exp. Med., (96), 409, 1952.

(139) Goebel, W. F., and Jesaitis, M. A. : J. Exp. Med., (96), 425, 1952.

(139a) Weidel, W.: Z. Naturforsch, (6b), 251, 1951.

(139b) Barrington, L. F. and Kozloff, L.M.: Science, (120), 110, 1954.

(139c) Mackal, R. P. and Kozloff, L.M.: L.M.: J. Biol. Chem., (209), 83, 1954.

(140) Watson, J. D. and Maaloe, O. : Biochim. et Biophys. Acta, (10), 432, 1953.

(141) Hershey, A. D. and Chase, M. : J. Gen. Physiol., (36), 39. 1952.

(142) Coons, A. H. ct al.: J. Exp. Med., (93), 187, 1951.

(143) Lahelle, O., and Horsefall, F. L.: Proc. Soc. Exp. Biol.\&Med., (70), 547, 1949.

(144) Friedewald, W. F., and Anderson, R. S. : J. Exp. Med., (78), 285, 1943.

(145) Weiss, E.: J. Infect. Dis., (86), 27, 1950.

(146) Price, W.: J. Gen. Physiol., (31), 127, 1947.

(147) Hook, A. E., et al.: J. Biol. Chem., (165), 241, 1946.

(148) Cohen, S. S.: J. Biol. Chem., (168), 511, 1948.

(149) Anderson, T. F.: J. Bact., (56), 403, 1948.

(150) Benzer, S. : J. Bact., (63), 59, 1952.

(150a) Hare, J. D. and Morgan, H. R.: J. Exp. Med., (99), 461, 1954.

(151) Taylor, A. R. : J. Biol. Chem., (165), 271, 1946.

(152) Cunha, R., et al.: J. Imm., (55), 69, 1947.

(153) Pfankuch, E.: Bioch. Z., (306), 125, 1940.

(154) Schramm, C.: Ber. D. Chem. Ges., (74), 532, 1941.

(155) Gullarnd, J. M. : Cold Spring Harbor Symp. Q. Biol., (12), 98, 1947.

(156) Jordan, D. O.: Ann. Rev. Biochem., (21), 209, 1952.

(157) Monné, L. : Arkiv Zool., (36 A), 1, 1945.

(158) Astbury, B.: Cold Spring Harbor Symp., (6), 109, 1938.

(159) Schmidt, W. J. : Die Doppelbrechung von Karyoplasma, Zytoplasma, Berlin, 1937.

(160) Greenstein, J., and Jenrette, W. : J. Nat. Cancer Inst., (1), 91, 1940.

(161) Carter, C. E., and Greenstein, J. : J. Nat. Cancer Inst., (6), 219, 1946.

(162) Heriott, R. M. : J. Bact., (61), 752, 1951.

(163) Barnum, C. P., and Huseby, R. A.: Cancer Res., (10), 503, 1950.

(164) Maaloe, O., and Symonds, N.: J. Bact., (65), 177, 1953.

(165) Price, W. H. : Proc. Nation. Acad. Scad. Sci. U. S. A., (34), 317, 1948.

(166) Hosoya, S., ct al.: Jap. J. Exp. Med., (20), 25, 27, 1949.

(167) Wittler, R. G., and Pillemer, L. : J· Biol. Chem., (174), 23, 1948.

(168) Dorner, R. W. and Knight, C.A.: J. Biol. Chem., (205), 959, 1953

(169) Hershey, A.D. et al.: J. Gen. Physiol., (36), 777, 1953.

(170) Wyatt, G. R. and Cohen, S. S.: Bioch. J., (55), 774, 1953. 


\section{PART III}

THE EVOLUTION OF VIRUSES AND THE GENERATION OF THE SECONDARY ORGANISMS 



\section{CHAPTER I THE ORIGIN OF VIRUSES}

\section{The Generation of Phage}

The possibility of the spontaneous generation of some viruses has long been argued by a number of authors. Among many viruses phage appears to have been discussed most frequently in connection with this problem. Phage is usually found in abundance in animal feces especially in those of chickens. The writer's colleague, Ohashi (1) (2), has studied the origin of phage in chicken feces and has come to the conclusion that the phage in chicken feces is originated from the chicken itself.

It may appear at first sight that the phage present in the feces may come from the phage taken by the chicken with food and that the phage, if enters undamaged the alimentary tract, may be able to multiply by affecting the phage-susceptible bacteria that may thrive there normally. However, it has been confirmed by Ohashi that phage experimentally fed not only failed to multiply in the chicken tract regardless of the presence of phage-susceptible bacteria thriving there, but was destroyed to such an extent that only its extremely small portion could be defected in the feces. The phage amount detectable in the feces was only of the order of $1 / 1,000$ to $1 / 10,0000$ of that given by the mouth, showing that almost all the phage particles, far from multiplying in the tract by affecting the bacteria, had been inactivated before excreted with the feces.

Thus, the conjecture that the phage in the feces may be originated from the one taken with the food does not appear to be correct, in spite of the fact that in the alimentary tract phage-susceptible bacteria are present abundantly. It cannot be expected that phage is continuously taken by chickens with their food in so large amounts. If phage is actually fed in any reasonable quantities, it will either be totally inactivated or, if its small portion can escape the inactivation, it will never reach the amount which can account for the large phage amount usually found in the feces.

In addition it was found by him that phage amount in chicken feces was intimately related to the kind of diet; thus, the feeding of diet, such as wheat bran, soy-bean, greens, and rice bran, resulted in phage release, while the release would cease almost totally on the 
administration of polished rice or wheat. Among the former group of diet, wheat bran was found the most effective. This effect was not destroyed even when the bran was exposed to $120^{\circ} \mathrm{C}$. for $15 \mathrm{~min}$., indicating that the effect was not attributable to phage contained in the diet, because no phage is so stable as to stand such high temperature.

The conclusion that phage present in the feces is produced by the chicken itself is supported strongly by the fact that phage was found in a variety of organs of the chicken, such as brain, liver, kidney, and lung; especially abundantly in mucous membranes of intestines. It is, however, a note-worthy fact that the demonstration of phage in organs was possible not only with chickens but also with mice, in whose feces phage could scarcely be found. Mackinley (3) likewise isolated phage from the organs of man and various other animals, and Tempe and Uhlhorn (4) from the blood of guinea-pigs and rabbits. These facts indicate that the presence of phage in animal organs is a general feature. It is strange to say, however, that usually phage cannot be found in the feces of animals such as mice, guinea-pigs, and rabbits, unlike in chicken feces. What is the reason then why phage is demostrated so readily in the feces of chickens while not in those of many other animals?

As above stated, the phages given by the mouth to the chickens were mostly inactivated in the alimentary tract, only their extremely small portion being excreted with the feces, while, on the other hand, it was confirmed that the degree of the inactivation in the alimentary tract was much greater in guinea-pigs than in chickens. In guinea-pigs usually the complete inactivation occurred and no phage was excreted in the feces even if extremely large amounts of phage were fed. This suggests that the absence of phage in the feces of the latter animals might be attributed to the complete inactivation of the phage which might be excreted by the organs into the alimentary tract.

This deduction is further supported by the following fact: Guineapigs given with castor-oil excreted phage markedly in their diarrhoeatic feces, showing that the phage produced by organs was excreted without being inactivated in the intestine owing to the diarrhoea. Besides castor oil, some chemicals such as ethyl alcohol and sodium bicarbonate could occasionally exhibit the same effect, but the phage demonstration in the feces was possible only when diarrhoea occurred.

It is said that phage was for the first time isolated from human feces suffering from dysentery. This might likewise depend upon the diarrhoea whicl prevented the inactivation of the phage. The phage isolated from $\iota$ dysentery patients is likely to be thought as being specific to the dysentery bacillus, but such appears not the case, generally no peculiarity being found in the specificity of the phage isolated from the 
patient feces (5).

The existence of an intimate relation between the kind of diet and the phage excretion into the chicken feces is apparently brought about by the difference in the digestibility or coarseness of the diet. Thus, if a diet consists of coarse, indigestible materials, the defecation will readily occur in favour of the phage preservation which will further be effected by the envelopment of the phage by these coarse, indigestible materials so as to be protected from the action of destructive agents which may be present in the alimentary tract.

Wheat bran, as mentioned above, was highly effective in the phage excretion but its water or alcohol-ether extract had no action, though the residue was somewhat effective. Moreover it was confirmed that a coarser bran could excrete phage more readily than finely smashed one. Further, wheat bran is the crust of wheat grains, but wheat grains themselves proved to be inactive. There seems no doubt, therefore, that the effectiveness of some diet in phage excretion was chiefly governed by its physical rather than its chemical properties.

In the feces of animals such as mice, rats, guinea-pigs, and rabbits, the phage detection was generally difficult, whereas in those of dogs or donkeys it was usually easy, a fact possibly dependent upon the property of feces which varies with the species of animals, the coarseness of the ingredient or the speed with which the food residues pass the digestive tract may play an important role. The digestive fluids likewise may vary with animals and may have some connection with it.

Phage seems, however, not always produced by animal organs, since the phage detection in chicken feces is only possible in summer or at least in warm seasons; in winter no phage is found even when the chickens are fed wheat bran or led to diarrhoea by the administration of castor oil. The cells of organs, therefore, seem to alter with the change of seasons in their character of producing phage; in winter may fall into a state unab!e to produce the phage. Thus the property of the cells of producing phage may not be constant, and may possibly be altered by proper agents other than the seasonal effect. The above mentioned action of castor oil or some diets such as wheat bran might be accordingly not only dependent upon their properties to hinder phage inactivation but also upon some other properties which may cause the cells to produce phage.

In addition to the phage having thus its origin in animal organs, there appears to be another group of phage. It is generally known that certain bacteria produce an agent capable of acting as a phage upon certain other bacteria. Such bacteria are termed lysogenic. There seems therefore two groups of phage, one of which is produced by lysogenic bacteria and the other by certain animal celis. 
That the phage detectable in animal feces is not originated from such lysogenic strains can be shown also in the fact that there are distinct differences between the phage found in feces and that produced by lysogenic strains. Feces phage is much less resistant to heat than the bacteriogenic phage: for example, whereas the former is inactivated to such an remarkable degree as $1 / 1,000$ to $1 / 10,000$ on the exposure to $65^{\circ} \mathrm{C}$. for $5 \mathrm{~min}$., the latter undergoes entirely no change even when heated at this temperature for 1 hour, and $75^{\circ} \mathrm{C}$. for $20 \mathrm{~min}$. is necessary for its inactivation to this degree; with several samples Ohashi (5) has found without exception this distinct difference between both groups. Moreover, he has confirmed that the plaques produced by bacteriogenic phage are commonly uniform in size, whereas those produced by feces phage tend to vary remarkably, indicating that there are much greater individual differences among feces phage particles than among bacteriogenic phages.

\section{The Reason for the Phage Production from Healthy Bacteria}

According to the writer's theory, a virus is an assimilase similar to the protoplasm or a portion of the protoplasm. A virus can act as an assimilase upon a certain cell when the cell protoplasm is weaker in the assimilase action than that of the virus. A virus exists usually in minute particles into which the protoplasm has been disintegrated and coagulated following the infection with the parent virus. Through this. infection the structure of the protoplasm is to be so changed as to become identical with that of the virus. If the structure of a particle is stable enough to retain the changed protoplasm structure the particle can behave as the virus towards another cell protoplasm, thus developing its template action.

This theory will naturally lead to the reasoning that normal protoplasm particles or fragments of some healthy cells must likewise be able to behave as a virus to some other cells having a weaker assimilase action, provided the structure of the former cell protoplasm capable of acting as a stronger assimilase is preserved in the fragments or particles. In such a case, the healthy cells may be said to have the property to produce a virus acting upon certain other cells. Lysogenic bacteria above cited should be recognized as such cells.

Thus, if certain bacteria are disintegrated into minute particles, or fragments and if the original protoplasm structure is held unchanged in the particles or fragments, then the latter will be able to act as a phage upon certain other bacteria which have a weaker assimilase- 
action than the former. All the particles, however, may not always be able to act as the phage, for it is expected that sometimes certain particles only, whose structure is especially stable and strong, can exert the template action. Virus particles produced by healthy cells, therefore, also should have an intimate connection with nucleic acids.

In this connection it should be noted the important fact that disruption of lysogenic bacteria does not liberate infectious particle (6), that is, lysogenic bacteria seem to contain no virus, but that if the bacteria are treated with certain chemical or physical agents, such as ultraviolet ray, phage liberation will occur (7). This may result from the need of a certain slight modification in the healthy protoplasm protein structure for the exhibition of the virus action. As detailed later, in the bacteria and animal cells the protoplasm protein threads appear normally to be present in a partially folded state and the particles consisting of such partially folded peptide chains cannot act as the template. Accordingly, for the virus liberation, healthy cell protoplasm proteins have to unfold the chains under appropriate conditions. Ultraviolet ray may lead the chains to unfold. As we shall have many occasions to consider in detail of this important problem later, for the time being it may be wise to put aside this problem and make a further advance on the question we are now facing.

Now, another reasoning led by the above theory is that the susceptible bacteria will become lysogenic when the bacteria are affected by the phage produced by lysogenic bacteria, since the structure of the susceptible bacteria is to be changed to become similar to that of the lysogenic bacteria through the infection, whereby the protoplasm of the susceptible bacteria is endowed with the strong structure able to act as a strong assimilase.

As is well known this is actually the case. Bacteria infected with a phage derived from a certain lysogenic strain are changed into the lysogenic strain which produces in turn the phage by which they have affected. Freeman (8) has isolated virulent strain of $C$. diphtheriae from avirulent strain cultures by incubating with a specific phage; the bacteria were found to become resistant to the phage, and even lysogenic.

Freeman was unable to find any immunological difference between the original avirulent and derived virulent strains, but since the avirulent strains got possession of the faculty to produce toxin besides becoming both lysogenic and phage resistant when inoculated with the phage, it is evident that the general property of the bacteria was altered, although the difference failed to be detected immunologically.

The manner in which phage-susceptible bacteria are changed into lysogenic is illustrated diagrammatically in Fig. 16. The faculty to induce such a change must be, of course, present also in the intact protoplasm 
or in the cell itself, since even in its fragments, $i . e$. , in the virus-like particles, the faculty can be proved. Smith (9) stated that non-lysogenic strain can be changed lysogenic through the incubation with the lyso-

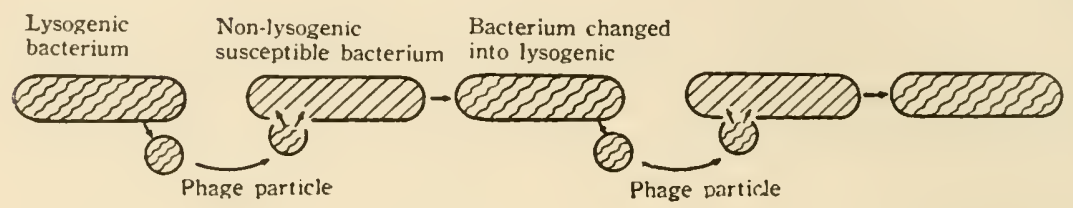

Fig. 16. The change of phage-susceptible bacterium irito lysogenic by the phage produced by a lysogenic strain.

genic strain; this change may not necessarily be ascribed to the action of phage freed from the lysogenic strain, because such a change should naturally to be expected in the interaction between two different strains without any participation of virus-like particulate agents.

If the above conception as regards lysogenic strains is correct, every strain of bacteria may be lysogenic to strains having weaker assimilase actions than it, provided that the mutual combination between them is established. Roustree (10) has proved that 27 out of 30 strains of staphylococci which have been chosen arbitrarily are lysogenic, and she stated that also with the remaining three strains lysogenicity may be proven if suitable indicator strains are found.

Such a phenomenon may not be confined to bacteria. If particles or fragments of the protoplasm of some animal cells can combine with certain bacterial protoplasm of weaker assimilase action the particles may be able to act as a phage upon the bacteria. The phage found in animal feces may belong to this category.

In this connection, however, a serious question may arise as regards the establishment of the combination between the two different cell protoplasm, since there should exist in a virus a structure which correspond to a structure of the host cell protoplasm for the establishment of the combination between the virus and the host cell. The relationship in the structures between a virus and the host protoplasm may be analogous to that existing between an enzyme and the substrate. Viruses can combine with host cells through such a structure, and after the combination, structures in the virus not in conformity with those of the protoplasm exert their action to rearrange the protoplasm structure.

Since the structure causing the combination distributed between a virus and the host protoplasm must be present, in general, between two protein molecules of a similar kind as already discussed in cletail in Part II, a certain strain of bacteria can act as lysogenic upon a certain 
other strain of the same kind of bacteria, that is, a certain strain of colon-bacteria or of staphylococci can be lysogenic to a strain of colonbacteria or of staphylococci, respectively. In this respect it may appear impossible that the protoplasm of animal cells share a structure which is in conformity with a structure in bacterial cells. Nevertheless, this must be the case in order that the combination between the fragments or the particles of the protoplasm and the bacteria upon which the fragments may act as a phage is established; and this seems actually to be the case. Bacteria are commonly parasitic on some animals, and since the correlation between bacteria and the animals is considered to be analogous to that between a virus and the host cell, there should likewise exist a structure in common between the bacteria and the animals.

If some bacteria combine with certain animal cells whose assimilase action is weaker than that of the bacteria, then the animal cell will be assimilized by the bacteria or, at least, will be disturbed in their protoplasm structure, thereby the bacteria may be able to multiply and the animal cells may fall into pathological conditions. In order to avoid such a disturbance the animal cells should be stronger than the bacteria in the protoplasm structure. If this were the case, also the fragments or particles of the protoplasm of such animal cells would also have a stronger assimilase action than the bacteria and would be able to act upon the latter as a virus. Such particles if excreted with feces would be called a phage.

As is generally known, various animal organs produce an agent known as lysozyme which can affect certain bacteria to lyse them. Fleming (11) who found first this agent emphasized the similarity of the agent to phage. There is, however, a striking difference between these two agents. Namely, lysozyme cannot multiply unlike viruses. Thus it seems possible that lysozyme can combine with some bacteria and disturb the structure of the bacterial protoplasm to cause disintegration or lysis as does phage, but without producing exact replica and accordingly fail to multiply, presumably because of its structure not fitted for acting as a template. Even phage, under some conditions, only can cause lysis of bacteria without multiplying as pointed out already.

On the other hằnd, as mentioned above, if the bacteria are stronger than some animal cells, the cells may be injured severely by the bacteria. In such a case particles of the bacterial protoplasm may likewise be able to affect the cells. Bacterial haemolysin may be regarded as one of such agents; haemolysin is apparently protoplasm particles of bacteria which can combine with red blood cells; the structure of the latter is disturbed by the particles (Part II, Chapter 
III), but without producing the replica corresponding to the bacterial template, so that haemolysin like lysozyme cannot be called a virus (12).

\section{Non-Pathogenic Viruses}

Bacteria, as a rule, undergo lysis when affected by a phage, but lysis does not always follow phage infection. Some phage can infect typhoid or coli bacteria without inducing lysis, though the phage can multiply through the infection (13) (14). Bacteria infected with a phage acquire the property to produce the phage like a genuine lysogenic strains; thus certain bacteria at least can only be altered in their character through the infection with a certain phage, without being lysed.

Since the multiplication of a virus consists in the replica formation in the host cells, the cell disintegration must be only a result of the multiplication, never an issue of necessity. The disintegration of bacteria into minute particles in case of the phage infection may be due to the destruction or the weakening of the mutual association of elementary bodies of bacterial protoplasm caused by the structural disturbance by the virus. Prior to the occurrence of lysis, bacteria infected with phage, as is well known, may become indistinct in their contour and swell up, a fact which is probably attributed to the appearance of lyotropic groups on the surface of elementary bodies as a result of the structural disturbance, most probably of the unfolding of the peptide chains. The disintegration may be thus caused by the accumulation of water molecules among elementary bodies. If some cells can endure such a change no lysis may follow. It is also conceivable that the degree or the rate of the liberation of lyotropic groups may vary with the kind of viruses which induce the change; thus certain host cells are disintegrated by some viruses while not by others.

It has frequently been noted that the virus multiplication is not always accompanied by the manifestation of any pathological disorder. The pathological disorder may be manifest when the changes lead to the disintegration of the cells. Certain strains of influenza virus multiply extensively in the mouse lung without producing pneumonia, whereas a closely related variant may, under the same conditions, produce pneumonic consolidation. Moreover, some strains of Coxackie virus multiply to an equal degree in a number of organs but fail to produce lesions except at certain specific sites (15).

On the contrary in some cases pathological changes are most remarkable, while virus multiplication scarcely occurs. Ginsberg (15) 
found, for example, that infectious Newcastle disease virus particles produce extensive pulmonary consolidation in the mouse in the absence of demonstrable virus multiplication, the lesion being indistinguishable from those of influenza $A$ virus infection. It may be possible to regard lysozyme or haemolysin as virus-like agent which, like Newcastle virus in this example, can cause a remarkable damage in the cell without multiplication.

The injurious action of viruses upon the host cells may probably be effective only during the process of the virus multiplication; with the finish of the process the host cells will be released from the suffering and will be able to behave like apparently healthy cells, unless the structure itself which has been provided by the virus is injurious to them. A virus as a rule can be detected in abundance in plant cells which have been recovered from the infection by the virus. Also in the case of animals, certain viruses are reported to be detected in some tissues long after the recovery from the virus infections.

The injurious effect, as above stated, is not necessarily associated with the virus multiplication, a fact which is clearly shown in the term of "inapparent" or "subclinical" infection, which is often regarded as a characteristic manifestation of viruses. Thus it is generally accepced that viruses tend to cause infections that do not give rise to clinically evident diseases.

It will, therefore, naturally follow that fragments or particles of some protoplasm capable of producing replica in other cells may fail to be realized of their existence because of their inability to cause any injurious effect, and that, even when their existence was acknowledged, they would not be called viruses. The well known principle found by Avery et $a l$ (16) with pneumococci can be regarded as one of such "viruses." This "virus" is produced from virulent, capsulated pneumococci and can convert avirulent, non-capsulated culture to the virulent type. The possible mode of action of this agent is illustrated in Fig. 17.

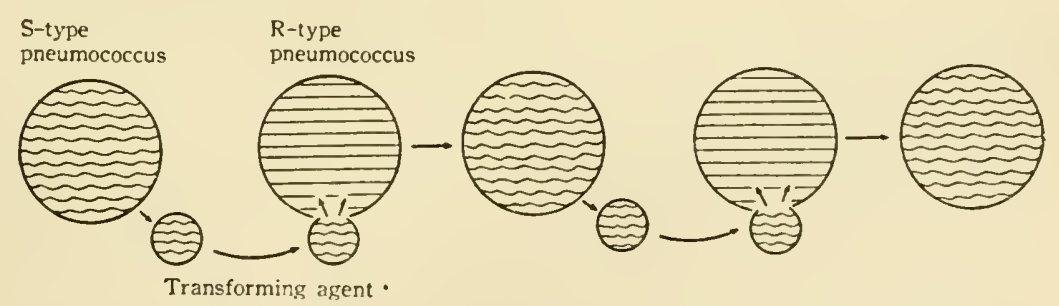

Fig. 17. The change of R-type pneumococcus into S-type by the transforming agent produced by S-type. 
It is most remarkable that this agent is reported to consist mostly of nucleic acid, and according to Hotchkiss (17) the protein content of the agent can be, at most, 0.2 per cent and is probably less. If this is true, it must be admitted that the template action is sometimes accomplished by nucleic acid only without being associated with any protein, although this possibility is not entirely inconceivable as dissussed previously (Part II, Chapter VIII).

The nucleic acid concerning this reaction is confirmed to be desoxyribonucleic acid, whereas this acid isolated in a pure form is reported to exist in forming virus-like particles; its molecular weight about $8,000,000$ and the length of the particle, $3000 \mathrm{~m} \mu$ (18). This suggests the possiblity that even the particles composed of nucleic acid only can behave like a virus.

From avirulent strain could be extracted similar nucleic acid resembling, in its chemical composition, to the agent isolated from the virulent strain, but it was wholly inactive in producing transformation (19), a fact which should be naturally expected, since the agent from the virulent strain can transform the avirulent only because the former structure is more powerful than the avirulent, and accordingly this avirulent strain itself or its particles should be unable to exert any influence upon the virulent.

The nucleic acid complex isolated from the avirulent strain ( $R-$ type), however, can transform a variant (ER-type), which produces very rough colonies, to $\mathrm{R}$-type, indicating that ER-type is still weaker than R-type. In this connection, it should be noted that transformation in the reverse direction, $R \rightarrow E R$, can be carried out with a transforming agent of the ER variant if anti-R serum is present (20). This fact shows that despite its weak structure the agent isolated from $\mathrm{ER}$ variant can overcome the $\mathrm{R}$-type owing to the assistant cooperation of the antiserum capable of disturbing the structure of R-type.

Similar transforming agents have been isolated from various other bacteria such as E. coli (21), Shigella (22), and Haemophillus (23). Furthermore, it has long been known that certain strains of bacteria, grown in the presence of culture filtrates or extracts of related organisms, acquire some of the properties of the latter. Thus, it was possible to convert colourless and nonproteolytic culture of $B$. pyociyameus into virulent and pathogenic form, by two consecutive passages in the culture flitrate of a virulent, pigmented and proteolytic strain of the same species. There are also claims that cultures of streptococci or of Gram-negative bacilli exposed to the cells or products of serologically different, but biologically related species, can develop the specific agglutinability of the latter (24). 


\section{Latent Viruses}

A vast number of examples of virus production by apparently normal cells can be shown also in animals. For instance the occurrence of Theiler's mouse encephalitis virus in the intestines of normal albino mice has been well established. As reported by Olitzky (25) and ascertained by other workers, whilst foetal and sucking mice up to 12 days of age are free from this intestinal virus, 20 to 25 days old mice harbour it irregularly, 30 days old mice invariably and old mice (6 or more months) again irregularly. Again, it is generally known that in the submaxillary gland of normal guinea-pigs a virus is contained, which can cause a meningeal symptom, when injected into the brain of guinea-pigs. It merits attention that this submaxillary-gland virus, like that of Theiler, is found only in the gland of adult animals, never in the gland of young ones. Furthermore, an extract of normal rabbit skin was confirmed by Daneal (26) to be able to induce serially transmissible papillomata on the skin of other domestic rabbits.

A series of neurotropic viruses, like that of poliomyelitis, have been isolated from various sources of animals, apparently having no association with the disease, such as feces or excretes of animals or men, and also from insect juices. A group of viruses having the name of Coxackie may be regarded as a type of such viruses. It is a note-worthy fact that non-biting flies are regarded as disseminating agents of both poliomyelitis and Coxackie virus, both viruses being frequently recovered from flies. There are striking similarities in the distribution of these fwo groups of viruses in nature.

A factor in brain tissue which induces acute disseminated encephalomyelitis, when injected into rhesus monkeys has been found by Kabat et al. (27) in human, monkey, rabbit and chicken brain. It is present in the spinal cord of 3 days old rabbits, but does not appear in the rabbit brain until about the 12 th day of life.

An infectious agent, which appears to be a virus has been isolated from the liver of a normal wild raccoon which has led to a highly fatal type of disease characterized by conjunctivitis and an elevated serum bilirubin, frequently accompanied by jaundice on inoculation of raccoons. Ferrets also appears to be susceptible to infections with this agent $(27 \mathrm{a})$.

Such examples appear to be too numerous to be cited, but are commonly regarded as evidences of detection of "latent viruses", i.e., viruses present in host cells causing an inapparent infection. Presumably, this may hold for some examples above cited, but it appears 
more reasonable to consider that most latent viruses are protoplasm particles of healthy cells stronger in the assimilase action than certain other cells on which they can act as viruses.

Similarly in plants the detection of latent or silent viruses is not unusual. A well known example of plant viruses, apparently belonging to this category, is produced by paracrinkle virus, which is found in every plant of the potato variety named King Edward, and which is actually regarded by some workers as a normal product of the metabolism of the plant with the characters of a virus when transferred to other potato varieties. Moreover, the so-called latent virus of potato mosaic is said to occur in almost all of the potato plants grown in America. It causes no damage to the potato plants, and, because of this fact and because of its almost universal presence in potato plants, it has come to be called the healthy potato virus. This has likewise occasionally been regarded as a normal constituent of potato plant. According to Smith (28), samples of apparently healthy sugarbeet and mangold collected at random from different farms in England and Scotland were all found to contain a virus, the presence of which can be rapidly demonstrated by inoculation of the sap to the first leaves of cowpea seedlings, which results in the production of characteristic lesions.

Whereas various plant viruses are, as is well known, transmitted by insects, it is believed that viruses are latent in a high percentage of apparently healthy caterpillars (29). Also there are many evidences that neurotropic viruses are present in normal insects which transmit commonly various animal virus diseases. Since the relation between insects and animals or plants, upon whose blood or sap they thrive, may be analogous to the relation between bacteria and animals, on which the bacteria are parasitic, there should be a common protoplasm structure between them. Therefore it seems a natural result that the protoplasm particles of some normal insects behave as viruses towards some animals or plants. The reason why the protoplasm particles of animal cells can act as a phage upon some bacteria can be explained in a similar way as already discussed.

The various facts above cited have naturally led a number of workers to the theory that viruses are originated from normal cell components. For example, Darlington (30) has postulated viruses originating from specified cytoplasmic units such as plasmagenes. Again, de Buy and Woods (31) have expressed the opinion that viruses are produced from plastids and mitochondria which have been altered in the form and function.

Since these cytoplasmic particles are known to contain nucleic acids in large quantities, also from the writer's theory it appears 
highly probable that such particles can act as viruses. It may, however, be unreasonable to postulate these particles to be only agents able to become viruses. Nucleic acid is contained in most abundance in genes themselves and there is a good reason to consider that these particles, the genes, have the greatest template action in the cell. Therefore, genes are most likely to behave as viruses when they are liberated from the cell. On the other hand, it has been suggested by a number of authors that viruses may be genes that have gone wild, acquiring in the process the ability to exist independently in the cell and to move from cell. Thus the "free gene" theory has been advanced by Muller (32) and Duggar and Armstrong (33). 


\section{CHAPTER II}

\section{THE GENERATION OF VIRUSES}

\section{Changes in the Struciure of Protoplasm Protein Leading to Virus Generation}

Many evidences cited in the previous chapter may indicate that the normal protoplasm particles of certain cells can sometimes exhibit their template action upon certain other cells as viruses. Although the existence of such normal particles never mean the generation of new viruses, it seems highly probable that new viruses can arise in some cells if the protoplasm is altered in its structure following the change of environmental factors, because the kind of viruses should be determined by the structural pattern of the protoplasm.

Bacteria sensitive to penicillin usually develop resistance to the antibiotic when grow in its presence. It is of most interest that the extracts of bacteria thus acquired the resistance are able to transmit this character to other bacteria (34). The effective agent prepared from a resistant strain has been reported to be involved in a nucleic acid fraction (35). This can be interpreted as indicating that the bacterial protoplasm undergoes a change in its structure under the influence of the antibiotic and that, as the newly formed structure is stronger than the original one, some particles of the protoplasm thus changed containing nucleic acid in rich amount can exert like a virus their structural influence upon the unaltered bacteria. This may, therefore, be regarded as an example of the generation of a new virus due to a certain stimulus given to the cell.

It is, however, unreasonable to expect that a newly formed structure is always stronger than the original. Voureka (36) has recorded the interesting observation that some strains of penicillin-resistant staphylococci and streptococci lost their resistance during exposure to a penicillin sensitive microorganisms or to the extracts of sensitive bacterial cells. It should be noted that the sensitizing principle was found in the ribonucleic acid fraction isolated from the sensitive bacteria (37) (38). This fact indicates that the newly formed structure is weaker than the original and, therefore, the original structure can overcome the newly formed one. The sensitizing principle must be nucleic acid rich particles, behaving like a virus towards the adapted bacteria. 
Virus-like agents seem to be produced occasionally during the adaptation process of microorganisms. It cannot be considered, however, that the generation of a structure capable of acting as a virus is necessarily associated with the adaptation. On the contrary, even if a structural change takes place which is unfavourable for the organism, a virus-like agent will be found in the fragments of the protoplasm thus changed in its structure, provided that the newly formed structure is stronger than the original.

As is generally known, viruses are liable to alter in their characters with the change of environmental conditions. For example, some viruses undergo variations when exposed to proper amounts of $\mathrm{N}$-mustard or of ultraviolet light. Such a variation is, as will be mentioned later, considered to be based upon a structural change in the virus protein caused by the environmental effects. These effects capable of giving rise to the change in viruses, $i$. $e$., in particles or fragments of protoplasm, can, as a natural result, exert their influence upon the protoplasm itself to cause changes in its structure, altering the character of the cell itself. Thus the variation or mutation of organisms will be brought about as a result of the alteration in the protoplasm protein due to the change in the environmental conditions.

In fact, microorganisms such as bacteria are known to undergo variation or mutation when treated with $\mathrm{N}$-mustard or with $\mathrm{X}$-ray. If the newly formed structure of the protoplasm is stronger than the original, its particles will be able to act as a virus. In short, it may be said that the mutation of organisms is, in general, liable to be associated with the generation of a virus.

However, as will fully be discussed in Part V, there are good reasons to suppose that newly produced structures are, as a rule, weaker than the original, so that it cannot be expected that mutation is always associated with the production of new viruses. On the contrary, it should be considered that the generation of new viruses will take place only on rare occasions, since a newly produced virus is always provided with a structure weaker than the original.

\section{Environmental Change and Virus Generation}

As stated in the foregoing chapter, there are many evidences suggesting that normal, healthy cells are producing viruses. The viruses appear, however, are not constantly excreted by the cells, but in most cases their production seems to be connected intimately with the environmental conditions. For example, Theiler's virus cannot be found in the feces of very young mice, though when the mice grow older to 
a certain age the virus is constantly excreted. The same is true for submaxillary-gland virus of guinea-pigs. These facts suggest that the animal cells may undergo a change in their protoplasm structure with the growth of the animal to yield the virus at certain periods of age. Such physiological change of the protoplasm structure to become capable of acting as a virus may be regarded as another example of virus generation.

The fact that phage can be detected in chicken feces in warm seasons, especially in summer, may also be explained by the assumption that the protoplasm structure of some chicken cells capable of acting as a phage can be provided only in warm seasons, in winter the structure being lost.

As emphasized by Bawden (39) the flowering-stimulating agent called florigen, present in the leaves of some plants, is remarkable in its virus-like properties; thus, like a virus, it moves from the cells in which it occurs initially and can be transmitted between plants by grafting. It is a fact worthy of note that this agent can be produced in the leaves of some plants when the latter are exposed to day-light of an appropriate duration. This may indicate that a certain structural change may be brought about in the protoplasm of the leaves on the exposure to day-light, the change being transmitted by a viruslike agent to another plant to stimulate the flowering. In this connection, it is of interest to note that Japanese encephalitis is believed to occur frequently when one's head is exposed to the violent daylight of summer. Again lysogenic bacteria which usually fail to liberate phage may be lysed with phage production on the irradiation with ultraviolet light (7).

These facts have led the writer to consider that the environmental effect may cause a change in protoplasm structure of some cells, the fragments or the particles of which are subsequently enabled to transmit the changed structure to other cells as a newly produced virus.

It should, however, be emphasized that for the production of a structure capable of acting as viruses, there should exist the cell protoplasm having the character to be altered into the virus structure by the environmental effect. In other words, for the generation of viruses certain cells are necessary possessing the character to generate the viruses; namely, certain cells with the predisposition to yield the virus are needed in addition to appropriate environmental factors. Viruses are yielded by such cells when the cells are exposed to certain stimuli involving environmental changes. Thus, a certain protoplasm having a certain predisposition and a proper simulus are essential for the generation of a virus.

In the case of Theiler's virus, for instance, the mice having the 
predisposition to produce the virus are necessary. It is known that there are virus-free strains of mice which never produce the virus throughout their life.

Cancer is, in a sense, a virus disease. As is well recognized, for its production above cited two factors, $i . e$. predisposition and stimulus, are indispensable. The important significance of predisposition has been well realized in the case of mammary cancer of mice, in the generation of which a virus is believed to be involved. Likewise in the case of fowl leucosis, a kind of malignant tumour caused by a virus, the predisposition of chickens plays an important role. It has been reported that in a certain line of chickens the disease is almost completely absent, whereas in some other lines over 50 per cent are afflicted by the disease (40).

Common cold is undoubtedly a virus disease, and as is well known the disease is established when a causative factor, chilling, is effected to man predisposed to catch cold. It merits attention that herpes febriles, known to occur following febrile discases such as pneumonia and malaria, may also be induced by artificial application of heat, such as infra-red ray or hot water bath. Moreover, poliomyelitis is liable to occur after trauma of various sorts especially following tonsillectomy. For the occurrence of these virus diseases it is needless to say that predisposition is also an essential factor. But it is commonly supposed that in such cases latent viruses are activated by the stimuli to cause manifest infections. This may indeed be an explanation more reasonable than to assume that viruses are newly generated by the stimuli, as will be fully discussed in Part V, but according to the writer's theory latent viruses are not the viruses themselves. They are present in a state of "provirus" before activation; "provirus" is the protoplasm structure able to be changed into virus structure by adequate stimuli. In short, by the term "provirus" is meant predisposition. In this connection, it should be mentioned that sometimes an active virus itself is enhanced in its activity by the presence of an adequate stimulus.

For instance, it is stated that when the leaves of tobacco piant are rubbed with carborundum the number of local lesions produced by tobacco mosaic virus increases approximately to such an extent as if the virus concentration were increased a hundred-fold. Potato virus $A$ is sap-inoculable to potato only when carborundum is used (39). It is also stated that when the virus of Rous sarcoma is injected into the blood of a fowl, the virus seems to become distributed throughout the blood and organs in the body but does not produce tumours. If, however, a muscle is injured immediately after the intravenous injection of the virus, then a tumour develops at the part of injury, but if the 
injury is made before injection of the virus, then no tumours are developed (41). A similar phenomenon is also observed with papillom virus: When a rabbit is injected intravenously with the papillom virus after smeared repeatedly with tar on the skin, a papillom develops only in the skin on which tar has been applied. The growth of the papillom is much more vigorous than the case when the virus only is applied, occasionally even developing to a cancer. Instead of tar, methyl-cholanthrene can be used to cause the same effect. Thus the infective faculty of the virus can be increased up to about a hundred-fold (42) (43).

These phenomena should not be compared to the above stated change of provirus into virus. These may only suggest that viruses can produce their replicas more readily in the cell protoplasm when the protoplasm is disturbed in its structure by proper stimuli. As discussed already, virus particles which have been inactivated to a certain degree can sometimes exert a favourable influence upon the intact virus to infect the host cells, showing that the inactive particles, though unable to produce their replicas, can disturb the protoplasm structure of the host cells contributing to the infection.

\section{The Seasonal Change in the Virus Infection}

Generation of viruses is apparently connected with the environmental change as above stated. Especially the connection seems intimate when the seasonal change is involved. The seasonal effect on the virus production is seen in the case of chicken feces phage as already mentioned. There are many other examples showing the same correlation. Poliomyelitis is prevalent in early autumn, common cold and influenza in winter, and smallpox from winter to early spring. German measles, according to our investigation, occurs preferably in early spring (44). Japanese encephalitis is epidemic only in summer.

In addition, seasonal factors affect the symptom picture of many plant virus diseases as well as the pattern of their occurrence. According to Smith (45), the bright yellow mottling characteristic of the aucuba type of tobacco mosaic does not develop under winter conditions in the glass-house but affected plants show an indistinct green mottle only. If, however, inoculations are made from a plant showing this indistinct mottle to other plants which are kept under artificial illumination, the characteristic bright yellow mosaic will develop in the illuminated plants.

Such seasonal effects may appear at first sight to be dependent upon the seasonal change in the resistance of host cells against the 
viruses not upon the generation of viruses themselves. However, as will be mentioned later, there are many good reasons to assume that effect is involved rather in the virus generation or in the provirus activation than in the alteration of the host resistance.

The writer made an investigation into the seasonal effect upon the incidence of measles, and obtained interesting data. The investigation was carried out with pupils of Japanese primary schools in Shanghai, when the writer was a member of the Shanghai Science Institute (46).

The seasonal effect upon the occurrence of the disease was found to be very striking as shown in Fig. 18. However, the curve shown

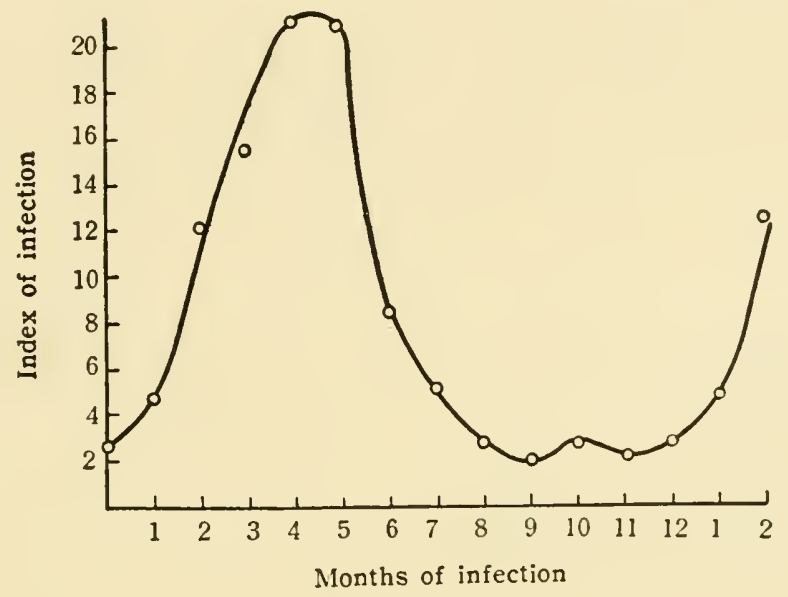

Fig. 18. The seasonal change in the incidence of measles in Shanghai.

in this figure representing the relation between the morbility and season is distinctly different from those obtained by other investigators at different districts as indicated in Fig. 19, in which it is also shown that the season effect varies considerably from district to district. In Shanghai and Cleveland the epidemic occurs only once in a year, in late spring or in early summer, while in other districts, especially in Wien and Lübeck, in addition to this peak, the second epidemic is seen in late autumn or in early winter, although in Shanghai there is some indication of this second peak.

On the other hand, it is of very interest that the seasonal change of impregnation of Japanese women bears a striking resemblance to that of the morbility as indicated in Fig. 20. The impregnation months cited in the figure are calculated by substracting 3 from birth 
months. Also with Europeans living in their native countries the same is the case. In most cases with Europeans two peaks are found as with Japanese in Aomori.

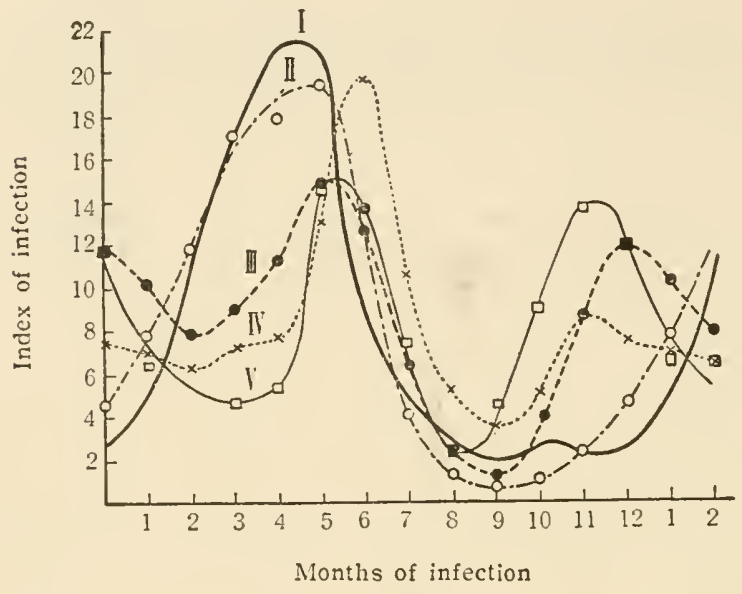

Fig. 19. The seasonal change in the incidence of measles in various regions. I : Shanghai. II : Cleveland. III : Wien. IV : Hamburg. $\mathrm{V}:$ Lübeck.

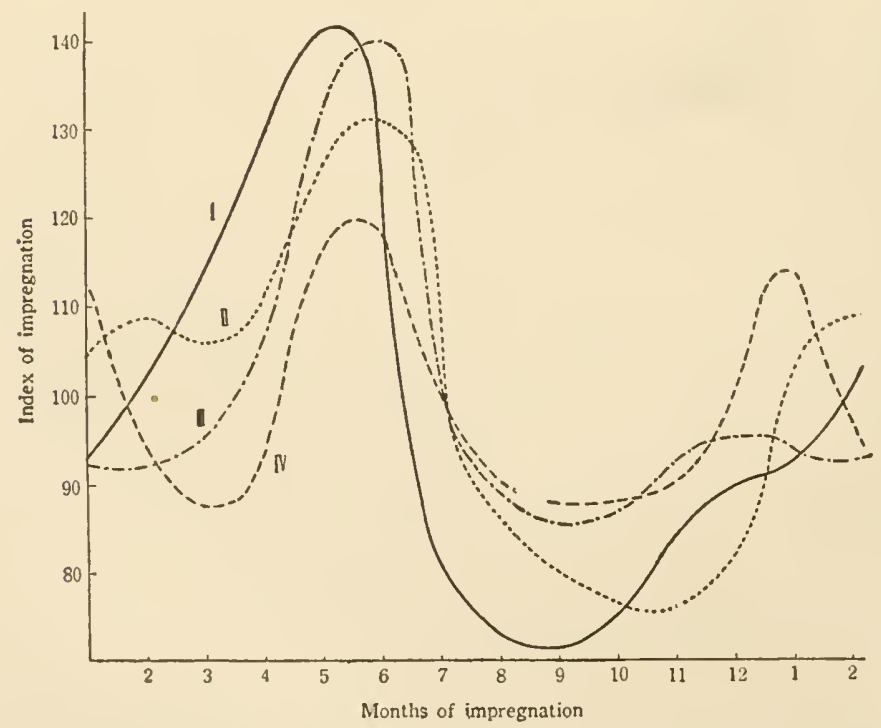

Fig. 20. The seasonal change of human impregnation in various parts of Japan. I : Nagasaki. II : Iwate. III : Hokkaido. IV. Aomori. 
This peculiar seasonal change in the human impregnation may be based upon certain metabolic activities mainly concerning with hormonal secretions, upon which the season may have great influences. It may be a reasonable explanation that the same seasonal influences are involved in the morbility change of measles, and the change in the susceptibility to the disease due to season may account for the phenomenon. However, if the susceptibility fluctuate regularly with season, measles virus should be present everywhere, always, and in abundance, in order that the susceptibility curve was precisely represented by the morbility curve, since the elevation of susceptibility would not be followed by the increase in the morbility unless the virus was present everywhere and in abundance. Can this actually be possible? As the time seems not ripe to discuss furhter of this problem, it will be left untouched until we have a proper occasion later.

In order to show how complicated is the problem concerning the virus generation, an experiment carried out by us (44) in connection with phage production will be discussed below. As above stated, phage is excreted from some animal cells, while flies appear likewise to excrete phage.

According to our investigation, common house flies almost constantly contained large amount of phage. However, phage could never be detected in the flies which had been raised from eggs in rearing boxes. If large amounts of phage were fed to such flies with foods, only small portions of the phage were found in the flies and even the detectable phage itself would disappear in a few days. It was quite impossible to make them carry phage as in naturally living flies. In addition, naturally living flies carry the virus only in summer, a fact comparable to the phage detection in chicken feces only in this season, suggesting that the virus is also excreted by the insect as by other animals.

Thus it was supposed that some factor or factors capable of stimulating the phage production might be lacking in the reared flies. We were, however, unable to find the supposed factor, although a variety of kinds of foods were given under various conditions in taking into consideration such physical factors as temperature and sun-light.

Though we failed to arrive at any definite conclusion on this problem, our finding may present some suggestions as to the origin of common cold. As stated above, for the occurrence of common cold the predisposition and the stimulus, $i$. $e$., chilling, appear to be necessary, but according to English workers (48), chilling neither induces nor favours common cold. The writer is inclined to think that such an unexpected conclusion, obviously contradictory to every man's personal experiences, has been arrived because of the isolated, unnatural living 
conditions under which the volunteers were left, for it is a very well attested fact that among a group of people isolated on a remote island, particularly if the community is small, common cold tends to die out. The experiments were reported to have been carried out in a solitary environment. The volunteers in this case, therefore, may be comparable to the flies bred by us in laboratory. In both cases some factor may be lacking against the virus production. According to the English workers, although common cold was induced in the volunteers when the virus was administered, the symptoms were unusually slight, mostly without fever, recovering in two or three days, and about 50 per cent of the volunteers did not show any symptoms. This may remind one of the fact above mentioned that phage harbours only for short periods, when the virus is administered to the reared flies. Thus the factor necessary for the virus production may be needed also for the thriving of the virus in the host cells.

It should be remembered in this connection that poliomyelitis virus and also Coxackie virus, which is closely related to the former, are very frequently isolated from flies, suggesting that these viruses, like phage, may be produced in flies. A number of evidences can be presented to suggest that epidemic poliomyelitis cannot be explained on the basis of person to person contact, but that the epidemic seems rather to be induced by a common source. Attention should be paid on the fact that the seasonal concentration of healthy virus-carriers, especially virus-carrying flies, is proved in the area where the disease is prevailed (49).

Like phage, both poliomyelitis and Coxackie virus fed to flies bred in laboratory not only fail to multiply in the flies but also fail to be retained in the insect body for long periods, suggesting that only naturally living flies can produce the viruses (50).

Rous sarcoma can be induced by the stimulus of tar, and it can be sometimes transmitted by a virus-like agent, whereas the action of the latter is remarkably enhanced by the simultaneous application of tar. A factor or factors like tar in this case may be lacking in the above case of common cold, whose virus may be unable without such a factor to exhibit its full action and at the same time the virus itself may not be produced. The virus, however, may be incapable of thriving indefinitely even when the factor is present, but gradually disappearing, and the virus may be generated de novo in succession. 


\section{CHAPTER III}

\section{IMPERFECT VIRUSES}

\section{Eczema or Allergic Dermatitis}

There is a large group of skin diseases called eczema, a dermatitis usually accompanying itching, erythema, vesicles or pustules. It is acknowledged that for the occurrence of this disease both the predisposition liable to be affected by this disease and adequate external irritants are needed.

The mechanism of eczema formation may be as follows: Skin cells having the predisposition may change in their protoplasm structure by the effect of a stimulus, and the change thus occurs may subsequently spread successively to surrounding cells followed by various pathological symptoms. The symptoms may be attributed in the main to the disturbance in autonomous nervous systems.

An abnormal reactivity of man to some agents is termed allergy, and eczema is commonly regarded as a kind of allergy. It should be borne in mind that allergy cannot exist without a peculiar predisposition which is occasionally hereditary and that the symptoms cannot be brought about without a proper stimulus. Common cold, as mentioned in the previous chapter, is also raised when a stimulus, chilling, acts upon the peculiar predisposition, although some other factor or factors appear to be needed, and hence sometimes common cold is also regarded as a kind of allergy.

If, in the case of allergic dermatitis, the fragments or particles of changed protoplasm were stable enough to retain its changed structure, they would be able to give rise to the same changed structure in other healthy cells as a virus. This is, however, never the case, presumably because of the lability or the weakness of the newly induced structure. The dermatitis cannot be called, therefore, a virus disease, although it may not be quite impossible to transmit the disease through the protoplasm fragment, if the latter is applied to a proper healthy region of the skin of the same individual immediately after the isolation.

The newly induced structure causing the dermatitis seems to be, on the one hand, stronger than the original, because the structure has to overcome the original structure at least for the period of its spreading, while on the other hand its strength seems to be lost 
promptly, as the pathological structure may soon disappear with the revelation of the normal. As a rule pathologically changed skin recovers its normal state in a few days when the causative agent is eliminated. Such a transient nature of the change may account for the failure of detecting the structure in the protoplasm particles. As will be stated later in greater detail, the change is transient because it is readily reversible.

\section{Cancers}

There are good many reasons that the structural change in protoplasm is generally reversible. In the case of the dermatitis above mentioned the reversibility seems very manifest, whereas there occur sometimes changes which appear to be irreversible, never to be expelled by the normal structures. Occasionally such changes may even overcome gradually the surrounding normal structure to proliferate still further at the cost of the latter.

Tumours may be involved in such irreversible changes. The high persistence of the changed structure may be ascribed to the transmission of the change to the genes themselves. The structural change in the gene, if occurs, should give rise to new cells which may be called tumour cells. The proliferation of the changed structure can be achieved by the multiplication of such cells. When the multiplicability is so striknig that the cells can proliferate indefinitely, the cells may be called malignant tumours, whose representative is cancer. The cancer cells multiply at the expense of normal cells so vigorously that the animals themselves affected by cancer are ultimately to be killed.

It is generally known that cancers are induced by a variety of physical or chemical agents, and it is also well realized that there is the predisposition to cancers. It seems, therefore, probable that the protoplasm having the predisposition must be changed in its structure by some stimulus to give rise to cancer cells. If cancerous structure was preserved in protoplasm particles, the cancer would be said to be transmissible by a virus. Cancers are usually readily transmissible by cancer cells but the transmission through the particles or a virus seems difficult to occur, suggesting the lability or the weakness of the structure.

One of the best known tumours proved to be transmissible by a virus is Rous tumour, a kind of cancers affecting fowls. The finding that this tumour could be induced by a virus might be shocking to those who regarded viruses as microorganisms like bacteria, but in 
the writer's concept of the nature of viruses it is never an unexpected result. In addition to this tumour, papilloma which affects cottontail rabbits is known to be transmitted by a virus, and which is commonly called Shope papilloma for it was described first by Shope as the fowl tumour is called Rous tumour after its first observer. Shope papilloma is characterized by the presence of warty or horn-like protuberances on various parts of the rabbit body, whereas human wart was also ascertained to be infectious through a cell-free filtrate long before the virus nature of Shope papilloma was confirmed. Mammary cancer of mice is likewise believed to be caused by a virus. This virus has been demonstrated in the milk and also in high concentrations in both the lactating mammae and mammary cancer of infected mice. The injection of so little as $10^{-5}$ to $10^{-6} \mathrm{gm}$. equivalents of lactating breast tissue has been sufficient to infect suitable host animals (51). The virus nature has been confirmed also with a kidney cancer of a frog, Rana pipiens.

Again, fowl leucaemia can be regarded as a kind of malignant tumours, and it was found that the infection could be transmittted by $0.000,0001 \mathrm{ml}$. of cell-free plasma, and that the virus could withstand drying for at least 54 days (52). Furthermore, both a myxoma and fibroma of rabbits can be transferred by filtrates of the tumour suspensions, and it is said that the virus of one of these diseases has a remarkable relationship to the virus of the other, and in view of the pathological changes caused by the viruses; these two diseases are considered to lie between tumours and usual virus diseases (53).

In these two tumours and also in fowl leucosis natural infections are said to be possible. Wound-tumour diseases of plants is likewise a tumour in which a virus is involved; this tumour is transmitted by an insect as in the case of usual plant-virus diseases (54). Nevertheless, in many other tumour diseases natural infections may fail to occur, although the transmission can sometimes be brought about artificially through cell-free filtrates; for instance, with Rous tumour it has been definitely confirmed that natural infection is entirely impossible despite the fact that a virus is involved in the tumour.

Carr (55) has emphasized the fact that the greatest recovery of infective virus from Rous tumour represented a yield of only one infective particle per 20 tumour cells. This appears to be surprising, since even when peculiar cytoplasmic particles only, containing large amounts of nucleic acid, could act as the virus, at least scores or hundreds of virus particles would be obtained per cell. This might be attributed to the requirement of a vast number of particles to cause a tumour because of the weakness or the lability of the tumour structure. 
Mueller (56) has shown that the infectivity of a filtrate of Rous tumour may vary from nil to such an effective dose as $0.001 \mathrm{ml}$, and moreover, it has been ascertained that the more slowly growing Rous tumours are less filtrable than the rapidly growing ones, and that occasionally the growth seems to lose its filtrability entirely, although still transmissible by cell inoculation (57). It has been found that the tumour of older chickens are tend to be more filtrable than that of younger ones. In addition to this effect of host age upon the filtrability, the age of the tumour itself, and whether the growth had been transferred by cell suspension or filtrate previously, prove to be important factors. It has been shown that non-filtrable growth becomes filtrable by the treatment with X-ray. In the case of Shope papilloma, although the virus is readily demonstrable with wild cotton-tail rabbits, if the tumour is transplanted to domestic rabbits, the demonstration of the virus becomes very difficult, usually impossible.

McIntosh (58) has reported that fowl tumours induced by tar can be transmitted to normal birds by cell-free filtrates, though this could not be ascertained by some other workers. On the other hand, it is known that if a fowl with a chemically induced tumour which cannot be transplanted by cell-free filtrates, is injected with Rous virus, then filtrates of this tumour will give rise to Rous tumour in other fowls (43), a fact which might suggest independent nature of the virus. This fact, however, can be interpreted as indicating the transmission of the strong structural pattern of Rous tumour to chemically induced one through the protoplasm particles of the former, thereby chemically induced tumour is endowed with the strong structure to be held in its protoplasm particles. Chemically induced tumours seem generally to have structures weaker than those of naturally occurring tumours, which may be the cause of the difficult demonstrability of the virus. Oberling and Guérin have proved filtrability of the tumour induced by methyl-cholanthrene in legs of chickens, but active filtrates were only obtained in the fifth and sixth passage (59).

\section{The Maintenance of Assimilase Action in Protoplasm Fragments}

Considerable damage of the assimilase action of protoplasm seems to follow even when the protoplasm is only slightly disintegrated, not so completely as to pass through porcelain filters.

On studying the number of metastases appearing in the lungs following the injection of tissue emulsion of a transplantable mouse tumour, Zeidman (60) claimed that, although the number of emboli produced is effected by such single factor as the initial size of the 
tumour inoculum, it depends to an even greater extent upon factors within the primary tumours as yet unknown. This may indicate that the structure of tumour-cell protoplasm varies with conditions under which the tumour grows, and that if it becomes stronger, the structure will remain intact to a greater extent in the emulsion, meanwhile the assimilase action itself becoming more effective, resulting in the increase in the number of metastases. If the structure became still stronger, it would be retained even in the filtrable particles; thus the virus would become demonstrable.

Gye and his associates (61) (62) have carried out a series of interesting experiments to demonstrate the virus etiology of animal cancers which cannot be transmitted with the filtrates. They have made extensive use of freezing and drying technics and have indicated the successful transplantation of cancer tissues after treatments which are considered sufficient to kill all the cells. Thus, normal embryonic tissue could not be successively transplanted, and no normal cells could be found by histological examinations after an hour's exposure to $-75^{\circ} \mathrm{C}$, and normal embryonic tissue was also killed when treated with glycerol and stored at $-40^{\circ} \mathrm{C}$, while fine emulsions of frozen tumour tissue made with distilled water, 40 per cent glycerol or 40 per cent glucose, caused cancer. Three mouse sarcomas, one induced chemically and the other spontaneously, were successively propagated with tissues dried completely after freezing, some mouse sarcomas even retained their activity after drying without preliminary freezing.

Hirschberg and Rusch (63), however, held the opinion that freezing technics alone are not suitable for the demonstration of virus etiology of the animal cancer, since there are a number of evidences for the survival of normal cells after freezing. Nevertheless, they acknowledged that the introduction of drying after freezing appears to provide a stronger evidence against the presence of intact cells.

According to the writer's opinion, it may be not impossible that the assimilase action can be retained in the tissue emulsion after the above stated treatments whether the tissue be normal or cancerous. Since such a treatment as freezing or drying may be less destructive upon the structure capable of acting as assimilase than the filtration procedure, even when the demonstration of the infectivity in the filtrate failed, tissue emulsions would sometimes stand the treatment and retain their infectivity. There are, however, a vast number of evidences that the structure of cancer cells are, as a rule, not so strong as to be capable of transmitting their structure in the form of protoplasm particles. If Shope papilloma is transplanted to domestic rabbits, not only do papillomas develop readily, but a considerable proportion of them undergo cancerous transformation, and at the same 
time the demonstration of the presence of the virus becomes impossible (64). It is a note-worthy fact that the demonstration of the virus always becomes difficult in this way, when tumours undergo cancerous transformation. This may indicate that the structure becomes weaker as it changes into cancerous one.

Such a weakness of cancerous structure may be based upon its juvenile and primitive nature. As will be fully discussed in Part V, there are sufficient reasons for supposing that non-differentiated, juvenile protoplasm structures are generally weak in their template action, so that they may be readily assimilized by other stronger, more differentiated structures. As is well known, chick embryos are extensively applied to cultivate various viruses, a fact which may be based upon their juvenile character to be readily assimilized by other protoplasm structures. In a similar way, it has been ascertained with a number of viruses that cancer cells can sustain the multiplication of virus like chick embryos (64a) (64 b).

Cancer cells are distinct in their embryonic nature. Although the cells having the embryonic nature are generally able to proliferate vigorously when existing as intact cells, their specific protoplasm structure may be lost because of its unstable character when the cells are decomposed into fragments. Even when the structure was retained in the fragments, the particles would be unable to act as the virus because of its inability to act as the template on account of its weak pattern.

The main reason why cancers are regarded as one of the most dreadful diseases may lie in their faculty to form metastasis in remote parts of the body. It is generally believed that for the metastasis the intact cells are indispensable. But it may be conceivable that the fragments of the cells may also transmit the structure, since the frägments can enter the blood-vessels in their fresh state and can reach, immediately after their liberation, healthy organs or tissues where prevails the high predisposition to the cancer.

Virus diseases are called as such because they are transmitted by protoplasm particles of the diseased cells, and hence they are regarded as infectious diseases, whereas ordinary tumour diseases, such as Rous sarcoma and Shope papilloma, cannot be called infectious, although they are sometimes transmissible by virus-like agents. They are not infectious because the infection cannot take place in natural ways, a fact which may depend upon the unstableness or the weakness of protoplasm structure of the tumour cells. As a rule the structure appears to be preserved only in the intact cells. Accordingly the disease can be transmitted generally by the intact cells, not by their fragments, and hence the structure cannot exist independently of the living intact 
cells. The structure shares its fate, therefore, with the organism in which it has developed. The structure has to cease to exist when the organism perishes.

The writer designates such structures as imperfect viruses. Nevertheless, it is impossible to draw definitely a line of demarkation between imperfect and perfect viruses. For example, as already referred to, natural infections are said to be possible with such tumours as fowl leucosis and plant wound-tumour. Moreover, a mutant strain of rabbit fibromatosis has been shown to cause acute inflammatory lesions similar to those of ordinary virus diseases instead of the usual proliferative change (65). Rivers (53) claimed that myxomatosis of rabbits, which has the remarkable relationship to this fibromatosis, mediates between tumours and ordinary virus diseases. 'The disease is transmissible to normal rabbits naturally by mosquitoes. Laboratory transmission of fibromas in rabbits by means of fleas and mosquitoes is also possible (66). Duran-Reynals (67) has made an investigation with a duck variant of Rous sarcoma and found that lesions induced in the central nervous system of ducklings by the virus is very similar to that observed in the infection by ordinary viruses.

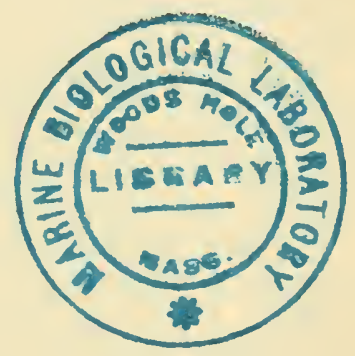




\section{CHAPTER IV}

\section{THE VARIABILITY OF VIRUSES}

\section{The Inheritance of Altered Structure}

Viruses are liable to alter in their properties, this being recognized as one of their characteristics. The altered property is occasionally transmitted to newly produced viruses, a phenomenon analogous to the mutation of organisms, though appearing to occur more frequently than the mutation of higher organisms.

Higher organisms may have acquired the prominent characters through their extremely long history of the struggle for existence. They seem to have elaborated wise mechanisms to maintain the good quality of these prominent characters, whereas, as this is not the case with viruses, viruses appear to change their structure abruptly and extensively with the change of environmental conditions.

For example, the virus of smallpox, normally infecting only man, undergoes variation to the virus of vaccinia when repeatedly transferred through the skin of rabbits. The virus has lost the capacity for causing smallpox, although antigenically it is still related to the variola virus, immunizing against it. Another variant of interest is a strain of yellow fever developed by passage through tissue cultures. This strain gives rise to excellent immunity to yellow fever and, since it is devoid of all tendencies to cause yellow fever in man, it can be employed as a live virus vaccine of high immunizing potency with safety. In a similar way, rabies virus changes its character when inoculated subcutaneously into a rabbit and passed in succession through a series of rabbits. The virus thus reduced in its virulence for man is likewise used as a vaccine.

For a virus, such a host change must be a great environmental change, so that viruses may be altered in their properties on continued passages in new host. A phage newly isolated from chicken feces is usually non-specific, being capable of affecting simultaneously several strains of bacteria, but when repeatedly transferred through a certain strain of bacteria, the phage will obtain the property to affect the strain specifically with the vigorous phage production. We (13) were able to reduce the virulence of phage by removing $\mathrm{Ca}$ and $\mathrm{Mg}$ from culture media of the host bacteria. "Virulence" designates here the 
rate of multiplication of the phage, which can be estimated from the plaque sizes.

For the variation of phage, however, the multiplication under the altered conditions is not essential. It can be induced, without any multiplication, by a mere addition of such substances as formaldehyde, mercuric chloride, or the antiserum against the phage (68) (69). In these cases the variation would occur in the reduction of the virulence, and the altered characters occasionally tended to be transmitted to new viruses produced from the altered virus. If these agents were added in proper quantities just enough to completely inactivate the phage, the antigenic specificity of the latter would be retained unaltered. The change in the virulence appeared to take place when the inactivation was imcomplete.

Similar phenomena have been observed with various viruses other than phage. For instance, Salaman (70) found that incubating tobacco roots infected with potato virus $Y$ for a week at various temperatures above $22^{\circ} \mathrm{C}$. produced less virulent forms. By keeping Rous sarcoma tissues for a long time at a temperature below $0^{\circ} \mathrm{C}$., Gheorghiu (71) has succeeded in reducing the virulence of the virus to be unable to kill the fowl, although active enough to produce the tumour. Bjorkman and Horsfall (72) found that a single treatment of influenza virus with lanthanium acetate or with ultraviolet radiation, followed by subsequent passage in chick embryos, altered the virus in its behaviour towards red blood cells. This altered property of the treated virus persisted through several passages. Miller and Stanley (73) have prepared the phenylureido derivatives of tobacco mosaic and found that although these derivatives produced as many lesions as untreated virus in Nicotiana glutinosa, they were less infective when treated in Phaseolus vulgaris.

Thus certain alterations in the structure of virus proteins appear to be accompanied by some change in the virus property, provided alterations are not so extensive as to entirely inactivate the virus. This should only be natural, since the property of a virus should be determined by the structure of the protein and the altered structure in turn should be transferred to the new viruses derived from it as the multiplication of a virus is nothing but the reproduction of the same structure. Newly formed structure of a virus therefore should always be inherited together with the altered characters. However, frequently newly formed character would soon be lost with the recovery of the original one. This is the most important phenomenon arising from the reversibility of protein structure, the detailed accounts of which will be given later. 


\section{The Variation of Newly Produced Viruses}

It may be not impossible to regard allergic dermatitis and cancers as virus diseases, but, since the pathogenic factors of these diseases, as a rule, fail to be carried by protoplasm particles, the pathogenic agents or viruses share their fate with the organisms in which they have been generated, and therefore become extinct without being transmitted to other organisms.

However, in the case of common cold, the virus of which may be generated under the influence of environmental changes, the situation is entirely different. If a man of the predisposition to the disease was exposed to chilling under some peculiar conditions, some change would result presumably in the cells of mucous membrane of the upper respiratory tracts, the change being able to spread successively in the protoplasm of the cells, accompanied by inflammation, which would in turn lead to the decomposition of the cells into fragments or protoplasm particles, and if the newly formed structure generated by the change remained intact in some of the particles, these latter, when transferred to another man, say, by coughing, would transmit the structure to cause common cold. It may be possible that further alterations would occur in the structure of the particles through some environmental changes during the periods they would affect successively different persons, and thus the virus would be able to undergo variations to become a more virulent virus entirely different from the one generated at the start.

This may also hold for viruses released from normal cells, $i . e$, normal protoplasm particles, capable of acting as a virus upon proper other cell. Thus, if a normal protoplasm particle of a certain organism capable of acting upon a man as a virus giving rise to symptoms like those of influenza undergoes variations during the successive passages through man to increase in its virulence for the latter, then it may be possible that the virus will acquire the properties entirely similar to those of influenza, thus becoming a strain of influenza virus itself.

Presumably each virus particle shows each individuality. This may be based upon the degree of precision in the replication which may vary with particles, and perhaps also upon the degree of damage which each particle may suffer during the course of the protoplasm decomposition into the particles or after their liberation from the cells. The difference in these degrees resulting from the dissimilarities in the structure of particles may cause the divergence in variations. 
In emphasizing of the virus individuality Schutz (74) stated that a striking feature of viruses is their heterogeneity when considered as a group. They are "individualist". They are not like in their resistance to heat, cold, drying, hydrogen ion concentrations, organic or inorganic chemical compounds or to the action of individual enzymes.

The occurrence of such a variation, however, seems to be a common feature of protein molecules, not being confined to virus particles only. There are a vast number of evidences that each molecule of proteins, such as toxins, antibodies, and enzymes, has each individuality.

It has been sometimes claimed that living matters are distinct from non-living matters in their variability as well as in their multiplicability. The variability, however, is merely a property of proteins, by no means a characteristic of living matters.

The individuality can be readily demonstrated with phage, for the individual property of this virus is clearly seen in the property of the plaques. Since a plaque is formed by a group of phages derived from a single particle, the degree or manner of the damage of bacteria due to the particle, derived from the single phage particle, is shown in it. Thus a large plaque will show the great multiplicability of the parent phage while a smaller plaque lesser multiplicability. If some parent particle has the property to lyse the bacteria completely a plaque of complete clearness will be obtained, whilst only a turbid plaque will be raised by a phage group derived from a parent particle causing an incomplete lysis.

Owing to such properties of plaques, we can easily demonstrate that even a phage sample, originated from a single particle, never consists of uniform particles. Some workers have attempted to divide phages affecting $E$. coli into several types, but it would be very surprising if there was no gradual transition among them.

The individuality of viruses is probably analogous to that of higher organisms, but presumably because of the easy occurrence of variation in viruses individual differences appear to be more distinct in them than in higher organisms. Virus particles having such distinct individuality may undergo further variations which may vary with each particle when exposed to various environmental effects. Thus the divergency in the individuality will become still greater, leading to the appearance of numberless strains.

Jensen (75) has isolated over fifty strains of tobacco mosaic virus from the yellow spots of the leaves. Some of these are apparently similar to tobacco aucuba mosaic virus, a strain commonly found in nature, but others differ from any strains previously described. Jensen distinguished the various strains by the ease with which they infect, 
and by the symptoms they cause on tobacco and tomato plants. All of them at normal temperatures produce only necrotic lesions on the latter, but some strains produce smaller lesions than others. All cause local lesions on tobacco plants, but some cause necrotic lesions and others chlorotic. Also, some give systemic infection and others do not. According to Kunkel (76), over 400 different strains of tobacco mosaic virus have already been recognized. And yet, still new strains are continuously being found to increase the number.

Of animal viruses the individuality or variability seems to be especially distinct in influenza virus. Sigel (77) stated that strains of influenza type A virus which originated in the same institutional outbreak from specimens collected on the same day, treated in the same manner, and isolated by individual procedures, exhibited certain biological differences; namely, dissimilarities in the capacity to agglutinate chicken and guinea-pig red cells, speed of elution from the red cells, and resistance to ultraviolet light. Even significant differences in antigenic composition were found.

As already mentioned, from various sources of animals have been frequently isolated agents that cause pathological symptoms, when transferred to proper other animals, like those of influenza or of encephalitis, suggesting that normal or pathological protoplasm particles of some animals tend to give rise to the pathological symptoms in other animals. Such agents may vary in their properties with animals or organs from which they have been generated, and even with the same organ of the same animal, considerable differences should be expected as with tobacco mosaic viruses or phages in chicken feces. If an agent is forthcoming having a striking affinity and a high virulence to a man, then a new epidemic of influenza or of encephalitis may follow. As is generally acknowledged, from every epidemic of influenza can be isolated a strain which differs in some or other aspects from any strains ever isolated from other epidemics. It is also known that new strains of neurotropic viruses causing symptoms like those of encephalitis or of poliomyelitis are continuously being isolated from various sources.

There is a rare disease called postvaccinal encephalitis, which may arise a short time after vaccination against small pox or rabies. It should be expected that agents above considered are sometimes contained in the vaccines, the emulsions of animal cells. It may be possible that by such agents postvaccinal encephalitis is caused in individuals with high susceptibility to the agent.

Following a variety of infectious diseases such as measles similar encephalitis is known to occur in which a virus causing the cencephalitis generated in certain cells of the patient by the stimulus of the 
infection may be involved. Margulis et al. (78) have claimed that they were actually able to isolate a virus from a patient of postinfection encephalitis. The agent causing postvaccinal encephalitis may either be present in normal animal cells or produced by the stimulus of the inoculated virus.

\section{Increase and Decrease in the Virulence of Viruses}

It is generally accepted that when a virus is propagated serially in a host different from the customary, it increases gradually in its virulence toward the host. For example, an influenza virus isolated from a man fails usually to produce no lung lesions in hamsters, although it can readily multiply in the animal, but on serial passage it will acquire the capacity to cause pulmonary consolidations and even the death of the animal (79). If rabies virus isolated from a dog is inoculated into a rabbit, the incubation period of the disease is about a fortnight, but if the virus is passed in succession through a series of rabbits, the incubation period gradually falls, till after 20 to 25 passages only to 8 days, and after further 20 to 25 passages it is no more than 7 days. Such a virus has a greater affinity for the nervous system of the rabbit than the virus isolated from the dog.

In the concept of the writer, such an enhancement in the virulence should result mainly from the increase in the combining force between a virus and the host cell. Namely, the virulence is increased because the combining force is increased when a virus is passed in succession through a series of the same kind of host. The reason for the increase in the combining force is considered as follows: The protoplasm of the host cell and the virus affecting the host are both assimilases, though the latter is stronger since it acts as a virus upon the host. However, the host protoplasm, though its assimilase action is weaker than that of the virus, will be able to exert some influence upon the latter, as the protoplasm is overwhelmingly larger in quantity than the virus, so that in the long run the virus itself may be assimilized by the host protoplasm to some extent in case when the virus is passed successively through the same kind of host, and as a result the protoplasm and the virus may come to share a structure or structures in common; namely, they may become gradually to resemble to each other. On the other hand, as already discussed, the development of common structure is nothing but the production of combining force between them, and therefore the affinity of the virus for the host cell may be enhanced with the increase in the virulence on continued passage through the same kind of host. 
The reason for the increase in the virulence during the passage can thus be elucidated. But in connection with this a more important problem has to be considered.

A virus can assimilize the host protoplasm, so that all the structures present in the protoplasm may become identical to the virus. Thus, since the parent and offspring viruses share all the structures in common, they can completely combine with each other, but without exerting any effect on one another because there is no differences in the structures.

In like manner, if a virus propagates continuously in the same kind of host until the degree of assimilation by the host protoplasm reaches to such an extent that almost all the virus structures become identical to those of the protoplasm, then the virus will have little influence upon the host in spite of its high affinity for the host. Thus, in order that a virus exerts injurious effect upon the host, the virus must have a structure or structures foreign to the host protoplasm, which may be lost when the assimilation by the protoplasm is advanced to an extreme. On the other hand, in order to combine with the host cell a virus must share a structure in common with the host protoplasm. This common structure can be increased or strengthened when the virus is assimilized by the protoplasm to a certain extent through the host passage.

It will be expected from this reasoning that, although a virus may increase gradually in its virulence against a certain host when passed successively through the host, the increase will come to cease sooner or later, and then a decrease will begin until at last the virus becomes entirely avirulent. In fact this seems actually true: An epidemic, for example, of influenza usually begins gradually, at first with only a slight indisposition, but in due course of time the virulence will increase in a striking way, the disease spreading vigorously and extensively; yet, after several months or a year or two, it will begin to lose vigour until entirely fades out and disappears.

In order to avoid the reduction in the activity, vaccinia virus is usually passed at times through rabbits apart from the customary cattle. We shall have later many occasions to return to this point, since this is one of the most important problems concerning life phenomena. 


\section{CHAPTER $\mathrm{V}$ \\ FIXED VIRUSES}

\section{Immunity against Viruses}

Usually an effective and lasting immunity is afforded by a single attack of a virus, a fact which is sometimes regarded as one of the characteristics of viruses. Small pox, yellow fever, varicella, measles, mumps, cattle plague, swine fever, and dog distemper, all these are common virus disease, and all confer an immunity that, in the majority of cases, appears to last throughout life. In some virus diseases, however, such as influenza, common cold, and poliomyelitis, the immunity is believed to be only transient.

As above mentioned, viruses such as those of common cold, influenza, and poliomyelitis, appear to be generated de novo. From this point of view, it may be able to explain the transient nature of the immunity against these viruses, because the immunity against a virus formerly produced would be ineffective to a newly produced virus. Not long ago foot-and-mouth disease would have been placed in this non-immunizing or poorly immunizing class, but we are now informed that there are at least 3 immunologically distinct types, and that animals that have recovered from infection with one type proves immune to further infection with the same type, but are readily infected by the other types.

Experimental results with one and the same strain of influenza virus showed, however, that the immunity is actually transient. For example, ferrets that have recovered from an attack of a strain of the virus are, for a time, resistant to the infection, but this persists only for about 3 months, and then gradually wanes. Again, the immunity which develops following an attack in man is said also not to last for long periods, usually becoming insignificant after 6 to 8 months (80). It must, therefore, be admitted that the immunity in itself is actually transient in some viruses, although sometimes the possibility of their successive generation may account for the apparent transient immunity as above pointed out. It is, however, a very remarkable fact that immunity appears incomplete in only the viruses that can be considered to be generated de novo such as those of influenza, and poliomyelitis. 
At present, influenza virus is divided into two distinct and immunologically unrelated serological types, termed A and B. However, it is of much importance that individual strains of influenza $A$ virus or of B virus are not identical immunologically with other strains of the same type. It is said that, in occasional instances, the immunological differences between individual strains which belong to one type are so great as to cause considerable difficulty in the proper identification and classification of a strain. It may be said that these two types may only be an artificial product of classification.

Various strains of virus causing symptoms like those of encephalitis are also known, and new strains are constantly increasing. Normal or pathological structure of the protoplasm of a certain animal may be called influenza virus when it is capable of giving rise to symptoms like those of influenza because of its special affinity for respiratory tract of some other animals, whilst when it is endowed with special affinity for central nervous systems, it may be called encephalitis virus. It seems that comparatively little is known of the durability of the immunity against encephalitis.

Poliomyelitis may be concerned with agents belonging to this category. It is certain that there are a vast number of different strains in poliomyelitis virus. Recent observations suggest that there are at least three main types (81) (82). As already pointed out, poliomyelitis-like viruses have frequently been isolated from various sources especially from flies apparently having no connection with the disease. It has been known that some immunity is established on the infection with this disease, but that it is only transient and inconsiderable (83).

The so-called Coxackie virus appears to be closely related to this virus. Its strains are known to be very numerous, and 16 antigenically distinct types have been reported (84) and like poliomyelitis virus it has frequently been detected in flies. Within the past decade, it has become established that during typical summer epidemics of poliomyelitis the causative agents, frequently also Coxackie-like virus, can be isolated from several different species of flies.

Whatever the reason may be for the presence of the temporary nature of the immunity, there seems no doubt that spontaneous generation is impossible in such viruses as those of small pox, mumps, measles, etc., which confer a lifelong immunity, since, if new viruses which could cause a similar disease were successively generated, the immunity would not appear to last effectively, for new viruses would be more or less immunologically different from the previous ones, though they could cause a similar disease.

It appears, therefore, reasonable to conclude that there are at least two groups of viruses. Those of influenza, poliomyelitis, eto. belong 
to the one group which may be produced de novo, and those of measles, mumps, small pox, etc. belong to the other; spontaneous generation may be impossible in this latter group.

\section{The Fixation of Viruses}

In general, viruses can be changed in their character, sometimes even inactivated completely, without losing their immunological specificity. For example, vaccinia virus is evidently different in some aspect from the variola virus from which it has been derived, but immunologically similar, so that it is capable of immunizing against the latter. Phage treated with such agents as formaldehyde, mercuric chloride, etc., may be reduced in its virulence, but may retain its immunological specificity even after the complete inactivation, although too severe inactivation may lead to the destruction of the antigenicity itself (68). This fact indicates that the structure involved in the immunological specificity is more stable than that concerned in virus activity. Consequently it is supposed that the variation in the activity may develop without difficulty in contrast to the variation in the immunological specificity.

It may, however, be possible that a virus generated de novo can be changed in its immunological specificity as well as in other characters to become in the long run a virus entirely different from the original. It is actually confirmed that also in immunological properties variations can occur. For example, evidence has been presented that antigenic variations take place in a strain of influenza virus propagated through continuous passage in tissue cultures and eggs as compared with a line of the same strain maintained in mice (85). Through such variations, a virus may acquire the properties differing in every respect from any other viruses which can be generated de novo.

In this way, the viruses that can induce lifelong immunity might have been developed. There are good reasons to suppose as will be detailed later that viruses such as those of measles and small pox, in contrast to those of influenza and poliomyelitis, are neither generated de novo nor lost in short periods. The writer proposes therefore the name "fixed viruses" to designate the former group of viruses. The reason why the viruses have acquired the fixed character and why they can produce rigid immunity will be fully discussed in later chapters.

It cannot of course be considered that these fixed viruses are all the same in their ages; some may be young, rather fixed recently, 
while the other old, generated much longer before. The particle size of virus may present some indication; the virus of foot-and-mouth disease and of poliomyelitis, and some kinds of plant viruses have been reported to be the smallest in size. Such viruses may be regarded as possessing comparatively primitive, undifferentiated structures, since "small size" may be interpreted as indicating that the virus or the assimilase can maintain its activity even when decomposed to this small size. Indeed, such small-sized viruses seem liable to develop de novo. Coxackie virus which bears a strinking resemblance to poliomyelitis virus has been reported to have the size of 10 to $15 \mathrm{~m} \mu$ (86) indicating that this virus is also one of the smallest viruses. On the other hand, the so-called lymphogranuloma-psittacosis group viruses are generally accepted to be most differentiated ones, and they are believed to be as large as 250 to $500 \mathrm{~m} \mu$. Nevertheless, there appears not always such correlation between differentiation degree and the particle sizes. For instance, influenza virus, spontaneous generation of which appears most possible, is generally acknowledged to be in size about $100 \mathrm{~m} \mu$, whereas with yellow fever virus which appears to be a fixed virus such small figure as $22 \mathrm{~m} / \ell$ has been reported (87). As already stated, since the size of a virus may be determined by the property of the protoplasm which the virus has affected, and also by the smallest protoplasm particles, in which the virus activity can remain, no great significance may be expected in the size.

However, fixation of the character of a virus as an independent entity seems to be accompanied by the establishment of character to form uniform particles, and moreover the complexity of the activity necessarily associated with the development of differentiation in virus may require large sizes because small particles may be impossible to contain the complex structure. Consequently, large uniform sizes may commonly be associated with old and differentiated viruses. In the case of Rickettsiae, as referred to in the next chapter, this seems especially the case. 


\section{CHAPTER VI}

\section{DEVELOPMENT FROM VIRUSES TO ORGANISMS}

\section{Rickettsiae}

Lymphogranuloma-psittacosis group of viruses is believed to be largest-sized, whilst there are much more differentiated, advanced viruses or virus-like agents, which are commonly called Rickettsiae. The Rickettsiae seem to occupy a place intermediate between the smallest cultivable bacteria and the largest viruses. The diseases caused by these agents are transmitted by arthropods, usually louse and ticks. They resemble usual viruses since they will multiply only in living cells, but differ from the former in that they fail, as a rule, to pass through bacterial filters and that their particles are distinctly seen under the ordinary microscope when stained properly.

This property of easily staining with proper dyes is note-worthy, because it may indicate the development of structure much different from that of the protoplasm of the host cell. With the usual viruses no staining technic have ever been found to make the virus particle distinct from the protoplasm, showing that the virus is alike in structure to the protoplasm. Moreover, specific therapy by chemical substances has usually been failed with virus diseases, whilst it proves effective in Rickettsiae, indicating also the development of peculiar structure in them. It is, however, known that specific chemotherapy is likewise effective to some extent in viruses of psittacosis-lymphogranuloma group, and that some viruses of this group sometimes stain like Rickettsiae. These facts show the presence also in these viruses of highly developed structures resembling to those of Rickettsiae.

The fact that these highly developed viruses as well as Rickettsiae are usually associated with rather uniform and large sized particles, suggests that the protoplasm, when changed in its structure by these agents, will decompose into large particles. Since the property to form large particles should be determined by the structural pattern peculiar to these viruses and Rickettsiae, and since this pattern is to be transmitted to the protoplasm they affect, newly formed particles will be always endowed with large sizes. The inheritance of the shape may be thus established. 
In order to form a particle larger than an elementary body of the protoplasm, a certain number of elementary bodies have to associate with one another to form a larger body, since the size of elementary body cannot be altered, and hence highly developed viruses including Rickettsiae have to be composed of a number of elementary bodies. In fact, this has been shown in the electron micrographs of Rickettsiae and of some large sized viruses.

\section{Indisputable Organisms}

The fact that Rickettsiae can be resolved microscopically by visible light, and that they are held back by membranes which allow most of the filtrable viruses to pass through, may bring them into line with the bacteria. However, their general failure to grow on ordinary culture media, and their predilection for intracellular multiplication, show that they are more akin to filtrable viruses.

It is, however, a fact worthy of note that although none of the pathogenic species of Rickettsiae has ever been cultured apart from living cells, the commensal species, $R$. melophagi, found in the sheepked, is said to be able to grow on blood agar (88).

The ability to grow without living cells may seem the indication of the appearance of mysterious nature, peculiar to living organisms and lacking in viruses. Nevertheless, there seems no need to postulate the appearance in this Rickettsiae of a mysterious nature, because the ability to grow without living cells may be interpreted as to be the faculty to produce its replica in blood proteins as well as in protoplasm proteins, whilst viruses and usual Rickettsiae are merely in the position to produce their replicas only in the protoplasm.

In addition, in various respects, blood may be looked upon as a large pool of a fluid protoplasm. If acetic acid is added to blood serum diluted with water, virus-like particles, composed of euglobulin and lipids, will be yielded as in the case of cell protoplasm treated in a similar way as was already stated in Part I. Moreover, intimate mutual association seems to exist among protein molecules in blood as in the protoplasm. In the protoplasm, owing to this mutual association among protein molecules, various changes, including coagulation process, can spread readily. In like manner, in blood the coagulation process can spread successively as will be mentioned later.

The mechanism, therefore, by which the Rickettsiae can accomplish the multiplication in blood agar can be regarded as being similar to that whereby viruses multiply in the protoplasm. However, since in its physical and chemical properties blood differs considerably from 
the usual protoplosm, in order to multiply in blood the Rickettsiae may require a quite strong assimilase activity not present in viruses. As will be fully explained later, such a strong assimilase activity, in the main, is apparently based upon its large particle size.

In addition to Rickettsiae, there are many other small bacteria-like bodies or organisms, found in higher animals on which they are parasitic. They are, like viruses, usually unable to multiply without cells of higher animals. The organism responsible for pleuropneumonia of cattle may be cited as one of the specimens. This twas once regarded as a kind of viruses, as it could pass coarse bacterial filters, but this undoubtedly cannot be a virus, for it can sometimes grow in nutrient media containing blood in the absence of living cells. It stains with proper bacterial stains and is microscopically visible.

The organism of contagious agalactia is also filtrable, and likewise once thought to be a virus, but at present known to be an organism akin to the above one, and can be also cultivated in the absence of living cells. Furthermore, various bodies have been described by different workers in close association with the red blood cells of man and animals suffering usually from certain types of anaemia. These bodies are believed to be definite organisms and called Bartonella. Non-pathogenic bodies similar to Bartonella are also found and named Grahamella.

It seems that these bodies or organisms occupy a position between the Rickettsiae and the ordinary bacteria. Generally they can multiply only in living cells as do viruses, but sometimes some of them can manage to utilize blood proteins to produce their replicas.

These organisms are distinct in the property to form specifically shaped bodies, so that it may be possible to distinguish them by their shapes in contrast to ordinary viruses. Primitive viruses may be able to exhibit their action only when the protein molecules are polymerized to a certain degree without being greatly concerned with their sizes and shapes. However, in highly differentiated viruses, such as those of lymphogranuloma inguinale and psittacosis, the property to form peculiar bodies is manifestly forthcoming, presumably as a result of the development of the structural pattern which may cause several elementary bodies to associate regularly into a larger body. A regularly associated product of the same molecules is a crystal, and its shape is determined by the structure of the component molecule. Since the assimilase is a liquid crystal of proteins, its shape should be likewise determined by the structural pattern of the proteins, again since such a structural pattern is to be transmitted successively to daughter assimilases, the shape should be inherited. Thus, when some protoplasm proteins, the structure of which has been changed to be 
ideritical to that of a Rickettsia, form a liquid crystal corresponding to the changed structure, the shape peculiar to the Rickettsiae will be developed in the protoplasm, with the multiplication of the peculiarly shaped body.

In case of viruses, protoplasm proteins, changed in structure by a virus, occasionally form large "crystals" under some conditions; the crystals are termed inclusion bodies. Viruses are able to exhibit their function in the form of a single elementary body or sometimes even in much smaller particles produced through the decomposition of the elementary body itself, so that such large crystals are never necessary for their function. However, if the function could not be set forth unless the proteins formed such a large "crystal", the inclusion body would be regarded as the only feature of the virus.

For the achievement of complicated functions, indispensable for usual organisms, large sizes are apparently necessary for the assimilase. Viruses therefore must have the property to form larger particles in order to advance on a line of indisputable organisms.

Of orgainisms called bacteria, there are many groups which are strictly parasitic, usually only capable of multiplying in the presence of certain cells, accodingly failing to grow in culture meida unless blood proteins are mixed. Neisseria Moraxella or the group of MoraxAxenfeld bacillus, and $B$. pneumosintes may be cited as such bacteria, which can be regarded as primitive bacteria evolved comparatively recently from viruses.

The writer proposes the name, "secondary organisms" to these organisms thus evolved from viruses, and accordingly the organisms in which the secondary organisms are advanced must be called "primary organisms". 


\section{CHAPTER VII}

\section{CAUSES OF THE EVOLUTION OF VIRUSES}

\section{Development of Instincts Indispensable for Organisms}

It has been described thus far that the viruses generated de novo can erolve into fixed viruses by variations, and that they can further advance into typical organisms through the stage of Rickettsiae. But, little discussion has been made on the problem why they have to evolve. In this chapter this problem will be fully dealt with.

Darwin attributed the cause of the organic evolution to the survival of the fittest set forth by natural selection; this likervise pos. sibly folds for the evolution of viruses, although there are some differences between usual organisms and viruses.

One of the principal differences between them may lie in the fact that viruses have no instinct for self- or race-preservation in contrast to the indisputable organisms. Even in this respect alone it seems impossible to regard viruses as organisms. This is the instinct indispensable for organisms; organisms cannot exist without this instinct.

Viruses are lacking in this important instinct, so that their existence is of quite evanescence; only some viruses which incidentally get possession of a property favourable for their existence, can continue to exist for some periods, while others are cancelled immediately after their generation.

The character most essential for organisms is their continuity in existence. This is secured by the instinct, owing to which they have been, and will be able to accomplish their evolution. For higher organisms, however, self-preservative instinct may be only of the secondary importance, merely answering the purpose of race-preservation the important necessity for organisms, and an individual of higher organisms having already fulfilled this purpose may be entirely of no use in itself; the existence of individual may always be ready to be sacrificed for the race-preservation.

On the other hand, in case of extremely primitive organisms existing at the stage of virus, there seems no distinct difference between the self and race-preservation. In most cases the race-preserva- 
tion may be accomplished by the self-preservation.

Newly formed viruses would start on the long journey of the evolution as a group of particles of various individualities. Of them some particles with high resistances against environmental influences might be able to escape from the immediate inactivation to continue their existence, and again some particles with strong assimilase action might be capable of achieving extensive multiplication, so that particles both with strong assimilase action and high resistance would be able to survive. Thus the survival of the fittest would be effectuated by a sort of natural selection.

Viruses thus having survived would undergo variations, with the yieldance of various particles differing from one another in their individual properties, and again some individual having a property more favourable for their existence would be selected out of these particles and would continue their existence. Variation, selection, and the survival of the fittest would thus be recited over and over again. There seems no doubt that the characters resembling those of higher organisms are most desirable for the continued existence, so that viruses that happened to possess more organism-like characters would be able to survive much longer than do other viruses, and therefore the characters would become continuously more and more alike to those of typical organisms.

The properties thus developed in selected viruses, resembling those of higher organisms, must be the properties favouring continuous existence, and thus the instinct for the self- or race-preservation would be developed gradually in the viruses until the stage of typical organisms was reached. The characters useful for the continuous existence, if became unusually strong and firm, should be called instinct. Therefore, even if we assumed that the instinct belongs only to indisputable organisms, it would be impossible to draw a line between living and nonliving.

The evolution of the shape or size of virus particles may be elucidated in like manner: In the case of tumour, such as cancers, newly formed structures can sometimes be transmitted to other normal cells by protoplasm particles, but commonly fail to be maintained in the particles, and accordingly as the structures have to share their fate with organisms in which they have generated they will be lost when the organisms are perished, and therefore their evolution will never take place; whereas since in the case of influenza or poliomyelitis the newly generated structures can be preserved in the particles, they are able to be liberated from the organisms, in which they have been generated, to be transmitted to other organisms. The continuity of their existence would thus be commenced, and the evolution would 
thus become possible.

Newly formed structure or a virus may be better maintained in larger particles, while readily lost in smaller ones as mentioned already (Part I, Chapter II), so that for the preservation of the structure larger particles may be more favourable. In addition, assimilase action is considered to be directly proportional to the particle size, that is, larger particles possess greater faculty for their multiplicability as also pointed out already (Part II, Chapter II). Larger sizes are, therefore, advantageous to the viruses for these two reasons, $i . e$, for the maintenance of the specific structure and for the enhancement of the assimilase action. Accordingly, viruses should have to secure the property to form larger particles in order to continue their existence.

This property like many other properties is determined by a structure of the protein; that is to say, the combination of many elementary bodies to form a large body, may be achieved when the elementary bodies have a peculiar structure favouring the large body formation. Hence, the virus which has incidentally secured such a structure by a variation may be in a better position to continue its existence, and the structure may be transmitted in succession. Thus the structure to form larger particles would be attained and strengthened, with the result that assimilases which can be called organisms might always get possession of the property to form bodies larger than ordinary virus particles.

\section{Difficult Situation of Newly Generated Viruses to Continue Their Existence}

Viruses seem to be constantly generated from a variety of cells, so that strains or kinds of newly formed viruses must be innumerable and thus numberless kinds of viruses must be constantly being thrown out on the earth. However, the kinds of fixed, typical viruses never seem to be numberless, but limited to a rather small number. This may indicate that the probability of newly formed viruses of becoming fixed is extraordinarily small. Most of the viruses generated de novo possibly disappear immediately after their generation, because they have no instinct of self-preservation.

If in some protoplasm a new structure is produced which is sufficiently stable to be retained in the protoplasm particles into which the protoplasm is decomposed, then such a particle may be able to transmit the structure to another organism as a virus. In order to evolve into a typical organism, however, the virus should be successively trans- 
mitted to other organisms with vigorous multiplication, only thereby variations in a desirable direction become possible. Remaining in the same host must result in its extinction together with the host as in the case of tumours.

On the other hand, In the case of common cold, for instance, the newly formed structure carried by particles may be able to spread easily through the coughing. The fact that common cold is always accompanied by coughing may enable the virus to exist for considerably prolonged periods and thus the virus may be able to become more and more virulent until it may come to appear even to be a kind of influenza virus. -

Like the virus of common cold, a large number of viruses, such as those of measles, mumps, poliomyelitis, and influenza, may be spread, though not so readily, by means of droplets of secretion from the upper respiratory tract. In the case of venereal diseases, such as lymphogranuloma inguinale, spread of viruses by direct contact can be afforded. In plant virus diseases, infections by direct contact is also conceivable.

Many other ways may be possible for viruses to spread to other host individuals, but in this connection a strong reason for which the continued existence of viruses may become most difficult can be considered. The reason is that, for the continued existence, viruses must be transmitted frequently to other organisms quite different from the customary host.

As mentioned already, when a virus continues for a long time to multiply by successively affecting a series of the same kind of host, the virulence in the long run has to decrease until the virus itself is lost, because the virus will be gradaully assimilized by the host protoplasm to become quite identical to the latter. In order to avoid such a fate, viruses have to change occasionally their host. Insects appear to be universally utilized by viruses for this purpose as will be detailed in the next chapter. 


\section{CHAPTER VIII}

\section{VIRUSES AND INSECTS}

\section{The Multiplication of Viruses in Insects}

Many virus diseases are transmitted by insect vectors, animal viruses usually by blood-sucking insects such as mosquitoes, lice, and sometimes by arthropods such as ticks, while plant viruses by aphides and leafhoppers. It is worthy of note that an insect which has been contaminated with a virus, as a rule, is endowed with no infectivity immediately after the contamination, but that only after a certain period of time becomes capable of transmitting the virus. In the case of yellow fever a kind of mosquitoes, for example, attains the infectivity 12 days after blood-sucking, and in dengue fever there is an incubation period of 7 to 10 or more days before the mosquitoes are infective to man. Also with a variety of plant viruses and their vectors the existence of similar incubation periods have been well established.

The presence of these incubation periods can be readily elucidated by the assumption that viruses multiply in the insect bodies thereby the properties of the virus are changed and activated. In corroborating this assumption, it has been confirmed by a number of workers that some viruses actually multiply in the insect vectors. For instance, Trager (89) has shown by cultivating the virus of equine encephalomyelitis in sterile mosquito tissue that the virus can under certain circumstances undoubtedly multiply in the mosquito. Merrill and Ten-Broeck (90), working with the same virus, macerated the bodies of infected mosquitoes, and fed the juices to non-infective mosquitoes, and found that after ten transfers the mosquitoes still become infective and since the dilution at each transfer was at least 1:100 it seems conclusive that multiplication must have taken place. Whitman (91) has presented evidences that a kind of mosquito is capable of multiplying yellow fever virus in its body. Following the injection of human blood, the content of virus falls for several days, reaching a minimum during the first week. It then increases rapidly until quantities of virus greater than those previously encountered can be demonstrated. 
Furthermore, working with the virus of aster yellows and its specific vector, Black (92) has confirmed that the virus could increase in the insect at least one hundredfold between the second and twelf th days of the inoculation. Maramorosch (93) has likewise ascertained the multiplication of corn stunt virus in its insect vector, a kind of leafhoppers. He has succeeded in transmitting the virus mechanically from an insect to another; the virus isolated from infected plant, when injected into insects, renders the latter infective and the juices of the insects can successively infect other healthy insects through mechanical injection. The minimum incubation period of the virus to become infective in the insects was found to be 6 weeks. A plant virus not mechanically transmissible from plant to plant can be thus mechanically transmitted between insect vectors.

In spite of such numerous positive evidences, virus multiplication in insect vectors are not fully admitted, because there are some facts known which appear to be inconsistent with the concept. For instance, if viruses multiply in insect vectors it would be reasonable to expect that all the vectors, when once become infective, will remain so for long periods and probably for the whole of their lives, but this does not happen, for it has been found that different individuals remain infective for varying periods, some soon losing infectivity and others not. Moreover, it has been confirmed that the length of time for which insects remain infective depends on the length of time they have fed on the source of the viruses. Vectors may become infective after a few minutes feeding but do not remain infective for long, whilst if they have fed for hours or days they remain infective for long periods, often for the remainder of their lives. Sometimes it was even confirmed that both efficiency of the vectors and length of time for which they remain infective increases with increased feeding time on the infected plant (39). These evidences may appear at first sight to indicate that a virus remains in the insect as such without multiplying, being contradictory to the above view, but in the theory of the writer these are by no means inconsistent with the view as will be argued in the following section.

\section{The Reversibility of Protein Structure}

The writer holds the opinion that the structure of protoplasm protein is springy or elastic, so that if the factor is removed which has changed to some extent the structure, the original structure may be recovered sooner or later. In short, protoplasm proteins can remember the structure they once possessed and will resume it if possible. 
A virus which has been inactivated by an agent can occasionally be reactivated when the agent is removed; this may result from the memory of the original structure. Numerous analogous phenomena are known and recognized in general as reversibility of protein denaturation.

Usually yeast cells cannot ferment galactose, but if placed in contact with the sugar, they will subsequently acquire the enzyme necessary to ferment the sugar. Such an enzyme is generally called adaptive enzyme. The adaptive enzyme is extremely unstable in the absence of the specific substrate, and as a rule the enzyme will come to show a loss of the specific activity shortly after the substrate is removed from the medium.

Spiegelman (94) held the opinion that adaptive enzyme is produced from the protein precursor by the action of the substrate and that since the change of the precursor to the enzyme is reversible, the enzyme will come back to the state of the precursor when the substrate is removed. This is almost in accord with writer's opinion, according to which the precursor is the protoplasm protein itself whose structure is altered by the substrate to become complementary to the substrate structure, thereby the specific activity as the enzyme is revealed, but as the change is reversible, on the removal of the substrate which have caused the change, the original structure is recovered (12).

In the case of allergic dermatitis, dealt with previously, it is supposed that the protoplasm protein of skin tissue cells is altered in its structure by some agent, the altered structure being able to spread successively in protoplasm and even from cell to cell, but that since the altered structure is unstable, having a high tendency to return to its original state, the skin tissue will soon recover from the disease when the agent is removed. The failure of the demonstration of any virus in such dermatitis is probably dependent upon such a lability of the altered structure.

Some workers claimed that the adaptation of yeast cells to a sugar could be transmitted by a virus-like agent, but some other workers are disinclined to accept this claim. Also in this case strong reversibility of the structure may render the detection of the "virus" difficult.

Protoplasm protein changed in its structure by a virus seems likewise to be inclined to recover its original structure. The recovery from virus diseases may be accomplished mainly owing to this reversibility (12). The recovery of the original structure is nothing but the disappearance of the virus itself.

Now we shall return to the problem regarding the insect vectors. As above mentioned, insect vectors which have once become infective by feeding on diseased plant do not always remain infective for long 
periods. This must be a most natural result since the normal structural pattern should be recovered sooner or later. The action of virus as already discussed is generally directly proportional to its quantity; the greater the quantity, the stronger the action. When the action is stronger, replicas produced will be more distinct and firmer. Thus, the above mentioned fact that the length of time for which insects remain infective depends on the length of time they have fed on the source of the viruses should naturally be expected, as the quantity of the virus taken by the insects must be greater when they have fed longer.

The writer is of the opinion that antibodies are produced in entirely the same way as the adaptive enzymes. Namely, protoplasm proteins altered in their structure by antigens are the antibodies (12). Also in the case of this antibody formation, it is known that both the amount of antibody produced and the length of time for which the antibody production persists are inclined to be directly proportional to the antigen amount administered. The protoplasm structure changed by antigens is likewise reversible, so that sooner or later the original structure is recovered with the cessation of the antibody production.

Kunkel (95) has found that a kind of the leafhopper carrying aster yellows virus is unable to transmit if exposed to high temperatures. Leafhopper exposed to $32^{\circ} \mathrm{C}$. for a day lost the ability to infect healthy plants, but on lowering the temperature to $24^{\circ} \mathrm{C}$. they quickly regained the ability without again feeding on a source of the virus. This may indicate that the structure of the virus is stable at lower temperatures, while at higher temperatures the normal protoplasm structure is more stable than the virus structure, so that at $32^{\circ} \mathrm{C}$. the virus structure may disappear but at $24^{\circ} \mathrm{C}$. it may reappear. Again, according to the above author the leafhopper carrying the virus, if heated for one day, regained the ability to transmit in a few hours at the lower temperature and those heated for a week required two days or longer, whereas those heated for more than 12 days never regained the ability to transmit. This is analogous to the above mentioned phenomenon that the length of time for which insects remain infective is determined by the length of time for which they have been fed on the diseased leaves. The stronger and the firmer is the change, the more prolonged period of time will be required for the recovery of the original structure, no matter whether the latter structure be normal or pathological. Thus the reactivation of a denatured virus would occur the more readily, the slighter and the shorter was the inactivation process. It may be possible to consider that, in the case of reversible inactivation of a virus, the inactivated structure is stable in the presence of the inacti- 
vating agent, whereas normal structure is stable in its absence, a view which will be dealt extensively in Part V.

\section{Heritable Changed Structure}

Although the reversible character of protoplasm-protein structure is generally revealed in such a remarkable way, profound distortions given rise to in the structure may render the recovery of the original structure extremely difficult. According to the structural pattern of virus the degree of distortion which will be raised in the protoplasm may vary, and some viruses can induce most profound distortions, while others only slight changes. If the distortion is slight, the distortion or the changed structure may be unstable, and the original structure may be promptly recovered with the disappearance of the virus and thus the disease will be cured, whereas if the change is striking the changed structure may be stable, and the virus will persist in the host cells as long as the host exists. Thus when a host is affected by a strong virus it may be unable to become freed from the virus for the remainder of its life.

In connection with this trend of thought an extremely important argument should be advanced. Strong viruses may thus be able to induce replicas in host firm enough to remain for the host's life. In the opinion of the writer, if the template action of the viruses is still stronger, the change can be spread even into the germ cells through which the structure is transmitted to the offspring. Such a transmission of the virus structure to the offspring must be the transmission of the virus itself to the offspring through the germ cells. Now we shall see next the verity of this concept.

It seems that the virus structures multiplying in insects commonly disappear sooner or later, yielding to the normal structures. It has been known, however, that sometimes viruses persist not only for the remainder of the host lives, but a!so can be transmitted to the progeny of the host. Fukushi (96) has found that the virus of dwarf disease of rice was passed from parent to offspring to the third generation without recourse to a fresh source of virus. Black (97) likewise has confirmed the transmission of the clover club-leaf virus from parent to offspring. He found that the virus can be inherited over a period of four years without the insect coming to contact with a fresh source of virus. Such hereditary transmission of viruses has been recorded also with animal viruses. The eggs of a kind of wood ticks apparently give rise to infective nymphs if the parent tick is infected with the virus of equine encephalomyelitis. Similarly, 
the virus of Nairobi sheep disease is said to be transmitted through the eggs of a brown tick, the vector (98).

Usually seed from infected plant gives healthy progeny, but a few plant viruses are believed to invade and survive in the seeds set by infected plants, thus making possible the transmission of the virus through seed, a phenomenon analogous to the virus transmission through the eggs laid by infective arthropods. Seed transmission is said to be more characteristic of viruses of leguminous plants than any others; a number of those have been described as seed-borne.

However, not all the seeds set by diseased plants are infected, and the proportion of infected seeds to virus-free ones depends on the length of time the parent plant has been infected (99). This may support the customary idea that the virus is mechanically carried by the seed. But in the opinion of the writer this should be regarded as analogous to the above cited fact that the ability of insects to cause infection depends upon the initial charge of the plant viruses, and also to the fact that both the duration of antibody production and the antibody amount produced tend to be directly proportional to the antigen amount administered.

Such transmissibility of virus to offspring through egg or seed involves, to the belief of the writer, the utmost important significance.

As will be discussed in great detail in Part $\mathrm{V}$, there are many good reasons to conclude that in germ cells the replicas given rise to by viruses are kept in an altered form. The virus in itself, accordingly, may unable to be demonstrated as such. The altered replica, however, may gradually recover its original form as the germ cell develops to an adult organism, so that the virus may be revealed at a stage of the development.

Usually young organisms having emerged from the eggs of the infected insects are not infective immediately after hatching, but will become so only after variable waiting periods. For example, according to Black (97) no insect raised from eggs laid by an insect vector infected by clover-club leaf virus can be infectious until three weeks after hatching, and most infections are obtained when the insects are between 6 to 12 weeks old. Such a failure of newly hatched insects in inducing infection may not be impossible to be attributed to too small virus amounts contained in the egg to cause the infection; if so, the waiting period may be the time required for the multiplication of this virus. The writer, however, regards this fact as an example of the reversion of the replica in the developing insect. It should be noted that plants generated from the seed inheriting a virus likewise never reveal the disease symptoms until the plants grow up to a certain extent. 
Such a phenomenon may never be confined to insects or plants only. Human beings may not be exceptional. It may be possible that children inherit the structure of a virus from parent through the germ cell, but that as the structure is transmitted in an altered form the reversion of the complete virus form requires some periods after the birth of the children, so that the virus appears when the children grow up to some extent. The virus of measles may be one of the viruses which can raise, in human protoplasm structure, a change strong enough to be transmitted to progeny. Children who have inherited the structure of measles virus from the parent may suffer from measles, without the infection from outside, when the structure recovers its complete form.

In this connection it should be mentioned that bacteria infected with phage may become lysogenic in which, however, the presence of the virus cannot be demonstrated, but the bacteria are bound to undergo lysis with the liberation of the virus if brought under peculiar environmental conditions (7). This fact must depend upon the presence of the virus structure in an altered, inactive form in the bacteria just as the structure of the measles virus may be in the germ cells or in the premature children.

In Chapter III in this Part is shown the fact that the seasonal change in the morbility of measles strikingly resembles the change in human conceptibility. Now, the writer is in the position to elucidate this phenomen clearly. Thus, the seasonal change in the human conceptibility may be represented in a smooth curve presumably because of a regular seasonal change in the activity of hormonal glands, while, on the other hand, the complete structure of measles virus may be developed in human cells just as harmones are excreted under the regular influence of seasons. It may be said, therefore, that the reversion of the virus structure tends to occur in a definite season when the children grow up to a certain age.

If the pattern of the structure of measles virus inherited from parent is developed in a child, the pattern will be engraved in the mother-germ cells of the child by the infection, and therefore the pattern will be transmitted from generation to generation. If, however, the normal structure of the protoplasm is sufficiently strong to reject the virus structure, or at least powerful enough to overcome the virus structure before it can engrave its pattern into the germ cell, the virus will not be transmitted to the offspring.

As is generally recognized, about 5 per cent of man do not suffer from measles throughout life, showing that some persons possess a strong predisposition to reject the virus. Even if the virus can afford to affect such a person of strong predisposition, the structure of the 
virus may not only be unable to remain for a long period but may be defeated and assimilized soon by the normal structure.

It is noted that not all larvae from eggs laid by insects carrying a plant virus become infective, suggesting those of a weak predisposition only admit the virus structure to develop. This appears to hold true also for viruses transmitted by seed. The proportions of infected seeds are reported to be highest with plants which are themselves the progeny of infected seeds (39), a fact which is to be expected since the predisposition associated with a weak structure may be heritable.

Not only the virus of measles but many other viruses of man may, more or less, be heritable. For instance, mumps, a virus disease of parotid gland, may be one of the heritable virus diseases. In this connection, it should be remembered that in the submaxillar gland of normal guinea-pigs can usually be demonstrated a virus, which, however, inay become detectable only when the animals grow up to a certain age.

Viruses such as those of measles and mumps may have extremely powerful structure, so that they may be able to pass though germ cells to progeny without being much concerned with the predisposition of man, but in the case of many other viruses, persons with a weak predisposition only may be affected and the structure may be transmitted to the next generation only when the virus affects most susceptible persons.

\section{The Cause of the Fixation of Viruses}

Some viruses confer the animals they affected high degree of specific immunity, occasionally lasting for the remainder of their lives. The writer designates such viruses as fixed viruses, and holds the opinion that the immunity to a virus is caused by the engraving of the virus pattern into the protoplasm of the animal and that the immunity continues as long as the pattern is present in the animal (12). In other words, when some cells in an animal are endowed with virus structure, the animal is said to be immune to the virus. Since such cells are endowed with the structure of the virus itself, the virus which may invade afterward from outside will be preferably combined with the cells and assimilized without exerting any injurious effect.

If this concept is correct, a virus will be detected in organisms as long as the organisms are immune to the virus. In fact this is the case with plants. It is confirmed that the persistence of active virus 
in plants recovered from virus diseases is an invariable and essential factor for the immunity. Thus, freedom from a second attack of an acute disease, or protection from the effects of virulent strains, persists only as long as tissues are infected. As for insects, after they become infectious on feeding on diseased plants, some remain capable for life of excreting the virus. Namely, the insects are "immune" to the virus for the remainder of their lives.

Likewise in the case of animals, it is known that, although they may apparently recover from infection with some viruses, the animals can continue to harbor the agent in their tissue long after recovery. Accordingly, some workers have postulated that active immunity in virus diseases is dependent on "infection-immunity", meaning that resistance to reinfection is due to the persistence of living virus in the body. The detection of viruses from recovered animals, however, is not always possible unlike with plants. It should be considered, therefore, that in the majority of animals, the structure of viruses in the host cells may be altered after the recovery from the diseases so as to be rendered unable to act as the virus itself but retaining the ability to combine preferably with the virus without being suffered from it.

This concept is strongly supported by the fact ascertained with phage; bacteria may become immune to phage after the infection with the phage but usually no phage is detected in the bacteria. However, the phage will be released when the bacteria are brought under peculiar conditions (7). It must be emphasized that the acquired character to produce the virus is transmitted to progeny. In like manner, human beings also may be able to transmit the virus-producing character acquired through the infection to the progeny.

It may appear strange that bacteria that have become immune to the virus will be lysed when the active virus is produced. As is already suggested, this may be attributed to an unfolding of the protoplasm protein structure which is necessary for the acquisition of active virus structure. The virus structure in the bacteria may be inactive because of the folding of the active group, and the unfolding of the structure may result in the striking adsorption of water to the surface of the elementary bodies leading to the dissociation of the cell into the elementary bodies. This may be the case also with human cells which inherited virus pattern from parent. The virus particles thus released from the cells may be scattered about throughout the body to cause systemic infections.

The persistent immunity must be based on the very powerful structure of the virus in question, because the host protoplasm will recover its normal structure by overcoming the virus structure unless 
the latter is purerful. Extremely powerful viruses can remain in the host not only for the host's life, but also can stick to its germ cells through which they can be transmitted to progeny.

Since such firmness of the structure must be associated with the invariability of the structure, fixed viruses with strong structures will hardly give rise to variants. The antigenic specificity of fixed viruses is, as a rule, constant, and generally every fixed virus has its own antigenic specificity. Therefore, usually various types may, be demonstrated in a single fixed virus. For example, the virus of measles or of mumps has each distinct immunological specificity and hence various types of measles or of mumps are never found. Also in this respect fixed viruses may resemble a typical organism.

On the other hand, nervly generated viruses, as for example, those of influenza are provided with an extremely weak structure so that they may readily be overcome by the normal structure. Consequently such weak viruses will persist only for short periods in host cells. This must be the reason why newly generated viruses can confer only transient immunities.

The weakness of the structure should necessarily be associated with the unusual variability. Indeed, influenza virus is noted for its marked variability. Newly generated viruses may have no constant immunological specificity because of this marked variability as well as of the successive generation of similar viruses having varÿing specificities. The presence of such various immunological specificities, therefore, must be one of the characteristics of newly generated viruses.

However, if a virus particle having a relatively rigid structure is incidentally generated, it will remain longer and multiply more abundantly than do other weaker individuals. Virus particles produced by such a virus should inherit this relatively rigid structure. And again a particle having the strongest structure will naturally be selected out of these relatively strong particles, as the strongest can remain much longer and multiply much vigorously. Thus the structure of a virus is to become naturally more and more rigid and powerful.

The acquisition of the strong structure appears to answer the purpose of race-preservation as well as of self-preservation of viruses. In short, viruses which can give rise to prolonged immunity are more fitted for existence than those which may confer only transient immunity and those which can spread to progeny through germ cells are still more fitted. As to plant viruses, the ones which can long persist in insect bodies are fitter, while the ones which can be transmitted through the eggs to the offspring must be still more fitter. 
Now we have to discuss the nature of the rigidity of virus structure, the most important fector determing the virus evolution. As already pointed out, protoplasm structure must be springy. A spring may readily resile to its original state after a slight bend, but do so only with a considerable difficulty after a heavy distortion. The viruses capable of exerting on protoplasm such a heavy distortion may be said to be strong and rigid. The degree of the distortion may be determined by the structural pattern of the virus probably by the manner of spatial arrangement of polar groups, especially of amino. acid residues.

Since the structure to cause a heavy distortion can multiply in the protoplasm through the infection with the virus having the structure, and since a heavy distortion must be the structural change which cannot easily spring back to the original structure, the virus which can cause a heavy distortion must be in itself stable and rigid. In short, the virus having a stable, rigid structure can cause a heavy distortion in the host protoplasm. The virus capable of producing a strong pattern, therefore, may accomplish vigorous multiplication, and at the same time the virus itself is stable and fitted for prolonged existence.

In additicr to the chemical structure of the component protein, the amount and the kind of nucleic acids contained in the virus particles may also play an important role in the determination of the virus strength. If a virus particle contains a nucleic acid in a richer amount than the others, it will act as a stronger virus owing to the hardening action of the acid already discussed. If, therefore, certain cells acuqire the property to synthesize nucleic acid more vigorously on the infection with some virus, strong virus particles will be produced by such cells. Thus it may be concluded that the property of a virus to make the nucleic acid increase in the host cells is essential to its evolution.

Particle size is considered to be another important factor for the virus evolution. As already pointed out assimilase action is, to some extent, directly proportional to the polymerization degree of the proteins, so that larger particles can reveal greater action.

To sum up, in addition to the specific structural pattern of the component protein capable of causing marked distortion in the host protoplasm, the properties to agglutinate into large particle and to combine with large amount of nucleic acid appear to be essential for the evolution of viruses, that is, for the increase in their template strength, although these properties may be governed also by the specific structural pattern of the component protein.

Even when the infected cells of the same kind are decomposed into virus particles, the particles produced may not be identical with 
one another either in size or in nucleic acid content. In such a case, large particles rich in nucleic acid only may be able to act as a strong virus, whilst small particles with little or no nucleic acid may be extremely unstable, scarcely capable of acting as a virus, and may therefore disappear immediately after the production.

Moreover, the replica corresponding to the virus pattern may not be uniformly produced in all the particles; in some particles complete replica may be formed but in others only incomplete replica. Particles endowed with such incomplete replica may be unable to behave as a virus. It can be said, therefore, that the demonstration of virus action can scarcely be achieved with all the particles produced by infected cells. However, the property to constantly produce similar large particles, rich in nucleic acid, having complete replica should be required for a virus in order to evolve into a typical organism. 


\section{CHAPTER IX}

\section{THE REJUVENATION OF VIRUSES}

\section{The Transmission of Viruses by Insects}

However strong the virus structure may be, it will gradually be assimilized by the host protoplasm if the virus continues to multiply in the same kind of the host. Since the protoplasm in which the virus multiplies is also assimilase similar to the virus, the protoplasm may always be striving to assimilize the virus though it is weaker than the virus in the assimilase action as to be assimilized, in the main, by the virus. In addition, as the protoplasm structure involves the reversibility, the protoplasm itself may be striving to recover its original structure even when it has been changed into a virus structure; that is to say, there must be a potential force in a virus to develop by itself the original structure of the protoplasm. It will be expected therefore that viruses themselves will be assimilized in the long run by the protoplasm of the host cells if they always adhere to the same kind of host.

In order to avoid this fate, viruses have to change frequently their host. Insects appear to be commonly utilized by viruses for this purpose. Various animal and plant viruses are transmitted by insects in which they can multiply, thus host change becoming possible. As for some plant viruses and their insect vectors, however, many cases are known in which virus multiplication in insects can never be considered, although the viruses are undoubtedly transmitted by insects. In such cases, insects can infect healthy plants immediately after they have acquired viruses, and they soon cease to be able to do so, sometimes becoming non-infective within minutes and always within hours after leaving the infected plants.

In view of these facts it may be said that the relationship between plant viruses and their insect vectors may be revealed in the following two ways: In the first, the insect acquires infectivity after a certain incubation period and the infectivity continues for long periods, frequently for the remainder of its life, as already described; in the second, the insect becomes infective immediately after leaving a diseased plant and the infectivity is rapidly lost.

Many workers may consider that in the latter case the insects act simply mechanically, transferring the virus as a contaminant on 
the outsides of their stylets. Nevertheless. Bawden (39) claims that there is much evidences showing that the vectors do not behave merely mechanically. Thus, if insects acted solely by getting their mouthparts contaminated externally with virus, it is difficult to see why all those with similar feeding habits should not be equally effcient vectors, except that those with larger stylets might perhaps be expected to carry a little more virus. However, this is not so, and insects with similar feeding habits will transmit some viruses but not others, and, with viruses that have a number of aphids as vectors, one species is often much more efficient than the other. Bawden states further that if transmission by insect were merely mechanical, it might be expected that virus most readily transmitted by artificial inoculation could also be most readily transmitted by insects. And, if the viruses were merely acquired as a contaminant on the mouth parts, those occurring in infected plants in the greatest concentration might be expected to be most easily aquired and transmitted. But it is not so. Tobacco mosaic virus and potato virus $X$ are more easily transmitted by rubbing or by needle inoculation, and occur in greater concentration in extracts of infected plants, than any of the viruses under discussion. Neither of these normally appears to be aphidtransmitted.

Moreover, Bawden emphasizes the fact that insects can cause infection with virus amounts much smaller than those needed by ordinary inoculation method, and he describes further the remarkable fact that the efficiency of insects as vectors is much increased if they are prevented from feeding for some time before they are fed on the source of the virus.

\section{The Mechanism of Rejuvenation}

There seems no doubt that plant viruses above mentioned which are rapidly lost in the insect vectors fail to multiply in the insect bodies. It seems, however, reasonable to consider that transitory activation of the viruses may take place in the insect bodies.

Prior to considering the mechanism of this virus activation, we have to discuss the nature of protein denaturation. Proteins, in general, when exposed to physical or chemical effects, may change their properties so as to become "denatured" through the alteration of their structure. In this process the closely folded polypeptide chains in protein molecules may unfold transitorily and subsequently again may refold to give some other folding pattern different from the original internal structure, as shown in Fig. 21 (102). 
The peptide chains in the native protein molecules are folded as shown in $\mathbf{I}$ of this Figure; in this state almost all the polà groups of the side chains may exist in mutual saturation, whereas, by the
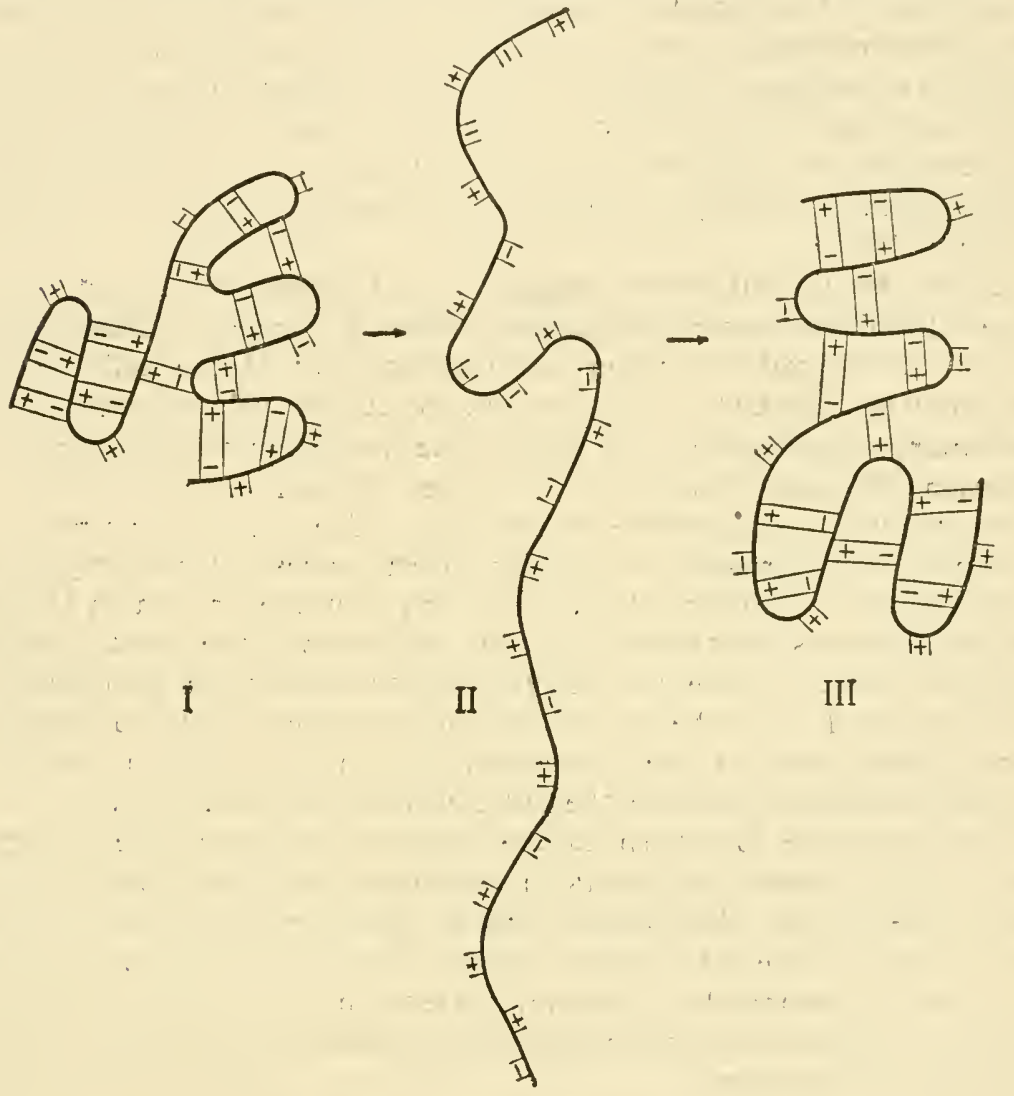

Fig. 21. Diagram of protein denaturation. After. Haurowitz: Chemistry and Biology of Proteins, 1950, (a little modified).

action of denaturating agent, the chains are unfolded as II, thereby polar groups of the side chains are set free, but as this form is unstable, refolding will follow soon to take the form III which is different from $I$.

At first, mention should be made as regards the phase II in Fig. 21 where peptide chains are unfolded and polar groups are set free. In this phase proteins may be in an activated state owing to this freed polar groups which enable them to combine with one another or to act upon other substances. For example, red blood corpuscles are 
agglutinated by the antiserum, probably because of the denaturation of the surface protein of the corpuscle due to the antiserum, whereby free polar groups causing the corpuscles to combine with one another are set free. If an agent named complement is present in this process, the phenomenon called complement fixation may occur, in which complement is combined with blood corpuscles owing to the same polar groups set free. Such a state of corpuscles to achieve agglutination and complement fixation is actually known to be only transient, showing that unfolding of the peptide chains is soon followed by the refolding (12).

Lesley et al. (101) have suggested that infection of bacteria by phage stimulates some mechanism whereby phage adsorbed subsequently to the cell is broken down extensively at the cell surface. This may be ascribed to the activation of the cell surface by the preliminary combination with phage; the activation resulting from the liberation of polar forces may enable the cell surface to cause a severe structural disturbance in the phage added at a later time.

Some plant viruses can readily infect leaves by rubbing if the leaves injured by carborundum, indicating that the protein in the surface of the leaves is activated by the application of the agent to adsorb the virus. In this case the activated state is likewise temporary, as it is confirmed that the leaves regain their resistance to infection within three hours of the procedure, though they remain permeable to salts presumably because the cuticle is not repaired (102).

Now we have to return to the problem concerning the transmission of plant viruses by insects in which no virus multiplication takes place. The writer claims that also in this case viruses may acquire the activated state in insect bodies. The agent causing such an activation is presumably a protein, present in the alimentary tract of the insect, which can combine specifically with the virus, like an antibody with the antigen.

As already mentioned, some insects can be parasitic on certain plants because of the presence of common structure between their proteins, whereas the same relationship must be present between a plant and a virus which can affect the former. Therefore, also between a virus and an insect living on the plant which the virus affects, there must likewise be common structure, through which the protein of an insect can combine specifically with virus. Thus, if the virus is taken into the alimentary tract of the insect, some protein present in the latter will combine with the virus. This combination should be analogous to that between antigen and antibody, and hence a kind of denaturation is to be raised in the virus, thereby the temporarily activated state will result in the latter. 
The presence of specific relation between insect and virus as well as the prompt disappearance of the infectivity can readily be explained by this concept. The efficiency of fasting of the insect upon the infection of the virus may be attributed to the increase by the fasting of the agent specifically acting upon the virus. It is fully expected that for the occurrence of the activation proper proportion of the virus to the activating agent will be required as in the case of antigen-antibody reaction. It is said that the optimum conditions for transmission are to use insects that have fasted for at least one hour, feed them on the diseased plant for about 2 minutes and then transfer them immediately to healthy plants (39). Increasing the length of time the aphids feed on the infected plant greatly reduces the number of infections obtained, indicating the decreased activation due to too much amounts of the virus.

Argument has been advanced thus far concerning the second phase of denaturation, whereas now we have to discuss the third phase shown in III in Fig. 21. If the environmental condition, under which protein molecules are present in the first state, is changed, thereby the structure of the protein being rendered unstable, then the protein will be changed in the structure to adapt itself to the new condition. It is considered that such a change is commonly accomplished by the refolding of the peptide chain.

Thus, if some protein is altered in its structure from form I to III in Fig. 21 by an environmental change, the structure represented by III must be stable under the new condition. Environmental change can be brought about by various physical or chemical agents, and hence, if some chemical agent causes the structural change from form I to III, it will be said that the form III is stable in the presence of the chemical agent.

The shift of the form, however, may often fail to occur when the environmental change gradually arises, just as water may be supercooled without being frozen, but when an adequate stimulus is applied to disturb the structure of the protein, the shift will immediately start to attain to the stable state just as super-cooled water is suddenly frozen by stir. For example, we have frequently observed that phage samples having been inactivated by some unknown causes are activated by such manipulation as the addition of inorganic salts or heating to a proper temperature (103). Such a phenomenon can be explained by assuming that the phage remained in an inactivated state despite of the removal of the inactivating agent, and that the manipulation served only to initiate the recovery of the original activated state.

In general, the mechanism of the reversible inactivation of physi- 
ologically active agents such as viruses, toxins, and ferments can be illustrated in the same way. Thus the nature of the reversibility of protein structure discussed in the previous chapter is also made clear.

As above discussed, some plant viruses may be activated in insect bodies, a phenomenon which has been explained by taking into account the second phase of the protein denaturation. This phase is considered to be brought about by some denaturating or inactivating agent present in the alimentary tract of insect, so that the third phase, reached by passing through the second phase, should involve the inactivated state. It is actually known that some agent capable of inactivating certain viruses are found in insect bodies.

However, if the inactivating action of the agent is not so strong as to completely inactivate the virus, the virus will be disturbed in its structure without being inactivated, and the disturbance may enable the virus to recover its original structure which the virus possessed before having been damaged by the host protoplasm from which it is now liberated.

Thus, if a virus which has been weakened by an unfavourable condition in the host cell, is removed from the host to an insect, a new host, in which no unfavourable condition is present and in which the virus can regain the original active state, then the virus can be said to have achieved "rejuvenation."

For the establishment of this rejuvenation in insect, some adequate stimulus to cause the shift towards the original active state may be necessary. Such a stimulus is possibly given to the virus through the contact of the virus with the insect protoplasm having a structure somewhat different from that of the virus. If such a stimulus is too strong, the virus will sooner or later be inactivated, although in the second phase of the inactivation or denaturation the transient activation may arise. In such a case plant viruses are transitorily activated in insect bodies without multiplication.

On the other hand, if the stimulus is mild and adequate the virus will be able to regain its original active structure in the insect body without inactivation, and in addition the virus thus activated is possibly capable of spreading its structure into the insect protoplasm, that is, the virus will be able to multiply in the insect body. Since different hosts must have different protoplasm consisting of proteins of different structures, although they may share some structure in common as they have the property to be affected by the same virus, the virus will possibly acquire some new structure through the multiplication in a new host.

The acquisition of a new structure on the multiplication in a new host means that the virus is assimilized to a certain extent by the 
new host, but by this process the virus will be able to get rid fully of the injurious assimilizing effect of the former host and thereby the rejuvenation will become quite complete.

The concept that plant or animal viruses can multiply in an insect, an entirely different organism, may seem at first sight unreasonable, but as already pointed out, the fact that an insect is parasitic on a certain animal or a plant shows the presence of common structure between the protein of the insect and that of the animal or of the plant; through this common structure the virus may be able to affect both the insect and the animal or the plant. In other words, the relationship analogous to that between an enzyme and its substate must be present between an insect and an animal or a plant on which the insect is parasitic, and a similar relationship must also exist between a virus and its host, and accordingly a virus can simultaneously affect both an insect and an animal or a plant.

\section{Various Means for Rejuvenation}

Whereas the host change appears to be most essential to the evolution of viruses, it cannot be said that all fixed viruses are transmitted by insects. For example, viruses of such diseases as measles, mumps, or small pox seem to have no insect vectors, a fact which appears contradictory to the writer's view.

Rejuvenation is, however, indispensable not only for viruses but also for every organism without exception. Organisms are generally supplied with a splendid tool to perform the rejuvenation. This tool is sexual reproduction. By means of this tool a protoplasm having a certain structural pattern can combine with another protoplasm having different structure, thus being able to achieve the structural exchange, a process which may be called mutual assimilation, and by this process rejuvenation is accomplished. This appears quite analogous to the rejuvenation of virus by means of the host change.

The writer holds the opinion that above cited viruses, such as those of measles and mumps which appear unable to perform any host change, can achieve rejuvenation by availing themselves of the sexual reproduction of the host. As fully discussed already, some fixed viruses can be transmitted to offspring through the germ cells of the host; in the case of insects the transmission is said to be usually established by eggs. Since egg cells are to be rejuvenated on the fertilization by the contact with sperm cells composed of proteins with somewhat different structure, the virus which has become a part of the egg cell should also be rejuvenated through this process. 
When the structure of a virus becomes the stronger, the longer it may remain in the host, until it can even be transmitted to the progeny. It may be said, therefore, that a virus can attain of itself the property to perform the rejuvenation as it evolves to a certain extent. Thus, it follows that the strong structure becomes indispensable for a virus to continue its existence not only for the reason of the necessity of the stable nature but also of the need of the ability to make use of the rejuvenation of the host.

Plant or animal viruses which can multiply in insect vectors may naturally avail themselves of the sexual reproduction of the customary hosts as well as of the insect vectors when the virus acquires structure strong enough to spread their pattern to the germ cells. Thus it should be concluded that most evolved viruses are always to be transmitted to the offspring through the germ cells of the host.

In order to acquire such a strong structure the majority of viruses might have attained to the habit of host change; therefore, a virus, which have bocome transmissible by a vector, may be said to have come up to the easy course leading to a typical fixed virus. The writer has already expressed the opinion that viruses such as those of influenza and encephalitis may be newly generated viruses, but some of them might possibly have already arrived at such a course and hence it may be unreasonable to regard all of them as being new viruses. Indeed, it is generally accepted that some encephalitis viruses do posses insect vector and further that they can give rise to considerably long lasting immunity in their host. Likewise, some strains of influenza virus may be half-fixed through a host change unknown to us. It has been reported that swine influenza virus is actually disseminated by a lung-worm parasitic to the dog (104). The so-called pneumonitis virus, highly specialized virus-group causing pneumonia, may be an advanced form of influenza virus.

It seems little doubt that insects or arthropods are generally utilized for the host change, but also other organisms may possibly be used for the purpose. For example, if a dog infected by rabies virus, it will become wild and maniacal and try to bite other animals, enabling the virus to be transmitted and at the same time to be rejuvenated. Some plant viruses, such as tobacco mosaic virus, known to have no insect vector, may have some means of rejuvenation, if they cannot use of the sexual reproduction of the host plant. It is believed that various plant viruses can contaminate the soil and persist in it for long periods so that many of the outbreaks in tobacco and tomato crops originate from virus surviving in the soil; in some favourable soils some plant virus could be ascertained to survive for more than 9 years (105). Such a fact led a number of workers to be- 
lieve that some soil-inhabiting organism acts as a vector, presumably a nematode (39)

Furthermore, it may be possible that some animal viruses multiply in certain bacterial cells to establish rejuvenation and subsequently to infect again the animals. If so, the bacteria would be thought pathogenic. The majority of workers believe that the causative agent of whooping cough is a kind of bacteria, whereas some others claim it to be a virus; the infection was reported actually possible through the filtrates of the patient excretes.

Again, scarlet fever is usually believed to be caused by streptococcus but repeatedly a virus has been suspected. Bringel (106) has claimed to have succeeded in isolating a filtrable agent which makes a strain of haemolytic streptococcus capable of causing scarlet fever. This agent when present in the filtrate is so unstable as to be inactivated by heating to about $60^{\circ} \mathrm{C}$. for $45 \mathrm{~min}$.

If a virus causes a venereal disease, it can be rejuvenated by being transmitted from one sex to the other. Such a rejuvenation may be as complete as that brought about by sexual reproduction, since the virus is always to be transmitted in turn between both sexes. It is known that the virus of lymphogranuloma venereum, a highly advanced virus causing a venereal disease, fails to leave any immunity in the host in spite of its highly fixed character. For achieving the rejuvenation, the establishment of the immunity must be unfavourable for such a virus, so that the character not to leave the virus pattern which can contribute to the immunity might develop, the individuals having this character being selected as the fittest. Such a virus might evolve into bacteria, such as gonococcus, which have also the character to cause no immunity. 


\section{CHAPTER $\mathbf{X}$ \\ THE SECONDARY ORGANISMS}

\section{Parasitism and Commensalism}

Since pathogenic filtrable agents are designated viruses, pathogenicity must be an essential feature of viruses, whereas pathogenicity is by no means needed by protoplasm particles to exhibit the assimilase action. Only when the particles cause an injurious effect on the protoplasm of cells on which they exert their assimilase action they may be called viruses. Their existence therefore may not be realized if no injurious effect follows. Plant viruses may be called so because they can cause deleterious effectes in plants, but when transmitted to insect no effect is exhibited, so that despite the occurrence of their multiplication, their existence is only demonstrated when they are given back to the plants. In general, no difference can be found between the insect carrying the virus and that carrying none. In such a case the virus may be called a, latent.

It seems highly possible as already discussed that protoplasm particles derived from normal, healthy cells act as a virus on some other cells, but it must be of most difficulty to distinguish a genuine latent virus from normal particles capable of acting as a virus. Presumably the term latent virus have been used up to the present without taking account of this problem.

Assimilase action is usually involved in the protoplasm having its full form, only rarely the action being preserved in the decomposed particles. But, for the development of an assimilase into an independent organism, it must be quite necessary for the assimilase to retain its function in the form of decomposed particles.

There is no reason to suppose, however, that pathogenicity is needed for the evolution of virus. On the contrary, it seems to be rather deleterious, because severe pathogenic action may have unfavourable influences on the multiplication of viruses.

If a virus possessed a virulence so violent as to infallibly kill the host, it would soon be perished together with the host. It may, therefore, be supposed that less virulent or non-virulent viruses can more easily pursue the course of the evolution than do more virulent. Thus the number of non-virulent viruses existing may be much greater than 
expected. However, we are unable to demonstrate their existence because a virus is detected always on the basis of its pathogenicity.

Nevertheless, when viruses have highly evolved as to arrive at the state of Rickettsiae, their existence may become demonstrable on account of the peculiar shape and size of their particles even if they have no pathogenicity. Thus, although only six or seven species of Rickettsiae have been found in association with disease of mammals, over forty non-pathogenic species have been described in various insects and other arthropods (88). In addition, isolation of various nonpathogenic pleuropneumonia-like organisms from the genitourinary tract has frequently been reported (107). Such organisms are considered to be intermediate between Rickettsiae and bacteria. It must be emphasized that the majority of the bacteria thus evolved from viruses are likewise non-pathogenic.

Some bacteria are not only non-pathogenic but sometimes appear to have favourable effect on the host. This may be the case also with some viruses, because in doing so the viruses may produce in the host more beneficial conditions for their multiplication and accordingly for the continuance of their existence, though it is also conceivable that some proper injurious effect upon the host may sometimes be desirable for their multiplication, for it is occasionally recognized that emaciated organisms are more readily affected by a virus, a phenomenon which is also well confirmed in some Rickettsiae.

Most workers have failed to find any significant differences between infective and non-infective individuals of insect vectors. Commonly infective individuals live as long as and breed as non-infective. However, a beneficial effect from feeding on celory and aster plants infected with aster yellow virus has been described by Severin (108), who found that several leafhopper species multiply more freely, pass through nymphal stages sooner, and live longer than those feeding on healthy plants. In most cases the difference was quite clear-cut, the insects dying off in a few days when put on healthy plants, but building up flourishing colonies when put on diseased ones. There are numerous leafhopper species which live on diseased celory and asters, but when transferred to healthy plant they die. Likewise with aphid and leaf-roll mosaic, Arenz (109) has found that the vector multiplies substantially faster on diseased potatoes than on healthy. These may show the favourable effect of the virus upon the host, but at the same time they may be regarded as being only the examples where injuries given rise to by virus upon the plant benefit the proliferation of the insect (110). However, in discussing numerous cases of an association between insects and microorganisms for mutual benefit, Leach (111) has claimed that there is no reason why association 
of insects and viruses may not have arisen in a similar manner.

Sonneborn (112) has found in a kind of paramecia a virus-like agent designated kappa; the individuals of this protozoan containing this agent produce a substance called paramecin which is able to kill another kind of individuals having no kappa, but when a large amount of this agent is present, the protozoan acquires the property not to be killed by paramecin. Protozoa not containing kappa or with only a few are readily killed by paramecin. The presence of kappa, therefore, confers a survival advantage to the protozoan. This agent is believed by some workers to be a kind of Rickettsia-like microorganisms parasitic on the protozoan. Likewise in various arthropods there are found Rickettsia-like bodies, known as bacteroids; they have rarely, if ever, been grown outside the living host. It is possible, however, to eliminate them in a certain insect by treatment with penicillin or with certain sulfa drugs, and in this way to show that bacteroid-free individuals invariably die.(113). This is no doubt an example of commensality. It is a noticiable fact that the bacteroids are said to be transmissible to progeny through the eggs like viruses.

Anyhow, it may safely be mentioned that numberless seeds of secondary organisms are still continuously being poured upon the earth, but that only exceedingly small portions of them are to evolve into organisms. The seeds are, of course, protoplasm particles with assimilase activity having become independent of the protoplasm. They are recognized as the virus when able to confer some injurious effects upon certain cells, but the majority of them may silently be pursuing the course of the evolution leading to the secondary organism without being recognized.

\section{Important Significance of Parasitism}

The primary organisms were presumably generated and developed in water. They were unable to leave the water until evolved into highly developed creatures, whereas the secondary organisms were produced in cells of other organisms already created, and developed in the cells, so that their parasitic nature would be of most obstinacy. For the second organisms the liberation from the parasitism, therefore, would be as serious and as difficult as would be the release from the water for the primary organisms.

In this respect any parasitic organisms, living outside the water despite their comparatively primitive nature should be regarded as the sccondary. Accordingly, at least the majority of bacteria and protozoa should be considered to be the secondary organisms. To be sure, 
bacteria are mostly parasitic, and all the major groups of protozoa, except the sporozoa and the sustoria, involve both free-living and parasitic forms. Sporoza are entirely parasitic and sustoria are sedantry, although they are often found attached to the surface of fresh-water organisms. Many parasitic protozoa, especiallly sforozoa, undergo extremely complicated life cycles, involving not only several successive hosts but also distinct evolutionary states that are mor phologically different.

It is noticeable fact that exceedingly vast number of species are known in protozoa, over 15,000 species having been described, and that most modern authors are inclined to consider many groups of them as so many phyla (114). This would follow naturally if protozoa were evolved from viruses continuously being generated de novo. Parasitism must be the most distinct characteristic feature of the secondary organism, but such an existence of a vast number of groups, between which no phylogenetic correlation appears to be fresent, must likewise be one of their chief characteristics.

The so-called mesozoa, which was once recognized to be intermediate between protozoa and metazoa, are totally parasitic, and there seems no doubt in their secondary nature. Notwithstanding their highly evolved state, animals belonging to platyhelminthes such as flukes and tapeworms are still sticking to their parasitic nature. These animals are not only parasitic but also still clinging to the habit of virus of changing host and unable to proliferate without the troublesome host change. Thus host change must also be one of the characteristics of the secondary organisms. Malaria-producing protozoa and a kind of mosquito, trypanosomes and a fly, spirochaetes of reccurent fever and a tic or louce,--these are all the examples of the attachment to the habit of host change.

In the case of viruses the host change may be indispensable for the purpose of rejuvenescence, but it is rather strange that the secondary organisms are frequently clinging to the habit still at present when they have acquired much more suitable means, namely sexual reproduction, for the purpose. The habit may be beneficial for the transmission as in viruses, but this cannot be supposed to be the only reason for the persistence. If eggs of a parasite such as a tapeworm or a flute acquired the faculty to develop in the host bodies without troublesome host change, it would be said that a remarkable progress was established for their proliferation, but the host should be perished because of the dreadful proliferation of the parasite, resulting in the extinction of the parasite itself. During the long course of evolution, parasite capable of developing without host change might appear, but it seems no doubt that the host of such dreadful parasites would soon 
be perished, followed by the extinction of the parasites themselves. In this way the habit of the host change might be left as an indispensable character.

This may hold also for the plant kingdom. Smuts parasitic on higher plants are known to perform most complicated host change or life cycle; not only smuts but generally pathogenic fungi are mostly confirmed to be transmitted by insects. Thus insect-fungi relationship is highly organized and has broad biological and evolutional significance. The insects known to be vectors of plant disease-producing fungi are grasshoppers, crickets, aphids, scale insects, beetles, true bugs, flies, wasps, and bees (115). Plant diseases caused by bacteria are also numerous, and in the dissemination of pathogens from field to field, insects have been recognized as vectors since the days of very early investigations (116).

Likewise nematodes such as ascaris and filaria belong most probably to the secondary organisms. Ascaris has no insect vector, but its eggs cannot develop unless they are exposed to low temperatures outside the host body, probably contributing to the prevention from its too vigorous proliferation as mentioned above, but also serving to the achievement of the rejuvenation as will be mentioned later. It has been established that certain mosquitoes are vectors of filaria, causing elephantiasis in man. Several other species of nematodes associated with insects possess likewise a heterogenic generation in their life cycle. In addition, each of the three fundamental groups of annelids also gives rise to parasitic species that show, in almost every case, definitely specialized characters of parasitism.

The arthropods, constituting by far the largest phylum of the animal kingdom, may be the most advanced secondary animals, although the majority of them have already succeeded in getting rid of parasitism. Of crustaceans of the arthropods, all stages of parasitism occur especially in copepods. Further, mites and ticks are the parasitic acarina. Nearly all the groups of insects contain species that fail as yet to become free from parasitism either in the larval or in the adult stages. However, only very few insects are totally parasitic in all stages of their life history and these are practically restricted to the true lice and sucking lice.

It is customarily believed that parasitic organisms have been evolved from free living ones on adapting themselves to parasitism. If this were true, parasitic organisms should have been more advanced than the free living. However, the truth is that the most primitive organisms such as bacteria and protozoa are almost parasitic, whilst the most advanced organisms such as vertebrates including man are entirely free-living without a single exception, presenting a fair con- 
trast to the fact that Rickettsiae as well as viruses are all parasitic. Is it really conceivable that man and bacteria share common ancestry and that the latter have waited and waited throughout a dreadful long span of time in the most primitive unicellular form for their brethren to be evolved into man, on which they were bound to be parasitic? Is there any doubt in assuming the parasitism as being the most primitive mode of life of organisms?

\section{The Limit of the Secondary Organisms}

The writer holds the opinion that free-living organisms can never enter parasitic life unless they have a phylogenetical experience of parasitism. Parasitic life involves too specialized and too limited environment to be newly adapted.

Certain animals especially some insects are known to become parasitic at their adult stage although free-living at the larval. As will be fully discussed later, this must be a kind of atavism, never the new acquisition of parasitism. This occurs generally much more frequently in females than in males; it can be supposed that parasitism is more profitable for females because of some physiological function particular to females such as egg production or egg laying, and hence females that could accomplish the reversible parasitism or atavism might have been selected as the fittest.

Parasitic botflies are known to show a complicated life cycle. Botfly eggs can hatch only under the combined influence of the horse's tongue and saliva with which they are carried into the mouth, whence they burrow into the mucosa of the tongue or the palate and tunnel their way through until they reach the pharyx. The second larval stage is now reached. The maggots emerge from the mucosa and attach themselves on the surface, close to the epiglottidean region. They finally pass down into the intestine and each species takes up its abode in a particular region, some in the stomach, the others in the rectum or the duodenum. They generally abandon their host before pupating and are evacuated with feces (117). The life history of ascaris is somewhat similar to this, indicating a similarity in their parasitic nature existing between nematodes and insects. Insects, in general, possess at their larval stage a form strikingly resembling that of nematodes, a fact in which a relic of parasitism is seen.

Metamorphosis, especially distinct in insects, may suggest the rapid phylogenetic occurrence of the liberation of themselves from parasitism just as the sudden release from water of a tadpole with the establishment of metamorphosis. 
It was said that the difference between a carnivore and a parasite is simply the difference between living upon capital and income, between the burglar and the blackmailer, but this view may not be legitimate, for there seems an essential difference between them. A carnivore may eat flesh of every kind of animals, whereas between a parasite and its host there is a specific relationship which should be found between a virus and its host or even between an enzyme and its substrate. Caterpillars feed on the leaves of plants, but there is a definite relation between a caterpillar and the sort of the plant. Again, there is also a specific relationship between a mushroom and the kind of trees on which it grows.

As to the plant kingdom, not only mushrooms but generally fungi can be regarded as the secondary organisms. Schizomycetes or bacteria may be regarded as the secondary as above stated. Likewise phycomycetes or slime fungi, at least plasmodiophorales may be so, since these are especially distinct in their parasitic nature. The groups of fungi proper, including phycomycetes, ascomyce'es and basidiomycetes, that constitute the overwhelming large phyla of the plant kingdom are all parasitic to more or less extent. They may possibly be the organisms having evolved chiefly from various plant viruses.

It should be noted that most of the fungi, though prefering moist locations, do not thrive if submerged in water; but their parasitic nature is marked, indicating clearly that they are never the primary organisms generated and advanced in water. Among them the rust fungi, protobasidiomycetes, are striking examples of obligate parasite, that is, organisms unable to live except in living tissues. They are incapable of living in culture media, even when the media are made from the host plant upon which they thrive.

Almost all the fungi are thus parasitic, but algae are free-living although many of them are extremely primitive both in form and function. For example, the cyanophyta, or blue-green algae, are so primitive that there is no morphological separation of nucleus and cytoplasm, chromatin being distributed throughout the cell. Non-parasitic nature, however, would be no gainsaying their possibility of being secondary organisms. It may be highly possible that the secondary organisms having been generated from higher plants, which can utilize the energy of sunlight by virtue of the catalytic properties of chlorophyll, may also be able to do so in their extremely primitive state because they may inherit the faculty from the plant.

The fact that phyla or classes in the plant kingdom are extraorainarily numerous and complicated, no phylogenetical correlation appearing to be present among them, suggests that at least the majority of them may be the secondary organisms. It may rather be 
possible to suppose that all the plants may be the secondary. The thallophyta, to which algae and fungi belong, include plants of great diversity, but many of which have little in common save the small size and simplicity.

On the other hand, it is a noteworthy fact that there is entirely no parasitic species in chordates, the most advanced animals, among the classes of which the most orderly phylogenetic relation can be found. This suggests that chordates are the primary organisms which might provide the first scaffolding to the secondary organisms for their evolution as well as for their generation. Moreover, it should be noted that no species of parasitic echinoderms is known, although certain kinds of echinoderms provide the host to parasitic snail, but they themselves are never parasitic, whereas it is generally accepted that there seems a closely related phylogenetical connection between echinoderms and chordates. This fact also suggsts that echinoderms as well as chordates are the primary organisms.

Now we must consider in this connection of mollusks, one of the most highly organized phyla of animals. It is remarkable that different groups of mollusks are parasitic. In unionidae parasitism is resorted to by larval forms only, known as glochidia, parasitic on fishes, whereas in parasitic snails the adults are parasitic and the larval forms free-living, the hosts being always provided by echinoderms (117). In contrast to arthropods, however; the parasitic nature of mollusks is rather exceptional, only being observed with some peculiar kinds. Nevertheless, since parasitism does exist in mollusks, if as relics, it may be not unreasonable to regard mollusks as the biggest senior of the secondary animals that were evolved from viruses in the oldest age.

Of course, if a secondary organism was developed to a certain extent, viruses would naturally be generated in it, and the viruses in turn would be evolved into organisms in this senior secondary organism, and hence it cannot be said that the secondary organisms are always generated and evolved in the primary organisms.

The writer thus has come to possess the strong view that viruses represent a transitional stage from non-living to living whereas many authors consider that viruses have evolved by a process of retrograde evolution from higher organisms, that is, present-day viruses are direct descendants of peculiar forms that were once free-living. This idea appears to be based upon the fact that saprophytic organisms sometimes lose the ability to synthesize certain essential growth requirements, involving the loss of structure and functions that are no longer needed, thus being degenerated both in their function and forms to become apparently very primitive organisms. They believe that in 
a similar way viruses have evolved from higher organisms as a result of degeneration due to the parasitism. It seems, however, that this idea might be based on a grave misunderstanding as regards parasitism and the basic principles of life phenomena as well.

It is true that parasites seem to be often very highly modified in their structure to meet the demands of their particular enviroment. Fixed parasites generally have neither sense organs nor well-developed locomotive functions, and intestinal parasites do not need highly organized digestive tracts. However, parasites must be specialized, ofter to a very high degree, to adhere to or to make their way in their particular host, or the particular part of the host in which they find suitable conditions for their existence. Still more remarkable are the specialization of parasites in their reproduction and life history to insure, as far as possible, a safe transfer to new hosts for succeeding generations. .Every structure, every function, every instinct of many of these parasites appears to be modified, to a certain extent, for the sole purpose of reproduction.

The apparent degenerancy associated with parasitism is, therefore, nothing but the highly developed specialization wonderfully adapted to the peculiar, extremely limited, environment. As above cited, viruses are distinct from typical organisms in their lack of self- or race-preservation instinct, whereas in ordinary parasites this instinct is found to be most manifest as just stated.

\section{Inclusion Bodies and Metamorphosis}

Many virus infections are characterized by the presence of peculiar bodies, usually referred to as inclusion bodies, in the nucleus or cytoplasm of parasitized cells. The bodies are rounded, oval, or irregular in shape. It has proved possible to photograph with the electron microscope inclusion bodies such as those of mouse ectromelia and human variola, and they are seen to consist of aggregations of virus particles clumped together. Thus it is difficult to regard inclusion bodies as other than intracellular aggregations of virus particles.

It has already been stated that plant-protoplasm particies are liable to aggregate into larger bodies sometimes resembling protozoa. This may be regarded as the production of inclusion bodies in vitro. The inclusion bodies may be produced in the host cells when virus structure is enveloped by the normal structure of the protoplasm; since both structures can not melt into each other because of their different structures, the virus structure may have to exist in forming a mass distinctly separated from the host protoplasm. 
If a virus structure was readily assimilized and destroyed by the normal structure when existed in separate particles, whereas in a large aggregated state it was stable and could resist the assimilase action of the protoplasm, then the large mass only would remain as the virus. In view of the virus nature already discussed such an occurrence seems highly probable.

The shape of inclusion bodies are said occasionally to tend to be peculiar to the kind of viruses and of the host cells. Since the bodies formed by the aggregation of virus particles may be regarded as a kind of liquid crystals like the protoplasm, the shape may naturally be exerted some influences by the kind of protoplasm as well as the sort of the virus. The shape accordingly may change with the host cell as well as with the virus. It should be remembered that the shape of crystals is, as a rule, determined by the solvent or the environment, in which they are formed, as well as by the component substance of the crystal.

If some viruses or some microorganisms could exist only in a form like that of inclusion bodies, they would vary their shape with the host or with the surrounding condition. This may be the reason why parasitic microorganism, existing in the form of virus-aggregate, alter their shape whenever they change the host. As already stated, the protoplasm can be regarded as a virus-aggregate or a mass composed of elementary bodies. The mechanism of metamorphosis may thus be developed.

The earlier workers regarded inclusion bodies of some viruses as protozoa, and pictured them as varying stages of an elaborate life-cycle. From the above point of view, it may be said that this supposition is, in a sense, correct. In fact, at present, the majority of workers considered that highly advanced viruses such as those of psittacosis and lymphogranuloma undergo a developmental life cycle, in which virus particles or elementary bodies enter a cell, and then increase in size to become initial bodies; a larger form of inclusion known as a plaque or morula is then formed from the initial bodies, and comes to contain large numbers of elementary bodies, which eventually rupture from the cell (118). This phenomenon can be interpreted as "crystallization" of virus particles or elementary bodies in the host protoplasm into larger bodies, which are decomposed when the environmental condition is changed.

It follows from the theory of the writer as regards the nature of protoplasm and virus that inclusion bodies should be produced by proper chemical or physical agents other than viruses, if structural changes similar to those induced by virus are given rise to in the protoplasm. In fact, it is known that a part of protoplasm having 
undergone a change by some injurious agent is separated from the normal protoplasm in forming an inclusion-like body which sometimes is expelled from the cell.

Yamafuji et al. (119) by applying peroxides have succeeded in producing in silk-worms inclusion bodies similar to polyhedral bodies peculiar to polyhedrosis. The generally confirmed mutagenic activity of peroxides in microorganisms shows that protoplasm is liable to be changed in its structure by these chemical agents. Of course, the formation of an inclusion body must be entirely different from the production of a virus. 


\section{CHAPTER XI \\ THE SUMMARY OF PART III}

\section{1}

Organisms may sometimes be changed in their properties when acted upon by proper chemical or physical influences. The change is termed mutation or variation and is probably caused by an alteration of the protoplasm structure including genes. If an altered structure thus produced is strong and retained in the protoplasm particles, the particles may be able to behave as a virus towards some other cells having the protoplasm of a weaker structure. Special case of such change involves adaptation, and the production of viruslike agents in the adaptive change of microorganisms is occasionally reported.

Even protoplasm particles of normal structure, provided the structure is preserved in the particles, may act as a virus upon certain cells having weaker protoplasm. Phage produced by some lysogenic strains may be regarded as such normal particles. Similarly, phage present commonly in chicken feces may be normal protoplasm particles of chick cells. Many other examples can be cited showing that normal protoplasm particles can behave as a virus.

Cells affected by a virus may acquire the faculty to produce the virus, even if they have not fallen into a pathological state, as a result of mutation or variation due to the virus. Since the protoplasm structure of a cell is to be changed into virus structure when affected by a virus, the cells affected by a virus naturally produce the virus.

\section{2}

If a new protoplasm structure, exhibiting assimilase action stronger than that of the original structure, is produced in a cell or cells of some tissues or organs the newly formed structure will spread into the surrounding cells by assimilizing the protoplasm. Allergic dermatitis or various tumours including cancer may occur in such a way. The general failure of virus detection in these cases is presumably due to the lability of the structure unable to be retained in 
the protoplasm particles.

If the protoplasm structure in question is preserved in decomposed particles of protoplasm, the structure will be transmitted to other cells or organs through such particles, and if the cells or the organs fall into a pathological state on the transmission of the structure, the latter particles will be regarded as a virus, and the pathological state will be called an infectious disease. Tumour diseases are commonly not infectious, as the structures cannot, as a rule, remain in the protoplasm particles. Although viruses are occasionally demonstrated in certain tumours, it cannot be said that the structures are stable enough to make tumours regarded as infectious diseases always caused by a virus.

The structure of virus can be altered by various causes. If a virus retains the faculty to act as such after a change is induced in its structure, the changed structure can multiply, so that a virus strain having the changed structure may be produced, because the only function of viruses is to confer their own structure to the protoplasm of cells which they affect, thus the altered structure being inherited to "progeny." Since the property of viruses is determined by their structure, the alteration in the structure is to be accompanied by the change in the property, and therefore the changed property is to be inherited with the altered structure. In such a case the virus is said to have undergone a mutation or variation.

Viruses may easily be generated and each particle of them may have individuality even immediately after their generation. In addition, they can variate in various possible directions. Among the particles with various properties thus having arisen, some ones with suitable property for the continuity of their existence may continue to exist in escaping the extinction. However, the probability of incidentally acquiring the property to become a virus able to continue to exist for prolonged periods is apparently extremely small, presumably far smaller than that of spermatozoa of higher organisms to meet an egg-cell to develop into a full individual. This may be attributed to the lack of the property in the newly generated viruses compatible with the self- or race-preservating instinct of higher organisms.

In order to escape the extinction, high stability of the structure is essential for the virus. If the structure is too unstable to be preserved in the protoplasm particles, virus may scarcely be found as in the case of eczema. 
Even when the structure can be retained in the particles, it will soon be destroyed if it is labile, whereas stable and firm structure may continue to exist, imparting the stable pattern to the host protoplasm, thus the virus with the strong and firm structure can multiply far from becoming extinct. The more stable and the stronger is the structure of a virus, the longer periods it will exist and the more abundantly it will multiply.

Viruses may affect certain cells because they are stronger in the assimilase action than the protoplasm of the cells; in other words, in order to multiply in the prototoplasm viruses must beat the protoplasm in the competition for assimilase action. The protoplasm on its part, however, may be making a continual effort to assimilize the virus. Accordingly, if a virus sticks to the same kind of cells for prolonged periods, it will gradually be assimilized until at last becomes identical with protoplasm, resulting in the complete disappearance of its action. Viruses with unstable structures will be soon assimilized and cancelled, whereas the ones with strong structures may resist the action of the protoplasm to continue their existence.

Organisms once affected by a virus may acquire the resistance to the virus, and become immune to it. Since the protoplasm structure of the organisms is to become identical in its structural pattern with the virus following the infection, it may only be a natural result that the organisms will become indifferent to the virus which shares the structural pattern in common with the organism. Such an immunity should last as long as the virus structure remains in the organisms. Strong viruses can remain long periods, so that immunity induced by a strong virus is of a long duration, whereas weak viruses may cause only transient immunities.

In many virus diseases, such as measles, mumps, and yellow fever, the immunity persists for prolonged periods, usually for life, while in some other diseases, such as poliomyelitis and influenza, it is only transient. It may be supposed therefore that the viruses of the former diseases are highly advanced and are endowed with strong structures, whereas the viruses of the latter diseases are probably newly developed and unstable. 
The structural change of protein is liable to be reversible. This reversibility is especially distinct in the protoplasm. Thus distorted protoplasm can spring back like a spring to its original state when an agent causing its distortion is removed; that is, the protoplasm, in remembering its former structure, is inclined to recover the structure it once possessed.

The recovery from virus diseases may chiefly be achieved owing to this reversibility of protoplasm. The reversibility tends to persist even when the protein is freed from the protoplasm, resulting in the fact that protein denaturation is generally reversible to some extent. Thus inactivated viruses may sometimes be reactivated when the inactivating agents are removed. A virus once subjected to a variation will tend to recover its original property if released from the causative agent leading to the variation.

The failure of virus demonstration in some inflammatory diseases, such as allergic dermatitis, may be attributed to a high reversibility of the distortion. On the other hand, the distortion induced by strong viruses may be so intensive that the recovery of the original structure is rendered impossible. Since such a distortion means a structural change of the protoplasm, a distortion induced by a virus must involve the structural patttern of the virus itself, and accordingly the irreversible distortion is nothing but the multiplication of the strong irreversible virus.

\section{6}

However strong and firm the structure of a virus may be, it will gradually be assimilized and annihilated by the assimilase action of the host protoplasm if the virus continues to multiply in the same kind of host, thereby the virus structure becomes identical with the protoplasm structure. In order to escape this fate virus has to change the host.

Host change enables a virus, which has already assimilized to some extent by the host protoplasm, to come into contact with the protoplasm of a new host having a structure different from the former host. Through this contact the virus will recover the original, nonassimilized structure, and at the same time structure of the new host will be introduced into the virus; thus the rejuvenescence of the virus is established. 
A virus can affect different individuals if these belong to a similar kind of organisms, since similar organisms share some protoplasm structure in common, through which the virus can affect them. A virus affecting customarily a certain animal, however, is occasionally able to infect a blood-sucking arthropod, parastitic on the animal, and multiply in it. This is probably due to the existence of common structure between the animal and the arthropod because it is considered that the relationship analogous to that between viruses and their hosts or between enzymes and their substrates is present between the host animal and the arthropods. This appears likewise to hold true between plants and their parasitic insects. Viruses make use of such arthropods for their host change not only to be saved from the extinction due to the assimilase action of the host but also to be rejuvenated with the enhancement in their activity.

Viruses having insect vectors are, as a rule, not mechanically transmitted by insects, but rejuvenated in them. To evade extinction newly generated viruses presumably have to acquire such means of rejuvenescence; those incapable of succeeding in the acquisition of such means would soon become extinct.

Whereas numerous viruses are transmitted by insect vectors, some viruses, which are never thought to be newly generated appear to possess no insect vectors. Such viruses are supposed to be capable of establishing rejuvenescence without insect vectors, presumably by making use of the sexual reproduction of the host.

If a virus has evolved highly by acquiring a strong structural pattern, it may become able to impart to the host protoplasm lifelong lasting distortion, giving rise to the immunity lasting as long. It is considered that the distortion caused by an extremely strong virus persists in the host for life but also may be transmitted to the progeny through the germ cells of the host. Many evidences are actually known indicating that some viruses are transmitted to offspring through eggs or seeds. Certain plant viruses can also confer a profound distortion of this kind on their insect vectors; as a result the viruses can be demonstrated in the offspring of the vectors.

The rejuvenescence of organisms is generally accomplished by sexual reproduction, in which a germ cell is rejuvenated by fertilization through the contact with a germ cell of the other sex, just as a virus is rejuvenated by a host change through the contact with protoplasm of a new host. If the structural pattern of a virus is in- 
volved in a germ cell, the pattern will be rejuvenated together with germ cell by the fertilization. The viruses, such as measles and mumps, can probably be rejuvenated in this way without insect vectors. Structural pattern of virus present in the germ cell, if ever present, possibly exists in an altered state, so that it may fail to act as the virus itself, but when the germ cell develops into an adult form after the fertilization, the pattern may also be developed into its original form, thereby becoming capable of acting as the virus; thus the virus may appear in the progeny only when germ cells have developed to a certain extent. As will fully be discussed in Part V, such is not a phenomenon restricted to virus pattern but may occur generally in various, normal patterns which may develop to produce adult forms.

Virus demonstration is commonly impossible in larvae hatched from the eggs deposited by an infected insect, but as they develop into adult forms the demonstration may become possible. In like manner, measles virus may not be demonstrated in a newly born baby, but the full pattern of the virus will develop as the baby grows, and thus the baby or the child will suffer from measles by the virus developed in its body.

The structural pattern of a virus will naturally be spread to the germ cells, when the structure of the virus becomes strong, and thereby the virus can acquire by itself the means of rejuvenescence. This must be the general course of the virus evolution. Thus, the virus possessing insect vector can be rejuvenated by both the host change and sexual reproduction of the host. Such an intimate correlation of a virus with the host results in the excretion of the virus from apparently normal organisms. In such a case the virus may be called "latent," but to distinguish latent viruses from newly generated ones may be difficult.

The increase in the strength of virus structure answers not only the purpose of self-preservation of the virus, but also that of racepreservation as just pointed out. Therefore, as the virus structure becomes the stronger the virus may become the fitter to continue to exist, and thus the property of self- or race-preservation will become more marked until it can be called instinct.

Both the strength of the virus action and the structural stability of the virus seem to be directly proportional to some extent to the polymerization degree of protein molecules, $i$. $e$, the size of virus par- 
ticles, so that the increase in the strength of virus structure will follow the increase in the particle size. Since virus particles as well as the protoplasm, the decomposed particles of which are virus particles, are presumably a kind of liquid crystals, the "crystal form" will be determined by the property of the protein component, which, in turn, will govern the structural pattern of the virus or of the protoplasm, and therefore the pattern of a virus producing large "crystal" will be transmitted to the host protoplasm; as a result a large sized virus will reproduce a similarly large sized virus.

For the continuity of the existence various properties similar to those of organisms, including size and shape, are essential for viruses. Therefore viruses mostly resembling organisms will become fitter and develop up to the stage of Rickettsiae until they come to get possession of the faculty to assimilize even proteins present in blood which can be regarded as a streaming protoplasm. Thus the faculty of multiplication without living cells will be obtained together with the stage of undoubted organisms.

\section{9}

When some protoplasm particles affect certain cells which are weaker than the particles in their assimilase action, and can make cells fall into pathological state, then the particles will be called viruses. However, particulate assimilases may not always exhibit the pathogenic action.

The presence of virus is acknowledged only by the pathogenicity; therefore the demonstration of the non-pathogenic virus must be most difficult, if not impossible. Latent viruses are usually non-pathogenic, but when environmental conditions are altered the pathogenic property may sometimes be revealed to prove their existence.

When a virus is developed up to the stage of Rickettsiae, its existence will be readily recognized, in contrast to undeveloped viruses, by its peculiar form even without pathogenicity. Thus, nonpathogenic groups of Rickettsiae known are much more numerous than the pathogenic ones.

For the evolution of particulate assimilase into organisms, any intensive pathogenicity may not be needed. On the contrary, in most cases it may prevent the evolution, because virus may be unable to find any favourable condition for their multiplication in the severely injured host, and, therefore, less pathogenic viruses may become fitter. From this point of view, non-pathogenic viruses existing are expected to be much more numerous than pathogenic ones as in Rickettsiae, 
although the existence of non-pathogenic viruses cannot be demonstrated so readily as in Rickettsiae. Moreover, the existence of viruses exerting favourable effects upon the host is conceivable, that is, the symbiosis between viruses and their hosts may be possible.

\section{0}

The writer designates the organisms having evolved from viruses, pathogenic or non-pathogenic, as the secondary organisms. The organisms having served as the first scaffolding for the secondary organisms are naturally to be called the primary organisms.

Whereas the primary organisms were generated and evolved in water, the secondary organisms depended on the protoplasm of senior organisms both for their generation and for evolution. Therefore, for the secondaty organisms, the liberation from the dependence upon the senior organisms might be a matter as difficult as the release of the primary organisms from the water. Accordingly parasitic, primitive organisms living outside the water should be regarded as the secondary organisms. From this point of view, at least the majority of bacteria, protozoa, and fungi, and also presumably more advanced parasitic animals such as platyhelminthes and nematodes may be secondary organisms. Furthermore, arthropods are considered to be likewise so, most of which have already succeeded in liberating from the parasitism.

If arthropods are the secondary organisms, it should naturally follow that the relationship between insects and plants or animals on which they are parasitic is analogous to that between viruses and their hosts; and this latter relationship may, in turn, be regarded as similar to that between enzymes and their substrates.

Some adult arthropods enter into parasitism after free-living larval stages, a fact which is to be interpreted as an atavism. It is considered to be impossible that free-living organisms can incidentally enter into parasitism, a most specialized, and accordingly extremely limited environment. Therefore, it may be proper to regard the organisms as the secondary ones if they reveal parasitic nature at any stage in their life. In this respect, mollusks may be supposed to be the secondary animals generated in the oldest age. It should be noted that there is no parasitic groups in chordates and also in echinodermates which the latter are generally believed to be intimately related phylogenetically to the former, suggesting that they are the primary organisms in the animal kingdom.

It is a remarkable fact that various secondary organisms are still 
clinging to the habit of cumbrous host change which was indispensable for the rejuvenescence at their viral stage but which has lost its essential significance now that the organisms have acquired the ability to perform the sexual reproduction, the better method for the rejuvenation. This shows how difficult is for the organisms the abandonment of their long abiding habitat. 


\section{REFERENCES}

(1) Ohashi, S.: J. Shanghai Sci. Inst., (4), 141, 1939., (5), 1, 34, 47, 1940.

(2) Moriyams, H., and Ohashi, S.: Kagaku (in Japanese), (11), 6, 1941.

(3) McKinley, E. B.: J. Laboratory and Clin. Med., (90), 185, 1923.

(4) Tempé, G., and Uhlhorn, M. : C. r. Soc. Biol., (109), 659, 1932.

(5) Ohashi, S.: Igaku to Seibutsugaku (in Japanese), (12), 261, 1948.

(6) Burnet, F. M. and McKee, M.: Aust. Jour. Exp. Biol. Med. Sci., (6), 277, 1929.

(7) Lwoff, A.: Bact. Revs., (17), 269, 1953.

(8) Freeman, V. J. : J. Bact., (61), 675, 1951; (63), 407, 1952.

(9) Smith, H. W.: J. Hyg., (46), 82, 1948.

(10) Roustree, P. M. : J. Gen. Microbiology, (3), 153, 1948.

(11) Fleming, A.: Proc. Roy. Soc., (B), (93), 306, 1922.

(12) Moriyama, H. : Immunity, in press.

(13) Moriyama, H., and Ohashi, S. : J. Shanghai Sci. Inst., (4), 51, 1939.

(14) Ohashi, S. : J. Shanghai Sci. Inst., (4), 51, 1939.

(15) Ginsberg, H. : J. Exp. Med., (94), 191, 1951.

(16) Avery, O. T., et al. : J. Exp. Med., (79), 137, 1944.

(17) Hotchkiss, R. D. : Colloques intern. natl. recherche Sci., (8), 57, 1949.

(18) Reichmann, M. E., ct al: J. Amer. Chem. Soc., (74), 3203, 1952.

(19) McCarty, M. : Bact. Revs., (10), 63, 1946.

(20) Taylor, H. E. : Compt. rend., (228), 1258, 1949.

(21) Boivin, A. : Cold Spring Harbor Symp., Q. Biol., (12), 7, 1947.

(22) Weil, A. J., and Binder, M. : Proc. Soc. Exp. Biol. Med., (73), 485, 1950.

(23) Alexander, H. E., and Leidy, G.: Proc. Soc. Exp. Biol. \& Med., (73), 485, 1950.

(24) Dubos, R. J.: The Bacterial Cell, 184, 1949.

(25) Olitzky, P. K. : J. Exp. Med., (72), 113, 1940.

(26) Daneel, R.: Naturwiss., (29), 364, 1941.

(27) Kabat, E. A. et al.: J. Exp. Med., (88), 417, 1948.

(27a) Kilham, L, and Herman, C. M.: Proc. Exp. Biol. \& Med., (85), 272, 1954.

(28) Smith, K. M. : Nature, (167), 1061, 1951.

(29) Smith, K. M., and Wyckoff, R. W. G. : Research, (4), 148, 1951.

(30) Darlington, C. D.: Nature, (154), 164, 1944.

(31) DeBuy, H. G., and Woods, M. W.: Phytopath., (33), 766, 1943.

(32) Muller, H. J.: Amer. Naturalist, (56), 32, 1922.

(33) Duggar, B. M., and Armstrong, J. K. : Ann. Missouri Bot. Gard., (10), 191, 1923.

(34) Wyss, D. : J. Bact., (54), 294, 1947.

(35) Hotchkiss, R. D.: Cold Spring Harb. Symp. Quant. Biol., (16), 457, 1951.

(36) Voureka, A.: Lancet, (1), 62, 1948.

(37) George, M., and Pandalai, K. M. : Lancet, (1), 955, 1949.

(38) Winner, H. I.: Lancet, (1), 674, 1948.

(39) Bawden, F. C. : Plant Viruses and Virus Diseases, 1950.

(40) Waters, N. F., and Prickett, C. O.: Poultry Sci., (25), 501, 1946. 
(41) Andrewes, C. H., ct al.: Lancet, (2), 893, 1937.

(42) Ahlström, C. G., and Andrewes, C. H.: J. Path. and Bact., (47), 65, 1938.

(43) Smith, K. M.: The Virus, Cambridge, 1948.

(44) Moriyama, H., and Tao, S.: J. Shanghai Sci. Inst., (10), 125, 1941.

(45) Smith, K. M. : Parasitology, (29), 70, 86, 1937.

(46) Moriyama, H., and Tao, S.: J. Shanghai Sci. Inst., (10), 111, 1941.

(47) Moriyama, H. : Sogoigaku (in Japanese), (8), 520, 1951.

(48) Andrewes. C. H. : Lancet, (1), 71, 1949; New Engl. J. Med., (242), 235, 1950.

(49) Melnick, J. L. : Ann. Rev. Micobiology, (5), 309, 1951.

(50) Melnick, J. L. and Penner, L. R. : J. Exp. Med., (96), 255, 1952.

(51) Barnum, C. P., ct al.: Cancer Res., (7), 522, 1947.

(52) Furth, J. : J. Exp. Med., (55), 465, 1932 ; (58), 253, 1933.

(53) Rivers, T. M. : J. Exp. Med., (51), 965, 1930.

(54) Black, L. M. : Proc. Amer. Phil. Soc., (88), 132, 1944.

(55) Carr, J. G. : Proc. Roy. Soc. Edingburgh, (62), 13, 234, 1946.

(56) Mueller, J. H. : J. Exp. Med., (45), 243, 1927.

(5i) Dible, J. H. : Recent Advances in Bact., 242, 1932.

(58) McIntosh, J., and Selble, F. R. : Brit. J. Exp. Path., (20), 49, 1939.

(59) Shrigley, E. W. : Ann. Rev. Microbiology, (5), 241, 1951.

(60) Zeidman, I., et al.: Cancer Res., (10), 357, 1950.

(61) Gye, W. E. : Brit. Med. J., (1), 511, 1949.

(62) Gye, W. E., ct al.: Brit, J. Cancer, (3), 259, 1949.

(63) Hirschberg, E., and Rusch, H. P.: Cancer Res., (10), 375, 1950.

(64) Rous, P., ct al. : J. Exp. Med., (79), 511, 1936.

(84a) Ackerman, W, and Kurtz, H.: Proe. Soc. Exp. Biol. \& Med., (81), 421, 1952.

(84b) Cheever, F. S. and Dickos, J.: Proc. Soc. Exp. Biol. \& Med., (83), 822, 1953.

(65) Andrewes, C. H., and Shope, R. E.: J. Exp. Med.. (63), 179, 1936.

(66) Kilham, L. and Woke, P. A.: Proc. Soc. Exp. Biol. Med., (83), 296, 1953.

(67) Duran-Reynals, F.: Yale J. Biol. and Med., (22), 555, 1950.

(68) Moriyama, H., and Ohashi, S.: Arch. Virustorsch., (1), 252, 1939.

(69) Moriyama, H., and Ohashi, S.: Zeit. Immun., (99) 282, 1941.

(70) Salaman, R. N.: Nature, (193), 924, 1947.

(71) Gheorghiu, J.: Acad. Roumaine Bull. Sect. Sci., (27), 399, 1945.

(72) Bjorkman, S. E., and Horsefall, F. L. : J. Exp. Med., (88), 445, 1948.

(73) Miller, G. L., and Stanley, W. M. : J. Biol. Chem., (141), 905, 1941.

(47) Schultz, E. W.: J. Amer. Med. Ass., (136), 1075, 1948.

(75) Jenson, J. H. : Phytopath., (27), 69, 1937.

(76) Kunkel, L. O.: Ann. Rev. Microbiology, 85, 1947.

(77) Sige1, M. M. : J. Imm., (62), 81, 1949.

(78) Margulis, M. S., et al.: Ann. Rev. Soviet Med., (1), 409, 1944.

(79) Friedewald, W. F., and Hook, E. H. : J. Exp. Med., (88), 343, 1948

(80) Francis, T., ct al.: Amer. J. Pub. Health, (34), 317, 1944.

(81) Wenner, H. A. and Rabe, E. F.: Amer. J. Hyg., (54), 243, 1951.

(82) Salk, J. E., et al. : Amer. J. Hyg., (54), 25j, 1951.

(83) Bridge, E. M., et al.: Amer. J. Dis. Child., (72), 501, 1946.

(84) Contreras, G., et al.: J. Imm., (69), 395, 1952.

(85) Francis, T.: Proc. Soc. Exp. Biol. and Med., (65), 143, 1947. 
(86) Himmelweit, F., et al.: Brit. J. Exp. Path., (31), 809, 1950.

(87) Rivers, T. M.: Viral and Rickettsial Infection of Man, 1848.

(88) Topley and Wilson's Principles of Bact. and Imm., III. Edit.. 928, 1946.

(89) Trager, W.: Amer. J. Trop. Med., (18), 387. 1938.

(90) Merrill, M. H., and Ten-Broeck, C. : Proc. Soc. Exp. Biol. and Med., (32), 421, 1934.

(91) Whitman, L. : J. Exp. Med., (66), 133, 1937.

(92) Black, L. M. : Phytopath., (31), 120, 1941.

(93) Maramorosch, K.: Phytopath. (4I); 833, 1951.

(94) Spiegelman, S.: Symp. of the Society for Exp. Biol., No. II. Growth, 1948.

(95) Kunkel, L. O.: Amer. J. Bact., (24), 316, 1937.

(96) Fukushi, T. : Proc. Imp. Acad. Japan, (11), 301, $193 \bar{\jmath}$.

(97) Black, L. M. : Phytopath., (38), 2, 1948.

(98) Smith., K. M.: Recent Advances in Plant Viruses, 11. Edition, 1951.

(99) Fajardo, T. G.: Phytopath., (20), 469, 1930.

(100) Haurowitz, F.: Chemistry and Biology of Proteins, New York, 1950.

(101) Lesley, S. M., et al.: Canadian J. Med. Sci., (29), 128, 1951.

(102) Kassanis, H., and Kassanis, B. : Ann. appl. Biol., (32), 230, 1945.

(103) Moriyama, H., and Ohashi, S.: J. Shanghai Sci. Inst., (5), 189, 1941.

(104) Shope, R. E. : Science, (89), 441, 1939.

(105) Mckinney, H. H.: Soil. Science, (61), 93, 1946.

(106) Bringel, K. F.: Z. Hyg. u. Infektionskrh., (127), 280, 1947.

(107) Morton, H. E., et. al.: Amer. J. Syphilis, Gono. Ver. Dis., (35), 14, 1951.

(108) Severin, H. H. P.: Hilgardia, (17), 121, 1946.

(109) Arenz, B.: Z. pfl. Bau pfl. Sch., (2), 49, 1951.

(110) Kenneby, J. S. : Nature, (168), 890, 1951.

(111) Leach, J. G. : Insect Transmission of Plant Dis., 1940.

(112) Sonneborn, T. M. : Cold Spring Harbor Symp. Q. Biol., (9), 236, 1946.

(113) Brues, C. T., and Dunn, R. C. : Science, (101), 336, 1945.

(114) Chandler, A. C.: Introduction to Parasitology, VIII. Edition, New York, 1949.

(115) Wolf, F. A., and Wolf, F. T.: The Fungi, II, New York, 1949.

(116) Burkholder, W. H.: Ann. Rev. Microbiology, (2), 389, 1948.

(117) Baer, J. G.: Ecology of Animal Parasites, Illinois Uni. Press, 1951.

(118) Rhodes, A. J., and van Rooyen, C. E. : Textbook of Virology, New York, 1949.

(119) Yamafuji, R., et al.: Enzymologia, (14), 120, 124, 1950. 


\section{PART IV}

\section{THE GENERATION OF}

THE PRIMARY ORGANISMS

AND THE FUNDAMENTAL PRINCIPLES

OF LIFE PHENOMENA 



\section{CHAPTER I}

\section{THE MATRIX FOR THE GENERATION OF THE PRIMARY ORGANISMS}

\section{The Sedimentation of Globulin and Lipids in Primeval Oceans}

As discussed in the previous Part, for the generation of the secondary organisms the living substances already produced are indispensable. It may be said, therefore, that the discussion on the generation of the secondary organisms has nothing to do with the problem as to the true origin of life, but as will be fully discussed later it should be considered that the primary organisms were generated principally in the same way as the secondary organisms.

The writer has advanced so far the argument into the problem of the possible manner in which organisms were newly generated in the living protoplasm. In like manner the primary life might have been created on the primeval, lifeless earth, if there were present any matrix acting as the protoplasm. On the other hand, there are actually good many reasons to consider that such a matrix was present on the lifeless earth.

As already stated in Part II, Chapter IV, protoplasm particles isolated from leaves of certain plants formed protoplasm-like masses when left in a weakly acid solution at the laboratory temperature. Usually the mass appeared homogeneous and tended to take a shape peculiar to the plant from which the particls were prepared. In most cases these particles fused by hundreds into a mass of a bacterial size, some forming a bacillar form and other a coccoid, and sometimes the mass grew further until it became a protozoa-like body.

The writer has succeeded by using protein particles isolated from castor beans in preparing beautiful protozoa-like bodies in which usually various granules were contained, some of which occasionally appeared to be nucleus. Such a protozoa-like body would coagulate or disintegrate into minute particles as does the ordinary protoplasm when pressed mechanically under cover-glass or added with chemical agents such as alkali.

This fact suggests that protoplasm particles produced by the coagulation of protoplasm.can solve the coagulation when stand in 
an aggregated state at the isoelectric point, thereby presumably folded polar groups of the protein are set free enabling the particles to combine intimately with one another. Thus a homogeneous mass is formed in which protein molecules are arranged regularly just as in the protoplasm proper. At the isoelectric point both $\mathrm{NH}_{2}$ and $\mathrm{COOH}$ of the protein molecules, constituting the surface of the particle, may become free enabling the particles to fuse and to arrange orderly as in the protoplasm. Accordingly, this may be regarded as the reversion of protoplasm in vitro.

In the primeval oceans various organic substances might be present abundantly as there were no microorganisms which would devour them up, and if proteins having the globulin-like property were synthesized by certain means which we shall have an oceasion to discuss later, the protein would be precipitated since the water had possibly a weakly acid $\mathrm{pH}$, the isoelectric point of the protein, on dissolving carbon dioxide which is considered to have been present in abundance in the air of the ancient lifeless world. Globulin in its nature may preferably involve lipids in its precipitate, and therefore, if lipids were present together with the protein, the globulin-like protein in the ancient ocean would precipitate with the lipids forming minute coagu. lated particles; as will be mentioned later, globulin molecules possess the character to form virus-like particles in combining with lipids at the isoelectric point.

Particles composed of the globulin-like protein and lipids would be thus formed and precipitated on the bottom of the ocean, where when they were standing in forming aggregates, the protein molecules would be unfolded to be fused into homogeneous masses, and thus protoplasm-like masses just referred to above would be produced.

If the globulin-like protein and lipids were synthesized successively in the primitive ocean, they might continuously be sedimented in such a way in forming protoplasm-like masses, which might be accumulated on the bottom, possibly presenting a picture as if the bottom were composed of protoplasm-like masses. The writer believes that the matrix for the generation of the primary organisms was thus established. All the organisms existing at present are, without exception, provided with the protoplasm composed of globulin-like proteins and lipids, suggesting that the primeval organisms were also created from such chemical components. In addition many evidences can be presented to show that life phenomena are revealed solely on the stage composed of such materials.

If only a part of carbon which constitutes all the organisms existing today was present in forming carbon dioxide, it might be suffcient to reduce the $\mathrm{pH}$ of the primitive sea water to about 5.5 at which 
globulin was most readily sedimented.

For the isolation of protoplasm particles, including viruses, by means of the isoelectric point precipitation method we used acetic acid, whereas carbon dioxide has been generally used by many workers for the isolation of substances related to globulin such as serum-euglobulin and the stroma of red-blood cells. The fact that both serum-euglobulin and stroma are precipitated at $\mathrm{pH}$ about 5.5 in the form of viruslike particles and that both can be regarded as a kind of protoplasm particles was already stated. The application of carbon dioxide is very convenient to adjust a globulin solution to its isoelectric point for the precipitation of the globulin. It may be a relic of this precipitating property of the primeval protoplasm in the ancient oceans that still now the substances related to protoplasm proteins are readily precipitable by carbon dioxide.

\section{The Properties of Artificial Cells}

As above stated, the writer has been able to prepare protoplasmlike masses from certain plant materials; especially from castor beans most, demonstable, beautiful masses were obtained. The preparing method was as follows:

The emulsion of castor beans ground up in a mortar is centrifuged to eliminate precipitable coarse materials together with lipids, which are raised to the surface. The $\mathrm{pH}$ of the emulsion from which the lipids and the coarse materials having thus been eliminated, is adjusted to 5.5 with acetic acid, whereby a precipitate is formed, which is subsequently separated by centrifugation, and washed with distilled water for several times, each time being separated from the water by centrifugation. The substance thus obtained proves to be composed of virus-like particles, which will fuse into protoplasm-like masses if stand in a solution of the weakly acid $\mathrm{pH}$ for several days at the laboratory temperature. It appears that peculiar conditions are necessary for the production of such masses, for frequently the experiment would fail. Presumably temperature at least is an important factor, and a good result was commonly obtained if the solution was lelt at a temperature near $10^{\circ} \mathrm{C}$.

At any rate, the masses may be called artificial cells or protoplasm, the properties of which are described in the following.

As shown in Fig. 22 the masses are globular with sizes of $10 \mu$ or more in diameter. Small sized particles similar to red-blood cells are sometimes abundantly produced, and if their size is thus small they are quite homogeneous, but in larger sized particles various granules 
are seen, some of which occasionally are of considerable sizes and have an appearance of nucleus. Consequently they strikingly resemble in appearance some primitive single-celled organisms. They are also
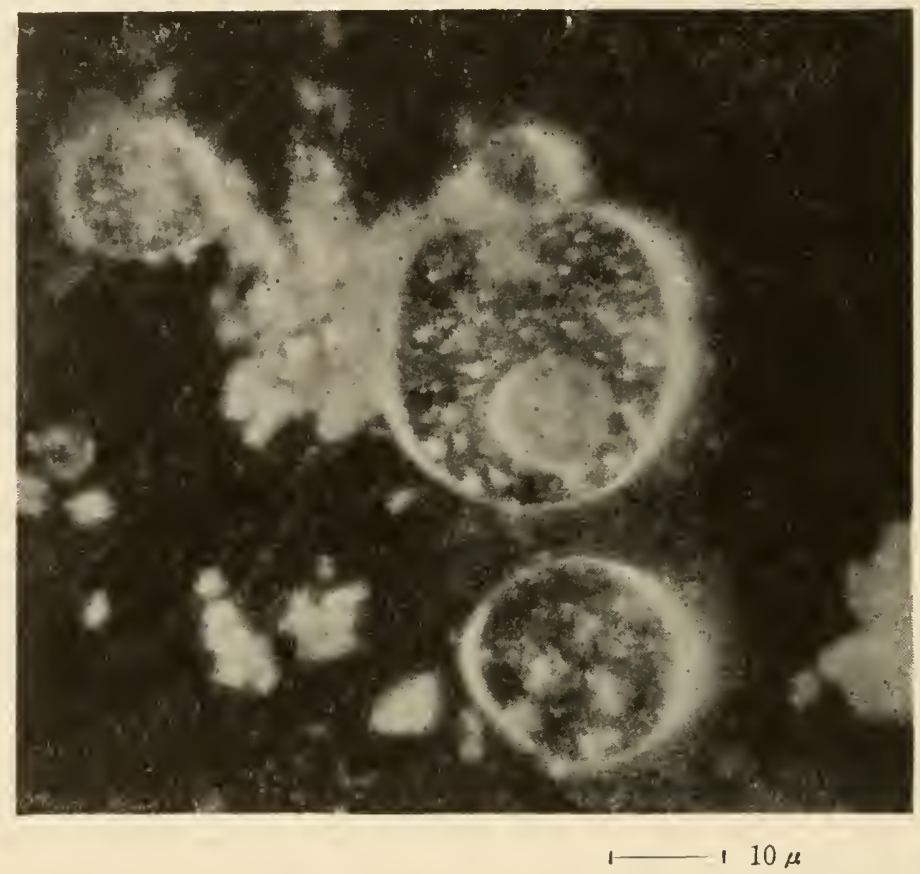

Fig. 22. Protozoa-like masses prepared from castor beans.

similar to the organisms in their chemical composition, and composed of globulin-like proteins and lipids, which the latter are contained in an amount about $1 / 4$ of the former.

Proper chemical or physical effects administered to the mass or the artificial cell caiuse to produce in the body minute particles which will again disappear gradually if the effects are removed. Thus, the reversible coagulation can take place as in the usual protoplasm, although the reversibility is very tedious. This fact indicates that the artificial cell can exhibit an irritability which is generally believed to be an essential character of living. If the stimuli are too severe the total body will be coagulated and then disintegrated into minute particles just as in ordinary cells.

If minute particles prepared from castor beans are given to the artificial cells, the former will be gradually fused into the latter, that is to say, the artificial cells eat the particles to grow, and when they grow to a certain extent, they will undergo fission into smaller bodies. 
Thus, they can accomplish both growth and multiplication, though the fission appears not to occur spontaneously, proper environmental changes, such as those of temperatire, or some mechanical effects appearing to be necessary for it.

From what has been described above, it will be evident that the mass is capable of exhibiting all the properties believed to be essential for living organisms, and therefore it may be said that a living organism is produced its vitro.

It should be a remarkable fact that such artificial cells can also be prepared from Merck's ricin preparation, in which no virus-like particles are contained. Therefore, it seems improper to regard the formation of the artificial cell as merely an example of reversible decomposition of protoplasm. The Merck's ricin preparation with which the writer carried out the experiment was composed of water soluble protein containing entirely no virus-like particles, but when its water solution was added with ethanol of $1 / 3$ of its volume and left in an ice box for several days after the $\mathrm{pH}$ had been adjusted to 5.5 by adding acetic acid, the protein would precipitate together with lipids contained in the preparation in forming virus-like particles. These particles after isolated by centrifugation were suspended in water of $\mathrm{pH} 5.5$ and left at the laboratory temperature, thereby the particles were fused into protoplasm-like masses.

This fact indicates that the globulin-like protein exhibits the property to polymerize with lipids to form virus-like particles, which in turn can fuse into larger protoplasm-like masses. As stated in Part II, elementary bodies of protoplasm are considered to be a parallel array of thread-like protein molecules among which lipids are inserted, and the protoplasm is assumed to be composed of such elementary bodies associating mutually also in parallel alignment. The virus-like particles prepared from ricin are comparable to the elementary bodies; the particles can combine with one another in a regular alignment to form larger masses which may in turn be comparable to protoplasm. The association among the particles in the mass, however, is not sufficiently strong to make the mass remain always in the same homogeneous state, so that upon the addition of a certain stimulus, the mass, like the protoplasm, will be decomposed into the particles, stable polymerization units of globulin-like proteins.

It should be considered, therefore, that globulin-like proteins possess in themselves the property to form protoplasm-like masses. Namely, globulin-like proteins possess the property to polymerize to virus-like particles by arranging themselves in parallel alignment in their stretched polypeptide chains in inserting lipids among them; these particles tend to combine further with each other in parallel arrangement to 
form larger masses. If proteins synthesized in the primeval oceans had a similar property, the formation of protoplasm-like masses would be only a natural result.

The property of proteins of globulin nature to form virus-like particles is also shown in an experimental result obtained by Gross (1), who has found that virus-like threads or filaments sedimentable by ultracentrifugation are produced in water solutions of crystalline trypsinogen added with a few crystals of trypsin preparation after incubation at $5^{\circ} \mathrm{C}$. for 3 to 7 days. Takahashi and Ishii (2) have isolated from plant tissues infected with tobacco mosaic virus a protein which formed at a weakly acid $\mathrm{pH}$ high molecular weight aggregates readily sedimentable, in the ultracentrifuge. This plymerization was reversible.

In short, it seems little doubt that proteins with globulin nature are able in a weakly acid solution, probably under an assistance of lipids, to form protoplasm-like masses. The primary organisms were most probably generated and evolved in the matrix composed of such masses, which on the other hand, may be regarded as the most primitive primary organisms. Oparin (3) stated that the primitive life might be originated from coacervates, while it is possible to regard the protoplasm-like mass above mentioned as a kind of coacervates composed of protein and lipids.

\section{The Evolution of the Primitive Organisms}

The Protoplasm-like masses produced in the primitive ocean might be similar both in their appearance and chemical composition to the protoplasm of the organisms existing at present. It is highly probable, however, that the component proteins and lipids of the primeval protoplasm, being not fitted for exhibiting the complicated life phenomena, were never similar to those of present organisms, and hence the ability to change the structure in accordance with the environmental effects might be insignificant as compared with the present organisms, and moreover if they could exhibit the property to rearrange the structure of weaker masses, $i$. e., the property to act as assimilase, it would also be insignificant.

Nevertheless, however trivial these properties might be, the masses might change their structure when certain stimuli were given, and the change thus raised would spread in the masses. Since the change produced in the protein structure may generally be reversible, the changed structure in the masses might also recover their original structure on the removal of the stimulus, but when some irreversible 
or difficult-reversible change was brought about and the changed structure was able to exert a physicochemical effect stronger than the original one, then the new structure would "assimilize" the surrounding masses. By the repetition of such a production of stronger structure the masses would become stronger and stronger in their structure, and more and more fitted to act as strong assimilase.

In short, the evolution of the primary organism at the primeval stage might be quite similar to that of the secondary organisms or viruses. In the case of viruses, however, the matrix in which they have been evolved is the preexisted, perfect protoplasm, which itself is the strong assimilase and in which various differentiated mechanism, as for example, that of the spreading of changed structure, are already provided, so that the stronger structure acting as the viruses would easily be produced, whereas in the case of the primeval organisms, as the matrix itself was quite incomplete, the enhancement of the strength of the structural pattern would be much more difficult; accordingly far longer span of time would be required for their evolution than for the virus.

As fully discussed in the previous Part, when a virus continues to multiply in the same kind of host cells, the virus structure is to be gradually assimilized by the host protoplasm until it becomes extinct. In order to avoid such a fate the virus must frequently change the host. This may account for the difficult situation in the continuance of their existence. The same might hold true for the primeval primary organisms.

The state in which the protolasm-like masses accumulated on the bottom of the ocean of the primitive age, exerting mutually structural influences upon one another, might be similar to the state of the viruses multiplying always in the same kind of cells. Therefore, even strong structures which might appear and spread in the accumulated massses would likely be influenced in the long run by the weaker masses existing in the overwhelming majority until it became identical to the weaker masses, or at least it became weaker in the structure.

In order to avoid such a decrease in the structural strength, the mass which might acquire a strong structure would find it necessary to move to another accumulation of the masses of a different type corresponding to a different kind of host cells in the case of viruses. In the movement of masses, the water in motion might play an important role. Thus, if the masses on the bottom of the ocean was carried near the shore, say, by a tidal current, they would always have good chance to contact with other masses of different structures by the constant motion of waves. If there was a structure in common 
between two masses thus brought into contact, they would combine with each other and the one with the stronger structure would overcome and assimilize the other. This must have been a struggle for existence, a struggle to eat or to be eaten. Thus the individuals with the stronger assimilase action could survive and prosper the more extensively.

The strength of the structure might be determined chiefly by both the arrangement and type of polar groups in the protein which constituted the mass, but of the masses of the similar proteins, the nucleic acid content might play an important part as in the case of viruses. The masses therefore capable of getting possession of great amounts af nucleic acids or nucleic acid-like substances would become powerful in their assimilase action, and accordingly would become more fitted for the continued existence.

In this problem, however, there seems to be involved a great dilemma. As stated in Part II, for the response to stimuli and for the spread of the structural change produced by the stimuli, lipids must be inserted among protein threads of the protoplasm, but in such a state the assimilase action of the protoplasm is extremely weak, and will be easily assimilized by other assimilases. On the other hand, if nucleic acids are contained in rich amount, the structure will become rigid and exhibit a strong assimilase action, but in this state, as a natural result, structural changes essential for life phenomena will scarcely occur. Primeval organisms had to find their way out of this dilemma in order to continue their existence, and did actually find a splended way as discussed in the next chapter. 


\section{CHAPTER II \\ THE PATTERN OF PROTOPLASM}

\section{Protoplasm as a Mixed Crystal}

A crystal is usually composed of similar molecules, occasionally different molecules in mutual connection can form a crystal, known as a mixed crystal. Molecules capable of forming a mixed crystal are different in their chemical composition, but similar in their arangement of polar groups and in their molecular sizes.

The protoplasm can be regarded as a crystal, but since it is by no means composed of the same molecules, it must be a mixed crystal. If the protoplasm is a mixed crystal, the proteins constituting it must be immunologically identical, if not uniform in chemical composition, since the component proteins should be identical in their arranment of polar groups.

It is generally recognized, however, that there exist several types of immunological different proteins, such as serum-albumin and serumglobulin, in the blood plasm which can be regarded as a pool of a liquid protoplasm. At first sight, this fact may appear to disprove the above concept. Nevertheless, the writer interprets this as being attributable to an artificial separation of protein fractions which are present in the plasm in a state of equilibrium exerting mutual influences to form a definite pattern specific to the plasm; the artificial separation may destroy the equilibrium to make each fraction reveal its own pattern.

Sörensen (4) has stated that proteins in biological fluids are never single chemical entities but systems of similar molecules in an equilibrium with each other and with other constituents of the solution, and that proteins isolated by the customary method are not identical with those originally present. Kleczkowski (5) observed that when pairs of proteins are heated, they combine in the initial stages of denaturation to form complexes. If one protein is present in larger amount the complex will not precipitate with antiserum to the minor component. The failure to precipitate with antiserum to the minor component could be reversed by peptic digestion of the major component. Addition of aniserum to the protein present in larger amount precipitates the entire complex. This fact may indicate that the in- 
fluence of the pattern of protein present in larger amount is greater in the establishment of the equilibrium. It has already been stated that a weak virus cannot exert its structural influence when present in a small amount, but can do so if present abundantly. This is the "dosis effect" recognizable in many other cases as detailed in Part II.

Again, according to Zoet (6), the antiserum against a heated mixture of horse and pig serum can react markedly with this mixture, but scarcely with a mixture of the two sera heated separately. McFarlane (7) stated that one protein in a mixture even can effect the molecular size of another.

It is well acknowledged that apparently similar serum-albumins from different animals exhibit markedly different antigenicities, whereas there is not so distinct immunological difference between two distinct proteins, $i$. e., serum-globulin and serum-albumin, if they are separated from the same animal, showing that proteins isolated from one and the same biological system cannot reveal immunological patterns so different as do those derived from different systems, although each protein may be able to recover its own pattern when separated from the system. It should be remembered in this connection that only substances with structural patterns almost similar can make a mixed crystal.

Various substances in protoplasm must be present in such a system of equilibrium. The main component element of the system may be the elementary bodies composed of globulin and lipids. These bodies may be fused into mixed crystal, exerting mutually structural influences to form a definite pattern specific to the protoplasm. When exist in the protoplasm they may be compelled to take this definite pattern, but if liberated from it, some bodies may change the pattern according to their own peculiar structure, for all the bodies constituting a cell cannot be identical in their chemical compositions. Bodies with high contents of nucleic acid may retain the pattern specific to the protoplasm, even when the particles with little or no nucleic acid undergo a change, since the former bodies cannot be altered in their structures on account of the presene of nucleic acid. Moreover, such bodies can act as strong assimilases for the same reason, and hence behave as viruses.

The same may occur when the bodies are present in protoplasm. Thus, when a stimulus is given to cell, most elementary bodies in it would change the structure in accordance with the stimulus, but not the bodies with high contents of nucleic acid, which, therefore, when the stimulus is removed, can bring back the other changed bodies to the original state by their strong assimilase action. The bodies thus capable of maintaing the original pattern of the protoplasm may 
be called genes when exist in the cells of higher organisms. Genes, therefore, are probably elementary bodies governing the structural pattern of the protoplasm.

If a primeval organism had a few number of such bodies, while it mostly being composed of bodies rich in lipids, having no or little nucleic acid, then the organism would be able to. respond readily to various stimuli without losing its original strong pattern. This must be the splendid means by which the organisms could escape the dilemma pointed out in the previous chapter. The most advanced means along this line must be the gene system of the higher organisms of the present day. Chromosomes which may be regarded as aggregates of genes are actually confirmed to contain uucleic acid in rich amount but without lipid (8).

\section{The Evolution of Protein Molecules}

Before discussing the problem of genes, it may be proper to make some argument regarding the above mentioned phenomenon that different proteins in a solution may exert mutual influences in the establishment of an equilibrium system. That each component protein in such a system can recover its own structural pattern on the isolation, may be a natural result of the reversibility of protein structure. However, it is noteworthy that adequate heating appears to render the change irreversible as indicated in the above cited both findings by Kleczkowski and by Zoet. If one component protein in a system exerts a particular strong influence upon the other the pattern of the system will be chiefly determined by the strong component. The effect of some agent, like heating, on such a system may render the change of the weaker components irreversible so that the change may become durable, that is, the weaker component proteins may be assimilized perpetually by the strong.

The writer assumed that assimilase action is given rise to only when protein molecules of the same type are orderly polymerized. However, from what has been mentioned above, it may be said that the assimilation is a general phenomenon occurring between different types of proteins, although the assimilase action may only become distinct on a, regular polymerization; in other words, it is considered that protein molecules can act in themselves as assimilase upon other molecules having weaker patterns.

The synthesis in the primeval oceans of high molecular proteins with a globulin nature might be possible on the basis of this property of proteins as assimilase. Thus it seems most probable that 
high molecular proteins were produced in the same way as primeval organisms; primeval organisms might be different from usual proteins only in their orderly polymerization. Possibly even at the stage of primitive proteins there might also be a struggle for existence, thereby weaker molecules were assimilized by the stronger, and thus the evolution of proteins were established just as in the case of primeval organisms.

There seems no doubt, however, that such a "struggle for existence" among the protein molecules, when the proteins had not yet been endowed with any highly evolved structure to produce a strong structural influences, was only trivial, and accordingly their evolution would be extremely tedious. The speed of evolution of assimilase or of protein would be directly proportional to the differentiation degree of the protein structure, and even when the proteins were able to form orderly polymerized masses, the speed would increase with the degree of differentiation of the polymerized mass, or assimilase. It should be said, therefore, that the highly evolved creatures of the present day may have the greatest speed of evolution and still now be evolving most rapidly. This reasoning may naturally lead to the view that the evolution of viruses which can make use of highly evolved protoplasm for their matrix for evolution might be much more rapid than that of the primary organisms.

The very important fact discussed above that proteins are able to exert their structural influences upon other proteins should be, on the other hand, interpreted as indicating that proteins themselves are enzymes, and therefore the evolution of proteins can be regarded as the evolution of enzymes. As regards this view further discussions will be made later.

\section{The Reason for the Presence of Optically Active Amino Acids in Protoplasm}

Organic substances synthesized by organisms are always optically active, as for example, amino acids constituting the proteins of protoplasm are as a rule laevorotary, whereas optically inactive racemic compounds are always yielded by artificial synthesis.

This fact has long been regarded as one of the biological enigmas, but this may naturally follow the crystal nature of the protoplasm. The fact that optical isomers are distinguishable by their different crystal shape has well been known since the brilliant finding of Pasteur. Substances of dissimilar optical activities can by no means exist in one and the same protoplasm. 
The writer is unable, however, to find any firm reason for which all the amino acids synthesized by organisms must be laevorotary. Presumably some individuals of primeval primary organisms were decomposed of L-amino acids, while at the same time the others were of D-amino acids, but there could be any synergetic connection between these two groups, as the one could not make use of the other because of the principal difference in their crystal shape, so that there might be a distinct line of demarkation, and perhaps an iron curtain hanged between them as in the case of the two groups of man in the present day.

There are actually ample evidences showing that $\mathrm{D}$-amino acids are not only useless but sometimes injurious to the present-day Lorganisms. The inhibition of growth of both gram-positive and gramnegative bacteria by D-amino acids has been well known (9) (10). Especially D-serine appears to be injurious, as for example, the growth of $E$. coli is injured even when D-serine is present in so small an amount as $5 r$ per ml. (11). Furthermore, D-serine exhibits a nephrotoxic action on rats (12).

It has become evident that D-amino acids occur occasionally in nature, particularly in products derived from certain molds and bacteria. Thus, D-proline occurs in a number of ergot alkaloids, ,whilst several of antibiotics, particularly penicillin and gramicidin are notable for their content of $\mathrm{D}$-amino acid residues. In the case of penicillin, the D-amino acid residue is one of critical structural features, since the L-analog is without activity. The high molecular polypeptides in the capusular substance of bacteria of the mesentericus group contains also D-amino acids.

It should be noted that all these substances are injurious to some organisms, indicating evidently that optically different amion acids are incompatible with each other. The supposed D-group of the organisms might have been extinguished by the injurious effect exerted by some products of L-group, leaving the latter alone on the primeval earth. If the L-group thus gained a victory over the D-group and left alone, all the secondary organisms generated from them should also be L-group and accordingly all the organisms have come to be L-group without exception. It is supposed, however, that some L-organisms existing at the present time have acquired the ability to synthesize D-amino acids to exert injurious effects upon the rivals in order to gain the victory in the struggle for existence.

It has been shown that lactobacilli, which have lost the ability to synthesize piridoxin, can grow in a medium which contains D-alanine even in the absence of piridoxin. Organisms grown in this medium are devoid of piridoxin and its derivatives, whereas organisms grown 
in media containing piridoxin but no $\mathrm{D}$-alanine are found to synthesize D-alanine. This finding has led to the discovery of a new enzyme called "racemase" which produces DL-alanine from L-alanine (13). Kögl and his collaborators (14) have repeatedly claimed that cancer cells contain D-amino acids in considerably high amounts, while Christensen et al. (15) have observed in vitro that a wide variety of amino acids, natural and unnatural, of the D- as well as L-configuration was accumulated in the free cells of mouse ascites carcinoma. The reason why cancers are injurious may be partly accounted for by this fact. 


\section{CHAPTER III}

\section{THE NATURE OF GENES}

\section{The Generation of Genes}

As referred to above, primeval organisms presumably could succeed in maintaining their structural pattern despite their easily changeable character which they had secured for the purpose to respond to stimuli, by making use of elementary bodies having high content of nucleic acid.

Such bodies or particles would decide the fate of a primeval organism when the organism combined with another type of organisms having a different structure; the one containing the nucleic acid-rich particles of stronger structure would overcome and assimilize the other, since the particles could govern the whole structure of the organisms. Owing to their strong structure due to the high content of nucleic acid, the particles even after freed from the organism would be able to transmit their pattern to the other organisms having weaker particles. In short, the same would result either when the particle combined in a free state with weaker organisms or when combined without being freed from the organism.

At least strongly active phage particles produced from lysogenic bacteria can be regarded as such representative bodies of the bacterial cell. Only one type of a certain virus can multiply when two different types simultaneously affect a host cell, a fact which is known as interference phenomenon, and which has been studied with phage especially in detail.

The multiplication of a phage in a bacterial cell is the transmission of the phage structure to the bacteria, whereby the bacteria are assimilized by the phage. Accordingly, it should necessarily follow that in a bacterial cell affected simultaneously by two different phages solely a strong phage can multiply, the weaker one being unable to exert any influence. If, however, the strength of two phages is alike both may multiply in a cell at the same time without interference; this is found to be actually the case.

In this connection should be mentioned a very remarkable fact. When a single bacterium is infected with a pair of phages like $\mathrm{T}_{2 \mathrm{r}}$ and $\mathrm{T}_{4 \mathrm{r}}$, one finds among the emerging viral progeny types which 
are different from either of the infection viruses, having peculiarities of both strains. In the example found by Delbrück and Bailey (16), the new types are such as $\mathrm{T}_{2 \mathrm{r}}$ and $\mathrm{T}_{4 \mathrm{r}+}$.

Similar phenomenon has been likewise observed by Burnet and Edney (17) with influenza viruses. From mixed infections in mouse brain of the neurotropic influenza strain NWS and the non-neurotropic strain MEL, virus strains which differ sharply from either of the originals have been obtained.

This phenomenon can be readily explained by the assumption that the strong structure is restricted to a portion of the protein molecules. If nucleic acid was inserted in a portion of the polymerization product of the thread-like protein molecules, only the portion would have the strong structure, although it is well conceivable that some portion of the protein threads themselves can possess the strong structure. At any rate, if two particles, one of which has the strong structure at portion $A$ and the other at portion $B$, affect simultaneously a single protoplasm, portion $A$ of the protoplasm protein will be changed by the former and portion $B$ by the latter independently from each other. If the portions in which the structural patterns of the two viruses are to be impressed are either identical or present very near, the interference phenomenon may occur, whereas if the portions are separated enough both patterns will be replicated without interference.

The formation of a replica of a strong structural pattern at a certain portion of the protoplasm protein may lead in the organisms to the appearance of property, which is determined by the structural pattern of the portion regardless of the kind of replica of the other portions. Namely, the particle having a strong structure at portion A will give rise to the property determined by the structure at portion $A$, and the particle having a strong structure at portion $B$ will reveal the property determined by the structure at portion $B$, and thus the new progeny having the peculiarities of both particles will be generated. In such a case the particles will be regarded as genes capable of determining the character peculiar to each particle. The mechanism for causing localized changes in limited portions of the protoplasm protein is apparently most advanced in the structure of genes of the present-day higher organisms.

\section{The Structure of Genes}

As the organisms advanced the higher, their character would become the more complicated, and at the same time the various loca- 
lized structures governing each of their differentiated and complicated characters would be arranged the more orderly in the protein molecules constituting genes.

The protein components in the nucleoprotein in the nucleus were believed to be basic proteins, such as histone and protamine, but at present a series of workers claim that the chief protein in the chromosomes is acidic one named chromosomin as already stated in Part II. In general, protoplasm consists of protein of globulin nature, which is also of acidic nature, and hence chromosomin may be a protein of globulin nature, forming genes by combining with nucleic acid, whose threads are presumably oriented parallel to the protein threads (18). Stedman (19) who was the first to find that the main protein component of chromosomes is acidic protein, objects to the opinion concerning the importance of nucleoprotamines and nucleohistones in heredity, because the structure of protamines is so simple that it could hardly account for their alleged importance as carriers of heredity.

In short, all the characters of organisms are subjected to the structures of protoplasm protein, which in turn are governed by the structure of genes. In the gene of higher organisms of most complicated characters, there must be present a great number of localized strong structures each of which may direct each component factor of the complicated characters; and a single gene may have only a single localized structure so that the number of genes may be equal to that of localized structures. If there are two genes which govern the structure of the same portion, the structure of the portion should be determined by the stronger gene, thus the reason why there are dominant and recessive genes may be readily explained; this must of course be an interference phenomenon between genes.

This concept is elucidated in Fig. 23, in which structures of the restricted portions are shown by various patterns; those given by dotted lines are weak structures, which are subjected to the strong patterns of non-dotted lines. Each gene in a pair is inherited from each of the parent and the structure of the restricted portion is determined by the stronger one, the dominant gene, which accordingly directs the character subjected to the structure.

Genes are, therefore, virus-like polymerization products of proteins, a portion of which is endowed with a particularly strong structure. Each of a certain number of the particles which are comparatively similar to one another may aggregate into each larger body at a stage of the cell division; such aggregates may be termed chromosames. Since different genes sharing the site of determination in common may repel each other, each gene in a pair may be distributed to different chromosomes so that every gene in a chromosome 
may have each site of determination. Mendel's laws of inheritance can be well explained by this concept.

However strong the reversibility of the protoplasm structure may be, the pattern will more or less be damaged when the protoplasm is repeatedly altered in its structure by various stimuli. The pattern thus damaged will be repaired by genes which are always able to retain the original pattern.
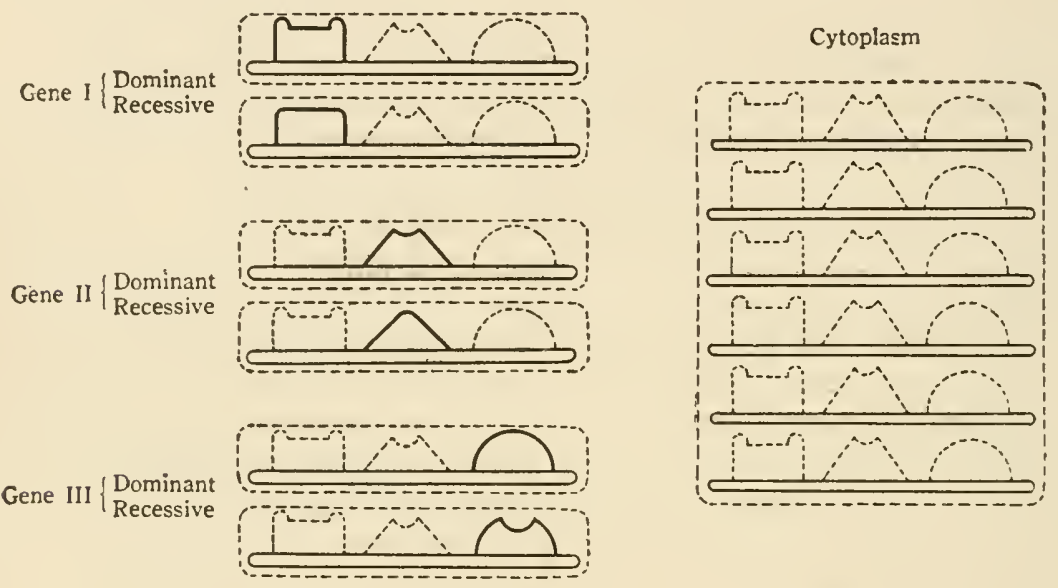

Fig. 23. Diagram of genes.

Chromosomes as above suggested may be coagulated aggregates of genes of relatively similar structures, so that in the chromosomes the active groups of genes may be present in a folded state. Accordingly, in this state genes may be unable to accomplish their function, which may develop only when the coagulation is solved and gene particles are set free. This may occur at a certain stage of the cell division, at which nuclear masses are mixed with the cytoplasm.

If genes always exerted their strong structural influence upon the cytoplasm, the latter would be unable to change freely its structure, and accordingly the exhibition of various vital functions would become impossible which must only be accomplished by movement of the protein molecules constituting cytoplasm.

It is said that chromosomes are present as such only at a certain stage of cell division, in the living interphase nucleus being optically homogeneous, and that chromosomal structures appear only after injury or treatment with histological fixations; in the living nucleus the chromosomes are in a greatly extended state, filling the nucleus homogeneously and upon injury the chromosome condenses and becomes 
visible (20). If isolated from the cytoplasm, the genes would be unable to exert their influences upon the latter even when their active groups were not folded. Anyhow, there should be in a cell a mechanism in action by which continuous effect of genes is prevented.

The mutation of genes may result from their irreversible structural change, and since chromosomes can be regarded as a kind of aggregates or even crystals of genes, the shape of the chromosomes composed of genes undergoing mutation may sometimes be altered. As is generally recognized, the shape of chromosomes is occasionally changed following a mutation of the organism.

\section{The Size of Genes}

The majority of workers consider that the dimensions of genes are not very different from those of viruses or of fibrous molecules. For instance, from genetic data it has been inferred that the shape of the genes is rod-like and their length is approximately $125 \mu$, their width being $5-20 \mu$ (21). An electron microscopic study of salivary chromosomes has shown that giant chromosomes were composed of series of granules of $210-330 \mathrm{~m} \mu$ in diameter (22).

On the other hand, a number of workers claim that genes must be much smaller than viruses, because small as they are virus particles seem to be endowed with many component characters each of which is inheritable independently, a fact already discussed in the beginning of this chapter.

If genes are regarded as entities as shown in Fig. 23, they must have a dimension of viruses, but if only unit structures directing each component character are taken into consideration, the dimension will naturally appear much smaller. However, since unit structures themselves cannot exist independently of the whole protein molecules, it should be proper to regard the genes as virus-like polymerization product composed of protein and nucleic acid.

In Fig. 23 only three unit structures are shown, but in reality the structures must, of course, be present in a much larger number. Since a single molecule of globulin may be composed of more than 1,000 amino acids, if a single amino acid residue can behave as a strong unit structure directing a character of an organism, more than 1,000 determinant structures may be able to exist and accordingly more than 1,000 kinds of genes may appear to be present in a cell, because in a single gene particle only one structure of them may act as a directive site.

Viruses can be regarded as genes, though not so differentiated. 
so that each unit structure in a particle can behave occasionally, like in genes, as each determinant site. For example, if a virus with three unit structures, $\mathrm{A}, \mathrm{B}$, and $\mathrm{C}$, affects a cell together with another virus having unit structures, $A^{\prime}, B^{\prime}$, and $C^{\prime}$, and if the degree of strength of each pair of the structures is as follows: $A>A^{\prime}, B<B^{\prime}$, and $C<C^{\prime}$, then newly formed virus will be $A, B^{\prime}$ and $C^{\prime}$, whereas if the former virus combines with a virus, $A^{\prime \prime}, B^{\prime \prime}$, and $C^{\prime \prime}$, and the degree of strength of the structures, being $A>A^{\prime \prime}, B>B^{\prime \prime}$ and $C<C^{\prime \prime}$, then the new virus will be $A, B$, and $C^{\prime \prime}$. In such a manner, each unit structure of the virus can be revealed separately appearing as if it were composed of many genes.

It may, however, be unreasonable to assume that in a gene particle only a single unit structure can always have the supremacy, directing a property without being influenced by other structures. There is a good reason to suppose, as will be mentioned later, that it is difficult to change a restricted portion in a protein molecule without altering other portions. Moreover, it is possible that the manner of arrangement or of combination of different structures may sometimes determine a character. A normal organism is said to be the resultant of action of some thousand of genes; for instance, in Drosophila about eight thousand of genes are believed to be in action (23). Presence of such a great number of genes cannot be explained by the assumption that a component character is always directed by only a single unit structure.

Antigens, like viruses, are capable of producing a sort of replica of their own in the protoplasm of certain cells, though the replicas produced by them are not so perfect as those produced by viruses, and moreover though the cells in which the replicas are to be formed are the cells which are generated for the purpose of antibody production. Anyhow, there seems no doubt that antigens are a replicaproducer just as viruses or genes, and in this respect genes may be regarded as the strongest antigens in the cell protoplasm.

By the famous researches of Landsteiner and his coworkers (24) it has become evident that the specificity of antigenic character of proteins resides in the chemical structure of their molecules. The average size of a specific group is said to be of the order of 600 to 1,000 in molecular weight, but occasionally very simple artificial chemical groupings may act as specific determinant (25). It seems, however, that the specificity of natural proteins is due not to a single type of group but to a specific arrangement of different polar groups in the surface of the protein molecule (26). The same may hold true for genes.

In general, the substances able to behave as antigens are proteins. 
This fact indicates that the property for replica formation appears to be a characteristic of proteins, at least proteins appearing to have the property most suitable for the replica formation. This is one of the main reasons why the writer has taken into considerations only proteins in the argument into the nature of virus or of protoplasm. Since substances such as sugars and lipids in protoplasm may merely be subjected to the pattern of the protoplasm directed by genes, the argument can be advanced without regard to their existence so far as the problem is concerned with the replica formation. 


\section{CHAPTER IV \\ THE SUPREMACY OF GENES}

\section{The Type of Nucleic Acids and Its Biological Significance}

In cells there are two kinds of nucleic acids, one of which is desoxyribonucleic acid (DNA) and the other ribonucleic acid (RNA). DNA is usually present in nuclei, and the nucleic acid in genes is believed to be DNA, whereas RNA is found as a rule in cytoplasm, mostly being contained in particles known as microsomes which therefore can be regarded as elementary bodies or their aggregates containing RNA in rich amount. In addition, particles called mitochondria are said also to contain this nucleic acid, but the particle present are much fewer in number than microsomes which the latter are reported to occupy even 15 to 20 per cent of the cytoplasm.

These particles must have directing influences upon the whole cytoplasm when genes are separated from the latter, since they contain nucleic acid in rich amount and form large sized particles. Most likely they, if liberated from the cell, may first and foremost behave as viruses, no matter whether the cell has the normal structure or the structure changed by a virus; in the latter case particles will be regarded as the virus having multiplied in the cytoplasm.

The nucleic acid in phage particles separated by ultracentrifugation are reported to be mostly DNA, while their lipid content is poor. On the other hand, chromosomes which may be regarded as aggregates of genes similarly contain DNA but no lipid, a fact which may enable one to consider the bacterial particles isolated as phage to be bacterial genes. Cytoplasmic particles such as microsomes and mito chondria contain nucleic acid in rich amount as mentioned above, but in contrast to genes the nucleic acid is. RNA and in addition, 30 to 40 per cent of the particles are composed of lipids (27) (28).

Such large lipid contents of these cytoplasm particles may partially account for their submission, in spite of their high nucleic acid contents, to the genes. The difference in the kind of nucleic acids, however, may have on this point a much more important significance.

According to the writer's concept, when inserted among protein 
molecules, nucleic acids may cause the polymerized product of the protein molecules to become rigid and strong, thereby the polymerized product is endowed with the ability to behave as strong assimilase. In this hardening action DNA may be more effective than RNA, since it is known that RNA is split by the action of solium hydroxide, whereas DNA is more stable and resists this treatment. Application is made of this difference for the quantitative determination of each nucleic acid in the presence of both of them; after the destruction of RNA by sodium hydrooxide, DNA is precipitated by the addition of proteins in acid solution (28). Presumably organisms make use of this difference and allocate DNA to genes which require the strongest Dattern, and RNA to cytoplasmic particles to be subjected to the genes.

In addition, owing to its rather weaker structure RNA may readily split off the energy-rich phosphate bond which may provide the energy necessary for the protein synthesis as will be discussed in greater detail in Chapter IX in this Part. This may be the more important reason why RNA is allocated to cytoplasmic particles where the protein synthesis may chiefly take place.

There appear to exist substances other than nucleic acids which may be able to exert hardening effect upon protein complexes. Divalent cations, such as $\mathrm{Ca}$, and polysaccharides, for instance, may be regarded as such substances, but it should merit attention that only nucleic acids seem to contribute the template action of viruses or virus-like agents including genes. This may be ascribed to the ability of nucleic acids, unlike other hardening agents, to stabilize the fine, specific pattern needed for the template action. It is claimed that there is as great a variety of nucleic acids as proteins (29); and again it is emphasized that the fundamental differences between groups of virus are correlated with their nucleic acid composition (30), and moreover that nucleotide composition of a viral nucleic acid is characteristic of the species (31). If nucleiotides are arranged according to the specific pattern of the protoplasm, nucleic acids with the specific composition will be produced. According to Hershey et al. (32), phage DNA can be distinguished by its hydroxymethylcytosine content from normal bacterial DNA, which contains cytosine, indicating that bacterial pattern changed by the virus is revealed in the pattern of DNA.

The analogies between genes and viruses have been discussed by numerous workers. Distinct difference between them seems to lie in that viruses can proliferate indefinitely in the cell, whereas genes appear to multiply only in an orderly and restricted way. However, the patterns of genes like those of viruses do multiply indefinitely in the cell, that is, the directing influences of the genes can spread 
throughout the cell to make the protoplasm identical in the structural pattern with the genes, although the multiplication of papticles itself capable of acting as a gene is restricted. Thus the multiplication or spreading of genic pattern must be equal in the nature to the proliferation of viruses.

One cannot regard the multiplication of the genic pattern in the same light with the proliferation of particle itself capable of acting as a gene. The indefinite multiplication of genic pattern is desirable for organism but the indefinite proliferation of gene particles rich in nucleic acid must be fatal to them, because, as was already discussed in detail, for the development of various vital phenomena protoplasm must be composed mostly of elementary bodies containing large amount of lipids without nucleic acid. Accordingly, if genes rich in DNA multiplied unrestrictedly, the cell would naturally be soon destroyed. This may hold true likewise for cytoplasmic particles such as mitochondria and microsome whose multiplication is also restricted like genes. In short, it is evident that the organisms have been able to continue their existence to be left on this earth as the fitter, only through the development of the mechanism by which the numbers of the particles, such as genes and microsomes, are held constant.

As stated above protoplasm is regarded as a mixed crystal. On the other hand, it is known that the crystal of a certain substance can take into it another substance to make a mixed crystal, but that the amount of another substance to be taken into it without effecting the original crystal pattern is limited. For example, $\mathrm{MgSO}_{4} 7 \mathrm{H}_{2} \mathrm{O}$ forms rhombic and $\mathrm{FeSO}_{4} 7 \mathrm{H}_{2} \mathrm{O}$ monoclinic crystal, and these two salts can form a mixed crystal with each other, but the quantity of each salt to be mixed with the other in order to form a mixed crystal without exerting any influence on the original shape of the other is limited. Thus the Mg-salt can dissolve at most the Fe-salt at 18.8 per cent to form a rhombic crystal. It may be not utterly impossible that similarly in the protoplasm the quantity of substances other than proteins, such as nucleic acid and lipid, which can be taken into it without effecting its original crystal shape may be limited.

At any rate, it may be reasonable to consider that the mixed proportion of various substances in the protoplasm may be determined by some physicochemical laws. The unbalance induced in the proportion of nucleic acid to protein by the nucleic acid increase may be the cause of the reduction division or meiosis, by which the proper proportion would be restored. Thus, the physicochemical force acting to maintain the mixed proportion constant might be best availed by organisms for the proper restriction in the multiplication of genes or 
other granules.

It is generally accepted that vigorous propliferation of cells is usually accompanied by a marked increase in nucleic acids. This may mostly be attributed to the unbalance in the mixed proportion caused by the increase of nucleic acid. It seems that some structural change in protoplasm may lead to the increased capacity of the protoplasm to combine with nucleic acids, as for example, mechanical injuries or chemical stimuli cause the increase in nucleic-acid content of the cell. Such nucleic acid increase would naturally bring about the vigorous synthesis of protein in order to alleviate the unbalance in the mixed proportion; this may account for the well known fact that various stimuli generally lead to the marked proliferation of cells.

The virus infection, in general, may bring about the nucleic acid increase in the cell. For instance, when bacteria are affected by phage nucleic acid, especially DNA, markedly increases (33). During the course of normal growth the cells of $E$. coli synthesize almost three times as much RNA, but susceptible cells suspended in a synthetic medium and infected with phage will show a rate of DNA synthesis approximately four times as great as that observed in normal cultures. This may suggest that the structural disturbance in the bacterial protoplasm caused by phage may alter the mixed proportion of DNA to the protein.

If a structural disturbance in the protoplasm caused by some stimulus resulted in the increase in nucleic acid, especially in DNA, the structural change, if occurred after the disturbance, would be well retained in the protoplasm particles, because these particles would be endowed with strong structure and accordingly with powerful assimilase action owing to the high content of the nucleic acid so that they might be able to act as a newly produced virus. When this virus affects some cells to produce the replica, in the protoplasm of the cells will be raised the structural disturbance which may cause the same nucleic acid increase, since the virus structure may have the property to cause the increase in nucleic acid. This may be the reason why nucleic acid is increased in the cells affected by a virus. A virus which failed to cause a change leading to a marked nucleic acid increase in the protoplasm would not be able to continue to exist.

Lysozyme can cause lysis of bacteria like phage, but fails to multiply. The characteristic chromatic granules of the bacteria are seen on the phage infection indicating the occurrence of striking change in the nucleic acid, whereas lysozyme fails to induce any similar change in the granules (34). The inability of lysozyme to become a virus would be accounted for partly by this failure to influence the nucleic acid content. In the presence of proflavine bacteria 
infected with phage undergo lysis with the liberation of phage-like particles which have no phage activity. The ultraviolet absorption coefficient of these particles revealed that their nucleic acid content, if any, is much lower than that of usual phage particles (35).

Bacterial strains having acquired increased resistance to the lethal effects of $X$-rays or ultraviolet rays have been found to synthesize several times as much DNA as do the sensitive strains (36). Such bacterial strains might have increased the resistance, because they have undergone a structural change to cause the increase in DNA.

In the presence of cobalt ions the growth of some bacteria is prevented but RNA content may be raised (37), and certain other bacteria, when grown under unfavourable conditions, may accumulate large amount of RNA (38). It is considered that also in these cases there might occur structural changes which caused the alteration in the mixed proportion.

\section{Plasmagenes}

A number of workers claim that the characters of organisms are determined not only by genes but also by cytoplasmic particles designated as plasmagenes. In the theory of the writer, it may be said that protoplasm is composed of a sort of genes, since all the elementary bodies of protoplasm are assimilases capable of exhibiting a function like a gene. However, as the bodies present in the nucleus possess the strongest assimilase action in the cell, the pattern of the whole protoplasm is usually directed by such bodies, which are accordingly called genes. It may be possible, therefore, that some cytoplasmic particles attain to the supremacy if the genes proper exhibit only a weak effect.

A factor called kappa found by Sonneborn (39) in Paramecium was regarded as a kind of plasmagenes. This factor can exhibit a strong influence on protoplasm structure of this protozoan and provides the protozoan with the faculty to produce a substance named paramecin. As this substance can kill a sensitive strain of the protozoan, the individuals producing paramecin are named killer. In short, kappa seems to be able to overcome the genes proper to direct the structure of the protoplasm. As a result the property to produce paramecin arises, but in the presence of some strong genes kappa fails to exhibit its influ= ence and is perished.

This factor, however, now appears to possess many characteristics of microorganisms such as a Rickettsia (40). The size of the factor is similar to that of Rickettsiae, being 0.2 to $0.8 \mu$. In addition, it should 
be noted that kappa particle contains a large amount of DNA. Its strong effect may be attributed to this nucleic acid in addition to its large size.

It is probably true to say that the gene system has been evolved amazingly in higher organisms because it is the most ideal system for their development. Therefore, presence of particles in cytoplasm such as kappa, which can strongly interfere with the gene, may not be regarded as a normal feature. Also from this point of view the concept of the parasitic nature of kappa appears to be reasonable.

The results obtained by L'Heritier and his collaborators (41) in their studies on the physical basis of sensitivity to carbon dioxide in the fruit fly, Drosophila, are in many respects strikingly similar to the results on kappa in Paramecium. Reciprocal crosses showed that sensitives to carbon dioxide differed from resistants, not in any nuclear gene, but in some cytoplasmic factor. This factor was named sigma and at first regarded as a kind of plasmagenes, but there are also some evidences for its parasitic nature, although the factor has not been seen or counted. Presumably it is much smaller than kappa, more alike in size to a small virus or a nuclear gene.

Likewise, in higher plants a similar cytoplasmic factor causing male sterility is known; the individuals having the factor fail to form functional pollen (42). This may also be of a parasitic nature. It has been demonstrated that this factor is inherited through the cytoplasm. Sigma is likewise inheritable; the flies that developed from the infected eggs are sensitive to carbon dioxide and transmit sigma to subsequent generations through the egg cytoplasm. Discussions have already been made on the fact that viruses are transmissible to offspring through germ cells (Part III, Chapter X). It should be borne in mind that not only viruses but also these parasitic Rickettsia-like organisms can be inherited to progeny through host germ cells.

Mitochondria are usually regarded as normal cytoplasmic particles, but some workers consider them to be parasitic microorganism. This is not an inconceivable concept, though nucleic acid in mitochondria is RNA, so that they are always subjected to the genes, not disturbing the beautiful control of the protoplasm by the latter.

To sum up, since the character of organisms having evolved to a certain degree is determined by genes, and since this mechanism of character determination is" considered to be most suitable for the organisms, cytoplasmic particles which can strikingly interfere with the function of genes may be, at least in the majority of cases, an abnormal existence.

However, it should be mentioned here that there is an interesting evidence showing that the normal cytoplasm can exert a considerably 
great influence upon the character of amoeba. Danielli (43), using two large species of amoeba, $i$. e., Amoeba proteus and $A$ discoides, has carried out transplantation of the nuclei of individuals of one species to those of the other species from which their own nucleus had been previously eliminated and found that the form of the amoeba and the diameter of the nucleus are mainly determined by the cytoplasm, although the nucleus, of course, has some influence and in the longrun it comes to exert a more pronounced influence, most of the amoebae becoming to have an intermediate form between discoid and proteus. 


\section{CHAPTER V}

\section{RELATIONSHIP OF GENES TO ENZYMES}

\section{The Nature of Enzymes}

Two protein molecules of different types do not combine with one another because of the presence of the repulsive polar forces resulting from the different structures, whereas if they have structures, complementary to each other, having strong attractive forces, the combination will be established. However, repulsive polar forces, if present, will exert mutually disturbing influences when the two molecules having the complementarily shaped structures are drawn near by the attractive force. For the establishment of the combination of two such protein molecules the attractive force must be stronger than the repulsive force, but the mutual repulsive action will become very manifest when the combination is established.

Now, if the repulsive force of protein $A$ is much greater than that of protein $B$, the structure of this latter protein will be changed on being defeated by the repulsive force of the former, thereby all the structures in $\mathrm{B}$ will be changed to become complementary to those in A. The structural change of the protein B to become complementary to $A$ in its structural pattern is nothing but to become identical with $A$ itself; accordingly through this change $B$ is assimilized by $A$. In this case $A$ is assimilase. When polymerized orderly, the structure of protein molecules will become stable with the enhancement of their structural influence. Assimilase is therefore usually polymerization product of protein molecules of the same structure, and can adsorb protein molecules through the complementary structure to assimilize and to eat the latter molecules, thus being able to grow. Accordinly assimilase is the primitive form of life itself, and at the same time it is a kind of enzymes.

When the influence of $A$ is not so great as to make the structure of protein $B$ identical with it protein $B$ will only be disturbed in its structure. If the protein is "denatured" as a result of such a disturbance and deprived of its faculty to combine with the protein $A$, the protein B will be liberated form A. A can thus combine again with another protein which will in turn be denatured and again liberated. 
In such a case, A can denature protein B successively and hence may be termed denaturase.

When $B$ has a structure to be easily decomposed into smaller molecules by the structural disturbance induced by $\mathrm{A}$, or when $\mathrm{A}$ can exert a disturbing effect which is specifically fitted for the decomposition of $\mathrm{B}$, the latter will be decomposed and liberated from $\mathrm{A}$ on account of the disappearance of the combining faculty due to the decomposition. Thus A can act as proteolytic enzyme upon B.

If $A$ and $B$ are similar in their disturbing action, both will be denatured by the mutual effect on the combination. This will occur in antigen-antibody combination. In such a case, as $\mathrm{A}$ is likewise to be denatured, it will lose its function as a denaturase, and consequently will be consumed in this reaction. A cannot be called on such an occasion an enzyme in a strict sense.

This is the general outline of the writer's idea as regards the nature of enzymes. From this point of view all the proteins may be regarded as enzymes.

All the protein molecules produced in the oceans of the primitive age might likewise be in this respect some kinds of enzymes. As above stated, the evolution or the production of protein molecules in the primitive age is considered to be established by the "struggle for existence" of the protein molecules, whereby weaker patterns were devoured by stronger patterns. Enzymatic nature can be said, therefore, to be the essential characteristic of proteins. It cannot be supposed, however, that the protein molecules in the primitive age were endowed with enzyme activities as elavorated as those of the enzymatic proteins existing in the present day. The present-day enzymes must be the proteins highly evolved for the purpose of acting as enzymes. The evolution of organisms might be involved in the evolution of proteins, which in turn might involve the evolution of enzymes. Proteins and enzymes, therefore, may be inseparable from each other.

\section{The Relationship between Genes and Enzymes}

According to the writer's concept, the protoplasm is a system composed of various substances especially proteins. In this system all the proteins must have a similar arrangement in polar forces, and the pattern of the arrangement is to be directed by genes. On the other hand, the action of a protein as an enzyme should be determined by the manner of arrangement of its polar forces, so that the functions of enzymes in a certain cell must be subjected to the genes of the cell. 
In fact, vast number of evidences are known to show the existence of intimate relation between genes and enzymes. In some lactobacilli, for example, loss of ability to ferment sorbitol and manitol is correlated with the loss of ability to produce the polysaccharides responsible for specific agglutinability (44). Such a loss of ability to produce the specific substance is ascribed to a change in the protoplasm pattern brought about by a change in the gene system which the latter is responsible for the change in the agglutinability. Furthermore, in the case of different mutable strains of colon bacilli, colonial dissociation, which shows a genic change, invariably occurs concomitant with the metabolic variation resulting in rapid fermentation of lactose (45). Again, passage of lactobacillus plantarum from the $S$ to the $R$ form is associated with the loss of ability to produce acid from certain sugars (46). Whereas the $\mathrm{R}$ form can grow in sugar-free peptone, the $S$ form does not grow in the absence of carbohydrates. It is also reported that the growth of staphylococcus in the presence of streptomycin is associated with the emergence of resistant mutants; at the same time, the emergence is linked with the ability of the cells to synthesize aspartic acid, phenylalanine, etc., in the presence of streptomycin (49).

It may be possible that each partial pattern of the protoplasm is connected with the action of each enzyme in a cell, so that it may be possible that a certain enzyme is directed chiefly by a certain gene. It cannot be considered, however, that one gene-one-enzyme hypothesis (48) holds always true; it may rather be reasonable to suppose that, though a certain gene tends to direct chiefly a certain enzyme, an enzyme is generally connected with several genes, or a gene has some connection with several enzymes, for it may rather be exceptional that the property of an enzyme is determined by a restricted partial structure only.

It is claimed that almost all the proteins present in a bacterial cell are provided with enzymatic action of various kinds (49), a claim which is also raised from the writer's view. The pattern of the proteins present in the protoplasm is subjected to the genes, and since an enzyme with a specific active group must be governed by a gene directing chiefly the specific group, the enzyme will be changed with the gene.

As stated in the previous section, the usual enzymes may not necessarily require for their action the regular polymerization of proteins which is indispensable for the assimilase action, but the polymerization may possibly favour the action of the usual enzymes, for the structural effect of proteins may, in general, be strengthened by the polymerization. Cytoplasmic particles such as mitochondria 
and microsomes appear to behave as assimilase and accordingly as viruses owing to their rigid polymerization resilting from the high content of nucleic acid, while at the same time they are known to have various enzymatic actions. For example, it has been noted that almost all the lactose activity in rat kidney homogenates can be sedimented with microsome fraction. Mitochondrial fraction, on the other hand, has been reported to have the major portion of the activity of a number of tissue enzymes (50) (51). It has already been stated that the structural pattern of these cytoplasmic particles is directed by genes, and hence their enzymatic actions must also be governed by the genes.

Since viruses can be regarded as pathogenic genes, which determine the pattern of the host cells after the infection, the enzymatic action of the cells may likewise be changed according to the kind of viruses. It has been found by Bauer (52) that chick eggs inoculated with yellow fever virus showed a marked rise in xanthine oxidase activity of embryo and of chorio-allantoic tissues. Both virus titer and enzyme activity reached a peak at about the 13 th day and then decreased. This may be a result of the change in the protoplasm pattern raised by the abnormal gene, namely, yellow fever virus, which may produce in the protoplasm protein the structure capable of acting as xanthine oxidase. Fredericq and Gratia (53) have described the phenomenon of stimulation of lactose fermentation by lactosenegative bacteria submitted to the action of a certain type of phage, a phenomenon indicating the production of the enzyme on the structural change by phage.

Further, Kun and Smith (54) investigating the respiration of the allantoic membrane infected with myxoma virus, found that zymohexase activity was increased and lactic acid production was correspondingly increased. On the contrary, if a virus pattern is incompatible with the pattern of some normally existing enzyme, the enzyme will be destroyed by the virus infection. According to Bauer (55) cholinesterase activity in mouse brain infected with yellow fever virus falls gradually during the incubation period and reaches a minimum value at the time when symptoms of encephalitis appear.

Paramecia affected by kappa, as already stated, produce paramecin. which can kill other paramecia having no kappa. This killing substance, paramecin, can likewise be considered as an enzyme directed by a gene or a virus named kappa. Since paramecin may be the protein of the protozoa endowed with the pattern of kappa, it will strive to give the pattern to normal paramecia, but paramecin itself cannot multiply probably because of its failure of producing its exact replica, although it can cause disturbance great enough to kill the protozoa. 
Even the kappa bodies themselves can neither cause the production of paramecin nor protect the protozoa from the killing action of paramecin, if they are contained only in small amount within the cell (56). If the pattern of the protozoa is changed into that of kappa, paramecin may be produced and, at the same time, the protozoa may become not to be killed by paramecin.

It has actually been confirmed that both kappa and paramecin contain desoxyribonuleic acid, both being present in the bodies of killer organisms, and both are unstable and inactivated at rates varying with temperature. Paramecin is inactivated by pepsin, chymotrypsin, and desoxyribonuclease, indicating that both protein and desoxyribonucleic acid are compounds essential for its killing action, and moreover paramecin particles are liberated from killer cells mechanically disrupted and they are easily centrifuged down (57).

Since most proteins present in a cell are synthesized under the influence of the pattern of the genes, their pattern may be originally identical with that of genes. But some proteins may reluctantly be submitted to the genic pattern because of their peculiar configuration which makes it difficult to form the pattern directed by the gene. Such proteins may take their own natural patterns, if freed from the control of the gene.

Various kinds of proteins which are somewhat different in their immunological specificity can exist in one and the same blood plasm. Similarly, proteins with different original patterns may be present in a single cell. But their original, peculiar patterns may be revealed only when they are liberated from the cell, in which they have been compelled to submit to the pattern of the gene. Recessive genes possess extremely peculiar pattern of their own, although in the presence of the dominant genes they are forced to change their patterns to be submitted to the direction of the dominant genes. However, when once freed from the dominant genes, they recover their original patterns and behave as independent genes in some cells where there are no dominant genes.

The cells especially those of bacteria are believed to consist of various antigenic substances varying in chemical nature and biological properties, and termed antigenic mosaic. However, according to the theory of the writer as above stated, the cells are never the antigenic mosaic; the substances contained in the same cell should be equal in the antigenic pattern at least as long as they are present in the cell. The isolation in a "pure form" may lead either to a damage of the pattern or to the recovery of the original pattern as in recessive genes. The protein components constituting bacterial cell bodies such as those of pneumococci are called the somatic antigen, the specificity 
of which is not so stiking as that of the capsular antigen which is believed to be type-specific and to be polysaccharide in chemical nature. The type-specific structure may be fine and unstable, so that the pattern may be lost in the somatic protein during the purification process, only the solid, stable pattern being left untouched, whilst the fine, type-specific pattern may be retained in the polysaccharide even after the isolation owing to the rigid nature of its structure.

The writer considers that the pattern of lipids is not only extremely labile but their existence results in the decrease in the action of assimilase as the rigidity of the pattern is lost by their presence. However, the fact that the pattern is not always labile in the substances called lipids is fairly demonstrated by the work of Yamakawa and Suzuki (58) who have shown that a glucolipid isolated from red blood cells is evidently different in chemical structure between man and the horse. On account of its peculiar chemical composition, presumably owing to the presence of sugars, the glucolipid may be able to retain its species specific pattern even after the isolation though it is called lipid. 


\section{CHAPER VI \\ FACTORS INTERFERING WITH GENES}

\section{Hormones}

The pattern of proteins in protoplasm is generally determined by genes, but there are many evidences showing that factors other than genes can also play an important role in the determination of the pattern. Hormones appear to be substances which are produced by organisms for the purpose to interfere with the gene action.

Insects will generally change in a remarkable way their shapes and functions during their developmental process, that is, they undergo metomorphosis. Such metamorphosis may be caused by the rapid development of the structural change in the protoplam protein. In other words, metamorphosis may be a result of a rapid change in "crystal shape" of the protoplasm; and the "crystal shape" may be altered by the change in the structure of the component substance. On the other hand, it is generally accepted that hormones are involved in metamorphosis. The removal from insects of endocrine glands, such as prothracic gland and corpora allata, can induce profound effects in the feature of matamorphosis. This fact strongly suggests that hormones may be able to change the structure of protoplasm proteins.

Such effects of hormones upon the "crystal shape" of protoplasm can generally be observed in animals. As is well known when fed thyroid gland, the metamorphosis of tadpoles is accelerated and pigmy frogs will result, whilst when fed thymus, the metamorphosis is delayed to make giant tadpoles. Likewise in man disorders in endocrine secretion lead to abnormal shapes and functions. It may be concluded, therefore, that hormones are factors capable of strikingly interfering with the action of genes. The effect of hormones on the "crystal shape" of protoplasm seems to be of extremely high physiological significance; organisms can establish their normal development and achieve various functions owing to this effect of hormones.

As we have seen above, enzyme systems are directed by genes, whereas it is believed that hormones constitute an important set of 
enzyme-regulating factors. It should only be natural that enzymes are regulated by hormones if hormones can interfere with genes. Definite patterns of changes in enzyme concentration occur in tissues and organs under the influence of hormones; this is well illustrated by the changes in the alkaline phosphatase, acid phosphatase, adenosine-triphosphatase, succinic dehydrogenase, malic dehydrogenase, and total glycolysis of corpora lutea of the rat during pregnancy and lactation (59). The action of hormones on enzymes has also been demonstrated with the effect of insulin and some adrenal and pituitary hormones on hexakinase. Insulin can increase glycogen formation and glucose utilization in rat diaphragm incubated in vitro, and further it also increases the incorporation of alanine into diaphragm protein $(60)$. Again, phosphorylase in the rabbit is inhibited by cortisone and activated by adrenalin administration (61). These facts may be attributed likewise to the influence of the hormone upon the enzyme systems.

An interesting finding throwing light on the nature of hormones has been reported by Scatchard et al. (62). They have found that albumin is stabilized remarkably by the addition of small quantities of substances such as sodium caprylate and sodium salt of acetyltryptophan. By the addition of these substances the protein becomes so stable as to stand heating at $69^{\circ} \mathrm{C}$. for 10 hours. It should be noted that these substances, which exhibit such a remarkable effect upon albumin, have no influence on $\gamma$-globulin, whereas this globulin is stabilized by glycine and by certain simple sugars which are in effective to albumin. This fact may indicate that the structure of a protein is stabilized by the presence of a certain substance. It is worthy of note that substances which can stabilize albumin fail to effect any change in globulin, while grobulin is stabilized by other substances which exhibit no effect on albumin.

Hormones may be regarded as an agent which can exert such specific effects on the structure of protoplasm protein. The deficiency of a certain hormone may result in the destruction of certain structure in the protoplasm on which the hormone exerts its specifically directive influence. Presumably hormones like genes can change the structure of the protoplasm proteins in various ways according to their specific properties and can maintain the structure. Further discussions on this subject will be made in detail in Part V.

Some organisms appear to produce hormone-like substances for the purpose of destroying some other organisms by taking advantage of the striking effect of the substances to change the "crystal shape" of protoplasm. The so-called antibiotics produced by microoganisms can be regarded as such substances. The phenomenon of synergism and 
antagonism which is generally recognized with hormones has been observed also with these substances (63).

Penicillin, like phage, can cause lysis in bacteria (64). It has already been stated that lysis of bacteria by phage may be attributed to the structural disturbance caused by phage in the protoplasm of bacteria. Penicillin may likewise be able to change the protoplasm structure of bacterial cell, wherein severe disturbance may be raised which may cause the dissociation of elementary bodies leading to the decomposition of bacterial cell into the elementary bodies. The lysis, however, may not account for the effectiveness of penicillin, for the lysis may thus only be a result of the disturbance of protoplasm structure due to the drug. The disturbance in the structure may lead to the universal derangement in enzyme systems which must be the main cause of the effect.

Antibiotics can produce specific resistance in bacteria, indicating the occurrence of specific change in protoplasm structure in response to the structure of the drug. Entirely a similar phenomenon will take place when phage affects bacteria. Thus, the bacteria become resistant specifically to the phage, and bacterial protoplasm particles can act as the phage following the infection. As pointed out in the previous Part, bacteria having become immune to a certain drug produce virus-like agent able to transmit the resistance to other organisms.

\section{Inorganic Salts}

Inorganic salts are considered likewise to have hormone-like action and can interfere with the genes.

Lehmann (65) has succeeded in controlling chorda formation by treating the gastrula of Triton or Rana with lithium chloride. Moreover, it was found by Stockard that when, at a certain critical period, the developing eggs of Fundulus, a common sea minow, were subjected to the action of various magnesium salts dissolved in sea water, a large percentage of them developed a single median eye instead of ordinary pair (66). A similar effect of salts has been also demonstrated with the cephalopod Loligo vulgaris and other species. The modifications were produced by lithium chloride and magnesium chloride. The most conspicuous feature of the modifications involves the differential inhibition of the head region, resulting in all degrees of approximation of eyes to complete cyclops, reduction in sizes, anophthalimia, and acephalic (67). Such a striking action of inorganic solutions on animal forms may only be explained by assuming the 
hormone-like function of the ions. We have found that the properties of virus-like polymerization product of proteins are remarkably influenced by inorganic salts: Protoplasm particles, including viruses, are agglutinated at weakly acid $\mathrm{pH}$ values and exhibit high turbidities, which are markedly changed by the presence of inorganic salts (68) (69). The isoelectric point of the particulate proteins, at which the turbidity is most manifest, is generally shifted to more acid sides with the increase in the salt concentration, whereby the turbidity at the $\mathrm{pH}$ values more acid than the isoelectric point becoming higher. These effects vary, in a most remarkable way, with the kind of ions.

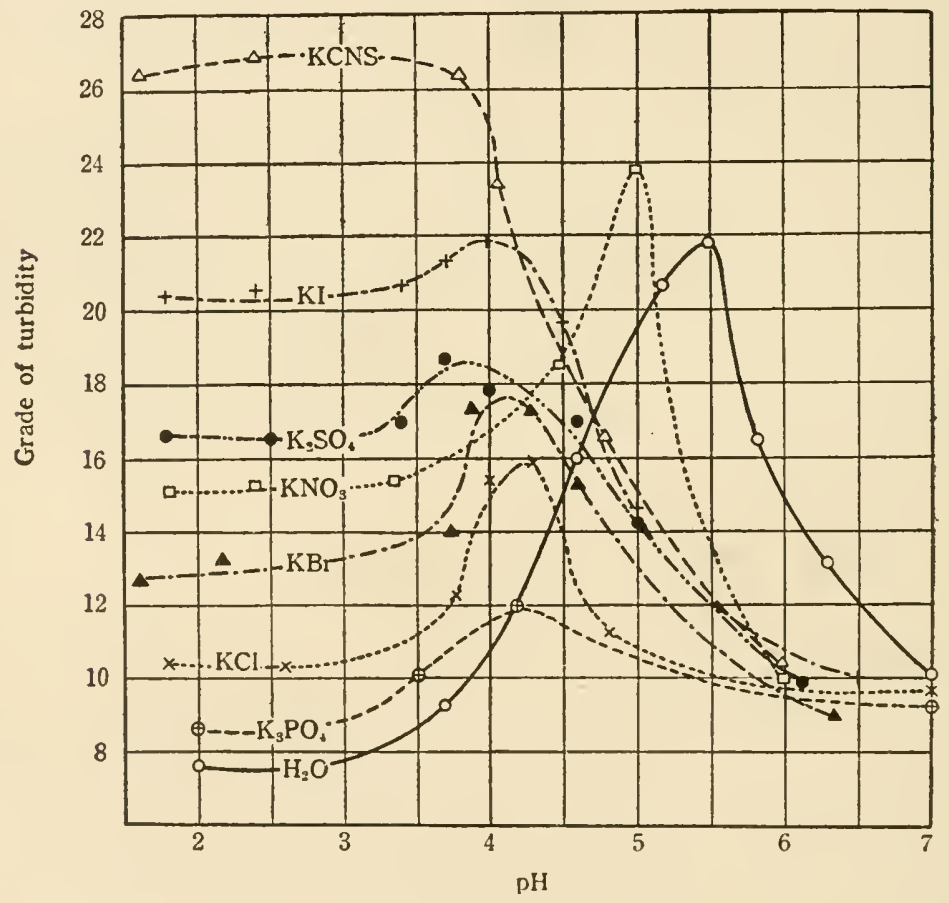

Fig. 24

The influence of inorganic salts upon the turbidity of vaccinia virus-protein solution.

Concentration of the virus protein : $0.1 \%$; concentration of salts : $0.2 n$.

The degree of the turbidity at pH 7.0 when no salt was present was taken as 10 .

An experimental result obtained with vaccinia virus is shown in Fig. 24. Although the influence of ions on the turbidity degrees is. 
somewhat different with the kind of particles, the gradient of the effect of various ions is almost similar regardless of the kind of particles, and so the result similar to that shown in this figure can be attained likewise with phage, rennin particles, and also with protoplasm particles having no virus action (70).

The inorganic salts which have particularly great effects upon the turbidity also exert remarkable deleterious influences upon the virus activity. Thus vaccinia virus particles are particularly injured by salts, such as $\mathrm{K}_{2} \mathrm{SO}_{4}, \mathrm{KI}, \mathrm{KNO}, \mathrm{KBr}$, ettc. while these salts have striking effects upon the turbidity. The virus remains active for the longest if preserved in water containing no salt.

Since a single active particle is regarded as producing a single plaque on agar, the deleterious action of inorganic salts on phage can be easily indicated in numerical values as seen in Fig. 25 . In this

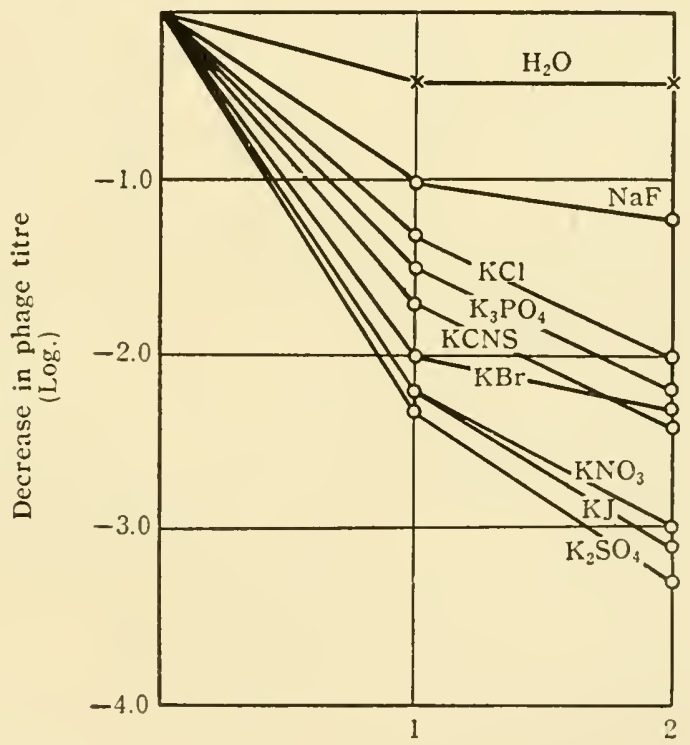

Hours

Fig. $2 \overline{5}$.

Influences of various potassium salts and also of NaF upon phage.

figure the degrees of the phage inactivation following the heating to $50^{\circ} \mathrm{C}$. in the presence of various salts are shown.

The rennin sample prepared by the writer consisted of virus-like 
particles, the activity of which was also easily shown with numerical values as in the case of phage, as the action was readily estimated by the length of time required by the enzyme for the coagulation of milk. As indicated in Tables $7 \& 8$, likewise with this enzyme it was clearly shown that inorganic ions exhibited striking effect, the gradient of ions in the effect being almost similar to that observed with the viruses.

Such remarkable influences of inorganic salts upon virus and rennin would be readily explained if we assumed that they could distort the structure of the particulate protein in various manners according to the kind of ions. This assumption seems reasonable, for the ions, like hormones, apparently interfere with the genes in changing the function of animals as above pointed out.

The production of phage by $E$. coli is remarkably decreased if the media, in which the bacteria are cultivated, contain neither calcium nor magnesium, or if a citrate or an oxalate is added to the media. According to our finding, the phage, yielded with difficulty under such conditions, would produce extremely small plaques only, its virulence to the bacteria being remarkably reduced. It is a noteworthy fact that this changed property was occasionally heritable; that is to say, the phage yielded from bacteria affected by such a changed phage tended occasionally to be of the same changed property, forming only small plaques. This property was inherited even when the bacteria were cultured in usual media, a fact which may show the occurrence of a "mutation" in phage (71).

Lack of the two-valent cations may cause a distortion in the pattern of the bacterial virus, giving rise to the "mutation" which is possibly induced by the alteration of protein structure in the protoplasm. As stated in Part II, Chapter VI, minute quantities of calcium can maintain the virus particles or protoplasm particles in a rigid form, so that the particles are photographed under the electron microscope in a complete shape, indicating that the lack of calcium results in a structural distortion of the particles.

Many evidences are known that calcium ions are involed in the transmission of structural change of proteins as in the case of blood coagulation. For example, it has been demostrated that calcium and magnesium ions in trace amounts are essential for haemolysis by antibdoy and complement, and possibly for bacteriolysis, as well as for enhancement of phagocytosis by antibody and complement (72). A number of the parthenogenetic agents lose their effectiveness when they are applied to eggs in calcium free sea water (73).

When one breaks the membrane of an amoeba or sea-urchin egg, the outflowing protoplasm soon forms a new membrane, but if the 
Table 7.

Effect of Inorganic Salts upon the Inactivation of Rennin due to the Neutralization.

\begin{tabular}{|c|c|c|c|c|c|c|}
\hline \multirow{2}{*}{ Medium } & \multicolumn{6}{|c|}{$\begin{array}{l}\text { The elapsed time (min.) after } \\
\text { the neutralization; } \mathrm{pH} 4,6 \rightarrow \mathrm{pH} 8,4\end{array}$} \\
\hline & 2,5 & 5,0 & 7,5 & 10,0 & 12,5 & 15, \\
\hline Water............ & 69 & 140 & 220 & 330 & 470 & 700 \\
\hline $\mathrm{BaCl}_{2} \ldots \ldots \ldots \ldots$ & 300 & 290 & 330 & 380 & 470 & 560 \\
\hline $\mathrm{CaCl}_{2}, \ldots \ldots \ldots \ldots$ & 220 & 250 & 270 & 280 & 290 & 310 \\
\hline $\mathrm{MgCl}_{2} \ldots \ldots \ldots \ldots$ & 225 & 180 & 170 & 170 & 180 & 180 \\
\hline $\mathrm{NaCl} \ldots \ldots \ldots \ldots$ & 1260 & 2220 & 一 & 一 & 一 & - \\
\hline $\mathrm{KCl} \ldots \ldots \ldots \ldots \ldots$ & 770 & 1320 & 2100 & 一 & 一 & - \\
\hline $\mathrm{LiCl} \ldots \ldots \ldots \ldots \ldots$ & 840 & 1320 & 1920 & - & - & 一 \\
\hline $\mathrm{NH}_{4} \mathrm{Cl} \ldots \ldots \ldots \ldots$ & 630 & 690 & 660 & 一 & 一 & - \\
\hline KCNS $\ldots . . . . . . .$. & 7500 & 一 & 一 & 一 & - & 一 \\
\hline $\mathrm{KI} \cdots \cdots \cdots \cdots \cdots$ & 2040 & 3600 & $\longrightarrow$ & 一 & 一 & 一 \\
\hline $\mathrm{K}_{2} \mathrm{SO}_{4} \ldots \ldots \ldots \ldots$ & 1660 & 2340 & 一 & 一 & 一 & 一 \\
\hline $\mathrm{KNO}_{3} \ldots \ldots \ldots \ldots$ & 1560 & 2520 & 一 & - & 一 & - \\
\hline $\mathrm{KCl} \ldots \ldots \ldots \ldots$ & 770 & 1320 & 2100 & - & - & 一 \\
\hline
\end{tabular}

The numerals indicate the time (sec.) necessary for the occurrence of the coagulation of milk. The concentration of the salts was $n / 10$.

Table 8.

The Influence of Inorganic Salts upon Rennet Action.

\begin{tabular}{|c|c|c|c|c|}
\hline $\begin{array}{l}\text { Medium in which the rennin } \\
\text { was dissolved }\end{array}$ & Exp. I & Exp. II & Exp. III & Average \\
\hline 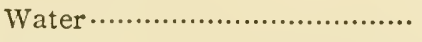 & 11 & 11 & 12 & 11 \\
\hline 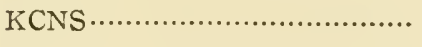 & 43 & 41 & 42 & 42 \\
\hline 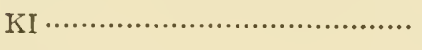 & 29 & 31 & 28 & 29 \\
\hline $\mathrm{K}_{2} \mathrm{SO}_{4} \ldots \ldots \ldots \ldots \ldots \ldots \ldots \ldots \ldots \ldots \ldots \ldots$ & 24 & 24 & 26 & 25 \\
\hline 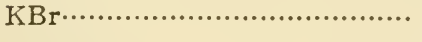 & 22 & 23 & 23 & 23 \\
\hline 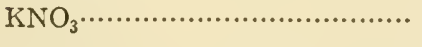 & 23 & 20 & 24 & 22 \\
\hline 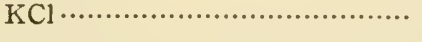 & 18 & 19 & 18 & 18 \\
\hline
\end{tabular}

Numerals indicate the time (sec.) necessary for the coagulation of milk, when $1 \mathrm{c} . \mathrm{cm}$. of the rennin solution $(0,01 \%)$ was mixed with $1 \mathrm{c.cm}$. of milk. The concentration of the salts was $n / 10$, and the $\mathrm{pH}$ of the solution being $5,4 \sim 5,6$. The experiment was carried out at laboratory temp. 
cell is kept in a solution completely lacking in calcium after its membrane is broken, all the protoplasm flows out of the cell and scatters through the surrounding solution, no new membrane being formed. Again, phosphatases require the presence of magnesium as coenzyme, and pyrophosphatase is optimally active only in the presence of definite ratio of magnesium and calcium ions (13). The action of such coenzym may be based on the ability of the ions to retain the structure of the enzymatic protein in a proper form.

A great number of evidences have been presented to show that the majority of vitamins behave as various coenzymes, suggesting that vitamins are also substances which have influences upon the structural pattern of proteins, especially that of enzymes. Anderson (74) has found that phage needs amino acids, tryptophan in particular, as cofactors for virus adsorption. These adsorption co-factors appear to combine reversibly with the virus, without them the virus being unable to combine with the bacteria. Presumably a distortion may occur in the virus structure when the amino acids are absent. It is said that D-tryptophan is inactive despite the marked effectiveness of L-tryptophan, indicating that this amino acid is involved in the determination of the "crystal shape."

The "crystal shape" of protoplasm may be influenced by the presence of various substances especially inorgenic ions, although the shape is, in the main, directed by the genes; the change in the mixed proportion of the ions will be followed by the alteration in the shape. Since the primary organisms were generated and evolved in the sea water, their protoplasm will retain its normal form only when their body fluid is similar, in the proportion of ions, to the sea water of the primitive age. An alteration of the proportion may, therefore,

Table 9 .

The Stabilizing Effect of $\mathrm{NaOH}$ upon Red Blood Cells.

\begin{tabular}{c|c|c|c|c|c|c|c}
\hline $\begin{array}{c}\mathrm{pH} \text { of the } \\
\text { cell-suspension* }\end{array}$ & $\begin{array}{c}5.5 \\
(\text { No ad- } \\
\text { dition) }\end{array}$ & $\begin{array}{c}5.5 \\
(n / 80 ; 1)\end{array}$ & $\begin{array}{c}5.6 \\
(n / 80 ; 2)\end{array}$ & $\begin{array}{c}5.8 \\
(n / 80 ; 3)\end{array}$ & $\begin{array}{c}6.0 \\
(n / 20 ; 1)\end{array}$ & $\begin{array}{c}6.4 \\
(n / 10 ; 1)\end{array}$ & $\begin{array}{c}9.2 \\
(n / 10: 2)\end{array}$ \\
\hline $\begin{array}{c}\text { Degree of hemo- } \\
\text { lysis by 0.7\% NaCl } \\
\text { solution }(\%) .\end{array}$ & 72 & 45 & 22 & 0 & 0 & 0 & 0 \\
\hline $\begin{array}{c}\text { Degree of hemo- } \\
\text { lysis by 0.41\% } \% \text { NaCl } \\
\text { solution }(\%) .\end{array}$ & 100 & 100 & 100 & 100 & 56 & 38 & 29 \\
\hline
\end{tabular}

* Figures enclosed by parenthesis indicate the concentrations of $\mathrm{NaOH}$ and number of its drops added to $0.5 \mathrm{cc}$ of the blood-cell suspension. 
lead to the destruction of the normal structure, causing severe injury in the organisms.

From what has been discussed above it should naturally be expected that the hydrogen ion has an effect upon organisms similar to, or rather more than that of the usual inorganic ions. It is of no use to emphasize the striking effect of $\mathrm{pH}$ in the body fluid.

We have found that the addition of minute quantities of alkali to blood-cell suspensions in vitro provides the cells with the faculty to resist the haemolytic action of hypotonic saline solution, probably because the structure of the stroma becomes stronger through this procedure (75). An experimental result showing this fact is cited in Table 9; rabbit's red-blood cells are enabled to resist markedly to hypotonic saline solutions by the addition of minute quantities of $\mathrm{NaOH}$. It may appear strange in this Table that $\mathrm{pH}$ of the cell suspension has the value of 5.5. Blood corpuscles washed and suspended in the physiological saline solution, always shows this $\mathrm{pH}$ value. This suggests that the cell stroma itself is of this $\mathrm{pH}$. Alkali salts other than $\mathrm{NaOH}$ exhibit the same effect, indicating that the effect is due to $\mathrm{OH}$ ion. Several minutes are required for stabilizing the cells. But the additions of hydrogen ion, on the contrary, instabilize the cells and render them liable to undergo lysis. The stabilization due to $\mathrm{OH}$ ion and the instabilization due to $\mathrm{H}$ ion are completely reversible.

It may be a natural result that the protoplasm, a mixed crystal, is influenced in its character, to a more or less extent, by the substances present in it. Inorganic salts are substances normally present in large quantities, and accordingly they have great influences upon the character. Hydrogen ion is also a normal component and its effect is compatible with that of heavy metals, having much greater effect than the usual inorganic ions.

As above stated, the embryonic development of some animals is much influenced by inorgnic ions; and similar effects are said to be induced also by the application of simple organic subatances, such as ether and alcohol, indicating that non-physiological substances, even if they have no peculiar, complicated structures, can cause a distortion in the protoplasm pattern when they enter the cell. It has been reported that trypan blue can induce in mouse embryos malformations, which bear a striking resemblance to those genically determined (76). This may indicate that the dye like a gene has the property to exert a peculiarly distinct distortion on the pattern.

To sum up, the pattern of protoplasm is in the main determined by the genes, but substances, such as hormones and inorganic salts, which are contained physiologically in the protoplasm may interfere 
with the genes and modify the pattern directed by the latter. Presumably higher organisms, by making use of these substances, are able to reveal different forms and functions that may vary with the varying parts of the body, even if the genes of different parts be equal. 


\section{CHAPTER VII}

\section{SEXUAL REPRODUCTION AND REJUVENESCENCE}

\section{The Origin of Sexual Reproduction}

The primary organisms in their extremely primitive stages were presumably able to come into contact with other individuals by virtue of the motion of the water in which they were generated. If two different individuals shared some structures in common they would combine with each other and the one having the stronger structure would overcome and assimilize the other. Such a combination might be a struggle for survival, but on the other hand the stronger individual might be able to establish rejuvenescence by this combination.

When the organisms were evolved to such a degree that some elementary bodies in them were provided with nucleic acid in rich amount and that the structure of such bodies was endowed with a strong reversibility, these bodies would be able to recover their original structure when they were liberated from the organisms which had been assimilized or devoured by some stronger individuals. Accordingly, such bodies, if liberated from the defeated, weaker individuals, would be able to assimilize the structure of still weaker individuals if they could enter the latter. The combination of two individuals at such a stage, therefore, may be regarded as a very primitive type of sexual conjugation. It may be said that the primary organisms at this stage had only a pair of genes, and the combination may be comparable to a phenomenon in which weaker bacteria are changed into stronger ones when these two strains are incubated together.

Thus a strain of bacteria producing phage, $i . e_{\text {., a lysogenic strain }}$ can change another strain, which is susceptible to the phage, into the lysogenic strain if these two strains are brought into contact, as was discussed already. Again, R-type of pneumococcus is changed to S-type when comes into contact with S-type. In these instances, nucleic acid-rich particles freed from the stronger strain can substitute the bacterial cell. These particles may be called free genes or viruses, 
but at the same time it may equally be possible to regard them as spores or primitive gametes, and so the combination may be said to be a primitive form of the sexual conjugation.

However, since in such a conjugation the weaker individual is completely defeated, the stronger one will be unable to adopt any specific character of the former, although it can anyhow accomplish rejuvenation through the contact with different structure. If a part of the structure of the weaker individual was intermixed with that of the stronger by this conjugation, an individual having a new character would be produced, with a much contribution to the advancement of the evolution.

This state of affairs would possibly result when protein structures of nucleic acid-rich particles of primeval organisms were differentiated to some extent. With certain bacteria the presence of such a differentiated structure is actually confirmed. As rvas already described, if two different viruses, $A$ and $B$, affect simultaneously a strain of bacteria, sometimes a new virus is produced which has peculiarities of both A and B. Instead of two kinds of viruses, two types of bacteria yield the same result; the conjugation of two strains of bacteria occasionally produces a new strain of bacteria which have the characteristics of both strains.

Thus Lederberg and Tatum (77) (78) have actually demonstrated such a sexual reproduction of bacteria. They have made use of biochemical mutant strain which are unable to synthesize certain essential substances and which consequently can grow only on media to which those substances have been added as nutrients. For example, one strain used was unable to synthesize biotin and methionine, another prolin and threonine. When two such double mutants are grown in mixtures in minimal medium, lacking the nutrients above mentioned, there appear appreciable numbers of structural recombinations which can synthesize all the enumerated substances and consequently survive. Lederberg has also made use of strains which differ in the ability or inability to ferment lactose, and in resistance or susceptibility to a specific bacterial viruses.

In this case also protoplasm particles can substitute the bacterial cells as has been demonstrated by Hayes (79) who has found that sexual conjugation of bacteria can be achieved by a virus-like agent. These particles, therefore, should be regarded as primitive gametes. However, both individuals may be unable to make use of such particles for their sexual reproduction, since the multiplication of the structure fails to be accomplished by the combination of two particles only, and so for the multiplication, one of them should be present in a cell or at least should be present in combining with large amount of 
cytoplasm into which the new structure is to be spread. For this reason sex had to be differentiated; the particles, active gametes, which are liberated from the one are called male gametes, or sperms, and produced in much greater numbers than larger female gametes, or egg cells, which are provided with great quantities of cytoplasm. In the case of the structural recombination of two viruses above cited, there are host cells in which the new structure can multiply; without host cells new structure, of course, cannot multiply.

If a weaker individual is devoured completely, its structure will be of no use, but if a part of the structure can be intermixed with the structure of the stronger individual, some character of the weaker one can remain in the combined structure to make the newly formed individual more fitted for existence; the part of the structure which can remain in the newly formed individual must be strong and so may give rise to a distinct character. Therefore, the individuals of assimilase or primeval primary organisms, which had succeeded in obtaining the faculty for the sexual reproduction would accomplish much more rapid progress in their evolution than those lacking the faculty, and consequently would become the fittest with the result that at present higher organisms without exception perform sexual reproduction. In short, the first aim of the conjugation may be the rejuvenation, but the yield of new structures may be another important aim.

It is true, however, that sexual reproduction is very troublesome, and the easiest and simplest way must be the reproduction by fission without sexual conjugation. This must be the reason why certain organisms choose the alternation of generation, or metagenesis, characteristic of plants, also occurring among a few animals. It involves an alternation of a sexually-reproducing with an asexually-reproducing generation; in this latter generation vigorous multiplication occurs because of its easiness, while in the former generation rejuvenation may take place with best chances to form new individuals more fitted for existence, although the multiplication in this generation is not so easily done as in the asexual generation.

Such a mode of multiplication must have been highly developed presumably because it was the best way to become the fittest for some plants and animals, whereas it must have been inconvenient for some other organisms exhibiting certain other forms and functions and therefore these organisms achieve sexual reproduction only.

Microorganisms, such as paramecia or other certain protozoa, which multiply usually by binary fission and have not yet acquired the undoubted, distinct mode of metagenesis, appear to accomplish rejuvenation by performing now and then an irregular conjugation. 
It is said that paramecium and other protozoa after a few hundred divisions apparently die out from senescence, unless conjugation takes place. Ciliate, uroleptus, after some two hundred or more divisions, in the absence of conjugation, the division rate slows down and the individuals pass into a decline followed by structural degeneration and death.

Since the organisms that cannot accomplish rejuvenation by means of conjugation must undergo senescence and be perished, the individuals having the property to perform conjugation can solely continue their existence. Individuals having the property to dislike the conjugation would certainly fail to produce their offspring. Therefore, the sexual desire has developed amazingly in the present day organisms, and has become the instinct for the race preservation.

The somatic cells of higher crganisms continuously perform cell division throughout the developmental process of the individual, and accordingly the division is comparable to asexual reproduction of microorganisms, so that it may be expected that the cells may undergo a senescence. This seems, however, not to be the case, for there is a mechanisn by which the cell is rejuvenated at every cell division, in which is involved the disappearance of the nucleus as a definite body, whereby the genes get into contact with the cytoplasm whose pattern has been deformed by the life activity during the inter-division phase, and the deformed pattern is to be adjusted to the original, normal pattern by the genes. This adjustment to the normal pattern must be the rejuvenation itself. Although somatic cells can be rejuvenated in such a way at each cell division, the individual itself, as a whole, cannot avoid a decade, the cause of which, however, should be explain-d from another point of view as will be considered later.

It should be noted here that in some strains of paramecium conjugation seems to be unnecessary. In such strains, a process of nuclear reorganization, termed endomixis, occurs every forty or fifty generations, in which the old macronucleus disintegrates and the micronucleus divides and redivides as if in preparation for conjugation. Then, there is the same disappearance of micronuclear material, and from the single micronucleus remaining new macro- and micronuclei are formed. This may be regarded as an extremely advanced feature of rejuvenation mechanisms which is seen in the mitosis of somatic cells.

The cyanophyta or blue-green algae are undoubtedly the most primitive of all green plants, and no evidence of sexual process has been observed in these algae, reproduction apparently occurring exclusively by means of simple fission. If so, since they have no para- 
sitic nature, they may have proper rejuvenation means like this paramecium.

In addition, certain organisms are apparently able to accomplish rejuvenation to a certain extent merely by the change of environmental condition under which they grow. According to our finding (80), when cultivated in broth for successive generations, typhoid bacilli will be reduced in virulence, changed to $\mathrm{R}$-type, and their resistance to heat is diminished, becoming liable to die out, whereas when the bacteria declined in such a way are transferred on agar-plate, they will be changed into S-type, whereby their virulence is enhanced and the resistance is increased. However, successive cultivation on the same agar-plates also lead to a decline in the virulence. The sole change in the temperature at which the bacteria are cultivated has a similar effect, the virulence tending to increase when the temperature is elevated over $37^{\circ} \mathrm{C}$.

The development of ascaris is accomplished when the eggs are exposed to low temperatures outside the host body, a fact which may also be an example that rejuvenation is established by the change of environment without contact with another individuals.

It can by no means be considered, however, that such a rejuvenation is perfect and complete. As is generally known, the enhancement in the virulence of bacteria, which have been decayed by the continuous cultivation under the same environmental condition, is accomplished usually by passage through proper animals, a phenomenon which must be analogous to the sexual conjugation as we shall see in the following section.

\section{Rejuvenescence of Microorganisms by Making Use of the Sexual Reproduction of the Host}

As seen in the preceding Chapter, the deformation in the pattern of viruses may arise if they continue to multiply in the protoplassm of the same kind of organisms. This deformation will be repaired by the host change with the achievement of the rejuvenation of the virus. It is considered that the host change answers not only the purpose of rejuvenation, but also the purpose of producing new species, since viruses through the host change may be able to get possession of the new protoplasm structure of the new host. The host change, therefore, is comparable to the sexual reproduction.

In many parasitic protozoa, for example, the parasitic amoebae and the intestinal and blood flagellates, no sexual process has been obser- 
ved with certainty. Protozoan cells tend to grow old after continued asexual multiplication and lose both their youthful vitality and reproductive power. The habitude of host change, like in viruses, is common among these protozoa, and therefore it seems possible that the host change can substitute the sexual reproduction in these lower animals. However, the fact that the faculty for sexual reproduction is fairly developed in certain parasitic protozoa, such as malaria plasmodium, indicates that the sexual process is much more advantageous for their evolution than the host change.

As already discussed in Chapter $\mathrm{X}$ in Part III, besides the rejuvenation by the host change, viruses can accomplish rejuvenation by taking advantage of the sexual reproduction of the host, that is, viruses penetrate into germ cells of the host and are rejuvenated there together with the germ cells through the fertilization. As we shall see in greater detail in Part V, germ cells of higher organisms are considered to have the undeveloped structure of the single celled creatures from which the organisms have been evolved. In other words, germ cells may be produced by the reduction of the developed structure of the somatic cells to their undeveloped pattern. Meanwhile, the pattern of a virus itself, engraved in the mother germ cell of the host, will be also altered during the reduction of the cell to the germ cell, and hence, in the germ cell, the altered pattern of the virus cannot behave as the virus. However, the pattern will recover its original form with virus activity, when the germ cell gradually recovers its developed original structure in the developmental process following the rejuvenescence by the fertilization. Such a rejuvenation of viruses may be quite perfect, because it is brought about together with the complete rejuvenation of higher organisms.

The stronger the structure of a virus, the longer period of time it will continue to exist, so that the viruses will become the stronger as they evolve the higher. Consequently, viruses may naturally acquire the faculty to engrave their pattern even into germ cells as they evolve to a certain extent; thus they may be rejuvenated to continue their existence still further.

A virus, which can already be rejuvenated by a host change, may still further be rejuvenated by penetrating into germ cells of the host. Certain plant viruses may be able to enter the germ cells of the host plant, while at the same time they may even engrave their pattern into the germ cells of their insect vector. This may hold true also for animal viruses.

Viruses, unable to change the host, will likewise acquire the ability to engrave their pattern into germ cells of the host, when their pattern is strengthened to a certain extent. Therefore, the rejuve- 
nation by means of the sexual reproduction of the host appears to be easily attainable for the viruses and accordingly to be most conventional for them to continue their existence.

As for the viruses affecting man, most of them can infect solely some particular individuals, though some of them, such as those of measles and mumps, are capable of affecting almost all the individuals. These latter viruses seems to be able to engrave their pattern into. the germ cells of almost all the human beings, whereas some other viruses appear to do so only in particular persons who have the predisposition liable to be afflicted by them. The pattern of the viruses will be transmitted to the children of such peculiar persons affected by the viruses, and the pattern will be developed into its full form when the children grow up to certain ages, leading them to the disease or rendering them virus-carriers, who will subsequently scatter about the viruses. Some of the viruses thus scattered about may affect individuals having the peculiar predisposition, and through such individuals they can be rejuvenated and transmitted to offspring. The viruses must be extinguished if there are no such predisposed persons.

Even in the case of measles, about 5 per cent of man are not infected with the virus for life. Such individuals must be completely freed from the pattern of the virus, since the virus cannot enter their germ cells. Measles would be expelled from this globe if all the human beings had such a peculiar character.

In addition, climatic or topographical conditions may play a considerable role in the development of virus diseases. Persons living in a certain region may have a peculiar disposition according to the climatic factors in the region and may be liable to be afflicted by some virus which cannot, or scarcely, infect the man living in other regions. In such a case the virus disease may be called an endemic disease, the pattern of the virus in question being engraved only in the germ cells of man living in such a region. This may also hold for animals other than man, and so it may occur that certain insects or other animals in a certain region always prove to be carrying a virus peculiar to the region.

The development, in a child, of the full pattern of the virus which is transmitted from parent appears to be effected mainly by the age of the child. However, many other factors are apparently involved in the development. It is a remarkable fact, as already discussed, that leaf hoppers carrying aster yellow virus lose the virus if exposed to a temperature of $32^{\circ} \mathrm{C}$. (81). This fact can be interpreted as indicating that the structure of the virus cannot develop at $32^{\circ} \mathrm{C}$.

The incidence of measles changes regularly with the change of 
season as described in Chapter III in Part III. This may be elucidated by considering that the pattern of measles develops in some seasons while disappears in other seasons. It seems probable that immature pattern of a virus present in some organisms cannot develop to a full form unless proper conditions are provided. Phage can be detected in chicken feces in warm seasons only, especially in summer, as stated already, a fact which suggests that the immature pattern of phage in chicken cells develops its complete pattern in summer. Even the virus pattern present in lysogenic bacteria can develop into complete form only when adequate conditions are provided (82).

In view of these facts it seems difficult to distinguish a newly generated virus from a virus inherited from parent. The discrimination of the two forms, however, may not be impossible, as in general in contrast to fixed, advanced viruses, the structure of newly generated ones must be weak and accordingly they are unable to give rise to a long-lasting immunity, that is, they cannot exist for long periods of time in host cells, not to speak of the transmission to progeny. Furthermore, newly generated viruses may be liable to undergo variations because of their weak structure, so that their immunological specificity is inconstant, whereas the pattern of fixed viruses is generally invariable, being transmissible unchanged in hosts from generation to generation.

Herpes simplex virus appears to be generated by various factors, especially by febrile diseases; the disease is said to occur also after artificial pyrotherapy. It seems reasonable, however, to regard this virus as a typical fixed virus. Burnet (83) has claimed that the most individuals acquire herpes virus in childhood, as in the case of measles, and carry it for the rest of their lives. After the first infection with this virus, individuals develop neutralizing antibodies in their serum which remain present for many years. Persons who have been infected with this virus can thus be detected by serum neutralization test, indicating the constancy of its antigenical specificity as well as the long duration of its existence in the host. The causative agent, such as fever, may therefore aid only the virus pattern inherited from the parent to develop into its complete structure. 


\section{CHAPTER VIII}

\section{REJUVENESCENCE OF MICROORGANISMS WITHOUT SEXUAL REPRODUCTION}

\section{The Microorganisms Parasitic on Insects}

If viruses can accomplish their rejuvenation in such a remarkable way as above described, it should be expected that the secondary organisms evolved from viruses may apply the same tool for their rejuvenation..

It is generally accepted that most of pathogenic Rickettsiae are inherited in arthropod vectors from generation to generation. It is unreasonable to consider that the secondary organisms choose the virus-like rejuvenation mechanism only up to the stage of Rickettsiae, and that the organisms evolved higher than Rickettsiae all abandon the mechanism. According to the writer's view the microorganisms, such as bacteria and protozoa, which perform no distinct sexual process are still succeeding to this remarkable habitude of their ancestors.

This view is strongly supported by the fact that in a large number of insect species, a special structure or organ is present, called mycetome, the principle function of which appears to be that of housing various microorganisms, some of which are of fungous nature while others are called bacteroid. Most of these organisms cannot be cultivated outside the insect body, only capable of proliferating in the host.

These microorganisms are looked upon as symbiotes, and in some cases the symbiotes apparently furnish their hosts with essential substances, such as vitamines, which are lacking in their regular diet; in some other cases they supply hormone-like substances which aid in the development of the ovaries. It appears quite possible that the symbiotes of many insects are capable of fixing atmospheric nitrogen. Certain insects cannot maintain their lives if the mycetome is mechanically removed, or the symbiotes are killed by drugs, such as penicillin, or by maintaining the insects for a prolonged period at higher temperatures (84).

Thus the mycetome, an aggregate of microorganisms, is regarded as an indispensable organ for the insects, and consequently the organ together with the microorganism are inherited from generation to 
generation through the eggs. The character to produce the microorganisms, therefore, must be the inheritable character essential for the insects. This fact strongly suggests that certain fungi and bacteria are still now succeeding to the habitude of viruses, their ancestors, to be rejuvenated.

Leach (85) has already expressed, the opinion that insects are not merely disseminators of inoculum in the case of pathogenic fungi, but that the insect-fungi relationship is highly organized and has broad biological and evolutionary significance. The fact that over 200 kinds of bacteria have been isolated from insects may show what a great number of bacteria are making use of insects for their rejuvenation. As we shall see later, spirochaetes are also regarded as microorganisms succeeding to this habitude; meanwhile about 20 kinds of them have been found in insects.

Many examples are known, as pointed out already, to show.that insects are not afflicted by viruses harbouring in them, in some cases the relationship between them being regarded even as a symbiosis. Such a relationship appears to be kept on even after the viruses have evolved into undoubted microorganisms. It cannot be said, however, that insects are always not afflicted by microorganisms. Numerous infectious diseases due to bacteria or fungi are well known.

\section{The Rejuvenescence of Tubercle Bacillus}

As already dealt with, a phenomenon related to sexual conjugation is occasionally observed with colon bacillus, but it cannot be considered that the bacillus is provided with the distinct habitude to be rejuvenated regularly by the sexual process. The observed phenomenon should be looked upon as only an extremely primitive form of the sexual conjugation, and presumably the bacillus is usually not rejuvenated by such a process.

Here again, it seems probable that this bacillus is also resorting to the means of rejuvenation which their ancestors used habitually. It has been reported that the normal human individual harbours two types of colon bacillus, residents and transients, $i$. $e$., persistent over long periods strain and limited to a few days strain, the former contains typically one or two strains (86). This pattern of bowell bacilli is shown by very young babies as well as adults. Babies as intimately associated as twins harbour the same resident.

Colon bacillus, however, may not be so adequate as tubercle bacillus to be dealt with concerning this subject. Almost all the human beings are infected with tubercle bacillus as with measles 
virus. The manner of establishment of the infection seems likewise very similar to that of measles; new born babies are scarcely infected, but the incidence increases rapidly with their growth until almost all of them are affected. This fact is usually explained to be due to the increase in the chance of infection as infants grow older, whereas it may also be possible to regard this as analogous to the phenomenon already discussed that larvae hatched from eggs of insects which were infected by a certain plant virus produce no virus when very young, but that as grow older they become infectious. As we shall see in the following, there are many good reasons to consider this interpretation to be much more reasonable than the former.

The concept that the tuberculosis is produced only by the infection from outside may be confused by the so-called Lübeck tragedy, in which 251 new born infants instead of the avirulent bovine bacillus $B C G$, a living human virulent strain was accidentally substituted in the laboratory. The bacilli were given by mouth in so large an amount as $400,000,000$ microorganisms, on three different occasions during the first ten days of life, with the result that 75 of them dead and 175 were living, eacaped death or even progressive tuberculosis. Moveover, in a study of children in tuberculous families, Puffer (87) has shown that of the contacts with sputum-positive parents infection came earlier than those of sputum-negative parents, but, by the time to come of ages of fifty, contact and non-contact cases with positive heredity showed the same percentage of infection.

According to Pottenger (88), the fact that the apex is not particularly susceptible in first infection, which so commonly takes place in childhood, but shows a particular predilection for reinfection in the adult, indicates that something occurs in the time between childhood and adult life which changes the localization of the bacilli; he stated that it might further indicate that the source of the bacilli and the route of invasion might be different. Such a phenomenon should be only natural if the bacilli are produced in human bodies.

For a long time it was known that tubercle bacillus can exist in a virus-like filtrable form, and numerous workers have claimed that this filtrable form constitutes a special stage in the life-cycle of the bacillus. This fact strongly supports the above concept, since, if the bacillus is transmitted by human germ cells, it should be reduced, at least during the transmission, into the form of a virus, from which subsequently bacillary form develops. Moreover, tubercle bacillus is known to be one of the acid-fast bacteria, but in young cultures it is common to find a certain proportion of non-acid-fast forms. In view of these facts numerous workers believe that the life-cycle of the bacillus consists in that the virus-like particles develop into acid-fast 
bacilli through the stage of non-acid-fast forms.

If tubercle bacillus can recover its complete form from such a virus-like particle, its penetration into germ cell like a genuine virus may be possible. To speak more precisely, the pattern of the bacillus, like that of a virus, may be engraved in the mother germ cell of man, and the pattern may be transmitted to the germ cell in a reduced form as in the case of a virus; as the pattern exists in the germ cell in this reduced structure, the original pattern of the tubercle bacillus cannot be proved in it, but when the germ cell develops to a human individual; the reduced pattern of the bacillus also develops to the complete, original pattern of virus-like particle having the original pattern, from which the acid-fast bacillus may be formed. The first infection may thus be established. The character of the bacillus thus developed may change further with the further development of the host individual, with the diminution in the virulence which may again increase afterwards to cause the reinfection.

The percentage of children of varying ages who have become tuberculin-test positive usually varies with regions, suggesting that the period of the development of the complete pattern varies according to the regions. In view of the climatic or topographical effect which may vary with regions, it seems natural that the disposition of the children also varies with them, resulting in the difference in the period of the development of the pattern. The infection from outside cannot be disregarded, but most of the first infections may result from the development of the pattern in the children as in the case of measles.

At any rate, tubercle bacilli, like microorganisms in insects referred to in the preceding section, appear to be entirely involved in the host, $i$. e., in human beings, with which they can be rejuvenated. The consumptive is born to a consumptive said Hypocrates. This must be the truth. It can even be said that tubercle bacillus is an inheritable pattern of the gene in the majority of human beings. The reason why such a mention can be made will become gradually evident as the description proceeds, especially in the next Part.

Since tubercle bacilli are connected with the mankind in such a firm way, if any alteration occurs in the latter, the former will be changed together with it. Some authorities regard tuberculosis as an epidemic disease with epidemic waves; the last one, having reached its peak about 70 years ago, is now declining. With the exception of war times, there has been an almost universal decline in the mortality of tuberculosis throughout the world (89). The writer is of the opinion that this may be attributable to the wave of the change in human disposition; it should be a very remarkable fact that human stature 
has meanwhile shown the tendency to increase steadily with the decline of tuberculosis. This very important and interesting problem will be discussed in most detail also in the next Part.

\section{The Significance of Filtrable Forms of Bacteria}

It seems probable that almost all the parasitic bacteria are being rejuvenated in the same way as tubercle bacilli, although the degree of the connection with the host may vary greatly with the species of bacteria. For example, the connection appears to be particularly firm in lepra as well as in tuberculosis, while in other diseases such as typhoid and dysentery it may be loose.

The filtrable form has been known and discussed for the longest time with tubercle bacillus. But the presence of related forms appears to be universal with bacteria, supporting the above idea that bacteria are generally inherited through germ cells of the host. It has long been known that dysentery bacillus can restore its full form even after decomposed into filtrable particles; that is, viable dysentery bacillus can be formed from lysed filtrate of the bacteria by phage (90). It is said that newly generated bacteria are phage resistant, which must be expected from the theory of the writer as already referred to. Again, Kasahara et al. (91) have found with many kinds of bacteria that original forms are actually produced from the filtrate of bacteria decomposed by ultra-sonic vibration.

On the other hand, Klieneberger (92) isolated from cultures of Strepto-bacillus miliformis a minute organism which she designated as L-form. This resembles the virus-like organism of bovine pleuropneumonia. They are isolated from many other bacterial species, and transformation into this virus-like forms is brought about apparently always by an injury to the bacteria, especially when treated with penicillin. Diens (93) is of the opinion that these virus-like organisms are part of a life-cycle not only in the streptobacillus, but in other bacterial species. In fact, he has succeeded in causing their appearance in cultures of E. coli, Haemophilis influenzae, Flavobacterium, the gonococcus and $F$. funduliformis. Some workers believed at least some of these $\mathrm{L}$-forms to be analogous to the so-called fitrable forms, but some others appear to hold the opinion that these two forms are different (94) (95). It has been reported that in penicillin-containing media $P$. vulgaris showed L-type growth. It is a note-worthy fact that L-forms thus produced could be changed into bacillary forms when transplanted to penicillin-free media or passed intraperitoneally through mice; the L-form appeared to be anaerobic and non-pathogenic 
for mice $(95 a)$.

At any rate, there seems no doubt that bacteria can recover their normal, complete form even after decomposed into smaller, virus-like particles. According to Dienes and Zamecnik (96) the presence of high concentrations of glycine in the media induces the transformation of some bacteria into L-form, glycin being the most effective among a variety of amino acids, although very few bacteriostatic chemicals induce the transformation. It is interesting that only one toxic bacteriostatic chemicals among many examined produced L-form, while three amino acids which are necessary constituents of the cell produces $\mathrm{L}$-form when they were present in high concentrations. This fact suggests that the L-form transformation is never a mere mechanical decomposition of bacterial bodies. but may be associated with a peculiar structural transformation like that in spore formation.

There is little doubt that sporogenesis is associated with considerable changes in protoplasm structure. Hardwick and Foster (97) have found that seventeen different enzymatic systems, abundantiy demonstrable in vegetative cellular extracts of $B$. mycoides and other aerobic sporeforming bacilli, could not be detected in spore extracts tested concomitantly. They regarded this fact as supporting their hypothesis that sporogenesis occurs at the expense of enzyme proteins preexisting in the vegetative cell. However, as stated already, any structural change in protoplasm should be accompanied by some change in enzymatic system, and hence this fact must only be indicating the occurrence of the structural change in the protoplasm. It is considered that the change leads to a primitive structure of the protoplasm, whose return to the original structure is to be accompanied by the recovery of the vegetative form, an important concept which will be discussed in great detail in Part V.

In order to accomplish the rejuvenation in germ cells of the host. bacteria must exist in a virus-like state at least at the early stage of the development of the host, not to speak when present in the germ cells. In virus-like state at such a stage, they may be unable to exhibit the function as bacteria even when incubated in vitro in much the same way as that the germ cells of higher organisms cannot develop into the adult in vitro. Therefore, failure of demonstration of the filtrable forms may not necessarily disprove the possibility of the bacteria to be transmitted by the host germ cells. Nevertheless, as above stated numerous authors believe that the various bacteria involve a virus-like stage, supporting strongly the above concept.

Almost all the human individuals seem to be utilized by tubercle bacillus. However, bacteria, like viruses, may be unable to utilize all 
the host individuals for their rejuvenation. Some individuals peculiarly susceptible to a certain kind of bacteria may be infected and utilized by them to establish the inheritance of their pattern to the offspring of the host, in which bacteria will subsequently develop and the offspring will be infected or become the bacterial carrier.

As in the case of viruses, the susceptibility of the host may vary with the regions where the host resides, leading to the establishment of endemic diseases. Namely, in a certain region there may live animals or man possessing extremely high susceptibility to a certain kind of bacteria, which the latter therefore can continue their existence particularly in such a region. In India cholera is almost always prevalent. The true home of cholera is said to be the delta of the Ganges. It spreads occasionally widely from this area, but in other areas it usually dies out, though it may be reimplanted from time to time. This may be accounted for by the non-existence, in areas other than India, of hosts in which the bacteria can harbour.

In addition to bacteria, a kind of yeasts (Schizosaccharomycetes filtrans) and of protozoa (Trypanosoma Brucei) are known to become filtrable at a period of their life-cycle. Moreover, spirochaetes have been reported to be able to exist in filtrable forms. According to Nicolle (98), spirochaetes go into a filtrable granular stage produced by fragmentation of the adult forms, and in the cycle of spirochaetal development a phase seems to occur in which the organism persists in the form of small granules. He considered that this form is apparently resistant and latent, becoming infective when it regenerates spirochaetes. Syphilis is caused by a kind of spirochaetes, Treporsema pallidum. It is a well known fact that the disease is transmitted from parent to infant; it should be noted that some authors believe that the transmission is established not only by mother but also by father. Numberous viruses have been confirmed to be transmitted through germ cells of the host, but in the majority of cases only egg cells are believed to be involved. Nevertheless, in the case of mammary cancer of mice, which is generally recognized as caused by a virus, it has been well established that males of cancer strains of mice may transfer the agent through the sperm (99) (100). Treponema pallidum may be transmitted in a similar way to the offspring.

As stated already, fungi in the insect are undoubtedly transferred through eggs from generation to generation, whereas many grass smuts are known to be seed-born; that is, the pathogenic fungi are carried by the seeds to progeny (101). The mechanical transport of the fungous spores by seeds may not be an adequate explanation of this fact.

It is worthy of note that in the gut of the termite, protozoa occur 
in so tremendous numbers that the protozoa constitute as much as one third of the weight of the termite (102). The protozoa is definitely benefitable for the termite and without which the insect cannot continue to live, suggesting that the termite harbours the protozoa as a heritable pattern of the gene.

As already referred to, phage is found in tissue cells of various animals, such as chickens, mice, flies, etc. It may not be impossible to regard such phages as the virus stage of bacteria parasitic on the animals. The appearance of such organ phages is under the influence of season as in the case of the virus of measles. Vincent (103) has studied the flora of the small intestine of normal and $X$-radiated rats and found that the coli-form and pathogenic staphylococcus increased markedly following irradiation. This effect of irradiation was observed not only with the bacteria in the intestine but with those in blood. Thus, bacteriaemia of enteric origin has been found to occur in a high percentage of mice following total-body X-irradiation (103a). This may be interpreted as indicating the revelation of the pattern by the irradiation. It should be remembered in this connection that active phage is readily revealed in lysogenic bacteria following ultraviolet irradiation (82).

In order to utilize the host effectively for the purpose of rejuvenation, microorganisms should not exert too great detrimental effect upon the host, and so mostly they may establish a commensal relationship with the host. This may be the case also with plants; it is well known that various non-pathogenic bacteria exist within plants, remaining viable and possibly multiplying, but not producing any visible disease symptoms (104).

The writer's concept discussed so far may seem erratic to some authorities. However, no one can doubt the fact that in our bodies there are normally produced cells named leucocytes which behave like protozoa able to move like amoeba and eat various substances which are injurious to us. Moreover, when a man develops to a certain extent, cells capable of acting as the gametes which can be regarded as an independent unicellular organism are raised in him. It is evident that these agents are not brought about by an infection from outside, although they have each immunological specificity like parasitic microorganisms. No one can deny the opinion that they should be attributed to respective heritable patterns of the gene.

A pathological agent belonging to this category may be cancer. When a man having the predisposition to cancer grows up to a certain age, occasionally a strange cell, termed a cancer cell, is produced which behaves like an independent microorganism, multiplying vigorously at the sacrifice of the normal cells until even the host itself is 
killed. It has actually been known that the mammary cancer of mice is induced by a virus-like agent which can be transmitted from parent to offspring through germ cells; that is, the predisposition to the cancer is inheritable.

The writer is of the firm opinion that at least the majority of the primitive secondary organisms can accomplish their rejuvenation by becoming thus patterns of the gene of their hosts, but it seems reasonable to suppose that they must abandon such means as they advance higher in the course of the evolution. As above considered, primitive secondary organisms such as bacteria, fungi, and perhaps protozoa can apparently invade the germ cells, but when they evolved into higher multicellular organisms, their structure might become too complicated to be reduced into a virus state with the result that they would become unable to make use of the host germ cells. 


\section{CHAPTER IX}

\section{METABOLISM}

\section{Energy Requirement by Extremely Primitive Organisms}

The constant renewal of substances and energy is often believed to be the essential feature of life, and consequently life is sometimes compared to a fountain or a flame, which has a constant shape but whose component is being continuously renewed.

Such a view, however, may be erroneous. Viruses exhibit every principal character of organisms, but there is no proof of metabolism involved in them; all the attempts by a number of workers to demonstrate the metabolism in viruses have been failed. Furthermore, it seems possible to present the evidence that no significant energy may be required even for the multiplication of viruses. Thus, it is generally recognized that viruses can multiply in dead cells. For example, as already stated in Chapter VII, Part II, influenza virus can proliferate in chick embryos killed by the exposure to a low temperature, and phage multiplies in bacteria killed by various agents, such as penicillin, mustard-gas, and ultraviolet ray.

In these cases, killing means to render them non-viable. For the proliferation of cells high metabolism of both substances and energy should be necessary, and accordingly viability may be lost by the destruction of the metabolic systems. The multiplication of viruses in the cells devoid of the reproducing faculty, therefore, may indicate that at least the energy so great as required for the multiplication of cells is not necessary for the rearrangement of protoplasm structure to answer the pattern of the virus. On the one hand, it is known that neither heat is produced nor energy is consumed when a protein is affected by proteolytic enzymes. On the other hand, multiplication of a virus may be looked upon only as the structural change of protoplasm protein by the action of a virus, which can be regarded as a special type of enzymes. Even in this respect it seems also probable that no peculiar energy is required for the multiplication of viruses.

In this connection it should be mentioned that there are numerous facts suggesting that virus multiplication is closely connected with the 
metabolic activity of host cells. For instance, as already described, phage is commonly produced in young, proliferating bacteria. Ackermann (105) was able to confirm that the yield of influenza virus was apparently directly proportional to the residual oxygen consumption of embryonated chick tissues. These facts may only show that young, active cells possess protoplasm which is particularly suited for the virus multiplication.

Ackermann and Johnson (106) found that 2,4-dinitrophenol inhibits completely the propagation of influenza virus in chorioallantoic membrane, while this reagent shows no virucidal effect in vitro. In minced preparations of chorioallantoic membrane the reagent was shown to have a pronounced stimulating effect upon adenosine-triphosphatase, and when this reagent was used with intact tissues, an excellent correlation was found between the inhibition of viral propagation and the stimulus of respiration and release of phosphate. From these facts they concluded that the energy required for viral synthesis was derived from the oxidative phosphorylative activity of the host tissue. However, this conclusion might be somewhat rash, since it can also be considered that the effect of the reagent upon the protoplasm may be unfavourable for the production of the virus pattern though favourable for the enzymatic action. Peculiar influences of a variety of such reagents upon the production of virus pattern will be discussed in detail in the next Part.

On studying the development of phage in bacteria "killed" by ultraviolet or X-ray, Labaw et al. (107) have found that although the phosphorus metabolism of the bacteria is greatly depressed by "killing," it will continue for a short time after the treatment, and it is during this time that phage can multiply; the yield of phage is large even for completely killed cultures, if infection occurs immediately after the treatment, but decreases with the time of incubation of the treated bacteria before infection. This fact, however, may not show that phosphorus metabolism is indispensable for the virus multiplication. It may be more reasonable to interpret this fact as indicating that for the virus multiplication the protoplasm structure could not be so severely damaged as phosphorus metabolism was completely lost.

The writer holds the opinion as discussed already that the primary organisms were generated and evolved by the mechanism by which viruses multiply. If energy is required for this mechanism the theory of the writer cannot be held, because no metabolic system is considered to have been present in the protoplasm-like masses in which the organisms might develop. The fact that no energy appears to be particularly needed for the action of the enzyme must be one of the main reasons for which life could be raised on this globe. 
As aleady stated, the primary organisms might have been generated and evolved by affecting other organisms or protoplasm-like polymerized proteins in rearranging their structure just as viruses multiply in the host cell. However, when such primitive primary organisms or the masses of assimilase evolved to a certait extent, they might have to rearrange the structure of free protein molecules or to synthesize protein molecules from smaller components to incorporate them into their bodies. If they evolved up to such a stage and were capable of synthesizing proteins, considerable quantities of energy would be necessary for the active and incessant structural change of protoplasm protein as well as for this function, and the energy required would be supplied by the decomposition of high molecular substances which might have been either synthesized by utilizing the energy of the sun or taken from other organisms.

Life may be nothing but the manifestation of the function of assimilase. If an assimilase was once generated and had to continue its existence, as a necessary consequence it would evolve and become to have complicated structure until the form and the function of undoubted organisms were obtained. It would be unable to become more fitted unless it was advancing continuously. Thus, assimilase might have to choose between the extinction and the advancement, and if the advancement reached to a certain extent the development of metabolic system would follow as a natural consequence.

In both cases of the primary and the secondary organisms, before they had enough evolved to synthesize proteins from smaller components, they might have to acquire the faculty to rearrange the pattern of free protein molecules, the structure of which was only slightly different from those of the organisms, in order to incorporate the molecules into them. It is well known that primitive secondary organisms, such as the organism of bovine pleuropneumonia, can sometimes multiply outside the host if blood-plasm proteins are added to the medium. Such organisms may be looked upon as having acquired this faculty. In addition, it seems possible that the cells of higher animals existing at present still adopt this easy way of assimilation. Thus it has been claimed that dogs can be kept in health when the only source of nitrogen is blood-plasm injected in their veins (108). The animal must, therefore, be able to form any required protein from the protein present in the plasm without preliminary hydrolysis by the enzymes of the digestive tract. Only a little energy, if any, may be required for such an easy assimilation procedure, in which are utilized complete protein molecules that have the structure not greatly different from that of the protein composing the assimilase itself. 
However, if the protein which is to be utilized has the structure entirely dissimilar to the pattern of the assimilase or the protoplasm of the organisms, incorporation following the sole rearrangement may be impossible; preliminary decomposition appears to be needed in such a case. Presumably reconstruction of a protein from decomposed components may be accomplished with more ease than the rearrangement of the pattern without decomposition; the rearrangement may be impossible because the protein would be disturbed in its structure so profoundly on the adsorption onto the protoplasm that the decomposition would take place before the occurrence of the rearrangement, as the pattern of the protein differs so greatly from that of the protoplasm.

Such a protein in its turn may exert some disturbing influence upon the protoplasm itself because of its foreign structure. This may be the reason why foreign proteins are generally toxic. The rapid decomposition of foreign proteins, therefore, may be needed even for the elimination of their toxic effect. Moreover, decomposed components would be favourable owing to their small molecular state to be transported to another part where the protein synthesis occurs. The function of the reconstruction of specific proteins from foreign ones through the decomposition appears to have already been developed in relative primitive organisms such as protozoa, since in a certain group of protozoa, for example in paramecium, primitive digestive system can be found.

A considerable attention seems to be paid on the fact that for the incorporation in vivo and in vitro of any one amino acid into protein, other amino acids are necessary (109). For instance, it has been conclusively shown that the essential amino acids are not able to sustain growth or even nitrogen balance in an animal when they are fed at intervals, whereas they are effective when fed simultaneously (110). This may be expected if each amino acid is to be adsorbed to the respective pattern of the protoplasm to form a complete peptide chain specific to the organisms.

It has frequently been suggested that the most primevai organisms might utilize the sunlight energy by chlorophyll. The writer claims, however, that organisms never acquired the faculty to yield chlorophyll until they evolved up to a considerably high stage. It is entirely inconceivable that primeval organism could synthesize anenzyme so highly developed as chlorophyll. Nevertheless, it may be possible that primeval organisms could synthesize proteins from carbon dioxide and ammonia or other $\mathrm{N}$-compounds by making use of the sun energy, even long before they could yield chlorophyll, but because of the non-development of the perfect synthesizing system, the syn- 
thesis of the protein might occur extremely slowly, so that there would be no significant flow of energy. In this connection, it should be realized that bacteria can, in general, make use of carbon dioxide for their growth regardless of their lack of chlorophyll.

Even before proteins which were synthesized in the primitive ocean could form polymerization product capable of acting as typical assimilase, carbon dioxide and some $\mathrm{N}$-compounds like ammonia might be condensed into protein molecules or amino acids by the sun energy. The first proteins might be synthesized in this way, but the primeval organisms, entirely disregarding such a synthesizing process, would utilize the preexisted proteins, which had been already produced by such a tedious way, just as the viruses utilize the protoplasm proteins; however, as they evolved higher and higher and the protein structure became complicated and advanced, the synthesyzing mechanism being gradually arranged until highly differentiated enzyme system, such as chlorophyll, were developed with the manifestation of evident flow of the energy and substances.

The organisms appearing to be primitive secondary ones, such as lower fungi and some protozoa, can produce chlorophyll to perform free-living despite their extreme primitiveness. This may be explained by assuming that these organisms have evolved from viruses which were parasitic on higher plants containing chlorophyll, succeeding to the highly developed enzyme system of the host, that is, chlorophyll, so that they might be able to accomplish free-living in utilizing the sun energy by means of chlorophyll despite of their most primitive nature. Some bacteria also can live independently of their host, suggesting that they might likewise obtain the enzyme system to utilize carbon dioxide by transfer from the host.

\section{Nucleic Acid as Energy Donor}

The incorporation of various amino acids into protein may require considerable amounts of energy, the majority of which may be produced by glycolysis whereby sugar is decomposed with the production of energy. The energy, however, is apparently not given directly in the form of heat, but it is preserved in the so-called energy-rich phosphate bonds which will be decomposed in case of necessary to liberate the energy. Most available energy-rich phosphate bonds are believed to be involved in adenosine triphosphate (ATP) which is one of the components of nucleic acids.

This may be regarded as one of the reasons why the cell proliferation is always connected with the increase in nucleic acid. The 
active proliferation of cells should be associated with the vigorous synthesis of proteins. Spiegelman and Kamen (111) have suggested that nucleoproteins are specific energy donors which make reactions possible leading to protein and enzyme synthesis. They have found that when protein synthesis is occurring in the presence of nucleoprotein containing $\mathrm{P}^{33}$, this latter element is liberated from the protein, while on the interruption of the synthesis by the addition of sodium azide or dinitrophenol the liberation of $\mathrm{P}^{32}$ is ceased, indicating the occurrence of the decomposition of nucleic acids solely in case of energy requirement. In addition, many facts are known suggesting that a certain correlation exists between the synthesis of proteins, particularly fibrous proteins, and the occurrence of phosphatase and nucleic acids both in the cytoplasm and in the nucleus (112). Nucleic acid in the cytoplasmic granules is considered to be involved in protein synthesis, and it is known from the work of Brachet and his colleagues (113) that these granules contain phosphatase. The tissue in which protein synthesis appears to be most active is also particularly rich in nuclear phosphatase.

On the other hand, numerous synthetic reactions driven by an influence of energy-rich phosphate bonds are known. Peptide formation, the synthesis of urea, transmethylation from methionine, the synthesis of $\alpha$ - and $\beta$-keto acids of fatty acids, and the process of bioluminescence appears to belong to this group (114). In most cases of such reactions ATP is apparently involved; and there are many evidences that ribonucleic acid is intimately connected with protein synthesis (29).

The synthesis of proteins can be regarded as the growth of protoplasm, a mixed crystal. Foreign proteins or various amino acids are adsorbed to the surface of the crystal or the protoplasm, and after the establishment of the rearrangement of amino acids or of polar groups in the protein molecules to answer the template pattern of the protoplasm, they may be incorporated into the crystal. Such must be the synthesis of proteins, whereby the growth of the protoplasm is achieved.

It has been already stated in Part II, Chapter VIII, that nucleic acids possess the faculty to strengthen the pattern of the protoplasm. Haurowitz (26) claimed that the role of the nucleic acids is apparently to maintain the template protein film in the expanded state, a state in which proteins do not remain unless exposed to the stress of interface forces. In addition to such an expanding action, if any, nucleic acids may have the hardening action by which the structure of the polymerization product of proteins is made firm.

Thus, the writer has come to possess the opinion that the role of 
nucleic acids in the protoplasm is firstly to strengthen its template pattern and secondarily to provide the energy for the protein synthesis.

As is well recognized, APT is involved in the energy metabolism in the contraction of muscles. The muscle contraction, on the other hand, is believed to be caused by expanding and contracting of the component proteins, mainly a protein called actin. According to SzentGyörgyi (115) ATP acts to expand the muscle protein and in case of contraction ATP undergoes decomposition, yielding inorganic phosphate and energy. Munch-Petersen (116) likewise showed that ATP expands monolayers of myosin, another chief muscle protein. Mommaerts (117) claimed that ATP is essential for the polymerization of actin. Polymerized actin can be separated from accompanying protein by ultracentrifugation. The separated actin, after dissolution and depolymerization in water, does not polymerize again upon addition of salt, unless ATP is present during the entire depolymerization process. He has further reported that in a pure system a stoichiometric reaction takes place between actin and ATP in which 1 mole of globular actin reacts with 1 mole of ATP, yielding actin in the polymerized form with the liberation of energy and inorganic phosphate (118). These evidences clearly indicate that ATP, the chief component of nucleic acid, has the function to maintain the protein molecules in a definite form while it can provide energy on the decomposition. The nucleic acid in the protoplasm may behave in a similar way.

Spicer and Rozsa (119) have found in an electron microscopic study that actomyosin tends to form fibrous aggregates in the presence of ATP. At a lower pH the tendency to form fibrous structures appears even in the absence of ATP, and needle-like or spindle-shaped aggregates are to be seen, but this tendency is intensified by the presence of ATP, showing that ATP contributes to the stretch of the actomyosin molecules.

There are two kinds of nucleic acids, $i$. $e_{\text {., desoxyribonucleic acid }}$ (DNA) and ribonucleic acid (RNA), but it seems probable that only the latter is used as the energy donor, as it is confirmed that the nucleic acid which increases in case of active protein synthesis is RNA, not DNA.

Histological and chemical studies on the distribution of RNA in different tissues and microspectrophotometric researches have led to the recognition of a constant coincidence in space and time of a high content of RNA and rapid protein formation. When protein formation or growth is taking place the RNA content is high, but when the same cells are not producing proteins, there is less RNA (29).

Davidson and Raymond (112) fed labelled ammonium citrate to 
pigions and rats, and found appreciable amount of $\mathrm{N}^{15}$ in the RNA, subsequently isolated from the livers, but only negligible amount in the DNA. It has thus been generally ascertained that at least RNA is metabolically much active than DNA. Griffin et al. (120) have even concluded that DNA is entirely inert.

The nucleic acid existing in the gene which always requires the most strong pattern appears to be DNA, whereas as pointed out already DNA is much more stable than RNA. It may be said, therefore, that DNA is fit for the establishment of strong pattern but unfit for the action as an energy donor for which some brittleness should be required. On the contrary, RNA is suitable for the purpose of providing energy owing to its brittleness although inferior to DNA in the action of pattern formation, so that while DNA is present in nucleus to provide the strong pattern, RNA exists in cytoplasm to deliver the pattern directed by the gene to proteins to be synthesized and also to provide the energy required for the synthesis. Schönheimer (121) has shown that proteins are continuously being synthesized and hydrolysed so that it is quite possible that part of the energy for synthesis of proteins is obtained from the hydrolysis of other proteins or peptides. Such proteins may possibly be nucleoproteins containing RNA.

As already considered, in case of protein synthesis, component amino acids are to be adsorbed onto the template, which is probably involved in cytoplasmic particles containing RNA in rich amount, in adapting themselves to the specific pattern of the template, but since they have both amino and carboxylic groups in a free state prior to condensation into polypeptide chain, they may exert a disturbing effect upon the template. This disturbing effect may lead to the partial decomposition of RNA with the liberation of the high energy phosphate bond. Meanwhile, amino acids adsorbed may also be disturbed in their structure and as a result they are activated to be readily united into a polypeptide chain.

It has been shown by using various isotopes that proteins composing animal tissues are constantly being renewed in a considerable speed. For example, liver tissue rapidly takes up amino acids following the injection or feeding, and the time for half replacement of the liver protein in the rat has been estimated to be about 7 days (122). Such a rapid renewal of tissue protein may partially be dependent upon the decomposition of the nucleoprotein as above mentioned, but there may be another important reason for the renewal. Since all the life phenomena involve the structural alteration of the protoplasm, the pattern of the protoplasm may be more or less deformed during the life process. As was mentioned earlier, the pattern thus deformed 
will be readjusted by the gene during mitosis, but it seems possible that part of the protoplasm or the proteins may be deformed too far to be repaired; such proteins must be expelled from the cell. This may be the other reason for the rapid exchange of the protein, which is known especilly manifest in tissues of active function.

The infection with viruses generally results in the nucleic acid increase in the cell as referred to already. Similar increase appears to be brought about by proper chemical or physical stimuli, followed by the active synthesis of proteins. It is considered that an adequate stimulus applied to protoplasm may enable the protein to acquire an active state leading to the temporary liberation of polar groups, as may occur in general in the protein denaturation; as a result capacity of combining with nucleic acids or the function of synthesizing nucleic acids may be enhanced. The enhancement in the action of phosphatase is apparently associated with the increase in the amount of nucleic acids (112), suggesting that in the active state of protoplasm the polar groups acting as phosphatase are also liberated. Phospha. tase activity thus enhanced may be favourable for the liberation of energy.

As mentioned previously, it seems necessary to hold constant in the protoplasm the amount ratio of nucleic acid to protein for the smooth establishment of life processes. If so, the increase in the protein amount will follow the increase in nucleic acid content in order to maintain the ratio contant. There is ample evidence that nucleic acid accumulation does occur before protein synthesis (29). In short, the increase in the amount of nucleic acid, which may be caused by the temporary liberation of polar groups in protplasm proteins following a stimulus, may lead to the enhancement of the template effect resulting in the active synthesis of protein, whereby the constant ratio of nucleic acid to protein is recovered, and thus the cells or the organism may be able to hold the state favourable for the continuance of their existence.

There seems no doubt that the constant ratio of nucleic acids to proteins is indispensable for organism. Therefore the mechanism to keep the ratio as constant as possible should be highly developed in organisms; and those failed to obtain the mechanism would surely become extinct.

There appears no particular reason why mainly RNA is increased on the application of certain stimuli which may activate the protoplasm structure, but for the rapid synthesis of protein which alleviates the unbalance in the ratio of nucleic acid to protein, RNA must be more adequate than DNA. Thus the accumulation of RNA would be favourable for the organisms if the organisms were brought under 
some conditions which might act as a stimulus to activate the protoplasm to accumulate or synthesize nucleic acid, and under which the vigorous multiplication was desirable for the organism. Therefore, the organisms which could accumulate RNA under such conditions might become fitter and left as such.

As is well known, the nucleic acid which will accumulate in bacterial cell after the infection with phage is DNA; the RNA content of the host remaining constant. This may result in the relatively long persistence of the unbalance of the ratio, and at the same time this should be favourable for the liberated protoplasm particles to act as the virus, if not for the bacteria themselves to act as living organisms. This property of bacteria to accumulate DNA on the virus infection might contribute not only to the virus multiplication but also to the evolution of the bacteria themselves, because the property to make the virus pattern rigid is to make the superior pattern of others spread easily.

\section{Dimensions of the Mass of Assimilase with Special Reference to Protein Synthesis}

The chemical analysis of the cytoplasmic particles, such as microsomes and mitochondria, shows that they contain ribonucleic acid in rich amount, and it is evident that these particles account for a very large proportion of cytoplasmic ribonucleic acid. It has been suggested that protein synthesis is mainly connected with such particles, which are therefore recognized as the fundamental units of living organisms having the power of selfduplication (8) (26).

It is evident, however, that each separate particle has no faculty of synthesizing protein and accordingly not selfduplicating, because as is generally accepted viruses cannot multiply outside the living cells. Nor any one has succeeded in making the cytoplasmic particles isolated from the cell multiply in vitro. Even the ability for incorporation of protein molecules is lacking in viruses, an ability which is not attained until they reach the stage of Rickettsiae. As already mentioned, a certain type of Rickettsiae can multiply outside the host if blood plasm is added to the media.

This ability seems intimately related to the dimension of the mass of assimilase or of the microorganisms, for as discussed in Part I assimilase action is, to some extent, a function of the size of the assimilase or the degree of polymerization of proteins. For the function of synthesizing the protein from amino acids may be needed still greater dimensions. Rickettsiae, pleuropneumonia-organisms, and re- 
lated primitive organisms can sometimes be cultivated in media containing blood plasm, but never in media containing merely amino acids or other simple protein components. The cultivation can only be achieved when the organisms or assimilase masses acquire the dimension of bacteria.

Liver slices are able to incorporate amino acids in vitro, but the ability is remarkably reduced by the mechanical grinding. According to Winnick (123) rat liver slices are 4 to 41 times as active as the homogenate in the uptake of methionine and 3 times as active in the uptake of glycine, indicating that synthetic ability is markedly reduced by the decomposition of the cell mass. In recent years numerous reports on the uptake of labelled amino acids not only by tissue slices but also homogenates have appeared (124), and considerable evidence has accumulated indicating that this uptake of amino acids represents a synthesis of peptide bonds (125).

It was found by Brunish and Luck (126) that the protein fraction, containing nucleic acids, in a liver homogenate, can incorporate the greatest amount of phenylalanine. On studying the uptake of radioactive alanine in vitro into the proteins of rat liver homogenate, Siekevitz (127) concluded that when the alanine was incubated with the homogenate, the highest specific activity in the homogenate protein was found in the microsome fraction. Significant incorporation did not occur when either isolated microsomes or mitochondria were incubated with radioactive alanine, but when mitochondrial and microsomal fractions were incubated together in the presence of the alanine incorporation occurred.

This finding suggests that the dimension of the assimilase is important not only for the reason that the dimension may determine the polymerization degree but also for the reason that the great dimension may provide the space for the association of a number of particles having a variety of properties, because it is conceivable that, in order to secure the energy supply for the synthesis of proteins, that is, for the uptake of amino acids, a variety of particles may be needed to cooperate for the common purpose, whereby each of the particles may play each specific part. A single virus-like particle may thus be unable to synthesize the protein even if the size of the particle is considerably great; only when numerous different elementary-bodies are united, protein synthesis becomes possible.

In the recent work on the incorporation of labelled amino acid into proteins in cell-free systems, Zamecknik and Keller (127 a) have shown that, in addition to a microsome-rich fraction, a soluble non-dialysable fraction and also an ATP generating system are necessary for the process. Thus it has been clearly indicated that a single virus-like 
particle cannot incorporate amino acid, but that it can only be achieved in the presence of complete energy supplying systems.

Since each type of protein molecules may be able to act as each type of enzyme systems as already suggested, such cooperation of a variety of particles for a common purpose may mean the establishment of an enzyme system for the protein synthesis. Rickettsiae are composed of a number of virus-like particles or elementary bodies, which may be different from each other in their physical and chemical properties, and hence the enzyme system may start to function and thus a certain type of Rickettsiae can proliferate outside the host.

The fact that some bacteria can recover their complete form after being decomposed into virus-like particles, may appear to show that each particle shares the faculty of synthesizing proteins. Yet, this seems not to be the case. Separated single particle cannot grow as above considered. In order to multiply, decomposed particles having various properties may have to unite with one another into larger masses; only in this way they may be able to accomplish the synthesis.

It has already been stated that virus-like protoplasm particles can be fused in vitro into cell-like masses. This may be looked upon as a restitution of the original cell mass. A remarkable example of the restitution of body form and function has been found with sponges and hydroids (66). These organisms were cut into small fragments and then pressed through the meshes of fine bolting cloth. In this way the flesh was broken up into very small fragments. Shortly after the operation many of the isolated elements were observed to have fused together into lumps or sheets of tissue. Soon larval stages arose very similar to the typical normal larva. Ultimately new complete adults developed.

Such a reversible decomposition of cells or organisms is probably based upon the general reversibility of protein structure. As repeatedly emphasized, the reversibility involved in the nature of protein is of the utmost importance in developing the various reversible characters in living substances. This reversible decomposition may be only one of these examples. It was found that haemocyanin, the coppercontaining respiratory chromoprotein of invertebrates, is split into subunits of one half or one eighth of the original molecular weight by exposure of the protein solution to $\mathrm{pH} 8.5$, and that reassociation by exposure of the protein solution to $\mathrm{pH} 8.5$, and that reassociation at $\mathrm{pH} 6.85$ to form the original units occurs quite specifically. Fragments of haemocyanin from Helix pomatia recombine with fragments of the same haemocyanin, but not with fragments derived from the haemocyanin of Littorina littorea (128). Various evidences of such 
reversibile decomposition have been shown with tobacco mosaic virus protein as already referred to.

The protein molecules of the same type tend to combine with one another. The protoplasm or the organism is produced by this property of protein, and the protein can reform organism even after the decomposition into the protein. However, since the protoplasm is a mixed crystal, the component or proteins are not necessarily of the same structure. Proteins having different structures can form one and the same protoplasm if the arrangement of their polar groups can be subjected to the original pattern of the protoplasm, and thus they may exhibit various functions according to their various chemical structures to accomplish a common purpose.

Although the primitive primary organisms were presumably provided from the beginning with the size and shape of ordinary cells of the present time, they would only be able to affect, like viruses, other weaker organisms to transfer their template pattern to the weaker. The faculty of protein synthesis might be obtained after an immense span of time following their generation, as a result of the proper differentiation of particles composing their body. Of course, individuals composed of particles thus differentiated might be much more fitted for the continued existence than those having undifferentiated particles, and so the differentiation of the particles or proteins and accordingly of the enzymes would be more and more developed.

The differentiation in the chemical structure, on the other hand, would result in the differentiation in the physical structure of the mass of assimilase or the organisms, since protein molecules or elementary bodies of the related chemical structure would preferably combine with one another, and thus various form-elements such as nucleus and cytoplasmic granules would be produced. As was already stated, even the artificial cells prepared by the writer from plant materials occasionally possess a variety of granules some of which has the nucleus-like appearance. It can be demonstrated that such granules stain particularly well when treated with a proper dye such as fuchsin, showing that they are the aggregates of proteins with related chemical structures different from those of other parts.

Thus, in the ordinary cells of the present day organisms, elementary bodies containing DNA may agglutinate into a body termed a nucleus, and those containing RNA may form various sized bodies, $i$. e. cytoplasmic particles such as microsomes and mitochondria, according to the different characters of the elementary bodies. Chantrenne (129) emphasized that the separation of the two fractions $i$. $e$. microsomes and mitochondria, might be arbitrary, and suggested 
that there might actually exist a more or less continuous spectrum of size and chemical composition. Indeed, there are reports that the microsomes and mitochondria may be separated into biochemically different fractions. Novikoff et. al (130) have separated the cytoplasmic particles of rat liver homogenates into as many as eight fractions by differential centrifugation, and have found evidence of biochemical heterogeneity among the isolated particles. 


\section{CHAPTER $\mathrm{X}$}

\section{THE CHANGE OF PROTOPLASM STRUCTURE AND THE REVELATION OF LIFE PHENOMENA}

\section{The Oscillation of Protoplasm Structure}

In studying the inactivating progress of phage or of rennin by the addition of inactivating agents such as antibody or tannin, we have found that the inactivation occasionally proceed in an extremely irregular way (131). In Fig. 26 is shown an example of an irregular

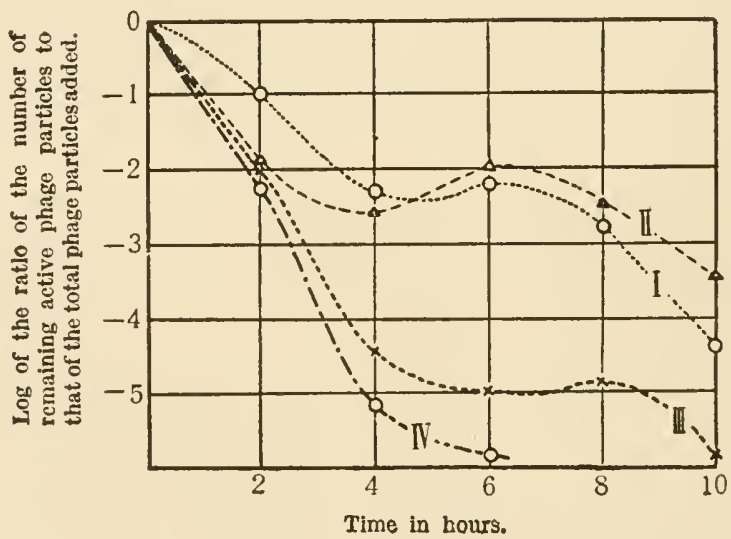

Fig. 26. The progress of inactivation of phage protein due to formaldehyde at pII 4,6. Formaldehyde concentrations : $0,022 \%$ (I), $0,044 \%$ (II), $0,088 \%$ (III), $0,175 \%$ (IV). - Concentration of phage protein (sample No. 3): $0,05 \%$ - Laboratorary temp.

progress of phage inactivation in a weakly acid solution by formaldehyde, and in Fig. 27 a similar irregular inactivation progress of rennin by tannin.

In these examples, it is clearly shown that the inactivation proceeds with remarkable oscillations as if it were a physical phenomenon, 
though such a phenomenon was rather rarely observed, in most cases only regular progresses being seen.

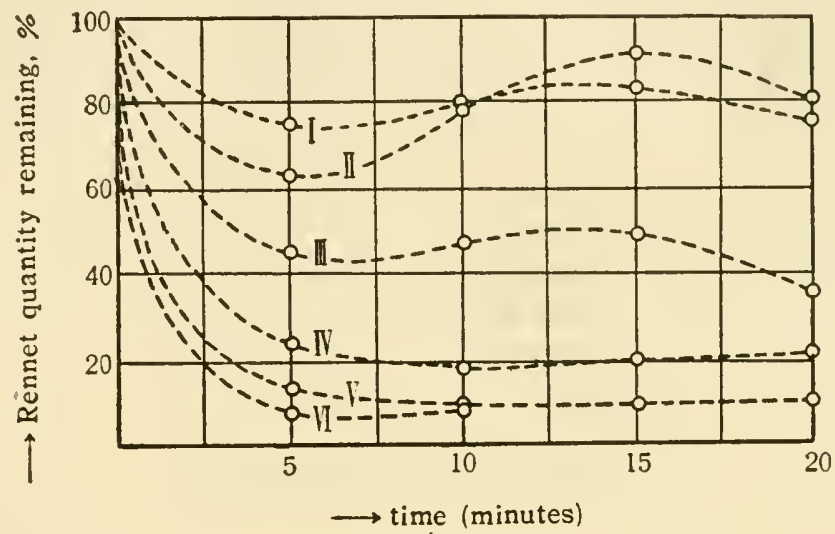

Fig. 27.

Decreasing progress of the rennet action due to tannic acid.

Tannic acid: Kahlbaum; used a month after its dissolving. Rennet solution $(0.01 \%), 1 \mathrm{cc}+$ tannic acid solution, $1 \mathrm{cc}+$ milk $\left(1 \% \mathrm{CaCl}_{2}\right) 1 \mathrm{cc}$.

Tannic acid concentrations :
I. $0.003125 \%$
II. $0.00625 \%$
III. $0.0125 \%$
IV. $0.025 \%$
V. $0.05 \%$
VI. $0.1 \%$

Even when freed from protoplasm, proteins of the same origin exhibit the property to combine mutually. On account of this property, as pointed out already, structural change of a protein molecule is likely to spread to other molecules existing in the same system. It is probable that such an oscillation is also brought about by a loose combination of protein molecules in a solution. If loose combination exists between protein molecules, each molecule may be prevented from behaving of its own accord, and accordingly all the molecules present in a solution may have to act as a single system. On the other hand, as stated repeatedly, since protein molecules possess the structural reversibility, phage or rennin inactivated to a certain extent may be striving to resume its original active structure in resisting the inactivating action of the added substance. These two opposite actions may cause the oscillation.

The writer has been able to prove that the turbidity produced in a solution of ovalbumin by the addition of tannin shows also an oscillation in the progress of time following the addition (132). It has also been found that the turbidity of a protein solution oscillates with the change of the added amount of sodium chloride (133). 
Such oscillation phenomena would not be revealed if each molecule changed its structure arbitrarily of its own accord and the mutual connection failed to prevent the arbitrary behaviour. Since, in the protoplasm, the component proteins are mutually associated in most orderly way, the oscillation phenomena must be much more manifest than in a mere solution of proteins. Indeed, this seems actually the case. Thus, oscillation or rhythmic contractibility appears to be an essential property of all forms of protoplasm as in heart muscle, intestine, the diaphragma, and in many unicellular organisms such as leucocytes, swimming protozoa, and myxomycetes. Its finest demonstration may be seen in the oscillating motion of cilia and flagella.

\section{Resorption and Excretion}

The writer has found a series of facts with which vital phenomena of resorption and excretion appear to be closely connected (134). In this section a discussion will be made on experimental results concerning the facts carried out with sugar and virus particles.

A heavy water suspension of vaccinia virus particles isolated by our isoelectric-point precipitating method is mixed with the same volume of sugar solution (glucose and fructose) of varying concentrations, and after left for 30 minutes at laboratory temperature the particles are precipitated by centrifugation. With the supernatant fluid the sugar concentration is measured, and at the same time a similar manipulation is carried out with the sugar solution mixed with the same volume of water instead of the virus suspension, and then from the difference between these two measured concentrations the rate of the penetration of the sugar into the particles is estimated.

There seems to exist in the particles a space into which sugar fails to penetrate, and this space is designated as sugar-insoluble space. Relations between such spaces and the sugar concentrations are shown in Fig. 28. Usually curves like GI or FI shown in this Fig. are obtained when virus particles isolated by an ordinary way are used, showing that both glucose and fructose can penetrate, to some extent, into the particles when their concentrations are high, the rate of penetration being directly proportional to the concentrations. As the water quantity combined with dried virus particles may be about 10 times as great as the latter, the sugar-insoluble space will be about 10 when no penetration occurs, and hence values smaller than 10 may indicate the occurrence of the penetration of sugars in 
the hydrated particles.

It seems natural that sugars can penetrate when their concentrations are high as shown in curves GI and FI; but very strange results are obtained if virus particles are used to which peculiar manipulations were preliminarily added. Ordinary curves such as GI

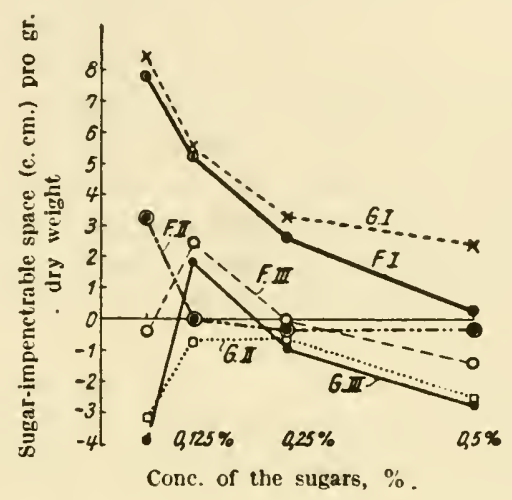

Fig. 28.

Sugar-impenetrable space of vaccinia particles. $G=$ glucose, $F=$ fructose.

Roman numerals indicate the No. of the preparations.

and FI are secured when virus particles are used which were prepared by merely washing with the water of a weakly acid $\mathrm{pH}$ without neutralizing the acid after each precipitation. However, when the experiments are carried out with the particles "purified" by repeated precipitations with acetic acid, which is neutralized by $\mathrm{NaOH}$ after each precipitation and centrifugation, the rate of penetration of sugars is not always proportional to the concentration as shown in many curves in the figure. Similar irregular results are yielded when the particles are preliminarily heated to an adequate temperature.

A mention will be made as regards the curve GIII in the figure, which shows that when the sugar concentration is $C .06$ per cent the sugar-soluble space takes a negative value, indicating that the sugar concentration in the particles is higher than that of the outer medium. whereas at the concentration of 0.125 per cent this is not the case. Thus, the sugar is absorbed when the particles are present in the sugar solution of a low concentration but excreted in that of a higher concentration. Similar results are obtained with both glucose and fructose, but not with lactose. This may account for the non-absorbability of the disaccharide. 
Such a phenomenon is not confined to virus particles, but is generally recognized with protoplasm particles without concerning virus activity. Even with coagulated casein particles similar results are obtained. In addition, not only with sugars but also with various inorganic salts related phenomena are demonstrated.

In view of these facts, it may be concluded that the absorption of a substance by a cell may be raised by a physicochemical combination of the substance with the protoplasm protein of the cell. The combination may be called adsorption, which may be established according to the structure of the protoplasm protein, so that absorption and excretion may take place in response to the change of the structure. On the other hand, as the protoplasm proteins are arranged so as to easily achieve structural changes, the absorption and excretion of a certain substance, if occurred in this manner, could be accomplished with ease.

The writer (131) once expressed the opinion that the oscillation phenomena referred to in the preceding section might be accounted for by such absorption and excretion of the virus inactivating agents in question, but it must be more reasonable to consider that this phenomenon of absorption and excretion is rather based upon the oscillation of protein structure; that is to say, the structure of the protoplasm particles, including virus particles, used in the experiments were presumably highly disturbed by the preliminary treatment, and the disturbed structure would be striving to resume its original state in an oscillating way during the experiments, taking a certain structure at a period and another structure at another, so that even so slight a manipulation as the addition of sugar or other substances might be sufficient to effect the phase of the oscillation according to the concentration, and in conformity with the change in the structure absorption or excretion might take place.

In any case, it may be said that a certain substance is adsorbed by, or eluted from, a protein, if the structure of the portein is properly changed. Absorption and excretion of substances by a living cell must be attributed to such adsorption and elution.

Being consistent with this concept, it has been found by klotz et al. (135) that there are structural specificities in the interactions of some organic ions with serum albumin. Human albumin undergoes major configurational changes with changes in $\mathrm{pH}$, making new sites available for interaction with ions of specific structural properties. Thus, it is obvious that albumin can absorb and excrete some organic substances when $\mathrm{pH}$ of the solution is changed. Moreover, it has been shown that human serum albumin combines preferentially with chloride ion in sodium chloride solutions, even when the net charge 
on the protein would be expected to favour the absorption of the sodium cation. Modification of the albumin by treatments which weaken the basic groups generally decreases the adsorbing properties, and it is generally accepted that the preference which albumin shows for anions is connected with the activities of the lysine and arginine residues, both of which possess single positive charges at physiological pH (136).

In the customary consideration of the absorption mechanism the existence of the so-called semipermeable membrane seems to be essential. The membrane is believed by some workers to be present even in viruses. For example, sedimentation of virus particles in solu tions of low molecular weight, such as sucrose, sodium chloride, and glycerol, has revealed a dependence of sedimentation rate on solute concentration, and further the rate was found to vary with time of contact with the solute. These facts seem to be often interpreted as giving evidences of the existence of a semipermeable membrane susceptible to osmotic effects of solutes of low molecular weight (137). However, these facts should be accounted for by the change in the rate of "absorption" responding to the concentration of the substances to be absorbed and responding to the time of contact. Membrane-like images shown in electron micrographs of some viruses may, as already pointed out, be interpreted as only an artificial product produced by the surface-protein molecules fused into a membrane-like aggregate through the heat of electron bombardment.

Bergold and Wellington (138) claimed that they could isolate from certain insect-virus particles particulate protein complex without DNA, which they believed to be the membrane of the virus. However, it is possible that this "membrane" may be the particles, which contain no DNA, contaminating the "virus particles" that contain a large amount of DNA. Insect virus particles were separated from inclusion bodies which had been formed in the insect after the virus infection. According to Bergold (139) the inclusion bodies consisted of about 95 per cent of a homogeneous protein and about 3-5 per cent of virus particles; these virus particles which contained DNA in rich amount could be liberated by dissolution of the inclusion bodies in weak alkali and separated from other component proteins by centrifugation. This fact suggests that the "virus particles" were the DNA rich elementary bodies which could withstand the alkali treatment owing to their high contents of DNA; the other component bodies might be decomposed and dissolved in the alkali so that virus activity might be left only in the undissolved particles containing DNA. It should be noted that the "membrane" was reported to dissolve in alkali like the main component protein of the inclusion body, suggesting thus strongly that 
it might be the main component itself.

To sum up, in order to elucidate the phenomenon of absorption and excretion the assumption of a semipermeable membrane seems not only unnecessary, but it is obvious that even if such a membrane could exist, the phenomenon would not be explained by the membrane.

If absorption took place always through the semi-permeable membrane, any penetration of macromolecular substances into cells would be impossible. However, this seems the case. Thus, according to Coons et al. (140) various protein injected intravenously into mice were clearly demonstrated in the unaltered form in the nuclei of certain normal cells, often even in higher concentrations than in the cytoplasm. If proteins were adsorbed by nuclei, they would naturally be found in the nuclei. The specific pattern of the protoplasm is expected to be strongest in the nucleus, and so the proteins, if the adsorptive force is involved in the specific pattern, will be most strikingly adsorbed by the nucleus. Again, there are evidences, as mentioned already (Part II, Chapter VII) that virus nucleic acid or its components can readily penetrate into the host cells. In this case, the specific pattern of the host protoplasm must involve the specific adsorptive force directing to the virus, and the pattern may be most powerful in the nucleus or in some cytoplasmic particles. Hence, the penetration into the cell of the nucleic acid in which the specific structure of the virus is retained should naturally follow.

Anderson (141) found that phage could be inactivated by suspending the particles in high concentrations of sodium chloride, and rapidly diluting the suspension with water. The inactivated phage was visible in electron micrographs as tadpole-shaped "ghosts." Since no inactivation occurred if the dilution was slow, he attributed the inactivation to "osmotic shock" and inferred that the particles possessed a semipermeable membrane. However, this phenomenon should be interpreted as based upon a structural derangement of phage protein due to the rapid release of adsorbed ions. As a result of this derangement, the association between nucleic acid and protein may be destroyed, as it is reported that DNA is released by this procedure (142). It is a noteworthy fact that the ghost could adsorb to bacteria and lyse them, though it cannot act as the active virus.

\section{The Movement of Organisms}

Protein molecules in protoplasm are mutually associated and orderly arranged, and can readily change their structure as a single system 
under the influence of various effects. It may only be natural, therefore, that such a change is sometimes revealed as the movement of organisms.

Amoeboid movement is said to be established by the sol-gel change of the protoplasm (143), thereby amoeba can move in pulsatory motion (144). If we assume that the protoplasm protein in amoeba are usually in a state somewhat contracted and that both the extension and contraction of the protein threads tend to occur too far, not exactly responding to the degree of the stimuli causing the change, the amoeboid movement can be easily explained. Thus, when a stimulus which may cause the extension of the protein threads is given at a site of the protozoan, the protein molecules at the site will be extended with the projection of the protoplasm, but as this extension may go too far, a compensatory contraction will follow. This contraction likewise may occur excessively, spreading to other proteins, with the successive coagulation of protoplasm into elementary particles; combining force between the proteins may then be increased and the coagulated elementary bodies will be attracted and drifted towards the site of the stimulus. If the stimulus extending the protein continues to be applied to the same site, the protein in the coagulated particles, thus attracted to the site, will be extended, and the protoplasm will be projected, followed by the contraction of the protein and subsequently by the gathering of coagulated particles.

On the contrary, if a stimulus causing a contraction is applied, the protein molecules at the site of the stimulus may undergo contraction, which may spread to the opposite side, where no contracting stimulus is present, so that soon the extension will follow at this side, with the projection of protoplasm. But this extension may be temporary if contracting stimulus continues to exist, so that contration may follow which will attract the coagulated particles to the side where no stimulus exists, as a result the protozoan can move to escape the stimulus as shown in Fig. 29.

If the protoplasm protein is stretched by the benevolent stimulus and contracted by the detrimental one, the movement will be said purposeful and the organisms having the protein of such a property can continue existence and well prosper. Since intense and unusual, physical or chemical stimuli generally cause the protein to coagulate or to contract, it should naturally follow that organisms will achieve the movement to get rid of such stimuli. However, occasionally the same stimulus may give rise to the different reactions among varying organisms, indicating that some stimulus is favourable for some organisms but unfavourable for others. The property to achieve such a purposeful movement must have been raised because only the 


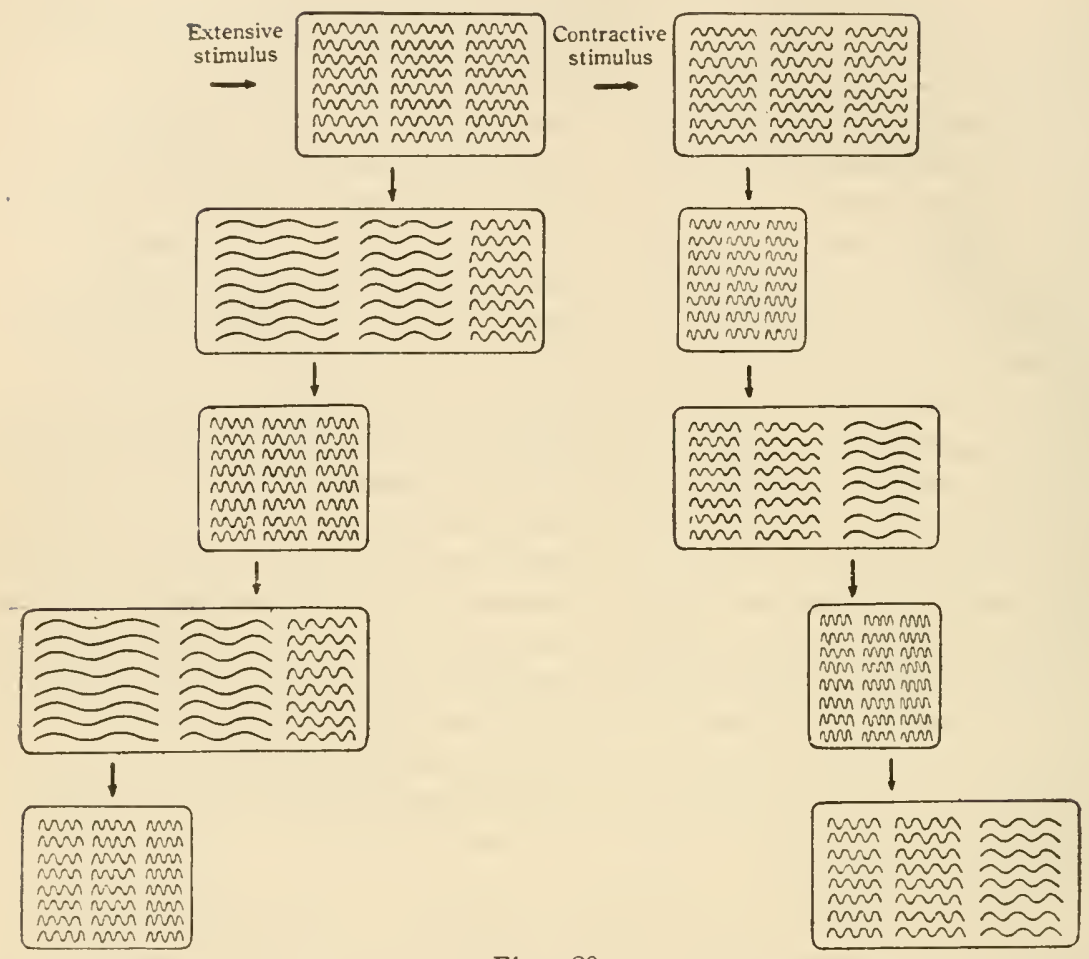

Fig. 29

Diagram of amoeboid movement.

organisms which would move purposefully were able to continue their existence.

The property of protoplasm protein to contract and expand excessively not precisely responding to the degree of stimulus must be required for the movement, such as amoeboid one and oscillation of cilia or flagella, and organisms which need movement must have the protein having such a property. If movement was favourable for certain organisms, this property would be developed in them, since the more developed the property, the organisms would become the more fitted and the more easily win the struggle for existence.

Monné (145) claimed that folding and unfolding of polypeptide chain seem to be the essential features of the protoplasm, and he stated that mobility and contractibility, which are due to active folding of polypeptide chains, are no doubt the characteristic life phenomena of all living fibrils. This view is quite compatible with the writer's. 
On studying the X-ray diagrams of bacterial flagella, Astbury (146) has found that films produced from the flagella show fine $\alpha$-keratin reflection and, upon squeezing, this goes over into the $\beta$-keratin pattern. Since it is known that $\alpha$-keratin pattern is formed when keratin molecules are expanded, while $\beta$-keratin pattern is revealed when the molecules are contracted, it is obvious that the movement of the flagella is brought about by the extension and contraction of the protein molecules.

However, whether or not the movement of flagella can lead to the movement of the bacteria themselves is another question. The writer holds the opinion that bacterial movement is not brought about by the flagella, but that bacteria can move by making use of the thermal motion of water molecules. As is well known bacteria show a very active Brownian movement which is caused by the bombardment of water molecules in thermal motion, it should therefore be expected that bacteria will be able to move in a definite direction if they have a specific form by which the bombardment of water molecules become effective only in one direction. It may not be difficult for bacteria to acquire such a form. Presumably flagella act as rodders and perhaps, in addition, play a role in making effective the energy of the water molecules. It is inconceivable that contraction and extension of the flagella, only frail bands of protein molecules, result in the active movement of the bacteria.

In this connection, it should be noted that pijper (147) claimed that bacterial flagella are not the locomotive organs of the bacteria, but are merely mucous twirls trailing from the cell surface. It seems, however, possible that the flagella themselves can achieve some motion and have some connection with the bacterial movement, and so it seems more reasonable to consider that though bacteria do not move by flagella, flagella contribute to the movement.

The writer has reached such a conclusion mainly from an observation on a kind of bacteria, perhaps a strain of water vibrio, which accidently contaminated a protein solution stored in an ice box. This strain of bacteria could vigorously proliferate at the low temperature in ice box, and move very briskly in a definite direction at a full speed as if flying in the air. Under the microscope of dark field illumination the speed appeared to have no connection with the temperature; they moved in icy water apparently at the same velocity as in water of laboratory temperature. If the movement is raised by flagella composed of protein molecules being enabled to move by the interposition of lipids, it should be very sensitive to environmental temperature just as the movement of cold-blooded animals. On the contrary, if the thermal motion of water molecules is directive, the temperature 
differences involved in the usual environments of animals should be insignificant, since the molecular motion is directly proportional to the absolute temperature not to the ordinary one.

Of course, organisms which may be able to utilize the molecular motion of water should be very small in size, and perhaps not larger than bacterial size. Animals larger than bacteria may be making use for their movement of the reversible contractibility of protein molecules. Muscle contraction of higher animals must also depend upon this property of protein molecules. Actin molecules, the main protein component of muscles, can exhibit even in vitro the reversible contraction as already pointed out.

The remarkable readiness with which impulses can be transmitted along nerve fibres may be based upon the most suitable arrangement of protein molecules for the spread of their structural changes. The transmission of an impulse must be the spread of the wave of the structural change, although this wave cannot be seen as a formal change such as contraction. It is shown in electron micrographs that protein threads are orderly arranged in nerve axon along the long axis of the fibre (148). Chambers (149) injected oil drops into the axoplasm of giant nerves of the squid and found that the drops assumed ovoid shapes along the long axis of the nerve. After agitating the axoplasm with a microneedle near the oil, the drop assumed a spherical shape, suggesting the occurrence of a contraction of protein molecules.

It has long been known that nerve tissues are especially rich in lipids, which may serve as lubricating oil enabling the protein molecules easily to alter the structure. It may be considered that phospholipids are involved, in addition, in the energy metabolism for the impulse transmission. Anaestetics or narcotics are generally lipid-soluble; the action of the drugs may depend upon their effect on the lipids, the lubricating oils, rendering the spread of the structural change impossible.

Neurotropic viruses, such as poliomyelitis virus, are said to travel preferably along neural paths. According to Sanders, mouse encephalomyelitis virus travels at about $0.2 \mathrm{~mm}$. per hour (150). If structural changes of the protein are readily transmitted in nerves, the structural change induced by a virus must likewise be easily spread in them. The spread of the structural change induced by a virus may be nothing but the travel of the virus itself.

Since the structural change of protoplasm proteins should accompany a change in polar groups, the spread of the change must be followed by an electric disturbance, which is known as action current.

On stimulation of muscles remarkable diffusion of $\mathrm{K}$ ion takes place. In nerves, as in muscles, there is a difference between the 
concentration of $\mathrm{K}$ ion in the inside and on the outside of the nerve fibre. The ratio of $\mathrm{K}$ ion in the inside to that on the outside is given as $29: 1$. However, during excitation this ratio is changed, the ion present in the inside being liberated. The occurrence of such an exchange of ions by stimulus can be demonstrated with the help of various isotopes (151). The exchange may be recognized as action current when examined electrically.

The existence of similar ratio of ions is also known between redblood cells and the serum: In the cells, $\mathrm{K}$ ion is present in a much higher concentration than $\mathrm{Na}$ ion, but in the serum the ratio is reverse as in the sea water. $K$ ion seems present in such high concentrations generally in the cell protoplasm, a fact which may depend upon the property of the protoplasm protein to adsorb preferably $\mathrm{K}$ ion. This adsorbed $\mathrm{K}$ ion, on the excitation, will be eluted by the structural change of the protein, giving rise to an electric disturbance.

It may, therefore, be concluded that action current is raised by the "absorption and excretion" of inorganic ions. On the other hand, it is known that action current is associated with the true absorption and excretion, a fact which can be expected, since this phenomenon may also be brought about by the structural change of protoplasm as discussed earlier.

The electric disturbance occurring in the brain may be called brain wave. The function of brain must likewise be given rise to by a similar structural change of the protoplasm protein. The fact that brain current is generally oscillating and wavy, suggests that the structure of the brain protoplasm tends to oscillate regularly.

The structural pattern of brain may be more or less disturbed and deformed during the constant change of the structure. Its mending may be accomplished by sleep during which no stimulus is given and sufficient time is provided for the recovery of the original pattern; presumably the reversibility of the protein structure accounts for this recovery.

Since all the life phenomena must be raised by the structural change of protoplasm, which is always to be accompanied by the electrical disturbance, this latter phenomenon should be "the sign of life" as Waller proposed a long time ago.

\section{The Mechanism of Blood Coagulation.}

The theory of the writer on life phenomena discussed so far is based upon the basic assumption that the structural change of proteins is transmissible. This assumption was made for the first time by 
taking into account of the view presented by Fischer (152) who found that the blood coagulation can be transmitted indefinitely in blood plasm. He found that the addition of coagulating agent, which is. being produced in a coagulating blood plasm, to another plasm, leads to the coagulation of the latter, in which cogulating agent in turn is produced, thus the agent multiplying indefinitely in the plasm. This coagulating agent, however, can be demonstrated only during the progress of coagulation, and so Fischer considered that the active radicals of the agent diappear by the mutual saturation with the completion of the coagulation.

It seems reasonable to consider that blood coagulation is caused by a kind of denaturation of plasm proteins, thereby as was previously mentioned protein molecules may unfold their polypeptide chains; the active groups of the coagulating agent may be produced by such an unfolding. Since the polypeptide chains are to be refolded upon the completion of denaturation with the disappearance of polar groups liberated by the unfolding, also the active groups must disappear with the finish of the blood coagulation.

According to the writer's concept, denaturation of proteins is generally infective because of such polar groups liberated during the denaturation process. The fact that the infection is particularly distinct in blood coagulation may suggest the presence, in the blood plasm, of the mechanism by which the transmission of the denaturation is readily brought about. Now, we shall consider in detail this mechanism.

It seems a general concept that the process of the blood coagulation consists of the following two phases (26):

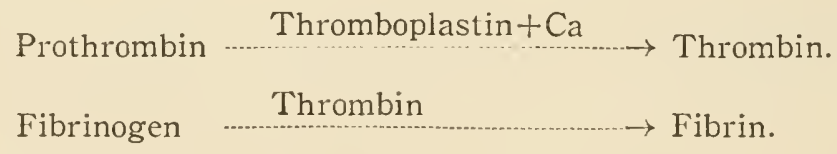

The factor termed thromboplastin or thrombokinase is usually found in blood platelets, and this factor appears to be vius-like protein particles containing both RNA and phospholipids (153), presumably a kind of elementary bodies of the protoplasm. When blood platelets are disintegrated into such particles, the particles will combine with prothrombin, a kind of serum globulin. By this combination the protein is disturbed in its structure just as host cells are disturbed by the combination of a virus. As a result prothrombin undergoes a denaturation, with the unfolding of polypeptide chains and the liberation of polar groups. The prothrombin affected by the thromboplastin particles can combine with other prothrombin molecules through these polar groups, causing the denaturation in the latter molecules and thus the 
denaturation spreads as a chain reaction as above mentioned.

The denaturated prothrombin is called thrombin, in which active group or groups are being produced as a result of the structural change due to the denaturation. Thrombin, the denaturated prothrombin, can combine with another kind of serum globulin, called fibrinogen, through these active groups to produce the structural disturbance in the latter. The fibrinogen thus denaturated is termed fibrin. During this change polar groups are likewise temporarily liberated, whereby the change is transmitted to other intact fibrinogen molecules. This is the general concept of the writer as regard the mechanism of blood coagulation, by which all the phenomena known concerning blood coagulation may fairly be elucidated.

Thus it may be clear that the blood coagulation as a whole is a chain reaction given rise to mainly by temporarily liberated polar groups. However, there are ample evidences that active group of thrombin is retained without disappearing long after its formation. Therefore, it must be considered as follows: The active group of thrombin is different from the polar groups which are liberated temporarily during the process of the denaturation, but their group may arise as a result of the denaturation and so can remain without disappearing. The reason why active group can be produced following denaturation will be discussed in the next Part.

Further it should be added that thromboplastin may not be present as such in the platelets, but that active groups capable of acting as thromboplastin may be produced upon the disintegration of the cells into elementary bodies just as the active groups of thrombin are raised by the denaturation of prothrombin. In fact, it is said that thromboplastin is present in cells as thromboplastinogen or prothromboplastin (154). This latter may be the natural state of elementary bodies present in platelets. During the conversion of prothromboplastinogen into thromboplastin transient liberation of polar groups may take place, leading to the transmission of the denaturation to accelerate the change; thus the phenomenon may appear the more complicated (155).

Throboplastin seems to be usually produced from platelets, but it can also be isolated from a variety of other materials such as brain, lung, and blood plasm, showing that it is elementary bodies not peculiar to the platelets. Its action is lost by the extraction of lipids; the lipids may be involved in the combination of the thromboplastin with prothrombin. Thromboplastin fails to act without $\mathrm{Ca}$ ion. As was already mentioned, Ca ion is generally necessary for the transmission of structural change of proteins because of its faculty to favour the liberation of polar groups in proteins, and hence the need of $\mathrm{Ca}$ ion is never peculiar to blood coagulation. 
Various denaturating agents involved in blood coagulation may be called enzymes in a wide sense. However, since it has been found that thromboplastin reacts with prothrombin in stoichiometric amounts (156), at least thromboplastin cannot be regarded as an enzyme in a narrow sense. On the other hand, it has been claimed that thrombin is not involved in the coagulation of the fibrinogen molecules, indicating that thrombin acts as a true enzyme, because a protein which can disturb the structure of another protein and which is not consumed in this reaction should be termed a true enzyme. Nevertheless, the fact that papain clots fibrinogen just as thrombin does is not sufficient to prove the enzyme nature of thrombin (157). Trypsin is also known to be able to convert prothrombin into thrombin. These enzymes may act only to disturb the structure of the protein as do the blood clotting agents such as thromboplastin and thrombin.

At any rate, blood coagulation may be regarded as a chain reaction brought about by a series of enzymes in a wide sense. The concept that a protein having a stronger structural influence overcomes a weaker by disturding the structure of the weaker, or, in a particular case, by converting the structure of the weaker to be identical with that of the stronger, has led the writer to the theory of enzyme function and further of the mechanism of the generation of life. It may be considered that life phenomena are based upon the combination of various kinds of proteins, that possess this fundamental property, joining together orderly as in this case of blood coagulation for a definite purpose in making an "enzyme system".

Presumably, enzyme systems present in living organisms are not necessarily composed of enzymes in a narrow sense. Protein molecules which can act as true enzymes may be rather exceptional, mostly behaving as enzymes in a broad sense as does thromboplastin in the blood coagulation.

\section{The Mechanism of Mitosis.}

In case of the cell division, the nuclear substances capable of determining the inheritable character are divided into two equal parts, each of which are subsequently distributed to daughter cells, by an apparently very complicated process termed mitosis. Though this process appears very mazy, since it is a basic phenomenon generally taking place in orgnisms, it must be a process raised by some simple, fundamental properties of protoplasm.

In the writer's opinion, as described in Part II, the protoplasm is composed of polypeptide chains, which make up elementary bodies in 
forming bundles; these elementary bodies are arranged in a regular manner associating loosely with each other. The bodies must be arranged in the protoplasm in an equal direction as otherwise the polarity of the cell or organism may not be raised. If the end-to-end association of the elementary bodies is tighter than the side-to-side association, the protoplasm may appear as if it were composed of protein threads in a parallel alignment, as shown in Fig. 30, but such threads may be invisible under the ordinary microscope.

E.

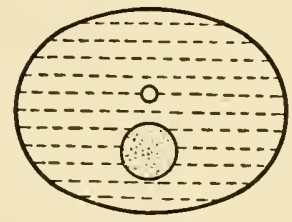

$\mathrm{D}$

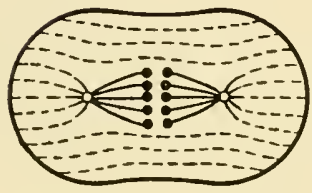

B

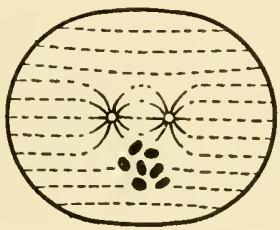

$\mathrm{E}$

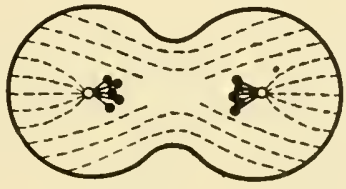

Fig. 30
$\mathrm{C}$

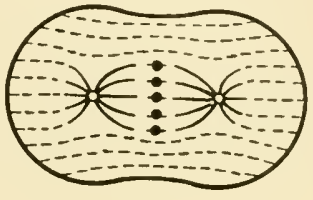

F

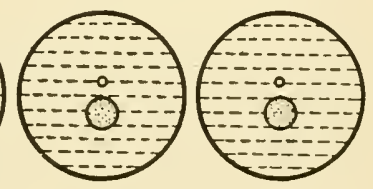

Diagram of mitosis

When the cell, after its full maturation, is brought under the influence of a certain stimulus, the protoplasm particle termed centriole may be activated by the liberation of some active groups, resulting in its vigorous growth until it becomes so large that it fails to exist any more as a single particle which therefore is to be divided. Through the division the structure of the proteins in the particle is disturbed severely, and a kind of denaturation follows with the temporary liberation of strong active groups which can attract the elementary bodies present nearby. The elementary bodies thus pulled and combined by the centrioles drag in their turn other elementary bodies successively, and moreover the denaturating process of the centriole is transferred to them, acting as a stimulus to contract the protein threads; as a result they are coagulated and thus the coagulation is spread centrifugally from the centrioles, so that radiating system of fibers, the so-called asters, become visible at least in some animal cells as shown in B in Fig. 30. This denaturating process is transmitted also to the nucleus 
which accordingly loses its normal appearance with the coagulation of nuclear substances.

Now, the centrioles and coagulated particles of nuclear substances, i.e. chromosomes, may have the-same electrical charge since they are composed of proteins of a similar nature. If the surface substance of the cell is equally charged, the centrioles and chromosomes will be situated as far as possible both from the cell surface and from each other. It is known that beneath the surface of cells there is a thin layer of gel called cortex, which may be the charged surface substance, since both the centrioles and chromosomes are considered to be also the gel of the same category.

At any rate, the divided centrioles are thus provided with the same charge, so that they have to be separated from each other when the division is accomplished; at the same time the chromosomes, $i . e$. the coagulated nuclear substances are shifted to acquire the most stable position in the field of the electrical force, and therefore finally chromosomes are distributed in a plane exactly between the two centrioles and to right angle to a line connecting them.

Meanwhile, this process may activate the chromosomes to increase in their mass until the division is unavoidable just as the centriole is activated by some stimulus to undergo the division. Through this division chromosomes are likewise disturbed in their structure with the production of free polar groups, at least a certain point of each particle, which can attract the protoplasm threads and thus each thread present close to each divided particle of chromosomes is attracted and combined with the particle and subsequently contraction occurs in the thread as the active group of the chromosomes acts as a stimulus to produce the contraction. Thus, each set of divided chromosme is pulled toward each centriole as shown in E in Fig. 30. The separation of the divided centrioles may be caused not only by their mutual expulsion but also by the contraction of the protoplasm threads between the cortex and the centriole. Now, between the pulled chromosmes a space is produced where no protoplasm substance is present, so that the cell is divided by the "furrow" formation around the equator of the cell.

Since all the changes above described are initiated by the temporary liberation of active groups following the denaturation of the protein, the normal state of the cells must be recovered with the disappearance of the active groups, owing to the reversibility of protoplasm structure, and accordingly two new cells with the quite normal structure are to be produced.

Mitosis, an apparently very complicated vital phenomenon, can be in this way easily explained by the theory of the writer. However, the assumption of existence of the specific particle termed centriole may 
be surplus, because it may be reasonable to consider that the elementary body of the protoplasm which by chance is situated in the center of the cell may somewhat be disturbed in its structure, because of its special position, by the physicochemical influence coming from the cell surface upon which a certain stimulus is added; the disturbence in the struc. ture of the body may give rise to the liberation of free groups which can fuse other proteins or protein components into the body leading to its vigorous growth until the division takes place. The growth and the division of chromosomes distributed between the two centrioles may be likewise attributed to their special position, $i$. $c$. the center of the electrical field produced by the centriole particles in a stimulated state.

The long effective distance of the charge of centrioles may be accounted for by the layer of water molecules attracted and regularly oriented around the centrioles as considered in Chapter II in Part II. The effect of the charge of both cortex and chromosomes must be likewise of the same nature, and therefore the pattern of the equilibrium of the effect at the phase when the separation of the centrioles is established may be as shown in Fig. 31. Hence, chromosomes are

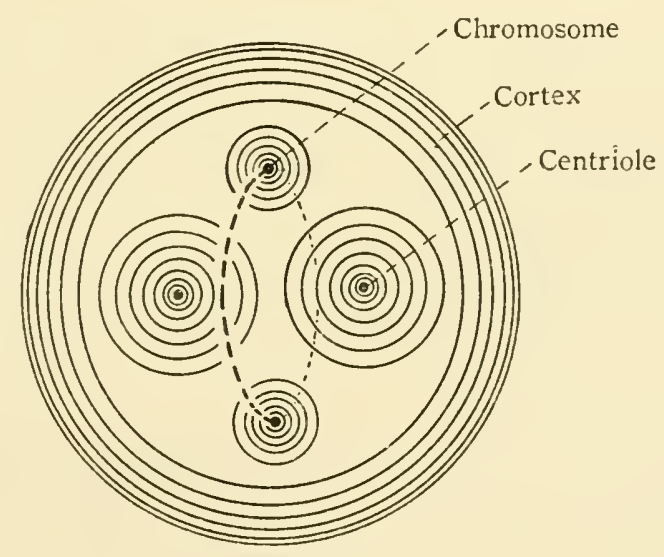

Fig. 31

Pattern of equilibrium of electrostatic forces in mitosis

not to be regarded as being distributed in a circular plane between the two centrioles, but distributed on the circumference of the circle. The protoplasm threads passing through the inside of the circumference may be either combined with the chromosomes or broken off at the middle point by the extensive pulling, thus contributing to the occurrence of the division of the cell mass. 
It is known that the mitotic spindle is birefringent and that the birefringency decreases when the chromosomes are separating (158). This demonstrates that the protoplasm threads between the two centrioles are pulled extensively so that they are arranged quite regularly in a parallel alignment, thus the birefringency revealing in the spindle, but that when the contraction of the threads takes place causing the separation of the chromosomes, the regular alignment is to be lost followed by the disappearance of the birefringency.

The fact that mitosis is the general, fundamental feature of organisms must depend upon that it is the mechanism easiest and most favorable for the organism to achieve the cell division. Thus the division of chromosomes without the preliminary fission of centriole may tend to cause only the increase of chromosomes without the cell division, because the divided centrioles only can produce the space where the essential protoplasm substance is absent, making the cell division possible. As is well known colchicine prevents the formation of the mitotic spindle, but do not affect the chromosomes, so that in the presence of colchicine chromosomes are increased without cell division, a fact suggesting that colchicine inhibits the growth or the division of the centriole.

The reason why the division of centrioles and chromosome is initiated normally is not clear. It may depend upon some basic property of proteins. Protoplasm-like masses prepared in vitro by the writer also cannot grow unlimitedly, suggesting that the fusion of elementary bodies can occur only to a certain limit. Both centrioles and chromosome may be the aggregates of elementary bodies, so that if they grow over a certain limit they may be bound to split. Colchicine may exert some influence on physicochemical property of the protein composing the centrioles to make the centrioles unable to grow or to divide if the growth is not inhibited.

An interesting phenomenon associated with neutron- or $\gamma$-irradiation of maize seeds has been found by Schwartz (159). The seedling irradiated at the higher dose levels were not only taller but also much healthier in appearance than those at the lower levels. These seedlings could not be differentiated from normal unirradiated young seedlings except that there was a cessation of farther growth after approximately 5 days. The finding concerning the phenomenon which must be noted here is that cytological examination of the root tips from plants which received high doses of irradiation revealed a complete absence of cell division. In other words, the growth was due entirely to cell elongation in these seedlings. Thus the conclusion drawn by Schwartz was that at high radiation levels the seeds were killed in the sense that no farther cell divisions occurred. According to the writer's view 
this may be attributed to a structural change in the protoplasm protein induced by the radiation, whereby presumably the division of either centrioles or chromosomes or of both becomes impossible. 


\section{CHAPTER XI}

\section{THE SUMMARY OF PART IV}

Organisms generated from the protoplasm of preexisted creatures are designated as the secondary organisms and those generated originally in the primeval oceans without living substances as the primary organisms. The former were evolved from viruses, while the latter were presumably developed from protoplasm-like masses, a model of which could be prepared by the writer using plant materials especially castor beans.

In general, coagulated elementary bodies are produced by the decomposition of protoplasm. These bodies yielded from plant materials are liable to fuse into a larger homogeneous mass when stand in a weakly acid solution.

Elementary bodies possess a character to aggregate readily in a weakly acid water solution. Under this condition, aggregated particles appear to liberate their folded polar groups to combine with one anotherand fuse into a homogeneous mass. In coagulated elementary bodies protein molecules may be in a contracted, folded state, while in a solution of weak acid, the isoelectric point of the protein, the stretching or unfolding of the molecules may take place, leading to the mutual combination of the elementary bodies.

Such a stretching appears to occur readily in plant elementary bodies in contrast to animal ones. Elementary bodies prepared from castor beans manifestly exhibit this property and occasionally produce fair protoplasm-like masses, which may be termed artificial cells. The most primitive feature of the primary organisms may be seen in such a mass.

In chemical composition the mass resembles the protoplasm, being composed of globulin-like protein and lipids, while also in its form it bears a striking resemblance to a kind of protozoa, having a globular form with a diameter of scores of $\mu$, granules of various sizes being included, some of which have frequently a nucleus-like appearance. On the application of a proper physical or chemical stimulus, the mass coagulates into minute particles just as the usual protoplasm, but may recover gradually its original, homogeneous state following the removal 
of the stimulus, a fact showing that it has a primitive feature of irritability. Furthermore, it can grow by incorporating the minute particles of lipoprotein yielded from castor beans, and the growth will be followed by fission if a suitable environmental change is provided. Thus it can accomplish both the growth and multiplication.

Such cell-like masses can be prepared not only from elementary bodies, but also from the constituents of the elementary bodies into which the latter was decomposed. Merck's preparation of ricin which is composed of globulin of a molecular state, combining with lipids, can form elementary-body like particles when its water solution is made weakly acid ( $\mathrm{pH}$ 5.5) by addition of acetic acid and left in ice box for several days. The particles thus formed can further fuse into protoplasm-like masses, when stand in the water of $\mathrm{pH} 5.5$ at laboratory temperature. Thus, under a suitable condition, globulin with lipids can form protoplasm-like masses following the formation of elementary-body like particles.

Since the assimilase, or the protoplasm including viruses, can be regarded as a kind of liquid crystals composed of globulin-like proteins polymerized with lipids, the fact just mentioned indicates that a liquid crystal closely resembling the protoplasm can be produced in vitro and that it is not entirely unreasonable to regard such a crystal as a very primitive organism.

The oceans of the primitive age might contain various substances as there were no microorganisms to devour them up, and the sea water might be weakly acid on dissolving carbon dioxide which might be present in rich amount on the surface of the earth. Consequently, when globulin-like protein having the property to sediment readily at a weakly acid reaction were produced in the ocean, they would be precipitated accompanied by lipids, and the sediments would form elementary body-like particles which in turn would fuse into protoplasmlike masses. These masses would be endowed with assimilase action by their specific structure and thus. primeval organisms might appear on this globe.

Such masses of a liquid crystal would accumulate on the bottom of the primeval ocean, and if a certain structural change capable of producing a structure stronger than the original one occurred in some of the masses, the newly formed structure owing to its stronger character would spread to surrounding masses. The structure thus could multiply just as viruses can in the protoplasm of host cells. The 
generation of a new structure would be brought about by an environmental change or a stimulus just as the generation of a new virus. The change of the structure might occur repeatedly, and whenever a stronger structure was produced it would spread and multiply. Thus the structure would become stronger and stronger, thereby the primitive organisms would evolve higher and higher with the enhancement of the assimilase action.

Some of the masses of the assimilase or the primeval organism might be carried to the shore by a tidal current, and in the active motion of the wave they might be able to come to contact with other masses generated in other regions. If they could then combine with each other, the individual with stronger structure would overcome the other and thus the stronger one would assimilize and devour up the weaker. Through such a struggle for existence, the structure would be more and more strengthened and the masses of assimilase would approach nearer and nearer the domain of the undoubted organisms.

When two different protein molecules can combine with one another, they would mutually exert influences arising from the different structures. On such a combination, if one of them is stronger in its structure than the other, only the structure of the weaker is disturbed, and if by this disturbance are lost the polar groups through which the weaker can combine with the stronger, this latter can be freed from the weaker and combine with another weaker molecule. In such a case the stronger protein is called an enzyme in a narrow sense. If the structure of the one is overwhelmingly stronger than the other, the structure of the latter would be changed to become identical to that of the former, that is, the latter would be assimilized by the former. In such a case the stronger one is called assimilase. The polymerization of numerous protein molecules is considered to result in the development of the strong structural effect and hence the assimilase is usually the polymerization product of protein molecules. Thus all the protein molecules may be enzymes in a wide sense, and the enzymatic nature may therefore be one of the characteristics of the proteins.

The protein molecules produced in the primitive oceans would be polymerized orderly owing to the weakly acid reaction of the water, with the appearance of assimilase action. However, even before they could polymerize thus orderly and act as a fair assimilase, protein 
molecules might have structural influences upon each other and molecules with stronger structure would more or less assimilize others. In this way the evolution of the protein structure itself would have had to continue to proceed for a dreadful span of time before the globulinlike protein which could polymerize orderly to exert strong structural effect could be produced.

Lipids are not necessary for the production of the strong structure, but if lipids are not inserted among protein molecules, these latter would not be able to change the structure freely so as to be assimilized by the stronger assimilase. The evolution of proteins as well as organisms would never be established without labile structures yielding to assimilation. In addition, free changes of the structure must be the essential feature of life, and thus where there is no lipid there is no life.

In order to strengthen its pattern and to become stable, the assimilase needs nucleic acids which may raise rigid structure in the polymerization product of proteins. On the other hand, the protein molecules in the assimilase are required to move or change freely for the achievement of various life phenomena, for which the rigidness of the structure is obviously unfavourable.

The organisms seem to escape fairly from this dilemma by mixing lipids in the greater part of the protoplasm, thereby the protein molecules being rendered easily movable, whereas in its smaller part nucleic acids are inserted instead of lipids to make the structure rigid. Since this smaller part of the protoplasm owing to its rigidness in the structure can remain unchanged even when the greater part containing lipids is altered in its structure, it enables the changed structure to return to its former state, as it can exhibit extensive structural effect by acting as a strong assimilase because of its rigid structure.

Such part of protoplasm rich in nucleic acid is in higher organisms usually located in nucleus and acts as the standard template of the cell, and is composed of minute particles termed genes. Thus the genes are a kind of elmentary bodies capable of preventing the deformation of the protoplasm pattern. As the pattern of the gene is, in general, unchangeable, the properties of the organisms can be retained unchanged.

Various genes are present in the cell of higher organisms and each gene seems to direct each specific, restricted structure of the protoplasm protein. In other words, a gene is composed of protein molecules, only 
a certain restricted portion of which is of strong structure, and it can accordingly direct only a restricted portion of the protoplasm protein corresponding to the restricted strong structure, other portions being governed by other genes.

\section{5}

The protoplasm is a kind of mixed crystals composed of a variety of molecules or molecular aggregates, but the polar groups of the molecules must be arranged in an equal way in order to coexist in one and the same protoplasm. The pattern of this arrangement is subjected to the genes, and therefore the "crystal shape" of the protoplasm is determined by the genes.

Principal components of this mixed crystal are elementary bodies, the chemical composition of which can not therefore be uniform eren in one and the same protoplasm. Because of this different chemical composition, some elmentary bodies can behave as genes and some others as specific enzymes.

There are two types of nucleic acids in protoplasm; one is desoxyribonucleic acid and the other ribonucleic acid. The former seems stronger than the latter in their stabilizing action of protoplasm and so the elementary bodies containing the former can behave as genes, whereas those containing the latter are present in cytoplasm to be controlled by the genes, and apparently participate in the synthesis of proteins to endow the specific pattern directed by the genes to newly formed proteins.

The genes thus control the pattern of all the proteins in the protoplasm, whereas all the proteins can be regarded as enzymes in a wide sense. All the enzymes, therefore, including enzymes in a narrow sense present in the cell, are to be subjected to the genes.

Hormones are looked upon as substances produced physiologically by organisms for the purpose of interfering with the action of genes. They may be capable of directing the pattern of the protoplasm in a certain direction according to their specific structure, thus giving rise to the shape and function, favourable for the organisms, which cannot be attained by the genes.

Various inorganic ions, like hormones, can exhibit similar effects upon the pattern of the protoplasm. However the ions, unlike hormones, are not the substances produced by organisms, but are the normal protoplasm components usually present there in large quantities, so that their effect must be cxtensive if not so strong in themselves as hormones. Organisms can develop various shapes and functions which vary with 
the various parts of the body, presumably by making use of inorganic ions as well as hormones and related substances, even if the genes were similar througout the body.

The so-called antibiotic agents, such as penicillin, and synthetic chemotherapeutic agents, such as sulphonamide compounds, may be effective because they can interfere with the action of genes of the pathogens as do hormones. However, in contrast to hormones, the alteration of the pattern induced by these agents is unfavourable for the pathogenic organisms, presumably because of the extensive and profound disturbances occurring in a variety of enzyme systems as a result of the deformation of the pattern. Similar changes in enzyme systems may occur in the cells following the infection with some virus which, as a free gene, can interfere with the original gene.

\section{6}

The primeval organisms generated in the oceans were advanced to certain extents with the complication of their structure, meanwhile the elementary bodies composing the organisms might differentiate to possess various properties, and thus some of the elementary bodies might become to contain nucleic acid in rich amount so that they would behave as primitive genes. Since such bodies were provided with stable and strong structures owing to the nucleic acid, they might be able to transmit their structure to other organisms even when liberated from the organisms. Thus such bodies would act as viruses or free genes.

Moreover, since the structure of such bodies might be reversible owing to the rigidness, they would not be deprived of the reversibility even when present in a weak individual, which had been assimilized or devoured by a stronger individual, although thereby the transient loss of their original pattern could occur, so that they might be able to recover their original structure when freed from the influence of the bodies or the genes of the stronger individual, and would be able in turn to affect individuals which were still weaker than themselves. On such a stage, the primitive organisms would be said to have only a pair of genes.

Further developments of the organisms would lead to further differentiations in the protein structure of genes, resulting in the development of strong structure in restricted portions of protein molecules; thus different genes would come to be composed of different proteins which have each strong structure at a different restricted portion of the molecule. If two organisms containing each of such genes combined 
with each other, the different strong structures of each gene would be left in the combined individual instead of the complete disappearance of one of them. The same would result if the gene itself, after liberated from an organism, combined with the other organism.

Thus, the splendid mechanism by which the characteristics of both individuals are combined and left in the progeny would be developed, and since such a mechanism would be the most favourable for the evolution of the organisms the differentiation of genes would be extensively advanced until the highly complicated gene system of the present higher organisms was attained.

The phenomenon in which two individuals having different genes combine or conjugate to produce new individuals that are endowed with the characteristics of the two is called sexual reproduction. In this case, one of them can achieve its function in a state of free gene and termed male gamete or sperm, whilst the other involves considerable quantities of cytoplasm in which combined pattern is to be spread or multiply and is designated female gamete or egg.

\section{7}

Viruses fail to retain their pattern unchanged, and consequently undergo senescence, when continue to multiply in the same kind of cell protoplasm because of their continual affliction by the assimilase action of the host protoplasm, though they are stronger than the protoplasm in the action. In entirely the same way, primeval primary organisms would be unable to keep their pattern when continue to multiply in the same group of protoplasm-like masses or primeval organisms. Therefore, they would need rejuvenescence for the continued existence. This rejuvenescence would be achieved by the contact with another group of the masses just as viruses are rejuvenated by the host change. The contact with another group is regarded as nothing but the sexual conjugation; the sexual process would arise for this purpose of reiuvenescence, but in addition to this, through this process a new character would be obtained by the combination of two different structures, and hence the sexual process would become essential for the evolution of the organisms both primary and secondary.

The secondary organisms, however, would apply a peculiar method for the rejuvenescence before they are enough evolved to achieve the sexual reproduction. The method consists in their taking advantage of the sexual process of the host with which they themselves are to be rejuvenated.

With the advancement in their structure, viruses become, as a rule, 
strong enough to be capable of spreading their pattern to the germ cells of the host, and thus they can be transmitted to the progeny. Primitive secondary organisms such as bacteria and protozoa which cannot as yet achieve the evident sexual reproduction, may succeed to the habitude of their ancestors, $i$. $e$. viruses. If the pattern of such secondary organisms, say, bacteria is transferred to the mother germ cell of the host, the pattern will be transmitted by the germ cell to the progeny of the host in a form of virus-like particles. It is considered that the pattern exists it the germ cell in an altered state, but with the development of the germ cell into a full individual, the pattern in the virus-like particles also develop into its original form, followed by the fusion of the particles into the bacterial form.

When the bacteria thus produced are injurious to the host, infection will follow, and in case of the continued production of the bacteria, the host will become bacterial carrier. On the other hand, it is possible that some produced bacteria exhibit favourable effect upon the host. In such a case the individuals harboured by the bacteria will be more fitted for their existence than those without the bacteria, so that the property to produce the bacterial pattern within its body will become an essential inheritable character of the host. The example belonging to this category is seen particularly in the relationship between insects and microorganisms.

Primitive secondary organisms can be rejuvenated in such a manner to continue their existence, and at the same time can evolve higher by taking advantage of the new structure which the host has acquired through the sexual process.

Life is often compared to a fountain or a flame, and it is claimed that life is a constant flow of both energy and substances. The advancement of the assimilase with complication and enhancement in its function would be followed by the requirement of energy for the active function, and since this energy must be provided by the expenditure of certain substances, active life phenomena should be always accompanied by the flow of both energy and substances. Such a flow is, however, only a by-product of life, never the life itself; presumably the distinct flow was revealed only after the assimilase had considerably evolved.

Viruses are known to be able to multiply in "dead" cells, that is, in the cells which have lost the faculty of multiplication. This fact indicates that for the multiplication of viruses at least not so 
much energy is required as for the multiplication of the cell, since the cells are "dead" presumably because the mechanism, whereby the energy required for the cell multiplication is produced has been destroyed. It is generally known that the action of proteolytic enzymes is not associated with neither significant expenditure nor recognizable production of energy, whereas viruses are regarded as a kind of enzymes capable of changing the protein structure. Also in view of this fact, it can be concluded that energy is not, or scarcely, needed for the mutliplication of viruses. This conclusion is compatible with the assumption that the primary organisms were generated by the mechanisms of virus multiplication in the protoplasm-like masses in which no energy-producing system was present.

\section{9}

The progress of inactivation of phage or of rennin by inactivating agents such as tannin or the respective antisera is occasionally found to be oscillating as if it were a physical phenomenon. Since the protein molecules of the same kind tend to combine with each other to make a single system, each protein molecule in a solution is unable to behave of its own accord but only permitted to act in concert with other molecules. This may be the main reason for the occurrence of oscillation.

Even if molecules in a free state oscillated in their chemical reaction, the oscillation would not come out as such because of their arbitrary behaviour. Protein molecules have a structural reversibility, that is, the changed molecules are striving to recover their original structure; on account of this property they may rebel against the action of inactivating agents resulting in the reaction which may reveal itself as the oscillation owing to the mutual combination of the molecules.

In the protoplasm, the association between the protein molecules is complete, so that oscillation is especially evident in the organisms. The protein molecules are able to freely change their structure owing to the existence of lipids in spite of their orderly association.

Protoplasm particles, including virus particles, when suspended in a sugar or inorganic salt solution after a manipulation which may cause their structural disturbance, can sometimes absorb and sometimes excrete the solute. This may depend upon the adsorption and elution of the solute according to the change in the protein structure. In this way, organisms can achieve absorption and excretion by changing the structure of protoplasm protein. 
When the change of the protoplasm protein is raised by in the contraction and extension of the molecules. the oscillation comes out as a rhythmic motion. In general, the movement of organisms may be achieved by such a contraction and extension of protein molecules even when it is not oscillating or moving rhythmically.

Not only the motion but also all the life processes are presumably given rise to by various changes of protoplasm protein. Since the changes should be associated with the adsorption and elution of a variety of ions and perhaps also with the appearance and disappearance of polar groups, life phenomena are always accompanied by electric changes as for example action current. 


\section{REFERENCES}

(1) Gross, J.: Proc. Soc. Exp. Biol. \& Med., (78), 241, 1951.

(2) Takahashi, W. N. and Ishii, M. : Nature, (169), 419, 1952.

(3) Oparin, A. I.: The Origin of Life, Trans. by Morgulis, New York, 1938.

(4) Sörensen, S. : Kolloid Z., (53), 102, 1930.

(5) Kletzkowski, A.: Brit. J. Exp. Path., (26), 24, 33, 41, 1945.

(6) Zoet, P.: Proc. Soc. Exp. Biol. \& Med., (32), 1469, 1935.

(7) McFarlane, A. S.: Bioch. J., (29), 407, 1934.

(8) Claude, A.: Advances in Proteinchemistry, (5), 423, 1949.

(9) Fox, S. W., et al.: J. Biol. Chem., (155), 465, 1944.

(10) Fling, M., and Fox, S. W.: J. Biol. Chem., (160), 329, 1945.

(11) Davis, B. D., and Maas, W. K.: J. Amer. Chem. Soc., (71), 18, 65, 1949.

(12) Arton, C., et al.: Proc. Soc. Exp. Biol. \& Med., (60), 284, 1945.

(13) Gale, E. F.: Chemical Activities of Bacteria, New York, 95, 1951.

(14) Kögl. F., et al.: Rec. trav. chim., (69), 822, 834, 841, 1950.

(15) Christensen, H. N. et al.: J. Biol. Chem., (198), 1. 1952.

(16) Delbrück, M., and Bailey, W. T.: Cold Spring Harbor Symp. Quant. Biol., (11), 33, 1946.

(17) Burnet, F. M., and Edney, M. : Aust. J. Exp. Biol. Med. Sci., (29), 353, 1951.

(18) Schmidt, W.: Naturwiss., (26), 413, 1938.

(19) Stedman, E., and Stedman, E. : Nature, (152), 556, 1943.

(20) Ris, H., and Mirsky, A. E.: J. Gen. Physiol., (32), 489, 1949.

(21) Muller, H. J. : Proc. Roy. Soc. London, B. (134), 1, 1947.

(22) Palay, S. L., and Claude, A.: J. Exp. Med., (89), 431, 1949.

(23) Gowen, J. W., and Gay, E. H.: Genetics, (18), 1, 1933.

(24) Landsteiner, K. : The Specificity of Serological Reactions, New York, 1936.

(25) Boyd, W. C. : Fundamentals of Immunology, II. Edition, 129, 1947.

(26) Hawrowitz, F.: Chemistry and Biology of Proteins, New York, 283, 1950.

(27) Davidson, J. N. : Ann. Rev. of Bioch., (18), 155, 1949.

(28) Schmidt, G., and Thannhauser, S. J. : J. Biol. Chem., (161), 83, 1945.

(29) Chantrenne, H.: The Nature of Virus Multiplication; The Sec. Symp. of the Soc. for Gen. Microbiology, 1953, 1.

(30) Mahkham, R. : Ibid. 85.

(31) Dorner, R. W. and Knight, C. A. : J. Biol. Chem., (205), 959, 1953.

(32) Hershey, A. D. ct al. : J. Gen. Physiol., (36), 777, 1953.

(33) Cohen, S. S. : Cold Spring Harbor Symposia Quant. Biol., (12), 35, 1947.

(34) Quersin, L.: Ann. Inst. Pasteur, (75), 522, 1948.

(35) Luria, S. E.: The Sec. Symp. of the Soc. for Gen. Microbiology, 1953, 99.

(36) Morse, M. L., and Carter, C. E. : J. Bact., (58), 317, 1949.

(37) Levy, H. B., et al.: Arch. Bioch., (24), 199, 1949.

(38) Bonnet, R., and Gayet, J. : Compt. rend., (230), 415, 1950.

(39) Sonneborn, T. M. : Cold Spring Harbor Symp. Q. B., (11), 236, 1946.

(40) Preer, J. R. : Genetics, (35), 344, 1950. 
(41) L'Héritier, P.: Heredity, (2), 325, 1943.

442) Rhodes, M. M. : J. Gen., (27), 77, 1933.

(43) Danielli, J. E. : Scientific American, (186), No. 4, 1952.

(44) Harrison, R. W.: J. Infect. Dis., (70), 69, 77. 1942.

(45) Hershey, A. D., and Bronfenbrenner, J. : J. Gen. Physiol., (21), 72, 1938.

(46) Tracy, R. L. : J. Bact., (36), 467, 1938.

(47) Sevag, M. G., and Rasanoff, E. I. : J. Bact., (63), 243, 1952.

(48) 'Beadle, G. W.: Chem. Revs., (37), 15, 1945.

(49) Virtanen, A. I., and Deley, J. : Arch. Bioch., (16), 169, 19.48.

(50) Meister, A.: Science, (115), 521, 1952.

(51) Schneider, W. C., and Hageboom, C. H. : Cancer Res., (11), 1, 1951.

(52) Bauer, D. J.: Nature, (161), 852, 19.48.

(53) Fredericq, P., and Gratia, A.: Compt. Rend. Soc. Biol., (143), 111, 1949.

(54) Kun, E. and Smith, M. H. D.: Proc. Soc. Exp. Biol. Med., (73), 628, 1950.

(55) Bauer, D. J.: The Sec. Symp. of Soc. for Gen. Microbiology, 1953, 46.

(56) Sonneborn, T. M. : Ann. Rev. of Microbiol., (3), 55, 1949.

(57) Van Wagtendonk, W. J. : J. Biol. Chem., (173), 691, 1948.

(58) Yamakawa, T., and Suzki, S.: J. Biochem., (38), 199, 1951; (39), 175, 389, 1952.

(59) Meyer, R. K., and McShan, W. H.: Recent Progress in Hormone Res., New York, 1950.

(60) Sinex, F. M., et al.: J. Biol. Chem., (198), 615, 1952.

(61) Kerppola, W.: Endocrinology, (51), 192, 1952.

(62) Scatchard, G., et al. : J. Clin. Investigation. (24), 671, 1945.

(63). Jawetz, E. et al.: J. Bact., (64), 29, 1952.

(64) Yanagita, T., et al.: J. Penicillin, (1), 34, 141, 331, 1947.

(65) Lehmann, F. E. : Naturwissenschaft, (25), 124, 1937.

(66) Guyer, M. : Animal Biology, III, Edition, New York \& London, 1941.

(67) Child, G. M. : Pattern and Problems of Develpoment, the Chicago Univ. Press.

(68) Moriyama, H. : J. Shanghai Science Institute, (3), 199, 1938.

(69) Ohashi, S.: J. Shanghai Sci. Inst., (3), 279, 1938.

(70) Moriyama, H.: Arch. Virusforsch., (2), 71, 1941.

(71) Moriyama, H., and Ohashi, S. : J. Shanghai Sci. Inst., (4), 51, 1939.

(72) Mayer, M. M. : Ann. Rev. Bioch., (20), 431, 1951.

(73) Brachet, J.: Chemical Embryology, Trans. by Barth, New York, 1950.

(74) Anderson, T. F.: Research in Med. Science, New York, 1, 1950.

(75) Moriyama, H. and Ohashi, S.: Medicine and Biology, (10), 135, 1947.

(76) Hamburgh, M. : Nature, (169), 27, 1952.

(77) Lederberg, J. : Genetics, (32), 505, 1947.

(78) Lederberg, J. : Proc. Nat. Acad. Sci. U. S., 178, 19.4.

(79) Hayes, W.: Nature, (169), 118, 1952.

(80) Moriyama, H. and Ohashi, S.: Medicine and Biology, (9), 326. 1946.

(81) Kunkel, L. O.: Amer. J. Bot., (24), 316, 1937.

(82) Lwoff, A.: Bact. Revs., (17), 269, 1953.

(83) Burnet, F. M., and Williams, S. W.: Med. Australia, (1), 637, 1937.

(84) Steinhaus, E. A. : Principles of Insect Pathology, 1949. 
(85) Leach, J. G. : Botan. Rev., (1), 448, 1935.

(86) Sears, H. J., and Brownlee, I. : J. Bact., (63), 47, 1952.

(87) Puffer, R. R. : Familial Susceptibility to Tuberculosis, Cambridge, 1944.

(88) Pottenger, F. M.: Tuberculosis, St Louis, 1948.

(89) Heaf, F., and Rusky, N. L.: Recent Advances in Resp. Tuberculosis, IV. Edition, 1948.

(90) Hauduroy, P· : Compt. Rend. Soc. Biol., (91), 1209, 1325, 1924.

(91) Kasahara, M., et al.: Medicine and Biology, (11), 331, 1947.

(92) Klieneberger, E. : J. Path. Bact., (40), 93, 1935.

(93) Diens, L. J. : J. Bact., (44), 37, 1943.

(94) Klieneberger, E. : Bact. Kevs., (15), 77, 1951.

(95) Dienes, L., and Weinberger, H. J.: Bact. Revs, (15), 245, 1951.

(95a) Silberstein, J. K.: Schweiz. Z. Path. u. Bakt., (16), 739, 1953.

(96) Dienes, L., and Zamecnik, P. C. : J. Bact., (64), 770, 1952.

(97) Hardwick, W. A., and Foster, J. W. : J. Bact., (65), 355, 1953.

(98) Nicolle, C., et al.: Arch. Inst. Pasteur de Tunis, (16), 207, 125, 1927.

(99) Andervont. H. B., and Dunn, T. B. : J. Natl. Cancer Inst., (8), 227, 1497.

(100) Mühlbock, O.: J. Natl. Cancer Inst., (10), 861, 1950.

(101) Wolf, F. A., and Wolf, F. T.: The Fungi, II, New York, 1949.

(102) Hungate, R. E.: Ann. Rev. Microbiology, (4), 53, 1950.

(103) Vincent, J. G. : Quarterly Progress Report, UCLA-15, 50, 1949.

(103a) Miller, C. P. et at. : J. Lab. and Clin. Med., (38), 331, 1951.

(104) Burkholder, W. H. : Ann. Rev. Microbiology, (2), 339, 1948.

(105) Ackermann, W. W.: J. Biol. Chem., (189), 421, 1951.

(106) Ackermann, W. W., and Johnson, R. B. : J. Exp. Med., (97), 315, 1953.

(107) Labaw, L. W., Mosley, V. M., and Wyckoff, R. W. G. : J. Bact., (60), 511, 1950; (65), 330, 1953.

(108) Madden, S. C., and Whipple, C. H. : Physical Revs., (20), 194, 1940.

(109) Borsock, H. : Physiological Revs., (30), 206, 1950.

(110) Geiger, E.: Science, (111), 594, 1950.

(111) Spiegelman, S., and Kamen, M. D. : Science, (104), 581, 1946.

(112) Davidson, J. N.: Ann. Rev. Biochemistry, (18), 155, 1949.

(113) Brachet, J. : Growth, (11), 309, 1947.

(114) Lipmann, F., and Kaplan, N. O.: Ann. Rev. Bioch., (18), 267, 1949

(115) Szent-Györgyi, A.: Science, (110), 411, 1950.

(116) Munch-Petersen, A. : Nature, (162), 537, 1848.

(117) Mommaerts, H. M. : J. Biol. Chem., (188), 559, 1951.

(118) Mommaerts, H. M.: J. Biol. Chem., (198), 445, 459, 469, 1952.

(119) Spicer, S. S., and Rozsa, G. : J. Biol. Chem., (201), 639, 1953.

(120) Griffin, A. C., et al.: Cancer, (4), 410, 1951.

(121) Schoenheimer, R., et al.: J. Biol. Chem., (130), 703, 1939.

(122) Shemin, D. and Rittenberg, D.: J. Biol. Chem., (153), 401, 1944.

(123) Winnick, T., et al.: J. Biol. Chem., (175), 117, 1948.

(124) Melchior, J. B., et al.: J. Biol. Chem., (189), 411, 1951.

(125) Simpson, M. V., and Tarver, H.: Arch. Bioch., (25), 384, 1950.

(126) Brunish, R., and Luck, M. : J. Biol. Chem., (197), 869, 1952.

(127) Siekevitz, P.: J. Biol. Chem., (195), 549, 1952. 
(127a) Zamecknik, P. C. and Keller, E. B. : J. Eiol. Chem., (209), 337, 1954.

(128) Tiselius, A., and Horsefall, F. L. : J. Exp. Med., (69), 83, 1939.

(129) Chantrenne, H.: Biochim. et biophys. acta, (1), 437, 1947.

(130) Novikoff, A. et al.: Histochem. and Cytochem., (1), 27, 1953.

(131) Moriyama, H., and Ohashi, S.: Z. Imm., (99), 349, 1941.

(132) Moriyama, H., J. Shanghai Sci. Inst., (3), 1091, 937.

(133) Holker, J.: J. Path., (25), 522, 1922.

(134) Moriyama, H. : Arch Virusforsch., (1), 430, 517, 1940.

(135) Klotz, I. M., et al.: J. Am. Chem. Soc., (72), 3972. 1950.

(136) Bailey, K., and Sanger, F.: Ann. Rev. of Bioch., (20), 103, 1951.

(137) Beard, J. W.: Ann. Rev. Microbiology, (5), 272, 1951.

(138) Bergold, G. H., and Wellington, E. F.: J. Bact., (67), 210, 1954.

(139) Bergold, G. H. : Ztschr. Naturforsch., (26), 122, 1947.

(140) Coons, A. H. ct al.: J. Exp. Med., (93), 187, 1951.

(141) Anderson, T. F. : Bot. Rev., (15), 464, 1949.

(142) Herriott, R. M. : J. Bact., (61), 752, 1951.

(143) Mast, S. O.: J. Morphol. Physiol. (41), 347, 1926.

(144) Seifriz, W.: Advances in Enzymology, (7), 35, 1947.

(145) Monné, L.: Advances in Enzymology, (8), 55, 1948.

(146) Blout, E. R., and Doty, P. M.: Science, (112), 639, 1950.

(147) Pijper, A.: J. Bact., (53), 257, 1947.

(148) Rozsa, C., et al. : Biochim. Biophys. Acta, (6), 13, 1950.

(149) Chambers, R.: Biol. Bull., (93), 191, 1942.

(150) Sanders, F. K. : The II nd Symposium of Soc. for Gen. Microbiology, 297, 1953.

(151) Haurowitz, F.: Progress in Biochemistry, 1950.

$(152)^{\circ}$ Fischer, A.: Bioch. Zeit., (279), 108, 1935.

(153) Chargaff, E. : Advances in Enzymology, (5), 31, 1945.

(154) Laurell, C. B. : Blood, (7), 555, 1952.

(155) Astrup, T.: Advances in Enzymology, (10), 1, 1950.

(156) Quick, A. J.: Science, (106), 591, 1947.

(157) Steiner, R. F., and Laki, K. : J. Amer. Chem. Soc., (73), 882, 1951.

(158) Schmidt, W. J.: Die Doppelbrechung von Karyoplasma, Zytoplasma, und Metaplasma. Berlin, 1937.

(159) Schwartz, D.: Science, (119), 44, 1954. 



\section{PART V}

\section{THE NATURE OF EVOLUTION}





\section{CHAPTER I \\ THE THEORY OF MEMORY}

\section{The Faculty of Protein to Memorize Its Structure}

As was described repeatedly, many evidences can be presented suggesting that protein molecules can return to the structural pattern, which they once possessed, when their structure is disturbed by some adequate agents. The writer designates such a phenomenon as the reversibility of protein structure or the memory of protein. Since this phenomenon is of the most importance to elucidate the mechanism of both the individual development or ontogeny and the organic evolution, it will further be discussed in this chapter in great detail.

In general, the reversible inactivation of physiologically active proteins such as enzymes and toxins can be looked upon as based upon this character of proteins. With viruses many phenomena apparently due to this character are also known. For example, a virus which has been changed in its property by an environmental change may recover its original property when brought under another environmental condition.

This phenomenon can be interpreted as indicating that a protein which can take two structures, $A$ and $B$, attains one structure, $A$, under a condition where $A$-structure is stable, while under another condition where the other structure, $B$, is stable it is shifted to the structure $B$ as follows :

$$
A \text {-structure } \underset{a}{\stackrel{b}{\rightleftarrows}} B \text {-structure. }
$$

$A$-structure is stable under the condition of " $a$ " and $B$-structure under " $b$ ", but in most cases $A$-structure seems not to be shifted to $B$ by a mere change of the environmental condition, $a \rightarrow b$, unless some proper stimulus disturbing the protein structure is provided. This may be analogous to the phenomenon that water can be coold under $0^{\circ} \mathrm{C}$. without changing into icc, but that the super-cooled water will be suddently frozen over on the application of an appropriate stimulus. This phenomenon was already discussed as we dealt with the denaturation of proteins (Chapter $\mathrm{X}$, Part III). 
It has been known that bacteria or phages damaged by ultraviolet light can be fairly reactivated by the subsequent exposure to visible light (1). This fact may be explained if we assume that reactivation occurs because reactivated structure is stable under the exposure to visible light which may cause the proteins to return to their original structure. Bawden and Kleczkowski (2) have found with Phaseolus vulgaris that irradiation for two to three minutes with ultraviolet light had not immediately obvious effects on the leaves, but that when kept in the dark for twenty-four hours after irradiation, became severely bronzed within the next two days; this was prevented when the irradiation was succeeded by exposure for some hours to day light. In this case day light presumably acted upon the leaves to make its protoplasm structure, which had been changed by the ultraviolet light, return to the original structure before the symptoms came to the forth.

It has been found that a strain of ultraviolet irradiated $E$. coli can be reactivated also by heat (3); the reactivation has been reported to be likewise possible by the addition of various metabolites (4). Frequently we observed that phage inactivated by unknown cause is reactivated by heating to an adequate temperature or by the addition of inorganic salts (5). Heat or salts in these cases may disturb nonspecifically the protein structure to initiate the returning change. Visible light may act in a similar way, but in addition it may exert a specific effect in favour of the original structure.

Herpes may occur following febrile diseases and poliomyelitis following tonsillectomy, and usually common cold is associated with chilling. These phenomena may be based upon the reversion of virus structures rather than upon the generation of the viruses themselves. Vago (6) has claimed that a virus which causes polyhedron disease is present in the majority of the insect, Bombyx mori, remaining latent through several generations, but it is activated and accordingly the disease is developed by the administration of fluorine compounds or by the feeding of insects on the leaves of the Maclura.

The recovery from virus diseases is possibly ascribed to this memory phenomenon. Thus, when an environmental condition, under which the structure of a virus is stable, is provided in the protoplasm of a certain organism, the virus will be generated or the organism will be readily infected by the virus, whereas if the environment is changed and a new environment is favourable for the original structure, the virus structure will be expelled with the cure of the disease.

On the one hand, as already mentioned, seasonal effect is striking on the incidence of virus diseases, while on the other hand functions of hormones are under the influence of seasonal factors. This suggests strongly that the hormones are involved in the environmental 
condition directing the virus structure.

In some particular cases, however, it seems that temperature alone can act as the directing factor. According to Kunkel (7) aster yellow virus is lost when the leafhoppers carrying the virus are exposed to $32{ }^{\circ} \mathrm{C}$. The insects exposed to this temperature for a day lose the virus, but on lowering the temperature to $24^{\circ} \mathrm{C}$, the virus appears in a few hours, whereas those heated for a week regain the virus only after two days or longer at the lower temperature; the virus is never regained when the insects are heated for more than 12 days. This fact indicates that the structure of the virus is stable at $24^{\circ} \mathrm{C}$. but unstable at $32^{\circ} \mathrm{C}$. at which the normal protoplasm structure is stable. It is highly suggestive, however, that the prolonged exposure to the high temperature renders the virus structure irreversible. This is considered to be dependent upon the "oblivion" of the virus structure, an extremely important phenomenon which should be discussed later in great detail.

Numerous facts indicating that the structure of plant viruses are apparently unstable at higher temperatures have been shown. For example, Vinca rosea and Nicotiana rustica infected with aster yellow virus are freed from it after growing for two weeks at $40^{\circ} \mathrm{C}$. and $V$. rosea is also cured by immersion in water at $45^{\circ} \mathrm{C}$. for a few hours. This $V$. rosea is also freed from potato witches' broom and cranberry false blossom virus by growing for 14 days or more at $42^{\circ} \mathrm{C}$. Potato tubers with diameters up to $2 \mathrm{~cm}$., infected with witches' brooms virus, are cured by storing at $36^{\circ} \mathrm{C}$. for 6 days (8).

This seems to be the case also with animal viruses. We may generally become feverish following the infection with a virus, a phenomenon which may be of significance from the point of the expulsion of virus structure. It has customarily been believed that sweating treatment with a sudorific or with a hot drink is effective to commoncold. It cannot be said, however, that high temperatures are always effective to make virus structures unstable. Since herpes febrils is generally raised accompanying with feverish diseases, the structure of herpes virus must be stable at higher temperatures in contrast to that of common-cold virus.

Thus, a virus structure may be lost when the environmental conditions become unfavourable for its existence, resulting in the cure of the diseases, but as the virus structure itself is also reversible the disease will return when the conditions become again favourable for the virus structure in so far as the structure is not "forgotten".

At present, chemotherapy is winning a brilliant victory over the campaign against some bacterial diseases. The effect of the drugs on virus diseases, however, appears to be insignificant, presumably 
because of the likeness of the virus structure to the normal structure of the protoplasm and because of the concealment of virus structure in the protoplasm, not existing as an utterly independent entity. This may also hold for bacteria; namely, chemotherapy may be ineffective against bacterial pattern which is present in the protoplasm in a latent state or in a state not yet developed into their full pattern. For instance, chloromycetin, according to our observation, can for a period of time expel dysentery bacteria from the carrier but the bacteria become soon demonstrable in the feces, indicating that the antibiotic is ineffective to the undeveloped pattern of the bacteria. Streptomycin may likewise be effective only to the tubercle bacilli having the completely developed pattern, so that the secondary infection by the bacteria themselves may be well effected by the drug, but with the incomplete pattern or the latent bacteria in the tissues from which the bacteria are being produced it may be powerless.

An accomplishment of radical therapy by physical or chemical agents, however, may not be impossible if it is attempted from the view point of the reversibility above suggested. Red-ray therapy is an old practice for small-pox, that is, red-ray exhibits beneficial effect upon the disease which will be cured readily if the patient is exposed to red-light. This should be comparable to the fact that bacteria or phages damaged by ultraviolet-ray are activated by visible rays with the recovery of the original structure. Similarly, tobacco-mosaic is known to be cured by the exposure to red or blue light, whereas some other plant virus diseases are said to be intensified by the visible ray (9).

Chemical agents may also be efficacious in this respect. The agents must have hormone-like action in order to expel the unfavourable structures. It has been found by Wright (10) that coli bacteria which underwent a mutation can recover their original character in the presence of certain organic acids having a particular configuration. Tartaric acid has this particular configuration and therefore proves to be effective, but it should be noted that only natural D-form is active, the L-form being inactive, suggesting its hormone-like action.

Again according to Hamre et al. (11) benzaldehyde thiosemicarbazone reduced the fatality rate of mice inoculated intranasally with vaccinia virus. This chemical agent does not appear to inactivate the virus as a result of prolonged contact in vitro. Derivatives with substitutions in the para-position of the benzene nucleus or in the 4-position of the thiosemicarbazone molecule produce little or no protection against vaccinia infection in mice (12). The $=\mathrm{N}-\mathrm{NH}-\mathrm{CS}-\mathrm{NH}_{2}$ group, as such, appears to be essential for antivaccinal activity (13). Ackermann (14) found that administration of sodium fluoroacetate to mice 
infected intranasally with influenza virus inhibits the growth of this virus in the lungs of the mice; further he (15) discovered that three $\alpha$-aminosulfonic acids cause marked inhibition of multiplication of influenza virus in tissue of the embryonated egg. Again, it has been reported that 2, 4-dinitrophenol inhibits completely the propagation of influenza virus in cholioallantoic membrane (16). These reagents showed no virucidal effect in virro.

As stated above, the so-called antibiotics may affect directly the complete pattern of pathogens and accordingly their effectiveness seems to be restricted. Shope (17) has shown recently, however, that a certain mould can produce an agent which may provide an unfavourable condition against the production of a virus pattern. He has isolated an antiviral substance from penicillium funiculosum, which is effective upon infection of mice with viruses such as SK encephalomyelitis virus. He believes that the agent causes an inhibition or interruption of multiplication of the virus presumably by interference with some stage in the developmental cycle of the virus.

However, not all the agents which prevent the virus-reproduction can be regarded as being capable of specifically acting to inhibit the development of a certain virus pattern. Some may non-specifically alter the protoplasm structure to render it inadequate for the establishment of the structural change due to some viruses.

Mirick et al. (18) claimed that urethane, given paraentrally or orally to mice, increased the severity of the infection with pneumonia virus of mice. Not only were the lesions more extensive but mice could be infected with smaller inocula of virus and the multiplication of virus in the lung was enhanced. It should be naturally expected that certain reagents are able to inhibit the development of a virus pattern, while others promote it as in this example.

\section{Training Effect and the Oblivion of Memory}

The reversibility of protein appears to be especially manifest in the protoplasm. Meanwhile, it should be noted that in the protoplasm the reversible change from the normal to a certain other structure appears to be facilitated by the repetition of the change. The socalled anamnestic reaction (anamnesis=recollection) may be cited as an example of this phenomenon. This reaction consists in that an animal, which previously produced antibody in response to a specific antigenic substance, will begin anew to produce the antibody vigorously when a minute quantity of the antigenic substance is injected. For the establishment of the reaction the repeated preliminary injection of the 
antigenic substance is generally necessary; this reaction may depend upon the recovery of the former pattern which was provided and strengthened by the repeated injection of the antigen, as the pattern produced by the antigen can be regarded as the changed structure of protoplasm protein of the antibody-producing cells.

An elastic substance, as for example a rubber tube, may become easily bent at a definite pcrtion at which a bending was previously applied. If the bending is repeatedly applied at the same portion, it will become more supple, and the bend will occur at the portion even when a force is applied at another portion. Anamnetic reaction is said to occur likewise even when a proper substance different from the former antigen is injected. The structural reversibility of protoplasm protein can thus be analogized with the elasticity of elastic substances.

On the other hand, there are many evidences to prove the presence of elasticity in the protein molecule itself. Organisms are without exception composed of protoplasm whose main components are proteins, while protoplasm itself is elastic and accordingly all the organisms are elastic, a fact which may be based upon the elastic nature of the protein. Strong elasticity can actually be demonstrated in isolated proteins such as gelatin or fibrin. As is well known, casein can produce an elastic paste if treated with alkali. Such an elasticity may be attributed to the thread-like form of the molecules which can combine end to end to form a long chain as in the case of other elastic substances. The elasticity of proteins appears to be not only revealed against physical forces but also against chemical agents.

A splendid application of this elastic nature of proteins, $i . e$. the reversibility, can be seen in the memory of nervous cells. When an animal is repeatedly subjected to the same kind of stimulus, the reaction to the stimulus will become more rapid and more exact, thus resulting in the training effect and in the strong memory. The protoplasm structure of our brain cells may undergo a change in response to a stimulus; the change will soon fade away, but when afterwards a related stimulus is given, the change will be revived owing to the reversibility of the structure. This must be the recollection of the stimulus. Every one knows that recollection is strong when the stimulus was previously given repeatedly or severely, and the recollection of a certain affair, when its "impression" was strong, is given rise to by some stimulus which has no direct connection with the affair.

Some memories are long-lasting, strong impressions being generally held in the brain cells for life. Since the protoplasm protein of the brain is to be renewed repeatedly during such a long period of time, the memory should be transmitted successively to newly formed pro- 
tein molecules. The renewal of the protein in the brain cells may be tedious as compared with that of other cells, but it seems little doubt that even the brain cells have to change occasionally the old protein molecules with new ones. If the pattern of the memory was not transferred to the newly synthesized protein, the memory of the cells would be lost on every renewal of the protein. Thus, the protein holding a memory must involve a specific structural pattern corresponding to the memory. In other words, the transmissible pattern of memory must be a structure produced in the protoplasm protein by the "impression".

Now, if we assume that structural factors which may prevent the change of a protein from $A$ to $B$-structure are removed when the protein achieves the change $A \rightarrow B$, it may be expected that the protein having recovered the original $A$-structure, will achieve the change more readily than before, and since the repetition of the change may result in the more complete removal of the preventing factors, the repetition of the change will facilitate the reaction the more. The protein structure resulting from the removal of such factors can be considered as the pattern of the memory. This may be comparable to a path beaten across a wilderness overrun with grass. If a man traverses the pathless wilds for the first time, he must have a hard walk, but on repeating the traverse the grass will be gradually removed until at last an easy path will be made. The above cited instance that the rubber tube is easily bent at a certain part on the repetition of the bending at that part, may also depend upon the removal of the structural factors which prevent the bending.

The path beaten across the wilds by repeated walk will be lost by being overgrown with weeds if the walk is given up for a long time. In a similar way, oblivion may occur in the brain by the reappearance of the preventing structural factors which were once removed. Thus it can be said that oblivion of the memory is also a result of the structural return to the original form.

The concept thus far formed of the memory phenomenon is illustrated in Fig. 32. Protein-I which is folded in the form-A under a certain environmental condition is converted to the form- $B$ when the environment is changed in favour of the establishement of B-form. During this change the protein is temporarily to be unfolded into the polypeptide chain; the side chains represented by $\oplus$ and $\Theta$ is assumed to prevent the change, $A \rightarrow B$. The repetition of this change leads to the removal of these preventing side chains, and as a result protein-I is changed in its structure into 11 .

When such a change is once established, the conversion to B-form will occur with facility, training effect being thus attained. Since the 
protein molecule having synthesized as a replica corresponding to such a template protein possessing the structure of a memory should have the same structure of the memory, the memory or the training effect can be "inherited". However, if the training is neglected, the side chains, $\oplus$ and $\Theta$, will gradually reappear, with the recovery of Istructure, on account of the reversible character of the protein struc. ture, thus occurring the oblivion of the memory.

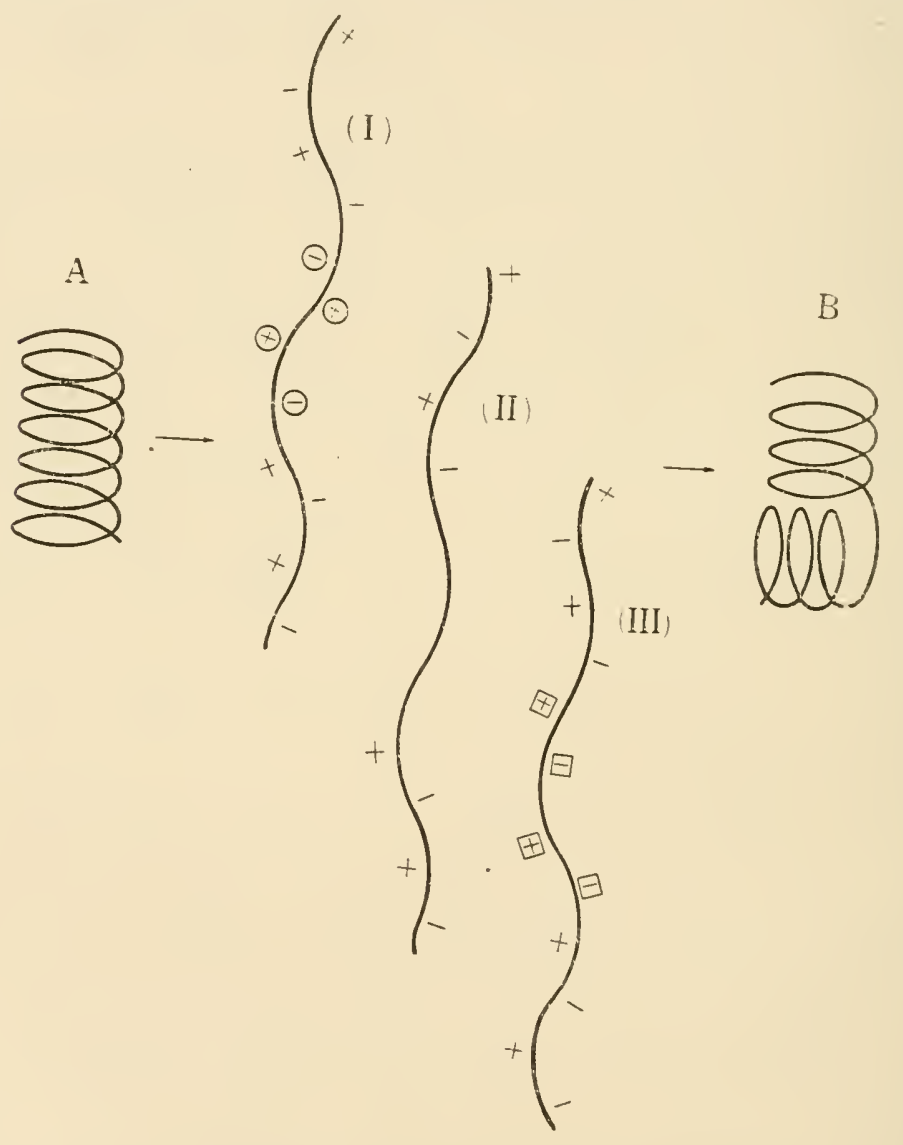

Fig. 32. Diagram of memory and oblivion in proteins.

The situation may be somewhat different with B-form; thus, when the protein long stands without returning to A-form, under the environment which favours B-form the side chains will reappear but not in the same arrangement as in I; the arrangement occurs so as to be favourable for the protein to take the B-form. As a consequence, 
the protoplasm structure is converted to III. Thus, both forms, A and B, possess each specific structure. If the protein acquires IIIstructure B-form becomes the normal state of the protein, so that the return to $A$-form becomes difficult, that is, when the protein stays for a prolonged time at B-form it will forget $\mathrm{A}$-form.

The newly production of the rearranged side chains in the protein left long under a new environment must be the result of the rearrangement of polar groups so as to become adaptive to the new form. The same rearrangement of polar groups will take place when the protoplasm is affected by a virus, in response to whose structure the polar groups of the protoplasm protein are rearranged. Such adaptive rearrangement of the structure appears to be a common character of proteins, but the character is never peculiar to proteins, since similar adaptation to a new environment can occur with usual elastic substances other than protein. A piece of elastic thread, for instance, whether it is composed of protein or steel, after standing in A-form for a prolonged period can be changed to B-form only with difficulty at first, but the repetition of the change will make the change easy and a long maintenance of the B-form will render the form normal, the return to the original form becoming difficult. Also in such a case, the adaptation may occur owing to the modification of the inner structure of the thread to adapt itself to the new form.

\section{The Cause of Aging}

It has been known for a long time that generally colloids including proteins grow old like livings. In the opinion of the writer, the process of just considered adaptation of the protein to environments leads in the long run to aging. During the adaptation process the fixation of the inner structures may be advanced so far that the forms of the protein molecules become hardly changeable to other forms. Such a hardening of the structure is considered to be aging.

We shall consider in detail of this phenomenon with Fig 33. Now it is assumed that a polypepide chain can take two forms, $a$ and $b$, according to the environment, $A$ and $B$, under which it stands. If the protein is left for a prolonged period under the same condition, the inner structure will gradually be fixed by the rearrangement or the shift of polar forces, until it attains the forms $a_{1}$ or $b_{1}$. The protein in such a form is in a well adapted state so that the change from one form to the other will be brought about only with difficulty, but if the change is repeatedly raised by the repetition of environmental change, the arrangement of the polar forces which may hinder the 
change will be removed or rearranged to return to the original state; as a result the change will come readily to occur.
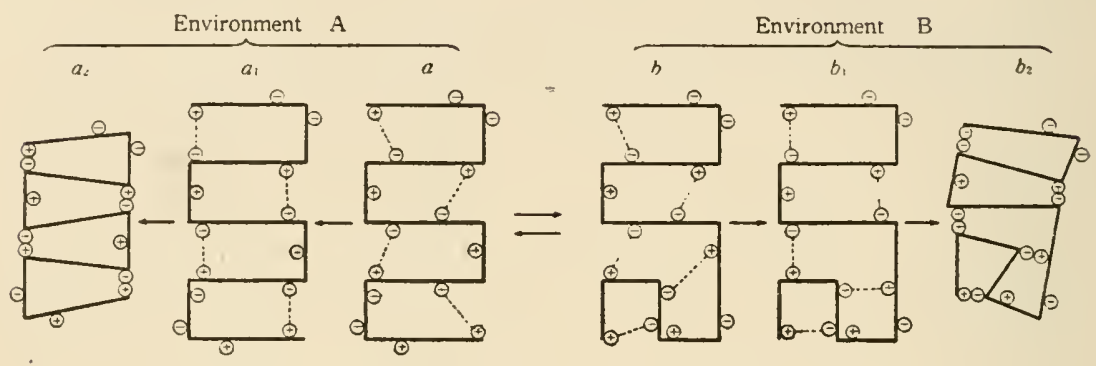

Fig. 33. Aging of protein molecule.

On the other hand, further progress of the fixation due to the persistent staying under the same environment will result in the establishment of the form $a_{2}$ or $b_{z}$. In such a state the polypeptide chain is folded very firmly by the mutual attraction of polar forces of opposite signs, leading to a shrinkage accompanied by a deformation and the loss of elasticity as well. This must be aging.

It is a well known fact that young tissues are tender and elastic, while old ones are hard and fragile. Similarly, as is generally recognized, colloids tend to change into gel when become aged, thereby the component particles becoming attached firmly and the contained water is pressed out as known as syneresis, a phenomenon which is also well acknowledged with living tissues. These phenomena may be expected from this figure. Instead of the long thread of polypeptide in this figure, many long colloidal particles may also be considered which are attached to one another in a colloidal system.

In the previous Part the writer has assumed that the senescence of viruses or of primeval organims may be mainly attributed to the effect of the assimilase action of the host or of the surrounding masses with which they are to be evolved. However, it has now become clear that another important reason for the senescence is involved in the nature of the protein itself. The senescence due to the assimilase action of the host is brought about because the viruses are persistent on the same host, that is, persistent on the same environment. Thus, it may be said that in both cases senescence, whatever the cause may be, results from the persistent staying under the same environment.

The senescence therefore, whether it be raised by the adaptive nature of the protein itself or by the assimilase action of the host 
will be prevented by the host change or in general by environmental changes. The host change as well as the sexual reproduction may be effective for the rejuvenation, as the individual is able to come into contact with other individuals by these processes, whereby the internal structures which are becoming fixed are unloosened and unfolded, and in addition the intermixing of the structural component of another individual can contribute to prevent the fixation.

As already stated, according to the writer's view, the cytoplasm is rejuvenated, presumably at every cell division, by the template action of the gene. The cells, however, must become senescent as above considered, a fact which seems to contradict with this view. But it should be realized that the gene itself gradually grows old. The cytoplasm recovers its proper form by the template action of the gene, but the template itself has to become aged with which the cytoplasm also must grow old, as the cytoplasm is to be endowed with the gradually aging pattern of the gene.

Individuals are thus bound to become old. As the aging is the general fate of the elastic substances composed of thread-like constituents, the senescence is the unavoidable fate of livings. Elastic substances such as gum, just like the living protoplasm, exhibits the phenomenon of memory and forgetting; at the same time they can adapt themselves to the environments until finally undergo senescence.

On the other hand, senescence makes a great contribution to the evolution of organisms; the evolution might be even impossible without senescence. In order to remove the old-patterned individuals for the promotion of more-advanced juniors, senescence is of course indispensable. But in addition, senescence of a proper degree plays an important part in the evolution. Since rigid structure was necessary for the continuance of the existence of the organisms, in order to achieve the evolution, to become rigid in their structural pattern would be required for viruses and primitive organisms including even primeval protein molecules. The writer considered previously that this rigidness might generally be acquired through the struggle for existence; but without this they would surely be able to become stronger in their structure, for the protein is provided with the nature in itself to become thus rigid in its structure.

Newly generated viruses are as a rule weak, but they may be able to be stabilized gradually without the struggle for existence owing to this nature. Thus, viruses and primeval organisms can acquire autonomically the rigid structure necessary for acting as strong assimilase; the rigid structure is raised without even increasing the amount of nucleic acid, the stabilizing agent of the protein.

Organisms can achieve the evolution thanks to this character of 
the proteins, a character leading to the adaptation and finally aging, and also to the recollection and forgetting, without which the evolution would be entirely impossible. The mechanism of the evolution can be fairly elucidated by taking into account of this character of the protein as will be fully discussed in later chapters. 


\section{CHAPTER II}

\section{ONTOGENY}

\section{The Principle of Individual Developmen:}

The development of a single cell into a complex, highly differentiated organism is one of nature's most marvelous pageants, a series of events that occurs in such an orderly fashion as to fill the observer with awe, and yet the writer believes that this marvelous event consists of nature's tricks only based upon the reversible nature of protein abore mentioned.

According to his concept, there is a mutually reversible relation between the protoplasm structure of germ cells and that of somatic, and somatic cells are converted to germinal cells under a series of proper stimuli under which the structure of the latter is stable. The ontogeny or the individual development is considered to be no more than the return of the germinal cell to the original, differentiated structure. Since the germinal cell can function independently of the somatic as a unicellular organism, its protoplasm must have the primitive structure of unicellular stage of the organism from which it has evolved, and the structure must have attained by the reversibility.

The spores of unicellular organisms may be analogous to the germinal cells of multicellular organisms and may be produced when unicellular organisms, such as some bacteria or protozoa, are reduced in their protoplasm structure to more primitive structure under a change of environmental conditions. A new environment under which the primitive structure is brought about must favour the reduction of the structure; as a result of the reduction the cells may be decomposed into virus-like particles, the more primitive forms of the organisms, thus producing spores, which, however, can return to the original structure under certain other environmental conditions, with the recovery of the form of unicellular organisms. As the protoplasm is looked upon as a kind of liquid crystals of proteins, the shape of the protoplasm should be determined by the structure of the protein. Therefore, the return of the structure to the original should be followed by the recovery of the shape.

As stated in the previous chapter, organisms are bound to grow old when they stand always under the same environment, but they 
can be rejuvenated by adequate environmental changes. The spores or germinal cells must be the splendid rejuvenated specimens produced by the environmental change. The spores or germinal cells may be comparable to the form $a$ in Fig. 33 which was cited in the previous chapter, and somatic cells to $b_{1}$, and accordingly $b_{1}$ is the differentiated structure of $a$, to which $b_{1}$ can be transformed if the environment is changed from $B$ to $A$. And further the form $c$, the spore or the germinal cell, thus recovered from $b_{1}$ can again be changed to $b_{1}$, the somatic cell or the vegetable form, when the environment is again changed to $B$, and if $b_{1}$ can take cell form while $a$ only virus-like particle, the cell composed of $b_{1}$ will be decomposed to the virus-like particles when brought under the condition $A$. Thus the spores or the germinal cells are looked upon as the completely rejuvenated form of the organisms.

As for higher plants, it seems generally accepted that germ cells can be directly originated from somatic cells, whereas in animals the cells, from which germ cells are to be produced, are usually believed to be distinctly separated from the somatic cells at their early stage of the development. It should be born in mind, however, that the cells which seem to be thus separated from somatic cells are not the germ cells themselves but cells from which germ cells are to be produced. Not only gonads but also other organs are generally developed separately from an early stage of the development. At present the majority of embryologists hold the view that the cells constituting gonads in which the germ cells become differentiated are not essentially different from usual somatic cells.

Many species of planarians have great power of regeneration, and when an individual of them is divided by cut into a head- and a tailpiece, each of which heals the wound and forms the missing parts by cell division, whereby germ cells are subsequently produced from the individual regenerated from the head-piece in which gonad was originally absent, indicating clearly that germ cells are produced from somatic cells.

In short, the germ cell thus produced from the somatic cell by the reduction in its structure to primitiveness can return to the differentiated structure of the somatic cell, if a proper stimulus is given under suitable conditions favourable for the development of the differentiated, original structure. This return process in itself is ontogeny. 


\section{The Mechanism of Regeneration}

Organisms can regenerate in general their lost parts, and sometimes even the whole individual is formed from a small piece. This regeneration process is particularly conspicuous in plants, although some animals such as sponges and hydroids can regenerate new individuals from cuttings, as do many plants, but in many animal species the restoration of lost parts is more limited.

It is a note-worthy fact that, in the case of organisms able to regenerate, sectioning of a structure is followed by a brief period in which the "blastema" forms, undifferentiated cells accumulating in the region of the wound (19). Thus, it appears that certain cells in the region of the wound are reduced in their structure to the primitive, undifferentiated, from which subsequently the complicated, differentiated structure is anew recovered. Regeneration must be, therefore, a reversible structural change of protoplasm just as ontogeny.

Anaerobic glycolysis is the metabolic process peculiar to undifferentiated, primitive cells and was discovered by Warburg in cancerous and in embryonic tissues. It shoud be noted that this primitive metabolic process was found in normal cells after injuries, indicating the occurrence of functional reduction in the wound regions which must be based upon the structural reduction (20).

Such a reduction of the structure to a primitive state may be caused by the stimulus of injury, and in each cut piece a primitive structure like that of germ cells may be produced, from which the original, differentiated structure will subsequently be restored, if each piece has the capacity to form a normal adult.

The remarkable process of reassociation of cells after dissociation occurs in some sponges and hydroids. For example, pieces of Microciona prolifera, the common red sponge of the Atlantic coast, can be squeezed through silk bolting cloth so that the cells are separated or dissociated. If these fragments are allowed to settle upon the bottom of a dish of sea water and remain undisturbed, they will become reassociated in small spherical masses within 24 hours and then develop as thin encristations upon the bottom (21). Attention should be paid to the fact that masses arising first are of a larval stage similar to the typical normal larva. This phenomenon may be also based on the formation of primitive structures by cutting.

Thus there is no doubt that somatic cells of certain organisms can recover their primitive structure when they are stimulated by such a simple factor as cutting. Germinal cells may develop into somatic, 
probably because the environmental condition under which the germinal cells were produced is changed to become favourable for the formation of somatic cells. However, the condition or the factor which formed the somatic cells may usually be removed when the somatic cells are once completed, so that somatic cells are existing normally under the condition favouring the primitive form, but without returning to the original, just as water can sometimes be present as such under $0^{\circ} \mathrm{C}$. without being changed to ice. Therefore, the completed somatic cells may be in a metastable state with a great tendency to change into the primitive structure. In this train of thought it is easily explained that a simple stimulus such as cutting is sufficient to cause the recovery of the primitive structure just as super cooled water is readily changed into ice on the application of a slight stimulus.

The capacity of regeneration seems to be especially great in plants. This indicates that some somatic cells of plants have particularly strong tendency to return to the primitive state. On the other hand, in higher animals regeneration occurs only to a small extent and even with difficulty. This may be due to the presence of some mechanism preventing the recovery of the primitive structure. In the majority of animals, gonad-forming cells are differentiated for the purpose of the production of primitive cells, $i$. e. germ cells, whereas, in plants, primitive cells including germ cells appear to be generated with ease from usual somatic cells not differentiated for the purpose. This fact also shows that in plants the structural reduction to the primitiveness can easily take place. Again, it is known that a variety of physical, chemical and biological agents readily induce pathological growth in plants; in general, cells of primitive structure have the capacity of vigorous growth, while pathological growth is associated with the primitive charactcr as with cancerous cells. Of physical agents inducing such a pathological growth mechanical injury is well known. Diseased growth considerably beyond that necessary for healing frequently occurs in plants following injury. A large number of chemicals are capable of inducing more or less diseased growth. This is so common that one can expect diseased growth to follow the application of almost all chemicals at a concentration which induces injury but does not kill. Also living agents, such as bacteria, fungi and nematodes, cause various kinds of pathological growth (22).

Thus, it may be concluded that plant cells are strongly inclined to be reduced to primitiveness following the application of a stimulus disturbing the protoplasm structure, in the same way as the supercooled water tends to be readily frozen on the application of a disturbing stimulus. This character is especially manifest in plants because it is a beneficial for them; namely, since this character enables plants 
to regenerate and the individuals having the stronger regenerative capacity might be able to survive the more, the character would be more and more advanced until the present status of plants was reached.

The occurrence of proliferative changes following injury, however, is observable also in higher animlas though not so manifest as in plants. Observations of Cooke et al. (23) on the changes which occurred in excised rabbit and human skin after injury have shown that even after removal from body certain tissue elements may retain the ability to react with proliferative changes in response to tissue injury if kept under artificially stimulated physiological conditions.

\section{The Formation of Organs}

Unlike the growth of usual crystals, in the development of organisms different shapes are developed on the different parts of the body with the formation of a variety of organs. Fundamental principles to induce such a dissimilarity was already discussed in Part IV, Chapter VI, stating that the main factors involved in it are considered to be hormones, which provide different stimuli to different parts of the body, according to which different restricted structures are developed, with the production of the complicated "crystal shape".

If $b$-stimulus is added to a germinal cell having $A$-structure, the structure will be converted to $B$ with the formation of the "crystal shape" corresponding to $B$-structure. In the same way, $c$-stimulus will induce $C$-structure and its "crystal shape". In this case, $B$ and $C$ are considered to be organs generated from $A$, the germinal cell, and $b$ and $c$ are the hormones inducing the respective organs. $A$ has the "predisposition" to be changed into both $B$ and $C$ in responding the respective stimuli, $b$ and $c$. In other words, $A$ recovers $B$-structure under $b$-stimulus, and $C$-structure under $c$-stimulus. As will be mentioned later $A$ is a structural complex, not a single structure. In the following the matter will be considered in a less abstract way.

As was mentioned already, protein structure is greatly influenced by environmental factors. On the other hand, the surface of an egg cell may not be similar with environmental factors, such as gravitation, light, and temperature. Whitaker (24) stated that in the egg of the brown alga, Fucus, the differentiation is clearly influenced by environmental factors before the first nuclear division occurs. Thus, gradients of visible light, ultraviolet radiation, metabolic products, hydrogen ions, and temperature are capable of determining polarity of an egg; even mechanical elongation of the egg influences its polarity. In addition, since the protoplasm consists of thread-like protein mole- 
cules arranged in parallel alignment, the cell itself should have a structural polarity. Harrison (25) believes that the polarity is based upon the dipole character of protein molecules.

Such a polarity, due to the asymmetrical character of protein molecules and numerous environmental factors as well, may control the manner of distribution of some components, such as yolk and nucleus, causing a dissimilarity of the cell mass, resulting in different structures according to different parts of the cell. The differentiation of a fertilized egg cell may thus start. At the beginning, however, the differentiation appears to be insignificant and easily reversible. As described already a newly formed structure is at first reversible and labile, but if the structure remains long under the same environment favouring the structure, it will "forget" the former structure becoming irreversible and stable with the establishment of the "adaptation" to the environment.

For example, at an early stage of development, when a piece of the tissue which is to develop into $A$-organ is transplanted to another part where $B$-organ is to be developed, the piece will develop into $B$ not into $A$. Thus specific differentiation seems not developed at an early stage, every cell of the embryo appearing capable of producing every organ according to the region into which it is planted. That is to say, each piece of tissue develops into the kind suited to the new region, and not in accordance with the place of its origin. The lack of specific differentiation is well shown by the fact that, if the first two cells of the frog embryo are separated, each gives rise to a whole embryo, although in some other animals, each of the first two cells will develop only half an embryo if separated.

However, as development progresses, certain cell masses are localized and differentiated into specific organs, and after localization is completely established in a region, differentiation is apparently independent of the influence of another region onto which it is grafted. At such a stage, many embryonic tissues, once started on their course of specialization, will continue the specialization as self-differentiating structures even when removed from the body and grown in artificial cultures.

Such a fixation of structure should be compared to the evolution of viruses. Newly generated viruses are weak and labile, but gradually become stronger and stable, acquiring a faculty not only to retain their pattern firmly, but to transfer their structural pattern to the surrounding protoplasm.

A group of cells, such as those of dorsal lip of the blastopore, known as an organizer, can be regarded as part of embryo which may first acquire a highly stable structure comparable to a strong virus. 
A piece of this part, when transplanted onto another part of embryo, is able not only to retain its specific structure but also to exert its structural influence upon surrounding tissues until a new embryo is formed around it. The ectoderm of the ventral and lateral surfaces of the frog embryos normally develops into epidermis, while ectoderm of the mid-dorsal region gives rise to the neural plate; however, when the dorsal lip of the blastopore, the portion called organizer, is transplanted beneath the ectoderm on the ventral or lateral regions of a frog at the gastrula stage, the ectoderm coverning such a transplanted dorsal lip gives rise to a neural plate, not to epidermis.

Such an action of organizer to induce a nervous system is apparently brought about by a hormone-like factor, which has proved to be a steroid. It has been shown that a variety of lipids induce experimentally a similar effect, but it cannot be said that the physiologically acting factor is entirely identical with the substances which may experimentally exhibit a similar action.

In addition to this inducing action, the organizer like viruses is capable of transmitting its structure to surrounding protoplasm. Thus, a portion of embryo which has no inducing action in itself, if transplanted onto a region near the organizer, will be endowed with the inducing action, becoming itself an organizer, indicating that the structural pattern of the organizer is transferred to the transplanted region.

In short, the organizer having developed under a specific influence of environment has a strong structure comparable to fixed viruses, and can transmit its structure like viruses. In addition it can produce a substance, a kind of hormones, which can induce peculiar "crystal shape" in its surroundings. This hormone may assist the virus-like action of the organizer, providing the specific structure to surrounding cells; as a consequence these latter cells may become the second organizer which in turn produces another hormone. Thus, differentiated, strong structures may be successively induced.

These successively induced organizers, however, may not be identical with the first organizer in their structure, even if they were induced by virus-like agents, for each portion of embryo is under each different environmental condition; each portion adapts itself to each environment to attain strong structure; and if a stronger structure is present near it, it will be influenced by the structure, but without becoming entirely identical with the stronger structure which may exert its influence as a virus. According to their different structures, each organizer may produce each different hormone-like substance, thus extremely complicated "crystal shape" being formed.

Since the substances behaving as hormones, whether steroid or 
protein, are determined in their structure by the protoplasm from which they were produced, they will vary in their structures if the cells from which they are to be produced are being changed as the embryo is developed. It has been found that the press juices of chick embryos of different incubation times acted differently on the growth of cultures of fibroblastic cell (26), indicating that the composition of the press juices which may act as hormones undergo changes during development of the embryos, and so it can be concluded that the composition of the body fluid which can exert hormone-like effect is gradu ally changing during ontogeny.

In a study on growth and mortality effects produced in early chick embryos by antiserum, results were obtained which point to the possibility that there are different protein complexes which make their appearance in the chick embryo concurrent with the embryo's development (26a). Crystals of the haemoglobins of the human adult and of the human foetus have different shapes (27); the difference is also demonstrable in their X-ray interferences (28), showing likewise that the protein structure is changing during the course of development. The change in protoplasm structure, which may vary with different regions of the body, will thus be advanced with the formation of various shapes and functions.

In higher organisms, hormones are, of course, distinct from viruslike agents, but at an early developmental stage of embryos or at an extremely primitive stage of organisms, the difference between the two might be subtle. If the protein, the main component of the protoplasm, is freed from the protoplasm in an orderly polymerized state, it may function as a virus, but when the protein or other components such as steroid having the pattern of the protoplasm are liberated from the protoplasm, not forming orderly polymerized masses and accordingly in a state unable to act as a virus, they may be able to act as hormones and are capable of exerting their specific structural influences, although unable to produce the exact replicas as do viruses. Consequently, primitive hormones may be regarded as incomplete viruses, only being capable of exerting on other protoplasm uncertain influences specific to their structures. At such a primitive stage, there might entirely be no essential differences between hormones and enzymes (see Part IV, Chapter V). The specialization of these factors must be advanced as the organisms are evolved or the emiryos are developed.

The differentiation of the protoplasmic structure, which may var: with different parts of the embryo, may not only lead to the produc. tion of different hormones or enzymes as well as of different virus-liki agents, but also may cause the different distribution of inorganic salts 
or other substances which may further promote the differentiation. As mentioned already in Part IV, Chapter VI, inorganic salt ions exhibit hormone-like function.

Since the famous researches of Vogt, numerous facts have been found by a number of workers to show the occurrence of considerably rapid movement of component cells in developing embryos (29). Through this movement some proteins with a certain pattern may be able to contact with certain other proteins having other patterns leading to the establishment of "rejuvenescence"; thus the cells may contribute to make the development the more complicated.

As discussed in the previous section, the mechanism of regeneration is identical with that of ontogeny, so that phenomena observed in ontogeny are also proved in regeneration. If, for example, the regeneration bud which is replacing the lost leg of a newt is grafted onto the base from which a part of the tail has been removed, the bud becomes tail instead of leg. That is, the bud tissue is in the state of very early gastrular cells which can readily conform themselves to the environments under which they are placed. Such a submission to a new position is based upon the juvenile character of the bud structure, weaker than that of the protoplasm upon which it is transplanted, accordingly it may be said that the bud is subjected to the organizer of the part.

However, the position effect is, of course, not alvays attributable to an organizer. Thus, if Tubularia, a simple marine animal, is decapitated it will regenerate its head, that is, the portion bearing mouth and tentacles. If, however, the animal is cut off at both ends and that which bore the head is buried in the sand, a new head is regenerated at the free or foot end and a foot at what was originally the head end. If both ends are buried in the sand none of them regenerates a head, and if the body is suspended free in the water both ends develop heads (30). Here, evidently, some external condition such as contact, or some other external chemical or physical factors determine whether the regenerative cells make a head or a foot.

In both cases of regeneration and ontogeny, the first direction of differentiation may be determined by external conditions and subsequently specific structures are developed, which, if developed into a certain extent, will become stabilized so as to be able to act as organizers.

To sum up, the individual development including regeneration appears to be a phenomenon in which the property of protein capable of changing its structure in conformity with the environment is revealed to the utmost; the changed structure is unstable at first but gradually becomes stronger and capable of exerting its structural influence upon 
others according to the general property of protein concerning memory and forgetting. The change will be continued until the differentiated structure is recovered from which the primitive structure of the germ cell was induced, thus the reversible change of the germ cell to the somatic being established.

Now, we have to consider this problem in connection with genes. Since protoplasm structure is controlled by genes, the change of the former should be involved in the change of the latter. It may be said, therefore, that genes are changed during ontogeny in every direction according to the various parts, or organs, of the body, so that every organ or tissue in a fully developed individual must have its own specific genes.

This conclusion may seem unreasonable to the majority of authors who believe firmly that genes are never changed except by mutation. Nevertheless, the evidences strongly suggesting the ununiformity of genes among various organs even in one and the same individual have generally been known. Namely, on the one hand, the immunological fact that organs have their antigenic specificity, that is, that organs contain their own specific proteins is well known; whereas, on the other hand, it is generally accepted that the protein structure in a cell is governed by the genes. It should naturally follow from these two facts that cells of every organ and tissue share their specific genes, never similar to each other.

The necessity of stressing the importance of chromosomes in the organization of plants and animlas, and their decisive role in inheritance, caused O. Hertwig in 1918 to formulate the "Law of constancy of chromosome number" which implies that all cells of a given organism contain the same somatic number of chromosomes. A great number of observers have, however, shown that his concept is in need of revision. Especially in insects and in plants, it has been established that certain cells and even whole tissues may represent different degrees of polyploidy. It has been found that a similar variation occurs also in the somatic chromosome number of man (31). This may be regarded as another evidence indicating the ununiformity of genes.

Thus it may be said that ontogeny is a phenomenon brought about by a complicated change of the gene in the germ cell, as is illustrated diagrammatically in Fig 34 . In this diagram, $A$ represents the gene or the gene-complex in the germ cell, $A^{\prime}$ and $A^{\prime \prime}$ the genes in somatic cells developed from the germ cell, and $A^{\prime \prime \prime}$ the gene of the mother germ cell developed also from the germ cell.

$A$ can be converted to $A^{\prime}, A^{\prime \prime}$ and $A^{\prime \prime \prime}$ respectively, by the action of the environmental factors, $a^{\prime}, a^{\prime \prime}$ and $a^{\prime \prime \prime}$. $A^{\prime \prime \prime}$ has the property to be readily reduced to $A$ by the effect of $x$, Also between $A^{\prime \prime \prime}$ and $A^{\prime}$ 
or $A^{\prime \prime}$, there is an intimate correlation, and both $A^{\prime}$ and $A^{\prime \prime}$ continuously exert structural influence upon $A^{\prime \prime \prime}$, an important assumption which will be discussed in great detail in later Chapter.

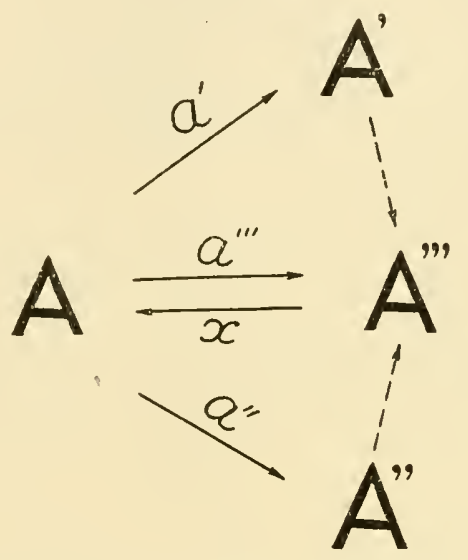

Fig. 34. Relationship between germinal and somatic cells.

$A$ : gene of germ cell. $A^{\prime}, A^{\prime \prime}$ : genes of jomatic cells.

$A^{\prime \prime \prime}$ : gene of mother germ cell.

That $A$ can reveal various structures under varying environmental conditions must indicate that $A$ consists of different proteins, each of which has different memory, since a single protein may have commonly only a single memory. Protein molecules having a similar memory may form a gene and the memory may be involved in a restricted, specific structure of the gene. Therefore, $A$ must be a gene-complex, consisting of many different genes. It is actually known that a single germ cell is provided with numerous different genes. $A$ can be converted to $A^{\prime}$ under the environment of $a^{\prime}$, because there exists in $A$ a gene having the memory to recollect $A^{\prime}$-structure under $a^{\prime}$-environment. If a gene in $A$ thus recovers the proper structure $A^{\prime}$, other genes remaining in their undeveloped structures must be subjected to this developed gene, and accordingly the protoplasm of the cell must have the structure of $A^{\prime}$. In a similar way, $B^{\prime}$-structure is established by another gene. "Predisposition" of the organism must be such a memory of genes.

A gene can change its structure in such an easy manner, but the direction of the change is generally fixed, and a changed gene should, as a rule, resume a definite structure under a given condition. Therefore, the structure of a gene is generally constant under a given condition. In this respect, the concept of the auther conflicts in no way with the customary view of the constancy of genes. 


\section{The Significance of Fertilization}

The germ cell with primitve structure returns to its differentiated state through the development and this developmental process is generally initiated by fertilization. Fertilization is comparable to the application of a stimulus to super-cooled water, which will be frozen by the stimulus; super-cooled water corresponds to the egg cell and the stimulus to the sperm.

Somatic cells or mother germ cells are reduced to their primitiveness to produce egg cells, but when factors are removed which caused the reduction and the eggs are brought under the condition which favours the differentiated structure, the egg cells may be in a metastable state, tending to return to the original, differentiated state. This is the reason why germ cells are compared to super-cooled water.

The sperm cell may act as a stimulus to initiate the recovering change because of its structure somewhat different from that of the egg. Similar effect can be provided by numerous forms of artificial means, such as subjection of the egg to certain chemicals, to changes in temperature or in density of the surrounding water, or even to mild shaking, or to the prick of a needle; all these can initiate fertilization as does the sperm. Natural parthenogenesis, which is common among invertebrates, must be caused by a proper stimulus provided by a natural process.

The development occurs in general only from the egg cell because the egg, in contrast to the sperm, contains a plenty of cytoplasmic substances, which will provide the energy and substances required for the development. Sperm cells like eggs must be in a metastable state, tending to return to the original specialized state, so that they can derelop like eggs if cytoplasmic substances are provided. It is actually known that if a sperm is inserted into an egg cell from which the nucleus was preliminarily removed, the development will take place from the sperm, a phenomenon known as merogeny.

If the returning process of a germ cell to its original state is once inititated by a stimulus of a sperm or of another factor, the gene in the germ cell will start on a gradual change towards the developed structure, and the protoplasm or the embryo will be compelled to take one form after another in succession, in response to the structure appearing successively in the returning process, since the form of an organism is determined by the "crystal shape" of protoplasm, which in turn is governed by the structure of the gene. The cell division may be required for the acquisition of the compelled form. 
A number of facts have been found that striking disturbances are raised in the egg cell following the fertilization like in super-cooled water or in super-saturated solution to which vigorous shaking is applied. Thus, unfertilized egg behaves like a cell in a "latent" state, all the metabolic exchanges being extremely slow, but on the fertilization they are suddently elevated. This may chiefly be attributed to the temporary liberation of polar groups. It should be remembered that a protein molecule in a metastable state will change into a stable state if a suitable stimulus is provided to initiate the change, and that at the first stage of the change the protein molecule is to be unfolded with the temporary liberation of free polar groups.

On the one hand, as was previously stated, the combination between proteins and lipids in the protoplasm becomes occasionally loose when the protoplasm structure is disturbed by the infection with a virus. On the other hand, it has been reported that lipids which cannot be extracted from unfertilized egg become easily extractable following the fertilization (32). It has been claimed, however, that a part of cephalin will combine firmly with protein after the fertilization (33); this may depend upon the liberation from the protein of polar groups capable of combining with cephalin. Such a liberation of free groups may cause the mutual association of proteins leading to the coagulation, which has been observed with eggs of the sea urchin to begin three minutes after insemination, obtaining its maximum in seven minutes, and being maintained thereafter unchanged for two hours (19). If the eggs of the sea urchin are frozen at $-77^{\circ} \mathrm{C}$. and then desiccated at $-25^{\circ} \mathrm{C}$, one obtains a fine powder that may be extracted at low temperatures with $\mathrm{KCl}$ solution at $\mathrm{pH}$ 7.3. Under this condition, 82 to 85 per cent of the proteins from the unfertilized egg dissolve, while for the fertilized egg this becomes 69 to 72 per cent (19). A portion of the egg proteins, more than 10 per cent of the total protein, thus becomes insoluble at the time of fertilization, presumably because of the mutual combination or the coagulation of the proteins due to the liberated polar groups.

It is generally known that a variety of enzymes are activated following fertilization. This activation may be attributed to the liberation of active enzymatic structures due to the disturbance by the sperm. During the coagulating process of blood a series of enzymes or enzyme-like factors are successively produced as stated in the last Chapter of Part IV. In the protoplasm of the egg the situation may become the same on the fertilization as in the coagulating blood plasm.

Serum protease, also known as plasmin and fibrinolysin, has been shown to be present in an inactive state as a natural constituent of mammalian blood. Numerous activating agents have been recorded 
for this enzyme. The treatment with chloroform or the addition of bacteria or starch paste are sufficient to activate the enzyme. More recently activators from tissue and serum have been recorded. The most rapid and potent activators have been prepared from bacterial sources, notably the so-called streptokinase from streptococcal culture filtrates, and staphylokinase from staphylococcal cultures. These activators may be comparable to a sperm or artificial stimuli that can initiate the development of the egg cell; the action of all these agents consists in the faculty of disturbing the protein structure.

The sperm cell may thus initiate the development of the egg cell as a mere stimulus, but besides this the sperm will play a decisive role in the establishment of rejuvenescence as already discussed in Part III. It must be desirable for the rejuvenescence that the sperm is as different as possible in structure from the egg. This may be the reason why hybrids are generally robust. However, two protein molecules cannot combine if their structures are entirely different from each other, so that for the establishment of the fertilization the sperm cannot be different from the egg too extensively. The development of the egg of a species of frogs or newts may sometimes be initiated by the sperm of some other species, but the embryos will perish earlier or later (34), presumably resulting from the difficulty of the mutual fusion on account of the too different structures. 


\section{CHAPTER III}

\section{THE DEVELOPMENT OF CANCERS}

\section{The Reduction of Protoplasm to Primitive Structure}

Since all the somatic cells have their origin in germ cells, all kinds of somatic cells, if their structure is reversible, should be reduced to primitiveness of germ cells when a suitable stimulus is given under a proper condition. In fact, as pointed out in Chapter I, the reduction tends to occur without difficulty in some lower animals and generally in plants, so that regeneration is common among these organisms. In higher animals, however, the reduction is not so common and it appears that some mechanism must have been developed because the reduction is unfavourable for these organisms, but occasionally this mechanism appears to get out of order, resulting in the occurrence of the reduction in some somatic cells.

The reason why cancer cells are developed from the somatic can be explained in this way. It is a well known fact, as will fully be described below, that cancer cells are of a primitive nature comparable to that of germ cells. The production of cancer instead of germ cells may be due to the incompleteness of the reversion of the primitive structure probably because of the presence of the mechanism to prevent the reversion.

Hormones, especially sexual ones, appear to be involved in the production of germ cells; sexual hormones may be the main factor providing the environment under which the reversible change of the somatic cells to gametes is established. In short, hormones may be factor $x$ which converts $A^{\prime \prime \prime}$ to $A$ in Fig. 34 . In this connection it should be mentioned that, as is well known, sexual or other related hormones are intimately connected with the development of cancer cells. This suggests strongly that the production of cancer cells is carried out by the same principle as that of germ cells. It is believed by the majority of workers that hormones or hormone-like substances may be produced in the wound regions of plants where the primitive cells are induced to achieve the regeneration. It is well conceivable that mechanical injuries will result in the formation of certain hormones which cause the production of the primitive structures comparable to cancers. 
The structure of a cancer cell or of a cell present at a precancerous stage is sometimes transmitted by virus-like protoplasm particles which are often called cancer viruses. The change of a somatic cell to a cancer must be associated with a change of the gene, and accordingly, if a change of a gene is to be called mutation, it may be said that cancer is raised by a mutation.

The primitive nature of cancer cell has been well established. For example, glycolysis peculiar to embryonic tissues is remarkable in cancer cells. The capacity of embryonic tissues to incorporate amino acids is much stronger than that of the ordinary ones, and being similar to that of cancer cells (35) (36). Again, it has been shown that in the conversion of normal hepatic cells to cancer there is almost total loss of certain highly specialized functions, including synthesis of fermentable carbohydrate from pyruvic acid and formation of acetoacetic acid from caprylic acid (37). The pattern of enzymes of foetal rat liver is said to be more closely related to that of malignant rat hepatoma than that of normal rat liver (38). As already pointed out, primitive structure is so unstable that it may easily be affected by viurses as evidenced by the fact that chick embryos are commonly used as the most suitable tissues for virus cultivation. It is note-worthy that cancer cells also allow viruses, such as those of poliomyelitis (39), St. Louis encephalitis (40), and influenza (41), to propagate in them.

Greenstein (42) has stressed the fact that all cancerous tissues show a strong resemblance to one another in their chemical pattern, and that the more autonomous they are, the more do they deviate from the chemical pattern of their tissues of origin, and the closer do they approach an apparently functionally undifferentiated type of tissue.

A series of hormones appear to be required in succession for the development of a germ cell to an adult organism as already mentioned. This must also be the case with the production of cancer cells as well as of germ cells, as the production of these cells is only the reversible reaction of the former, the ontogeny. Sexual hormones may be required at the finishing stage for the completion of the change. Substances commonly termed carcinogenic may be regarded as a type of hormones or hormone-like substances to initiate the change. Many carcinogenic substances are known to exhibit an inducing action like that of the organizer (43), suggesting that they are related to the primitive hormones produced by the organizer.

Rous and Kidd (44) stated that when carcinogenics are applied to the skin of man, the rabbit, or the mouse, they nearly always elicit benign growth some while before cancer appears, and the latter 
frequently takes origin from one or another of them. Tar cancers usually come about by successive, step-like deviation from the normal, and so also do the cancers which derive from virus-induced papillomas as well as many human cancers. After cells have become cancerous they frequently undergo further changes, some apparently step-like in character, and all taking the direction of greater malignancy.

Sexual hormones may presumably play a role at the last stage of such step-like deviation, although most carcinogens are in themselves closely related to sexual hormones in the chemical structures. It has been stated that acute fowl pox develops in practically every bird painted with methylcholanthrene, one of the well known carcinogenics; if one continues to paint the bird with this carcinogen, chronic inflammatory reactions develop, as well as angiomas and discrete, poliferative, epithelial lesions. Upon inoculation of the bird with testosterone, a sexual hormone, all of the lesions flare up and the epithelial growths become squamous-cell carcinomas (45).

Further, if a single application of 3,4-benzpyrene, a carcinogen, is made to the skin of mice, it does not induce cancer, but if the single application is followed by repeated treatment with croton oil, then tumours develop (46) (47). The croton oil treatment alone will not induce tumours. Cholestrol exhibits a similar accelerating effect; in contrast to croton oil, cholesterol itself has a feeble carcinogenic activity (48). In such a case, croton oil and cholesterol may behave like sexual hormones.

Greenstein (49) believes that carcinogens lower the threashold of spontaneous malignancy in the tissues, so that malignant growth is brought about by the sexual hormones, which as such are not carcinogen. There are numerous evidences showing that estrogens enhance the development of mammary tumour of mice. The inhibitory effect of testrosterone in this connection is also well known. According to Kirkman and Bacon (50) male golden hamsters will produce almost constantly renal cortical cancers if they are treated with estrogen.

If cancer cells are produced by the structural reduction of somatic cells, and if the structural change of the protoplasm is reversible, cancer cells must return to the original, differentiated structure as do germ cells. In fact this seems actually the case. Thus, if renal carcinoma of the frog, Rana pipiens, is transplanted onto the base of the fore limb of a newt, and the greater part of the limb being cut off, when the cancer cells begin to multiply, leaving some of the cells in the tissue of the newt, then the cells are changed to normal frog cells during the regeneration of the limb. The frog cells can be easily detected owing to the small size of the nucleus (51). Thus the return of the cancer cells to their original state is established under the 
peculiar environment of the regeneration, in which strong factors are at work to develop the primitive structure into differentiated. Similarly, chicken sarcoma cells implanted on to chick embryos are reported to be developed into normal cells under the strong influence of organizer.

The recovery of the original, normal structure is common in benign tumours, but the normal structure which was recovered from the tumour appears to have the memory of the tumour, tending to return again to the tumour structure. Thus, according to Mackenzie and Rous (52), where tumours once were one finds only epidermis of ordinary appearance, yet if tarring is resumed some of them not infrequently reappear, even after months, and by reapplying tar at intervals they may be made to recur again and again, clear proof that some cells in the apparently normal epidermis have retained their neoplastic potentialities, that is, their memories of the tumour structure. Usually each successive period of tarring brings out more growth than before, and they appear very soon, sometimes almost at once instead of after several months. It is as if new tumour cells had been merely waiting for encouragement. The stimulus of wound healing will suffice to make some of them multiply and form tumours.

The malignancy of the cancer may depend upon its primitive nature associated with the character of the vigorous multiplication at the sacrifice of the host. It seems strange, however, that germ cells, inspite of their primitiveness, fail to exhibit such a malignancy. This is presumably because there is some mechanism by which the malignancy is inhibited, since the malignancy is, of course, unfavourable for the organisms, but the mechanism may be destroyed by the fertilization to the revelation of the malignancy, that is, the character of vigorous multiplication. Leucocytes may be looked upon as another group of primitive cells whose malignancy is usually inhibited; the destruction of this inhibiting mechanism may lead to leucosis. The structural pattern of leucocytes which thus acquire the malignancy can sometimes be transmitted by protoplasm fragments, and consequently the disease is considered to be induced by a virus as in avian leucosis.

\section{The Predisposition to Cancer}

Cancers are liable to develop in certain individuals, that is, certain individuals possess the predisposition to develop cancers. This predisposition is inheritable. As mentioned already, predisposition is a memory of the gene, or a genic structure capable of recovering a 
former pattern.

Animals and plants, infected with viruses, appear to transmit frequently the viruses to offspring through germ cells. Avian leucosis is looked upon as a kind of malignant tumours and considered to be induced by a virus. It has been established that the virus is likewise sometimes inherited to progeny through the eggs (53) (54). In such a case the pattern of the virus is memorized by the germ cell and recovered when the egg develops into the adult. The pattern of a virus, which has been impressed into a mother germ cell, must be changed during the reduction of the cell to a gamete, so that the pattern is present in the gamete only as a "memory" not as a complete pattern. However, when the gamete develops to a certain extent following fertilization, the pattern is also developed to the revelation of the virus. In such a case, the pattern of the virus may be impressed in, and carried by, a certain gene, just as a normal pattern which determines a normal character of the organisms.

Every morphological and functional character, including organs and tissues, are developed by numerous patterns memorized by each gene in the germ cell. But peculiar environmental factors are required for their development. In the same way, a certain factor or factors must be necessary for the development of a virus pattern. In the case of cancers, sexual hormones may be at least one of the factors. It was already stated that seasonal factors are important for the development of the virus of measles (Chapter III, Part III). The coincidence of the seasonal incidence of measles with that of the conception of man, may suggest the involvement of hormones in both cases. As previously detailed, hormones are, as a rule, required in ontogeny for the development of organs and tissues.

The predisposition to cancer must also be a memorized pattern of a certain gene present in the germ cell, and the full pattern will be raised in the individual developed from the germ cell when the proper conditions are provided. When the pattern is revealed in the protoplasm structure of a cell, the cell is called cancer cell, and when the pattern is stable enough to be retained in protoplasm fragments or elementary bodies whereby the transmission of the structure to another protoplasm is possible, the fragments may be called cancer virus. It is considered, as above mentioned, that cancer cells may be raised by the destruction of the mechanism by which the reduction of somatic cells to primitiveness is inhibited, If this is the case, the character tending to lose the mechanism are also to be borne by some gene in the germ cell as a memorized pattern which will later develop into the full pattern with the revelation of the character to lose this inhibiting property in favour of the development of the proper pattern 


\section{of cancer.}

The property to produce germ cells must likewise be impressed in a gene as a pattern, for whose development sexual hormones are required. This property is thus inherited, and is, of course, one of the most important characters of the organisms, in contrast to the propertr to produce cancer cells, notwithstanding that cancer cells are also of a primitive nature and sexual hormones apparently are likewise concerned in their production.

Not only germ cells but also various other cells or organs should be determined and produced by respective pattern transmitted through the genes of the gamete. If some microorganisms succeeded in engraving the patterns into some of the genes carrying such various normal patterns, the organisms would develop as one of the patterns, and if the microorganisms were farourable for the host, they would become organs or cells indispensable for the host as the mycetoma in insects.

Symbiosis or commensarism between microorganisms and insects is well known. An example of this is the commensality between the corn borer, Pyrausta mubilalis, and a flagellata discovered in this insects by Paillot (55). The protozoan occurs in the Malpighian tubes of the corn borers as well as in the alimentary tract. In the midgut the flagellates attach themselves by their anterior ends to the epithelium, standing side by side in a regular fashion as if they were ciliated epitheliums of higher animals. It may be not quite impossible that symbiotic microorganisms become more and more indispensable for the host until they come to be the essential cells or organs, such as leucocytes and ciliated epitheliums, appearing as if they were belonging to the host from the beginning.

The pattern of the microorganism, however, must be very strong and must be continuously applied to a host in order to become the inheritable pattern of the gene of the host. Unless a new pattern becomes normal and the previous one is forgotten, the new pattern will not become a predisposition of the host. 


\section{CHAPTER IV}

\section{THE MANNER OF THE GRADUAL CHANGE OF GENES}

\section{The Explanation of the Biogenetic Law or the Theory of Recapitulation}

The biogenetic law or the theory of recapitulation states that each organism tends in its individual life history (ontogeny) to recapitulate various states through which its ancestors have passed in their racial history (phylogeny). This law or the theory should be expected from the above concept that the germ cells are the reductive form of the somatic and that the change is reversible.

To begin with, we shall consider of the case of primitive unicellular organisms such as Sporozoa. If a primitive organism like Sporo$z o a$ is brought under a certain environment, the structure of the cell may be changed to produce spores, whereas this latter can recover the original cell structure if the environment is again properly changed. Since the structure of protoplasm is always governed by the elementary bodies, rich in nucleic acid, also the change between the cell and the spore must be subjected to such elementary bodies, which can be looked upon as the genes. Thus, the change can be expressed as. $A \rightleftarrows B$, in which $A$ is the structure of the gene of the cell and $B$ that of the spore.

Now, if this primitive organism advances to have $A^{1}$-structure, the change is : $A \rightarrow A^{1}$. Since the evolution of both morphological and functional characters of an organism is given rise to by the advancement of gene structure, the advancement of the gene structure from $A$ to $A^{1}$ should be accompanied by the evolution of the characters of the organism corresponding to the structural advancement in the gene.

On the other hand, as the genes possess the structural reversibility, when $A^{1}$ is reduced to $B$-structure of the spore, or of the germ cell, it must take the course: $A^{1} \rightarrow A \rightarrow B$, whereas when $B$ is developed to $A^{1}$ the course should be $: B \rightarrow A \rightarrow A^{1}$. This course is to be repeated in every change from the adult form to the spore, or the germ cell, and from the latter to the adult form. Through this repetition the 
reversible course will become a beaten track; in other words, the memory of the course will be held completely by the repetition as discussed in Chapter I of this Part. Since the memory is a type of the structural pattern of the gene, it should be inherited generation after generation.

If the organism evolves further and advances successively to $A^{2}$, $A^{3}$, and so on, the course will become longer and longer, but as the repetition is to be continued without stopping, the track continuously being beaten and the memory is as sure as ever. In such a manner, the organism can evollve upto an extremely high state as $\mathrm{A}^{\mathrm{n}}$. Even in this case, in its reduction to the germ cell, it must take the course : $A^{n} \rightarrow A^{n-1} \ldots \ldots A^{3} \rightarrow A^{2} \rightarrow A^{1} \rightarrow A \rightarrow B$; and $B$, the germ cell, must take the entirely reverse course in its development to $A^{n}$. It is impossible to take another course. Thus, the germ cell must take the course of phylogeny when it develops into the adult form.

\section{The Liberation of Active Groups}

Proteins may consist of two types of structures, one of which is stable back-bone structure and the other may be involved in polar forces in side chains which are to be liberated by the unfolding of polypeptide chains. There are numerous evidences that each of two structures determines each different character of the proteins (71). For instance, as already discussed, immunological character of viruses is determined by the stable back-bone structure, while virus action cannot be developed without the labile structure.

In a similar way, the manner of changes in the genic structure governing both ontogeny and phylogeny may also consist of two types due to these two structures. The main change of chief importance may be involved in back-bone structure, but the development of some functional and morphological character responding to the back-bone pattern may not take place without the transient, unstable structure.

This view is supported by the following fact: Lysogenic bacteria do not liberate phage by a mere disruption, while an adequate change in their environmental conditions, for example, irradiation with ultraviolet light results in phage production (56). And the susceptible bacteria infected with this virus become lysogenic and at the same time resistant to the phage, but usually they do not produce the virus unless proper stimuli are given. This fact can be readily explained by the assumption that the virus consists of two types of structures, stable and unstable. Namely, bacteria may acquire the immunity against a phage following the infection with the virus, because they 
are endowed with the back-bone structure of the virus. However, in order to act as the virus unstable structure is necessary, which may consist of polar forces that will be liberated by the unfolding of the protoplasm protein.

The manner of arrangement of these temporal polar forces may be determined by the back-bone structure. In other words, bacterial protoplasm proteins are normally in a partially folded state so that polar forces necessary for the virus action are also enclosed in the folding, but the protein in this folded state can retain its specific pattern which determine the immunological character. In order to prodice the virus, however, an appropriate stimulus is necessary whicn can cause the unfolding of the protein.

Actually it has been proved that after irradiation with ultraviolet light lysogenic $B$. megatherium shows increased capacity in its respiration; the synthesis of desoxyribonucleic acid is blocked during approximately the first $1 / 3$ of the latent period, but rapid synthesis then commences and continues until the moment of lysis (57).

When the virus is produced the bacterium is always bound to undergo lysis. This fact may be interpreted as due to the virus action itself. In the opinion of the writer, however, lysis has no concern with the virus. Polar forces liberated by the unfolding of the polypeptide chain may subsequently combine with great quantities of water molecules leading to the quelling of the bacterial body and accordingly to the dissociation of elementary bodies, thus resulting in the lysis of the bacteria. The bacterial lysis due to phage is also attributable to the liberation of polar forces attracting water molecules; the stimulus given by the virus may cause the unfolding of the peptide chain.

The phages released upon lysis of bacteria infected with a cofactorrequiring strain possess a nascent activity, which makes possible their adsorption in cofactor-free medium. It has been shown that this nascent activity is lost gradually, indicating the temporary liberation during lysis of active group which enables the phages to combine with the bacteria without cofactor (58).

Many evidences are known that lysis can occur without producing any virus. According to the writer's view, any bacteria can produce virus which affects other bacteria having weaker patterns. However, if the bacteria cannot liberate the free active group, no virus would be produced. Lysogeny may therefore be no more than the bacterial character of unfolding the peptide chain readily. Usually the irradiation of lysogenic bacteria with ultraviolet ray is followed by lysis and by phage formation, but the bacteria would undergo no lysis when they became non-lysogenic, showing that lysogeny is nothing 
but the property of readily liberating the polar forces.

Lysogenic bacteria, having the property to unfold the polypeptide chain easily on the application of ultraviolet light, cannot produce phage following the irradiation if the bacteria are not present under the appropriate environment. For instance, a culture grown in yeast extract and irradiated will produce phage and lyse if left in yeast extract, whereas it will not produce phage if suspended in broth after irradiation. Again certain bacteria in a manganese deficient medium will not produce phage after irradiation, but if supplemented with manganese after the irradiation the virus is produced. Furthermore, the inducing effect of ultraviolet light is inhibited by visible light (59). These evidences may show how important are the environmental conditions even for the liberation of the polar forces.

The so-called latent viruses may, in the majority of cases, be the virus pattern lacking this polar forces. For example, almost every man shares the pattern of herpes virus but the virus is developed only after some febrile diseases or after heat application which may cause the unfolding. As considered already, common-cold is a disease whose main causative factor appears to be chilling, but sometimes chilling is not sufficient to cause the disease; only persons who are under an appropriate condition seem to become common-cold following chilling. Chilling is therefore comparable to the irradiation, and the peculiar condition or X-factor in common-cold may be comparable to the yeast extract in the case of phage production. Persons apart from the peculiar condition may not catch cold even if exposed to chilling; in a similar way, bacteria suspended in broth may not produce the rirus even after the irradiation.

If common-cold is a disease which occurs in such a manner, its virus must be a fixed one and accordingly it must cause a longlasting immunity; but no immunity appears to be left behind the infection. This must be attributed to the virus structure tending to readily liberate the active group which, if liberated, makes the cells fall into a severe pathological state as in the case of herps virus, a phenomenon comparable to that concerning the phage against which bacteria can become immune but the bacteria are occasionally shocked by the liberation of the active group. If a man carrying the pattern of common-cold virus is chilled under a peculiar condition, the active group of the virus will be unfolded resulting in the severe shock in the cells in which the unfolding takes place, thus common-cold being developed.

The pathological symptoms designated iuflammation may be caused by the liberation of the polar forces; the liberation is caused not only by microorganisms including viruses but also many other physical or 
chemical factors; if caused by a virus, active group for the virus will be raised. In short, the liberation of polar forces may result in the shock to the cells, and the shock is revealed as inflammation. No virus can usually be found following the inflammation due to stimuli other than viruses, a fact which must be attributed to the transient and labile nature of the polar forces. But normal structure of the cell may sometimes become capable after inflammation of acting as a virus upon other cells having weaker patterns.

Lysogeny can be raised by the infection with phage, but certain bacteria must originally be lysogenic without infection. The pattern determining the lysogeny is controlled by the back-bone structure, and so it should also be formed under the appropriate conditions and be lost under some other conditions. For example, it is possible to 'cure' at will a certain strain of bacteria from its lysogeny by serial transfers in synthetic media (59).

Human children may produce measles virus presumably because the pattern of the virus inherited from the parent may become completed as the individual development is coming to its completion. The individual development must be brought about step by step by the gradual change of back-bone structure of the gene; the development of a virus pattern also must be raised entirely in the same way, but in order that the complete pattern acts as a virus the liberation of the active group is needed. The seasonal effect upon the occurrence of virus diseases is probably due to the liberation of the active group which is effected by some hormones or a hormone whose secretion is under the influence of seasonal factor. As the active group is of a transient nature, it will disappear when the season is over; but will reappear in the next year when the same season comes.

Such a transient group may likewise be involved in the revelation of the pattern of cancers and also of that of parasitic bacteria. The fact that the virus demonstration of Rous tumour of chickens cannot be achieved constantly may be mainly based upon the inconstancy of the liberation of active group rather than that of the maintenance of the pattern in the particles. As already stated, animals irradiated with X-ray may excrete in feces and in blood many types of bacteria in abundance. This fact may be interpreted as due to the liberation of active group of bacterial pattern by the irradiation as in the case of the pattern of phage which will be activated by the ultraviolet irradiation. The release of active group may probably be needed also for the activation of normal pattern, that is, organisms may not develop a function or a shape corresponding to a certain back-bone structure of the gene unless the active group is released.

Successive appearance of enzyme-like factors in the case of blood 
coagulation and the rapidly occurring activation of a variety of enzyme systems after the fertilization of egg may be ascribed to the activation of latent enzymes by the liberation of active group. In these cases the unfolding of the active group must be caused also by proper stimuli.

The long lasting immunity against viruses is based upon the persistence of the virus patterns in the host but in animals only the back-bone structure is remaining, so that virus detection in the host is hardly possible. In contrast to animals, however, the virus is detected without difficulty in plants having become resistant to the virus after the infection, showing that there is a considerable difference between animals and plants in their protoplasm protein, that is, plant protoplasm protein may exist usually in an unfolded state, or a slightly folded state to be readily unfolded unlike that of animals.

In this respect bacteria are similar to higher animals, a fact which may depend upon the parasitic nature of the bacteria on higher animals from which they are inherited the protein. If so, the bacteria parasitic on plants may have the protein similar to that of the plants, although the writer cannot find any report corroborating this supposition. The view that plants are thus different from animals in the character of protoplasm protein is also supported by the already mentioned fact that plant protein can fuse readily into protoplasm-like masses and that plant viruses are inclined to be obtained in crystal shapes, indicating the strong tendency of the protein to combine mutually. This is probably due to the presence of free polar forces mutually attracting. Further, there is another evidence corroborating this view. Namely the reduction to young primitive structure is readily raised in plant tissues, that is, cancer-like proliferative cells and germ cell-like regenerative ones and even germinal cells themselves seem to be produced in every tissue of plants, thus their regenerative faculty being striking and no peculiar cells being needed for the production of germinal cells, whereas in animals specific cells are necessary for the germ-cell production and the regenerative faculty is insignificant.

In order that the differentiated pattern is reduced to the primitiveness the unfolding of the protein structure is indispensable. Therefore, it may naturally follow that the return to the primitive structure takes place only with difficulty in animals in which the unfolding of the protein hardly occurs.

In the preceding chapter the writer assumed the presence of the mechanism in animals which can prevent the return to the primitive structure; the character of difficult-unfolding must he regarded as being this mechanism. The predisposition to cancers, therefore, must 
mean the possession of the protoplasm protein tending to unfold. Precancerous stage is commonly associated with inflammation, which as above pointed out means the unfolding of the protein.

\section{The Establishment of the Strong Reversibility in Gene Structure}

The biogenetic law would never be established if the gene failed to have strong reversible character. In this section, the reason for the establishment of such a strong reversibility in genic structure is considered.

If the combination of two primitive organisms, $A$ and $B$, takes place, and the strongest elementary body in $\mathrm{A}$ is stronger than that in $B$, then the strongest body in $B$ as well as other elementary bodies will be changed to become similar to $A$. But if the change is reversible and the strongest body in $B$ can recover its original structure when freed from $A$, the body can behave as a recessive gene. Thus, in order to become a recessive gene, the elementary body in B must have the complete structural reversibility, that is, it must have the faculty to recover completely its structure when the stress is removed. This holds true also for dominant gene, since if it had no structural reversibility, it would lose the independent character when it fell in with a gene stronger than itself. The reversibility must, therefore, be the indispensable character for elementary bodies to become genes.

If the reversibility of a gene is strong, the organisms having the gene can recover its original characthr even when it has been changed to primitive structure under some environmental conditions. The reproduction by means of germ cells cannot be achieved without this reversible character of genes. When the organisms were advanced in their evolutional course upto a certain degree with the acquisition of complicated forms and structures, the mutliplication by fission would become very inconvenient or rather impossible. Consequently, the organisms having evolved to a certain extent would find it necessary to fold their complicated structure into a mass as small as posisible in order to discharge this folded structure out of their bodies to expand again the orginal structure from this small mass.

This small mass is the germ cell, and the expand of the folded structure, a process termed ontogeny, can be achieved by the reversibility of the gene. Therefore, the reversibility is the indispensable character for organisms in so far as they continue the reproduction 
by means of germ cells, and accordingly only the individuals having the genes, which can exactly expand the original structure after the reduction to primitiveness, could survive and continue their existence. Thus the reversibility of the gene have been amazingly advanced. 


\section{CHAPTER V}

\section{GRADUAL ALTERATION OF GENE AND ITS RELATION TO MUTATION}

\section{Orthogenesis}

If the miniature copy of phylogeny is seen in ontogeny, phylogeny must have been raised by the same continuous changes as ontogeny. In ontogeny the destination is settled preliminarily, the destination bound to reach is the original, advanced structure, and since the development is only the recovery of the original structure by means of the reversibility, it can occur rapidly, whereas in phylogeny as the destination is unsettled and a new course has to be made, its progress must be very tedious.

Nevertheless, the change of genes in phylogeny, like in ontogeny, would occur under the influence of environmental factors, wherein hormones would play an important part as will be considered later. This change of genes, as shown in ontogeny, would proceed in a certain direction and therefore organic evolution would occur gradually along a certain course. In fact, it is stated that evolution has occurred in straight lines or definite directions as indicated by the term "orthogenesis."

The term "orthogenesis" has been used to indicate a straight genesis or evolution directed by internal factors. It is claimed that lines of evolution were not haphazard but were determined, not by natural selection but by law of organic growth. True, in some cases evolution seems to have proceeded along a certain course. For example, the increasing size of such forms as the horses, elephants, and dinosaurs, the reduction of digits in the horse series. Many fossil series suggest that types begin simply and evolve into complexities, such as overgrown spines and tusks, the overdevelopment being followed by extinction. Some zoologists and a larger number of paleontologists claim that evolution of this general sort has occurred so often in widely different types of animals that it can be explained only as a result of fundamental forces inherent in a race and carrying it along a straight or orthogenetic course. 
Such evolution along an orthogenetic course must have depended upon the gradual change of a gene in a certain direction. If some gene began to change its structure gradually, the organisms having the gene would be compelled to change the character according to the change of the gene. However, as seen also in ontogeny, the direction of the change would be not always straight, although in the majority of cases it appears to have occurred in straight lines.

This supposed property of genes to alter the structure gradually must be of the utmost importance for the establishment of erolution. Full discussions on this problem will be made next.

\section{Phage as a Free Gene}

The gradual alteration of genes must be reversible since the individual development from the germ cell is given rise to by this change. owing to the reversibility the developed structure can be reduced again to the germ cell. On the other hand, genes may frequently undergo a sudden, irreversible change. The change of organisms due to such an abrupt, irreversible alteration in a gene is called mutation. There seem, therefore, two types of genic changes, one of which is this irreversible, abrupt change, the mutation, and the other a gradual, reversible change. The nature of these two types of changes will be considered below.

Phage among many viruses can be regarded especially as a free gene, for it contains a large amount of desoxyribonucleic acid, the nucleic acid peculiar to genes. Here, it should be noted that phage thus possessing a free gene nature will change gradually in a certain direction under the influence of formalin as shown in Fig. 35 . If phage is regarded as a gene, formalin should be compared to an environmental factor having an influence upon the gene. In so far as the factor is present the change appears to proceed in a certain direction.

The evolution along an orthogenetic course may thus be attributed to the existence of some environmental factor or factors which, like formalin, caused the gradual change of a gene in a certain direction. Orthogenesis, therefore, would come to a stoppage, if the environmental factor in question was removed. Thus it may be concluded that for the establishment of orthogenesis continued existence of a certain stimulus or an environmental factor which caused the change of a gene would always be needed.

The proteins of our somatic cells are bound to undergo senescence since the time of birth, a phenomenon which may be due to the 


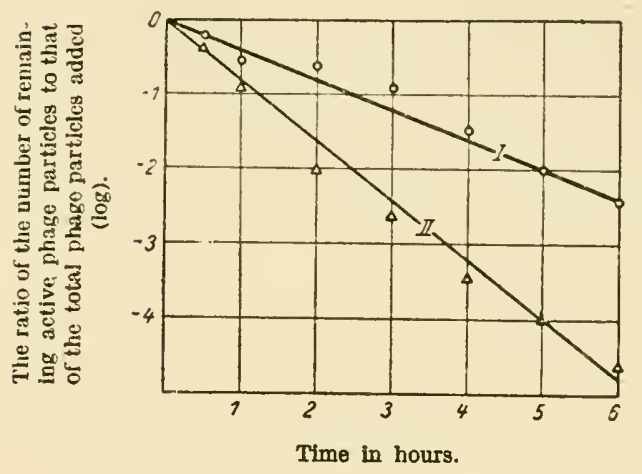

Fig. 35. The progress of the inactivation of phage protein due to formaldehyde. Concentration of formaldehyde $0,05 \%$ (I) and $0,1 \%$ (II). Concentration of phage protein : $0,05 \%: \mathrm{pH}=7,5$.

presence of a factor or factors making the change leading to senescence proceed continuously. As already discussed, senescence is a phenomenon occurring when the proteins or organisms continue to exist under the same environmental condition. Proteins having biological actions, such as toxins, antibodies, or enzymes, will achieve a gradual change, known as denaturation, when preserved in vitro after being separated from organisms. The change is usually very gradual and frequently many years may elapse before complete inactivation occurs. Such a change is likewise attributable to the continued presence of some factors which cause the denaturation. Furthermore, the production of cancer from a somatic cell as well as the development of adult from a germ cell is considered to be brought about by a constant influence of a series of proper stimuli as already discussed.

Here again, we have to consider the problem of reversibility. It is true that sometimes formalin-inactivated phage is reactivated following the removal of formalin, but the reactivation occurs only seldom, usually being irreversible. The same can be said with phage inactivated by chemical or physical factors other than formalin. However, the reduction of the virulence of phage, without inactivation, due to extremely small amounts of formalin is usually reversible (60). Namely, the virus reduced in its virulence by formalin may produce small plaques on agar plate, but after a period of incubation following the removal of formalin the original property to produce plaques of ordinary size will generally be recovered. Such a reversible change can be demonstrated not only with formalin, but also with such agents as antibody, mercuric chloride, and tannin (61) (62). In general 
severe changes raised abruptly appear to be irreversible, while slight changes given rise to gradually may be reversible.

The recovery of the original structure of protein, as previously considered, is dependent upon the memory of the path along which the change proceeded. If one goes without hurry tramping on the overgrown grass through a pathless field, he will be able to return easily along the beaten path, but if he goes hurriedly in long strides the return will be difficult. In like manner, if proteins are changed in their structure gradually, the return to the original structure will occur with ease, as the structural factor resisting the change may be removed by the gradual change, whereas in an extensive change raised abruptly the recovery of the original structure may be unable. This latter change must be mutation, while the former the reversible alteration of the gene.

It will be expected from this concept that in mutation the recovery of the original structure may be never impossible but only difficult. In fact, it has gradually become acknowledged that reversible mutation, commonly called back mutation, is by no means a rare occurrence. As pointed out previously, even so great a change as that of somatic cell into cancer is reversible if the most favourable condition for the reversion is applied. It has been claimed that exposure to $\mathrm{X}$-ray and ultraviolet-light irradiation increases the back mutation rate of microorganisms very substantially (63), indicating that appropriate stimuli can also necessary for the promotion of the back mutation.

The frequency of back mutation of bacteria from streptomycindependence to non-dependence is greatly increased by the treatment with ferrous compounds. According to Catlin (64) streptomycin-dependent colon bacilli show a considerably higher frequency of reversion to streptomycin-nondependence after exposure to ferrrous compounds at $1^{\circ} \mathrm{C}$. than after comparable treatment at $37^{\circ} \mathrm{C}$. This suggests that in this case ferrous compounds may only contribute to initiate the return to the original structure which may be stable at lower temperatures. It should be remembered in this connection that besides ferrous compounds, ultraviolet and X-rays, and a number of chemicals such as manganeous compounds, also effective in the promotion of this back mutation.

According to our observation, phage is frequently changed in its property by unknown causes to produce small plaque, which indicates the reduction of its virulence, and the changed property is sometimes inherited, showing that the change is a mutation. The maintenance of the changed property appears to be connected with the medium in which the phage affected the host bacteria. For example, as shown 
in Table 10, the changed property is maintained in a usual broth, but in a broth prepared with Liebig's meat extract it will soon return to the original property; this may indicate that back mutation is induced by some factor present in the extract. When phage is developed in a medium containing neither calcium nor magnesium, the property to produce plaques of extremely small size is given rise to, and this property tends to be retained when the phage is incubated in an or-

Table 10.

Inheritance of Acquired Character in Phage

\begin{tabular}{|c|c|c|c|}
\hline & $\begin{array}{r}\mathrm{Te} \\
\text { (Phage and ba } \\
\text { Agc } \\
\text { (Both large and }\end{array}$ & $\begin{array}{l}\text { tube } \\
\text { are incubated) } \\
\text { plate } \\
\text { mall plaques exist) }\end{array}$ & \\
\hline Larye & laque & Smal & aque \\
\hline $\begin{array}{l}\text { Extract-broth } \\
\text { test tube } \\
\text { (Incubated } \\
\text { with bact.) }\end{array}$ & $\begin{array}{l}\text { Ordinary broth } \\
\text { test tube } \\
\text { (Incubated } \\
\text { with bact.) }\end{array}$ & $\begin{array}{c}\text { Extract-broth } \\
\text { test tube } \\
\text { (Incubated } \\
\text { with bact.) }\end{array}$ & $\begin{array}{c}\text { Ordinary broth } \\
\text { test tube } \\
\text { (Incubated } \\
\text { with bact.) }\end{array}$ \\
\hline $\begin{array}{c}\text { Agar plate } \\
\text { (Small plaques as } \\
\text { well as large } \\
\text { plaques appear) }\end{array}$ & $\begin{array}{l}\text { Agar plate } \\
\text { (Large } \\
\text { plaques only) }\end{array}$ & $\begin{array}{c}\text { Agar plate } \\
\text { (Large plaques as } \\
\text { well as small } \\
\text { plaques appear) }\end{array}$ & $\begin{array}{l}\text { Agar plate } \\
\text { (Small } \\
\text { plaques only) }\end{array}$ \\
\hline
\end{tabular}

dinary medium, although frequently the original property is recovered (65). Thus it appears that there is no fundamental difference between the irreversible and reversible change in phage.

\section{The Production of Fitted Characters by Adaptation}

In addition to the reversibility, there may be another great difference between mutation and the gradual, reversible alteration. The difference exists in that the gradual alteration will generally give rise to favourable effects upon the organisms, whereas the characters raised by mutation are mostly harmful. For example, although the possibility of using radiation to induce useful mutations has been extensively investigated, especially in crop plants, almost all of the experimentally produced mutations have proved to be deleterious in character (66). Further, it has been generally recognized that muta- 
tions induced in the fruit fly, Drosophila, by X-ray are mostly associated with the production of lethal factors.

Every existing organism must be the fittest or at least must have the characters of a survival value and accordingly its genes must have the structures well fitted for survival. Therefore, the probability for producing more fitted structures by a random change may be extremely small and so it will naturally be expected that the changed structures are mostly deleterious far from being useful. This may be the reason why mutation produces, as a rule, deleterious characters.

However, strange to say, the gradual, reversible alteration of the genes, as pointed out above, seems commonly to produce characters favourable for the organism. This may depend upon that the reversible change is induced by naturally existing factors such as temperature, light, and food, to the changes of which the organisms must always be exposed. If organisms could not endure the change of such common environmental factors, they would surely become extinct, and as a natural consequence only the organisms capable of enduring the change would be able to continue the existence.

As discussed in the previous chapter, the proteins including the organisms composed of the proteins are provided with the property in themselves to become adapted to the environmental factor under which they are placed for long periods; in other words, the protoplasm and accordingly the gene can acquire the structure which is most stable under an environment to which the organisms are exposed for prolonged periods, so that the environment may become the most suitable one to the organisms. Thus the changed environment which acted at the beginning as a stimulus upon an organism becomes normal having no influence upon the organism. If an organism achieves a change in its character in such a way according to the alteration of an environmental factor, it may be said that an adaptation farourable for the organism has took place.

Naturally existing environmental factors may usually change gradually, and so the protoplasm structure may also undergo the structural change responding to this environmental change gradually, thus gradual, reversible change being established. If the structural pattern thus induced by the gradual environmental change was unfarourable for some organisms, the organisms would be perished; the organisms for which the newly produced pattern was favourable or at least uninjurious would be left as the fitted. This must be the reason why reversible alteration of the gene always produces characters favourable or at least uninjurious to the organisms.

On the other hand, the structural pattern induced by unusual stimuli such as X-ray or some synthetic chemical agents, even if the 
pattern is induced gradually and accordingly is reversible, must be unfamiliar to the organism and the organisms are not accustomed to the pattern; and since, as above pointed out probability of a pattern produced at random of becoming favourable for the organism must be extremely small, patterns produced at random by unnatural factors must, as a rule, be injurious to the organisms. In addition, unnatural factors are usually applied suddenly in experimental researches, the changes are generally irreversible, resulting in mutation. 


\section{CHAPTER VI \\ SELECTION AND ISOLATION}

\section{The Effect of the Selection}

As already pointed out, protein molecules appear to have the individuality. Every protein molecule is a replica of a template molecule, but it may be impossible to make a replica entirely equal to the template; presumably every replica may differ slightly from the latter on some point or other. The degree and kind of such a difference may give rise to the individual character in protein molecules.

It is well recognized that the molecules of even carefully prepared samples of a single protein from a single source may differ perceptibly in such diverse properties as molecular weight, charge density, composition, molecular configuration, solubility, and biological activity. The conclusion is drawn that all protein preparations prepared so far represent populations of closely related members of a family, not collections of identical molecules (66a).

Since genes consist of protein molecules, they should likewise possess the individuality, and again since organisms are determined in their character by the genes, they should likewise have the individuality even when they belong to one and the same species. Individual variations may thus arise.

Proteins will be changed in their structure by environmental effect but the degree and perhaps also the direction of the change may vary with the difference in the structure. In like manner, each individual may change the character in different ways even under the influence of the same environmental factor, and as a consequence variations will disperse.

As stated above, organic evolution is considered to be raised by the gradual change of genes. However, since even the individuals of the same species are thus not endowed with entirely the same series of genes, each may tend to undergo different changes, advancing into different directions. An individual exhibiting an extreme character, therefore, may have a gene tending to undergo an extreme change, and extreme character may be further advanced if the environnental effect, which has given rise to the character, continues to exist. 
Here, the well-known experiment of Castle carried out with hooded rats should be mentioned. He was able, through selection in successive generations, to extend or diminish the black of the colour pattern of the rats. In his experiment it was clearly shown that the selected rats having the extreme colour-pattern tend to push the extremity in successive generations, the colour pattern becoming more and more conspicuous. His observation was spread over about 20 generations of the rats. Another well known example of this kind is Tower's experiment with colorado beetles in which he likewise succeeded in increasing the peculiar colour pattern by selection.

Many other evidences can be presented to show the effect of the selection, but it appears that they were not adequately interpreted, presumably because of the general acceptance of Johannsen's pureline theory, which, however, the writer is unable to accept as being legitimate.

The beans with which Johannsen carried out the experiment and also the paramecia with which a similar result was obtained afterwards by another author might have been taking place a gradual change so slowly that the progress of the change was overlooked. It should be noted, however, that even during the experiments of Johannnsen, two mutations, one for higher and one for lower seed-weight, were detected; this may show that his selection would produce high and low seed-weight respectively. Granting that such mutations were only rare, it may also conceivable that the peculiar condition under which the observations were made might remove the factor which was causing the gradual change. It should be remembered that the observations were made with the descendants from a single homozygous organisms exclusively propagating by self-fertilization. Whatever might be the reason why apparently unusual result were obtained for which the pure-line theory was derived, there seems little doubt that his findings were exceptional since as just stated there are many evidences showing the striking effect of the selection.

However, most examples in which the selection appeared to be effective were commonly interpreted as being due to the accumulation of minute gene mutations occurring in a certain direction. Such an interpretation, on the other hand, may be proper as it may not be entirely impossible to consider the gradual alteration of the gene to be an accumulation of minute mutations.

At any rate, the genes in each individual even under the same environment may perform a change peculiar to the individual according to the individual difference in the structure of the genes, giving rise to a peculiar character. When such a peculiar character becomes to have a survival value, it will be selected by natural selection. Since 
the individual thus selected must have the property to develop still further the character in question, the character will be advanced unlimitedly in so far as the environmental effect causing the change continues to exist, thus resulting as a natural result in overspecialization or overgrowth, in which once useful parts are carried entirely beyond the point of utility until they become iujurious and possibly even instrumental in the extinction of the species. The tusks of the saber-toothed tiger, the antlers of the extinct Irish elk, the huge size of certain dinosaurs, and the tusks of the Columbian elephant are often-cited examples of such disadvantageous development.

As already discussed, although the genes seem to direct each particular restricted structure of protoplasm protein, the change at a certain structural portion of a protein molecule by a gene tends to exert influences upon other portions, and accordingly a development of a useful, selected character is inclined commonly to be associated with a development of certain other characters which may not be so useful or even utterly useless. In addition, as will be mentioned later in detail, hormones play a predominant part in the evolution in developing many characters by their peculiar effect upon genes. These may be the reasons why organs appearing to be of no use are also of ten overspecialized.

In fact, useless characters, along with those that are useful, seem to be common in organisms. This is particularly true for the structural features of animals. In recent years a number of mass-selection experiments directed at increasing, as well as decreasing, the magnitude of a given quantitative character have been reported. A constant feature of such experiments has been the occurrence of correlated changes in characters other than the one being selected (67). The difficulty of the independent occurrence of a change restricted to a portion of the protein molecule is shown also in the fact that the influence of genes is believed to be usually dependent upon their association with certain other genes. Evidences for this interaction of genes is clear-cut. At least twenty five pairs of allelomorphic genes are believed to be concerned with eye-colour. Conversely, a single pair of genes may influence more than one character. In many instances it is known that the three or more allelic states of the genes for a particular character exist. A single pair of genes may also influence the appearance of more than one character. It has also been known that genes have somewhat different actions according to what neighbours they possess. This is the so-called position effect.

Anyhow, the individual having an extreme character shares the gene which will more and more extend the extreme character; conse 
quentiy-the effect of the selection is enormous. Thus the wonderfu! variety of domestic animals and cultivated plants were produced by artificial selection, whereby the individual that seems most disirable would be selected out of numerous individuals from generation to generation.

\section{The Effect of Isolation and the Origin of Species}

As repeatedly described, newly acquired patterns of protein structure are so unstable and liable as to return readily to the original patterns. This may hold true also for a selected character. A newly appearing character will be determined by a gene having a structural pattern newly acquired, and therefore, when an individual having an extreme character which has been adranced by selection is crossed with an unselected individual, the extreme character will soon be lost. Thus the new pattern of a gene of a selected individual is much weaker than that of the original.

This fact was clearly shown in Castle's experiment above cited. He found that the extreme color-pattern obtained by selection was lost when the selected individuals were crossed with the normal. Entirely similar phenomenon was likewise observed in Tower's experiment with the beetles (68).

Notwithstanding the fact that mutation is given rise to by irreversible change of a gene, it is generally accepted that the overwhelming majority of characters resulting from mutations are recessive, indicating that newly acquired characters are weak even when they are irreversible, and so it should be expected naturally that characters. newly acquired by the reversible, gradual alteration of the genes are always weaker than the original. This must be the reason why isolation is regarded as the necessary frerequisite and the inevitable cause of specialization.

Isolation is considered thus to be indispensabie for the development of new characters, but when a new pattern can deviate from the original so far that the crossing between them becomes impossible, isolation is no more necessary and the new pattern is then a new species. There are thus commonly distinct discriminations between species, no intermediate types existing.

As accepted generally, ecological factors may play the most important role in the isolation, and when the deviation goes far enough the reduction in fertility will result, thus new species being established. The reduction in fertility involves interspecific sterility and hybrid sterility. Interspecific sterility may be based upon the produc- 
tion of an inviable zygote. Hybrid sterility prevents the reproduction of hybrids that have reached the developmental stages at which the parents normally breed. Sterile hybrids produce either no functional gametes or gametes that give rise to inviable zygots.

It should be noted, however, that while wild species, as a rule, thus cannot be crossed, domestic species can usually be bred among themselves. This indicates that wild species cannot exist as such unless the crossing with the original species becomes impossible, whereas domestic species can be preserved without the reduction in fertility owing to the isolation by artificial selection. 


\section{CHAPTER VII}

\section{THE MECHANISM OF ADAPTATION}

\section{The Adaptation as a Reversible Change}

The close adaptations of animals and plants to their individual stations in life, fitting them to secure food and protect themselves, are the most striking features we find in them. Since environment is usually not constant, it is plain that if living things are to continue to live, they must have the capacity for readjustment directly or indirectly to changed environment.

It is a matter of common observation that plants of the same species, even if grown from seeds of the same fruit, may be quite unlike when grown in very different habitats. Thus, when plants of moist valleys are grown from seed or by transplantation in alpine regions, their internodes are generally shorter, their leaves are smaller and thicker and their flowers are larger than when they grow in their natural habitat. If, however, the seeds of these plants are planted in the natural habitat again, it will be found that the modifications which the species displayed when grown in an alpine habitat have not been inherited. The reversibility of the change is thus definite.

If we continue to use some of our muscles in excess, by and by the muscle will become hypertrophic so that we may be able to use it with facility. Such an adaptation is especially striking with bones and blood vessels as well as with muscles, but the change is reversible and the removal of the stimuli will soon be followed by the recovery of the normal state.

Some animals like chameleons of the tropics can change color promptly according to the color of the background. Some of the most remarkable instances of variable concealment coloration are met with in the flounders and various other bottom-dwelling species of fish which change their colour pattern in conformity with the colour shade of the background. According to Kammerer (69) the salamander, Saramandara maculosa, could change their colour pattern in a similar way, but the change proceeded very gradually during the prolonged breeding periods with the background of certain colours. The observation was continued for several years, but the change was not definitely proved to be heritable. As is generally recognized, the colour 
of some pupae can be altered by the colour of background on which the caterpillars are reared.

Sumner (70) was able to crange the tail length of rats by changing the temperature at which they were breeded. High temperatures induced long tails and low temperatures short tails. In this connection it should be remembered that, as is well known, cold climates reduce body surfaces such as ears, tail, and neck, resulting in the development of a generally more compact build of body. This tendency is particularly well exemplified by the size of ears in foxes as one passes from warmer to colder climate. This kind of adaptation may be valuable on the point of the thermal regulation of the body.

It is said that the red primrose had red flowers if kept at a temperature ranging from $15^{\circ}$ to $20^{\circ} \mathrm{C}$. A plant with the same genes but reared at a temperature of $30^{\circ}$ to $35^{\circ} \mathrm{C}$ with other environmental conditions unchanged, produced white flowers. If a plant with white flowers is brought into a room at $15^{\circ}$ to $20^{\circ} \mathrm{C}$ the flowers that develop later will be red. This phenomenon may be likewise significant in the regulation of heat effect.

Rabbits of a certain kind, when grow adult, have black hair on such heat-losing surfaces as ears, tail, and the ends of extremities, but the bandages to keep warm the part from where the black hair has been removed, will cause white hair to grow, whereas black hair will result on the back or the abdominal region when hair is cut to cool the region.

A certain case of the fruit-fly, Drosophila, is distinguished from the normal by the fact that these are very few black bands on the abdomen. When this race is reared on a rich supply of moist food, the abdominal bands are almost completely absent in all individuals. The same stock raised on scant, dry food exhibits normal banding of the abdomen.

Thus organisms can readily change their characters according to the change of environment. Such a change is always reversible, and' remarkably enough the change mostly appear to be favourable for the organism, at least not deleterious. The last mentioned example of the fruit-fly may not be regarded as a favourable change, but at least harmless.

\section{Dauermodifikation}

The reversible, adaptive change observed in higher organisms can likewise be seen in unicellular organisms, such as bacteria, protozoa, and fungi. Especially with bacteria this phenomenon has been well 
investigated. Bacteria can usually acquire the resistance against a variety of chemical and physical agents, which exert some injurious effects upon them, when cultivated under their influences. The change is, as a rule, reversible, acquired characters being lost when the influence is removed.

Attention should be paid, however, to the fact that usually some periods of time are required, after the removal of an injurious agent, for the complete disappearance of the resistance, that is, for the recovery of the former character, so that the change is often called "Dauermodifikation" or durable modification. This shows that the changed character is inherited through several generations although meanwhile the character will become less and less distinct until at last completely disappears.

In higher organisms, acquired characters may appear not to be transmitted to progeny, but since in the majority of cases the characters tend to continue for some periods after the romoval of the stimulus, it must be admitted that the character is transmitted from the somatic cells to other newly formed ones in the region. Accordingly, in this respect, the adaptation of higher multicellular organisms should likewise be regarded as "Dauermodifikation" of somatic cells. On the other hand, as the structural pattern of a cell is to be determined by the genes contained in it, it should be considered that Dauermodifikation is caused by a temporary change of the genes in the somatic cell.

As already described, the genes have the property to alter their structure responding to the environment or stimulus under which they are placed, but as the change is reversible the removal of the stimulus will lead to the recovery of the original structure. However, a considerable period of time is usually required for the complete recovery. Dauermodifikasion is thus raised.

\section{Various Types of Adaptation}

As already mentioned, a new environment may act upon an organism as a stimulus but when the organisms is exposed for a prolonged time to the same environment, the organism will complete the change induced by the environmental factor, that is, it will acquire the structure correponding to the new environment, which therefore comes to have no influence upon the organism. The adaptation thus depends upon the basic character of the protein itself, If the adapted structure, that is, structure which had completed the change induced by the new environment was favourable for the organism, the organism would be more fitted to continue its existence; therefore, the adapted struc- 
tures are in the majority of cases favourable for the organism having been able to continue their existence up to the present.

Now, if a factor causing such an adaptation is a chemical agent having a definite structure, the organism has to be changed to become complementary in their structural pattern to the chemical agent in order to become adapted to the latter, as the adapted structure of the organism must be the structure completely complementary to that of the agent. When such an adaptation is accomplished, the organism can readily combine with the agent through the complementary pattern but without being exerted any influences. As a good example of this type of adaptation will be mentioned the so-called adaptive enzymes.

A certain strain of colon bacteria will gain the faculty to utilize lactose when cultivated in the medium containing this sugar. It is believed that in such a case an adaptive enzyme to ferment the sugar is produced. In order to utilize the sugar, the bacteria are to combine easily with it, and the combination will be achieved by the production, or rather reinforcement, of the combining group. The appearance in the bacteria of the structure complementary to that of the substrate as a result of the adaptation must account for the reinforcement of the combining group. The bacteria cannot utilize the sugar presumably because they are not provided with the strong combining group for the sugar. In like manner, antibody production is also regarded as this type of adaptation of certain cells against the antigen. Again virus is produced by a virus because the complementary structure is formed in the protoplasm. Thus it may be said that virus-formation is also involved in this type of adaptation. The production of such complementary structure in response to chemical structures of the stimulants appears to be based upon the general and essential character of the protoplasm. Viruses, antibodies, and adaptive enzymes are nothing but the structures freed from the protoplasm thus adapted to respective stimulants.

Anyhow, if a complementary structure given rise to in the protoplasm of a cell or of an organism by a certain agent is transferred to the gene, or the structure is raised directly in the gene, then the cell or the organism can be regarded as having adapted itself to the agent with the acquisition of the property to utilize the agent. This type of adaptation may be designated as attractive adaptation, as attractive or combining structure is to be strengthened as a result of the adaptation. However, in addition to this, there seems to be another type which may be called rejective adaptation. Next a mention will be made of this type of adaptation.

An agent, in order to act as a stimulus on an organism, must be received by the protoplasm of the organism. The reception may be 
established by some combining group present in the protoplasm or on the cell surface. In the case of the attractive adaptation, such a combining group is strengthened by the adaptation. A sugar or an antigen may combine with bacteria or antibody-producing cells, respectively, through such a combining group to act as a stimulus causing the adaptation; and after the establishment of the adaptation the combining group will be reinforced so much that the group, if liberated from the protoplasm, can act as the enzyme or the antibody, respectively. Also a virus can affect a cell because a combining group directed to the virus may be present on the cell surface, and after the infection the protoplasm structure will become complementary to that of the virus; as a consequence the cell will combine readily with the virus but without being exerted any injurious effect by the latter. Thus the cell will become,immune to the virus as a resuit of the attractive adaptation.

However, such a complementary structure may fail sometimes to be produced following a virus infection as in the case of influenza virus affecting red-blood corpuscles, presumably because of the character of the cells unable to produce the pattern completely complementary to that of the virus. The failure in the production of complementary structure may give rise to only the disturbance in the protoplasm structure by the virus, leading to a type of coagulation of the protein, whereby various free polar forces including the combining group for the virus will be lost. As a natural result of this disappearance of the combining group, the virus will be released from the cell and at the same time the cell will become immune to the virus, thus the rejective adaptation being established.

For instance, when a virus such as that of influenza combines with a red cell, only a structural disturbance will result, causing the disapearance of the combining group, because the red cell is not susceptibie to the virus and so cannot reproduce the virus, that is, the cell cannot form the complementary pattern so as to establish the attractive adaptation. As a consequence the cell may acquire the property to reject the virus and the virus will be released from the cell. If such a change is given rise to by an agent other than a virus, it may be said that the cell is adapted itself to the agent not to be combined by it. The mechanism by which an enzyme can liberate from a molecule of a changed substrate to affect another unchanged substrate is elucidated in a similar way as discussed in chapter V, in Part IV.

In the majority of cases, a number of combining groups directed respectively to each group of agents may be involved in some constituents present on the cell surface. If such a constituent combines with an agent to be disturbed in its structure and undergoes denatu- 
ration, the substance will be removed from the cell as it will become, as a result of the denaturation, a substance foreign to the cell. If the removal of such a substance is accompanied by the destruction of the enzyme systems concerning the synthesis of the substance, the substance is not only lost but also its synthesis will come to cease, leading to the rejective adaptation of the cell against the agent.

On the other hand, the inability of producing certain substances may be involved in the corresponding pattern of the protoplasm and accordingly also the gene itself may come to possess the pattern following the establishment of a rejective adaptation. If so, the pattern may be transmitted to newly produced cells in so far as the gene retains the pattern thus induced.

The mechanism of adaptive colour changes, such as those of salamander above cited, may be elucidated in this manner. If the structure of a gene is changed so as to reject the visible ray of a certain wave length, under which the cell or the organism is always exposed, the cell or the organism will come to have the colour of the wave length. Thus organisms will become white, when they are exposed to the light of every wave length, since all the visible rays will come to be rejected, whereas they will become black when kept in a dark place as no light will be repulsed. Of course, such a change is valuable as a concealment colouration. In higher animals, hormone systems are amazingly advanced and protoplasm structure changed by environment may be liberated in the forms of hormones to effect the pattern of the protoplasm and also even the genes of the other cells. Thus colour change is usually achieved by special pigment cells called chromatophores the function of which is under the control of hormones. In the case of temporary adaptation as in this case of concealment colouration, only the pattern of the protoplasm may be involved without being accompanied by the corresponding change of the gene.

The rejective adaptation like the attractive one must be involved in one of the basic characters of the protein. Thus, on the one hand, physical of chemical agents which exert deleterious effect upon organisms may tend to cause, as a rule, coagulation of proteins. On the other hand, various polar groups of proteins are likely to disappear when they are coagulated. It appears, therefore, to be a natural result that organisms may adapt themselves to deleterious agents to reject them. In so far as an agent causes the coagulation whereby combining groups may disappear, an organism will achieve a rejecting adaptation to the agent, while if an agent has no action of coagulation, the protoplasm may produce the complementary structure in response to the structural influence of the agent with the establishment of the attractive adaptation. 
Organisms which can establish rejective adaptation to injurious agents are, of course, being fitted for existence and, will continue to exist, and therefore this adaptability has to be highly advanced.

In higher animals including man various hormone systems are amazingly developed for their evolution, and adaptive evolution appears to be mainly achieved by hormones as will be described later. Hormones, as mentioned above, can be regarded as protoplasm structures changed by environmental factors. Namely, changed structures of the protoplasm liberated as free forms may be looked upon as hormones, but as the influence of the environment may be greatly modified through hormones, and as the types of hormones are restricted in number in higher animals, the direction of evolution tends likervise to be restricted, not very specifically controlled by environment. 


\section{CHAPTER VIII}

\section{THE INHERITANCE OF ACQUIRED CHARACTERS}

\section{The Forgatting of the Original Structura}

As pointed out above, adaptive change of genes, as a rule, can be regarded as "Dauermodifikation" or durable modification. Since the gradual, reversible change of genes takes place gradually under the influence of environmental factor, the return to the original structure on the removal of the environmental factor, which induced the change, should take place also gradually, with the result that the changed character continues to exist for some periods of time after the removal of the factor.

Durable modification, however, is found mainly with unicellular organisms. In the case of higher, multicellular organisms the modification is, as a rule, restricted to one generation, not being inherited to progeny, although its transmission from soma to soma appears to be possible. This is apparently not to be attributed to the failure of the transmission of the change of the somatic cell to the germinal, since, as will be discussed below, there are good reasons to consider that the transmission is quite possible. The general non-inheritance of acquired characters in higher organisms should be ascribed to the fertilization in which the changed pattern of the germ cell is to return to its original state through the structural disturbance by the germ cell of the other sex. This return to the original pattern is nothing but the rejuvenation caused by the fertilization as already stated.

This is also the case with unicellular organisms. It has several times been reported that the occurrence of conjugation or autogamy was particularly likely to result in rapid reversal of an adaptive change. In every case described the adaptive change can take place only during periods of asexual reproduction, in cells originally derived from single ancestral individual.

For example, by culturing for long periods in the presence of sublethal amounts of arsenious acid, a strain of paramecium was obtained which was several times as resistant as its parent (72). This 
strain retained its resistance during hundreds of vegetative divisions in an arsenic-free medium before beginning to revert gradually to its former sensitivity. However, if conjugation of two resistant cells occurred, the progeny became completely sensitive. It should be noted that irregular fluctuations of temperature and other changing conditions hastened the de-adaptation, though not so conspicuous as conjugation. As was already emphasized, the rejuvenation or the recovery of the former structure is caused generally by the disturbance of the structure; the effect of the conjugation or the fertilization is based upon this disturbing action.

A similar phenomenon is also kown with trypanosomes, rendered resistant against arsenophenylglycine. The resistance can be retained for considerably long periods, but a passage through a rat louse and then to a healthy rat the resistance is lost completely, thereby the trypanosomes become highly susceptible to the drug (73). As was previously discussed, in the respect of the rejuvenescence the host change is analogous to the fertilization.

However, inspite of such rejuvenating action of fertilization, whereby the recovery of the original pattern will be facilitated, there are numerous evidences showing that acquired characters even of higher organisms are transmissible to progeny, though of a transient nature. The wheat of a south country can shorten the period required for reaching the maturity, when removed to the north and cultivated there for prolonged periods. Such an acquired character is likely to be retained for several generations even wheh the wheat is removed to the south. It is well acknowledged that various characters, based upon habitats, of cultivated plants tend to continue even when the plants are transplanted to entirely different places, although the characteristics will diminish gradually. The same has been reported with water-flea having the shape varying with the habitats.

It has been confirmed that the acquired character to produce antibodies to some antigens can sometimes be transmitted to progeny. Thus, domestic doves and chickens immunized with some viruses transmit the character to produce the virus-neutralizing antibody through the egg to their offspring, although immunity conferred on the offspring is short, lasting a maximum of 12 weeks in dores and of 4 weeks in chickens (73a). This may be regarded as the transmission to the progeny of the antigenic pattern impressed in the antibody-producing cells by the virus.

The transmission of the very pattern of bacteria or of the viruses to the offspring through germ cells of the host, a phenomenon which was repeatedly discussed, should be likewise regarded as a kind of a durable modification. The pattern is to be lost sooner or later eren 
if its transmission to the progeny is possible unless it is repeatedly engraved by infection.

The Lamarckian theory of the inheritance of acquired characters is not generally accepted because of the failure to secure any definite evidence for the theory. However, since the change of the genes is reversible, the evidence demanded has never been, and will never be, obtained. The organism can develop a new character under a new environment, but, when it is returned to the original environment, the change is not lasting. For the continuance of a new character, the new environment inducing the new character is always needed, and in so far as the new environment continues to exist, the new character is not only lasting but also will gradually be stabilized.

Here we should remind the most remarkable character of proteins already discussed. Namely, proteins can recover their previous structure, because the previous structure is remaining in their memory, but if it is forgotten the recovery will become impossible. This very forgetting is to occur when proteins exist for prolonged period away from the previous pattern. Therefore, a new character produced under a new environment will be fixed when the new environment becomes constant, and as a result the character will not to be Iost so soon even when the organisms are removed to the previous environment.

Thus adapted characters of organisms to environmental factors, such as temperature, light, and nutrition, will not only remain in existence as long as the factor continues to exist, but will be gradually stabilized in being accumulated little by little in the genes, until the acquired pattern becomes the ordinary one with the oblivion of the previous pattern.

If an environmental factor is again altered in a new direction after the former pattern has been forgotten or the return of the former pattern has become difficult, then adaptive change will begin anew on the basis of this acquired pattern without returning to the previous one. In such a way the pattern of the genes will continuously advance leading to the evolution of the organisms. Newly formed pattern is always unstable, and so the isolation is indispensable for the evolution.

In the case of individual development or ontogeny, as previously stated, newly formed structures are likewise unstable, but they will gradually become stable and irreversible as a result of the oblivion of former structures. Also in this respect, ontogeny recapitulates phylogeny.

In short, it may be concluded that the oblivion of the former structure is necessary for the evolution. However, for the function of the germ cell to recapitulate the phylogeny, an extremely strong 
memory must be required. For this purpose higher animals may possess a certain group of cells, in which the memory is well kept by the repetition of the reversible change, to produce the germ cells.

\section{The Transmission of Acquired Pattern to Germ Cells}

In the case of unicellular organisms, the transmission of the change, given rise to in the protoplasm, to the gene may readily take place, but in multicellular organisms, its transmission to the genes of the germ cell appears at first sight to be impossible, although for the establishment of the inheritance of acquired characters the transmission of the pattern from soma to germinal seems indispensable. The writer holds the opinion, however, that this is actually possible, and factors involved in this process are both hormones and virus-like agent as will be mentioned below.

For a multicellular organism, to exhibit various functions as a single living organism, that is, to behave as an individual, a variety of cells or cell systems involved in the organism must be coordinated, or must work in harmony. This coordination is established usually by two ways. One is by means of substances that circulate in the blood, namely hormones, and the other is by means of impulses that pass along the nerves. As the result of secretions which enter the blood from endocrine glands, what is known as chemical coordination is possible.

It has been known for a long time that various external influences, especially deleterious agents, applied to higher animals lead to a striking change in the function of endocrine glands. At present, it is generally believed that the endocrine system plays a prominent part in the adaptive reactions which occur irrespective of the specific nature of the damaging agents. The sum of all these non-specific, systemic reactions of the body which ensue upon long-continued exposure to stress has been termed the "general-adaptation-syndrome" (74). It is characterized by a number of morphological and functional change. Among the most prominent of these is the enlargement of the adrenal cortex with increased corticoid-hormone secretion, involution of the thymus and of other lymphatic organs.

A number of deleterious effects such as reduced atmospheric pressure, extreme heat or cold, severe muscular work, hemorrhage, burns, traumatic shock, and a large variety of drugs, cause histological signs of increased adrenaline and corticoid hormone secretion. All these phenomena run closely parallel with the general damaging effects of agents and must be regarded as part of the general-adaptation- 
syndrome which they elicit. Their influence upon the cortex is mediated by the anterior lobe of hypophysis and prevented by hypophysectomy.

It is considered that the effect of such damaging agents or the stress is first received by the anterior lobe of hypophysis with the increased secretion of adrenocorticotrophic hormone which induces the adrenal cortex to produce corticoid-hormone; the production of gonadtrophin and thyrotrophin, on the contrary, may be diminished by the same stress.

Of the various environmental factors, climatic ones seem to be most closely connected with animal and vegetable life, whereas it is supposed that also the climatic effects are received mainly by hypophysis or pituitary gland, and that seasonal cycles of pituitary activity that are reflected by cytologic change or bioassay are known to occur throughout animals especially in vertebrates. It is reported that a combination of environmental factors, including amount of rainfall, mean temperature, and a food supply, affects the pituitary cycle of some animals (75). Moreover, it is stated that the breeding season rhythm of all amphibia is under the anterior pituitary control. which is in turn influenced by nervous factors, and the final release of pituitary hormones is thought to occur in response to complex climatic changes. Internal sexual rhythms are usually adjusted to externa seasonal change.

It is generally acknowledged that water metabolism of man is extensively effected by climatic factors, wheras it is known that the water metabolism is subjected to the function of adrenocorticotrophic hormone of hypophysis. On the other hand, the features of plant activities such as flowering and fruit bearing are, as is well known, governed by climatic factors, such as temperature and light, whilst it is believed that in this connection hormones or related substances play an important part. Some animals such as the chameleon can promptly change the colour-pattern in response to the colour of back ground. Such a colour change in several vertebrate species depends upon stimulation of the eyes which is transmitted to the endocrine glands, which in turn produce hormones acting directly upon the piginent cells, chromatophores.

The interfering action of hormones upon genes is remarkable as already emphasized, and consequently changes in the feature of hormone secretion induced by environmental factors may be able to have influences peculiar to the changed feature upon the genes of germ cells leading to the formation of peculiar characters of the organisms. However, the effect of hormones cannot be so specific as can produce the amazingly specific adaptation of organisms. The specific 
change corresponding to a given environmental factor will be produced in the cells or tissues upon which the factor is directly supplied; and such a specific change may be unable to be transmitted to the germ cells by hormones. However, if the specifically changed structure of the protoplasm is liberated from the cell in the form of a "virus" the structure will be easily transmitted to the germ cells.

In ontogeny, as was already considered, the development of protoplasm structures inducing the advancement of various characters are apparently achieved by both hormones and virus-like agents. It may, therefore, be reasonable to suppose that in phylogeny also virus-like agents, in addition to hormones, are involved, since the miniature of phylogeny is seen in ontogeny.

It is most interesting that this concept is well in accord with Darwin's pangenesis theory. Darwin considered that minute particles termed gemmules may be distributed throughout the body of the organism in order to accept external effects and subsequently to transmit them to germ cells. Of course, gemmules are no more than "viruses" in the writer's concept.

It was already pointed out that primitive structures of the protoplasm are unstable and weak, as the primitive structures are the newly produced as compared with the differentiaied ones. Chick embryos have primitive structures and so can readily affected by various viruses. In a similar way, since germ cells or mother germ cell are of primitive nature, their structural pattern will be readily changed by the "viruses" or gemmules. Consequently, environmental effect may be easily transmitted to germ cells or mother germ cells through virus-like agents.

The structural correlation between soma and germ cells or mother germ cells, suggested in Chapter I, 3, in this Part, can be thus achieved by "viruses." The genes in germ cells are considered, therefore, to be always accepting the structural pattern of every part of the body through the "viruses"; and accordingly the genes in the germ cell must be the representatives of all patterns of somatic cells. The patterns engraved in the genes will be developed during ontogeny when the conditions become fitted for their development.

To sum up, if certain somatic cells are changed in their protoplasm structure by a certain environmental factor, the changed structural pattern will be transmitted by the "virus" to the germ cell; and the character corresponding to the pattern will be developed in the progeny, if the pattern is strong enough not to be returned to its original during the development.

Hormones may be able to induce striking changes in the structure of the genes, but their action is rather non-specific as compared to the 
highly specific effect of the "virus." Presumably hormones may act as helpmates of viruses, assisting the development of the specific structure determined by the latter. As was already pointed out, mammary cancer virus or leucosis virus can act as such only when sexual hormones or related factors are present. At an early stage of the individual development, hormone-like substances may assist the function of organizer, which can be looked upon as a strong structure comparable to a virus.

During pregnancy, striking changes take place in the feature of hormone secretions, especially leading to hypertrophy of the hypophysis associated with its increased function, indicating the requirement of unusually increased activity of hormones for the development of the foetus.

\section{The Effect of Use and Disuse}

It is a familiar fact that during the life of an individual, new characters can be acquired by use, and that disuse leads to the disappearance of the characters. For example, the use of muscles increases their development, while disuse results in the deficiency or even complete loss of the function. According to the Lamarckian theory the characters thus acquired in an individual are inherited by its offspring. Although the majority of authors claim that this theory has no support from modern biology, the writer is able to provide the theoretical basis to this doctrine.

The development of a structure by use can be regarded as a sort of the general phenomenon that the cells are increased in their activity upon the reception of stimuli, a phenomenon which in turn may be based upon that the protein molecules may be activated by a proper physical or chemical factor as a result of temporary liberation of free polar groups as previously stated. The change in certain somatic cells thus produced by use may be transmitted to the germ cell through the "virus", as in the case of other structures changed by environmental factors. There is, of course, no reason to suppose that the change brought about by use is different from that induced by usual environmental factors. The change induced by use must likewise be reversible, and so disuse will result in its disappearance.

Also in the development of the pattern induced by use, just as in that caused by usual environmental factors, hormones appear to be involved. As above stated a severe muscular work leads to the increased corticoid-hormone secretion. It is a well-known observation that cows may be brought into full lactation some time before partu- 
rition, if they are milked regulary, and lactation may be induced in virgins, or women many years past the menopause, by repeated stimulation of the nipples through sucking. In this connection, many workers believe that nervous impulses from the nipple affect pituitary function. Further, it is stated that the acquisition of new modes of behaviour as a result of experience cannot occur in the absence of hormonal influences (76).

Thus the pattern developed by use in the local somatic cells can be transmitted to the germ cells by the "virus" whose function may" be strengthened by the cooperation of hormones. If the pattern transferred thus to a certain gene in the germ cell is strong enough to be preserved, even if partially, until the individual developed from the germ cell begins to use the organ in question, the pattern will be accumulated in successive generations resulting in a striking development of the organ. However, since such a development may be also reversible, disuse will lead to the disappearance of the character, although the disappearance will become more and more difficult if the use extends over generations. 


\section{CHAPTER IX \\ EVOLUTION AND MUTATION}

\section{The Significance of Mutation}

As discussed in Chapter $\mathrm{V}$, environmental effects may give rise in organisms to adaptive changes, which are usually advantageous, as a result of the gradual, structural changes of the genes. The environmental effect is gradually transferred to genes through the cytoplasm or hormones. Such a change of genes is reversible, so that the change will disappear when the environmental condition is returned to the original state.

On the other hand, occasionally, strong physical or chemical agents can act directly upon the genes, producing sudden, irreversible changes. A change belonging to this category is called mutation. It is usually deleterious to the organisms in contrast to the gradual, reversible change. Up to the present, this latter change has been disregarded presumably because of its reversible nature, whereas the former, irreversible change, namely mutation, has attracted universal attentions and is generally regarded as one of the main causes of evolution.

However, such a random change can by no means be considered to be significant for the evolution, as was already emphasized by Osborn. In fact, as just mentioned, characters raised by mutation are mostly deleterious to the organism far from being useful.

The mechanism, by which the abrupt change of the gene is not only prevented but on the contrary favourable gradual change is induced in the gene, must be of the utmost importance for the establishment of the evolution, and consequently such a mechanism might have been considerably developed even when the organisms were in rather primitive stages. It should be remembered that the adaptive, reversible changes are common even in the primitive organisms, such as bacteria and protozoa, whilst mutation is rather unusual. In higher animals, environmental effects may be modified and unspecialized by hormones, thus abrupt changes being completely prevented. But, at the same time, the reaction modus of higher organisms against common environmental factors may become restricted, and the direction of the evolution is accordingly confined in a narrow scope.

It is believed that Australia and New Zealand have been separated 
from the Asiatic continent probably ever since the Cretaceous period, and nowhere else in the world do we find so many old-fashioned relics of earlier mammalian forms. At the time of the Australian region was cut off from the continent of Asia, marsupials were probably the highest mammals in the region. In their isolation they were not brought into competition with the higher types of mammals which were evolving on the other continents; hence they have lingered along the path of development at the lower levels of mammalianism. Evolution, however, has not been at a standstill among them, for we find that special types have arisen to occupy many of the available physical and nutritional stations of animal existence. Thus, there are carnivorous forms such as the so-called Tasmanian wolf, and herbivorous forms like the kangaroos; or in terms of dwelling place there are land forms (Tasmanian wolf, kangaroo, wallaby), burrowing forms (bandicoots, marsupial mole), arboreal forms (phalangers, Koala, Dasyurus) and volplaning forms (flying phalangers).

It should be noted that these forms of divergency, commonly called adaptive radiation, are very similar to those of other continents where various higher types of mammals have been developed, indicating that the direction of the evolution of mammals is strikingly restricted. Presumably this is due to the highly developed mechanism by which environmental influences are reduced to limited forms, and the mechanism may consist of the hormones.

Organisms especially higher animals appear to be able to avoid abrupt mutations by such elaborate means. It may be said, therefore, that organisms liable to undergo mutations are only defective specimens, never fitted to continue their existence.

\section{Genic Changes due to Chemical Agents}

Typical, gradual changes in higher animals are considered to be induced by physical environmental factors, such as climatic ones, which are transferred to the genes after greatly modified by hormones, whereas chemical agents appear to affect the genes directly and abruptly; accordingly, the change due to chemical agents is rather a mutation, occurring rapidly and tending to be irreversible.

Viruses afford a good example of the mutation of this category. The specific structure of viruses is transmitted directly to the genes, and the change occurs rather rapidly, tending to be irreversible and usually even injurious to the organism. The action of pneumococcustransforming principle, as discussed repeatedly, may belong to this category. This principle causes no injurious effect unlike usual viruses. 
On this point the change raised by this principle is different from mutation more than is the change raised by usual viruses.

This tendency is more manifest with the production of the so-called adaptive enzymes. Adaptive enzymes may be produced as a result of the adaptation of an organism to chemical substances whose specific structure is transmitted to the genes of the organism. The production of antibodies can be likewise regarded as a type of such adaptation (71). In these cases, although the specific structure may be taransmitted to the genes rather abruptly, the change can no more be called mutation, since it is not only completely reversible but very useful to the organism.

Such a change is called adaptation because it is useful to the organism, and such a usefulness has been undoubtedly established because the organisms capable of performing such a change could continue to exist, and therefore the character to adapt themselves to chemical agents have been advanced amazingly in the organisms. In this respect, it bears a striking resemblance to the gradual change due to physical agents, both being purposeful and have been developed by organisms for their existence. It should be realized that local changes in somatic cells would never be transferred to the germ cells without virus-like agents. This must be a very useful function of "virus", a mutagenic agent.

This seems the case not only with a single individual, but also with an organized colony of gregarious animals such as termites and honeybees, as various individuals in such a colony may be regarded as so many cells; the colony may be equivalent to an individual of multicellular organisms, and so the kings and queens may correspond to germ cells, and workers and soldiers to somatic cells.

The soldiers of termites have greatly enlarged mandibles, guard the king and queen and defend the nest against the attacks of intruders. Again, the specialized structures and habits of the workers of the honeybees is remarkable, food is collected and stored, the young are tended and fed. Each individual groups of these insects exhibit such amazingly instinctive reactions, which must be the result of inherited reflexes. How can have arisen and then inherited these characteristics of each individual group, since the workers and soldiers leave no descendent, unless the characteristics are transferred by virus-like agents to the kings or queens?

Such a transmission of characteristics by virus-like agents appears to occur even among fruit flies which are kept in laboratory. In an observation with a certain strain of the fruit fly characterized by a high mutation rate, Mampell (77) found that the mutability is increased not only in this species but also in the individuals of another species 
raised in the same cultures. He has suggested that the characteristics may be transmissible through a virus-like agent. Again, L'Heritier (78) has published a full account of the behaviour of the "viroid" or cytoplasmic particle responsible for $\mathrm{CO}_{2}$ sensibility in Drosophila. The agent is capable of transmitting the sensibility from an sensitive individual to other normals as do usual viruses.

As already stated, besides virus-like agents hormones are involved in the communication of characters among different cells or organs in a multicellular organsism. Similarly, the balance of castes in a termite society is believed by some workers to be maintained by means of certain special hormones (79).

The castes of termites develop according to the needs of the colony, just as the embryonic cells of a single animal differentiate into blood cells, bone cells, muscle cells and so on. Thus a colony of a termite is a kind of superorganism. The caste of a termite is not fixed; any nymph in a colony of termites can develop into a soldier or a king or queen of the supplementary type, developing on what the situation demanded. Such a development is controlled by means of some kind of the so-called social hormone, produced by the differentiated adults and acted upon the undifferentiated nymphs. When the colonies were separated by a screen which prevented any contact, the orphanized colony was produced and supplemented its own king and queen in the normal way. An active principle transmitted by contact, that is, a social hormone, is responsible for the development of differentiation.

As fully discussed in Chapter II, at an early stage of the development embryonic cells are endowed with unstable structures and so can change in every direction according to the change of environmental factors, but as the development advances, the structures will become gradually fixed and unchangeable. Entirely the same phenomenon is recognized in the development of the castes of a termite society. Thus, probability of change in a nymph decreases with time after molt. With the proper stimuli all nymphs that have just molted will change, but of the nymphs that molted 19 days earlier, only 50 per cent will change and 50 days earlier less than 20 per cent.

These facts clearly show that the castes of termites correspond to cells or organs in an ordinary multicellular organism, which the latter in turn is equivalent to a colony, and that the development of the castes is achieved by the same principle as that of the cells of an embryo. Therefore, the above concept that the transmission of the characters of soldiers and workers to the king and queen is achiered by "virus" seems to be only natural.

It is well known that numerous varieties of flowering plants are attributed to virus infection. The most usual effect of a virus infection 
on the flower is to cause a characteristic change in the colour. The oldest known example is tulip break due to an aphis-transmitted mosaic virus. Another common virus variegation occurs in the flowers of wallflowers and stocks and other cruciferous plants (80). These examples should be regarded as mutations due to viruses rather than as virus-diseases.

Many breeders believe that domestic animals will sometimes breed the young resembling on some point to the male animal which is not the true father of the young but with which the mother animal was once crossed, a phenomenon called telegony. This phenomenon is, of course, never consistent with the modern theory of genetics, and accordingly it has been dismissed as a mere bigotry. Nevertheless, this appears possible according to the writer's concept.

It has been well established that males of cancer strains of mice may transfer the virus-like agent capable of producing the cancer at the time of copulation to infect females of either susceptible or relatively resistant strains and that these infected females may propagate and pass the agent to their progeny (81). If this is caused by "infection", not by the transmission of the pattern through the germ cell to the progeny, such a female mouse may be able to transfer the character of the male to the young of another male. In case where the sperm of a male after the fertilization developed in the body of a female into the foetus, the influence of a male pattern upon the female would probably be far more striking. Moreover, it may not be quite impossible that every married couple tends to become Jack and Jill after a prolonged cohabitation not only through the mental connection but through virus-like agents. 


\section{CHAPTER $\mathrm{X}$}

\section{THE MECHANISM OF EVOLUTION}

\section{The Evolution without Natural Selection}

An alteration of the environment results in a change in the character of the organism. The change thus raised is mostly favourable for the organism and will be advanced as long as the environmental factor to induce the change continues to exist, until at last the changed character becomes fixed and inheritable. For example, if a mammal is kept under the environment of a high temperature, its tail and ears will become long and expanded as shown by the Sumner's experiment with rats as above cited. Such a change is indeed significant for the temperature regulation, and hence the mammal is said to have adapted itself to the high temperature. If the mammal is kept under such a high temperature for successive generations, the acquired character will become hardly reversible and fixed so as to be inheritable.

According to the experiment of Sumner, some of the rats exposed to high temperatures for only one generation tended to transmit the character to the progeny. The phenomenon, known as Allen's rule, that mammals of the warmer climates expand body surfaces by the development of parts, such as ears and tails, may be the result of this adaptation fixed by Nature. Here, it should be realized that such adaptive evolution is possible to occur without any natural selection, that is, organisms can evolve without natural selection in adapting themselves to environments.

The evolution must be far more rapid and easier if the effect of use is added to the environmental factor. Thus, if a tail, lengthened by a high temperature, can be used to grasp objects and if its use is required, the animal will use it repeatedly and this effect of use will be added to the influence of the temperature to derelop the tail extensively. In such a way, tails of certain animals such as monkeys and rats have probably developed.

The stimulus resulting from the use would become greater, the higher the organ was advanced, because of the more unusual and incessant use, and therefore the development of the organ would be advanced unlimitedly unless it ceased to be used. Orthogenesis may be thus established. Natural selection may help more or less the 
derelopment, but there is no doubt that the evolution is quite fossible without selection.

Every character of organisms is wonderfully fitted for the purpose of their existence. Since the character to achieve the evolution must be of the most importance for the organism, extensive advancement should be expected also in this character. In addition, since the character must be advanced with the progress of the evolution, it must be the more advanced, the higher the organimsms are evolved.

Nevertheless, at present the majority of authors seem to believe that the organic evolution was advanced mainly by virtue of random mutation and natural selection, a wonderfully clumsy means which might only be adopted by extremely primitive organisms as mentioned in the next section.

\section{The Evolution of Evolution Mechanism}

New protein molecules are considered to be syntheszied as replicas of the template protein constituting the protoplasm or assimilase, but the newly formed replicas may not be precisely identical to the template because of partial failure of the replication, thus arising the individual differences in the structure of protein molecules. Such an individual difference of the structure may in turn give rise to the difference in the behaviour of each protein molecule or each polymerized product of molecules towards the environmental factor, and accordinly each may tend to undergo each peculiar change or mutation. Variations of primitive organisms might be thus raised and become distinct. An individual having a structure tending to be changed in a definite direction under the effect of a certain environmental factor might continue to be changed in its structure in the direction under the influence of the factor.

Among the individauls thus coming to have different structures, only those most fitted to exist would be able to survive, that is, the fittest would be selected by natural selection. As a result of this: selection, the faculty to evolve spontaneously into a well adapted direction might be gradually advanced.

Although the protein itself possesses the basic character to adapt itself to environments as alrcady stated, the character might be insignificant when it was not yet developed enough, and presumably random mutation and selection might play a predominant part in the evolution. of protein molecules.

The evolution brought about thus mainly by mutation and selection might continue until the proteins were polymerized into complete form. 
of assimilase, or until the assimilases were endowed with characters as primitive organisms. The progress of the evolution in such a manner, however, was most probably highly tedious, and hence a dreadfully long span of time might pass between the appearance of assimilase-like protein aggregates and the production of the organism-like assimilases. The very remarkable evolution mechanism belonging to the present organisms was presumably advanced thereafter, and since the mechanism might be advanced the more, the more evolved the organisms, the rate of the evolution of the modern organisms may be extremely great as compared with that of primeval organisms.

It seems reasonable to regard the acquisition of resistance of bacteria to several antibacterial drugs as a type of the adaptation seen in higher organism, but a number of authors claim that the resistance is attributed to spontaneous mutation and natural selection, that is, the emergence of a population resistant to a given drug results from the selective effect of that agent upon mutants arising spontaneously from the original population (82). Since in primitive organisms such as bacteria a highly advanced mechanism of the evolution cannot be expected, adaptation may be not so distinct as in higher organisms. But it should be borne in mind that adaptability is one of the basic characters of protein itself and so the protein molecule itself can be adapted to environments though in an undeveloped way.

Mutation may be looked upon, in a sense, as a generation of a virus as discussed already. Hence, the primary organisms in extremely primitive stages may be said to have been evolved by the successive production of viruses. Accordingly the primary organisms may be considered as being originated from viruses like the secondary. In this respect, there appears no fundamental difference between the primary and the secondary organisms.

In fact, it may be difficult to draw a distinct line of demarkation between these two main groups of organisms. It may be said, however, that the secondary organisms were generated on the basis of preexisted protoplasm or organisms, whilst the basis on which the primary organisms were developed was the incomplete protoplasm or the aggregation of protein molecules which had been produced without organisms. Therefore, the secondary organisms unlike the primary have their host at least when they are in their primitive stages. Nevertheless, in the case of the secondary organisms that developed on the basis of the primary organisms existing at the most primitive stage, there might be no distinct difference between the parasite and the host, and so the parasitic nature would be insignificant in such secondary organisms.

In this respect it may be suggested that the Mollusca may be the oldest secondary organisms, because their parasitic nature seems only 
trifling, if exists, and the Echinodermata which may be the oldest primary organisms are being adopted by them as the host (83).

\section{The Survival of the Fittest}

As we have seen above, natural selection appears not so important for the evolution of the higher organisms as customarily believed, but the selection may play sometimes a prominent role in the determination of the direction of evolution.

The genes are provided with the property to achieve the gradual change in a certain direction. However, as the genes have the individuality, even under the same environmental effect every gene will behave differently, changing in different rates and in different directions. Nature will select the gene which has been changed in the most fitted way to the environment. As the gene thus selected may have the property to continue the change favourable for the organisms, the organisms having the gene will continue the favourable change as long as the environmental effect in question remains in existence.

For example, if a colour change brought about in an insect under a certain environment becomes significant as a mimicry, the insect will be selected and separated from other individuals, and since the insect thus selected has the property to advance the colour change and, in addition, since the selection will continue without resting, the mimicry will sooner or later be completed, although it may be possible that the change may go so far as becomes useless as mimicry.

If herbivorous animals need fleetness in order to escape from their enemies, by which the slower-footed individuals are to be devoured up, the swifter-footed individuals will be selected and separated from the original type. Since the selected individuals may have the tendency to develop the character, the fleetness will gradually increase even without further selection, but the continual enforcement to the use and the successive selection in each generation will no doubt accelerate the development. Thus, swift-footed animals like horses have presumably been created.

The phenomenon known as vernalization can be regarded as resulting from the natural selection by which a character fitted to a peculiar environment has been adopted and developed. As is well recognized, spring rye, in common with other spring cereals, requires no low temperatures during germination for normal flowering, whereas winter rye, unless exposed to low, temperatures during germination, as is known as rernalization, shows a great delay in ear formation and emergence. 
As was already discussed in detail, germ cells in general, whether they are of animals or regetables, may develop various characters in response to the environments to which they are exposed. The seed of the winter rye is to be always exposed to low temperatures during germination and so if in some individuals a character favourable for the subsequent development was produced by this stimulus, the individuals capable of acquiring such a character under the effect of low temperatures would be selected as the fittest, and as a result the individuals thus selected would fail to show usual development without preliminary exposure to the low temperature.

\section{The Degeneration of Organs and Atavism}

Organic evolution is induced by the structural evolution of the genes, whereas the structural change of the genes is reversible. Therefore, organic evolution itself must likewise be reversible.

Our eyes are no doubt one of the most advanced organs. The development of the eyes is probably mainly brought about by continuous and exceeding uses, so that the cessation of the use will lead to the return to the original, undeveloped state, that is, the eyes will become atrophied by disuse. Thus, many species of fishes living in deep seas or in caves are known to be blind. Horses kept in mines and man kept in dungeons are said to have had their eyes so impaired that they could be restored to sight only by gradual exposure to light, showing the wonderful swiftness by which the eyes would degenerate. This suggests that their evolution has occurred rapidly and comparatively recently.

The theory of orthogenesis holds true in so far as the environmental effects including the stimulus from use are constant and unchanged. Any change in the effects, of course, would lead to a change in the direction of the evolution. In an extreme case, the direction may become entirely reverse. For example, when human ancestors might live on trees like apes, their feet might have been developed so as to be able to grasp objects, but their leaving from the trees followed by the cessation of the use of the feet to grasp, might result in the return to the original form. Again, whales might be able to walk when they lived on land, but their return to water was followed by the degeneration of the legs.

The reversibility of genes is attributed to the memory of the original structure. Hence, the diminution in the memory will lead to the difficulty in the manifestation of the reversibility. Since newly acquired characters may have strong memories, their disappearance may occur 
readily. Thus a character acquired during one generation may disappear rapidly on the removal of the causative effect. However, when the effect continues to exist for generations to stabilize the acquired character, the memory of the former structure will become faint, while the new one being fixed. It may be supposed, therefore, that organs like eyes having the tendency to degenerate rapidly are newly produced organs retaining still the fresh memory of the original pattern.

Attention has been paid for a long time to the fact that old organs which were useful in the old environment but which have ceased to have a function in the new, linger on in a reduced form with astonishing persistence, the grip of ancestry still upon them. Various land birds, particularly among those of oceanic island, have lost the power of flight. Externally no trace of wing is visible, but close inspection reveals a minute rudiment of wings still persisting under the long hairlike plumages, although wholly without significance as an organ of flight. Familiar examples of vestigial organs are presented.by the degenerative eyes of many cave animals.

The writer believes that such vestigial structures were produced as a result of a random change of the genes, or, more precisely, were produced involuntarily being associated with the development of a peculiar structure of a gene causing a certain useful character, and when a certain structure thus produced involuntarily came to have the value of use, the use would be commenced with the rapid development. Therefore, the portion which is to be degenerated by the disuse must be the structure that was developed rapidly by the use, vestigial structure thus being left unchanged.

Many useless structures are more fully developed in the embryo than in the adult, thus presenting an instance of the recapitulation of phylogeny by ontogeny. Some of the most striking cases are those of functionless organs which develop in the embryo but which disappear again before birth. For example, in whales both anterior and posterior limbs are formed, though the latter subsequently atrophy and in some species wholly disappear. Likewise the embryo is densely covered with hair, although the adult whale is devoid of hair. Furthermore, it was found in general that blind cave fishes develop what appear to be normal eyes in their early life history, but later the eyes are lost again through atrophy.

If the individual develpoment is interrupted halfway, some character may remain in an embryonal state, a phenomenon called atavism. The well known salamander, axolotl, shows atavism and remains for life in an immature form, performing respiration by gills instead of by lungs as in the larvae. This atavism is known as neoteny. Under a peculiar condition associated with a food change or water deficiency, 
however, this salamander may develop into the adult form of lungbreathing. The administration of thyroid gland substance seems most effective to lead the salamander to the adult form, suggesting that the inability to recover the adult form may be involved in the deficiency of thyroid hormone.

Environmental effects, as previously stated, appear to exert their influences upon genes mainly through hormones. Accordingly, hormones only, without accompanying any environmental effects, may naturally be able to induce certain changes in genes. As discussed previously, hormones play an important role in the establishment of individual development. The metamorphosis of tadpoles is accelerated by the administration of thyroid-gland substance and also, as is well known, that of insects is subjected to the function of endocrine glands. The pattern of individual development of the stag antler may show the pattern of its phylogenical development, but its development is interrupted by the castration, showing also the involvement of hormones. Thus certain environmental alterations having influences upon hormones may cause atavism as in axolotl.

The primary organisms were generated and evolved in water, so that their parting from water might be achieved only with unusual difficulties. In like manner, for the secondary organisms the departure from the parasitism may also be not an easy task. However, the return of land animals to water or of free-living secondary organisms to parasitism may occur comparatively easily, because the return must only be atavism or the recovery of the former structure. To be parasitic at the larval stage and free-living at the adult may be a natural form of the individual development recapitulating the phylogeny. The reverse is, however, unnatural, and so may be a type of atavism.

The advancement of parasitic animals into free living may be thus possible. On the other hand, the entry of free-living into parasitism appears to be impossible, because any spontaneous production of characters which are suited to parasitism, a most particular and extremely restricted environment, cannot be considered. From this point of view, the writer regards animals which are parasitic at any stage of life as the secondary organisms. 


\section{CHAPTER XI}

\section{THE CHANGE OF HUMAN CHARACTER BY ENVIRONMENT}

\section{Climate and mankind}

Sörensen (84) has concluded from his experimental results that proteins in biological fluids are composed of a variety of components in a state of equilibrium depending upon the environmental conditions, and that this equilibrium is shifted reversibly and readily through changes in the composition of the solution. According to the writer's concept, organisms by themselves are such systems of protein components, their state of equilibrium changing reversibly through the alteration of the environment. On the other hand, as the state of protein comlexes in organisms is governed by the genes, it can be considered that changes of organisms are induced by the genes changing reversibly in response to the varying state of environment.

Of such environmental factors, climatic ones appear to be of the most importance for us humans, for we are on all occasions exposed to the climatic factors which are always changing, and accordingly our genes are expected to be always changing with them. In the extensive investigation into the relation between civilization and climate, Huntington concluded that a stimulating climate is the main condition which promotes civilization.

His contention, as set out in "Civilization and Climate" is that a certain type of climate, now found mainly in Britain, France and neighbouring parts of Europe, and in the Eastern United States, is favourable to a high level of civilization. This climate is characterized by a moderate temperature, and by the passage of frequent barometric depressions, which give a sufficient rainfall and changeable stimulating weather. Now it is well known that the great centers of civilization in the past lay in more southernly latitudes than those of to-day, beginning in Egypt, Mesopotamia, and the Eastern Mediterranean and then passing to Greece and Roma. Huntington attributes these changes of the centre of civilization to climate change associated with the northward shifting of the belt of cyclonic activity. In this connection, he stated as follows in "Principles of Human Geography": 
"To understand the relation of climate to civilization, let us compare the province of Ontario, where the climate is one of the best in the worid, and the Bahama Islands, which have a warm, monotonous, tropical climate. The original white settlers in both places were of the same stock. They were English colonists, many of whom left the U. S. at the time of the Revolution because of their loyalty to England. Today the descendants of the Loyalists in Canada are one of the strongest elements in causing that country to be conspicuously well governed and progressive. In the Bahama the descendants of similar Loyalists are also one of the finest elements, but many of them are relatively inefficient. Among the Canadians practically every one has a fairly good education. Among the Bahamans a large number have never been to school, and many who learned to read and write in their childhood have forgotten these arts because they do not practice them.

"The main cause of these differences is the climate. As the Bahamans themselves say "This climate is rery healthful and pleasant. The only trouble is that it doesn't make one feel like work. In winterit's all right, although even then we can't fly all very well for you Americans to think we're lazy, but try living here a year or two yourselves, and you'll be as lazy as we are'. The regular routine of daily life can be carried on without much difficulty, but when a new kind of work is to be done, he says, 'Wait till tomorrow'. It must not be forgotten that a stimulating climate is only one of the conditions which promotes civilization".

We, the writer and Ohashi, observed the same tendency in Shanghai, while staying there for several years, and after the inquiry was made of Japanese students of the Dobunshoin University, a conclusion similar to that of Huntington was reached, although we were ignorant at that time of his splendid work. The climatic conditions in Shanghai were found to be comparable to those in Bahama in the example cited above. According to our investigation into the difference of climatic factors between Shanghai and Nagasaki, a Japanese city situated at the opposite side of the sea about in the same latitude, there was no significant difference in the average temperature or humidity, but it was noted that the extent of deviation of the factors including atmospheric pressure were far greater in Nagasaki than in Shanghai.

We expressed the opinion that in China, including Shanghai, as well as in tropical countries there is some deficiency in climatic factors, a deficiency causing a disorder in the function of endocrine glands especially adrenal contex (85). The lack of mental and physical activities and abnormal pigmentation common in the inhabitants in these countries may be accounted for by the hormonal disorder. The symptoms appear to be similar to those of Addisonism, a diseased state 
due to the disfunction of adrenal cortex. As we have seen above, climatic factors exert influences upon hypophysis, which in turn governs adrenal cortex. We beleive still at present that our conclusion was reasonable.

The lack of stimulating climatic factors proposed by Huntington may induce a disturbance of the pituitary function followed by a change in the feature of hormonal secretion, especially by a disorder of adrenal cortex resulting in the change of human characters. The continued lack of the factors for successive generations may lead to the fixation of the characters peculiar to the climate. The increase in the strength of the factors may, on the other hand, change the characters in the opposite direction.

There has long been a strong conviction among anthropologists that head form is the most reliable element in the classification of race, but Boas (86) in measuring with many immigrants and their children in New York City proved that even the cephalic indexes which were formerly considered permanent racial traits are changeable by environment. Thus, the children of broad-headed Jew from Poland, born in New York, proved to be less broad-headed than the parents. Moreover, the reduction in breadth of head of the children increased in accordance with the duration of the residence of the mothers in New York. Another group of immigrants consisted of long-headed people from southern Italy. In their cases, also, there was a slight but significant difference between the American-born children and their parents or brothers and sisters born in Europe. This difference, too, increased as the time elapsed since the immigration of the parents. In this case, however, the change was towards greater instead of less breadth of head. In other words, each type of children tended somewhat away from the standard physique of the parent, and towards a less extreme middle type.

This middle type might be, therefore, the standard physique in New York, determined by the environmental factors, probably chiefly those of climate, prevailing there. It should be noted that the change in the children was increased with the duration of residence of the mothers. This fact indicates that the influence of the prolonged residence exerted on the mother was transmitted to the children, proving clearly the inheritance of the acquired character.

\section{The Inheritance of Habitude}

The environmental effects which change the human characters may not be involved in climate only as will be considered later, but the fate 
of civilization can be easily explained even by taking only climate into consideration. It is true that the people living in the regions of the climate lacking in the stimulating factors, especially in tropical regions, seem to be born tired, whether or not it may be due to a disorder in endocrine glands, and seem to dislike to use their brains. On the other hand, disuse of the brain will result in its atrophy. Thus, disuse of the brain for successive generations may lead to the production of mentally inactive people, whilst people live in climate full of stimulating factors may become more and more mentally active by the constant use of the brain.

The change in the brain pattern thus produced may be transmitted to germ cells by virus-like agents. In addition, hormones may beconcerned with the transmission as in the pattern of other organs. The majority of authors are inclined to believe that hormones exert their most direct and pronounced effects upon behaviour through the induction of changes in the central nervous system. As pointed out' already, the acquisition of new modes of behaviour as a result of experience cannot occur in the absence of hormonal influences, although. all evidences point to the conclusion that capacity to learn is not directly and immediately dependent upon the secretion of a singleendocrine product.

In addition, certain hormones appear to exhibit pronounced effects. upon emotion in human (87). The conspicuous difference in human emotions due to the difference in sex can, of course, be attributed to. the sexual hormones, indicating how great are the hormonal influences upon the emotion. On the other hand, certain endocrine functions. appear to be strongly influenced by psychic factors. Observing that repeated gentling of rats changes their reactions towards man from. flight or pugnacity to relaxation and docility, Hammett (88) reports that 79 per cent of "non-gentled" rats die within forty-eight hours. after parathyroidectomy, while among "gentled" individuals mortality within the same period amounts to only 13 per cent. There is a widespread impression that thyroid secretion may in certain instances. increase general irritability and contribute to the intensity of emotional. reactions. Administration of thyroid to Plymouth Rock hens is said to result in a change from a gentle and phlegmatic to a highly nervous temperament (89). In this connection, Rickey (90) claimed that the offspring of rats which have been fed thyroid gland substance are more nervous and erratic than the progeny of normal parents. On the other hand, as will be mentioned later, adrenal extract exhibits the function to release the nervous temperament in contrast to thyroid. Thus it may be concluded that a change in the function of hormonal glands due to the effect of environment, including climate, can induce 
the change in the brain pattern even if the effect of use or disuse is not involved, Therefore, the addition of the effect of use and disuse to that of the hormones may result in a striking change in the mental pattern, and when the effects continue to have their influences in successive generations, the mental characteristics will become strong and fixed and accordingly inheritable.

As to the inheritance of memory, a detailed consideration was made in the beginning of this Part. The memory in our brain is considered to be a structural change in the protoplasm protein of brain cells. Such a structural change can be transmitted to the newly formed proteins; thus the memory can be transferred successively to newly formed proteins or protoplasm. If this change is transmitted to germ cells by protoplasm particles, namely by virus-like agents, and if the changed structure does not return to the original during the development of the germ cell to the young individual, the memory can be inherited by the young. The memory of phylogeny has been inherited from generation to generation for a dreadful long span of time as the ontogeny. Moreover, every instinct of creatures must depend upon the memory thus inherited for generations.

One may say that not only do English children have to learn their own language, but they learn it no quicker than they would learn French if brought up from the first in a French-speaking household, and that this fact demonstrates evidently the failure of the inheritance of memory. However, if this is true, it can be considered that minute structural differences corresponding to the memory of difference between French and English cannot be transmitted, but that the faculty to learn words as a whole must be inherited and strengthened when man continues to speak a language.

A number of experimental evidences suggesting the possibility of the inheritance of habit has been presented by several workers, but mostly have been regarded as very doubtful like other experimental data concerning the inheritance of acquired character. Since the structural change in the protoplasm in central nervous system must also be reversible, it should naturally follow that the clear demonstration. of the inheritance of habitude likewise failed when observations were made only for a few generations. From the theory of the writer thus far described the inheritance of habitude seems to be a natural consequence. The splendid advancement of animals, especially of mankind as seen in the present day, might not have occurred without the inheritance of habitude.

Domestic animals such as dogs and horses are very faithful as well as obedient to their masters. This may probably not only due to the artificial sclection, but also to the inheritance of habitude. Thus, habit does become a nature. 


\section{CHARTER XII}

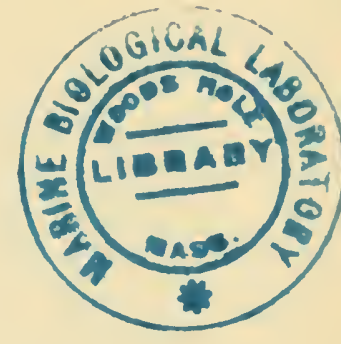

\section{THE EVOLUTION OF MANKIND AND ITS FUTURE}

\section{The Orthogenesis of Protein Molecules}

The denaturation or the structural change of proteins appears to proceed without stopping in so far as the change-inducing agents are present as seen in the change of phage by formalin. The process is compared to the gradual alteration of the genes by environmental factors. It is worthy of note, however, that the velocity of the process or the velocity constant, if calculated as a monomolecular reaction. tends to become smaller as the reaction proceeds. It is reasonable to regard the process as a monomolecular reaction, but if calculated as a bimolecular reaction the decreasing rate of the velocity constant is lessened, and therefore the writer attributed this phenomenon to the infection of denaturation, that is, he considered that the denaturation was autocatalytically accelerated by the transmission of denaturation from the protein undergoing denaturation to other intact molecules (61). Frequently, however, the decrease in the progress is so manifest that it cannot be explained even by this assumption and there seems to exist still another cause to lessen the velocity.

At present the writer believes that this must be based upon the tendency of the protein to recover its original structure, that is, upon the reversibility. As previously discussed, protein denaturation sometimes proceeds in oscillation like a physical phenomenon. This is interpreted as resulting from the competition between the effort of the protein to recover its original structure and that of denaturating agent to promote the change. If so, the velocity of the change would be the more lessened as the change progresses the more, since the repulsive force or reversibility should become stronger with the progress of the change. This appears actually the case. As every one knows an elastic substance can be bent easily at first, but further bending may become difficult on account of the increase in the force to spring back to the original shape. Protein molecules can be looked upon as such an elastic substance.

In Figs. 36 and 37 the progress of inactivation of rennin respectively by its antiserum and tannin is illustrated (91). In both cases 


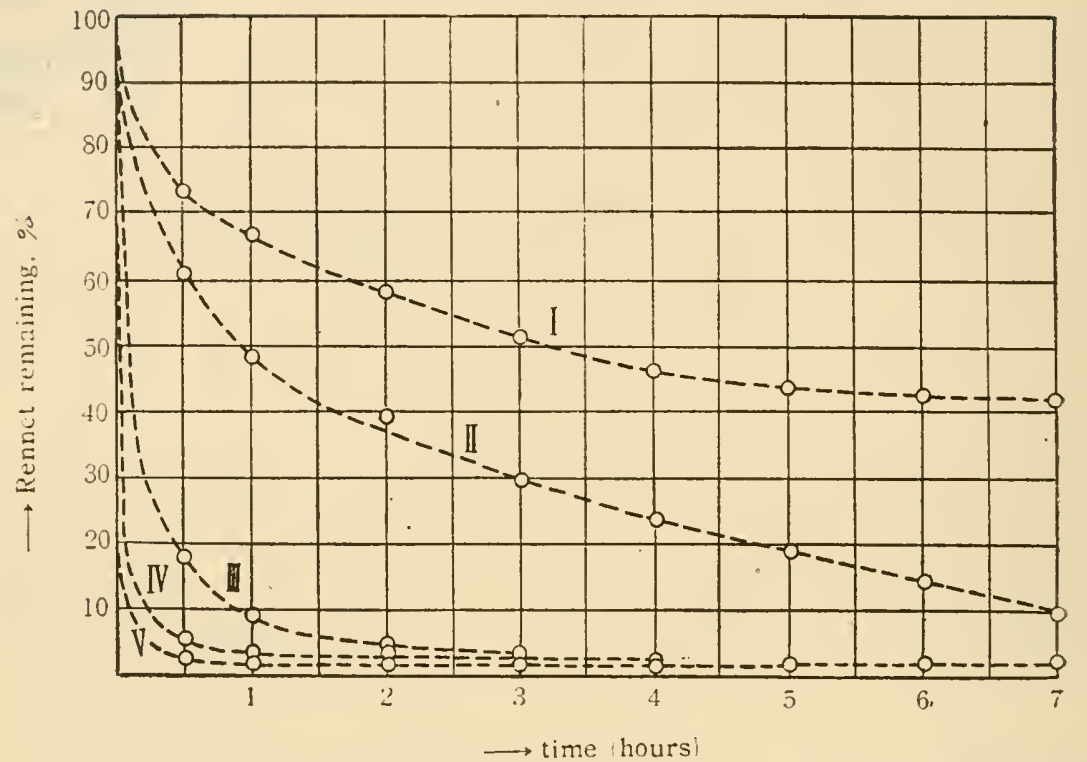

Fig. 26 Decrease in progress in the action of rennet due to anti-rennet serum. Antiserum : anti-rennet rabbit serum.

Rennet solution $(0.01 \%), 1 \mathrm{cc}+$ antiserum $0.9 \% \mathrm{NaCl}$ solution, $1 \mathrm{cc}+$ milk $\left(1 \% \mathrm{CaCl}_{2}\right)$ lcc

Dilution of antiserum: $I=1: 160, I I=1: 80, \quad I I I=1: 40, I V=1: 20$, $\mathrm{V}=1: 10$

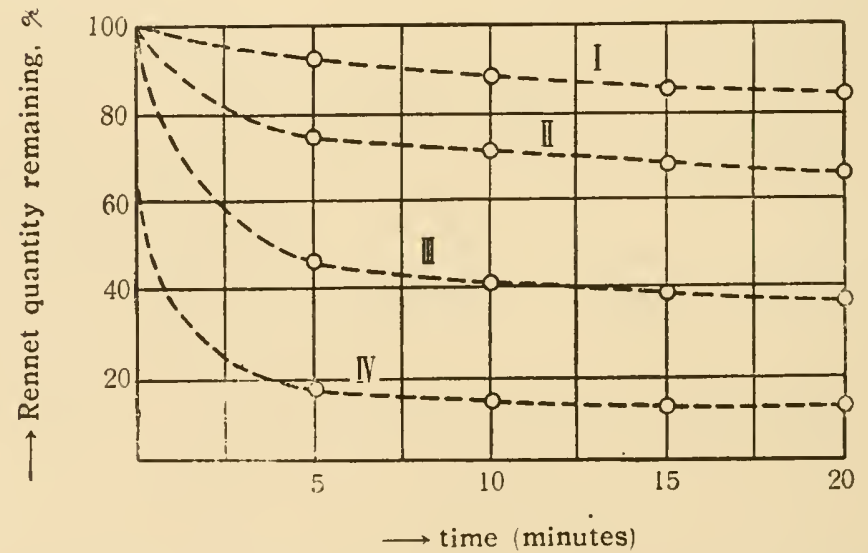

Fig. 37 Derease in progress in the action of rennet due to tannic acid. Tannic acid: Kahlbaum, used about a month after its dissolving. Rennet solution $(0.01 \%), 1 \mathrm{cc}+$ tannin $0.9 \% \mathrm{NaCl}$ solution, $1 \mathrm{cc}+$ milk (1\% $\mathrm{CaCl}_{2}$ ), $1 \mathrm{cc}$.

Concentration of tannic acid: I : $0.003125 \%$, II : $0.00625 \%$. III : $0.0125 \%$, IV : $0.025 \%$. 
the inactivation progresses rapidly at first, but later distinctly slows down presumably because of the increase in the intensity of the repulsive force.

The bending force of the inactivating agents, on the other hand, should be determined by the kind of the agents, and in one and the same agent, the higher the concentration the more rapid and the more extensive may be the change. The change of rennin due to antiserum or tannin in low concentrations appears usually to cease to proceed after some periods of progress, whereas the inactivation due to heat proceeds without diminishing the velocity until the inactivation becomes completed. Thus heat is regarded as an agent strong enough to be able to ignore the repulsive force.

In the case of phage, formalin acts as a strong agent, so that almost no diminution in the velocity is observed as already shown in Fig. 35, whereas some diminution is seen with $\mathrm{HgCl}_{2}$ or with heat, and in both cases of antiserum and tannin diminution is usually manifest (62). It may be said, therefore, that, if the force to push forward the change is much greater than the repulsive force, the change will proceed without decreasing, whilst if the former force is weak, the repulsive force will be revealed to diminish the changing velocity.

Presumably this holds true for the change of genes. The effect of environment including climate seems to exert its action comparatively rapidly on human genes as shown in the above cited observations of Boas, but a considerable span of time may be necessary for the immigrants from various parts to become an entirely similar type, if possible. which is to be determined by the environment of the settlement.

Here a great question may arise. If the repulsive force of the genes becomes greater as the change proceeds, the change is expected to be suspended sooner or later. If so, evolution is impossible unless the environmental factor is strong enough to disregard the force. Is this ever the case?

Here again we should remember the nature of the reversibility of protein structure. The reversibility depends upon the memory of the former structure. Therefore, the loss of the memory may result in the disappearance of the repulsive force, and consequently if a gene is left for a long time in the same changed state under the same environment causing the change, its former structure will be forgotten with the disappearance of the repulsive force, the change thus being gradually pushed forward. This seems not only the case with genes, but also with elastic substances in general. The organic evolution as already stated cannot take place unless the memory of the former structure is lessened or lost.

Proteins can thus be looked upon as an elastic substance and the 
elasticity is revealed most strikingly in the genes. As previously mentioned, there are actually many other evidences that protein molecules are elastic in themselves. The organisms having genes provided with only weak rigidity or feeble elasticity must be very sensitive to environmental changes, by which will be induced profound and extensive alteration in their characters. Therefore, if the rigidity in the structure of the genes is reduced by some or other causes, the organisms will commence an or thogenetic changes advancing rapidly with the extensive specialization until they become extinct due to the overspecialization.

The progress of phage inactivation by formalin does not decline as stated above, showing the relative weakness of the reversibility, but in a weakly acid solution the diminution of the velocity is manifest, and the progress frequently oscillates. Thus it can be said that phage as a gene-model had a strong reversibility in a weakly acid solution, but that in a neutral or an alkaline solution the reversibility is lessened and accordingly formalin will cause an rapidly-advancing orthogenetic change leading to the extinction of the phage.

\section{The Orthogenesis of Mankind}

As we have already seen, environmental factors including climate appear to exert the influence mainly through the endocrine glands especially hypophysis. Shapiro's work (92) on Japanese immigrants to Hawaii has led to the same conclusion as that of Boas on migrants from various Eeuropean countries to New York City. The Japanese migrants are taller than their stay-at-home relatives, and the children of the migrants depart further than do their parents. The children of Japanese in Los Angeles are also known to be taller than their kindred in Japan, the increase in size being especially distinct in legs and arms as compared to that in the trunk. Such an increase in stature may be readily explained by the assumption that the climate or other environmental factors in Los Angeles and Hawaii stimulate the function of hypophysis as will be detailed below. A similar change is reported to occur also in New York in the immigrants of Central European people, such as Bohemians. Slovaks, Iungarians, Poles, and Hebrews, suggesting the presence of the same environmental factor or factors also in New York.

It has long been known that pathological hyperfunctions of hypophysis result, on the one hand, in the gigantism in man, while, on the other hand, the experimental gigantism in rats or dogs was actually demonstated by injection of an extract of hypophysis. The final proof for the existence of the growth hormone comes from the 
isolation of the hormone in a crystalline form from anterior pituitary glands (93) (94). The administration of this hormone leads to weight gain, muscle hypertrophy, increased skeletal dimensions, and skin thickening in hypophysectomized rats. Climatic factors in general appear to stimulate the function of pituitary gland, and hence people living under a stimulating climate will increase in stature owing to the increasd secretion of this growth hormone.

On the contrary, people live in the climate lacking in the stimulating factor may become smaller because of the want of the growth hormone. In addition, it has been believed that adrenocorticotrophic hormone (ACTH) of hypophysis whose production is considered to be increased by the stimulating agent is antagonistic to both gonadotrophic and thyrotrophic hormones which are also produced by the anterior lobe of pituitary gland (95) (96), so that the absence of stimulating climate may increase the production of these latter principles.

In man, hyperthyroidism is often followed by degeneration and atrophy of adrenal cortex. There is an evidence of a decrease in cortical function after long-continued increase in thyroid activity. Moreover, adrenal extract counteracts the effect of thyroxin administration even in normal animals, reducing the increased loss of nitrogen and lowering the increased pulse rate. In addition, it is observed that corticosterone and desoxycorticosterone counteract the effects of mild hyperthyroidism of the liver (97). Corticosterone is known to be able to modify or correct the symptoms of hyperthyroidism.

There are also many experimental evidences that administration of cortisone or ACTH will suppress thyroid function (98). Similar findings have been obtained under experimental conditions which are known to be accompanied by increased secretion of cortical hormone including injection of epinephrine or formalin and expasure to abnormal temperature (99).

On the other hand, it is well recognized that animal metamorphosis fails to occur after thyroidectomy. Further, gonadotrophic principles are known to lead to the development of precocious sexual maturity. In this connection, it should also be mentioned that metamorphosis of a South African frog is retarded by the administration of adrenal extract or desoxycorticosterone acetate (100). Thus, if metamorphosis is promoted by thyroid hormones small precocious animals may result, whilst this may be prevented by adrenal cortex. Stockard, (101) in his extensive studies of dogs and their hybrids, showed that dwarfs, such as the dwarf bulldogs and other dwarf breeds, have a larger thyroid per weight of dog than such normal breeds as the fox-hound while the giant breeds have a relatively small thyroid. During pregnancy, a remarkable hypertrophy of pituitary gland is forthcoming, 
followed by the demonstrable acromagaly or gigantism, that is, enlargement of hands and feet, and the thickening of the lips and nose, probably due to the hypersecretion of growth hormone, whereas the gonadotrophic content of the pituitary is found to be reduced (102).

It may be concluded, therefore, that the absence of stimulating climate makes a precocious, small man tending to Addisonism, owing to the hyperfunction of both gonadotrophic and thyrotrophic hormones together with the insufficiency of growth hormone and ACTH, accompanying the dysfunction of adrenal cortex. This is the feature of man, common in tropical regions. A similar phenomenon may be observable also in animals. Thus, each group of garter snakes is found to show independently dwarfing as the distance increases from the center of dispersal in the Mexican plateau, no matter whether the group is pushing into the tropics or temperate regions (30). This may be ascribed to the degree of deficiency in climatic or other factors, which can stimulate the secretion of growth hormone in the snake, resulting in the hyperfunction of thyroid gland.

As we have seen above, our genes possess rigid structure with strong elasticity, so that they can show a resistance to environmental factors which are able to induce a change in them. If any change is induced, usually repulsive force will become stronger as the change proceeds, so that the progress of the change may come to be very slow, if not stopped, a consequence which may not issue when the genes have weak structures with feeble elasticity. A loss of the rigidity in genes, therefore, may lead to a rapid, unceasing change of the characters of the organism having the genes. In such a case the organism under a climate or other environments that stimulate the hypophysis will becoine continuously bigger to attain a gigantic figure which may result in its extinction. As is known as Cope's law, overspecialized animals usually would become extinct with gigantism. The extensive specialization results from a rapid change of a gene, which would be in turn a result of a loss of the structural rigidity in a gene.

Here a very remarkable fact should be pointed out that a fearful tendency to this gigantism is most manifestly being revealed in mankind. The statistics indicating the increase in stature of man go back a century and a half in Switzerland and fifty years or so in many other places. Among European countries data covering one or two generations are available for Norway, Siveden, Denmark, Germany, the Netherlands, Italy, and Spain. People in the United States also share this trend. Japanese date from 1878 onwards are partcularly significant because they show that an Asiatic people is undergoing the same lengthening process as those of European extraction. The 
stature of army recruits or others is known to have increased almost steadily since 1792 in Switzerland, 1836 among Harvard students, 1811 in Sweden, 1852 in Denmark, 1855 in Norway, 1863 in Holland, and 1885 in Japan (103). From measurements of about half a million males of twenty years from 1892 to 1926 , the mean height of Japanese was found to have increased by $3.23 \mathrm{~cm}$, the increase being steady and uniform from year to year (104).

In this connection, another most remarkable fact should be cited. During almost the same period of time the resistance to tuberculosis has been increased steadily in man. The disease has shown since 1849 a steady decline, along with approximate mortality rate, for the United States and for England and Wales, during the same period (105).

The dramatic change in the relation of the tubercle bacillus to man took place over most of the Western World during the same 100 -year period. In other words, mortality rates began to decrease in Europe and North America around 1850 and they have continued to decrease steadily eversince, in conformity with the increase of the stature, except for short and local interruptions in the downward curve during and after the first and the second World Wars as shown in Fig. 38. It should be noted that the rate of decrease in tuberculosis mortality had already reached its maximum slope around 1990.

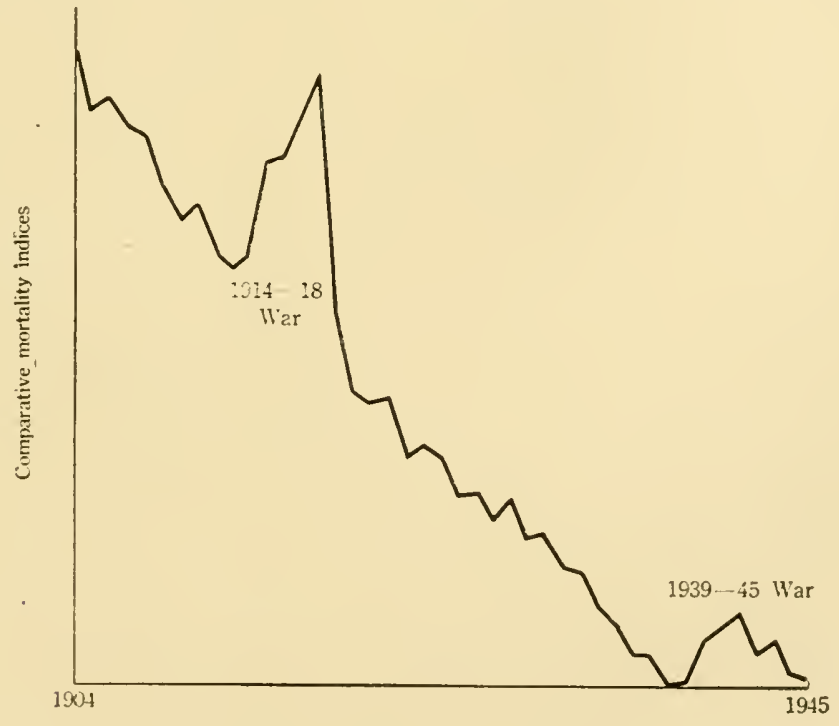

Fir $\therefore$. Tuberculosis all forms, under 15 years, England and Wales. 1904-19:5. Reproduced from Dubos, R. J. : Science in Progress, Sixth Series, $19 \div 9$. 
long before any specific immunization or therapeutic measures were available.

The writer claims that these two amazing phenomena are closely related with each other. As mentioned above, adrenal cortex is controlled by hypophysis, and environmental factor stimulating the anterior pituitary gland causes the secretion of adrenocorticotrophic hormone, which in turn induces the secretion of cortex hormone, whereas the same factor leads to the involution of thymus and other lymphatic organs. On the other hand, as is well known the so-called "status thymicolymphaticus" is a status liable to be affected by pulmonary tuberculosis. Its characteristics are an excessive development of the thymus, tonsils and other lymphatic organs, with a concomitant hypoplasia of the adrenal cortex. Moreover, intimate relation between tuberculosis and Addisonism, known as a morbid state associated with a dysfunction of adrenal cortex, has also been generally accepted. It can be concluded, therefore, that the hyperfunction of hypophysis caused by hypophysis-stimulating environment may result in the increase in the growth-hormone secretion, thereby man obtains a gigantic figure, and at the same time, it may remove the status thymicolymphaticus and Addisonism, thus rendering man insensitive to tuberculosis.

The average span of man's life is said to have been lengthened steadily during the same time in civilized nations. This must be a natural result of the increase in man's stature, since in general the bigger the animal, the longer its life. On the contrary, the hyperfunction of thyroid gland, which seems to be antagonistic to the action of adrenocorticotrophic hormone and which may be brought about by the want of the stimulating factor may result in the shortening of life span because of the premature growth due to the hormone.

It is little doubt that the remarkable morphological change in man, above mentioned, has begun to occur comparatively recently. According to Schwidetzky (106) as illustrated in Table 11, the head form of man has shown a great change at least after the year 1200 ; it should be noticed that almost no significant change occurred during the period ranging from 1200 B.C. to 1200 A. D. Moreover, it is reported that the actual increase in the stature in a generation among Yale University students has been about $1 \frac{1}{2}$ inches (103). If man had continued in the past to become bigger at this rate, only 1,000 years ago he wrould have been a dwarf of only about 2 feet; such was of course never the case. It is evident, therefore, that the change began recently and is continuing to proceed with a frightlful speed. Of course, the period of time extending over only a few 
Table II.

Change in Human Head Form.

After Krogman, W. M.: The Science of Man in the World Crisis. Columbia Univ. Press. 1946.

\begin{tabular}{r|r|c|c|c|c|c}
\hline \multirow{2}{*}{ Date } & \multicolumn{3}{|c|}{ Nordic } & \multicolumn{3}{c}{ East European Type } \\
\cline { 2 - 7 } \cline { 5 - 7 } & $\begin{array}{c}\text { Cranial } \\
\text { index }\end{array}$ & $\begin{array}{c}\text { Cranial } \\
\text { length }\end{array}$ & $\begin{array}{c}\text { Cranial } \\
\text { breadth }\end{array}$ & $\begin{array}{c}\text { Cranial } \\
\text { index }\end{array}$ & $\begin{array}{c}\text { Cranial } \\
\text { Iength }\end{array}$ & $\begin{array}{c}\text { Cranial } \\
\text { breadth }\end{array}$ \\
\hline 1200, B. C. & 69.2 & & & 76.1 & & \\
300, A. D. & 69.6 & & & 77.1 & & \\
1200, A. D. & 73.5 & 189.0 & 137.6 & 78.6 & 182.3 & 141.8 \\
1935, A. D. & ca 81.0 & 183.3 & 157.1 & ca 86.0 & 176.5 & 152.7 \\
\hline
\end{tabular}

generations of man must be solely one moment as compared with the awfully long history of organic evolution.

Mankind seems to have begun to make a dash at the fate of extermination with a dreadful speed like many other animals that have already become extinct because of rapidly developing orthogenesis.

\section{The Cause of the Orthogenesis of Man}

What is the cause of this dreadful orthogenetic change of man? Since any gain in height seems neither to have occurred nor to be occurring in tropical countries or in polar regions such as Greenland and Alaska, where tuberculosis is still increasing far from showing any decline, the main cause of the change must be attributed to climate. It was believed that a gradual lowering of temperature was the primary factor for the gain in height (107), but the writer claims that what has changed and is now changing is the character of genes of mankind rather than climate.

It may be reasonable to consider that the genes of man have changed by some causes so as to be rendered sensitive to climatic factor and that still at present are being rendered more sensitive to the factor, that is, the genes are changing to lose the rigidity or the elasticity in their structure.

Many causes are considered by which genes may lose the rigidity, but the most important one may be found in that man becomes well nourished with the advancement of civilization. The writer believes that the effect of deficiency in food counteracts the stimulating action of climate on hypophysis. There are many evidences that inanition injures the pituitary function. Chronically underfed rats 
shows a decrease in weight of the adrenals, and it has been suggested that this is a result of insufficient adrenocorticotophic hormone, since implantation of pituitaries increases the weight of the adrenalis in such animals (108). Such adrenal atrophy is by no means attributed to the general deficiency of calory, for the administration of carbohydrate cannot alleviate the symptom. Presumably, protein deficiency plays a predominant part, since it is said that a deficiency of certain amino acids in the diet causes a severe atrophy of cortex, although it is also reported that a fat-free diets produce a relative atrophy of both cortex and medulla (97).

A series of symptoms following hunger can be well interpreted as the hypofunction of adrenocorticotrophic hormone of hypophysis. The so-called "famine oedema" or "hunger swelling" can be regarded as the manifestation of the pituitary hypofunction. War oedema has appeared in epidemic form in many of the recorded wars of history among both the troops and the civil populations. A similar oedema occurring in a malnourished infants or in those fed principally on a high carbohydrate diet has long been recognized. A considerable nocturia is seen in mild chronic cases, as we ourselves miserably experienced during and after the War, whereas as generally accepted water metabolism is severely affected by adrenal insufficiency, thereby, despite the negative water balance, water diuresis is extremely limited. In addition, albumin-globulin ratio of the blood is greatly altered in hunger as in Addisonian syndrome, all globulin fractions usually showing some increase, while albumin decreasing (109). It is well established that such a change in albumin-globulin ratio in the blood, which also characterises some cases of rheumatoid arthritis and other diseases of mesenchymal tissue, is frequently returned to normal during administration of cortisone or adrenocorticotrophic hormone (110). Again, a number of investigators have reported that, as a result of hypophysectomy in animals there is a decrease in the albumin content of the blood (111). It has actually been confirmed with rats that inanition has a similar effect (112). According to a recent studies by Ulrich $e_{i}^{i}$ al. (113), treatment of hypophysectomized animal with growth hormone results in a great stimulation of albumin synthesis, while ACTH has no such effect. Moreover, it should be remembered that fatigability and muscular weakness peculiar to Addisonian syndrome are also manifest in inanition, as we had likewise rich experience of it thanks to the War.

In addition to the want of food, an irritable, uneasy life associated with nervous tenseness of animality, peculiar to a barbarous life, may contribute to the prevention of the loss of the rigidity in gene structure. As already described, there appears to be a closely 
related connection between hormones and temperament; hormones effect the latter, while the latter effects the development of endocrine glands. Such a relation is especially obvious in the relation between thyroid gland and temperament, and general irritability goes parallel to the increased function of the gland. Therefore, an uncivilized life requesting beastly tenseness and nervousness may lead to the development of thyroid gland, or hyperfunction of thyrotrophic hormone of the pituitary gland, which in turn exerts antagonistic influence upon the function of adrenocorticotrophic hormone and probably also on growth hormone, thus reducing the stimulating action of climate. On the other hand, civilized life makes man feel at ease resulting in the loss of the beastly strain, contributing to the effect of climate, and may thus promote the loss of the rigidity. Environmental factors are considered to affect hormonal glands mainly through autonomous nervous system, and hence it may be a matter of course that psychic conditions such as irritability and nervous tenseness are capable of exerting a similar effect through the nervous system.

In this connection, it shoud be mentioned that adrenal cortex exhibits the function of releasing the nervous irritability in contrast to thyroid. Also in this respect these two hormonal glands act antagonistically with each other. Thus, it has been reported that adrenal extract produced improvement in some cases of pathological irritabi-

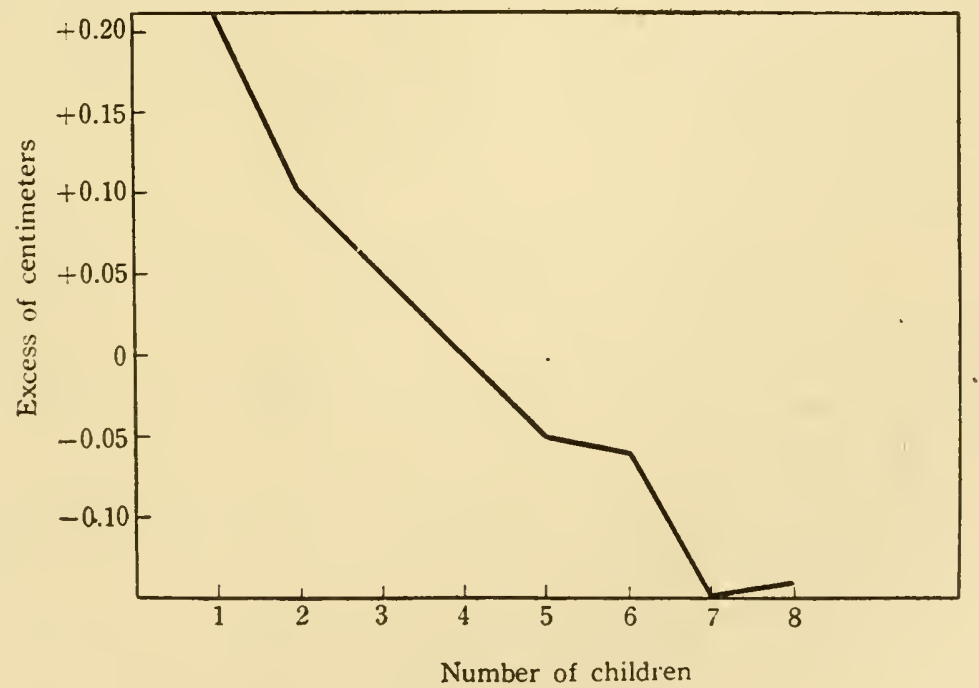

Fig. 39. Excess of stature over averge stature for families of various sizes. Reproduced from Boas: Race, Language and Cultures, New York, 1949. 
lity, and a sense of well-being, even euphoria, replaced depression, and abnormal irritability disappeared (114). Even normal individuals at times respond, the immediate effect being drowsiness and release of nervous tension, if present. These effects are not due to suggestion, because sheep, in a state of neurosis due to overtaxing the nervous system in conditioned reflex experiments, showed marked improvement under adrenal extract therapy (115).

In corformity with this concept, according to Boas (86) the increase in the stature of children is greatly influenced by their number in the family, and the increase is the more manifest the fewer the number, as shown in Fig. 39. It can be expected that nervous tenseness of animality may be necessary the more and the chance of hunger may be the more frequent, the larger the size of the family.

This is most clearly demonstrated in the investigation of the Department of Education of Japan into the bodily change of Japanese children during and after the Second War. It is reported that the stature of the children was remarkably reduced during and after the War and that the reduction was distinct in the length of legs as compared with the almost unchanged trunk (116). It should be realized in this connection that the increase in Japanese stature in Los Angeles occurs chiefly in legs not in trunk. Such a reduction of stature is, of course, mainly ascribed to the want of food which might not only lead to the insufficiency of ACTH and growth hormone, but also might stimulate the production of thyrotrophic hormone. However, in addition to this, irritable, uneasy life during the war might play a prominent part in this reduction in stature.

On the other hand, the removal of uneasiness will result in the increase in stature, as evidenced actually by an experiment with animals. Thus, according to Weininger (117), albino rats, gentled for three weeks for ten min. a day following weaning, would show significantly greater mean weight. The difference in weight between experimental and control groups was related to a significantly greater proportion of adipose tissue and to a significantly greater skeletal length for the former.

Porter (86) found that students with high scholastic standing are generally above the average in height. Entirely similar phenomenon was afterwards confirmed by Sargent (103), They considered that the growth of bright boys was more rapid than that of dull boys, but it may be more reasonable to suppose that the children able to be endowed with a higher education are rather civilized and better nourished, and perhaps need less nervous tenseness of animality. The Provident Mutual Life Insurance Co. of Philadelphià surveyed 270,000 men to see if there was any relationship between height and 
success. The company chose as its barometer the size of the policy each man held, long considered an excellent key to earning power. As the result it was found that an amazing parallel was present between the average policy and height, indicating clearly that the richer a man the taller his stature (118). It is well known that the increase in the stature of Japanese is more striking in towns than in the country. Dwarf horses have arisen on various islands where a limited food supply was probably the most effective cause; the pygmy elephant of Malta and some other cases in animals may have had a similar origin.

Another probable cause of lessening in the rigidity of the genes may be the growth hormone itself. Overspecialization of animals appears to be usually connected with the increase in the bodily size, suggesting that growth hormone is required for the specialization. The specialization, on the other hand, may not be achieved without the decrease in the rigidity or the elasticity of a gene. Growth hormone, therefore, may be essential for the lessening in the rigidity. The effect of the hormone revealed in the establishment of unusual growth may be a manifestation of the reduction of the genic rigidity itself, since unusual growth may be unable without the loss of the rigidity.

A stress causing a rapid change in animal characters appears to exert stimulating effect upon hypophysis. Growth hormone may be yielded in response to such a stress in order to achieve with ease an adaptive change. If this conception is true, when a hypophysis-stimulating factor continues to exist with the continuous production of growth hormone, the decrease in the rigidity will be unlimitedly pushed forwards, thus the speed of a change directed to the destruction being accelerated.

\section{Viruses, the Fatal Enemy of Man}

The loss of the rigidity in the genes will be revealed in the protoplasm as the acquisition of an easily changeable character. Such a character of the protoplasm, on the other hand, may be nothing but $\exists$ high susceptibility to various viruses, since the structural change of protoplasm by a virus is no more than the occurrence of the infection with the virus. If the protoplasm is provided with a strong reversibility, virus structure will soon be expelled and the protoplasm will recover its normal state even when the protoplasm is once affected by the virus.

Cancer is a disease presumably resulting from the loss or the reduction of the rigidity in the protoplasm including genes, a disease in which genes are coming out of order leading to the abnormal reduc- 
tibility to their primitive structure. Sometimes it is regarded as a type of virus diseases. Now, it is a noteworthy fact that cancer is prevailing extensively among civilized nations. For example, in the United States present cancer mortality is over 200,000 per year and it is increasing at the rate of approximately 3 per cent or about 5,000 per year. Specifically, 207,721 deaths from cancer and leucaemia were reported in 1948. If cancer will continue to increase at this rate, in only a few hundred years it will so happen that all the people in United States are doomed to die of cancer. This fact strongly suggests that the decreasing process of the structural rigidity in human genes is rapidly proceeding in civilized people, presumably because of hypohysis increasing in its function. Moon et at. (119) have actually confirmed that hypophysectomy does exhibit inhibitory effect upon cancer of the rat induced by methylcholanthrene. Moreover, the significance of pituitary function in neoplastic diseases including cancer is indicated by the many and diverse tumours that develop in the rat following the prolonged administration of growth hormone (120).

During pregnancy, as was already pointed out, a remarkable hypertrophy is observed in hypophysis; at the same time, attention is paid on the increased incidence of poliomyelitis during pregnancy. Domestic animals and cultivated plants have been highly specialized probably by the lessening in the rigidity of the genes. Their character highly susceptible to viruses must be based upon this; as is well known the more specialized, the more susceptible they are to viruses. Civilized people showing the tendency to increase in stature and becoming more and more resistant to tuberculosis may be regarded as highly specialized species. At the same time, they are very susceptible to some virus diseases including cancer as seen above, whereas among small uncivilized people inclined to be severely afflicted by tuberculosis, the incidence of virus diseases including cancer appears to be much fewer than among civilized.

Poliomyelitis is extensively prevalent among civilized nations especially in United States and Canada, whereas low incidence of the paralytic disease, even among children, of the tropics and subtropics, such as Africa or Far East, has been reported (121). In Japan, recently poliomyelitis is becoming not a rare disease, while the decrease in tuberculosis has become conspicuous. In 1916 there was an explosive outbreak of poliomyelites in America, and in was then impressed by the fact that patients so often were of the same physical type, usually large, overgrown children (122).

On the other hand, many experimental results suggesting that the hyperfunction of hypophysis is disavantageous to virus infection have been recently accumulated. Thus, adrenal cortex hormone (cortisone) 
or adrenocorticotrophic hormone (ACTH) has been found to affect the course of virus infection to the disadvantage of the host. This has been demonstrated for influenza and mumps virus in chick embryo and in mice, for poliomyelitis and Coxackie virus in mice and hamsters, vaccinia in guinea pigs (123) (124).

The administration of these hormones, however, is known to be inhibitory to inflammatory or allergic reactions. For example, it has been reported that $\mathrm{ACTH}$ and cortisone reduce the febrile response of rabbits to typhoid vaccine and that cortisone markedly reduces the temperature of patients with typhoid fever (125) (126). Moreover, it has been shown that these hormones can suppress the febrile response of rabbits to bacterial endotoxin (127). Cortisone and ACTH are known also to inhibit cellular inflammatory reactions to a wide variety of other irritants including burns, trauma, and various chemical agents (128).

Inflammatory reactions against bacterial infection may thus be prevented by the administration of cortical hormones. However, replication of the bacterial pattern in the host, like that of the viral pattern, seems to be accelerated by these hormones. Thus, cortical hormones have been shown to increase the susceptibility in a number of hosts of many unrelated bacterial diseases (129). Effective doses of ACTH or cortisone are usually associated with evidence of increased multiplication of the pathogens and more wide spread dissemination, although adrenal cortical hormones are not stimulatory to bacterial growth in vitro (128). This seems the case even with tuberculosis. Clinical experience suggests that administration of cortisone may reactivate an apparently healed tuberculosis infection in man (130). Laboratory studies with various animal infected with tubercle bacilli have shown that the administration of cortisone causes a more extensive diseases than that exhibited by controls.

The favourable effect of the heightened function of hypophysis upon tuberculosis may, therefore, be not dependent upon the hypersecretion of cortisone, but upon the involution of thymus and other lymphatic organs which favour tuberculosis as already pointed out. It should be mentioned, however, that although cortisone exerted its deleterious effect upon tuberculous animals, corticotrophins of hypophysis could be administrated in excess of human dose levels without harmful effect and was deleterious only at levels so high as 20 times human doses (131).

Not only tuberculosis but the infectious diseases of bacterial origin in general appear rather rare in civilized people, in contrast to the high prevalence of some virus diseases among them, despite the above mentioned fact that the susceptibility of hosts to bacterial diseases is 
enhanced by the cortical hormones. This may be dependent on the prevention of the infection of the diseases presumably owing to the hygienic life modus of the civilized people. Civilized life may thus prevent the infection of not only bacterial but also of some viral diseases.

However, in general, adrenalectomy has been found to diminish the capacity of the body to withstand such stresses as infection, and a more or less complete return to previous resistance is achieved by the administration of sufficient amounts of adrenocortical hormones. This is the case also with Addison's disease. The value of adrenocortical hormones to the patients with Addison's disease hardly requires no comment (128). In view of this fact, it may safely be concluded that if disfunction of adrenal cortex is prevailed in some barbarous life, infections by various pathogens including tuberculosis should occur strikingly arnong the uncivilized people.

Whooping-cough is similar in many respects to an ordinary virus disease, and the incidence of the disease is much higher in United States and European countries than in Japan. Even in Japan it is more frequent in towns than in the country. According to the investigation of Nukada (132) in our laboratory, the incidence of Whooping-cough in Japanese children is the lesser, the greater their number in a family, provided that the number is greater than three, as shown in Fig. 40.

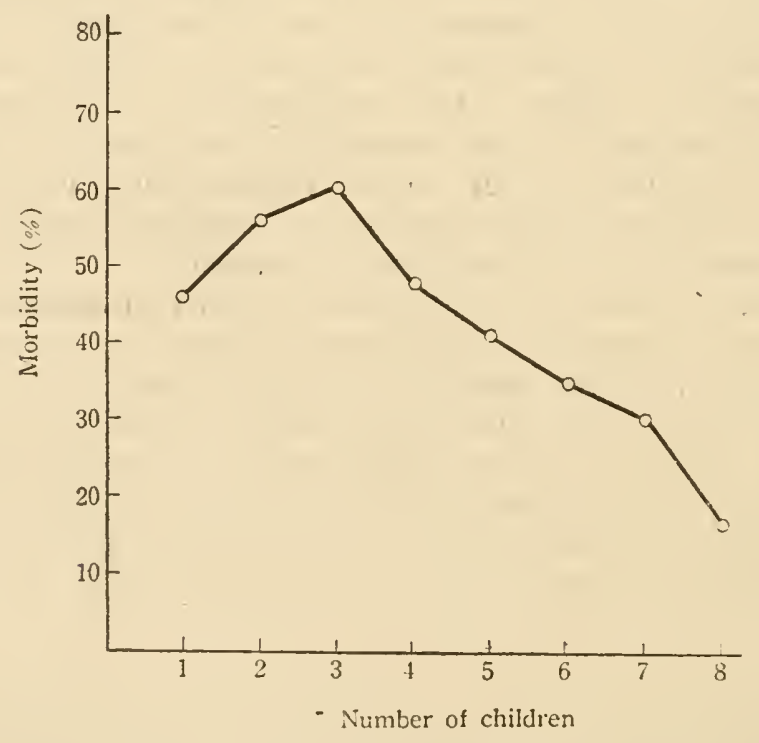

Fig. 40. Mobidity of whooping-cough in families of various sizes. 
On the view point of easy infection, the increase in the number of children must result in the increase in the incidence; this is shown when the number is less than three, but the reverse is the case when the number is greater than three, showing the overwhelming influence of another factor acting in the reverse direction. This reminds us of the fact, already shown in Fig. 39, which indicates that the stature of the children is the taller, the fewer their number in a family. Thus there is little doubt that the easy going life of children of smallsized family increases in them the sensitivity to whooping-cough as in more civilized nation.

It has been confirmed by us (133) that the incidence of measles and whooping-cough in Kanagawa Prefecture in Japan was reduced remarkably at the end of, or immediately after, the War; this fact indicates that the uneasy life of the children at the end of the War bears a striking resemblance to the usual but irritable, and food-insufficient life of the children of a large family. In addition, it is generally accepted that also intestinal toxic disorders of children and appendicitis almost entirely ceased to occur in Japan during the War. Even common bacterial infectious diseases such as dysentery and typhoid fever were said to have been likewise remarkably diminished. The development of the pattern of causative agents of these diseases, whether they were viruses or bacteria appeared thus to have been inhibited by the same factors. As is generally accepted, tuberculosis, on the contrary, increases strikingly during wars, a phenomenon most probably due to the want of nutrition which causes a hormonal unbalance including the dysfunction of adrenal cortex favouring the disease.

It is needless to mention that inanition is generally accepted to have an intimate connection with tuberculosis. However, if one considers that inanition causes a general decrease in the resistance to infectious diseases he commits a great mistake, because it is well established that an ill-fed animal is less susceptible to virus diseases including cancer than a well-fed one, as is readily expected from the theory above stated.

One of the earliest demonstrations of this phenomenon was that of Rous (134), who in his experiments on fowl sarcoma virus, emphasized that healthy, well nourished fowls were more susceptible to the virus than the thin and ill. Olitzky, et al. (135) found that guinea pigs suffering from malnutrition were resistant to infection upon inoculation with the virus of foot-and-mouth disease than were normal, healthy animals. Recent reports dealing with nutrition and poliomyelitis have contribute further data on the way in which nutritional deficiency can decrease susceptibility. It is a note-worthy fact that cancers are very difficult to transplant in otherwise susceptible animals, 
if the food intake of the animals is restricted severely. Even with the tumours growing well, the animals survive longer on restricted amounts of food (110).

Thus it may be concluded that the nutritional defficiency and perhaps uneasy life requesting nervous tension of animality during the war were the cause of the reduction both in stature and in the incidence of some infectious diseases such as whooping-cough and measles. The same cause may increase tuberculosis.

However, attention should be paid to the fact that, as shown in Fig. 38, although the decline in mortality index of tuberculosis was interrupted during the war, compensative, extensive decline occurring after the war. Again, as already stated, Japanese children were reduced miserably in their stature during the War, but now they have already not only recovered their former status but have become even bigger, showing also the compensatory alteration. These facts may suggest that the factor pushing forwards the orthogenetic change was exerting its influence without stopping during the war, and that the mode of social life may have only a superficial influence. However, the more reasonable explanation is that the genic pattern having gradually been attained by the orthogenetic change is so strong that it cannot be altered permanently by the transient uncivilized life modus with both nutrient deficiency and nervous uneasiness which may only temporarily alter the pattern in the opposite direction, and that therefore on the removal of the factors involved in the uncivilized life modus the former pattern can be readily recovered. Of course, if the War lasted much longer, the former pattern would have been forgotten and as a consequence the compensatory alteration would have never or hardly occurred.

Huntington claimed that the only factor which promotes civilization is a stimulating climate. Indeed, under a stimulating climate man may become active both mentally and physically owing to the stimulating effect of the climate upon hypophysis, and may tend to use extensively the brain and related organs, which may subsequently be developed, contributing to the promotion of civilization. The advanced civilization will enable man to live an easy life with sufficient food, thereby the factor arresting the stimulating action of climate exerting on hypophysis is removed. Consequently, stimulating climate can exert its full action, thus growth hormone being produced abundantly ; at the same time, for the development of the brain or other organs growth hormone may be required, and unusual use of the brain or other organs may stimulate hypophysis. Thus man may increase in his stature followed by the rapid promotion of civilization, which may further lead to the more sufficient supply of nutrition with the 
diminution in the requirement of nervous strain of animality, contributing to the more extensive production of growth hormone.

In this way, causes becoming results, results becoming causes, the speed of the orthogenetic progress of man towards gigantism will be increased acceleratively.

As described above, the average increase in height in a generation of Yale University students has been reported to be about $1 \frac{1}{2}$ inches. Swedish stature has increased $9 \mathrm{~cm}$ in the last hundred years (104). If man will continue to become bigger at this rate, after 10,000 years he will be a giant over 40 feet. On the other hand, as the body weight is directly proportional to the square of height, animals becoming bigger over a certain extent would fail to resist the terrestrial gravitation, thus there being no choice but to become extinct. The whale can exist notwithstanding its gigantic size owing to its aquatic life as water alleviates the gravitation.

However, more dreadful foresight may arise from the point that man is losing rapidly the structural rigidity in the genes. The frightful speed of specialization may be dependent upon this point, which seems to be accelerated by the growth hormone; at the same time, this loss of rigidity in genes may give rise to an extremely changeable character of protoplasm with profound and incessant production of various malevolent viruses including cancers. Thus mankind is bound to become extinct in a near future as did the gigantic reptiles.

As is well known, a great many reptilian orders, including those containing the dinosaurs and pterydactyls, are entirely extinct, but still at present a number of small reptiles are existing. In like manner, some of the mankind may be left behind on the earth even when the majority of them will be extinct, and presumably those who are to be left are the people who are living in a climate lacking in the stimulating factor and who are compelled to live in a nutritious deficiency with a nervous tenseness of animality on account of their uncivilized life, which must always be associated with the climate.

Irritability and uneasiness as well as the want of food peculiar to uncivilized life may result in the precocious sexual maturity through the hormonal pattern induced by the situation, presumably involving the hyperfunction of thyroid and gonadal glands, and this may cause the uncivilized, small people to have many children, in contrast to civilized, specialized people. This seems to be a phenomenon common to the animal kingdom in general. The smaller the animal, the more numerous enemies it must have, and accordingly the more nervous tenseness must be required, and again therefore the peculiar hormonal pattern mainly concerned with thyroid and gonadal function should be developed the more. Thus the smaller the animal, generally the more 
numerous its youngs, a consequence which must be most purposeful for the survival of small animals, since numerous victims may always be demanded by their enemies. This may likewise contribute greatly to the natural selection of the fittest, so that the character will bocome more and more fitted for survival. The reverse may be true when the animal increases in its size, there being no room for the natural selection on account of its poor procreative power, and also in this respect there is a strong reason for the extinction of large-sized animals.

Since there may be no natural selection in such animals, their evolution should be orthogenetic and animals bound to be extinct by their overspecialization are always to advance on an orthogenetic course. It appears, however, that Nature has prepared an excellent mechanism to check this terrible orthogenesis. The mechanism may be based upon the want of food, which is expected to occur when animals greatly increase their size. Inanition may not only inhibit the active function of hypophysis, but may stimulate thyroid or related glands to make the evolutionary course entirely reverse. Reptilians might accomplish the orthogenesis until they became extinct presumably because there were too plentiful food to make them hungry.

Orthogenetic, unlimited increase in size, therefore, may occur rather rarely. In general, an animal having increased in its size to a certain extent may cease to become bigger through the want of food, as the bigger an animal, the food may be required the more. On the contrary, an animal having become smaller and smaller may sometimes begin to increase in size owing to the sufficiency of food-supply because small animals need only a small quantity of food. In this way, the quantity of food may always control the size, accordingly, the specialization rate of animals.

However, civilized man is able to escape this want of food, however big he becomes, owing to the high civilization, and therefore, for the future, the character difference between civilized and uncivilized man will become amazingly great, and when the overspecialized, civilized man is extinct, the latter will represent the mankind, but those who escape the extinction will follow the fate of the former after migrating into the stimulating climate.

When one surveys the entire field of animal evolution, the significant fact becomes apparent, not only from the geological but from the facts of comparative anatomy, that each great distinctive new group in animals has always sprung from the more primitive member of the preceding group, [not from those highly specialized. Mankind may not provide an exception.

The extinct Cro-Magnon people are known to have a stately stature 
comparable to the present and live a considerably high civilized life in the old stone age nearly 20,000 years ago. Their cranial capacity appears to have been even somewhat greater than that of modern Europeans. Their extinction might be caused by the loss of structural rigidity in the genes, followed by the prevalence of some severe virus diseases. In their days, the ancestors of the present Europeans must have been only a barbarous, insignificant existence, but now they are the representative of mankind and apparently are going to follow in the steps of Cro-Magnon.

How fatal are some viruses to overspecialized people may be understood if one remembers the dreadful prevalence of influenza which outbroke during the Ist War; the victims of the disease were far greater than those of the War. Human being have presumably achieved since then a great advance in their specialization by losing remarkably the rigidity of genes; who can warrant you there is no chance of outbreak of an influenza or other virus diseases so severe that almost all the civilized people will be sacrificed to it?

However, the writer's forecast concerning the future of man thus far described will be failed if his theories are accepted generally and counter-measures are taken against the human orthogenesis. Not only the prevention of human extinction as a race but also as an individual may be not utterly impossible. Since the individual devolopment is raised by hormones, administration of a proper hormone or hormones may be able to stop or retard the advancement; even to accomplish the rejuvenescence may be not imppossible as seen in the production of germ cells. As already stated, thyroid hormones will promote maturation leading to premature decay, while certain pituitary hormones appear to be involved in prolonging the life span. Carlson and Hoelzel (136) compared the effects of omnivorous and herbivorous diets upon the life of rats, and confirmed a favourable influence of the diet rich in meat. This may be accounted for by the positive effect of the protein rich diet upon the pituitary function. Moreover, in most experiments recorded in the literature the female of a species such as the rat lives longer than the male, a phenomenon which has long been recognized also in the case of man. This must be attributed to the difference of hormones due to the difference of sex. 


\section{CHAPTER XIII \\ THE SUMMARY OF PART V}

Protein having $A$-structure may be converted into $B$-structure under the effect of $b$-factor, whereas the protein thus converted to have $B$-structure will return to $A$-structure under $a$-factor. The writer designates such a phenomenon as reversibility of protein structure, and regards it as the "memory" of the former structure. This phenomenon appears to be common in usual proteins but especially manifest in the protoplasm, and may be indicated as follows:

$$
A \text {-structure } \underset{a \text {-factor }}{b \text {-factor }} B \text {-structure, }
$$

wherein both $a$ - and $b$-factor are various chemical or physical agents, such as temperature, light, or hormones. $A$-structure may be stable under the effect of $a$-factor, and $B$-structure under $b$-factor.

However, protein having $B$-structure frequently fails to be converted into $A$-structure by the mere change of the environmental factor. In such a case, some "stimulus" is needed to induce the change, the change being prompted by a disturbance in the protein structure in duced by the "stimulus". This may be compared to the conversion of supercooled water into ice by a certain "stimulus" like a mechanical agitation.

If unfolding and refolding of polypeptide chains are required for the structural conversion of a protein, "stimulus" may favour the unfolding, that will be followed by the refolding, which the latter is specific to the factor or environment under which the protein is placed.

The change from $A$ to $B$ structure and from $B$ to $A$ structure appears to take place easily when the change is repeated; in other words, the repetition of the reversible change may result in the facility of the change, the memory being thus strengthened.

The intensification of the memory is presumably raised by the removal of structural factors which prevent the reversible change. The removal of such preventing factors may be achieved the more 
completely, the more frequently occurs the repetition of the change. The memory is, therefore, a structural pattern of proteins, given rise to by the removal of the structural factors which prevent the reversible change.

Proteins produced as replicas of memory-carrying proteins, which constitute the protoplasm acting as a template, must consequently have the memory. The memory in our brain may thus be established. As the structural pattern specific to a memory is transferred to newly synthesized proteins, the memory can continue for life.

On the other hand, the structural pattern involving a memory also has the tendency to recover its original pattern to produce again the preventing structural factors, and therefore the persistence to the original pattern render the reversible change difficult leading to the oblivion of the memory. Hence, for the maintenance of memory is needed the repetition of the reversible change, namely continuous training, thereby the production of preventing structural factors is inhibited.

In addition, if a protein remains under the same environmental condition in taking the structure determined by the environment, the structure will be gradually altered so as to be more fitted to the environment, so that the structure is fixed and its change in any directions becomes difficult, thus the original structure being forgotten.

The gradual alteration of protein structure to be fitted to the environment is the adaptation of the protein to the environment, and if the adaptation goes so far as the structure becomes too fixed to lose its elasticity it may be called senescence.

Individual development may be based upon the reversible character of proteins constituting the genes of germ cells. It is considered that a germ cell can act as an independent, unicellular organism because of the primitive structure of its protoplasm and that this primitiveness is brought about by the structural return of a certain somatic cell to its primitive stage, from which the organism producing the germ cell has been developed. Such a return to the primitive structure must be brought about by the reversible character of protoplams protein. Since the structure of the protoplasm is governed by the genes, the return of the protoplasm to the primitive structure must be raised by the structural change of the genes. The structure of the genes may be reduced to the primitiveness under the influence of a factor or factors that make the primitive structure more stable than the developed. 
The gene of a germ cell thus reduced to the primitive stage can again recover its previous, advanced structure owing to the reversible character, when factors or environmental conditions are provided which favour the development of the advanced structure. The recovering change is generally initiated by fertilization, but the fertilization can be substituted by a proper physical or a chemical stimulus, which may act like a mechanical stir in the case of the transformation of supercooled water into ice.

Various characters are revealed in a developing individual with the progress of the recovering change of the genes, because the protoplasm is looked upon as a kind of liquid crystal, whose crystal shape is determined by the structure of protein components, which is in turn controlled by the genes. The change is continued until the complete recovery of the advanced structure is established, whereby various morphological and functional characters are successively revealed with the progress of the change.

Regeneration, which is common to plants and some lower animals, can be explained in the same way. The somatic cells in the site of cutting are reduced in their structure to a primitive, undifferentiated state, from which subsequently the differentiated structure is anew recovered. Regeneration, therefore, is dependent upon the reversible character of protoplasm protein just as ontogeny.

The formation of organs or tissues in the various body portions during the ontogeny is apparently due to the environmental effects varying with the portion. Since, as above cited, protein structures are determined by environmental factors, different environmental effects produce different structures which in turn iuduce different organs or tissues.

Different structures thus produced in the different places of developing body are unstable at the start, and tend to change to other structures if the environments are changed, but by a prolonged exposure to the same environment the structures are gradually fixed and become so stable that they can retain their specific structure even when transplanted to another part. The so-called organizer is an embryonal part whose structure has been thus fixed.

The protein of organizer, if liberated as protoplasm particles, may be able to transfer its specific structure to other unfixed parts of the embryo as a "virus", but the protein molecules, which fail to form virus-like particles, or other components, such as steroid, liberated from the organizer may behave as hormones by exerting on the surrounding embryonal parts their structural effect which is peculiar to the organizer, though they are not so powerful as being capable of transmitting the specific structure itself as a "virus". Thus, if a 
local structure is so fixed as to be called an organizer, a series of peculiar structures are formed around it, with the formation of complicated shapes and functions.

The structures thus rarying with the portion of an individual are produced because the local genes have been changed in their structure by the environmental effects which vary with the portion. Therefore, every organ and tissue must have peculiar genes. This conclusion seems natural, since, as is generally accepted, every organ is provided with protein antigenically specific to the organ, while it is also believed that what determine the protein structure are genes.

\section{3}

The so-called biogenetic law that ontogeny recapitulates phylogeny can also be readily explained by the assumption that the germinal cells are the reductive form of the somatic cells, and the change giving rise to reduction is reversible. Since the structural reversibility of a protein is considered to be based upon the memory of the course along which the protein has attained to the present structure, the somatic cells, in the course of reduction to the germinal, have to pursue reversely the course of phylogeny, whilst, in the course of development of the germ cell, the course of phylogeny itself is to be followed. This reversible change must be repeated in each generation, and hence the memory is correct, and ontogeny recapitulates phylogeny without fail.

In higher animals, it seems that the memory is retained only in the particular cells involved in the production of germ cells, and in ordinary somatic cells there appears to be some mechanism by which the reduction of the structure is made difficult. Ordinary cells are, however, also inclined to be reduced to the primitive structure when the preventing mechanism is rendered weak, and in such a case cancer cells, the structure of which is primitive like germ cells but different in many respects from the latter presumably on account of this abnormal reduction, are produced. Sexual hormones play an important role in the production of germ cells, whilst the same hormones or related substances appear to be involved in the production of cancer cells. The reduction of somatic cells to cancerous is an abnormal process and so the return of the cancerous to somatic cells appears to be not an easy occurrence as in germ cells, but it does sometimes occur.

The structural reversibility of genes is extremely strong. The genes have the property to alter their structure according to the 
environment under which they are exposed, as in general do proteins, the main component of the genes. Owing to this strong reversibility a gene can return to its original structure when the environmental effect which has caused the change is removed.

The recovery of the original structure, however, is difficult, if a profound change arises abruptly in a gene. The genes can recover their original structure because the course leading to the original structure is remembered, but the memory of the course may not be retained clearly, if the change occurs too rapidly and extensively to remove the structural factors which obstruct the progress of the change. In such a case the change is inclined to be irreversible and called mutation.

Mutation is uncommon, and usually the genes undergo extremely gradual changes, which continue to proceed by degrees in directions which are determined by the environmental factors. Such a change is completely reversible, and can be regarded as the normal change of the genes.

The factors, which induce directly or indirectly the gradual, reversible change, involve the stimulus coming from organs which are continuously used excessively, as well as ordinary environmental factors such as temperature, light, and diet.

The change of a gene leads necessarily to a change of character of the cell or of the organism which is governed by the gene. Organisms may alter their characters when brought under a new environmental factor. Since such a change is reversible, the acquired character is lost when the environment returns to the original state.

However, if organisms are exposed always to a new environmental factor without returning to the original environment, the previous structure will be gradually forgotten with the fixation of the new character responding to the new environmental factor. Thus acquired characters will become fixed, not easily to be lost by the change of the environment.

Fossils forming an evolutionary series suggest that evolution proceeds in definite directions, from the directing tendency of internal structures, and this phenomenon is called orthogenesis. If a certain environmental effect continues to exert its influence upon a gene or genes, in producing a gradual change in the gene in a definite direction, the organism having the gene is to undergo orthogenesis.

Bacteriophage can be looked upon as a free gene, and when acted on by an inactivating agent such as formalin or antiserum, it tends to change gradually in a definite direction until completely inactivated. This change is usually reversible if the change does not proceed too far and too rapid, the original character of phage being recovered on the removal of inactivating agent, which can be compared to the 
stimulus coming from the use of organs or from a changed environment. In this case, phage is of course a gene-model.

The gradual change of genes is completely reversible, but some periods of time are necessary for the reversion to the original structure after the removal of the changing factor, as the reversion occurs also gradually. Consequently acquired characters, which have not yet been fixed enough, usually remain for some periods after the removal of the factor, a phenomenon known as "Dauermodifikation."

Characters given rise to by mutation are generally injurious to the organisms, whereas those given rise to by the gradual, reversible change of genes are usually favourable.

This may depend on that the reversible change, unlike mutation, is induced by naturally existing environmental factors to which the organisms have to be always exposed. If organisms could not endure the change of usual environmental factors, they would inevitably become extinct; as a natural result only the organisms capable of bearing the change would be able to continue the existence. In order to become the fittest, the organisms would have to acquire the faculty not only to endure the change but to obtain a favourable character through the change, so that the present-day organisms, which must be the fittest, can attain characters adapted to the changed environments.

The change of genes thus raised by the environmental change is gradual and reversible, and leads to the production of favourable character, whereas the abrupt action of some unnatural chemical or physical agents upon genes may cause random changes; thus mutation may arise. The genes of an organism existing in the present day must have structures most fitted to the existence of the organism, so that the majority of random changes taking place in the genic structures would naturally result in the production of less fitted or unfavourable characters. Thus, mutation is usually injurious.

The adaptability of organisms to a new environment is based upon the gradual change of genes producing the character suitable for the new environment. The adaptability must have been developed because it is an indispensable character for the organisms. The organisms without this character would surely fail to continue their existence.

Such a favourable character, however, is presumably not yet sufficiently developed in extremely primitive organisms, but as they were evolved higher, the mechanism of the gradual change of genes might 
be developed so elaborately that evolution is sometimes possible even without any natural selection.

\section{5}

The principle of adaptation may exist in the nature of protein itself. Protein molecules are usually inclined to be coagulated by agents which appear to be injurious to organisms, in the coagulation polar groups being folded and lost, while the injurious agents presumably can act upon the protein by combining with it through certain polar groups of the protein, and therefore the disappearance of the polar groups can be regarded as the adaptation of the protein not to be further combined by the agents or to reject the agents.

On the other hand, when the environmental factor is not injurious, the protein adapts itself to the new environment in rearranging its structure to become stable under the environment. And, if affected by some chemical agents possessing certain structures which are not injurious, the polar groups of the protein may be rearranged instead of being folded in response to the structural arrangement of the agent, thus the protein becoming able to combine more easily with the latter with the establishment of adaptation to utilize the agent. The transmission to genes of such structural changes occurring in the protoplsm protein may result in the adaptation of cells to environmental factors.

The adaptive change thus raised in local somatic cells can be transferred to the germinal by both virus-like agents and hormones. If the changed structure is liberated in the form of protoplasm particles or virus-like forms, the structure can be precisely transferred to the geminal cells. On the other hand, if the changed structure is released in the form of a certain hormone or a hormone-like agent corresponding to the structure, some influence related to the structure will be exerted on the germ cells through the agent. In higher organisms, the environment appears to exert usually its effect on hormonal systems, whereas it is known that hormones have great influences upon the character of organisms.

In the transmission of a precise structure of somatic cells to germinal, a virus-like agent is involved whilst hormones may act as carriers of inexact structures.

The continued effect of a certain environmental factor on germ cells may cause a fixed change in the genes of the germ cells, with the result that the original structure of the genes is forgotten, thereby the acquired character becoming inheritable.

In like manner, structure corresponding to the character resulting 
from use or disuse of an organ can be transmitted to the genes of germ cells and will gradually be fixed in them to become inheritable. The influence of the use of an organ upon genes appears to be so intensive that it leads to a swift and extensive development of the organ.

The atrophy of an organ following disuse is similarly rapid, which should, however, be naturally expected, since the memory of the previous structure must be strong and fresh if the development has occurred swiftly by the use. Vestigial organs, remaining persistent after a prolonged disuse, may therefore involve the structures whose deveiopment was never raised by the use. Probably, vestigial organs were produced aimlessly by an orthogenetic change of a gene not directly involved in any use, and when they came to have a useful value, the use was commenced leading to a rapid development.

A gene is usually concerned with a restricted, peculiar structure of piotoplasm protein, but a change in a restricted portion of a protein molecule cannot, as a rule, occur without exerting any influence upon other portions of the protein. Hence, an extensive development of a certain character by the change of the corresponding gene is to be associated more or less with developments of other related characters, thlis overspecialization of useless organs being sometimes established. The production of primitive organs, which may be left as vestigial organs, might also occur in this way.

\section{6}

A gene can undergo a gradual change in a certain direction under a given environmental factor, because it has the property to achieve the change, that is, because it has the predisposition to undergo the change. Such a predisposition may somewhat vary with the difference in individuality of the genes. Individuals with genes having each particular predisposition may accordingly change the character in different ways or at different rates even under the same environment, thus individual variation becoming more manifest. Out of them the fittest will be selected by natural selection. Since the fittest thus selected has the predisposition to proceed further in the change which will produce the fitted character, the individual will continue to evolve in an orthogenetic direction in so far as enrironmental factor persists.

Newly acquired structure of a gene is unstable and may readily return to the previous structure as the memory of the previous structure is fresh, and accordingly a new structure of a gene, produced by a certain gradual change, may be put back readily to the previous 
state when the gene falls in with some other genes having the original structure. Therefore, in order to establish a peculiar, gradual change the gene must be isolated from other genes having the original structure. This may be the reason why isolation is needed for the evolution.

When a gene has achieved a gradual change to such an extent that the genes having the original structure can no more exert their influence upon it, the organism having the gene may be called new species. Since changes of a gene, not great enough to reject the interference of the unchanged, original gene, are always to be returned to the previous structure by the latter, there should usually be a distinct line of demarkation between any two species, no gradual transmission from a species to another being found among individuals existing without separation.

\section{7}

It seems probable that the fate of civilization is subjected to climate as Huntington believed. The want of stimulating effect in climate may cause the loss of mental and physical activity in man, leading to the disuse of brain and related organs, resulting in the degeneration of the organs.

Stimulating climates exert their influence on hypophysis, probably in the main through autonomic nervous systems, and as a result the secretion of corticotrophic and growth hormones may be enhanced at the expense of thyrotrophic and gonadotrophic principles. Consequently, under a stimulating climate man may tend to be increased in his stature owing to the growth hormone, and at the same time his susceptibility to tuberculosis may be reduced, since status thymico-lymphaticus and cortical insufficiency, both of which raise the susceptibility, are removed by the pituitary hyperfunction.

It is known that civilized people have been showing the tendency to be increased in their stature since about a hundred years, while on the other hand, during the same period the resistance to tuberculosis has been increasing steadily in them. These two amazing facts can be readily explained by the assumption that the stimulating effect of climate on hypophysis was heightened rapidly.

This heightening of the effect, however, is probably not attributed to the increase in the stimulating action of climate itself, but to the increase in the susceptibility of man to the climate. The promotion of civilization may enable man to live an easy life with a plenty of nutrition. The cause of the increase in the susceptibility to climate 
may lie in this, because the deficiency of food and also the irritable, uneasy life peculiar to uncivilized life presumably exert upon hypophysis counteraction against climatic factor.

The want of food may result in adrenal insufficience by counteracting against stimulating climate, and in addition nervous tension and irritability attending on uncivilized, barbarous life may also counteract against climate by stimulating some hormonal systems mainly thyroid. Because of the presence of such preventing factors, uncivilized people may neither increase their stature nor become insusceptible to tuberculosis even under the stimulating climate. However, with the development of civilization these preventing factors are removed to enable the stimulating climate to exert its full influence.

During and immediately after the Second World War, Japanese children were reduced in their height remarkably, suggesting strongly that the want of food and nervous tension inhibited the production of growth hormone. Even in peace time, country man is smaller than town man, and students with high scholastic standing are generally above the average in height. Moreover, children of a large family are remarkably below in height than those of a small family.

Excessive use of certain organs may lead to the enhanced secretion of growth hormone, which may presumably contribute to the rapid development of organs continuously used. The speedy development of an organ would be achieved by a swift change of the genic pattern, and this swift change may be favoured by growth hormone. This hormone, on the other hand, would promote the growth by causing a swift change in genic pattern. Therefore, the vigorous production of growth hormone resulting from the pituitary hyperfunction may cause the lability of the structural pattern of genes, rendering the protoplasm very unstable so that the susceptibility of the organism to some viruses including cancer may be strikingly increased.

It has actually been established that adrenocorticotrophic and cortical hormones affect the course of some infectious diseases mainly those caused by viruses including cancer to the disadvantage of the host, whereas nutritional deficiency which inhibits the production of these hormones is known to increase the resistance to viruses and cancer. During a war, the incidence of tuberculosis may be strikingly increased, but that of some virus diseases is declined. Children of a large family show a great resistance to some viruses, and children in the country are also less susceptible to the viruses than those in towns. The same is the case with uncivilized and civilized people.

The specialization of civilized people as revealed in the increase in the stature may be ascribed to the structure of genes becoming unstable as shown in the fact that their susceptibility to some viruses 
and cancer is also increasing strikingly.

Overspecialized animals, in general, appear to have become extinct with the unusual development of bodily size. Likewise mankind seems to be bound to become extinct in a near future as did the reptiles. However, uncivilized people who can remain small in stature with a high resistance to virus diseases owing to the absence of stimulating climate in their abode, which inhibits the promotion of civilization, may escape the extinction and will represent the mankind when the civilized are extinct. 


\section{REFERENCES}

(1) Kelner, A.: Proc. Natl. Acad. Sci. U. S., (35)), 73, 1949.

(2) Bawden, F, C., and Kleczkowski, B.: Nature, (169), 90, 1952.

(3) Tatum, E. L., and Perkins, D. D.: Ann. Rev. Microbiology, (4), 129, 195 c.

(4) Heinmets, F. ct al.: Jour. Bact., (67), 511, 1954.

(5) Moriyama, H., and Ohashi, S.: J. Shanghai Sci. Inst., (5), 189, 1941.

(6) Vago, C.: Rev. Canadienne Biol.: (10), 299, 1951.

(7) Kunkel, L. O.: Amer. J. Bot., (24), 316, 1937.

(8) Bawden, F. C. : Plant Viruses and Virus Dis., 1950.

(9) Fukushi, T.: Plant Viruses, Tokyo, 1952.

(10) Wright, B. E. : Nature, (168), 1087, 1951.

(11) Hamre, D., et al.: Proc. Soc. Exp. Biol. \& Med., (73), 275, 1950.

(12) Thompson, R. L. ct al.: Proc. Soc. Exp. Biol. \& Med., (78), 11, 1951.

(13) Thompson, R. L., et al.: J. Imm., (70), 229, 1953.

(i4) Ackermann, W. W. : J. Exp. Med., (93), 635, 1951.

(15) Ackermann, W. W.: Proc. Soc. Exp. Biol. \& Med., (80), 362, 1952.

(16) Ackermann, W. W., and Johnson, R. B. : J. Exp. Med., (97), 315, 1953.

(17) Shope, R. E. : J. Exp. Med., (97), 601, 627, 639, 1953.

(18) Mirick, C. S., et al.: J. Exp. Med., (95), 147), 1952.

(19) Brachet, J.: Chemical Embryology, Translated from the French by Barth; New York, 1950.

(20) Haurowitz, F.: Progress in Biochemistry, New York, 1950.

(21) Curtis, W. C., and Guthrie, M. J.: General Zoology, IV. Edition, 1947.

(22) Riker, A. J., and Hildebrandt, A. C. : Ann. Rev. Microbiology, (5), 223, 1951.

(23) Cooke, J. V., et al.: J. Exp. Med., (97), 651, 1953.

(24) Whitaker, D. M.: Growth, (4), 75, 1940.

(25) Harrison, R. C. : Trans. Carn. Acad. Arts and Sciences, (36), 277, 1945.

(26) Gaillard, P. J.: Symposia of the Society for Exp. Biol., (11), Growth, 139, 1948.

(26 a) Nettleship, A.: Proc. Soc. Exp. Biol. \& Med., (84), 325, 1953.

(27) Drabkin, D. L.: Arch. Biochem., (21), 224, 1949.

(28) Kendrew, J. C., and Perutz, M. F. : Proc. Roy. Soc. London, A, (194), 375, 1948.

(29) Nicholas, J. S. : Thə Chemistry and Physiology of Growth, Princeton, 187, 1949.

(30) Guyer, M. F.: Animal Biology, III. Edition, New York and London, 1941.

(31) Timonen, S., and Therman, E. : Eature, (166), 995, 1950.

(32) Oerström, A.: Z. Physiol. Chəm., (271), 1, 1941.

(33) Oehman, L. O.: Arkiv Zool., (36 A) 1, 1945.

(34) Baltzer, F.: Naturwiss., (29), 177, 196, 1940.

(35) Zamecknik, P. C., ct al.: J. Biol. Chem., (175), 299, 1918.

(36) Friedberg, F., ct al.: J. Biol. Chem.: (173), 437, 1948.

(37) Dickens, F., and Weil-Mahlherbe, H.: Cancer Res., (3), 73, 1943.

(38) Burk, D., and Winzler, R. J.: Ann. Rev. Biochm., (8), 1944. 
(39) Scherer, W. F, et al.: Jour. Exp. Med., (97), 695, 1953.

(40) Cheever, F. S. and Dickos, J. : Proc. Soc. Exp. Biol. \& Med., (83), 822, 1953.

(41) Arckermann, W. and Kurtz, H.: Proc. Soc. Exp. Biol. \& Med., (81), 421, 1952 .

(42) Greenstein, J. P.: Biochemistry of Cancer, New York, 1947.

(43) Needham, J.: Biochemistry and Morphogenesis, Camb. Univ. Press, 1942.

(44) Rous, P., and Kidd, J. G. : Exp. Med., (73), 365, 1941.

(45) Shrigley, E. W.: Ann. Rev. Microbiology, (5), 241, 1951.

(46) Mottram, J. C. : J. Path. Bact., (56), 181, 391, 1944.

(47) Berenblum, I. : Brit. J. Cancer, (1), 383, 1947.

(48) Dickens, F., and Weil-Mahlherbe, H.: Cancer Res., (6), 161, 1946.

(49) Grecnstein, J. G. : Ann. Rev. Bioch., (14), 643, 1945.

(50) Kirkman, H., and Bacon, R. L.: J. National Cancer Inst., (13), 745, 757, 1952.

(51) Rose, S. M., and Wallingford, H. M.: Science, (107), 457, 1948.

(52) McKenzie, I., and Rous, P. J. : Exp. Med., (73), 391, 1941.

(53) Durant, A. J., and McDougle, H. C. : Missouri Agr. Exp. Sta. Res. Bull., (304), 1. 1939.

(54) Walter, N. F., and Voegtlin, C. : Amer. J. Cancer, (34) 375, 1938.

(55) Paillot, A.: Inter. Corn Borer Insect Sci. Repts., (1), 77, 1928.

(56) Lwoff, A.: Bact. Revs., (17), 269, 1953.

(57) Siminovitch, L. and Rapkine, S.: Biochim. et Biophys. Acta, (9), 478, 1952.

(58) Wallman, E. L. and Stent, G. S. : Biochim, et Biophys. Acta, (9), 538, 1952.

(59) Lwoff, A.: The Nature of Virus Multiplication, 1953, 149.

(60) Moriyama, H., and Ohashi, S.: Arch. Virusforsch., (1), 252, 1939.

(61) Moriyama, H., and Ohashi, S.: Z. Imm., (99), 282, 1941.

(62) Moriyama, H., and Ohashi, S.: Arch. Virusforsch., (2), 205, 1941.

(63) Kolmarky, G., and Westergaerd, M.: Hereditas, (35), 490, 1949.

(64) Catlin, B. W.: J. Bact., (65), 413, 1953.

(65) Moriyama, H., and Ohashi, S.: Shanghai Sci. Inst., (4), 51, 1939.

(66) Dodson, E. O.: A Textbook of Evolution, Philadelphia and London, 1952.

(66a) Colvin, J. R. et al.: Chemical Revs., (54), 687, 1954.

(67) Sokal, R. R. and Hunter, P. E. : Science, (119), 649, 1954.

(68) Tower, W. L. : Carnegie Inst. Publication, (48), 1906.

(69) Kammerer, P.: Inheritance of Acquired Characteristics, Trans. by Maerker-Branden, 1924.

(70) Summer, F, B.: J. Exp. Zool., (18), 3, 1915.

(71) Moriyama, H. : Immunity, 1955.

(72) Jollos, V.: Arch. Protistenk. (43), 1, 1921.

(73) Gonder, R. : Z. Imm. For., (15), 257, 1912.

(73a) Reeves, W. C. et al.: J. Infect. Dis., (95), 168, 1954.

(74) Seyle, H.: Ann. Rev. Med., (2), 327, 1951.

(75) Weisman, A. I., and Coates, C. W. : Rev. Bull. N. Y. Biol. Res. Foundation, 1944.

(76) Petersen, W. E. : Physiol. Rev., (24), 340, 1944.

(77) Mampell, K. : Genetics, (31), 589, 1946.

(76) L'Héritier, P. : Heredity, (2), 325, 1948. 
(79) Lüscher, M. : Scientific American, (188), No. 5, 1953.

(80) Smith, K. M. : Recent Advances in Plant Viruses, London, 1951.

(81) Mühlbock, O.: J. Natl. Cancer Inst., (10), 861, 1950.

(82) Lederberg, J., and Leđerberg, E. M. : J. Bact., (63), 399, 1952.

(83) Baer, J. G.: Ecology of Animal Parasites, The 1llinois Univ. Press, 1951.

(84) Sörensen, S.: Kolloid, Z., (53), 102, 1930.

(85) Moriyama, H.: Yacho, (S), No. 2, 1941.

(S6) Boas, F.: Race, Language and Culture, New York, 1949.

(87) Beach, F. A.: Hormones and Behavior, New York, 1948.

(SS) Hammett, F. S. : Amer. J. Physiol., (56), 196, 1921.

(89) Martin, J. H. : Biol. Bull., (56), 357, 1929.

(90) Rickey, E. : Comp. Psychol. Monogr., (2), 1, 1925.

(91) Moriyama, H.: J. Shanghai Sci. Inst., (3), 93, 109, 1937.

(92) Shapiro, H. L.: Migration and Environment, New York, 1939.

(93) Li, C. H., and Evans, H. M. : Recent Progress in Hormone Res., (3), 3, 1948.

(94) Wilhelmi, A. E., et al.: J. Biol. Chem., (176), 735, 1948.

(95) Mellgren, J.: Acta Path. Microbiol. Scand., (25), 284, 1948.

(96) Tepperman, J., and Tepperman, H. M. : Ann. Rev. Physiol., (12), 503, 1950.

(97) Hartman, F. A., and Brownfell, K. A.: The Adrenal Gland, Philadelphia, 1949.

(98) Money, W. L., ct cl.: Endocrinology, (48), 682, 1951.

(99) Williams, R. H., et al. : Am. J. Physiol., (159), 291, 1949.

(100) Gasche, P. : Helv. Physiol. et Pharmacol. Acta., (3), 10, 1945.

(101) Stockard, C. R. : Cold Sping Harbor Symp. Q. Biol., (2), 118, 1934.

(102) Grollman, A.: Essentials of Endocrinolgy, II. Edition, 1947.

(103) Huntington, E. : Mainsprings of Civilization, New York, 1945.

(104) Gates, R. R. : Human Genetics, 1313, 1946.

(105) Dubos, R.: American Scientist, No. 7, 1949.

(106) Krogman, W. M.: The science of Man in the World Crisis, Col. Univ. Press, $19 \frac{1}{1} 6$.

(107) Mills, C. A.: Climate Makes the Man, New York, 233, 1942.

(108) Mulinos, M. G., et al.: Endocrinology, (31), 276, 1942.

(109) Youmans, J. B.: Nutritional Deficiencies, II. Edition, 1946.

(110) McCay, C. M.: Vitamines and Hormones, (7), 147. 1949.

(111) Goldberg, I. : Compt. rend. Soc. biol., (128), 1135, 1938.

(112) Levin, L. : Amer. J. Physio1., (138), 258, 1943.

(113) Ulrich, F. et al. : J. Biol. Chem., (209), 117, 1954.

(104) Hartman, F. A., et al.: J. Nervous Mental Dis., (77), 1, 1933.

(115) Liddell, H. S., t al.: Arch. Neurol. Psychiatry, (34), 973, 1935.

(116) Takeuchi, A.: Nihon-Ishikai-Zasshi, (28), No. 8, 1952.

(117) Weininger, O.: Science, (119), 285, 1954.

(118) Lieber, L. : Reader's Digest, Sept., 109, 1953.

(119) Moon, H. D., et al.: Science, (111), 331, 1952.

(120) Moon, H. D., ct al.: Cancer Res., (10), 297, 364, 549, 1950.

(121) Sabin, A. B. : Amer. J. Publ. Health, (41), 1215, 1951.

(122) Gray, G. W.: The Advancing Front of Medicine, 1941, 30.

(123) Southam, C. M., and Babcock, V. I. : Proc. Soc. Exp. Biol. and Med., (78), 
105, 1951.

(124) Kilbourne, E. D., and Horsefall, F. L.: Proc. Soc. Exp. Biol. \& Med., (76), $116 ;(77), 135,1951$.

(125) Woodward, T. E., et al.: Ann. Inst. Med., (34), 10, 1951.

(126) Kass, E. H., and Finland, M. : New Eng. J. Med., (243), 693, 1950.

(127) Duffy, B. J., and Morgan, H. R.: Proc. Soc. Exp. Biol. \& Med., (73), 687, 1951.

(128) Kass, E. H. and Finland, M.: Ann. Rev. Microbiology, (7), 361, 1953.

(129) Thomas, L.: N. Y. Acad. Sci., (56), 799, 1953.

(130) King, E. Q., et al. : Jour, Amer. Med. Ass., (147), 238, 1951.

(131) Sidney, H. ct al.: Jour. Bact., (67), 264, 1954.

(132) Nukada, A.: Medicine and Biology, (12), 136, 1948.

(133) Nukada, A., Moriyama, H., and Ohashi, S.: Medicine and Biology, (12), $89,1948$.

(134) Rous, P.: J. Exp. Med., (13), 397, 1911.

(135) Olitzky, P. K., et al. : Rept. Foot-and-Mouth Dis. Comm., U. S. Dept. Agr. Tech. Bull., (76), 93, 1928.

(136) Carlson, A. J., and Hoelzel, F. : J. Nutrition, (34), 81, 1947. 



$\frac{649}{407}$

Dis 